\title{
DeVELOPING GateWAys as TOURISt Destinations: FERRY SERVICES AND NOdAL Functions IN Wellington AND Picton
}

By

\section{Gui Lohmann}

\begin{abstract}
A thesis
submitted to the Victoria University of Wellington in fulfilment of the requirements for the degree of

Doctor of Philosophy in Tourism Management
\end{abstract}

Victoria University of Wellington 2006 


\section{ABSTRACT}

This thesis discusses the relationship between transport and tourism with a particular focus on how to improve the destination function of gateways. The case study chosen for this research is the Cook Strait ferries and the ferry ports of Wellington and Picton, in New Zealand. With the absence of academic literature dealing with the impacts of tourism in gateways and the fact that the concept of gateway has not been operationalised yet, the thesis proposes, via empirical analysis, a classification of ferry passengers according to four different functions: gateway tourists; overnight gateway visitors; stopover visitors and destination tourists. These nodal classifications basically comprise two variables: the absolute and relative length of stay in the nodes and the main reason for going there. The analytical framework also takes into consideration the passengers' place of origin: those living within the gateways' catchment areas (the Centre Stage of New Zealand Region CSNZ); those living in New Zealand but outside the catchment areas; and passengers living overseas.

Apart from secondary sources, on-board surveys with ferry passengers and semistructured interviews with operators in Wellington and Picton are used to collect primary data. What the results show is that some segments of passengers are interested in extending their stay in Wellington and Picton if they had been offered more information about these two nodes or a special deal including the ferry crossing and accommodation in one of the gateways. International passengers are the group of passengers most likely to take advantage of these opportunities as those living in New Zealand are more interested to reach their final destinations and perhaps more familiarised with both nodes, so the incentives would not be very appealing to them. However, local operators were not able to suggest offers/arrangements to engage passing travellers to stop and visit these gateways. This can be attributed to lack of funds and relationship issues between tourism operators, particularly in Picton, and one of the ferry operators.

In comparison to Picton that has a smaller destination function, results from this research suggested that gateways with a more developed destination function, such as the case of Wellington, are better prepared to convince traffic passing by to stop and visit them as tourist destination. The existence of more tourist attractions and activities is certainly an incentive to persuade travellers to stay longer in gateways.

From the operationalisation of the concepts proposed in this thesis and the results obtained from the questionnaires and interviews, this research concludes that not only nodal functions vary from place to place (e.g. Picton as a small resort town and Wellington as a capital city), but also that these functions evolve throughout time, with seasonal 
variations also occurring (high vs. low seasons). With regards to the direction passengers are travelling and the influence on travellers' functions, variations are identified only among overnight gateway visitors, with the common pattern being passengers staying overnight before catching up the ferry the following day. 


\section{ACKNOWLEDGEMENTS}

I am grateful to a number of organisations and people that contributed to this thesis. First of all, I am very much in debt to the Brazilian Ministry of Education for granting me a scholarship from CAPES Foundation. I also wish to acknowledge the financial support received from the Victoria Management School (VMS) for field trips and data collection. Special mention needs to be made of the Waikato Management School who, while employing me as a lecturer in the final stage of my thesis, allowed me to use part of my research allowance to pay for my trips to Wellington and for the maps presented here.

This research would not have been possible without the generous support of both ferry companies, The Interislander and BlueBridge, which gave me free access to passengers and to confidential data about their markets. I am especially thankful to Peter Monk (former Marketing and Sales Manager) and Campbell Moore (Research Coordinator) from The Interisland Line and Nicole Campbell from BlueBridge (Wellington Terminal Manager). I would like also to acknowledge all the passengers (over one thousand) who took time from their trips to answer questionnaires and the twenty managers and owners of businesses who kindly accepted to be interviewed for this research.

My sincere "thank-you" goes also to my supervisors, Doug Pearce and Mondher Sahli. I am glad you have not given up supporting me through the "ups and downs" that a PhD involves. Doug, I am particularly grateful for keeping me focused throughout the whole research process and for insisting on the importance of making the necessary changes to improve the quality of my work. Mondher, your professional and personal support has been vital so I could reach the end of this long journey. Merci!

I am also grateful for the support I received from other PhD colleagues and staff from the VMS and the Tourism Management Department. In particular, I have to mention Hassanal Bagul and Davina Stanford for sharing the joys and pains that are part of a PhD. Thank you for your unconditional support. I am also glad for the opportunity of meeting Dr Brad Jackson, former head of the Victoria Management School, who was very supportive not only to me, but to most PhD students at the VMS. Linda Walker, Administrative Assistant of the Tourism Department, also looked after all of us very well. Ta!

While a VMS PhD candidate, I spent many years in different offices located in the School of Government. Despite being an "outsider", I have been well treated by staff and other PhD candidates, particularly to Patrick Nolan, who has proven to be a great kiwi friend.

Last, but not least, I would like to acknowledge the help I got from my family back in Brazil. I am also grateful for them and for my in-laws who were able to visit us in New 
Zealand in crucial moments of our lives in Aotearoa. I also have to thank Maria, my sisterin-law, who helped me fold and staple over 1,500 questionnaires. Above all, I am in debt with my wife Anna Luiza, for her patience, encouragement and support, and for tolerating my absence from the family. This thesis is dedicated to her. 


\section{TABLE OF CONTENTS}

1. INTRODUCTION

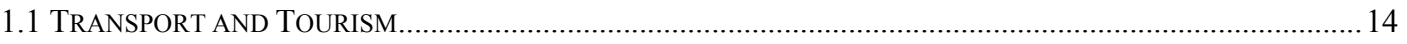

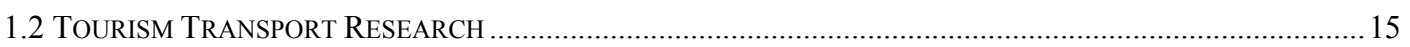

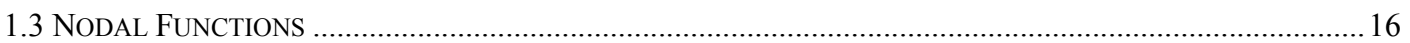

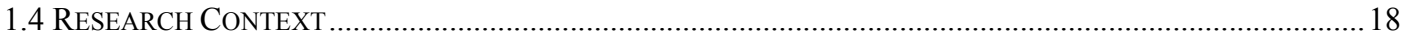

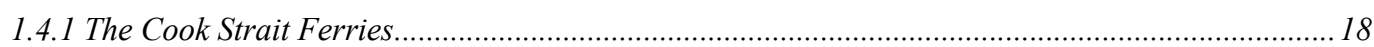

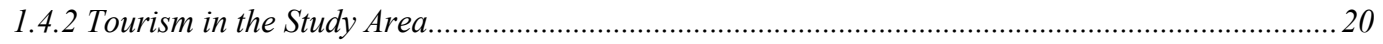

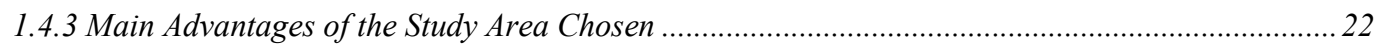

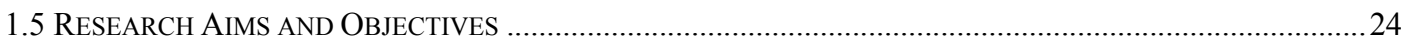

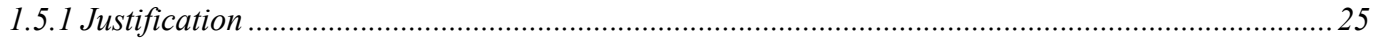

1.5.2 Scope

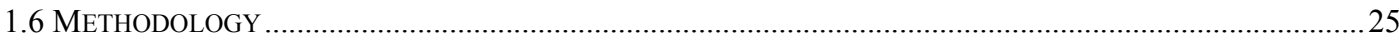

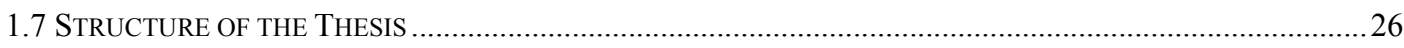

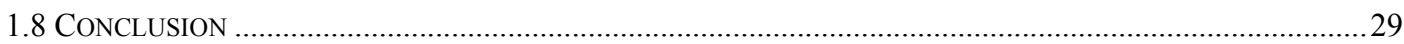

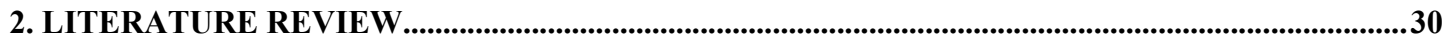

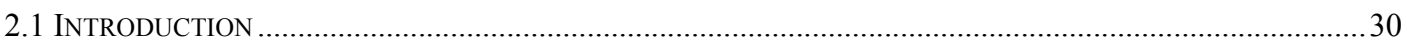

2.2 TOURIST TRANSPORT: EXISTING RESEARCH ……………................................................................. 31

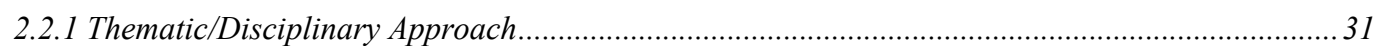

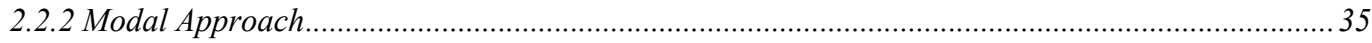

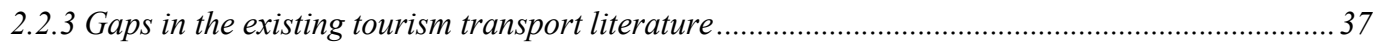

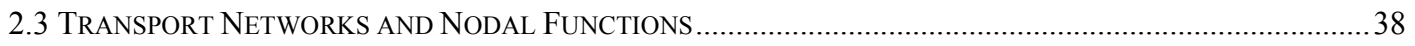

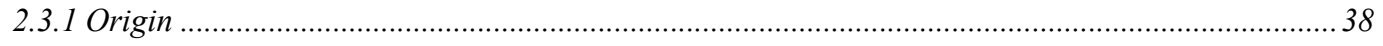

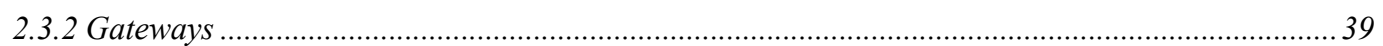

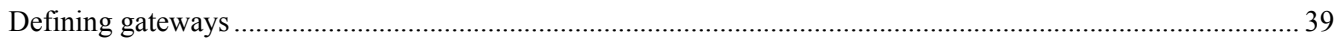

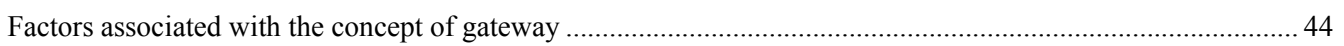

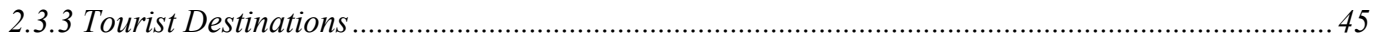

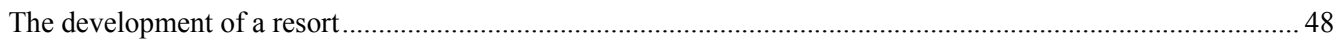

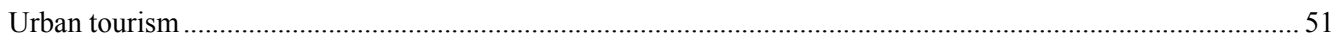

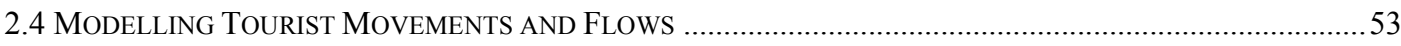

2.5 Other Pertinent IsSues to Destination Gateways....................................................................6 61

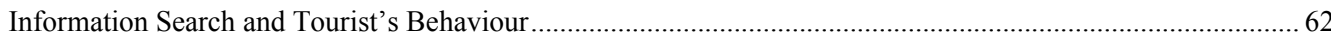

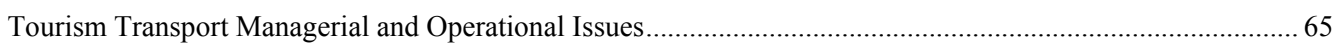

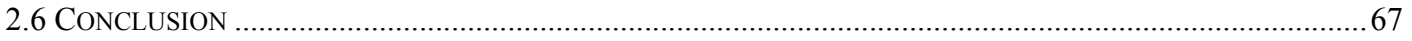

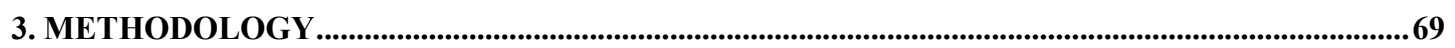

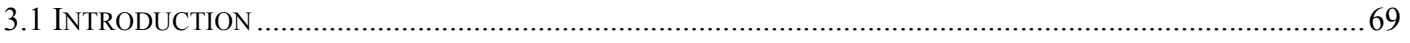

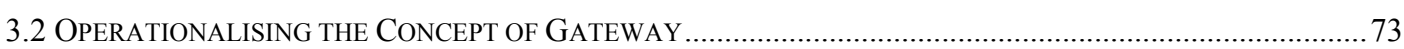

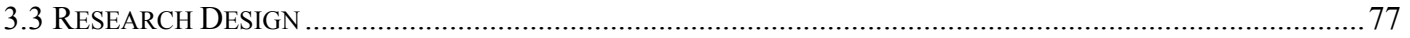




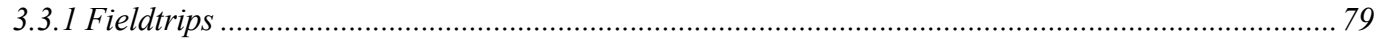

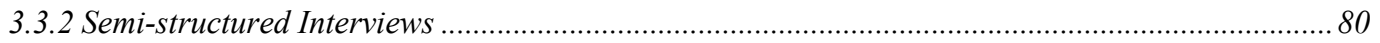

3.3.3 The Questionnaire ……………………………………………………………………. 81

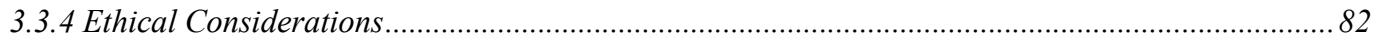

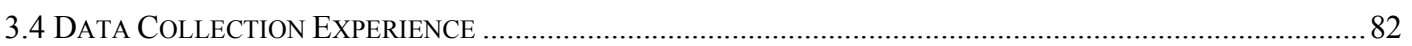

3.4.1 Primary Data Collection................................................................................................. 83

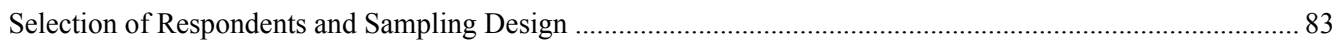

Ferry Passengers' Self-completion Questionnaires ....................................................................................... 84

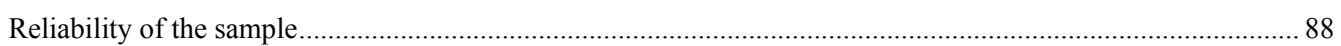

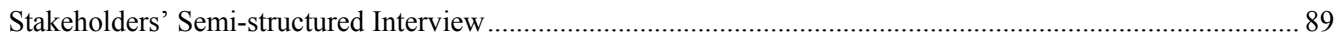

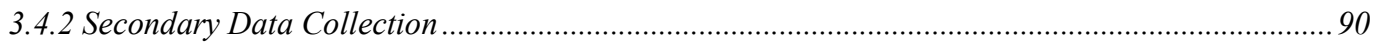

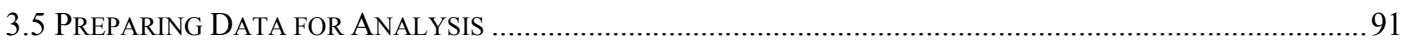

3.5.1 Questionnaire Coding and Data Cross Validation ................................................................ 91

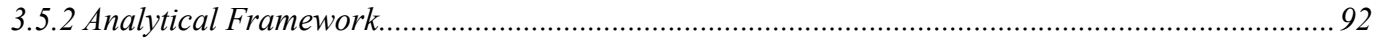

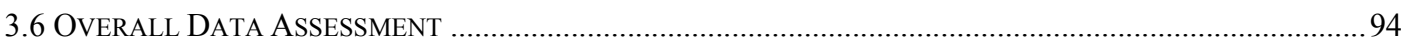

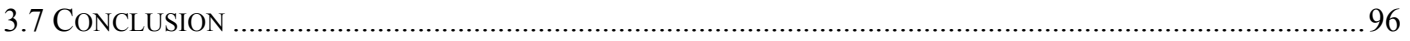

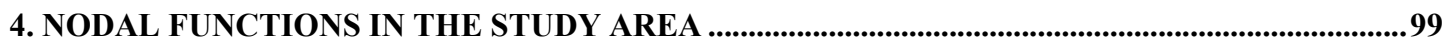

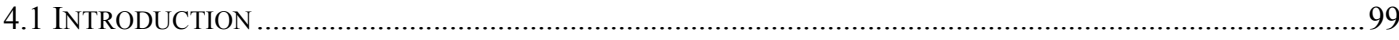

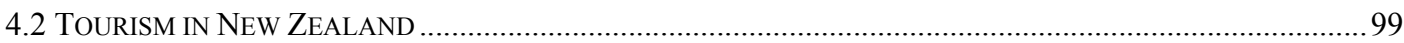

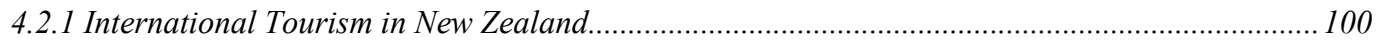

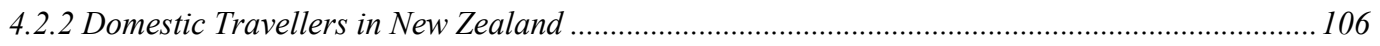

4.3 The Hinterland of the Ferry Gateways: the Centre Stage of New Zealand Region ........... 109

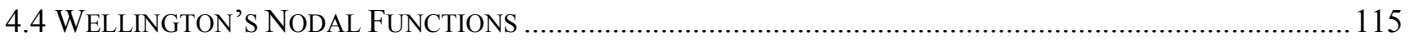

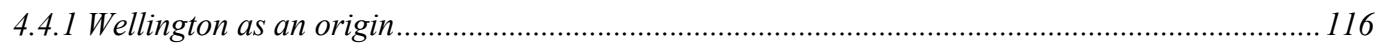

4.4.2 Wellington as a tourist destination ....................................................................................... 116

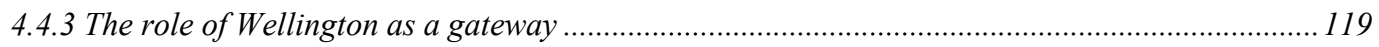

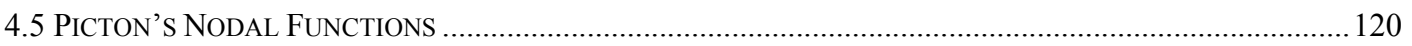

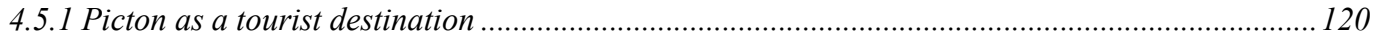

4.5.2 The role of Picton as the ferry gateway ...................................................................................... 125

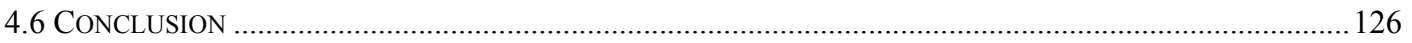

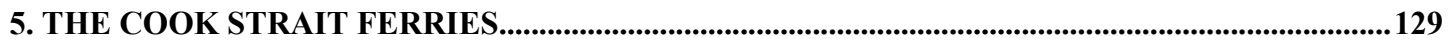

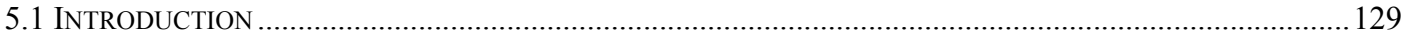

5.2 The ReCEnt DeVElopment of FerRy SERVICES In COOK STRAit .................................................. 130

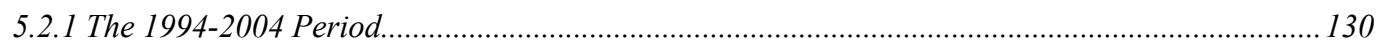

5.2.2 The Cook Strait Market during the Data Collection Process ................................................... 137

5.2.3 Fast Ferries and Environmental Issues.................................................................................. 140

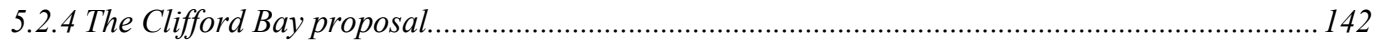

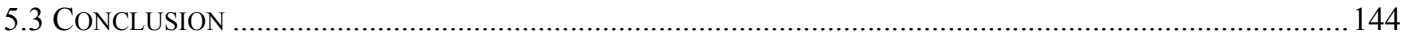

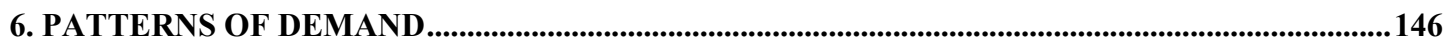




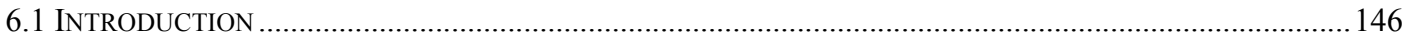

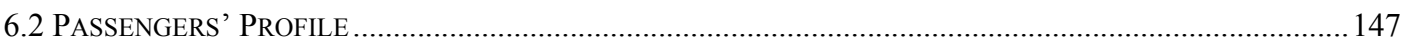

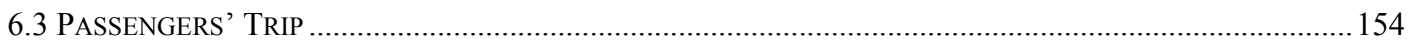

6.3.1 General Information about the Passengers' Trip ................................................................ 155

6.3.2 The Travellers' Flow Pattern around New Zealand ................................................................... 159

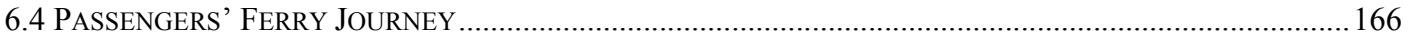

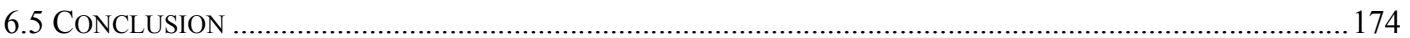

7. FERRY PASSENGERS IN WELLINGTON AND PICTON ............................................................176

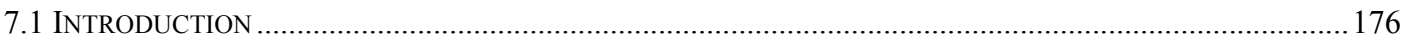

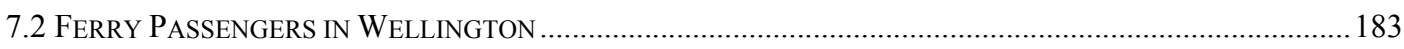

7.2.1 Changing passengers' length of stay in Wellington ........................................................... 193

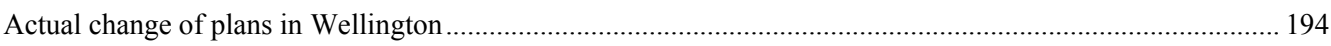

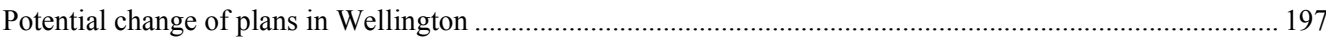

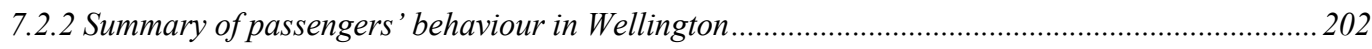

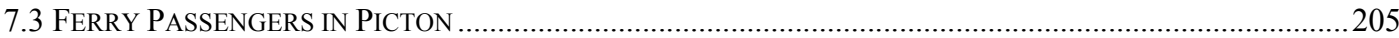

7.3.1 Changing passengers' length of stay in Picton .........................................................................2 214

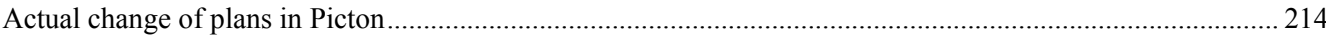

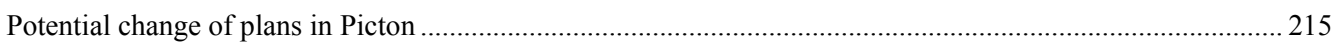

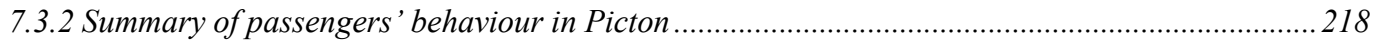

7.4 COMPARISON BETWEEN PASSENGERS’ BEHAVIOUR IN WELLINGTON AND PiCTON...............................223

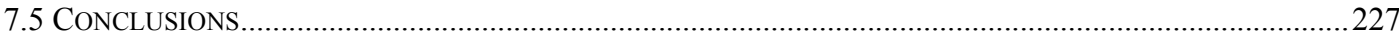

8. GATEWAY AND DESTINATION ISSUES: THE SUPPLIERS' PERSPECTIVE ..........................2230

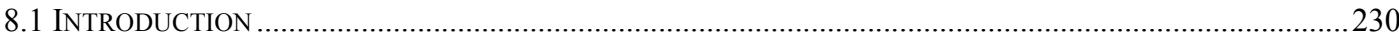

8.2 CURRENT SITUATION AND FUTURE TRENDS FOR DEVELOPING THE DESTINATION FUNCTION.................233

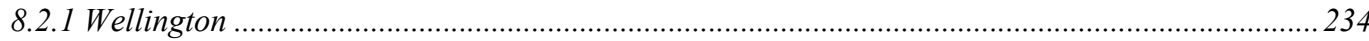

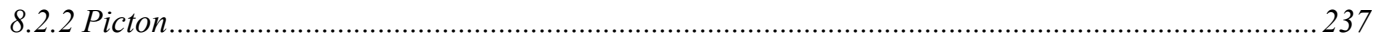

8.3 MAJOR CONSTRAINTS AND LIMITATIONS TO DEVELOP THE DESTINATION FUNCTION............................242

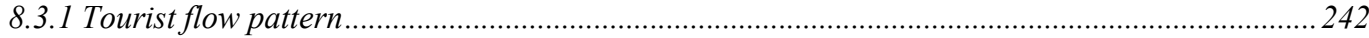

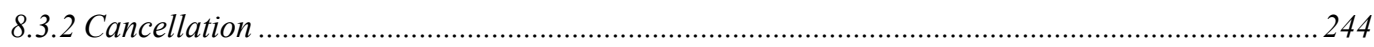

8.3.3 Speed Restrictions and Environmental Impacts ................................................................246

8.3.4 Relationship between Tourism and Ferry Operators.................................................................248

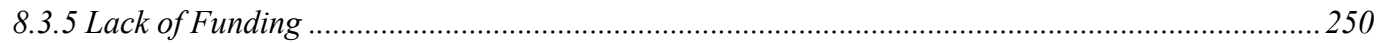

8.4 CLIFFORD BAY PROPOSAL: THREATS AND OPPORTUNITIES …......................................................252

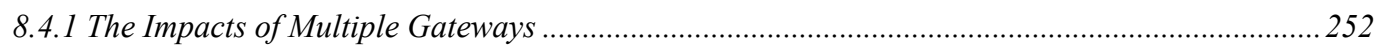

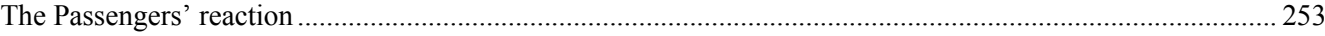

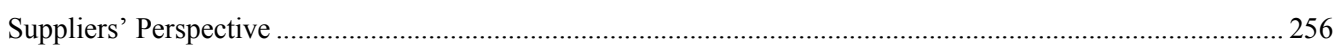

8.5 CONCLUSIONS

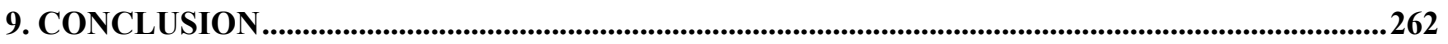

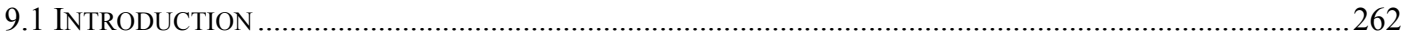


9.2 THEORETICAL CONTRIBUTION .

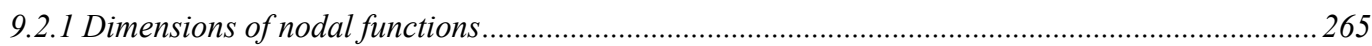

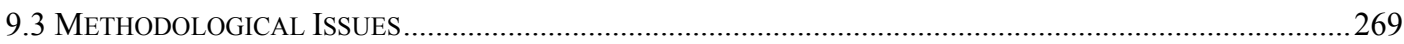

9.3.1 Operationalisation of travellers' functions ......................................................................2270

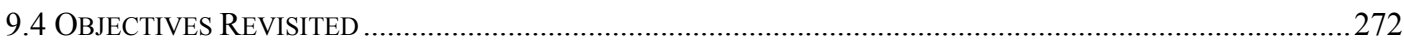

9.4.1 How can passing by passengers be persuaded to visit and stay in gateways?..........................2273

9.4.2 How can partnerships among transport and tourism suppliers improve tourism in gateways? .. 275

9.4.3 How would passengers and business react if a second gateway existed? What would happen to

Picton if the Clifford Bay proposal becomes a reality? ...............................................................2276

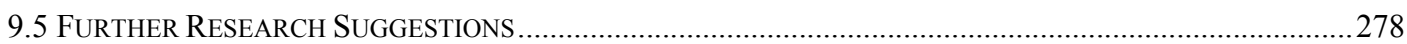

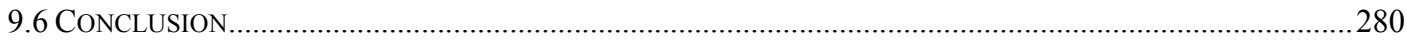

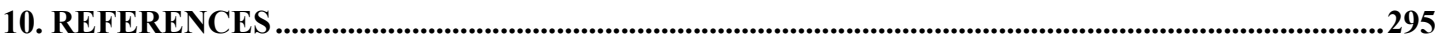




\section{LIST OF TABLES}

Table 1.1. The main features of each Centre Stage of New Zealand sub-regions. 21

Table 2.1 Dimensions/attributes determining the perceived destination image. 48

Table 2.2 The Resort Development Spectrum Framework. 50

Table 2.3 Forms of relationship of places to route itineraries. 60

Table 2.4 Classification of tourism information sources. 63

Table 3.1 Stakeholders interviewed by sector and area of influence. 84

Table 3.2 Information about questionnaire data collection - July 2003 to January 2004. 87

Table 3.3 Passengers' place of origin - comparison between the research and the Ka Pai samples.

Table 3.4 Cross-tabulation of passengers' place of origin and nodal function. 93

Table 4.1 Characteristics of the major international markets to New Zealand - Year Ended 2003.

Table 4.2 Regional distribution of international and domestic tourism expenditure (1998). 108

Table 4.3 Inter-Regional Overnight Trips 2001. 111

Table 5.1 Ferry services comparison - May 1999. 136

Table 5.2 Ferry services comparison - Spring 2003 and Summer 2004. 140

Table 5.3 Positive and negative impacts of large, high-speed vessels on Marlborough Sounds._142 Table 6.1 Country of origin for international ferry passengers and total number of international arrivals - year ended September 2004. 150

Table 6.2 Respondents' occupation. 152

Table 6.3 Number of passengers in respondent's group. 153

Table 6.4 Relationship of respondents' accompanying passengers. 154

Table 6.5 Main purpose of respondents' trip. 155

Table 6.6 Number of nights spent in trip (in New Zealand for international visitors). 158

Table 6.7 Major international gateways to enter and leave New Zealand. 159

Table 6.8 Means of transport to arrive in or depart from Wellington and Picton. 167

Table 6.9 Means of transport to arrive in or depart from Wellington and Picton by place of origin.

Table 6.10 Frequent use of ferry for New Zealand resident passengers. 169

Table 6.11 Main reason for travelling by ferry. 170

Table 6.12 Ferry bookings' distribution channels. 172

Table 6.13 How far in advance ferry ticket was booked before the day of crossing. 173

Table 7.1 Cross-tabulation of nodal function in Wellington and Picton. 180

Table 7.2 Cross-tabulation between passengers' nodal functions and time of ferry journey. 181

Table 7.3 Nodal functions in Wellington by place of departure. 182 
Table 7.4 Nodal functions in Picton by place of departure. 182

Table 7.5 Main reason for going to Wellington. 185

Table 7.6 Number of nights while in Wellington. 185

Table 7.7 Trip index for Wellington. 186

Table 7.8 Type of accommodation used in Wellington. 186

Table 7.9 Reason for gateway travellers and destination visitors not staying overnight in

Wellington. 190

Table 7.10 Sources of information used to find out more about Wellington. 191

Table 7.11 Activities undertaken and attractions visited in Wellington. 192

Table 7.12 Number of passengers that changed original length of stay in Wellington. 196

Table 7.13 Reasons for passengers changing original plans in Wellington. 196

Table 7.14 Change of plans in case passenger had more information about Wellington. 200

Table 7.15 Change of plans in Wellington in case passenger was offered a special deal. 200

Table 7.16 What should be offered to make passengers stay longer than planned in Wellington. 201

Table 7.17 Main reason for going to Picton. 208

Table 7.18 Number of nights while in Picton. 208

Table 7.19 Reason for gateway travellers and stopover visitors not staying overnight in Picton. 209

Table 7.20 Trip index for Picton. 209

Table 7.21 Type of accommodation used in Picton. 211

Table 7.22 Sources of information used to find out more about Picton. 212

Table 7.23 Activities undertaken and attractions visited in Picton. 213

Table 7.24 Change of plans in Picton. 217

Table 7.25 Reasons for change of plans in Picton. 217

Table 7.26 Change of plans in Picton in case passenger had more information. 220

Table 7.27 Change of plans in Picton in case passenger was offered a special deal. 220

Table 7.28 What should be offered to make passengers stay longer than planned in Picton. 221

Table 8.1 Reasons for passenger travelling via Picton. 255

Table 8.2 Reasons for passenger travelling via Clifford Bay. 256

Table 9.1 Impact of more information, a special deal or both strategies would have on visitor numbers in Wellington and Picton. 274 


\section{LIST OF FIGURES}

Figure 1.1 Theoretical example of origins, destinations, hub and gateway. ___ 17

Figure 1.2 Aratere in the Interislander ferry terminal in Wellington. 19

Figure 1.3 The Centre Stage of New Zealand Region. __ 21

Figure 1.4. The different ferry services and the gateways across the Cook Strait. ___ 23

Figure 2.1 Diagram of the gateway as a node and as a link._ 42

Figure 2.2 Chained destination region.

Figure 2.3 The TALC model showing the relationship between evolution and capacity. __ 49

Figure 2.4 Mariot's model of tourist flow. 55

Figure 2.5. Leiper's geographical elements in a tourism system with two destinations. ___ 55

Figure 2.6 Spatial and nodal possible configurations of gateways with origin and destination

functions. 56

Figure 2.7 Lue et al. 's model of alternative spatial patterns of pleasure vacation trips. ___ 58

Figure 2.8 Oppermann's tourist flow patterns.

Figure 2.9 Process framework of information sourcing. __ 64

Figure 3.1 Dimensions and elements of the concept of gateway.___ 74

Figure 3.2 Operationalising the concepts of gateway travellers, overnight gateway visitors,

stopover visitors and destination tourists.

Figure 3.3 Distribution of the number of questionnaires collected (a) and trips done (b) by day of week. 88

Figure 3.4 Primary data collection methodology and target samples. 97

Figure 4.1 International visitors' intended length of stay. 101

Figure 4.2 Intra-national flows in New Zealand by country of residence - 1991. 106

Figure 4.3 Distribution of domestic tourism in New Zealand in regional scale - three year average $1987 / 88-1989 / 90$. 108

Figure 4.4 Domestic travel flows in New Zealand - three year average 1987/88 - 1989/90 112

Figure 4.5 The Centre Stage of New Zealand Region. 113

Figure 4.6 Map of Wellington Central Business District. 119

Figure 4.7 The ferry terminal infrastructure (front) and the town of Picton (back). 121

Figure 4.8 Map of Picton Region. 124

Figure 4.9 Transport integration in Picton. 126

Figure 5.1 The Porirua-Picton fast ferry Te Hukatai. 134

Figure 5.2 Aratere arriving in Wellington. 135

Figure 5.3 Incat 050, Fast Cat Ferries' TopCat ferry. 135

Figure 5.4 The Bluebridge ferry, Santa Regina. 138

Figure 5.5 The Lynx ferry, The Cat, in Wellington-September 2004. 139 
Figure 5.6 Proposed route to Clifford Bay. 143

Figure 6.1 Place of origin for ferry passengers living in New Zealand. 149

Figure 6.2 Age group of respondents. 151

Figure 6.3 Respondents' total annual household (in NZ\$). 153

Figure 6.4 How far in advance the decision to travel was made. 156

Figure 6.5 Respondents' trip flexibility and previous bookings. 157

Figure 6.6 Regions where respondents have stayed at least one night. 160

Figure 6.7 Number of different regions in which passengers stayed at least one night. 161

Figure 6.8 Area where passengers stayed overnight before departing from Wellington. 163

Figure 6.9 Area where passengers stayed overnight after having arrived in Wellington. 163

Figure 6.10 Area where passengers stayed overnight before departing from Picton. 164

Figure 6.11 Area where passengers stayed overnight after having arrived in Picton. 165

Figure 6.12 Average importance of five main attributes when using Cook Strait ferries. 171

Figure 6.13 Place where ferry ticket was booked. 174

Figure 7.1 Distribution of nodal functions for respondents in Wellington and Picton. 178

Figure 8.1 Passenger choice of route - comparison between Picton and Clifford Bay. 254

Figure 9.1 Hypothetical nodal function variation: evolvement through the time and seasonal change. 


\section{Introduction}

\subsection{Transport and Tourism}

Transport is a fundamental component of the tourism industry. It is a precondition for travel because it facilitates mobility and the movement of tourists from their places of origin to tourist destinations; within and between tourist destinations; and later on back to their places of origin (Page et al., 2001). Among other factors, technological development in transport has led to the expansion of tourism at various scales. According to Chew (1987), three principal tourism epochs can be identified with three successive transport revolutions. The first began with the creation of the steamships and steam trains in the middle of $19^{\text {th }}$ century, which laid the foundations of modern tourism. Next came the invention of cars and coaches which transformed lifestyles and travel in the 1930s. The third transport revolution, having the greatest impact on international tourism, was the arrival of the wide-bodied jets in the 1960s. Therefore, it is not a coincidence that the tourism industry always experiences a massive growth after the introduction of new transport technology.

From the 1950s onwards, the worldwide growth of both road and air transport provided immense competition with existing rail and sea transport. Air transport, for example, offering the advantage of speed, which is often a critical factor in the choice of long-haul travel, has largely supplanted sea travel for long intercontinental journeys (Stubbs \& Jegede, 1998). Despite this, transport by water-borne vessels of all kinds continues to make an important contribution in the industry. Cruising, in particular, is enjoying a popularity boom, providing total relaxation and an all-inclusive price, which allows the passenger to be carried from one destination to another in comfort and safety, without the inconvenience of constantly packing and unpacking. Using a different example, short-sea ferry vessels have achieved new levels of comfort and speed on many routes, to a point where they now attract tourists not just in order to travel from one point to another, but also to enjoy a 'mini-cruise'. What is more, technological developments have helped to reduce high operation costs, while new forms of water-borne transport have been developed, such as the catamaran ferry (Holloway, 2002).

Transport, while an integral sector of the tourism industry, also provides services that are not dependent upon tourist demand. Road, rail and air services, for example, owe their 
origins to government mail contracts, and the carriage of freight, whether separate from or together with passengers (Holloway, 1985).

\subsection{Tourism Transport Research}

Although most of the tourism literature acknowledges transport as one of the most significant factors to have contributed to the development of tourism worldwide, there has been relatively little research undertaken on the precise relationship between transport and tourism (Prideaux, 2000a).

According to Lumsdon and Page (2003), the tourism literature since the 1970s has discussed the significance of transport as an integral component of the tourism system. Nonetheless, so far the literature has not been able to produce a coherent body of knowledge to understand and explain the relationship between transport and tourism. These two authors made an interesting and detailed analysis of the articles published in mainstream journals of both transport ${ }^{1}$ and tourism ${ }^{2}$ fields. It is worth quoting their comments (Lumsdon \& Page, 2003: p. xiii-xiv):

In the case of the Journal of Air Transport Management, it published six articles between 1994 and 2003 with a direct reference to tourism in its abstracts. [...] Similarly the journal Transport Policy makes no mention to tourism and some 52 articles it published have referred to travel, with some elements relevant to tourism but not explicitly discussed. In the Transportation Research collection of journals, only four articles are listed under "tourism" which appeared between 1981 and 2003 in Transportation Research A, while one in Transportation Research D. Similarly in the Journal of Transport Geography of the three articles listed under "tourism", only one of these actually addresses tourism issues explicitly. [...] A search of the journal Tourism Management using the term 'transport' yields 41 items, but only 20 of these are articles published between 1982 and 2003, less than one article for each year of publication. For a journal which publishes around 30-40 articles a year as full length research articles or research notes/case studies this will give some indication of the limited number of papers in this area. Similarly the journal Annals of Tourism Research lists 54

\footnotetext{
1 Transportation Research A-F series, Transport Policy, Journal of Transport Geography and Journal of Air Transport Management.

2 Tourism Management and Annals of Tourism Research.
} 
items under "transport" but only four can specifically be deemed to be full-length

research articles.

Although there has been comparatively little research undertaken on the detailed relationship between transport and tourism, an analysis of the existing literature reveals that most of the research has been conducted from a single disciplinary perspective. In addition, studies usually concentrate on only one mode of transport, predominantly air transport and more recently cruise tourism. Ferries and trains are the modes of transport with least research undertaken with regards to their relationship with tourism. Another characteristic of the current literature in tourism transport is that the main focus is on the provision of accessibility to certain destinations and its impacts on tourism. Tourism businesses, planners and managers are, in most cases, keen to increase the transport accessibility to their destinations in order to enhance the opportunities to receive more tourists. In various cases, transport networks already provide places with an increased number of travellers that pass by their doors on route to their destinations who could be converted into tourists to the gateway regions.

\subsection{Nodal Functions}

Tourism requires that travellers leave their place of origin making use of a mode of transport to reach a certain destination. Commonly the modes of transport considered for travelling from the place of origin to the destination are part of a transport network. These networks consist of a set of links (highways, railways, air routes etc) and nodes - terminals or interchanges - that are interconnected to each other. The more links a certain node has, the more accessible it becomes. The nodes can have several associated functions. Apart from the origin and destination functions, the other two types of nodes that exist are hubs and gateways. They are associated with the connecting (flow-through) traffic, being points on the network where several routes converge and often act as the focus for transport services or for the exchange of traffic between two modes of transport. Such nodal points often have their location predetermined by the industrial or settlement geography of the region that the transport system was built to serve.

Hubs can be defined as a centrally located point that a flow of passengers passes through in order to integrate a set of origin-destination points (O'Kelly \& Miller, 1994). Fleming and Hayuth (1994) use the words centrality and intermediacy as spatial qualities that enhance the traffic levels of transportation hubs, and hence indicate that they are places strategically located within transportation systems. Gateways, on the other hand, can be 
defined as "major entry/exit points for travellers into or out of a national or regional system" (Pearce, 2001f: p.30). Lew and McKercher (2002: p.609) state that "a travel gateway is a place that provides access to (and often travel services for) a destination place or region".

Figure 1.1 presents a theoretical example of the four different types of nodes (origin, destination, gateway and hubs). All the eight nodes illustrated there ( $A$ to $H$ ) can be considered, in principle, as potential origins or destinations. Point $\mathrm{H}$, however, has a special location on the network, because through it passes most of the traffic that links the various points in the system. Therefore, it can be considered as a hub. Point $G$ also has an advantaged position with respect to point $F$ as any traffic to or from $F$ needs to pass through $G$, making it the gateway to point $F$. Due to the privileged position they have in the transport network, hubs and gateways have a huge potential to attract visitors, as all they need is a good reason to stop and visit them. In contrast to non-hub and non-gateway nodes that need to attract visitors to travel to them, hubs and gateways already have potential visitors passing by. This thesis focuses particularly on the case of the gateway function. More precisely, it aims to analyse how to convert gateway travellers into destination tourists.

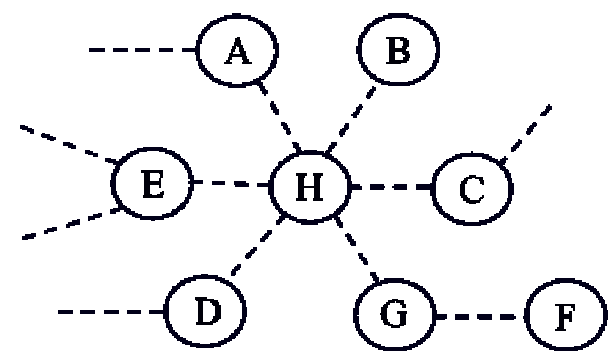

Figure 1.1 Theoretical example of origins, destinations, hub and gateway.

The extent to which places can establish themselves as something greater than a gateway - that is, as destinations in their own right - will depend on their ability to provide facilities and attractions that will appeal to the connecting traffic to stop for a few hours or even to spend some nights there. For example, many cities that have traditionally acted as gateways are recognising the potential to increase their tourism earnings by enhancing their image and taking advantage of increasing visitor interest in urban experiences (Warren \& Taylor, 2003).

The next section briefly introduces the study area chosen for this research, i.e. the ferry gateways of Wellington and Picton, located in the Centre Stage region of New Zealand. 


\subsection{Research Context}

The study area chosen to undertake this research consists of the ferry ports of Wellington and Picton, in New Zealand. Apart from flights linking the main cities and towns in both islands, ferry services are the only other available alternative to cross Cook Strait. Ferries link Wellington, the capital city, to Picton, a small town in the South Island. As these two locations are the only available options to arrive and depart by ferry in the two main islands, Wellington and Picton are, respectively, the gateway to the North and South Island. The next two sections briefly describe the Cook Strait ferry services and tourism available in Wellington and Picton. Chapters 4 and 5 of this thesis will deal more extensively with these two key topics.

\subsubsection{The Cook Strait Ferries}

Due to the fact that the Cook Strait ferries are able to transport trains and vehicles, the main trunk rail line in the country and the State Highway One are brought together. The regular sailings of the Cook Strait ferry service transport an estimated 1.265 million passengers, 253,000 vehicles and over 2.5 million tonnes of freight per annum (Marlborough Economic Development Trust, 2001). Currently, two ferry companies - The Interisland Line and Strait Shipping - provide services linking Wellington and Picton.

The Interisland Line provided two services, The Lynx and The Interislander. The Lynx was a high-speed service and the vessel used is a comfortable wave-piercing catamaran built in 2000. It crossed Cook Strait in two hours and fifteen minutes. The Interislander service is provided by two conventional vessels: Arahura and Aratere ${ }^{3}$. The former was specially built in 1983 to operate in the strong winds, heavy swells and wave motions across the Cook Strait. These two ferries are able to link the ferry ports in three hours. Figure 1.2 shows the freight and passenger infrastructure in the Interislander Wellington terminal. On the left it is possible to see the vehicle (above) and rail (below) bridges that link the terminal to the vessels. On the right is the passengers' bridge, which gives access to foot passengers onboard the ferries.

\footnotetext{
${ }^{3}$ All references made about the ferry companies, their services and vessels refer to the period of data collection, with ended on August 2004. On 17 April 2005, The Lynx service came to an end, with the fast ferry catamaran being replaced by a conventional vessel named Kaitaki. As a consequence, the Cook Strait fast ferry era came to an end, after nearly 11 years. The Interisland Line was then rebranded to "Interislander".
} 


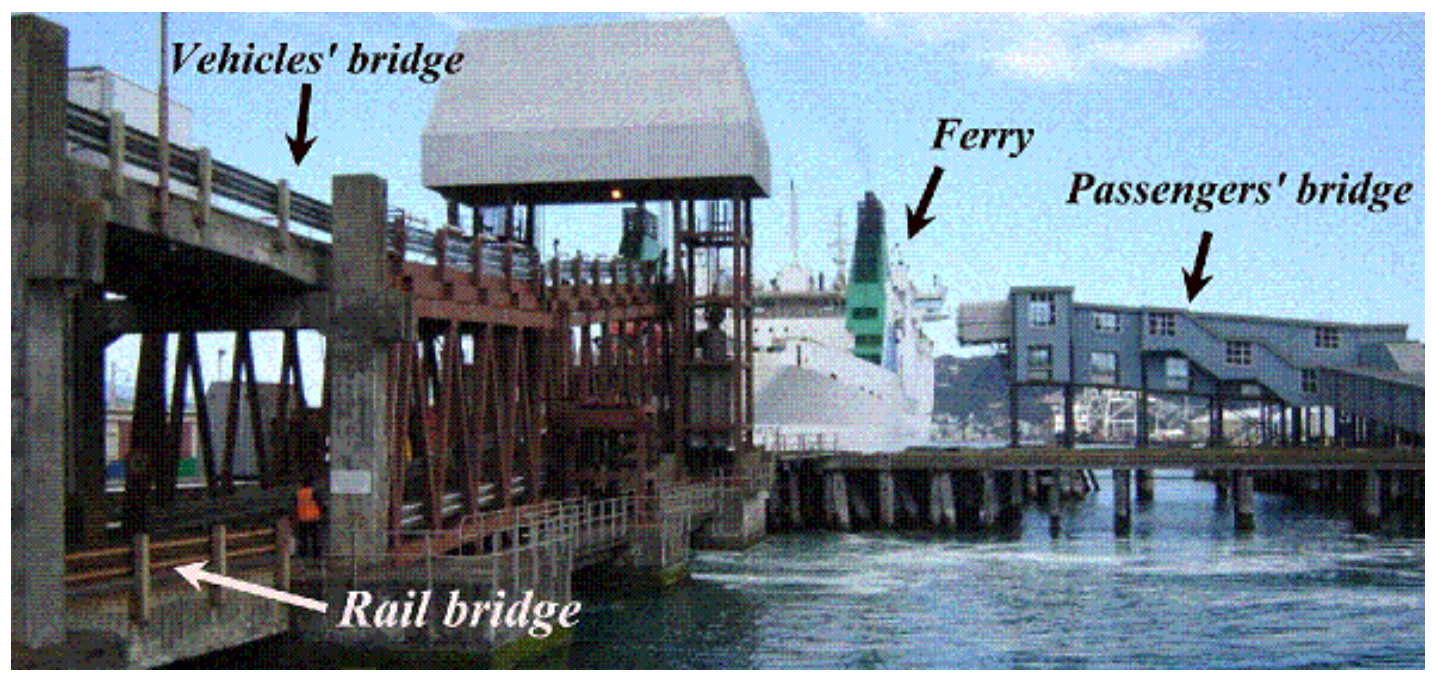

(Photo by Gui Lohmann)

Figure 1.2 Aratere in the Interislander ferry terminal in Wellington.

In the beginning of 2003, Strait Shipping Company introduced a passenger and vehicle service called Bluebridge. The 137-metre-long Santa Regina is an eighteen-knot conventional ferry that has a crossing time of three hours and twenty minutes. As a result of the competition, prices went down and the consumers gained more options in terms of services and timetable.

Apart from the ferry operations, another relevant issue for this research regarding the study area is the proposal to build a ferry terminal in Clifford Bay (South Island). Although a ferry terminal in Clifford Bay would bring some advantages, inter alia reducing crossing time by sea and road to the main destinations, its construction would require a substantial amount of investment with some environmental issues. However, for academic purposes, it is an interesting topic to consider, especially to analyse what would happen to Picton in the case where there were two ferry gateways in the South Island (Picton and Clifford Bay). In the theoretical example of Figure 1.1, this is the same as analysing what would happen if a link between $C$ and $F$ was created. Considering this new scenario, $F$ would have two gateways, C and G. As the new gateway (C) would probably offer some advantages for the traffic to and from $F$, it would be interesting to see what the passengers' reaction would be and the impacts on G's tourism sector. 


\subsubsection{Tourism in the Study Area}

In addition to the transport component, it is also appropriate to briefly introduce the context of tourism in the nodes studied, as well as in their surrounding areas.

Tourism plays an important role in New Zealand's economy and its potential has been recognised since the first European arrivals. International and domestic tourism has enabled the country to diversify its economy from over-dependence on the export of agricultural products. Major tourism products include nature, adventure tourism and cultural experiences.

Internationally, New Zealand is marketed as a touring destination and most trips are multidestination journeys. The country's geography (two main islands) and the location of the main international gateways in the country - Auckland in the top of North Island and Christchurch in the middle of the South Island - make mobility and circuit travel a key feature of tourism in New Zealand.

With well-known destinations located near the two main international gateways Auckland, Rotorua and Waitomo Caves in the North Island; Christchurch, Mount Cook and Queenstown in the South Island - the central part of the country is usually neglected. In order to promote the regions located in the heart of the country, a brand for international tourism marketing was developed in 1998 by four regional tourism organisations. Called Centre Stage of New Zealand (CSNZ) ${ }^{4}$, it represents four macro regions (see Figure 1.3): Wellington, Wairarapa, Marlborough and Nelson (Totally Wellington, 2000). Some of the key attractions and main tourism features in each of these regions are presented in Table 1.1.

In the North Island, the Wellington and Wairarapa regions are the hinterland for the city of Wellington. Wairarapa is a rural region sometimes also considered as part of the greater Wellington area. Wellington Region is, from a different perspective, the most important sub-region within the CSNZ. It is the political capital of the country, attracting a number of businesses and public services. In addition, it is one of the most populated regions in New Zealand, with nearly 400,000 inhabitants; its population has the highest national average weekly income (\$515).

In the last few years, Wellington City has transformed itself from a monotonous government centre into a vibrant urban destination. It is considered New Zealand's capital

\footnotetext{
${ }^{4}$ This organisation ceased to exist in 2005.
} 
of arts and culture, being the home of the Museum of New Zealand Te Papa Tongarewa, New Zealand Symphony Orchestra, Royal New Zealand Ballet Company and the National Art Gallery. Every two years, it holds the country's largest cultural festival, the New Zealand Festival of the Arts. On 1 December 2003, Wellington hosted the world premier of the movie the Lord of the Rings: the Return of the King. Peter Jackson's Trilogy has in fact been extensively used as a way to promote and market the city worldwide (Ruggia, 2002). Wellington has also a beautiful harbour and other attractions such as the Botanic Gardens and the cable car. In addition, its compact downtown area allows visitors to explore its main attractions on foot. For these reasons, Wellington is by far the main tourism destination in CSNZ and also its key tourist generating area.

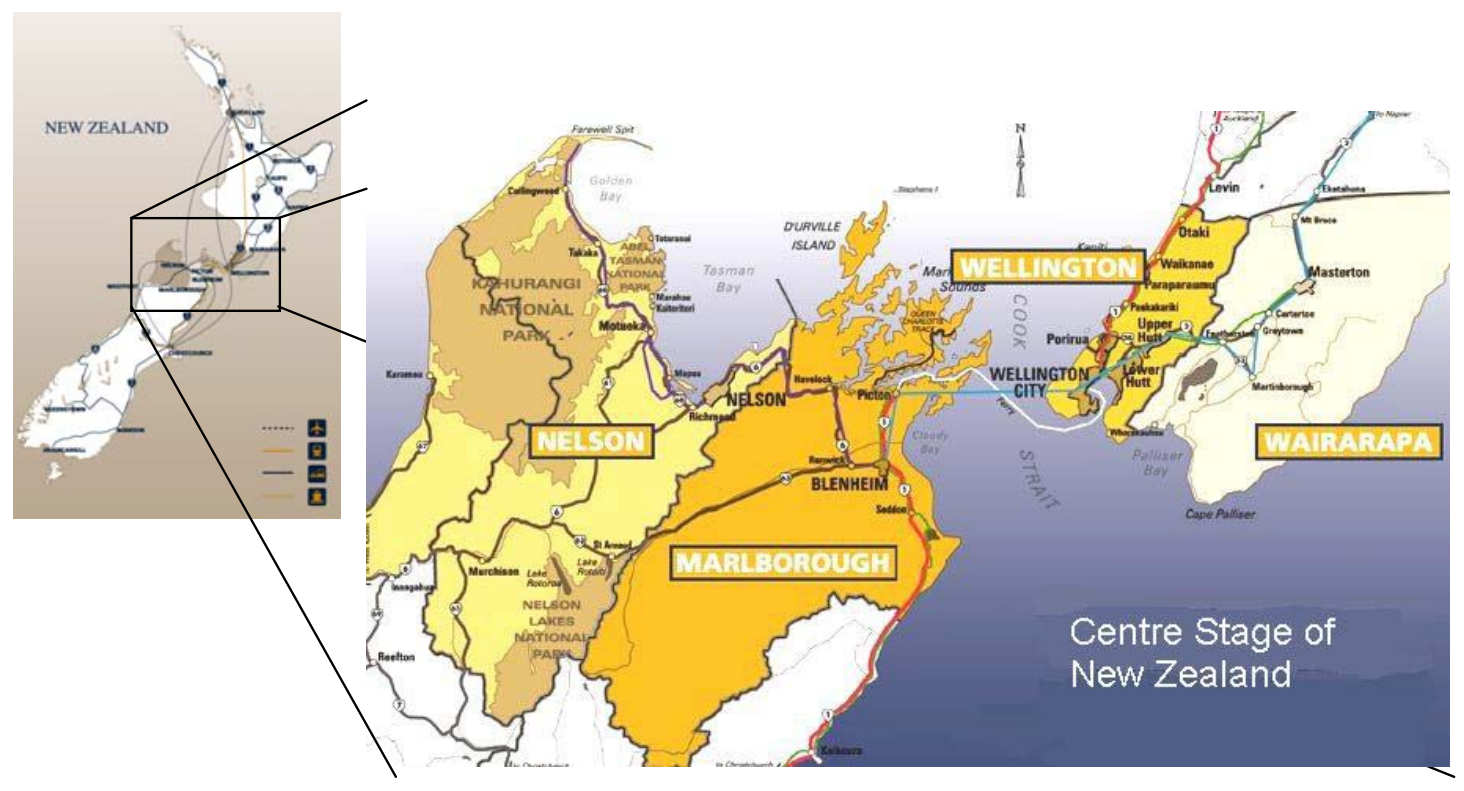

(Source: Centre Stage of New Zealand, 2003)

Figure 1.3 The Centre Stage of New Zealand Region.

Table 1.1. The main features of each Centre Stage of New Zealand sub-regions.

\begin{tabular}{|c|c|c|c|c|}
\hline & \multirow{2}{*}{ Marlborough } & \multirow{2}{*}{ Nelson } & \\
\hline & & & Wairarapa & Wellington \\
\hline 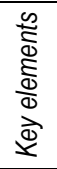 & $\begin{array}{l}\text { Maritime / Marlborough } \\
\text { Sounds / Wine and food }\end{array}$ & Art / Culture / Environment & $\begin{array}{l}\text { Mt Bruce / Rural retreat / } \\
\text { Wine }\end{array}$ & $\begin{array}{l}\text { Arts / Café / Capital / } \\
\text { Culture / Government / } \\
\text { Heritage / Restaurants / Te } \\
\text { Papa Museum }\end{array}$ \\
\hline $\begin{array}{l}\frac{a}{0} \\
\text { d } \\
\frac{d}{5} \\
\frac{2}{2} \\
\text { क }\end{array}$ & $\begin{array}{l}\text { Boutique accommodation / } \\
\text { Climate / Dolphin watching / } \\
\text { Gateway to South Island / } \\
\text { Gourmet food / Maori product / } \\
\text { Queen Charlotte track / Sea } \\
\text { kayaking }\end{array}$ & $\begin{array}{l}\text { Clear days / Crafts / Maori } \\
\text { culture / National parks / } \\
\text { Performing arts / Pristine } \\
\text { environment / Sea kayaking / } \\
\text { Wearable arts / Wine \& food, } \\
\text { café }\end{array}$ & $\begin{array}{l}\text { Adventure activities / } \\
\text { Boutique accommodation } \\
\text { / Forest parks / Gardens / } \\
\text { Tiny population / Wide } \\
\text { open spaces }\end{array}$ & $\begin{array}{l}\text { Compact city / Events / } \\
\text { Gateway to North Island / } \\
\text { Harbour / International } \\
\text { airport / Nightlife / } \\
\text { Parliament / Shopping / } \\
\text { Wildlife }\end{array}$ \\
\hline
\end{tabular}

(Source: adapted from Ateljevic, 2002) 
In the South Island, the Marlborough Region is a major wine-producing centre. In the past, the region was the second major area in New Zealand for high-country sheep farming, after Wairarapa. The main urban areas are Blenheim and Picton. The Marlborough Sounds Maritime Park, where the Queen Charlotte track is located, offers forest walks, fishing and boating activities, among others. The Nelson region is located in the northwest corner of the South Island and the Kahurangi and Abel Tasman National Parks occupy the great majority of its lands. Tourism in Nelson consists of rural activities such as adventure, national parks and wineries. White-water rafting and kayaking are popular tourist activities, mainly in the city of Nelson and in the Abel Tasman National Park.

Nestled among high hills, at the heart of Queen Charlotte Sound, Picton is ideally placed as a holiday resort, although industries such as boat building, fishing and forestry share its beautiful setting. From a base of about 4,000 people, thousands more visit Picton during the peak summer season. With tourism the main industry during summer time, apart from the holiday homes, Picton provides well over 1,000 tourist beds in cabins, motels, hotels and motor lodges. Tent and caravan accommodation at campsites in the area brings in yet hundreds more. The last few years have seen an array of development initiatives being undertaken in Picton and neighbouring Waikawa (arguably, as a response to Tranz Rail's proposal to relocate its inter-island ferry operations from Picton to Clifford Bay). The most striking of these has been the foreshore redevelopment project, which saw Picton's foreshore area transformed into an attractive recreational area for both tourists and local residents (Marlborough Economic Development Trust, 2001). This redevelopment has been extended to incorporate the main street in Picton's CBD.

\subsubsection{Main Advantages of the Study Area Chosen}

The appropriateness of the case study region for the problem to be examined is based on several factors, including:

1. Wellington and Picton are, by far, the most important ferry gateways in New Zealand, with a significant amount of passengers, vehicles and freight crossing the Cook Strait by ferry each year;

2. The Centre Stage of New Zealand region has been neglected by travellers while touring in the country, hence the need to improve tourism in this area;

3. Ferries depart and arrive in only one city/town on each side of the Cook Strait (Wellington and Picton), making the gateway function even more evident in each island. In addition, as these two places have different political, geographical and 
economic scales (capital city vs. small town), this creates a possibility to explore comparisons in terms of tourism and transport impacts;

4. The proposal to build a new ferry terminal in Clifford Bay, South Island, gives a chance to analyse and discuss what would happen to Picton in case it lost the exclusive function of being the ferry gateway to the South Island;

5. The location of the study area. Being closer to where the researcher and the university are located, it allows easier access to analyse the demand and supply. Although this research required some expenditure in terms of transport and accommodation costs, this was cheaper than choosing a study area further away;

6. Both ferry operators, The Interisland Line and Strait Shipping, agreed to participate in this research giving free access to passengers and information related to the ferries' operation.

Figure 1.4 illustrates some of the above-mentioned arguments to support the chosen case study region.

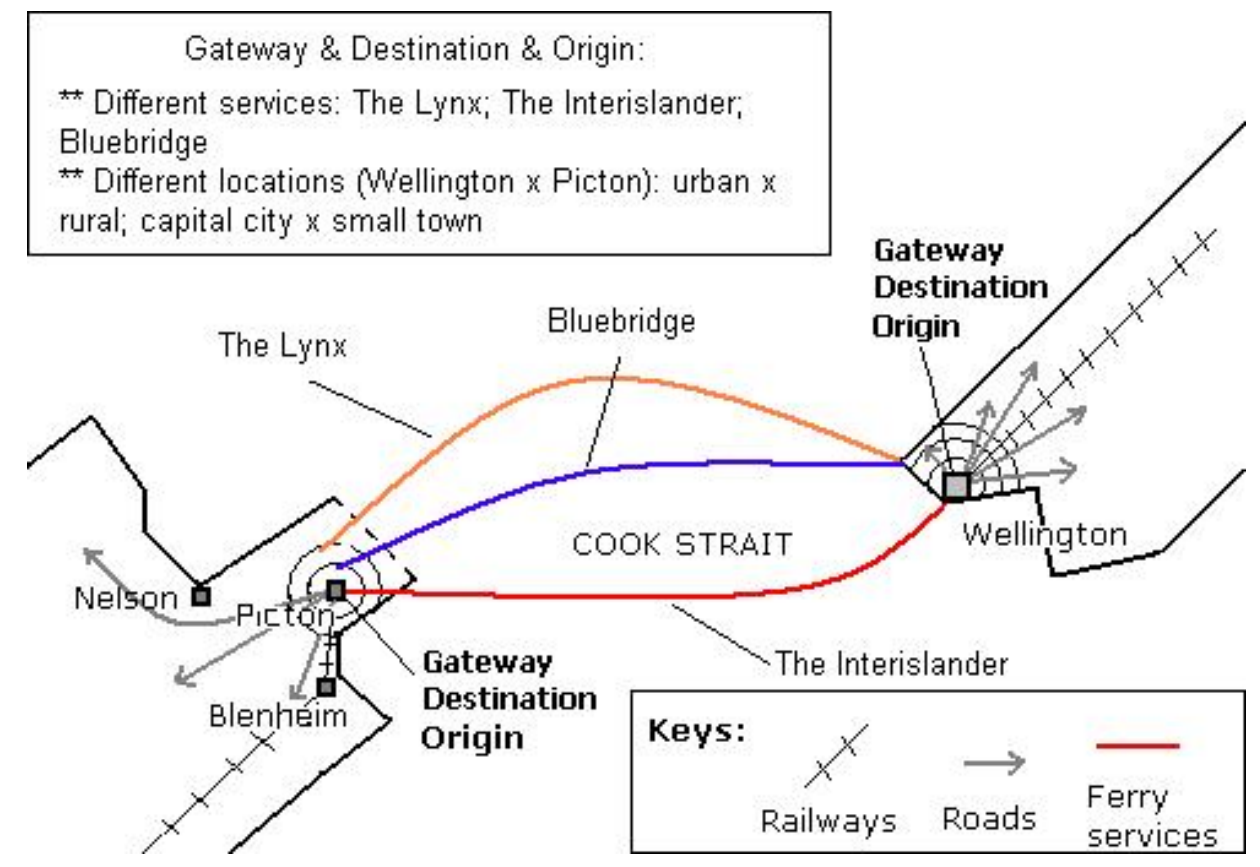

Figure 1.4. The different ferry services and the gateways across the Cook Strait. 


\subsection{Research Aims and Objectives}

With the significant number of passengers using the Cook Strait ferries (over 1.2 million yearly), associated with the fact that both Wellington and Picton are still trying to emerge respectively in New Zealand's international and national tourism context, capturing part of the passing through flow of potential tourists seems to be a good strategy.

Therefore, this research aims to understand how gateways can be better developed into tourism destinations. To this end, the main research questions of this project are:

- How can passing by passengers be persuaded to visit and stay in gateways?

- How can partnerships among transport and tourism suppliers improve tourism in gateways?

- How would passengers and businesses react if a second gateway existed? What would happen to Picton if the Clifford Bay proposal becomes a reality?

The research questions suggest there is a need to obtain data from two different types of stakeholders: the ferry passengers and transport / tourism providers located in Wellington and Picton. Even though passengers are the ones who have the ultimate decision to stop and visit the gateways, they are also influenced by the management and marketing strategies established by the suppliers, hence the need to collect data from these two different sources. Some more specific questions related to these two major stakeholders include:

\section{Passengers' perspective:}

1.1. Is the passenger visiting/staying in Wellington or Picton during the current ferry crossing? Why? Why not?

1.2. What is the main reason for the passengers to go to Wellington and Picton?

1.3. What is the nodal function of Wellington and Picton for each passenger?

1.4. What would make the passenger visit Wellington or Picton? What would make the passenger extend their stay in Wellington or Picton?

1.5. What is the passengers' behaviour in the nodes for the different places of origin? What is the passengers' likelihood to stay longer in the nodes for the different places of origin?

1.6. From the questions mentioned above, what are the differences existing between Wellington and Picton? 


\section{Suppliers' perspective:}

2.1. What is the importance of the ferry services to tourism in Wellington and Picton?

2.2. How can tourism providers and ferry operators create more opportunities to convert passengers into tourists? What are the major constraints and limitations to achieve this?

2.3. What would be the impacts on Picton if a new ferry terminal was built in Clifford Bay? Which businesses and destinations would benefit? Which would lose?

\subsubsection{Justification}

Answering these questions will contribute to clarifying how transport can be important to develop and promote tourism in gateways. In addition, transport companies, tourism organisations, regulation policy makers and academic researchers will also benefit from a better understanding of the transport management practices and their impacts on tourism development.

\subsubsection{Scope}

The size, variations and characteristics of gateways around the world are such that a comprehensive study embracing a wide variety of gateways is beyond the scope of the research undertaken for this thesis. However, in terms of New Zealand's transport and tourism industry, Wellington and Picton are by far the largest sea passenger gateways.

As a consequence, the scope of this thesis is limited to a study of port gateways in New Zealand. Patterns observed in the New Zealand context may have parallels in other regions and types of gateways and some general points which can be applied elsewhere are going to be draw out. However, it is always important to keep in mind that the concepts developed in this research and the results obtained are primarily applicable to the context of the study area.

\subsection{Methodology}

As seen before, the research problem can be summarised as how to develop the destination function of gateways. More precisely, the main aim is to investigate how passengers might take advantage of their trips to the gateways to stop and visit them as a tourist destination. While transport and tourism businesses and organisations have a significant impact to create incentives and opportunities for passengers to become tourists, ultimately the decision to stop, visit and stay longer in the gateways is made by 
the travellers. Therefore, there is a need to collect data from both the suppliers and the consumers who demand these services.

In addition, primary and secondary data are required. Secondary data are important in order to bring together tourism and transport issues of the study area, especially in the gateways (Picton and Wellington). Primary data are necessary to collect information from different players - passengers and tourism / transport providers - so that their various perspectives can be addressed. For the passengers, self-completion questionnaires were delivered onboard the ferries between July 2003 and January 2004. A total of 1,076 questionnaires were collected. Although most of the data collected were quantitative, they also had some qualitative aspects. In the case of the supplier, twenty semi-structured interviews were conducted with stakeholders. They were carried out with transport and tourism businesses and organisations in Wellington and Picton from October 2003 to August 2004. The interviews had a qualitative approach and dealt especially with management aspects of tourism and transport business in order to attract travellers to stay and visit Wellington and Picton.

Finally, it is worth mentioning that the analytical framework adopted in this research was designed to analyse the passenger data from the nodal function and place of origin points of view. The actual nodal function provides the chance to understand the current perception of the passenger towards the two nodes. The proximity of the passenger's place of origin to the gateway can also be related to the familiarity of the passenger to the node and this can influence the likelihood of converting the passenger into a destination tourist. Passengers living far away from the node may be more likely to take advantage of the opportunity that they are passing by the gateway to visit it as a destination. In addition, the place of origin also allows setting up appropriate marketing strategies to attract these passengers according to the differences identified for the several markets (passengers living in the CSNZ region, domestic passengers living outside the CSNZ region and international passengers).

\subsection{Structure of the Thesis}

This thesis is divided into nine chapters. This chapter has introduced key issues regarding transport and tourism and presented the research aims and objectives, the methodology used in this research and the study area chosen.

A review of the literature is done in Chapter Two. It starts by considering the current tourism transport research. Two different approaches are used to deal with the topic. The 
first takes a thematic perspective, trying to identify the different disciplines, sciences and fields that have already contributed to the tourism transport debate. The second focuses on the individual modes of transport, as well as the cases of multimodality. From this approach, it is possible to highlight the themes and modes of transport that have been neglected by previous studies. The chapter continues conceptualising the different nodal functions presented by the literature that are relevant to this research, i.e. origins, gateways and destinations. Next, several models of tourist movements and flows are introduced. This aims to provide a broader outlook trying to understand the gateway as part of a link in the travellers' itinerary around New Zealand. Finally, the chapter concludes by presenting aspects of tourism and transport related to the research topic that are relevant to the suppliers.

Chapter Three explains the methodology used in this thesis. The first section operationalises the concept of gateway. Four main nodal functions are established as being appropriate for this research. They are the gateway traveller, the overnight gateway visitor, the stopover visitor and the destination tourist. With this classification established, it is then possible to collect data that will allow not only a better understanding of the passengers' behaviour while at the gateways, but also to classify them according to these four different nodal functions. The chapter continues explaining the choice of research method, above all the type of qualitative and quantitative data required for this research. Then the research design is presented, explaining, among other things, the need for secondary and primary data in a research with this scope. Secondary data are gathered in order to give a state of the art presentation of transport and tourism in the study area, notably Wellington and Picton. Primary data were obtained from self-completion questionnaires delivered to passengers onboard the ferries and semi-structured interviews conducted with key transport and tourism stakeholders. Furthermore, this chapter also describes the data collection experience, explaining how the respondents were selected and the research was conducted. The final section gives a picture of the analytical framework of the data analysed in Chapters Six to Eight.

Presentation of the study area is made in the two following chapters. In Chapter Four the focus is on the nodal functions of the study area. This chapter is divided into four main sections. The first section gives a general view of tourism at a national level, comparing domestic and international travellers. The second section points to tourism in the Centre Stage of New Zealand (CSNZ) region, particularly to the four macro-regions: Wellington, Wairarapa, Nelson and Marlborough. The last two sections describe the key nodal functions existing in Wellington and Picton. 
Chapter Five describes the development of ferry services in Cook Strait in order to better explain and highlight the current main issues about this mode of transport. Apart from considering the competition existing in the ferry market, it also discusses some key topics that have an impact on tourism, such as the Clifford Bay proposal and the speed restriction imposed on the fast ferries travelling in Tory Channel.

A general overview of the passengers' characteristics is presented in Chapter Six. This chapter is divided into three main parts. The first presents the passengers' profile, including information such as their place of origin, age, occupation and income. The second describes and interprets aspects related to the passengers' trip, including the main purpose of the trip, number of nights away from home and the regions in New Zealand where the traveller has spent at least one night. The third focuses on the passengers' ferry journey, presenting data such as means of transport to arrive in and depart from the gateways, frequent use of the Cook Strait ferries, main reason for travelling by ferry and the booking patterns (distribution channel, time and place of booking).

Chapter Seven examines the travellers' behaviour while at both gateways and the likelihood of them stopping and visiting Wellington and Picton or simply extending their stays. Two similar sections - one for Wellington, the other for Picton - present information such as the main reason for going to the node, the number of nights travellers are staying there, the type of accommodation used, the activities undertaken and attractions visited. Both sections then analyse the actual and potential change of plans in Wellington and Picton. The chapter concludes comparing the results obtained between Wellington and Picton.

Chapter Eight, "Gateway and destination issues: the suppliers' perspective”, discusses the key issues that transport and tourism companies / organisations regard as important in order to develop the destination function to ferry passengers. The chapter is divided into three main sections. The first considers how the suppliers perceive the current situation and the future trends for developing the destination function in Wellington and Picton. The second section deals with the major constraints and limitations involved. Several issues are considered, such as: tourist flow pattern; ferry cancellation; speed restrictions and environmental impacts; relationship between tourism providers and ferry operators; and lack of funding. The third section focuses on the Clifford Bay proposal and its impacts on tourism in Picton. 
The thesis concludes in Chapter Nine. The major theoretical contribution of this research is summed up with new aspects of gateways being discussed there. The chapter also briefly reviews the methodological issues and revisits the thesis' objectives. Finally, the chapter proposes some suggestions for further research.

\subsection{Conclusion}

This chapter provided an introduction and overview of this thesis. It outlined the tourism transport research, briefly exploring the lack of research that exists in the body of literature about this topic. It noted the fact that most research tends to focus on transport as a link that provides accessibility to a certain destination. This research, however, takes a different approach as it focuses on how to make travellers passing through the gateways stay longer and visit the nodes as tourist destinations. Gateways have a privileged position in the transport network that is not always explored by tourism businesses and organisations to boost tourist from this particular potential market. In the case of the study area chosen for this research, the relevance of the gateway role is emphasized by the fact that tourism in Wellington and Picton is still trying to be recognised, respectively, on international and national levels.

From this introduction it is also possible to conclude that in order to convert passengers into tourists, two major perspectives need to be taken into consideration; i.e. the passengers and the suppliers (tourism and transport businesses / organisations located in both gateways). From the passengers' perspective, the analytical framework will analyse data taking into account the place of origin and the nodal function in relation to each node. From the suppliers' point of view, issues will be considered separately for Wellington and Picton in most cases, and later on compared. In order to achieve this, it is necessary to better analyse the concepts of gateway and destination functions, which will be discussed in Chapter 2. 


\section{Literature Review}

\subsection{Introduction}

Chapter 1 presented the primary research objectives of this thesis. They can be summarised in the following research question: "How can gateway travellers be converted into destination tourists?" In other words, "What needs to be done by destinations and businesses to persuade travellers to stop and visit gateways?" In order to achieve this aim, a theoretical consideration of the key concepts associated with this research is necessary so that the differences existing between the gateway and destination functions and how to make gateway travellers consider the node as a tourist destination can be understood. In the context of the chosen study area, the two nodes to be analysed Wellington and Picton - present differences regarding their scales, nodal functions and stage of tourist destination development. For example, Wellington is an urban destination that also plays an important political role as a capital city. This contrasts radically with the small resort of Picton. Tourism in Wellington and Picton is discussed in Chapter 4, but prior to that it is necessary to examine, among other things, what the key characteristics of an urban destination and of a resort are. Diverse approaches to persuade travellers to stop and visit these two gateways may be required.

Apart from focusing on the nodes, it is also indispensable to consider that the gateway involves just one part of the travellers' journey. Therefore, persuading ferry passengers to take advantage of the gateway and explore it further as a destination will depend on other variables related to their whole trip. For example, the length and the purpose of the trip are just two of the variables that can influence the decision to stop and visit a gateway. What is more, attracting travellers to gateways will most likely impact on other destinations since the extra time allocated to the gateway will be taken from another destination, unless travellers do not have a fixed length of stay for their trips. Hence, understanding tourists' flow and movements can help to contextualise and explain the attitudes passengers have in the gateway node from the broader perspective of their whole trip.

Finally, it is relevant to consider the issues that can affect the ability of the suppliers to offer conditions to make the node more attractive to ferry passengers. Transport and tourism suppliers can influence the passengers' behaviour in two different ways. Firstly, in terms of how the information provided can persuade travellers to include the gateways 
among their primary destinations. Secondly, in relation to how transport and tourism businesses and organisations can work together in order to create incentives for passengers to stay longer in the gateways. Gateways are particularly sensitive to the impact of transport and it is important to understand what the key transport variables are that influence passengers to visit the gateways. Moreover, transport providers and tourism businesses need to have a good relationship so that some collaborative initiatives can be set up (e.g. marketing and product development) in order to promote the destination side of gateways.

This chapter will consider these topics starting with a review of the existing tourism transport literature.

\subsection{Tourist Transport: Existing Research}

Tourism transport research can be analysed from at least two different perspectives. The first considers the various themes of research in transport and tourism. For example, economics, geography, management, psychology and sociology have influenced the theme of tourism and transport. The second focuses on the various modes of transport, because air, water, and land transport have their own peculiarities. The following two subsections present a general overview of the subject from these two perspectives.

\subsubsection{Thematic/Disciplinary Approach}

From an analysis of the existing tourism transport literature, some characteristics can be identified. Firstly, studies that attempt to understand issues of tourism transport are usually based on a single theme/discipline, e.g. history, economics, geography, management, psychology and sociology. Very few studies have attempted to undertake a multi-thematic approach. Despite this, it is interesting to note that some scholars have published in more than one theme. As an example, Prideaux has dealt with tourism transport from historical (Prideaux, 2000a), economic (Prideaux, 2000c) and tourism (Prideaux, 1999b) perspectives. This section summarises some of the findings obtained by research based around single disciplinary studies.

One of the first attempts to understand a given topic is to consider its historical evolution. In the case of tourism transport, it is clear that a certain degree of development has occurred. As Prideaux (2000a) emphasizes, in the first quarter of the $19^{\text {th }}$ century, transport was a bimodal system comprising roads and sea. Pearce (1989) found that it is not a coincidence that the tourism industry always experiences a massive growth after the 
introduction of new transport technology. Nelson and Wall (1986) conducted a more specific study where they analysed the relationships between cars and accommodation in Vancouver Island from 1890 to 1980 . According to their findings, the widespread adoption of the automobile in the 1920s stimulated a rapid growth in the number of accommodation establishments. However, the affluent tourists who frequently stayed on the island for weeks at a time and engaged in sporting and social activities were replaced by less wealthy tourists who were more likely to tour and only spent a few days. Greater mobility allowed tourism to become a mass activity, transforming tourism in Vancouver Island quite suddenly.

Another way to measure the importance of transport to tourism is to analyse the economic benefits that the former can bring to tourist destinations. This type of study usually refers to the change in economic activity in the region associated with a certain mode of transport being provided, measuring the 'value added' to the economy in terms of wages paid, employment created and tax revenues collected. Among these studies are those by Mescon and Vozikis (1985), Dwyer and Forsyth (1998), Braun et al. (2002) and Dwyer et al. (2004) about cruise tourism; Haitovsky et al. (1987), Raguraman (1997) and Morley (2003) with regard to air transport.

Apart from economic impacts, other studies have focused on the impact of transport costs on the development of tourism. Abeyratne (1993), for example, discusses the consequences of increasing air transport tax on tourism. Prideaux (2000c), in a more interesting study, outlines a transport cost model that identifies the significance of transport as a factor in destination development as well as in the selection of destinations by intending tourists. He developed a model that identifies Transport Access Costs (TAC) as one of the key categories among Total Holiday Expenditure - THE (the others are Discretionary Spending [DS] and the Accommodation Costs [AC] at the destinations). In his model, Prideaux assumes that tourists usually have a certain pattern of accommodation and discretionary spending; TAC is considered the one that varies according to the destination chosen. The further the destination is from the touristgenerating region, the higher the TAC will be. In the author's words (Prideaux, 2000c: p.61),

The relationship between origin and destinations points based on transport access price indicates that transport is a significant factor in both destination development and the type of markets that destinations compete in. 
Downward and Lumsdon (2004) take a different approach when they consider the relationship between visitor spending and mode of travel. In their study they identified a difference in the pattern of visitor spending between car-borne and public transport visitors, with the former spending higher amounts. This source of information can be an important consideration for planners seeking to encourage a modal switch of visitors, especially in the case of national parks where their research was conducted.

Studies from a geographical perspective are usually concerned with the spatial interaction between places. In order for spatial interaction to exist, a transport system is required, which includes the four elements of transport, i.e. the way, the unit of carriage, the terminal and motive power. Among these four elements, transport geographers in general have concentrated their research on the terminal (or node) part of the transport network (Boniface \& Cooper, 2001). As Hoyle and Knowles (1998: 2) state "nodal "situations" change and the spatial qualities of centrality and intermediacy enhance the importance and traffic levels of strategically located hubs within transport systems'. In fact, hubs and their implications and location on transport network and design have been consistently studied by transport geographers (Bowen, 2000; Dennis, 1994; O'Kelly, 1998; Rutner \& Mundy, 1996). Among these, however, only Bowen (2000) briefly mentions how tourism can be developed in a transport hub. However, he does not explicitly describe how this might happen.

Another type of nodal function that is often studied is the gateway. As in the case of hubs, most of the research about gateways is from the air transport perspective (Caruana \& Simmons, 1995; Matthiessen, 2004; Page, 2001). Other studies do not focus specifically on one particular mode of transport and tend to analyse the importance of the gateway role to tourism in general. Mistilis and Dwyer (1999), for example, show that gateways are in a better position to host meetings, incentives, conventions and exhibition (MICE) events than non-gateways. Others deal with some specific gateway destinations, such as Singapore (Low \& Heng, 1997), Hong Kong (Lew \& McKercher, 2002) and Southeast Asia (Pearce, 2001f).

Apart from the analysis of nodes, the geography of transport and tourism also analyses the patterns of tourist travel and tourism travel models. This means considering the whole trip undertaken by travellers and tourists in a certain region or country, including the number of nights and places visited. Researchers have proposed several models which will be presented in Section 2.4 . 
As in other disciplines, aviation dominates the research about tourism transport from a management point of view. Turton and Mutambirwa (1996), for example, consider transport management effects on tourism destinations. They draw some interesting conclusions on how Air Zimbabwe's marketing, management and operational policies can impact tourism development in Zimbabwe. Lafferty and Fossen (2001) explore management issues, i.e. horizontal and vertical integration between transport and tourism companies, although most of their analyses are focused on the airline industry and hotel chains. However, other types of partnerships among tourism and transport companies are still to be explored, e.g. operational, marketing and strategic alliances, involving many other tourism (travel agents, tour operators, distribution channels, restaurants, tourism organisations etc.) and transport (cruise, ferry, coaches, rental car, taxi, train etc) sectors.

Most of the psychology research undertaken on the tourism transport subject is associated with travellers' behaviour and choices. Punter (1999) focuses on tourist behaviour about transport choices regarding different patterns of travel (intra-regional and inter-regional). He concluded that the choice of holiday transport results in distinct holiday location and activity patterns for each modal group. Eby and Molnar (2002), analysing the factors overnight automobile drivers choose on their routes, identified that in the US they are more concerned with directness, safety, congestion and distance rather than whether the route is entertaining or pleasant to drive. The scenic appeal on byways in route choice is not a major issue for automobile drivers. However, what can be said about other modes of transport, especially sea, rail and road transport, when the passenger can relax and enjoy the trip rather than worrying about driving their own vehicles? Is scenic appeal important in these other situations?

Finally, sociological and anthropological studies of tourism transport remain practically non-existent. Wood (2000) describes many sociological aspects of the cruise tourism sector, emphasizing its global character. Foster (1986: 219), an anthropologist, describes a personal trip where he observes "his fellow passengers with a more professional eye".

Apart from the individual themes previously considered, another aspect to identify in the literature is the research about tourism transport undertaken by 'tourism scholars'. An analysis of the literature shows that two different approaches are usually considered. The first is a more general consideration of the tourism transport debate, dealing with the importance of transport to tourism (Page, 1994, 1999; Palhares, 2002; Prideaux, 2000a, c). The second set of scholarly sources tends to emphasize the role of a particular mode of transport to tourism, e.g. cruise tourism (Douglas \& Douglas, 1999, 2001a, b, 2004a, b) 
or rail tourism (Prideaux, 1999b). In these latter cases, the tourism component is more evident as these types of transport include some aspects of hospitality, catering facilities, scenic trips and leisure. The next section reviews the tourism transport literature considering a modal approach.

\subsubsection{Modal Approach}

Another way of analysing the tourism transport literature is according to the different modes of transport. Most of the research on this subject is based on a single mode of transport, with very few undertaking a multimodal perspective (González-Savignat, 2004; Owen, 1991; Phang, 2003; Stubbs \& Jegede, 1998). Particularly interesting is the contemporary view of competition and complementarity among the different modes of transport presented by Stubbs \& Jegede (1998). In their study they suggest that although rail-road and sea-air competition still exists, with road and air transport being more relevant in the case of passenger transport, complementarity between transport modes has changed during the 1990s. Traditionally modes of transport that were complementary to each other were rail-sea and road-air, such as in the cases of boat-train and airport car parks. Nowadays, roads tend to complement sea transport (e.g. Ro-Ro ferries) and rail does the same with air transport (e.g. trains linking airports to the CBD).

In terms of the individual modes of transport, aviation is probably the most studied facet of tourism transport. One explanation for this phenomena can be attributed to the growth of airline networks and airport infrastructure, the decrease of the real costs of air travel and the increase of capacity and speed, which have all contributed to the development of the international tourism industry over the last decades (Palhares, 2002; Sypher:Mueller International Inc, 1990). In addition, the airlines' ongoing contribution to tourism expansion goes far beyond providing the essential transportation links. During the 1970s and 1980s, vertical integration with accommodation and tour operating sectors as well as other tourism-related activities, such as restaurants and rental cars, was a reality within the aviation industry (Lafferty \& Fossen, 2001; Pyle, 1985). What is more, airlines also contribute to the promotion of destinations which has a significant impact especially in the case of small island developing states and developing countries (Grech, 2000; Kissling, 1998a; Turton \& Mutambirwa, 1996). Other studies, however, have focused on the negative impacts of air transport and tourism, particularly the environmental aspects such as aircraft noise, waste, atmospheric pollution, accidents and congestion (Abeyratne, 1999; Janic, 1999; Wheatcroft, 1991). 
Perhaps the most studied aspect of the relationship between aviation and tourism is deregulation (Boberg \& Choy, 1988; Shaw, 1982; Wheatcroft, 1994). Government regulation restricts competition, accessibility and capacity to airlines to operate to or from a given destination. Tourism benefits from the removal of these barriers because the accessibility for passengers to fly to a certain destination increases, providing opportunities for the tourist industry to grow. This has happened in different countries and regions of the world, such as the US deregulation of 1978 (Shaw, 1982), the EU liberalisation process during the 1980s and 1990s (Simons, 1992; Subrémon, 2000) and that of Pacific countries in the mid-1980s (Boberg \& Choy, 1988; Kissling, 1998b).

Although water transport has lost its appeal for tourists with the ocean liners substituted by wide-body jets as the main long-haul transport of choice, cruise tourism has been a rapidly growing segment over the last twenty years, with a resultant increase in research on this topic. Some of these studies take a general approach, usually describing the cruise industry as a whole (Cartwright \& Baird, 1999; Dickison \& Vladimir, 1997; Douglas \& Douglas, 2001a, 2004a). Others are more specific, analysing a particular aspect or segment, for example, describing problems, trends and issues associated with a particular country or region (Hobson, 1993; Lawton \& Butler, 1987; Wood, 2000), or passengers' behaviour and motivations on-board (Douglas \& Douglas, 1999; Prosser \& Leisen, 2003; Qu \& Ping, 1999) or in ports of call and their regions (Douglas \& Douglas, 2004b; Henthorne, 2000; Jaakson, 2004). Wood (2000; 2004) considers aspects of globalisation that have impacted on cruise tourism, such as the structure of the cruise sector, labourforce, destinations and onboard multi-culturalism.

Ferries, which transport more passengers than cruise liners, are usually not as well researched possibly because they are operated only in some specific areas/regions around the globe (e.g. Europe - the Baltic, France, Italy, the Irish Sea, Spain and the UK; Oceania - Tasmania and Cook Strait). Some studies describe the passenger ferry market in particular countries and regions, such as Japan, UK, Indonesia and Europe (Baird, 1999, 2000; Dunlop, 2002; Rutz \& Coull, 1996). One particular issue that deserved some attention was the impact of the Channel Tunnel on the North Sea / Channel ferry routes (Garnett, 1993; Peisley, 1992, 1997). As water transport has always been the slowest mode of transport, Wang and McOwan (2000) consider the development of high speed craft technology as an alternative way to make sea travel more competitive.

Despite the current importance of road transport to tourism, there is very little research undertaken about this mode of transport. This neglect is even more relevant when it is 
considered that some forms of road transport, such as cars, buses and coaches, are one of the most used by tourists to reach their destinations. In Europe, the USA, New Zealand and Brazil, among other countries, at least $80 \%$ of domestic tourists use cars, buses and coaches (Palhares, 2002; Ward, 1987). In the case of private vehicles, one of the explanations for the lack of research in road transport can be attributed to the absence of a formal, globally established industry in order to gather and collect information in a systematic, coherent way. Another reason is that road transport accessibility can disperse travellers over a wide geographic area, making access to them more expensive and difficult (Hensher et al., 1991). Some of the research which associates road transport with tourism tends to analyse particular forms of travel or vehicles, such as taxis (Waryszak \& King, 2000), recreational vehicles - e.g. campervans and caravans (Fidgeon, 1983; Gnoth, 1999; Janiskee, 1990; Jobes, 1984) or coaches (Dean, 1993). Meanwhile others focus on the road and highway perspective, such as the studies of Tyrell and Devitt (1999), Smith et al. (1986) and Wallis (2001). Some research also describe drive tourism in a particular country, for instance Australia (Carson et al., 2002; Prideaux et al., 2001) and Bermuda (Teye, 1992).

Finally, among the main types of transport, rail transport is probably the least used by tourists. This can explain why there is so little research published on this topic. Usually the existing research tends to emphasize services in regions where rail transport is finding some prominent niche markets, which has occurred in the case of Europe (Hollings, 1997; Kosters, 1995; Russell, 2000), especially with the High Speed Trains, North America (Loverseed, 1999) and Australia, where some long-distance passenger trains have been used as the focus of the tourism experience (Prideaux, 1999b).

\subsubsection{Gaps in the existing tourism transport literature}

From the analysis of the two previous sections, it is possible to state that current research on transport and tourism not only has some gaps, but is also not well balanced in terms of modes of transport and disciplines used. This review highlights the following trends:

- There has been comparatively little research undertaken on the detailed relationships between transport and tourism;

- Most of the existing research has been conducted from a single disciplinary perspective, without an interdisciplinary approach;

- Studies usually concentrate on only one mode of transport, predominantly air transport and more recently cruise tourism. In addition, the literature rarely 
considers intermodalism. Ferries and trains are the modes of transport with least research undertaken with regards to their relationship with tourism;

- Other types of partnership (marketing, promotion, alliances, loyalty programs, CRS) between transport and tourism companies are still to be considered, principally with other suppliers rather than airlines and hotel chains;

- Transport networks and their impacts on tourism development can be better understood, notably in relationship to the gateway nodal function. According to Lew and McKercher (2002), the concept of gateways has rarely been critically examined and is marginally understood from theoretical and empirical standpoints. This is one of the issues this research aims to tackle.

With few exceptions, such as cruise tourism, the usual research problem associated with the tourism transport literature is how to create or improve transport accessibility to a given destination (Prideaux, 1999a, 2000c) or how the use of a particular type of transport affects the tourist experience (Punter, 1999). The main focus in this thesis is not the tourist destination directly, but its gateway function. Transport in this research is seen as the element that creates opportunities for the traffic passing by to decide to stop and visit a destination. One of the issues not usually presented in the literature is how to take advantage of the chosen itinerary to persuade travellers to stop and visit places located along their journeys. More precisely, this research tries to understand how gateways can benefit from the passing through traffic to develop its tourist destination function.

Gateways have a privileged position to attract the passenger traffic passing through and, from a tourism perspective, this could be an incentive to boost the interaction between passengers and the whole tourism industry, more precisely the accommodation sector, attractions and events.

\subsection{Transport Networks and Nodal Functions}

The next subsections deal with the key nodal functions associated with this research, i.e. origins, gateways and tourist destinations.

\subsubsection{Origin}

Defining the concept of a node as an origin is very straightforward, being associated with the tourist's 'place of permanent residence' or the 'traveller generating region'. Other similar terms are "tourist generating region" or "tourist generating markets". In Leiper's 
words (1990) it is the tourist's home region. Apart from the number of people living in a certain area and the level of income presented by its population, other factors can influence the origin function of a node. These include, for example, the economic (cost of living), political (e.g. in the form of visa restrictions to leave the country) and environmental (weather and pollution) factors in the generating region (Pearce, 2001f).

\subsubsection{Gateways}

Defining gateways

The notion of a gateway is usually associated in the literature with an entry/exit point to reach a given place (Pearce, 2001f). Other authors also make similar points, without being as direct and succinct as Pearce. Burghardt (1971: 269), for example, states that:

the word "gateway" gives a fairly clear image of the unique positional characteristic of a gateway city. It is an entrance into (and necessarily an exit out of) some area. The entrance tends to be narrow and will probably be used by anyone wishing to enter or leave the tributary area "behind".

Timothy (2000: 246), alternatively, defines gateways as

a port of entry or exit that functions as an international access point. Border crossing points, seaports and airports are the most common examples of gateways. Urban centres through which tourists enter or exit a country are referred to as gateway cities.

Timothy's definition links gateways to international tourists entering a country, although this is not a sine qua non situation as domestic gateways are also possible as in the case of the study area chosen for this research. Moreover, this definition embraces a quite common concept also found in other research, in which gateway cities become synonymous for large metropolitan centres (Caruana \& Simmons, 1995; Ley \& Murphy, 2001; Page, 2001; Short et al., 2000).

In fact, Burghardt (apud Pearce, 2001a: 937) outlines four key attributes of gateway cities:

One, they are "...in command of the connections between the tributary area and the outside world" and develop "... in positions which possess the potentiality of controlling the flows of goods and people". Two, they often develop in the contact zones between differing intensities or types of production. Three, although local ties are obviously important, gateways are characterized best by long distance 
trade connections. Four, they are heavily committed to transportation and wholesaling.

One aspect of gateways is the notion of intermediacy, which can be "augmented by an associated staging-post role whereby visitors are sent onwards to other centres or resorts" (Pearce, 2001f: p.31). According to him, these functions are particularly evident in the case of New Zealand where circuit tourism is predominant.

Burghardt (1971) discusses the idea that gateway cities, in opposition to central places, are located eccentrically toward the end of a tributary area. Wellington and Picton are, in this sense, perfect examples of gateways, as they are situated, respectively, at the bottom of the North Island and the top of the South Island. Van Klink and van der Berg (1998: 8) go further and suggest that gateways are in "an excellent position to stimulate intermodal transport" and that in the case of seaports, this can be a way to enlarge their hinterlands ${ }^{5}$ (Weigend, 1958). In this regard the change of transport can happen within the same mode, but on a different scale, or from one mode of transport to the other; this being the case of the Cook Strait ferries. Especially in the case of seaports, land transport such as trains, cars, buses, coaches and trucks are important for feeding the ships with passengers or freight. In the case of the accessibility created by the transport infrastructure in gateway destinations, the change of mode can provide an opportunity for passengers to stop and visit or stay there. In addition, the existence of a multimodal transport network enables residents to have more access to other destinations contributing to the origin function. While the intermodal aspect of a gateway may be one of its characteristics, it is not an essential one (Janin, 1982).

Although Low and Heng (1997: 246) comment that there are no stated, formal conditions relating gateways to tourism, they summarised two for the case of Singapore:

1. One set of conditions would be related to the infrastructure, including communication and telecommunication facilities, accommodation, entertainment and recreation and others, such as its manpower support and conducive sociopolitical environment for foreign visitors;

2. Another set of conditions concerns how willing the neighbouring countries are to accept the place as a gateway.

\footnotetext{
${ }^{5} \mathrm{~A}$ seaport's hinterland is the interior region served by the port.
} 
Regarding the first condition presented above, it is appropriate to mention that this is more relevant for large cities that act as gateways. It is true that metropolitan areas have an intrinsic role as gateways, mainly due to their importance to the surrounding areas in terms of the trade, people and business that exist there. This explains why the studies developed by Ley and Murphy (2001) and Mistilis and Dwyer (1999) identify, respectively, the potential of gateways to receive immigrants and to hold MICE events.

The second condition is more related to the fact that Singapore's Changi airport is an international hub that attracts passengers mainly from Asia and Australasia on long-haul trips. Although to some extent it is true that the hub role must be accepted by other countries in order to exist, it is also a fact that Changi became a hub due to the intense investment made by the government of Singapore despite all the efforts of places like Kuala Lumpur and Hong Kong to attract airlines to their main hubs. For the case of a gateway dependent on the hub concept, mainly those associated with air transport, there is a greater possibility of existing competition, as airports can be built almost anywhere and can attract airlines by giving them incentives through feeless policies. However, this may not be the case for non-hub gateways, which exist due to the presence of a rail or road network, for example. It is definitely easier to alter air routes than road and rail ones. Although some of these studies focus on the particular situation of gateways in large metropolitan cities and areas, gateways can be developed on different scales as well.

Pearce (1995) is probably one of the few authors to conceptualise gateways in a broader perspective. In fact, he suggests that two basic types of gateways may be identified:

1. Those that simply act as point of entry/exit for tourists en route to a destination;

2. Those that also are major destinations in themselves.

As can be noted in the second point, gateways can also enjoy a multiple nodal function, being gateway, destination and origin at the same time. In the case of gateways that have a strong destination function, Lew and McKercher (2002) use the term gateway destination. In the case where a place has only the role of a gateway, it could be named as a 'pure gateway'. However, because of all the socio-economic benefits that tourism can provide to a certain area/region, some 'pure gateways' may want to develop themselves as gateway destinations.

This research is particularly interested in contributing to the theoretical and empirical development of the notion of gateways. Therefore, the remaining part of this section 
examines the various concepts that have been presented in the literature, in order to elaborate a more holistic definition of gateway.

One idea connected to the theoretical example of Figure 1.1 that is rarely considered in the literature is the exclusiveness and dependence that a gateway may impose on the regions or destinations that it provides access to. Gateways can then be compared to a funnel where travellers converge from different routes to have access through a certain point and from where they can disperse or not, depending on the nodal function existing on the other node.

Another way of illustrating the gateway concept (see Figure 2.1) is through the diagram proposed by Burghardt (1971), where it is classified as a node in a system of traffic flows (I) or as a link between two matrices of interconnections (II).

1. The gateway as the connecting node in a system of traffic flows
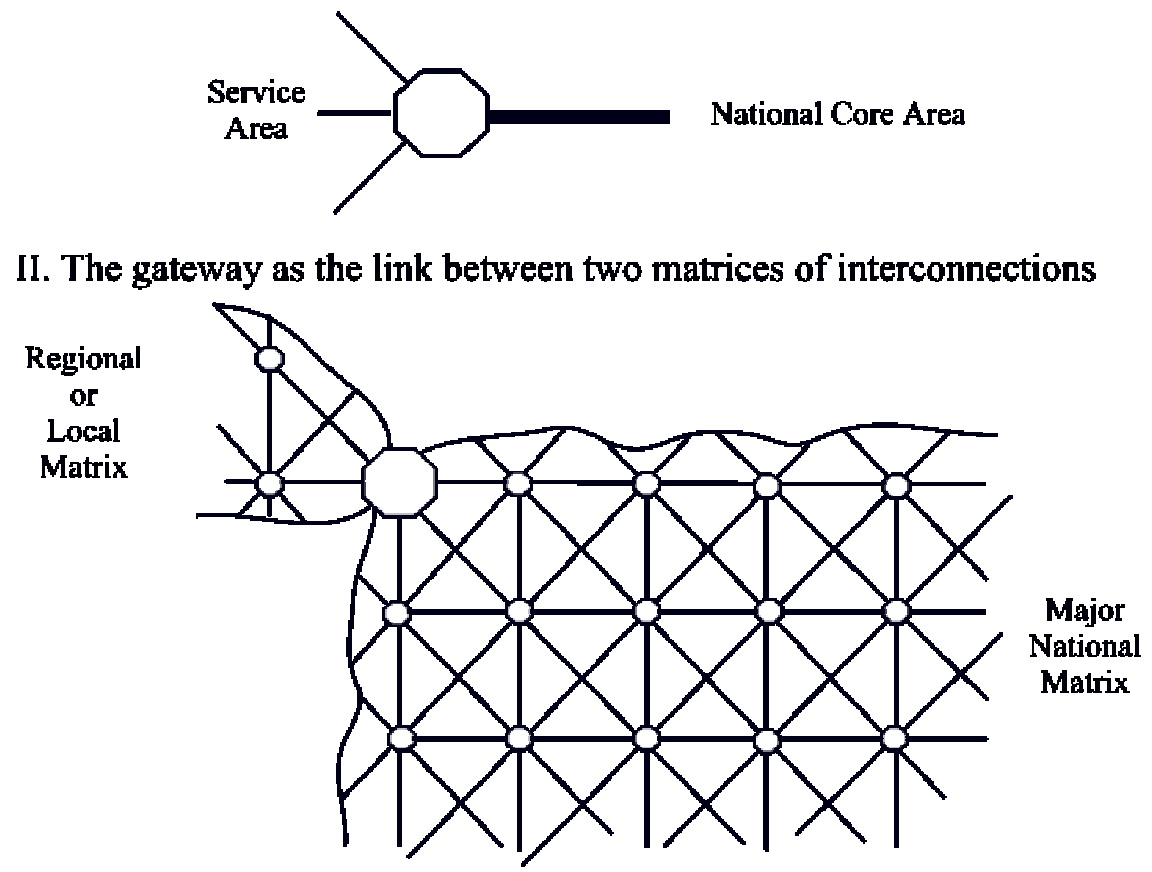

(Source: Burghardt, 1971)

Figure 2.1 Diagram of the gateway as a node and as a link.

Dredge (1999b) proposes the chained destination region model which is constructed by connecting either single and/or multiple node destinations (see Figure 2.2). Links from the tourist generating regions to destinations or within the various destinations can be made through two types of gateways: those that are "sharply focused at a particular point along 
a route" or those that "involve a gradual transition from one destination to the other" (Dredge, 1999b: p.786). The first type of gateway seems to apply better to this research, notably due to the mode of transport change in the ferry terminals. In addition, this model offers the possibility of graphically visualising the function of gateways as entrances or arrival zones to the destination region, being located along inter-regional circulation routes. One of the problems associated with her model is that it does not consider the multiple function aspect previously discussed, with the origin, destination and gateway functions not integrated within the same node.

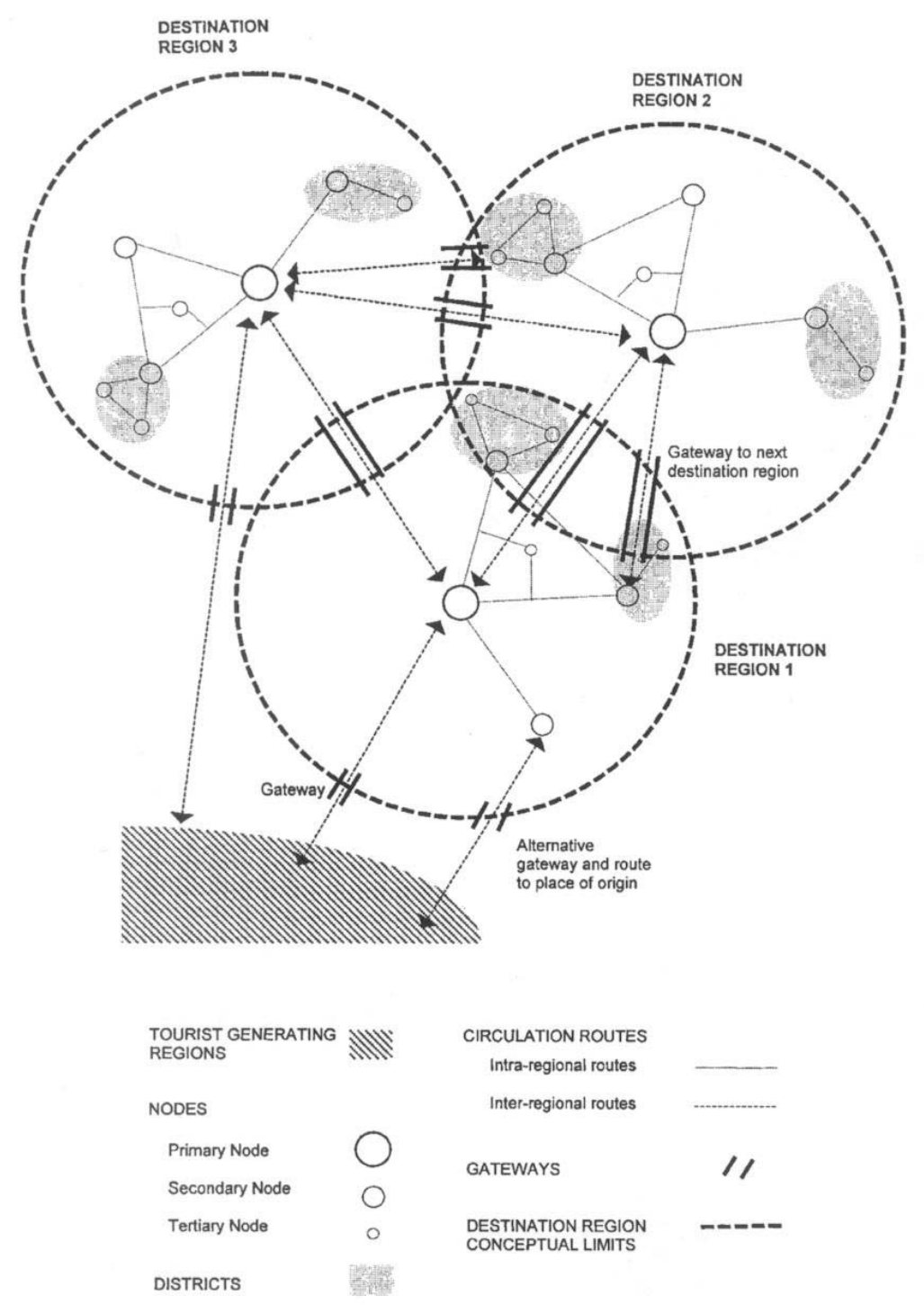

(Source: Dredge, 1999b)

Figure 2.2 Chained destination region. 
Apart from this conceptual analysis of exclusiveness and dependence of gateways, it is possible to identify some more practical aspects in order to analyse a gateway. They will be presented and discussed in the next sub-section.

Factors associated with the concept of gateway

Apart from the definitions of gateway, the literature also mentions some factors which influence and characterise gateways, notably in relation to tourism. They are the catchment area or hinterland, the place of origin, the purpose of trip and the intermediate role of places, measured by the Trip Index. These factors are considered in this section and they help to operationalise the concept of gateway, which is done in Chapter 3.

Due to gateways' privileged position, there is usually a certain geographic area around it from which the gateway is more likely to attract passengers or freight. This area is usually called the catchment area or hinterland (Burghardt, 1971; Caruana \& Simmons, 1995). For residents living outside the catchment area, as well as travellers not passing by it, the appeal of a particular gateway may not be as intense and they may prefer to travel through a different gateway. This choice may also include the decision to use a different mode of transport, which in some cases may represent a direct route that will not necessarily pass through any gateway.

Janin (1982), in an interesting study about travellers' behaviour in the Aosta Valley (Italy), found a relationship between the number of vehicles passing through and the nationalities recorded in the various accommodation establishments in the valley. This author observed that the proportion of motorists staying there increased with the distance from the country of origin.

If distance is one attribute which contributes to the decision to stop in a certain gateway, other variables such as country / region of origin and the purpose of trip can also have an impact on this decision-making. Travellers on a business trip may be less inclined to stop, for example, than holidaymakers on a long vacation. Despite this, Mistilis and Dwyer (1999) present a piece of research where gateway cities attract more MICE tourists than non-gateway destinations. However, as Pearce (1995) suggests, obtaining precise purpose of visit figures is not always an easy task, particularly in gateways.

Finally, in terms of the length of stay, the simple consideration of the number of nights may not necessarily indicate the use a traveller is making of a given node. Consider the case of two different visitors staying one night in the same destination. If one of them is spending only one night away from home (single-destination trip), the night spent in this 
destination will have a different meaning compared to another visitor who is travelling for several weeks and staying one night in the destination simply because s/he arrives late at night and is continuing his/her trip the next morning (destination with a stopover or gateway function). The Trip Index (Ti) developed by Pearce and Elliott (1983) is used as a means of operationalising the function of places. It relates the number of nights spent in a particular destination $(\mathrm{Dn})$ to the total number of nights spent on the whole trip (Tn), according to the formula (Pearce, 1995):

$$
\mathrm{Ti}=(\mathrm{Dn}) * 100 /(\mathrm{Tn})
$$

In their study (Pearce \& Elliott, 1983), the authors made use of the Trip Index to compare the relative importance of Christchurch and Westland National Park in tourists' overall trips. In their analysis they classified the Ti according to ranges varying from transit or day trip (no nights spent in the destination); various degrees of stopover destinations; and sole destination, in the case all the nights are spent in only one place (single destination). The range below has been adjusted in order to allow fractional numbers (e.g. 10.57) as in the original proposal of these authors only whole numbers were considered between the different classifications:

- Transit or day trip: $\mathrm{Ti}=0$

* Short stopover: $0<\mathrm{Ti} \leq 10$

- Medium stopover: $10<\mathrm{Ti} \leq 20$
* Long stopover: $20<\mathrm{Ti} \leq 50$

* Principal destination: $50<\mathrm{Ti}<100$

* Sole destination: $\mathrm{Ti}=100$

Splitting travellers in terms of their place of origin (North Islanders, South Islanders and overseas travellers) the authors could identify different functions in both places studied. In the case of Christchurch, for example, almost $50 \%$ of South Islanders spent their whole trip there, whereas international travellers were using it as an urban gateway to the South Island or to New Zealand.

The use of different variables may prove to be a useful tool to help understand the concept of gateway. Particularly relevant to this research is identifying the various uses travellers make of the node so that an analysis can be done in terms of what would make them stay longer and visit Wellington and Picton. The operationalisation of the concept of gateway will be done in Chapter 3.

\subsubsection{Tourist Destinations}

Tourist destinations, on the other hand, are the places that tourists visit and stay in during their trip. Geographically, destinations can be a self-contained centre, a village, a town, a 
city, a region, an island or even a whole country. Furthermore, it can be a single location, a set of multi-destinations as part of a tour (as discussed in the models proposed in the next section), or even a moving destination, such as the case of cruises, scenic and heritage rail trips (Page, 1999). Several elements can influence tourists to choose one or various destinations. Cho (2000) considers that there are five main types of attractiveness in a destination:

- Attractions: natural, built, cultural and social;

- Amenities: basic infrastructure, accommodation, transportation, catering services, entertainment, shopping facilities and visitor information;

- Accessibility: relative to how easily or not tourists can reach the destinations and move within and among them;

- Images: the ideas and beliefs that tourists hold about the destination;

- Price: it varies by type and class of service (accommodation, transport and activities), seasonality and distance to a destination.

From the considerations above, it becomes clear that the provision of accessibility is only one of the factors that attract tourists to a destination. Therefore, although the transport network alone is enough to develop and maintain a gateway, this is not the case with destinations. Other attractiveness factors - attractions, amenities, images and price - are perhaps equally or more important than transport to stimulate the interest of tourists to visit and stay in a given place. In other words, transport on its own and the flow of passengers that may pass through a gateway will not be sufficient to develop it as a tourist destination.

Attractions, defined as "a named site with a specific human or natural feature which is the focus of visitor and management attention" (Pearce, 1991: p.46), are a factor in persuading tourists to visit a given destination or to improve their perception of the destination visited. In some cases, attractions work as a sort of icon to destinations, making the visitor realise that s/he is really "there", such as the cases of Sydney's Opera House, the Eiffel Tower and the Statue of Liberty, among others. Attractions can range from cultural institutions (museums, concert halls, art galleries, theatres); buildings; historical, sporting and religious sites; parks; scenic outlooks to political borders (Shoval \& Raveh, 2004; Timothy, 1995). 
Cho (2000) suggests a wide range of services and facilities that can be considered as amenities, including basic infrastructure, accommodation, catering services, entertainment, shopping facilities and visitor information. Particularly relevant to the overall success and development of a destination is the capacity and the quality of the accommodation provided (Sharpley, 2000). Shopping is also increasingly becoming a tourist activity and in the case of many destinations an essential aspect of the choice for travel (Heung \& Qu, 1998; Yuksel, 2004).

Image has been proven to be a pivotal factor in travellers' decision process and destination selection behaviour. The ever-increasing competition among tourist destinations highlights the need for an effective marketing plan and strategy (Baloglu \& Mangaloglu, 2001). From a cognitive point of view, tourist destination image is assessed on a set of attributes that correspond to the resources or attractions that a tourist destination has at its disposal (Beerli \& Martin, 2004b). Therefore, having a good range of attractions and amenities is not enough to attract visitors if these products do not reach potential consumers. Various channels help to promote destination information and marketing campaigns, such as tour operators and travel agents (Baloglu \& Mangaloglu, 2001), friends and relatives' recommendations or word-of-mouth (Baloglu \& McCleary, 1999), distribution channels, media, films and literature or previous experiences. Table 2.1 presents the attributes and dimensions determining the perceived destination image.

According to Dwyer et al. (2000), price factors related to a given destination include the cost of transport services to and from the destination and the cost of ground content (accommodation, attractions, tour services, entertainment, food and beverage). One factor that can influence these prices is seasonality, notably in coastal resort destinations and recreational areas, which is exactly the case of Picton. Price can also characterize the type of destination, attracting different profiles of tourists, such as luxurious or budget, exclusive or popular. Finally, as in the case of image, price is also an important aspect of the competition among destinations.

The two destination gateways analysed in this research have different characteristics, mainly due to their sizes. Picton is a small resort and Wellington is a capital city. Even though these two destinations will be analysed in Chapter 4, it is appropriate to explore some of the key concepts associated with resorts and urban destinations. 
Table 2.1 Dimensions/attributes determining the perceived destination image.

\begin{tabular}{|c|c|c|}
\hline Natural Resources & General Infrastructure & Tourist Infrastructure \\
\hline $\begin{array}{l}\text { Weather: temperature, rainfall, } \\
\text { humidity, hours of sunshine } \\
\text { Beaches: quality of seawater, sandy } \\
\text { or rocky beaches, length of the } \\
\text { beaches, overcrowding of beaches } \\
\text { Wealth of countryside: protected } \\
\text { nature reserves, lakes, mountains, } \\
\text { deserts } \\
\text { Variety and uniqueness of flora and } \\
\text { fauna }\end{array}$ & $\begin{array}{l}\text { Development and quality of roads, } \\
\text { airports and ports } \\
\text { Private and public transport facilities } \\
\text { Development of health services, } \\
\text { communications and commercial } \\
\text { infrastructures } \\
\text { Extent of building development }\end{array}$ & $\begin{array}{l}\text { Hotel and self-catering } \\
\text { accommodation: number of beds, } \\
\text { categories, quality } \\
\text { Restaurants: number, categories, } \\
\text { quality } \\
\text { Bars, discotheques and clubs } \\
\text { Ease of access to destination } \\
\text { Excursions at the destination } \\
\text { Tourist centres } \\
\text { Network of tourist information }\end{array}$ \\
\hline Tourist Leisure and Recreation & Culture, History and Art & Political and Economic Factors \\
\hline $\begin{array}{l}\text { Theme parks } \\
\text { Entertainment and sports } \\
\text { activities: golf, fishing, hunting, } \\
\text { skiing, scuba diving etc. Water parks, } \\
\text { zoos, trekking, adventure activities, } \\
\text { casinos, nightlife, shopping. }\end{array}$ & $\begin{array}{l}\text { Museums, historical buildings, } \\
\text { monuments etc. } \\
\text { Festival, concerts etc. } \\
\text { Handicraft; Gastronomy; Folklore } \\
\text { Religion; customs and ways of life }\end{array}$ & $\begin{array}{l}\text { Political stability and tendencies } \\
\text { Economic development } \\
\text { Safety: crime rate and terrorist attacks } \\
\text { Prices }\end{array}$ \\
\hline Natural Environment & Social Environment & Atmosphere of the Place \\
\hline $\begin{array}{l}\text { Beauty of the scenery } \\
\text { Attractiveness of the cities and towns } \\
\text { Cleanliness } \\
\text { Overcrowding } \\
\text { Air and noise pollution } \\
\text { Traffic congestion }\end{array}$ & $\begin{array}{l}\text { Hospitality and friendliness of the } \\
\text { local residents } \\
\text { Under privilege and poverty } \\
\text { Quality of life } \\
\text { Language barriers }\end{array}$ & $\begin{array}{l}\text { Luxurious; Fashionable } \\
\text { Place with a good reputation } \\
\text { Family-oriented destination } \\
\text { Exotic, mystic } \\
\text { Relaxing vs. stressful } \\
\text { Fun, enjoyable } \\
\text { Pleasant vs. boring } \\
\text { Attractive or interesting }\end{array}$ \\
\hline
\end{tabular}

(Source: Beerli \& Martin, 2004a)

The development of a resort

According to Prideaux (2000b), a number of models have tried to explain the development of tourism destinations from different perspectives, such as modifying spatial relationships, changes to the psychological needs of visitors and evolutionary cycles. Among the approaches developed to explain destination area growth, the Tourism Area Life Cycle (TALC) proposed by Butler (1980) has received the most attention and has been tested by numerous researchers (Douglas, 1997; Russell \& Faulkner, 1999; Tooman, 1997).

Butler's model proposes that tourist destinations tend to experience five distinct stages of growth: exploration, involvement, development, consolidation and stagnation. Depending on the response of destination managers to the onset of stagnation, various scenarios are then possible, including continued stagnation, decline or rejuvenation (see Figure 2.3). 
The model is essentially descriptive and, according to Williams and Patterson (1998: p.138), lacks "adequate explanatory power".

The TALC model is long-term in outlook and traces changes in visitation rates and the development of tourist related facilities such as accommodation and attractions. In theory, the stages postulated by Butler should be identifiable on the basis of number of tourists, rate of change and visitor types.

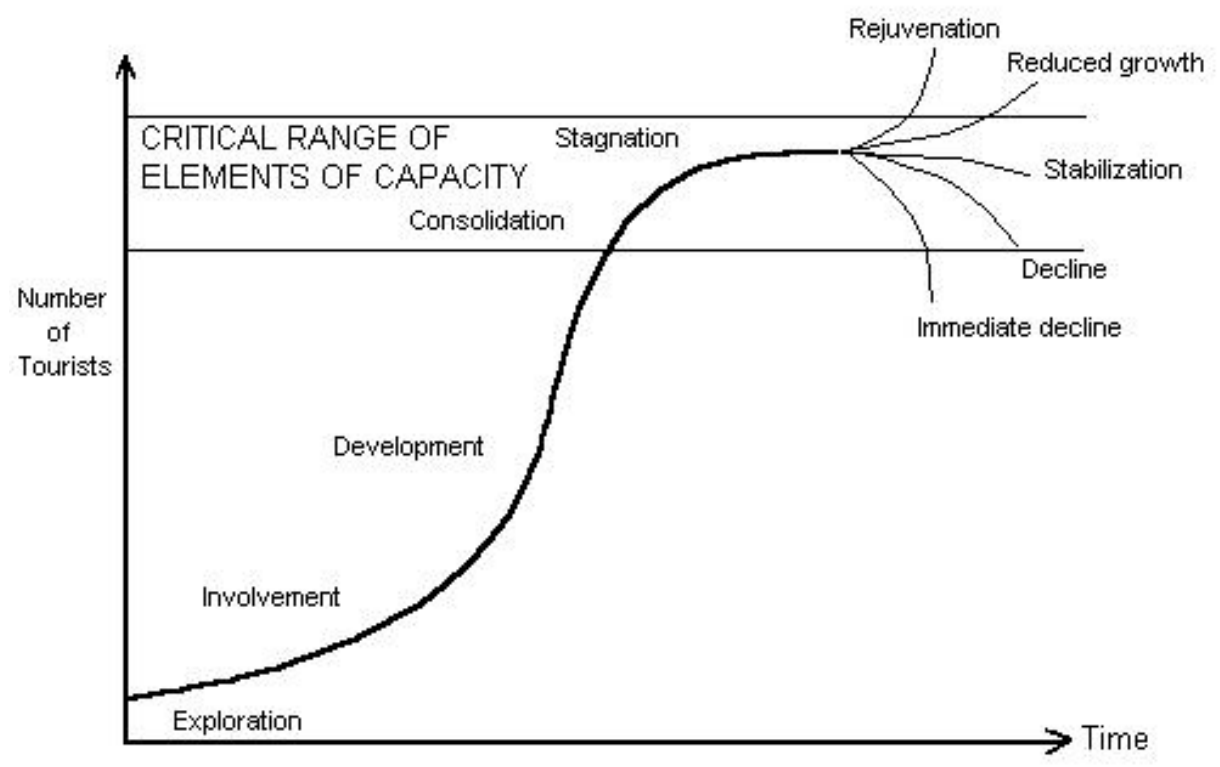

Source: reproduced from Pearce (1995), after Butler (1980).

Figure 2.3 The TALC model showing the relationship between evolution and capacity.

Prideaux (2000b) builds upon previous destination development models and proposes the Resort Development Spectrum. According to his model (Prideaux, 2000b: p.233),

Growth occurs as a result of expansion of the resort's infrastructure and parallel development of new market sectors. The key components determining the shape of the growth curve are the willingness of suppliers to invest in new tourist facilities, the rate of construction of new or expanded transport infrastructure designed to transport additional tourists from new markets, capacity of the resort with respect to physical and ecological constraints and success in attracting new market sectors. The role of external political and economic forces will also become evident as the resort moves into the international market place. 
In fact, some of the factors presented by Prideaux can also be applied to Butler's model in order to explain what determines progression from one stage to the next. The characteristics of each phase in the Resort Development Spectrum are outlined in Table 2.2 .

Table 2.2 The Resort Development Spectrum Framework.

\begin{tabular}{|c|c|c|c|c|}
\hline $\begin{array}{c}\text { Major } \\
\text { characteristics } \\
\end{array}$ & $\begin{array}{c}\text { Phase 1: } \\
\text { Local tourism }\end{array}$ & $\begin{array}{c}\text { Phase 2: } \\
\text { Regional tourism }\end{array}$ & $\begin{array}{c}\text { Phase 3: } \\
\text { National tourism }\end{array}$ & $\begin{array}{c}\text { Phase } 4: \\
\text { International tourism }\end{array}$ \\
\hline $\begin{array}{l}\text { Principal } \\
\text { Markets }\end{array}$ & $\begin{array}{l}\text { Locals } \\
\text { People from } \\
\text { nearby towns }\end{array}$ & $\begin{array}{l}\text { As per stage one plus: } \\
\text { Tourists travelling from } \\
\text { areas within the state or } \\
\text { region } \\
\text { Possibly limited interstate } \\
\text { tourist traffic passing } \\
\text { through the area en route to } \\
\text { a larger resort }\end{array}$ & $\begin{array}{l}\text { As per stage two plus: } \\
\text { Tourist who travel long } \\
\text { distances from all parts of } \\
\text { the nation } \\
\text { State capital cities } \\
\text { become primary markets }\end{array}$ & $\begin{array}{l}\text { As per stage three plus: } \\
\text { Emphasis on international } \\
\text { tourism }\end{array}$ \\
\hline $\begin{array}{l}\text { Accommoda- } \\
\text { tion }\end{array}$ & $\begin{array}{l}\text { Beach houses } \\
\text { Caravan parks } \\
\text { Licensed hotels } \\
\text { (not resorts) } \\
\text { Inexpensive } \\
\text { motels } \\
\text { Backpacker } \\
\text { hostels }\end{array}$ & $\begin{array}{l}\text { Unit and apartment } \\
\text { development occurs } \\
\text { 2-3 star resort motels } \\
\text { appear } \\
\text { Caravan parks still important } \\
\text { Outside investment } \\
\text { commences in hotels }\end{array}$ & $\begin{array}{l}\text { 3-4 star hotels } \\
\text { Integrated resorts } \\
\text { Internationally known } \\
\text { hotel chains commence } \\
\text { hotel development i.e. } \\
\text { Hilton, Ramada }\end{array}$ & $\begin{array}{l}\text { International hotel chains } \\
\text { establish resort hotels } \\
\text { Numerous } 5 \text { star hotels } \\
\text { which may incorporate } \\
\text { golf courses, casinos and } \\
\text { stage shows }\end{array}$ \\
\hline Promotion & $\begin{array}{l}\text { Local area and } \\
\text { surrounding towns } \\
\text { Undertaken by } \\
\text { local progress } \\
\text { and/or tourist } \\
\text { associations } \\
\text { Limited funds and } \\
\text { professionalism }\end{array}$ & $\begin{array}{l}\text { State wide } \\
\text { May attract government } \\
\text { funds } \\
\text { Business operating in the } \\
\text { resort advertise on an } \\
\text { individual basis } \\
\text { Increasing professionalism } \\
\text { of advertising campaigns }\end{array}$ & $\begin{array}{l}\text { Establish professionally } \\
\text { staffed promotion body } \\
\text { Joint campaigns with } \\
\text { state and local } \\
\text { government and local } \\
\text { business } \\
\text { Hotels and major } \\
\text { attractions fund significant } \\
\text { campaigns in national } \\
\text { media }\end{array}$ & $\begin{array}{l}\text { Very professional } \\
\text { approach } \\
\text { May attract significant } \\
\text { government funds } \\
\text { Corporate advertising } \\
\text { very significant }\end{array}$ \\
\hline $\begin{array}{l}\text { Tourism } \\
\text { Infrastructure }\end{array}$ & $\begin{array}{l}\text { Limited to beach } \\
\text { and nearby scenic } \\
\text { areas such as } \\
\text { parks } \\
\text { Limited } \\
\text { restaurants and } \\
\text { shops catering for } \\
\text { tourists }\end{array}$ & $\begin{array}{l}\text { Commencement of a } \\
\text { commercial sector based on } \\
\text { tourism } \\
\text { Additional specialist shops } \\
\text { Tour coach operators } \\
\text { commence } \\
\text { First man-made attractions } \\
\text { built, generally on a small } \\
\text { scale }\end{array}$ & $\begin{array}{l}\text { Establishment of first } \\
\text { theme park } \\
\text { Expansion of shopping } \\
\text { and dining opportunities } \\
\text { specialising in tourism } \\
\text { Construction of a range of } \\
\text { sporting facilities such as } \\
\text { golf courses }\end{array}$ & $\begin{array}{l}\text { Focus of tourist interest } \\
\text { shifts from the beach to } \\
\text { non-beach activities } \\
\text { Development of specialist } \\
\text { shopping precincts and } \\
\text { establishment of Duty- } \\
\text { Free shopping } \\
\text { Construction of } \\
\text { Convention Centre and } \\
\text { possibly Casino }\end{array}$ \\
\hline Transport & $\begin{array}{l}\text { Very limited in } \\
\text { scope } \\
\text { Main mode is road } \\
\text { Possibly some } \\
\text { traffic from rail if } \\
\text { the resort is } \\
\text { located close to } \\
\text { rail services } \\
\text { No scheduled air } \\
\text { services }\end{array}$ & $\begin{array}{l}\text { Road access is significantly } \\
\text { enhanced } \\
\text { Other modes may be } \\
\text { assisted by infrastructure } \\
\text { development } \\
\text { Limited (if any) scheduled air } \\
\text { services operated by local } \\
\text { airlines }\end{array}$ & $\begin{array}{l}\text { Scheduled interstate air } \\
\text { services commenced by } \\
\text { national operators or } \\
\text { affiliates } \\
\text { Road access continue to } \\
\text { be improved, i.e. } \\
\text { freeways. } \\
\text { Other modes may be } \\
\text { significantly redeveloped, } \\
\text { i.e. sea terminals and rail } \\
\text { services }\end{array}$ & $\begin{array}{l}\text { International air services } \\
\text { commence } \\
\text { Other modes continue to } \\
\text { be developed } \\
\text { Depending on distance to } \\
\text { source markets air may } \\
\text { become the dominant } \\
\text { mode }\end{array}$ \\
\hline
\end{tabular}

(Source: Prideaux, 2000b)

Although Prideaux's model may contribute to the analysis of resort destination development, particularly offering a detailed list of aspects that can be identified for the major characteristics in the four different phases, when it comes to analyse the destination aspect of a gateway node, the model has limitations, especially in relation to transport characteristics. The reason for this is that in the case of gateway destinations, the 
transport component may be in an advanced stage when compared to the other major characteristics. This is particularly true in the case of Picton. As it is discussed in Chapters 4 and 5 , its transport facilities and services are disproportional to the scale and stage of development of Picton as a tourist destination. In fact, one the key limitations of the TALC model is that it does not take into account external connections and its impacts to the destination development.

\section{Urban tourism}

According to Pearce (2001a), urban destinations are typically associated with a wide range of multiple products, sectors, functions, dimensions, purpose, scale and actors. This section emphasizes notably the multiple products and functions related to the particular case of Wellington.

Urban destinations play a notable role in tourist travel as in many cases they serve as gateways for tourist entry to the country, as centres of accommodation and a base for excursions to rural areas as well as destinations in their own right (Page \& Hall, 2003). In fact, apart from the gateway function, cities are characterised by other tourism functions, such as origin, destination and staging post (Pearce, 1981). Cities offer some usually accepted qualities, such as "high physical densities of structures, people, and functions; social and cultural heterogeneity; an economic multifunctionalism; and a physical centrality within regional and interurban networks" (Ashworth, 1992: p.5).

The promotion of tourism has become a vital element in contemporary planning for urban economic development, with cities creating tourism demand through enhancing the tourism resources and services (Rogerson, 2002). In many cases architectural and urban design / planning are used as means of developing cities as urban destinations. On the other hand, tourist attractions can also lead to changes in the land use of a destination, as new businesses may be set up to take advantage of the existence of tourists in one particular place (Pearce, 2001d). For example, Gospodini (2001a) mentions the case of Bilbao (Spain), where large scale urban design interventions, including the avant-garde design of the Guggenheim Museum, led to an increase in Bilbao's capacity to attract tourists and also improved the image of the city. Pearce (2001e) provides the example of the Christchurch tourist tramway that was redeveloped as part of an urban exercise to revitalise some areas of the CBD and also to improve and promote tourism. 
According to Blank and Petkovich (1987), there are a number of important points to consider when attempting to assess why visitors seek urban tourist destinations, including:

- Urban areas are often the focal point of tourist-transport interchanges and termini, which is particularly true in the case of Wellington, which has the busiest domestic airport in New Zealand, and where the country's main highway ( $\mathrm{SH}-1)$ begins due to the city's position at the bottom of the North Island. Wellington also has the trunk rail line and is where the Cook Strait ferries are located;

- Cities are places of high population density, with the result that there is a high propensity to visit friends and relatives (VFR);

- The concentration of commercial, financial, industrial and service providers in urban areas acts as a focus for different people to visit cities for employmentrelated purposes such as conferences, exhibitions and business travel;

- Cities provide a wide range of cultural, artistic and recreational experiences.

In the context of urban tourism, a well-established approach developed by JansenVerbeke (apud Page, 1995) is to view the urban area as a leisure product. This comprises primary elements, secondary elements and additional elements.

Primary elements include cultural facilities (concert halls, cinemas, exhibitions, museums, art galleries and theatres), sporting facilities (indoor and outdoor), amusement facilities (bingo halls, casinos, festivities, night clubs and organised events), physical characteristics (harbours, interesting buildings and ecclesiastical buildings) and sociocultural features (folklore, liveliness and the ambience of the place).

Secondary elements consist of the supporting facilities and services which tourists consume during their visit that shape the visitor's experience of the services available in the city. They include accommodation, catering outlets and shopping facilities. Additional elements are the tourism infrastructure that conditions the visit, such as transport infrastructure, visitor information centres and tourist signposting.

Particularly in the case of the urban destination analysed in this thesis (Wellington), two main functions can be identified: the 'culture city' and the 'capital city'. In the first instance, there are three major components that comprise the cultural tourism product: 'high tourism', e.g. the performing arts and heritage attractions such as museums (JansenVerbeke \& van Rekom, 1996) and art galleries; 'folk and popular culture', e.g. gastronomy, 
sport, architecture and crafts; and 'multiculturalism', which refers to cultural and racial diversity and language. The status of capital city provides an administrative and political base of government operations with spin-off effects for business travel. In addition to business-related travel, capital cities are also important for tourism because of their cultural, heritage and symbolic roles. They are frequently home to some of the major national cultural institutions while also tending to have a significant wider role in the portrayal, preservation and promotion of national heritage, which showcase national culture (Page \& Hall, 2003).

In terms of image, Law (2002) suggests that there is an anti-urban bias in society, with rural areas usually seen in a more positive way: quiet, peaceful, healthy, uncrowded and beautiful. However, in some cases the positive features of a city can outweigh the negative ones, making it easier to attract visitors. This can be supported, in the case of large metropolitan areas and capital cities, by the presence of media usually based there, causing these places to receive much more attention than other areas (Avraham, 2000). Although advertising destinations to attract visitors is not a new concept, the manner in which large, multi-functional cities are now being reimaged is becoming more sophisticated (Hall et al., 1997). The image creation aims to sell their tourism, locational, residential and business features as part of the place-making competition which cities are engaging in (Page, 1995).

The gateway and destination theoretical contributions seen here and in the previous section will be used in Chapter 4 to better explain the characteristics of the nodes chosen in this research (Wellington and Picton), as well as to identify their current stage of development as tourist destinations.

\subsection{Modelling Tourist Movements and Flows}

The previous section defined and conceptualised the nodal functions related to this research. Apart from the nodes, which comprise the static component of the spatial structure of tourism, it is also relevant and appropriate to analyse the dynamic part that involves the movements and flows of tourists (Williams, 2002). This is even more necessary in the case of New Zealand where tourists tend to visit various destinations while touring the country, which increases competition among the various destinations. In their spatial analysis of tourism in Spain, Pearce and Priestley (1998) also applied a systematic and analytical treatment using both flow and distributional patterns. 
Tourist travel pattern studies usually consider domestic and international flows. It is appropriate to identify the main generating and destination regions in order to analyse the flows between and within regions. In most cases, major transport routes will be recognized as those heavily used by tourists into, out of and within the country. In addition, these routes will also integrate the various nodes and shape them in terms of their nodal functions, especially in the cases of hubs and gateways. In Wellington and Picton the land transport network (roads and trains) converges at these two nodes, which are then linked by the Cook Strait ferries. Therefore, the design of the transport network and the flow of passengers using it establish the gateway function in these two places. Classification of tourist flows can include the nature, volume, direction, duration of trip and distance travelled. Figure 4.2 shows the volume of intra-national ${ }^{6}$ flows for international tourists in New Zealand in 1991.

Various models have been developed in order to understand tourist movements and flows. They include those proposed by Mariot; Campbell (Pearce, 1995); Lundgren (Pearce, 1989); Leiper (1990); Mings and McHugh (1992); Lue et al. (1993); Oppermann (1995); and Lew \& McKercher (2002).

Mariot's model considers that the tourist can have three different routes to go from his/her place of residence to a tourist centre: an access route, a return route (that may be the same or different from the access route) and a recreational route (see Figure 2.4). The first two provide a direct link between the place of residence and the tourist centre, while in the recreational route tourists "will make use of various tourist facilities along the way, even if the intervening area does not constitute the main goal of the journey, entering or leaving it at some stage en route between the origin and destination" (Pearce, 1995: p.3). The recreational route offers some attractions to the traveller in a way which catches his/her attention and in different degrees competes with or completes the tourist centre. In this case the concept of touring emerges based on tourists' desire to visit multiple destinations in order to fulfil multiple motivations (Dredge, 1999a).

\footnotetext{
${ }^{6}$ Movement of international travellers within the receiving country.
} 


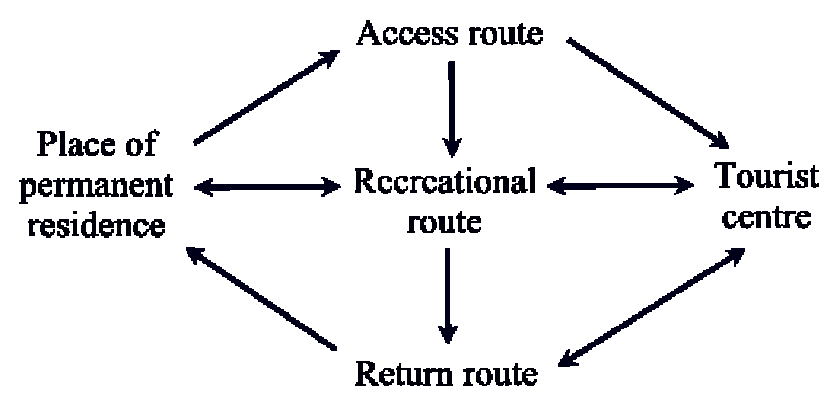

(Source: Pearce, 1995)

Figure 2.4 Mariot's model of tourist flow.

Pearce (1995: p.3) notes that Campbell's model of recreational and vocational travel "...portrays different patterns of movement away from an urban centre". The model distinguishes three groups of travellers based on motivations for travel. First, the recreationist travels radially from the city and is motivated by the desire to participate in recreational pursuits. Second, the patterns of travel which best define the vacationist are linear or highway oriented. The journey or excursion is the primary activity and incorporates a number of stopovers on a round trip. Third, elements of both forms of travel characterise recreational vacational travel.

In a certain manner, Leiper's model (Figure 2.5) is similar to Mariot's model presented by Pearce (1995). The notion of a transit route region through which travellers pass during the course of their travel is developed by Leiper (1990: p.22):

in order to visit the places they regard as appealing, tourists must travel through intermediate places. Sometimes this travelling stage is very short and sometimes it can span the world. But it always exists; there is always an interval in a trip when the traveller feels they have left their home region or country but have not yet arrived in a region or country they choose to visit. This element can be called the transit route.

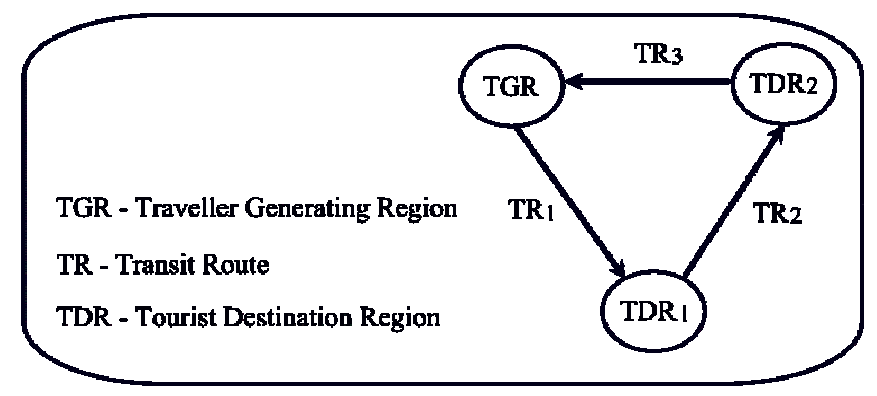

(Source: Leiper, 1990)

Figure 2.5. Leiper's geographical elements in a tourism system with two destinations. 
Structural models, such as those of Lundgren and Britton presented by Pearce (1995), are particularly useful to explain the concentration and dispersion of travellers in the origindestination spatial interaction. As seen in the definition of gateways, these are precisely some of the characteristics of this type of function. Lundgren's model is particularly useful in explaining the different functions existing in a particular place. It also illustrates how the various types of flows are associated with these functions. Lundgren proposes four types of tourist destinations: metropolitan destinations, peripheral urban-destinations, peripheral rural destinations and natural environment destinations. In order to contextualise the possible existing cases across Cook Strait, Figure 2.6 adapts the concepts of Lundgren's model presented by Pearce (1995) to propose seven different types of origin-destinationgateway situations. The case of hub-gateway status (Matthiessen, 2004) was not included as this is not being considered in this research.

(a)

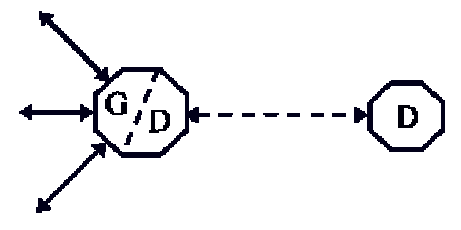

(b)

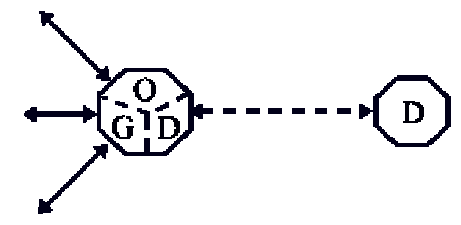

(c)
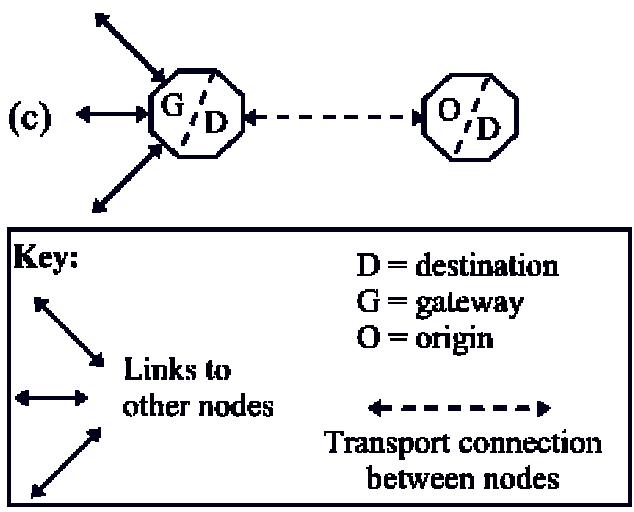

(d)

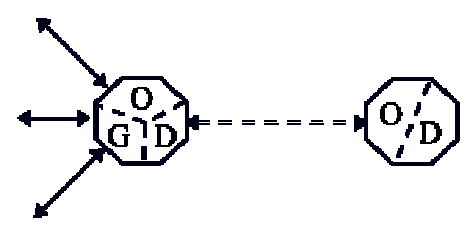

(e)

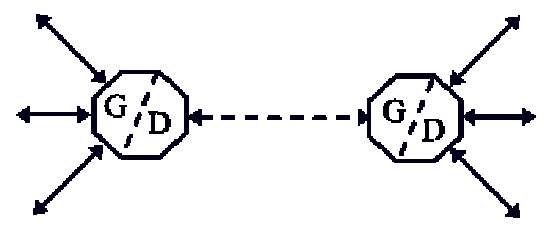

(f)

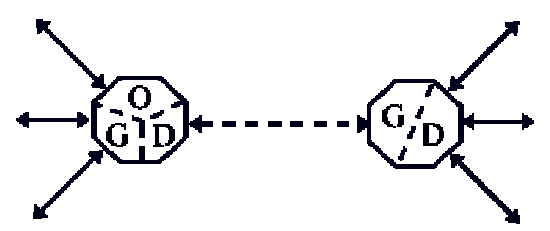

(g)

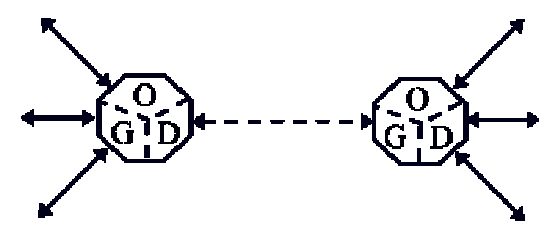

Figure 2.6 Spatial and nodal possible configurations of gateways with origin and destination functions.

Each of the seven cases ( $a$ to $g$ ) proposed in Figure 2.6 is explained below: 
(a) One regional destination gateway linked to a peripheral rural destination. A strong net inflow from the rest of the network connected to the gateway towards the peripheral rural destination is usually expected;

(b) One centrally located metropolitan destination linked to a peripheral rural destination. In some cases the latter may also work as a sort of a short break destination for residents in the metropolitan area;

(c) One regional destination gateway linked to a satellite town with associated origin and destination functions. Depending on the flow of traffic originated by the satellite town, the same amount of reciprocal traffic can be expected;

(d) One centrally located metropolitan destination linked to a satellite town. Due to the gateway and origin traffic originated by the metropolis, a higher volume of traffic should be expected in one direction;

(e) Two regional destination gateways that have a high volume of reciprocal traffic and function. No major generating area is identified between them;

(f) One centrally located metropolitan destination linked to a regional destination gateway that has a high volume of reciprocal traffic. A major generating area is identified only in the metropolitan destination. The destination gateway node may also be seen as a short break destination for residents living in the metropolitan area;

(g) Two centrally located metropolitan destinations that have a high volume of reciprocal traffic and function both as a generating area and a major destination.

Mings and McHugh's (1992) spatial model of tourist movements proposes four possible travel route types. These include the direct route, partial-orbiters, fly-and-drive and fullorbit routes. While direct route visitors travel in a direct fashion both to and from the selected destinations, partial-orbiters more or less travel directly to the destination areas, before touring the surrounding region staying in a number of destinations. Fly-and-drive visitors follow a direct route except that air transport is utilised. Finally, the full-orbit includes a number of destinations within a travel itinerary.

Lue et al. (1993) propose five spatial patterns of tourist movements (see Figure 2.7): single-destination, en route, base camp, regional tour and trip-chaining. The authors consider that if there were five (A1, A2, A3, A4 and A5) similar destinations that are equidistant from the home origin, the last four would probably be preferred as they provide extra opportunities ( $B, C, D, E$ and $F$ ) for relatively small increments of cost. More than 
analysing the various spatial patterns proposed by Lue et al.'s model, this research is interested in the possibility that multiple destination visitors can be "intercepted" on the way to or returning from the primary destination. This is even more evident in the case of ferry port gateways where travellers have to stop at least to change to a different mode of transport, the ferries. The main problem is that adding a new destination can increase the competition among the existing destinations, notably if the traveller has a fixed and short timeframe for the trip.

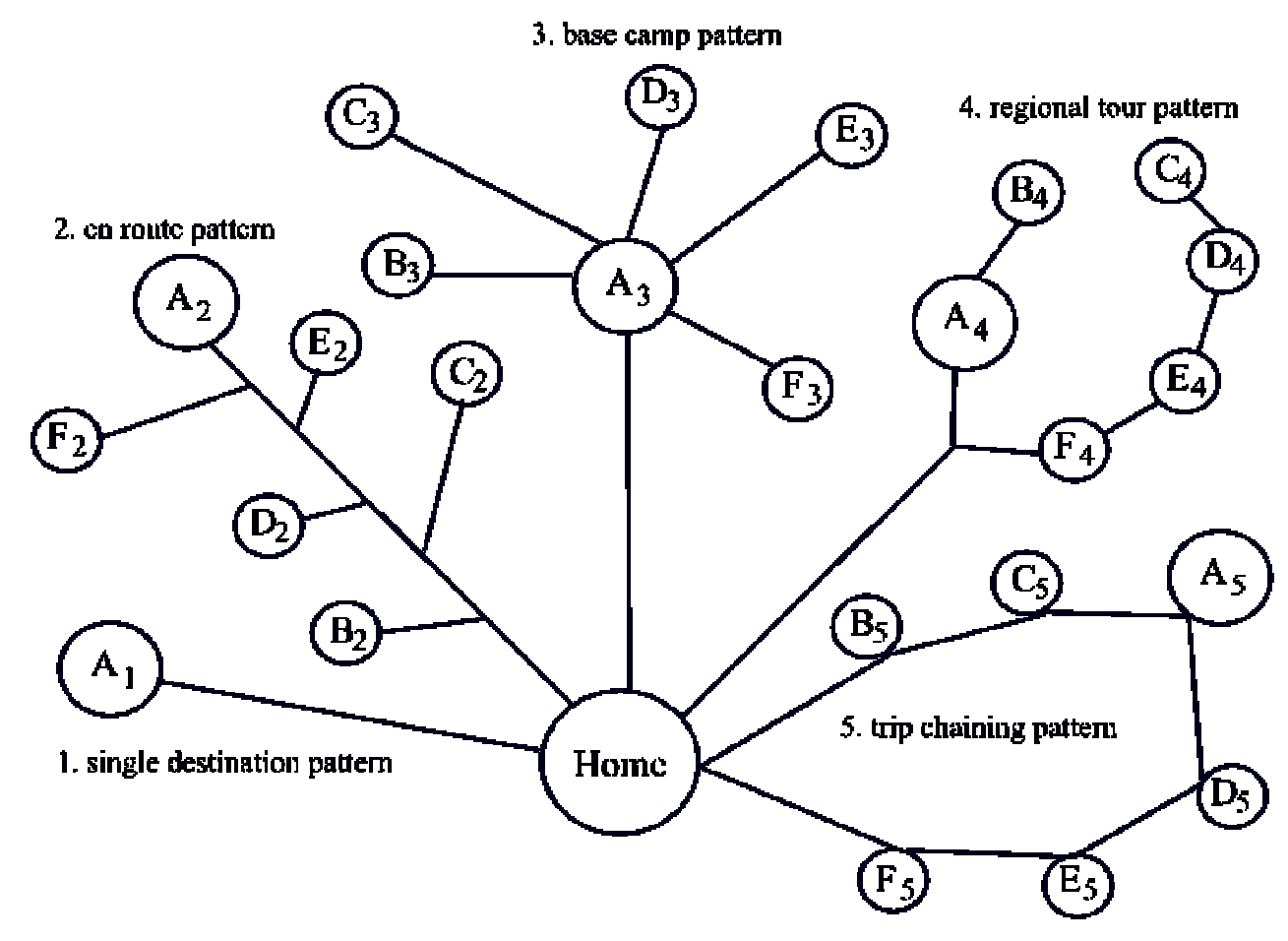

(Source: Lue et al., 1993)

Figure 2.7 Lue et al.'s model of alternative spatial patterns of pleasure vacation trips.

Oppermann (1995) builds upon the previous models, especially those from Lue et al. (1993) and Mings and McHugh (1992), by proposing a model of travel itineraries which is divided into two parts: single and multiple destination patterns (see Figure 2.8). The first consists of single destination (S1) and base camp (S2). The author also proposes five types of multiple destination: stopover pattern (M1), full loop (M2), destination area loop (M3), open jaw loop (M4) and multiple destination areas loop (M5). The single destination patterns (S1 and S2) and the first three types of multiple destination patterns (M1, M2 and M3) correspond to patterns identified by Mings and McHugh (1992) and by Lue et al. (1993). The open jaw loop (M4) is a type of long-haul travel that is very common in New 
Zealand, when the arrival and departure points in the country are not identical. As explained in more detail in Chapter 4, many international travellers arrive in New Zealand using one point of entry, usually either Auckland or Christchurch, tour around the country either northbound or southbound and leave the country from a different gateway. The multiple destination loop (M5) is a combination of the destination area pattern (M3) and the open jaw loop pattern (M4). According to Oppermann (1995: p.58) in the multiple destination loop "the tourist visits completely different regions and travels to a number of places within each region". The most typical example of this type of pattern is the round the world trip, with travellers going to different countries and visiting several regions within them. Being a long haul-destination, some international travellers to New Zealand do undertake round the world trips and the travel they do within New Zealand can be considered as part of a multiple destination areas loop (M5c or M5d).

\section{Single Destination Patterns}

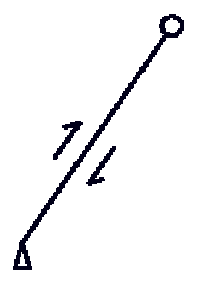

Sl "single destination"

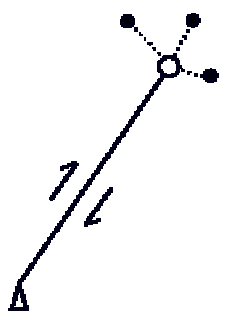

S2 "bast camp"

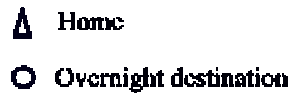

- Day trip dostination

Multiple Destination Patterns

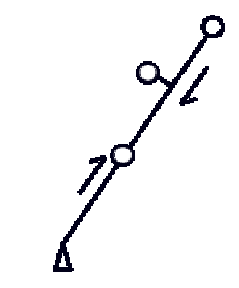

MI "stopover pattern"

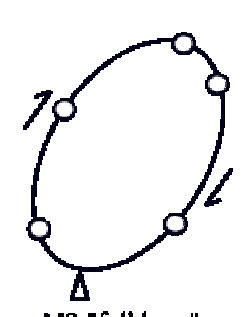

M2 "full loop"

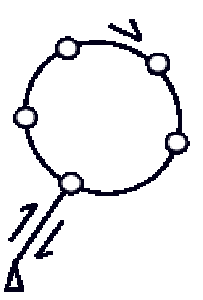

M3 "destintation atea loop"
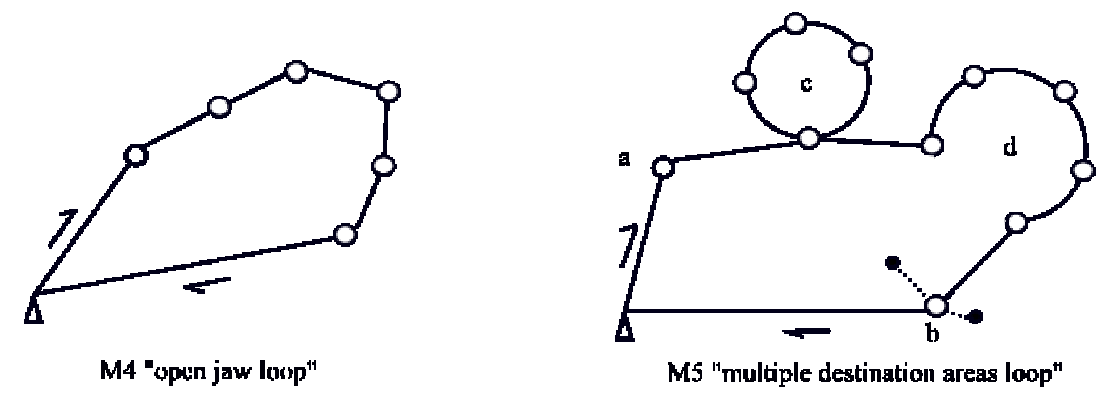

(Source: Oppermann, 1995)

Figure 2.8 Oppermann's tourist flow patterns. 
Finally, Lew and McKercher (2002) propose a model that combines aspects of the previous three models. Two main differences exist in their model. Firstly, they discuss the importance of knowing where the destination lies within the larger tour itinerary and the significance of being in one part of the itinerary versus another part. Secondly, they take into account the places' nodal functions, notably travel gateways and transportation hubs. Using Oppermann's tourist flow patterns, Lew and McKercher developed five forms of relationship of places to route itineraries (Table 2.3): single destination, gateway destination, egress destination, touring destination and hub destination. Their model is elaborated more from the perspective of the international traveller and they focus primarily on the case of Hong Kong. They associate the notion of gateway destination with a place of access/entry; the first place encountered in the country whose importance can potentially influence the rest of the itinerary. The gateway destination acts in opposition to the egress destination, the last point visited before travelling home, which provides a sense of closure. Even though this model proposes the notion of different nodal functions in various destinations, the idea of gateway is quite different from the one applied in this current study and also established by Burghardt (1971), Pearce (2001f) and Timothy (2000) where the gateway works as a passing through point (entry and exit).

Table 2.3 Forms of relationship of places to route itineraries.

\begin{tabular}{l}
\multicolumn{1}{c}{ Type of Destination } \\
$\begin{array}{l}\text { Single Destination } \\
\text { Multiple Destinations }\end{array}$
\end{tabular}

Keys:

延 = trip origin place, including transit stopovers.

= destination focus

w, = other destination places

$\downarrow$ travel link
${ }^{a}$ See Figure 2.8.

b Any destination that is visited more than once in a multiple destination itinerary can be considered a hub destination.

From the analysis of these models, it is possible to note that they all have some aspects that enrich the concept of gateways considered in this study, even though none of these models fully explains it. Some of the general comments to be made are: 
- In some cases travellers will use a more direct route (access route) and in others they will make use of an indirect one (recreational route, more appropriate in the case of road travel), whereby they can visit some places on their way to their final destination;

- In fact, most of these models focus on the recreational aspects of travellers, which are quite appropriate to explain the Cook Strait ferry passengers' flows and movements before and after taking the ferries;

- The notion of touring presented in some of these models, which are characterised by multidestination trips, are the primary type in New Zealand (Oppermann, 1995);

- These are models that focus on the travellers' perspective rather than models of tourism transport systems. As the travellers are the ones who have the decision power to stop and visit other destinations during their trips, considering the traveller's point of view is a necessary procedure to take into account. This will surely shape the methodology used in this study (Chapter 3);

- The multiple destination touring aspect of most of the models presented in this section is relevant to this thesis as it is exactly the type of travel pattern presented by domestic and more notably by international tourists while travelling in New Zealand. Travellers presenting this type of pattern usually do not concentrate all their stay in one particular destination, spreading the number of nights they spend in destinations located along their travel routes. This allows destinations located in the travellers' circuit to attract visitors at the expense of an increasing competition among the various destinations;

- There is a spatial hierarchy within the travellers' itinerary, with some nodes attracting the travellers' attention more than others, in addition to the different roles they provide in the passengers' trip.

\subsection{Other Pertinent Issues to Destination Gateways}

In the same way that the structure and shape of transport networks are vital for establishing a gateway, other factors can also help to enhance its intensity and importance. In order to develop themselves as tourist destinations, portal communities need to persuade transiting passengers to visit them and stay overnight (Wansink \& van 
Ittersum, 2004). This requires well co-ordinated actions involving transport providers and tourism companies and organisations. However, as Pearce (1995: p.170) suggests in the case of gateways:

the potential for retaining visitors may be limited [...] the number of stays in the area might be increased by improving the distribution of information and systems of hotel reservation for passing motorists.

The literature presents at least two major themes related to this topic. The first considers how tourists search for information and how information can influence them in the decision making process. This seems particularly crucial when choosing which places will be visited during their trips. The second theme to be analysed is the managerial and operational issues relating to transport and tourism that can impact on the traveler's decision to stay longer in the gateways. These two topics will be considered in this section.

Information Search and Tourist's Behaviour

Information can play an important role in attracting travellers to a given destination or to a particular tourism business. To inform potential travellers about their businesses and destinations, many private tourism enterprises and public tourism organisations advertise in the media (e.g. magazines, newspapers, TV and radio). In addition, travel writers can also be invited so they can write about a particular destination or business. Other ways of travellers getting information include travel consultants (i.e. travel agents and tour operators), the internet, family and friends. Apart from the types of sources of information used by travellers, two other key aspects need to be addressed. They are the information search process and the various "facets" or sub-decisions that planning a travel involves. The sub-decisions include the choice of destination, companion, length of stay, among others (Fesenmaier \& Jeng, 2000).

In a general way, the sources of information can be categorised into internal and external sources. According to Gursoy and McCleary (2004), internal information is based on knowledge from memories, including personal experiences either with the specific destination or with a similar one, and the knowledge accumulated through an ongoing search (e.g. advertisements, newspaper/magazine articles and TV programming). External sources consist of information collected from the marketplace, including family and friends, destination specific literature (e.g. maps, brochures and travel guides), media, internet and travel consultants. The search for external sources usually implies time spent, 
financial costs and effort required (Gursoy \& McCleary, 2004). Sources of information can also be classified into commercial or non-commercial and personal or impersonal (Fodness \& Murray, 1997). Table 2.4 presents these classifications for eleven types of sources of information.

Table 2.4 Classification of tourism information sources.

\begin{tabular}{|c|c|c|c|}
\hline & & \multicolumn{2}{|c|}{ Type of information } \\
\hline & & Impersonal & Personal \\
\hline \multirow{2}{*}{ 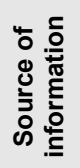 } & Commercial & $\begin{array}{l}\text { Brochures; guide books; local } \\
\text { tourist offices; state travel guides }\end{array}$ & Auto clubs; travel agents \\
\hline & $\begin{array}{l}\text { Non- } \\
\text { commercial }\end{array}$ & Magazines; newspapers & $\begin{array}{l}\text { Friends and relatives; highway } \\
\text { welcome centres; personal experience }\end{array}$ \\
\hline
\end{tabular}

(Source: Fodness \& Murray, 1997)

According to Snepenger and Snepenger (1993) the use of different types of information depends greatly on the type of trip, distance to be travelled and expense. The information search process aims to reduce the risks and uncertainty of a tourist product or destination (Bieger \& Laesser, 2004). In addition, they also consider that those taking routine trips, visiting family and friends or a favourite destination often utilize only past experience and information obtained from family and friends. The use of travel consultants as a source of information increases when travellers undertake longer trips, trips requiring the use of the air transport mode, first time trips to a destination and overseas travel (Snepenger \& Snepenger, 1993). Therefore, in the case of a destination like New Zealand, isolated from the rest of the world, it can be expected that travel agents and tour operators will play a vital role of disseminating information to international visitors.

Research done on the process of information search for leisure trips identify at least three different stages in looking for information. Bieger and Laesser (2004) present two of them: the information source before the trip decision and the information source after the trip decision (before the trip departure). Travellers also search for information after they have departed for their trips and they are considered as "en route" decisions (Fesenmaier \& Jeng, 2000). Figure 2.9 shows the three stages when travellers can obtain information for their travel. It also indicates the importance of the information changes during this process. In fact, research conducted by Fesenmaier and Jeng (2000) identified not only that the degree of planning changes throughout the information sourcing process, but also that certain types of decisions are usually associated with particular stages. For example, they identified that pre-trip planning includes five key decisions: date(s) of trip, primary destination, travel route, lodging/accommodations, and travel party. Budget and trip 
activities were also consistently considered as decided upon prior to the onset of the vacation. Those facets left to be decided en route (unplanned) included secondary destinations, food/restaurant choices, rest area stops and gift items to be purchased.

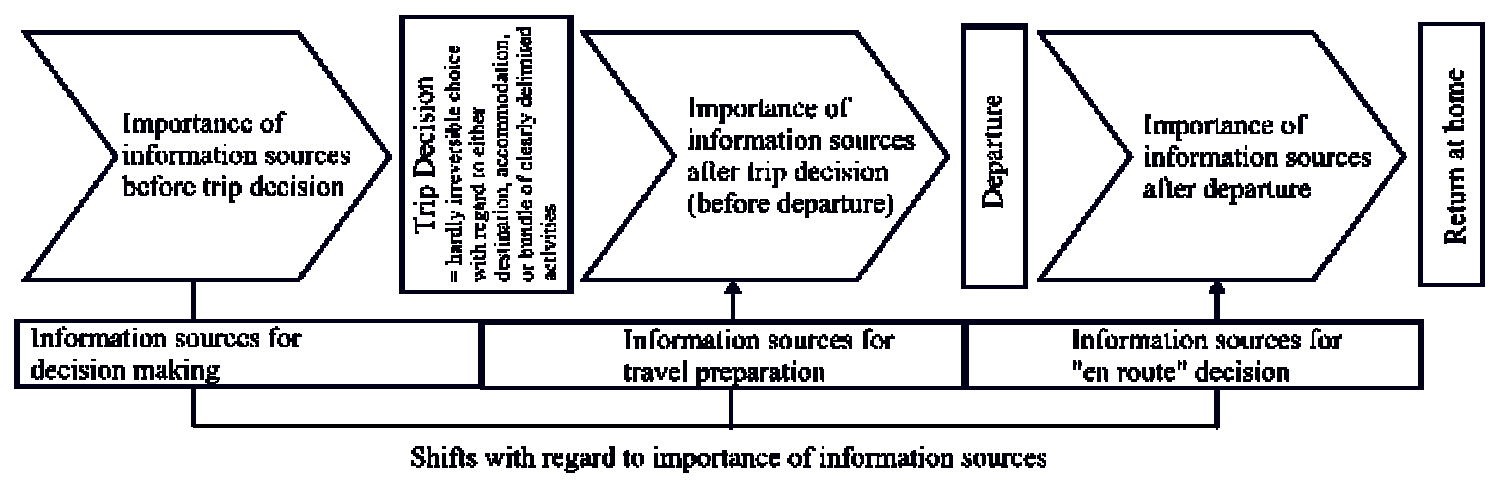

(Source: adapted from Bieger \& Laesser, 2004)

Figure 2.9 Process framework of information sourcing.

From the brief analysis presented here about information search and tourists' behaviour it is possible to contextualise some aspects that can be usefully applied in the case of this research. Firstly it is important to consider that in order to have passengers using the Cook Strait ferries, which ultimately will lead to travellers passing through the gateways, a combination of choice of destination(s) and mode of transport are necessary. Tourists not crossing Cook Strait or doing it by air transport will not be considered in this study. For that reason, in order to attract travellers to the ferry gateways, it is important to disseminate information about the ferries, preferably in a coordinated way. Secondly, it can be expected that domestic and international travellers will use different sources of information to find out about Wellington and Picton. Most likely domestic travellers will be more familiar with the ferries and the gateways in comparison to international visitors. Therefore, the former group will probably make use of previous experiences in order to decide whether or not to visit the gateways, while the latter will tend to rely on travel consultants. Thirdly, as will be discussed in Chapter 4, only recently, travellers - notably international ones - have become more aware of the CSNZ region. As its destinations are not as famous as others in New Zealand, such as Auckland, Rotorua, Christchurch and Queenstown, there is a risk of passengers considering the central part of the country just as a passing through area and neglecting it in their plans. In this case, especially considering the touring pattern that characterises tourism in New Zealand, the en route decision process can play an important role in persuading travellers to visit and stay in the 


\section{LITERATURE REVIEW}

gateways, especially if they have not booked the ferry journey before their arrival in New Zealand.

Tourism Transport Managerial and Operational Issues

Even though few studies have tried to explain which managerial and operational issues can create incentives to develop tourism in gateways, two general considerations can be made. They involve those aspects specifically related to transport operators, the ferry service providers, and those that exist between the ferry companies and tourism firms/organisations. This section briefly presents some of the aspects related to this topic, which will be further developed in Chapter 4 .

Although the literature does not establish how transport management and its operations can impact on the decision of travellers to stop at gateways, some possible factors identified in previous transport studies can be considered, such as: speed, frequency of service (Bertazzi \& Grazia Speranza, 1999), capacity, competition and environmental problems (Yang, 1998). Other relevant variables specifically related to ferries are: price (including special rates/discounts), level of comfort, ferry arrival and departure time, sea crossing time, delays and cancellations and journey enjoyment (Mangan et al., 2002; Wang \& McOwan, 2000). Although these variables are not directly related to the gateway concept, some of them can help to understand the use ferry passengers make of the gateways and how they can influence the passengers' decision to stay or not in the gateways.

For example, the variables of speed, frequency of service, ferry arrival/departure time and sea crossing time may or may not affect the need for passengers to stay in the gateways. As will be mentioned in Chapter 4, there are a range of daily services between Wellington and Picton and the crossing itself is a short one, taking on average three hours. In contrast to the long ferry crossings that usually provide overnight services (e.g. the ferry to Tasmania in Australia), the Cook Strait ferries are not a major competitor for the accommodation market in the gateways. In a three-hour crossing, there are no cabins for passengers on board the ferries. The combination of various services provided in a short trip crossing allows passengers to easily drive from nearby areas of one gateway to nearby areas of the other gateway during their journey. On the other hand, travellers coming from destinations further away may be inclined to drive long hours and then either stay overnight in the gateway before catching the ferry the day after, or to take the ferry and after arriving on the other side of Cook Strait to stay overnight there. In this case 
passengers can catch the ferry straight away, even if they are tired after a long trip because they can relax on board for a few hours.

The level of comfort and the journey enjoyment can also have an impact on the decision to stay in the gateways. Comfortable journeys where the passenger can also experience a cruise style with a scenic trip have a two-fold characteristic. Firstly, they will tend to encourage journeys during the day, which can make passengers stay overnight either before or after their crossing. On the other hand, if the transport experience is perceived as more interesting than the attractions provided in the gateways, passengers may be less inclined to spend time in the gateways as the ferry journey is considered as the main tourist attraction in the region.

Service disruption, such as cancellation due to bad weather, can make passengers stay longer than planned in gateways, allowing them extra time to visit and stay in a given place. However, this can imply that travellers will spend less time in other destinations than previously planned. Competition can help to decrease fares and increase total capacity and frequency, stimulating traffic through the gateway and enhancing the chances of passengers having a stopover there. The higher the flow of passengers, the higher the chance of capturing visitors. Finally, authorities may impose some regulations, resulting in a decrease of capacity offered if the transport operation is damaging the environment previously used for tourism and recreational activities.

In terms of the possible management initiatives between the transport providers and tourism firms/organisations, the research already undertaken on this topic has been mentioned earlier in this chapter. Even though the studies related to an aviation perspective, aspects of marketing, management and operational policies were correlated with improvement of tourism at a destination (Turton \& Mutambirwa, 1996). Promotion strategies (Bonnett, 1982) and product development can also be considered, particularly when the transport providers are directly linked to few destinations, such as is the case of the Cook Strait ferries. Even though the ferries' catchment areas can go beyond the gateways of Wellington and Picton, they have a special relationship with these two destinations, as these are the places where operations are based. Therefore, tourist information and products offered by the transport operator can influence passengers' decision to stay or not stay in the gateways. 


\subsection{Conclusion}

Traditionally, transport has been seen as a way to create accessibility to tourist destinations. For this reason, it is possible to state that transportation is an intrinsic element of tourism. In the case of this research, the main focus is not on how to create accessibility to a given destination, but on how to persuade passing through travellers to stop and visit a gateway. Consequently, it is not a surprise that the literature does not provide much evidence of the specific relationship between transport and gateways. What is more, even though previous research identifies some variables associated with the gateway function and the likelihood of travellers stopping there (e.g. place of origin and purpose of trip), in general there has been no attempt to bring together all these individual variables to establish a more practical approach to operationalise the concept of gateways.

This fact has several implications for the body of knowledge related to the research topic. Clear evidence of this can be noted in the other main conclusions obtained from this chapter. Among them, there is a gap in the literature in terms of studies related to some particular modes of transport (e.g. land transport and ferries).

Another main conclusion from this chapter is that nodes can have multiple nodal functions and these functions - origin, destination and gateway - are influenced by several factors. From Butler's (Figure 2.3) and Prideaux's (Table 2.2) models, it was possible to identify different stages of destination development, which imply different types and scales of attractions and amenities provided, the image perceived by visitors and the prices asked by local businesses (Cho, 2000). The different characteristics of the gateways studied in this research (urban destination vs. small resort) represent different challenges that can facilitate or hinder the attraction of potential visitors. This presents some methodological implications, with data being collected from passengers and suppliers in both gateways, in order to consider the two separately. From the theoretical aspects discussed in this chapter, it is possible to conclude that an analysis of the various functions presented by Wellington and Picton might be necessary in order to identify what exactly contributes to the existence of their origin, destination and gateway functions (Chapter 4).

In terms of the methodological implications of the conclusions obtained here, two major aspects have to be considered. The first is the need to operationalise the concept of gateway in a way that includes at least some of the variables previously identified by the literature, such as intermodality (Burghardt, 1971; van Klink \& van den Berg, 1998); 
influence on catchment area or hinterland (Burghardt, 1971; Caruana \& Simmons, 1995; van Klink \& van den Berg, 1998); and the relationship between the travellers' place of origin and their propensity to stop in gateways (Janin, 1982). The second aspect to be considered in the methodology (Chapter 3 ) is the importance of demand in the decision to stop part-way to their final destination (Wansink \& van Ittersum, 2004). Even though there is a possibility of transport and tourism businesses/organisations influencing travellers which will be developed within this research - the models of tourist flows and itineraries presented in this chapter suggests that the research design needs to focus primarily on the passengers as they are the ones that ultimately make the decision to stop and visit a destination. As seen in this literature review, understanding the travellers' behaviour throughout the whole trip and their information search processes is vital for answering the research questions outlined in Chapter 1. 


\section{Methodology}

\subsection{Introduction}

Tourism research can be conducted using various theoretical paradigms. Jennings (2001) lists and discusses six paradigms: positivism, interpretative social sciences, critical theory, feminist perspectives, post modernism and chaos theory. Traditionally, a significant proportion of tourism research is located within the positivism domain, as is evidenced by statistical and quantitative analysis of a high proportion of journal articles. In contrast to positivism, interpretivism focuses on understanding and interpretation (Decrop, 2004). For critical theorists the world can be described by "oppression, subjugation and exploitation of minority groups who lack any real power. The social world is perceived as being orchestrated by people and institutions in power who try to maintain the status quo and subsequently their positions of power" (Jennings, 2001: p.41). Feminism's prime criterion is research related to women. For some it is based on the assumption that women are disadvantaged in some way (Olsen, 2000). Postmodernist research methodologies have a strong reliance on intuition, imagination, personal experience and emotion. They seek to deconstruct or tear apart surface appearances to reveal internal hidden structures - structuralism and constructivism (Nueman, 2000). Finally, chaos theory assumes that reality is 'out there', it exists outside the human mind (also known as ontological realism), but this reality tends to instability, disorder, disequilibrium and nonlinearity. The current social world is seen to be characterised by an accelerated pace of technology driven change, leading to the destabilisation of social relationships and increasing levels of uncertainty (Faulkner \& Russell, 1997).

As Chapter 2 has shown, much of the transport and tourism literature has been written within a positivist and/or an interpretative paradigm. Research on passengers' choices, behaviour and impacts is commonly based on statistical and quantitative analysis (Douglas \& Douglas, 2004b; Dwyer et al., 2004; Dwyer \& Forsyth, 1998; Eby \& Molnar, 2002; Henthorne, 2000; Punter, 1999). There are also a substantial number of studies that undertake an interpretivism approach, usually describing a given market (Lawton \& Butler, 1987; Nelson \& Wall, 1986; Prideaux, 1999b; Prideaux et al., 2001), management issues and the strategies used by transport providers (Douglas \& Douglas, 2001b; Morley, 2003; Turton \& Mutambirwa, 1996) or the impact of transport regulation on tourism (Kissling, 1998b; Shaw, 1982). In the case of this thesis, that aims to understand how ferry passengers can be made to stay longer in the gateways, the research paradigm is 
predominantly positivist as a quantitative method is preferred in order to collect and analyse aggregate data from a large passenger sample. To a lesser extent, an interpretivist paradigm is also relevant, particularly in terms of exploring and getting an "insider's" perspective of the suppliers' point of view about the research topic.

Positivists assume that human nature (and behaviour in turn) is determined by the situation or environment that the person is in. They are quite pragmatic in orientation when approaching human relationships and situations. Research is often characterised by a problem-oriented approach, seeking to provide 'practical' solutions to 'practical' problems. This is exactly the case of this research. The problem proposed here is how to encourage ferry passengers to extend their stays in Wellington and Picton. What is more, it is expected that 'practical' solutions will be given in order to solve this 'practical' problem. The view of science that underlies this paradigm emphasizes the possibility that objective enquiry is capable of providing true exploratory and predictive knowledge of external reality (Burrell \& Morgan, 1979). This assumes that social science theories can be assessed objectively by reference to empirical evidence.

Empirical studies in tourism can be predominantly classified within the positivist paradigm (Riley \& Love, 2000; Walle, 1997), reflecting the industry prerogative that views tourism as a business. Key defining characteristics of scientific-positivist research are (Deshpande, 1983; Tribe, 2001):

- quantitative methods are preferred;

- the facts or causes of social phenomena are sought;

- the emphasis is on positive data, that is, on facts that can be verified and can survive attempts at falsification;

- a rigorous scientific method is used, based on hypothesis formulation and testing against empirical evidence;

- researchers adhere to the principle of value neutrality; they distance themselves from the data.

Of particular interest to this study is the spatial behaviour of travelers not only in the gateways, but also along their journeys (Chapters 1 and 2). Walmsley (2004) discusses aspects of spatial behaviour, making a distinction between aggregate behaviour and tourists' individual behaviour. The analysis of behaviour in the aggregate has the underlying principle that there are behavioural characteristics that are recognizable 
irrespective of the individuals that are studied. This is directly related to what this thesis aims to identify: the behaviour of ferry passengers in gateways while aggregating them according to their nodal functions in Wellington and Picton. On the other hand, studies of spatial behaviour at the individual level, also known as actor-oriented approaches, have been used primarily to infer tourist behaviours. According to Moore and Golledge (apud Walmsley, 2004, p.52), 'the underlying philosophy behind these approaches is transactional constructivism'. Because they should be considered more as a point of view rather than a rigorous paradigm, they have been used to complement rather than to supplant other approaches.

In the case of interpretivism, the ontological basis is not objective reality 'out there' but multiple subjective mental constructions. For them, reality comes from the mind. Any one of a range of multiple realities is not more or less 'true' in an absolute sense but simply more or less informed and/or sophisticated (Guba \& Lincoln, 1998). These realities and their construction can change over time. This relativism can lead to conflicting social realities not only between researcher and informant but also for the individual researcher if his/her constructs change as he/she gets more informed and experienced over time. The starting point of this research was not in the mind of the researcher but a real phenomenon: ferry passengers and tourism and transport business in Wellington and Picton, which exists independent of the researcher's investigation. Yet independent existence is no guarantee of objective interpretation.

In examining the assumptions of human nature for the interpretivism paradigm, the focus is on the essentially complex and problematic nature of human behaviour and experience (Burrell \& Morgan, 1979). The human being has a free will and a rational nature that is often influenced subjectively by the emotions and the senses. This is crucial when collecting data from the transport and tourism suppliers in Wellington and Picton. A descriptive approach can capture some of the problems identified by these stakeholders in a more exploratory way, aiming to synthesise their views about the challenges and opportunities to tackle the research objectives. Qualitative research methodologies, rather than quantitative, are preferred by researchers in this paradigm. The researcher undertakes an inductive approach to the research by getting involved with the data or the participants in order to develop explanations for the phenomena.

In terms of the research methodology, a number of challenges were set out in the conclusion of Chapter 2. As Walle (1997) mentions, tourism scholars and practitioners deal with a complex phenomenon, requiring the use of scientific and qualitative methods. In terms of data collection, obtaining information from different players (supply and 
demand), in two contrasting gateways, i.e. Wellington and Picton, can be quite challenging. As the ultimate decision of considering the node as a tourist destination comes from the traveller, data collected from ferry passengers provide key elements to understand the use they make of the nodes and how it would be possible to change it in order to extend their stay in Wellington and Picton. These methodological challenges also involve the need to use concepts in a way that has been done infrequently before. The most obvious need is the operationalisation of the concept of gateways. Even though previous research has elaborated on different aspects of the definition of gateways, in general the concept has not been developed so it could be applied in a practical way.

Apart from the demand data, information collected from transport and tourism suppliers is also required as they can set up strategies to influence the passengers' decision to visit the gateways. Even though the literature has identified some of the aspects that can impact on travellers' response to visit a destination (e.g. information), it seems appropriate to look at particular issues related to the Cook Strait area. Hence, there is a need to also collect secondary data associated with tourism and transport in the study area.

In order to tackle the aims of this research, a multiple method approach is required. Firstly, primary and secondary data are necessary. Primary data were collected from different players so that their various perspectives could be considered. They included the supply (tourism and transport businesses and tourism organisations) and the demand (travellers). Secondary data also needed to be obtained in order to contextualise transport and tourism issues in the study area, highlighting the key aspects related to the research problem. A description of tourism in the gateways (Picton and Wellington) as well as in the catchment area (Centre Stage Region of New Zealand) is presented in Chapter 4 . The historical evolution of the ferries is described in Chapter 5. Some of the trends and problems identified in these two chapters contributed not only to better understanding some of the transport and tourism aspects of the gateways, but also to structure the instrument to collect data from the suppliers. Bibliographic sources used in these two chapters include newspapers, magazines, books, tourism and transport organisations reports, as well as the fieldtrips done before and during the data collection process.

Secondly, the nature of the problem in this thesis is intrinsically related to both passengers and tourism transport providers, which requires a research design that combines qualitative and quantitative methods. Mixing quantitative (positivism paradigm) and qualitative (interpretive paradigm) has become more commonplace in tourism research (Goodson \& Phillimore, 2004; Walle, 1997) and can enrich the results obtained in a study with a scope like this. Interviews, that are one of the most commonly used instruments by 
humanistic researchers, were used to collect data from transport and tourism providers. On the other hand, quantitative data were collected employing a large scale questionnaire survey with ferry passengers. The use of both types of data also produces challenges, as integrating mixed methods at the analysis stage is not always easy.

Apart from the methodology used in this research and the techniques applied to collect data, this chapter also describes the data collection experience. Before doing this, the next section will attempt to operationalise the concept of gateway to be used in this research.

\subsection{Operationalising the Concept of Gateway}

According to Pearce (2001a: p.937), the operationalisation of the gateway concept requires "more than the analysis of routes, services, and [...] networks that underpin the work of transport specialists", it being more important to consider travel patterns as they form "one of the most direct and tangible links". Some of the travel patterns were identified in the literature review and will be reconsidered here.

In order to operationalise a concept, Cavana, Delahaye and Sekaran (2001) developed a model which involves a series of steps. In their model they establish that a given concept is made up of a set of broad characteristics, which they call dimensions. Although breaking the concept into dimensions reduces its level of abstraction, the operationalisation of the concept is done by breaking each dimension into elements. Figure 3.1 schematically illustrates the dimensions - main characteristics - and the elements of the concept of gateway. The dimensions are the four previously mentioned for the concept of gateways: 'transport intermodality', 'catchment area', 'purpose of trip' and 'length of stay'.

In the case of transport intermodality the elements are: mode of transport used to arrive in one of the gateways (Wellington or Picton), mode of transport used to depart from the other gateway, main reason for travelling by ferry (in the case of Cook Strait ferries, the ability to take vehicles onboard the ferries could be one of them, which helps to emphasize the intermodality issue), and other ferry transport variables identified in the literature: total travel time, fare, smooth crossing, scenic trip and cancellation.

Two elements comprise the catchment area dimension: place of residence and area where passenger stayed the previous night. In some situations they are the same, such as when domestic travellers stayed the previous night at home. 


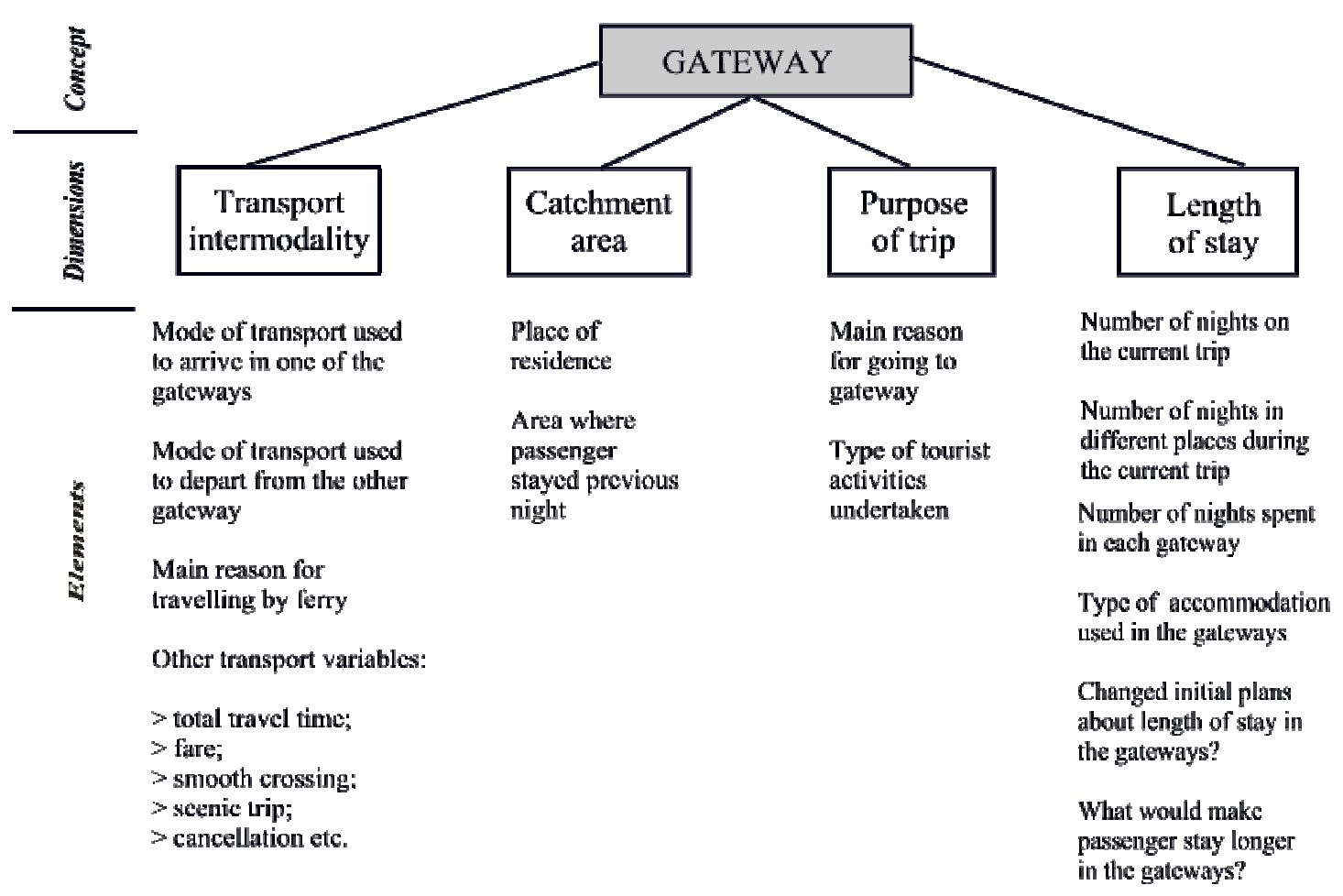

Figure 3.1 Dimensions and elements of the concept of gateway.

The purpose of trip dimension is formed notably by the main reason for the passenger to go to the gateway. Among the possibilities is the 'inter-island connection', which emphasizes the gateway as the primary reason to go to the node. Other reasons such as leisure/holiday and business are more related to the tourist destination function. Even though the main reason for going to the gateway can be an important aspect of this dimension, the activities undertaken by the tourists while at the gateways also indicate a certain level of interaction between the visitor and the tourist sector while passing by or staying there.

The length of stay dimension is a more complex one. It involves a series of elements; some basic ones such as the number of nights on the current trip and the number of nights spent in each gateway. These two variables are relevant in order to measure the Trip Index for each gateway. Other elements include the type of accommodation used in Wellington and Picton - depending on the type of accommodation a greater or lesser involvement with the tourism sector can be identified, regardless of the length of stay in the gateway. It is also relevant to recognize the changes in the length of stay in the gateways and the reasons for this change. This element is particularly pertinent to analyse whether the passenger after arriving in the destination gateway changed his/her perception of it, deciding to stay longer (if s/he realised that the gateway had also a role 
as a destination) or shorter (if s/he realised that the destination gateway did not have the appeal s/he was expecting and decided to use it only as an inter-island gateway) than planned.

Some of the indicators proposed by the literature seem to be more easily applied to operationalise the concepts of nodal functions in a way it is appropriate to this research. They include the main reason for going to the gateway and the length of stay in the gateway, which also relates to the Trip Index. Catchment area and intermodality are dimensions that can distinguish the level of importance that a given gateway has, influencing the use travellers make of the gateway node. However, they do not have a straightforward relationship that can help to operationalise the nodal functions of Cook Strait ferry passengers ${ }^{7}$ in Wellington and Picton.

Before operationalising these concepts, it is appropriate to consider that not every passenger will necessarily be inclined to stay longer in the gateways even if they are induced to do so through some sort of incentive. For example, business travellers on a short trip may be less inclined to stay and visit Wellington and Picton. Classifying passengers only into gateway travellers and destination tourists could perhaps not provide the best analysis from the data, as gateway travellers may, in general, be less inclined to stop and visit the nodes in comparison to destination tourists. Therefore, one possibility is to make use of intermediary nodal functions. In fact, this was done in the Trip Index classification (Pearce \& Elliott, 1983) where various degrees of stopover visitors exist between the two extremes when considering types of travellers: transit/day trippers $(\mathrm{TI}=0)$ and sole destination $(\mathrm{TI}=100)$.

As in the case of the Trip Index, a useful approach to consider when classifying passengers in terms of their nodal function is to analyse their length of stay. Passengers staying some days in a node are more likely to get involved with the tourism industry, than those that are only spending a few hours before catching the ferry. In addition the passenger's statement of what is their main reason for going to the gateway can also reveal the use made of the node. For the purpose of classifying passengers in terms of their nodal function, the most relevant reason to be considered is whether or not the interisland connection is the main reason for passengers going to the node.

\footnotetext{
${ }^{7}$ Hereafter, the terms Cook Strait ferry or Cook Strait ferries will be designated simply as ferry / ferries. Unless otherwise mentioned, the terms ferry / ferries will refer exclusively to the Cook Strait market. The same applies to the term "passenger(s)".
} 
The classification of gateway travellers, overnight gateway visitors, stopover visitors and destination tourists proposed in Figure 3.2 basically makes use of a combination of the length of stay and the main reason for going to the node. Three different possibilities for the number of nights spent in the node were considered. They include those passengers that do not stay overnight, those that spend one night and those that stay two or more nights. Regarding the main reason for going to the node, two major possibilities influence this classification, i.e. whether or not the inter-island connection is the principal motive for the traveller going to the node.

According to Figure 3.2, gateway travellers are defined as passengers that do not stay overnight in the node and whose main reason for going there is the inter-island connection. In this case it becomes clear that the major reason for going to the node is the gateway function and no major involvement with the tourism industry might be expected.

How many nights is the passenger spending in the node before/after taking the ferry?

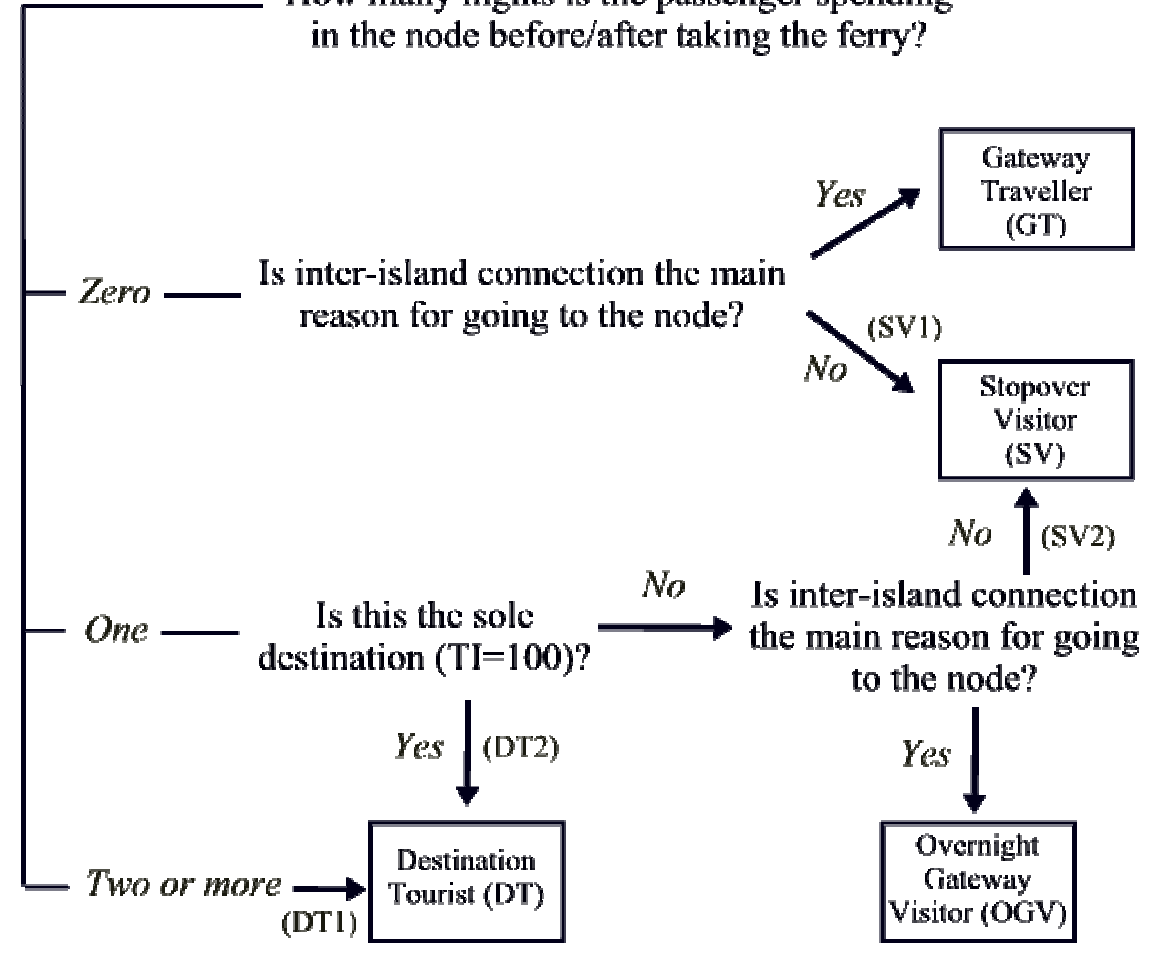

Figure 3.2 Operationalising the concepts of gateway travellers, overnight gateway visitors, stopover visitors and destination tourists.

There are two situations where passengers can be classified as stopover visitors. In the first case, the traveller is not staying overnight in the node but has another important reason for going to the node apart from the inter-island connection $\left(S V_{1}\right)$. The second 
situation is when a traveller is spending only one night in the node, as long as $s /$ he is staying more than one night away from home $(T \mid \neq 100)$, and having another important reason for going to the node rather than solely the inter-island connection $\left(\mathrm{SV}_{2}\right)$. Stopover visitors can be characterised by the fact that they have some sort of interaction with the node apart from the gateway function. Even though major involvement with the tourism industry may not be expected, especially for those that are not staying overnight, at least the existing interaction is stronger than in the case of gateway travellers who are simply passing by.

Overnight gateway visitors are made up of those travellers who are spending only one night in the node, as long as they are more than one night away from home ( $\mathrm{Tl} \neq 100)$, and having the inter-island connection as the main reason for going to the node. The only difference between gateway travellers and overnight gateway visitors is that OGV stay one night in the node.

Finally, passengers staying two or more nights are classified as destination tourists regardless of their main reason for going to the node. Even though some travellers may state that their main reason for going to Wellington and Picton is the inter-island connection, the fact that they are staying two or more nights is an indication that there are other important reasons for going there. An exception would be the case of passengers that could not get a ferry crossing when they wanted. Even in this situation, the fact that the travellers end up staying two or more nights in the node increase their chances of engaging in tourist activities and visiting tourist attractions, hence their classification as destination tourists $\left(\mathrm{DT}_{1}\right)$. Among destination tourists are also included those travellers who are only staying one night in the destination, with the condition that this is their single destination - $\mathrm{Ti}=100\left(\mathrm{DT}_{2}\right)$. This traveller is classified as having a single destination pattern, according to Oppermann's (1995) model seen in Chapter 2.

The classification proposed for gateway travellers, overnight gateway visitors, stopover visitors and destination tourists on Figure 3.2 will be used in Chapter 7 to analyse the possibility of changing the passengers' plans according to their nodal function in Wellington and Picton. The analytical framework section at the end of this chapter provides further details in terms of how this will be done.

\subsection{Research Design}

In order to collect data from two different types of sources (passengers and business managers), two different types of research were designed. 
In the case of the ferry passengers a survey was considered the most efficient way to collect a large amount of data (passengers' opinions, behaviours and profiles) from a large number of respondents. Surveys have been used in a number of transport (Brown et al., 1998; Feyer \& Williamson, 1995; Oborne, 1977) and tourism (Finn et al., 2000; Veal, 1997) related studies. In the case of this research, it was decided to conduct the survey on board the ferries, rather than at the ferry terminals. There were two major reasons for this. Firstly, passengers spend at least two hours and a quarter on board the ferries travelling between Wellington and Picton, making them potentially more available to answer a 10-15 minute questionnaire. Secondly, the main problems with applying the questionnaires in the ferry terminal were that passengers are too busy before the arrival of the ferry to be inclined to answer questionnaires, they are usually spread over a large area and the questionnaire would target only foot passengers, as those travelling with their own car drive inside the vessel and wait for their crossings outside the ferry terminal. Other transport surveys have also undertaken research onboard, such as in-flight (Chen \& Gursoy, 2000; Gursoy \& Chen, 2000; Jang et al., 2004) and cruise (Petrick, 2003, 2004) surveys.

The need to have a large sample of respondents led the researcher to opt for the use of a self-completion questionnaire as it does not require the researcher to spend time with the passenger in order to complete the questionnaire, making him available to approach a larger number of passengers. On the other hand, the main disadvantage of this type of data collection is that there is a chance of passengers not understanding or misinterpreting the questions or the instructions, particularly among passengers whose mother tongue is not English.

In the sampling design, one aspect that was considered was the structure of the analysis planned (Singleton Jr et al., 1988). As evidenced by the literature, place of residence is an important aspect in the use made by travellers in gateways (Janin, 1982). It was decided that the sample would be divided into the various types of nodal functions, i.e. gateway travellers, stopover visitors, overnight gateway visitors and destination tourists (see Figure 3.2). For marketing purposes, each of these nodal functions would then be broken down in the three place of origin subcategories: international travellers, domestic travellers living outside the Centre Stage Region of New Zealand (CSNZ) and domestic travellers living within CSNZ. Due to the timeframe available and the fact that the researcher did the data collection process by himself, it was possible to undertake 35 trips onboard the ferries from which a total of 1,076 useful questionnaires were obtained (see Table 3.2). 
In order to collect data from the supply and tourism organisations, semi-structured interviews were used. The main advantage of this type of qualitative data collection is the possibility of gathering data in a structured way, although leaving space for some other issues that could be raised during the interviews (Finn et al., 2000). Interviews are a very common technique of investigation to collect qualitative data in tourism studies (Dann et al., 1988; Veal, 1997). Semi-structured interviews with tourism suppliers have been done by Fallon and Kriwoken (2003), Li (2000) and Okumus et al. (2005). The interviews were designed to last between 30-45 minutes, depending on the availability of the interviewee, and a total of 20 interviews were conducted (see Table 3.1).

This section explains in more detail both the structure of the questionnaire and interviews used in this research. It also briefly considers the fieldtrips undertaken in the early stage of this project.

\subsubsection{Fieldtrips}

Two fieldtrips were undertaken for familiarization with the study area. The first took place in July 2002 and was a day trip to Picton. Its main purpose was to use the Cook Strait ferry service and also to have a better idea of Picton. This was particularly useful in order to set up a meeting with the marketing and sales manager of The Interisland Line, the only ferry operator existing at that time, in order to explain the project and ask permission to interview passengers onboard. A second and longer field trip was undertaken at the end of October and beginning of November 2002. This took seven days and its aim was to visit the gateways' catchment areas. Some visits and informal meetings with tourism organisations took place in Blenheim, Kaikoura, Nelson and Motueka (South Island) and Wairarapa (North Island). Clifford Bay, the potential site of a future ferry terminal in the South Island, was also visited.

The fieldtrips influenced the research design in several ways. From them it became clear that the catchment areas comprised a huge area and that it would be hard to conduct research with businesses located in the entire CSNZ region. In addition, it was evident that the ferry has a huge impact on Picton, due to its small scale, and that concentrating the data collection with businesses in Picton would be sufficient to gather the necessary data required for the research. In the case of the North Island, it was possible to note that the Wairarapa Region was more of a rural area adjacent to Wellington where the Cook Strait gateway function of Wellington did not impact much. Wairarapa was seen more as a place for short break trips for Wellingtonians rather than a passing through region for Cook Strait travellers. 


\subsubsection{Semi-structured Interviews}

Qualitative semi-structured interviews were chosen as the technique to collect data from stakeholders. The main characteristic of the qualitative interview is that it is concerned with establishing common patterns or themes between particular types of respondents (Warren, 2002).

The interviews with stakeholders were conducted face-to-face. Although there was a set of pre-established questions about transport and tourism in Wellington and Picton that were usually covered, according to the progress of the interview and the comments made by the interviewees, other questions were added in order to obtain additional relevant information. Examples of this type of interview in tourism and hospitality research include Okumus and Hemmington (1998), Rowley and Purcell (2001), Yang (2004) and Yang and Wan (2004).

There were eleven pre-established questions covering the following topics (see Appendix One):

- Questions 1 to 3: importance of the ferries and the gateway role of tourism in Wellington and Picton;

- Questions 4 to 7: current interaction and possible partnership between transport and tourism companies and organisations that are / would be helpful increasing the destination role from the gateway node;

- Question 8: comparison of the role played by both ferry companies in this process;

- Question 9: analysis of previous and possible future packages between ferry operators and tourism players;

- Question 10: impacts of the Tory Channel speed restriction on tourism, particularly in Picton;

- Question 11: about the proposal to build another ferry terminal in Clifford Bay and its impacts on CSNZ and notably Picton.

In the case of the ferry operators, another version of the interview checklist was developed with 15 questions. This was done in order not only to adapt the questions to the ferry operators' perspective, but also to include some of the data collected during the passenger survey. This explains why the interviews conducted with the two ferry operators happened a few months after the others. 


\subsubsection{The Questionnaire}

As discussed in Chapter 2, the data required for this research must not only identify the ferry passengers' behaviour in Wellington and Picton, but also their whole trip. In addition, information about the ferry journey (including intermodality issues, factors influencing the use of the Cook Strait ferries, booking procedures etc.) was also relevant in order to better understand how the option to use the ferry could induce passengers to stay and visit the gateways. Other type of information needed included the scenario of the proposed ferry terminal in Clifford Bay and also some general information about the passenger profile. The questionnaire (see Appendix Two) contained questions arranged in the following seven sections (sections $B$ and $C$ are mutually exclusive):
A. About the passenger's trip;
B. Profile of passengers living in New Zealand;
C. Profile of passengers living overseas;
D. About current ferry journey;
E. About Wellington and Picton;
F. About the proposal of a new ferry terminal in Clifford Bay;
G. About the passenger and his/her family.

The twelve-page self-administered questionnaire included 47 questions. The first page introduced the researcher, the project and the questionnaire. The contact details of both the student and supervisors were also given in case passengers required further information.

A map of New Zealand was available on page 2 to help passengers to identify the 18 regions in the country. These regions did not match the political regional division of New Zealand, being identical to the one used by The Interisland Line in their passenger survey questionnaire. This proved useful not only as a way to compare data between the current research and the research conducted by the main ferry operator, but also because on this map the areas near the gateways were divided into more sub-regions than those further away from them. As seen in Chapter 2, the gateways' catchment area is one of the relevant aspects when analysing a gateway and dividing the areas near Wellington and Picton in smaller sections than the traditional political boundaries allowed data to be analysed in more detail. 
Different types of questions were used in the questionnaire. They include closed questions, 5-point scale questions (Likert scale ranging from "not important" to "very important" or from "strongly disagree" to "strongly agree"), scenarios and open-ended evaluations (Jennings, 2001). Closed questions, for example, were used to ask passengers the activities and attractions they have visited both in Wellington and Picton. Offering a certain number of limited options helped the respondent to easily remember whether or not a particular attraction was visited or a given activity was undertaken. In addition, this type of question is also more easily coded as each answer has its own predefined code. In cases where it was not possible to predict the answer the passenger will give (e.g. why some passengers decided to stay shorter or longer in the gateways), an open-ended response set was provided so passengers could explain in a few lines what their personal reason for this change of plans was. Finally, scenarios were also considered in the case of questions associated with the Clifford Bay proposal. As currently there are no ferry services to Clifford Bay, passengers were asked whether or not they would travel via Clifford Bay if they had this option.

Two different questionnaires were produced; one to be completed by passengers travelling northbound (Picton to Wellington), the other on southbound trips (Wellington to Picton). The reason for this was to make passengers answer questions about the place they had just departed first, so they could recall more easily what they did there. Apart from the order of the questions, both questionnaires were identical. In order to make reference to questions presented in the questionnaire, the one used for travel departing from Wellington was chosen as reference (see Appendix Two).

\subsubsection{Ethical Considerations}

In 2003, two separate applications were submitted and approved by the Human Ethics Committee of the Victoria University of Wellington. The first was for the passenger questionnaire and the second for the stakeholder interviews. In the case of the passengers the data collection process was strictly anonymous. For the stakeholders, consent was given for the data collection and for the attribution or non-attribution of the information provided. Most of the interviewees gave consent to attribute the provided information to them or their organisations.

\subsection{Data Collection Experience}

After the questionnaire and the interview schedule were designed, data were collected. This section describes the experience of collecting primary and secondary data. 


\subsubsection{Primary Data Collection}

This section describes the primary data collection experience for this project, i.e. the ferry passenger self-completion questionnaires and the stakeholder interviews.

\section{Selection of Respondents and Sampling Design}

The selection of respondents, passengers and suppliers, was done in two different ways. In the first case, the target population was the passengers travelling on Cook Strait ferries. They were chosen randomly on board the different ferry services, making sure that passengers located in different areas of the vessels had the chance to be approached. In practice, however, it was not always possible to deliver the questionnaire to every passenger (e.g. those that were sleeping, in the movie theatre, travelling in seats where other passengers blocked the access of the researcher to them and foreign travellers who did not feel confident to fill in a self-completion questionnaire in English). In addition, some passengers initially agreed to take part in the research, but later on did not reply to the questionnaire or did not drop it in the appropriate box.

Although each questionnaire was designed to be filled in by only one passenger, in the case of passengers travelling together (couples, families and small groups), it was considered that one questionnaire would represent the travel behaviour, itineraries and activities undertaken for the whole group. For large tour groups, more than one questionnaire was delivered, usually to two or three passengers, depending on the size of the group.

In the case of the suppliers, interviewees were chosen among those directly related to tourism and the Cook Strait ferries. The research aimed to include a wide range of tourism sectors such as accommodation providers in Wellington and Picton (three motels, one holiday park and three backpackers); the two ferry operators and other transport companies (e.g. one regional airline and two rental cars), government and support organisations (one tourism organisation, the Department of Conservation, visitor information centres in Wellington and Picton and one non-governmental organisation); one tourism operator; and two attractions (a large one in Wellington and a small one in Picton).

Table 3.1 presents the sectors and the area of influence of the 20 stakeholders interviewed for this research. For the large companies and organisations the manager or the marketing manager was interviewed. For the small tourism operators usually the owner was contacted. In terms of the number of interviews conducted, in Picton it was reasonably easy to identify tourism businesses that are directly affected by the Cook Strait 
ferries. Managerial and operational aspects of the ferries heavily impact most of the businesses located in the town. Ten interviews were conducted with businesses that operate in Picton and this number seemed to be adequate as after a few interviews it became evident what the key issues were. In Wellington as the ferry gateway function is just one of the functions existing in this node, it was more difficult to identify businesses that are particularly affected by the Cook Strait ferries. Most of the tourism businesses in Wellington seem to rely on other sources of visitors, particularly the domestic business travellers who go to the capital city in order to deal with the government organisations that exist in the capital city. Another possibility would have been to include other businesses located outside Wellington that could also rely on the ferry passengers. They could include, for example, motels located on $\mathrm{SH}-1$ where ferry passengers might potentially stay overnight in order to get cheaper accommodation in comparison to the capital city. Again, the difficulty of this task would be to identify these businesses. It was then decided to choose a few businesses located in Wellington where the influence of the ferries were more evident, rather than spend too much time trying to identify other potentially relevant interviewees. Two backpackers, one of them located very close to two of the ferry terminals, a major tourist attraction and the visitor information centre were selected, making a total of four businesses (see Table 3.1). Six other interviews were conducted with businesses and organisations that have an influence both in Picton and Wellington and throughout their catchment areas (CSNZ). They included, for example, rental car companies, the ferry providers, one regional airline and the CSNZ marketing organisation. The interviews with these remaining businesses and organisations helped to enhance the analysis of the ferries in Wellington, complementing the other four interviews.

Table 3.1 Stakeholders interviewed by sector and area of influence.

\begin{tabular}{|l|c|c|c|c|c|}
\cline { 2 - 6 } \multicolumn{1}{c|}{} & Accommodation & Transport & Government / Support & Tour Operators / Attractions & Total \\
\hline Picton & 5 & & 3 & 2 & 10 \\
\hline Wellington & 2 & & 1 & 1 & 4 \\
\hline CSNZ $\left.{ }^{*}\right)$ & & 5 & 1 & & 6 \\
\hline Total & 7 & 5 & 5 & 3 & 20 \\
\hline
\end{tabular}

${ }^{(*)}$ It includes transport providers, such as the ferry companies, regional airline and rental car companies, and the tourism organisation responsible for marketing the CSNZ region.

Ferry Passengers' Self-completion Questionnaires

\section{Pilot Test}

In order to be familiar with the data collection process on board the ferries as well as to ensure that passengers understood the questionnaire and were able to complete it within the expected time, a pilot test was conducted. Before doing this, a meeting with The Interisland Line's research co-ordinator was arranged in order to get some tips about 
doing research aboard the ferries. The Interisland Line undertakes monthly research with its passengers, with the specific purpose of measuring their level of satisfaction with the transport service. A sheet of paper with instructions regarding the method of distribution of the questionnaires and maps of Aratere and Arahura were provided. Some of the points that were particularly useful were:

1. Go to the information counter and check-in with the crew. Although the staff were aware that a survey was being conducted onboard, it was always prudent to remind them about it;

2. Take 30 minutes to allow passengers to settle down onboard before beginning to hand out questionnaires. This was a very useful piece of information because it was possible to observe that on arriving onboard many passengers went to the restaurant, bar, play area or just for a walk to familiarise themselves with the vessel before they sat down and relaxed;

3. Try to distribute questionnaires equally in different areas of the vessels. Some parts of the vessels tend to gather similar types of passengers, such as the bar, the dining area and the playground - notably families with children. This procedure increases the chance of getting a sample more representative of the ferry demand.

The pilot test took place on the $8^{\text {th }}$ and $11^{\text {th }}$ of July 2003 with two return trips undertaken. To some passengers, usually those that looked more available and willing to talk, it was explained that this was a pilot test and some of them provided feedback about the questions and the questionnaire as a whole.

Overall, the pilot test was very successful. A total of 104 questionnaires were collected (see Table 3.2). Passengers were able to complete the questionnaire easily. As no major change in the questionnaires was required, questionnaires collected during these two days were included in the research sample. The next section explains how the sample was collected for the survey.

\section{Sample collecting}

Following the pilot test, data collection took place between August 2003 and January 2004. During this period it was possible to cover both the low and high seasons.

A total of 1,076 questionnaires were collected in 35 trips (18 northbound and 17 southbound). Access to The Interisland Line and Bluebridge vessels was obtained through previous arrangement with both companies that agreed to support the research giving free access to passengers. Nine return trips were done during the low season 
period, between August and October, with 390 questionnaires (36\% of total) being collected. This included one long weekend (Labour Day). The remaining 686 questionnaires were collected between $16^{\text {th }}$ December 2003 and $22^{\text {nd }}$ January 2004 (peak season). Coincidently, as seen in Table 3.2, the same number of questionnaires (538) was collected in each direction (Wellington-Picton and Picton-Wellington).

Apart from the time of year, other factors such as day of the week, ferry service (The Lynx, The Interislander and Bluebridge), time and place of ferry departure were also taken into consideration so that the sample would be representative of passengers travelling in various situations. Questionnaires were not delivered during the dawn sailing because in most cases passengers were asleep the whole journey, and therefore not available to be interviewed. In addition, according to the ferry operators, the percentage of passengers travelling during that time of the day is very small, with ferries transporting mainly freight.

The response rate, that is, the percentage of passengers who agreed to take part in the survey and whose questionnaires were returned, was considerably high (85\%). To a certain extent, this reflects the methodology where questionnaires were only given to passengers who had previously agreed to take part in the research. As shown in Table 3.2, however, five questionnaires had to be rejected because either the questionnaire had not been completed in full or the passenger forgot to complete some of the key sections.

Table 3.2 also presents some other information related to the questionnaire data collection process. In terms of ferry services, twenty trips were undertaken on The Interislander (57\% of total), nine on The Lynx $(26 \%)$ and six onboard Bluebridge (17\%). Conversely, when the number of valid questionnaires obtained in each service (number of questionnaires collected minus number of questionnaires rejected) is considered, 53.8\% are from The Interislander, $38.6 \%$ from The Lynx and $7.6 \%$ from Bluebridge. On average 30.7 questionnaires were collected per trip. Nonetheless, the mean on each service varied widely between the three services (The Interislander 28.9; The Lynx 46.1; Bluebridge 13.7). The low mean for Bluebridge can be attributed to two reasons. Firstly, this service started to operate in January 2003, a few months before the data collection process. It is possible that by that time this service had not yet been sufficiently advertised to the general public and was still gaining market share. Secondly, until October 2003, the Santa Regina vessel had capacity constraints, being able to carry only one hundred passengers. Later on a refurbishment process increased the number of seats to 370 (see Chapter 5). 


\section{Methodology}

Table 3.2 Information about questionnaire data collection - July 2003 to January 2004.

\begin{tabular}{|c|c|c|c|c|c|c|c|c|c|}
\hline Date & $\begin{array}{l}\text { Day of the } \\
\text { week }\end{array}$ & Ferry Service & $\begin{array}{l}\text { Place of } \\
\text { Departure }\end{array}$ & Departure & $\begin{array}{l}\text { \# Quest. } \\
\text { Delivered }\end{array}$ & $\begin{array}{l}\text { \# Quest. } \\
\text { Collected }\end{array}$ & $\begin{array}{l}\% \text { of } \\
\text { respond } \\
\text { ents }\end{array}$ & $\begin{array}{l}\text { \# Quest. } \\
\text { rejected }\end{array}$ & $\begin{array}{l}\text { Total } \\
\text { valid }\end{array}$ \\
\hline \multirow{2}{*}{ 08/07 } & \multirow{2}{*}{ Tuesday } & \multirow{2}{*}{ The Interislander } & Wellington & 09:30 & $\left({ }^{*}\right)$ & 26 & -- & & 26 \\
\hline & & & Picton & 13:30 & $\left({ }^{*}\right)$ & 29 & -- & & 29 \\
\hline \multirow{2}{*}{$11 / 07$} & \multirow{2}{*}{ Friday } & \multirow{2}{*}{ The Interislander } & Wellington & $14: 00$ & 27 & 18 & $66 \%$ & & 18 \\
\hline & & & Picton & $18: 00$ & $\left(^{*}\right)$ & 31 & -- & & 31 \\
\hline \multirow{2}{*}{$13 / 08$} & \multirow{2}{*}{ Wednesday } & The Lynx & Wellington & $11: 00$ & $\left({ }^{*}\right)$ & 24 & - & & 24 \\
\hline & & The Interislander & Picton & $17: 15$ & 17 & 14 & $82 \%$ & & 14 \\
\hline $22 / 08$ & Friday & Blue Bridge & Wellington & 13:00 & $\left(^{*}\right)$ & 13 & - & & 13 \\
\hline $24 / 08$ & Sunday & Blue Bridge & Picton & 08:00 & $\left(^{*}\right)$ & 6 & -- & & 6 \\
\hline $02 / 09$ & Tuesday & The Interislander & Wellington & 09:30 & 29 & 23 & $79 \%$ & & 23 \\
\hline \multirow{2}{*}{ 03/09 } & \multirow[b]{2}{*}{ Wednesday } & \multirow{2}{*}{ The Interislander } & Picton & $10: 00$ & 33 & 28 & $85 \%$ & & 28 \\
\hline & & & Wellington & $17: 30$ & 11 & 10 & $91 \%$ & & 10 \\
\hline $04 / 09$ & Thursday & The Interislander & Picton & 10:00 & 24 & 20 & $83 \%$ & & 20 \\
\hline \multirow[b]{2}{*}{$12 / 09$} & \multirow{2}{*}{ Friday } & \multirow{2}{*}{ Blue Bridge } & Wellington & 13:00 & 17 & 13 & $76 \%$ & & 13 \\
\hline & & & Picton & 19:00 & 10 & 7 & $70 \%$ & & 7 \\
\hline \multirow[b]{2}{*}{$24 / 10$} & \multirow[b]{2}{*}{ Friday } & \multirow[b]{2}{*}{ The Interislander } & Wellington & 09:30 & 38 & 29 & $76 \%$ & 1 & 28 \\
\hline & & & Picton & 13:30 & 43 & 33 & $77 \%$ & & 33 \\
\hline \multirow{2}{*}{$27 / 10$} & \multirow[b]{2}{*}{ Monday } & \multirow[b]{2}{*}{ The Interislander } & Wellington & 09:30 & 54 & 42 & $78 \%$ & & 42 \\
\hline & & & Picton & $13: 30$ & 35 & 26 & $74 \%$ & 1 & 25 \\
\hline $16 / 12$ & Tuesday & The Interislander & Wellington & 09:30 & 44 & 38 & $86 \%$ & & 38 \\
\hline \multirow[b]{2}{*}{$17 / 12$} & \multirow[b]{2}{*}{ Wednesday } & The Lynx & Picton & $11: 30$ & 49 & 43 & $88 \%$ & 1 & 42 \\
\hline & & Blue Bridge & Wellington & 19:00 & 18 & 14 & $78 \%$ & & 14 \\
\hline $18 / 12$ & Thursday & The Lynx & Picton & $11: 30$ & 47 & 45 & $96 \%$ & & 45 \\
\hline \multirow[b]{2}{*}{$23 / 12$} & \multirow[b]{2}{*}{ Tuesday } & \multirow[b]{2}{*}{ The Lynx } & Wellington & $15: 30$ & 75 & 69 & $92 \%$ & 2 & 67 \\
\hline & & & Picton & 19:00 & 67 & 62 & $92 \%$ & & 62 \\
\hline \multirow{2}{*}{$27 / 12$} & & & Wellington & $15: 30$ & 57 & 46 & $81 \%$ & & 46 \\
\hline & Saturday & The Lynx & Picton & 19:00 & 39 & 36 & $92 \%$ & & 36 \\
\hline & & & Wellington & 09:30 & 60 & 47 & $78 \%$ & & 47 \\
\hline 05/01 & Monday & The Interislander & Picton & 13:30 & 26 & 25 & $96 \%$ & & 25 \\
\hline & & & Wellington & $17: 30$ & 47 & 44 & $94 \%$ & & 44 \\
\hline 09/01 & Friday & The Interislander & Picton & $21: 30$ & 23 & 18 & $78 \%$ & & 18 \\
\hline $14 / 01$ & Wednesday & The Lynx & Wellington & 08:00 & 47 & 42 & $89 \%$ & & 42 \\
\hline $17 / 01$ & Saturday & The Interislander & Picton & 13:30 & 39 & 37 & $95 \%$ & & 37 \\
\hline $20 / 01$ & Tuesday & The Interislander & Wellington & $17: 30$ & 50 & 43 & $86 \%$ & & 43 \\
\hline $21 / 01$ & Wednesday & Blue Bridge & Picton & 08:00 & 44 & 29 & $66 \%$ & & 29 \\
\hline $22 / 01$ & Thursday & The Lynx & Picton & $11: 30$ & 57 & 52 & $91 \%$ & & 52 \\
\hline & & & TOTAL & & & & $85 \%$ & 5 & 1,076 \\
\hline
\end{tabular}

${ }^{(*)}$ The researcher forgot to count the number of questionnaires delivered.

In terms of time of departure (see Table 3.2), questionnaire delivery can be divided into three groups: those whose trips started in the morning, in the afternoon or in the evening. For the first group, fourteen trips ( $40 \%$ of the total number of trips) had their journeys starting in the morning (from 8am to $11.30 \mathrm{am}$ ). Fifteen trips (43\%) were in the afternoon 
(between $1 \mathrm{pm}$ and $5 \mathrm{pm}$ ) and six trips (17\%) in the evening (ranging from $6 \mathrm{pm}$ to $9.30 \mathrm{pm}$ ). The number of questionnaires collected during each of these periods of the day was 510 ( $47.4 \%$ of total) for services departing in the morning, $416(38.7 \%)$ in the afternoon and $150(13.9 \%)$ in the evening.

Finally, regarding the days of the week that the survey was conducted, Figure 3.3 presents data for the number of questionnaires collected on each day (Figure 3.3a) and the number of trips done (Figure 3.3b). This information was obtained from Table 3.2. As can be seen from both charts in Figure 3.3, the number of trips and questionnaires collected on Sundays are underrepresented when compared to the other days of week. This may have affected the sample especially neglecting the weekender market that tends to depart on Fridays and return home on Sundays. To compensate for this, the number of trips undertaken on Fridays, which can still catch this particular market on their way to their destinations, has the highest percentage, nearly one in four trips. In addition, the number of questionnaires collected on this particular day accounts for $19 \%$ of the sample, being the second highest, just below Tuesday with $26.7 \%$.

(a)

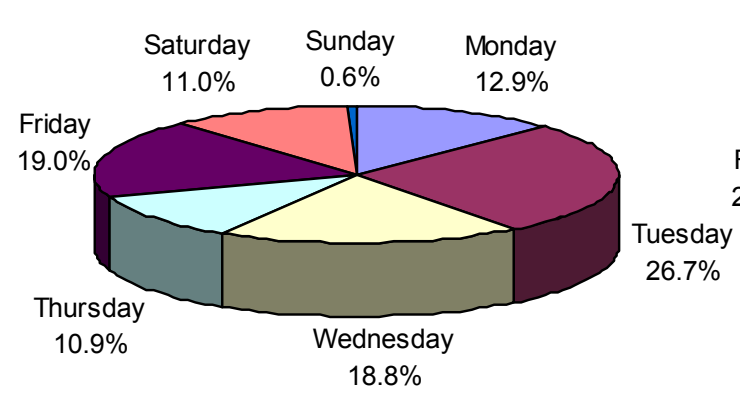

(b)

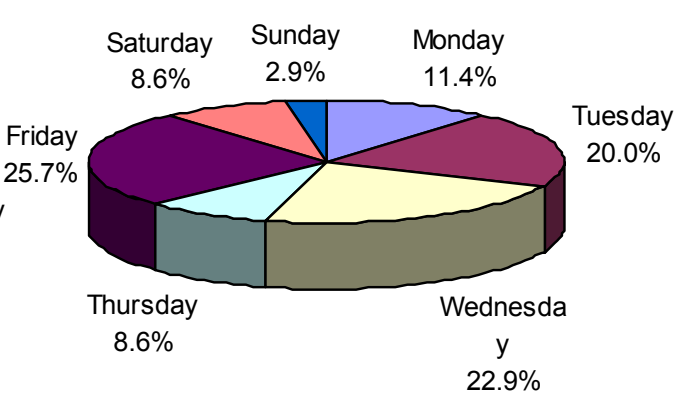

Figure 3.3 Distribution of the number of questionnaires collected (a) and trips done (b) by day of week.

Reliability of the sample

Different statistical techniques can be applied in order to test the reliability of a research sample. In the case of this research, as the researcher had access to data of a survey done with nearly 7,000 ferry passengers throughout the year of 2003, it was opted to compare these two sets of data. In order to facilitate future references to this larger sample and to guarantee the confidentiality involved in the use of these data, this other survey is labelled as Ka Pai. 
When the research data are compared with those from the Ka Pai sample, it is possible to identify some similarities. Table 3.3, for example, compares the passengers' place of origin in terms of the three main groups that will be used in this research (CSNZ, domestic and international passengers). As can be noted, very similar results were obtained in both surveys for the passengers' place of origin. In Chapter 6 other comparisons between these two sets of data are made and they also suggest that the data used in this research is reliable, despite the considerations of sampling made in the previous section.

Table 3.3 Passengers' place of origin - comparison between the research and the Ka Pai samples.

\begin{tabular}{|l|c|c|}
\cline { 2 - 3 } \multicolumn{1}{c|}{} & $\begin{array}{c}\text { Research sample } \\
(\mathrm{n}=1,076)\end{array}$ & $\begin{array}{c}\mathrm{Ka} \mathrm{Pai} \\
(\mathrm{n}=6,820)\end{array}$ \\
\hline CSNZ & $28.6 \%$ & $28.1 \%$ \\
\hline Domestic & $30.5 \%$ & $31.3 \%$ \\
\hline International & $40.9 \%$ & $40.6 \%$ \\
\hline
\end{tabular}

\section{Stakeholders' Semi-structured Interview}

Most interviews were conducted between August and December 2003. They started in Picton because, as a small town during off-peak season, it would give the required time and experience to the researcher in order to conduct interviews later with the big players in Wellington. These interviews usually took place after the sailings to Picton in order to collect data from passengers onboard the ferries. In most of the cases there was no need for a previous appointment, as most of the managers/owners were available to arrange an interview immediately or at a later convenient time.

In the case of Wellington, companies and organisations were contacted by email or telephone in order to arrange an interview. If further information was required, the interview questions and the information sheet about the research were emailed. In Wellington, interviews took place in October 2003 and January 2004. Interviews with the ferry operators took place in July and August 2004, as in both cases part of the passengers' questionnaire was analysed so that the managers could comment on the data provided by their clients. In the case of both Wellington and Picton, at the end of the interview the interviewees were asked to give the contact details of a person or organisation that would be useful to interview and whose point of view could be relevant to the research.

The interview was designed to take between 30 and 45 minutes. It usually started with two sheets of paper being given to the interviewee (see Appendix One); the first was the 
information sheet; the second was the consent form. The interviewee was then asked if $\mathrm{s} / \mathrm{he}$ agreed to have the interview tape recorded to be transcribed and analysed later. All of them accepted, although in some cases they were more likely to talk about delicate issues after the interview was over and the tape recorder was turned off (Warren, 2002). While the information not recorded was not included in the data analysis, this attitude reflects that there are some issues - notably the relationship with the ferry operators - that the interviewees felt more comfortable to talk about without the conversation being recorded.

\subsubsection{Secondary Data Collection}

Answering the research questions for this research also required secondary data collection. Its main purpose was to better understand the background of the study area, notably transport and tourism aspects of the two gateways (Wellington and Picton) and their surrounding areas (CSNZ).

A description of tourism in the study area is presented in Chapter 4. Apart from introducing tourism in New Zealand, in the CSNZ region and in the two nodes considered, it is particularly relevant to describe and analyse the multi-nodal aspects of Wellington (origin, destination and gateway) and Picton (destination and gateway). This helps to contextualise the study area as well as to understand which the main characteristics that comprise each nodal function are.

The evolution of the ferry services is considered in Chapter 5. Particularly relevant is the introduction of the fast ferry technology in 1994, which produced some impacts on other sectors, including tourism. Also relevant to this study is the proposal to build a ferry terminal in Clifford Bay (South Island) which even though it is currently unlikely to go ahead, allows an interesting hypothetical discussion about what would happen to Picton in case it lost its role as the only ferry gateway to the South Island.

Further knowledge of transport and tourism aspects of the study area will help to better understand passengers' behaviour and choices while at the gateways, as well as the main issues raised by the supply side during the interviews. Some of the key aspects identified after describing the study area were also used to formulate the questions for passengers and business managers/owners.

Secondary sources used in this research included newspapers, magazines, books and reports from tourism and transport organisations. Particularly important was access to the Wellington Central Library (WCL), which holds key publications about maritime transport 
in Wellington and New Zealand. What is more, consultation of Index New Zealand (INNZ) provided access to abstracts from articles published in selected New Zealand newspapers. With the key articles identified, microfilm copies of the newspapers were then obtained at the WCL, the National Library and at Victoria University of Wellington's library. Some tourism and general information about the four CSNZ's macro regions were provided after contact with the various regional tourism organisations (RTOs). Other information about the current ferry operators and the markets they operated was gathered during various informal conversations with managers of both companies.

\subsection{Preparing Data for Analysis}

\subsubsection{Questionnaire Coding and Data Cross Validation}

After data from the questionnaires were collected, parts were coded so the data could be analysed by computer (SPSS). Some of the questions were pre-coded, with the codes shown beside the boxes (e.g. questions 1, 2 and 3). In the case of open-ended questions (e.g. question 31), quite an elaborate procedure was followed to devise a coding system. All answers were then written down and similar ones were gathered in groups. In other cases (e.g. question 41), they were separated by themes so they could be analysed.

In order to make sure that data obtained from the passengers was consistent, some checks were done, before proceeding with the data analysis. Some of these checks were very basic such as making sure that there were no typing mistakes when entering data. This can be easily done with SPSS because if a value was added which had no corresponding label, the incorrect value is shown in the Frequency Descriptive Statistic mode. Some other verification was conducted in order to certify that the information provided by the passenger was consistent. In this case, the tool Data $\rightarrow$ Select Cases was used. Some examples of the latter situation include:

1. Verifying that the areas included in questions 4 and 5 of the questionnaire were also ticked in the respective boxes of question 6 . If either the area of question 4 or 5 matched with the area where the passenger lives (question 7) they were not added in question 6 ;

2. Checking whether passengers that had stayed overnight in Picton or Wellington during their trip (questions 26 and 34) had also ticked boxes 11 or 12 on question 6 ; 
3. When passengers mentioned that $s /$ he lived in Wellington (there are three opportunities for this in questions 7,13 and 25), it was ensured that the other two boxes corresponded to this. In most of the cases, passengers living near Wellington (e.g. Hutt Valley or Kapiti Coast) ended up in one of these three questions saying that $s /$ he lived in Wellington. Some passengers living in Wellington also ticked in question 13 the box corresponding to the mode of transport used to arrive at the ferry terminal rather than selecting the first option ("I live in Wellington"). There were 35 questionnaires in this situation and all of them were considered as passengers living in Wellington. The same type of crossconfirmation was made for passengers living in Picton, although in this case the number of passenger in this situation was less significant.

\subsubsection{Analytical Framework}

The analytical framework of this research encompasses the analysis of data from different sources. In order to join these different sources of information, the approach used was to analyse them separately and later discuss them together.

Secondary data about tourism in the research area, presented in Chapter 4, are analysed in a way to give support to the concepts presented in the literature review, including the nodal functions (origin, destination and gateway) and the travel pattern presented by both domestic and international travellers. The development of the ferries is presented in Chapter 5. Due to the lack of literature about the influence of managerial and operational issues on tourism, Chapter 5 aims to fill in this gap and present the key ferry issues that have influenced tourism in Picton and Wellington. In general, the analytical framework presented in Chapters 4 and 5 aims to provide a link between the concepts and models presented in the literature review (Chapter 2) and the primary data analysis (Chapters 6, 7 and 8 ) in a way to better understand the key tourism and transport topics associated with the study area.

The results of the primary data analysis are presented in three chapters. The main characteristics and patterns of the demand are presented in Chapter 6, providing a general overview of the sample. Basically, the analyses are done separating the sample by travellers' place of origin in a three-fold approach: international travellers, travellers living outside the CSNZ (hereafter to be considered as domestic passengers) and those living in the CSNZ region (hereafter to be considered as CSNZ passengers) (see Table 3.3). According to the literature, it is likely that groups of travellers from different places of origin will present different travel patterns and uses in relation to the gateways. One 
hypothesis, for example, is that passengers living in the CSNZ region are less likely to be interested in staying overnight in the gateways as they live within their catchment area. International travellers, on the other hand, might be more inclined to as their trips to Wellington and Picton to catch the ferry might be seen as an opportunity to visit a new place.

Once general aspects of the demand have been presented (Chapter 6), the analysis then focuses specifically on issues related to the gateways and the travel patterns and activities undertaken in Wellington and Picton by ferry passengers (Chapter 7 ). In order to understand what makes passengers likely to stay longer than planned in the gateways, the sample was subdivided in the four nodal functions presented in Figure 3.2 (gateway travellers, overnight gateway visitors, stopover visitors and destination tourists). For each nodal function, data are presented segmenting the passengers according to their place of origin, i.e. CSNZ, domestic and international. Table 3.4 presents a cross-tabulation for the passengers' place of origin and their nodal functions for Wellington and Picton. The origin function will not be considered in Chapter 7 , as there is no point in trying to persuade residents to stay longer in their own place of origin.

Table 3.4 Cross-tabulation of passengers' place of origin and nodal function.

\begin{tabular}{|c|c|c|c|c|c|c|c|}
\hline & & Origin & $\begin{array}{c}\text { Gateway } \\
\text { Traveller (GT) }\end{array}$ & $\begin{array}{c}\text { Overnight } \\
\text { Jateway Visito } \\
\text { (OGV) }\end{array}$ & $\begin{array}{l}\text { Stopover } \\
\text { Visitor (SV) }\end{array}$ & $\begin{array}{l}\text { Destination } \\
\text { Tourist (DT) }\end{array}$ & Total \\
\hline \multirow{8}{*}{ 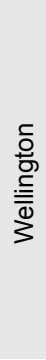 } & \multirow{2}{*}{ CSNZ } & $182(100.0 \%)$ & 39 (13.9\%) & $6(5.1 \%)$ & 21 (9.9\%) & $46(18.3 \%)$ & 294 (28.2\%) \\
\hline & & $17.4 \%$ & $3.7 \%$ & $0.6 \%$ & $2.0 \%$ & $4.4 \%$ & $28.2 \%$ \\
\hline & \multirow{2}{*}{ Domestic } & & 157 (56.1\%) & 41 (34.7\%) & 55 (25.9\%) & $63(25.1 \%)$ & 316 (30.3\%) \\
\hline & & & $15.1 \%$ & $3.9 \%$ & $5.3 \%$ & $6.0 \%$ & $30.3 \%$ \\
\hline & \multirow{2}{*}{ International } & & $84(30.0 \%)$ & $71(60.2 \%)$ & $136(64.2 \%)$ & $142(56.6 \%)$ & 433 (41.5\%) \\
\hline & & & $8.1 \%$ & $6.8 \%$ & $13.0 \%$ & $13.6 \%$ & $41.5 \%$ \\
\hline & \multirow{2}{*}{ Total } & $182(100.0 \%)$ & $280(100.0 \%)$ & $182(100.0 \%)$ & $2(100.0 \%)$ & $182(100.0 \%$ & $1,043^{(*)}(100 \%)$ \\
\hline & & $17.4 \%$ & $26.8 \%$ & $11.3 \%$ & $20.3 \%$ & $24.1 \%$ & $100.0 \%$ \\
\hline \multirow{8}{*}{$\frac{.}{0}$} & \multirow{2}{*}{ CSNZ } & $8(100.0 \%)$ & $210(30.2 \%)$ & $4(3.4 \%)$ & 42 (31.1\%) & 31 (38.3\%) & 295 (28.4\%) \\
\hline & & $0.8 \%$ & $20.3 \%$ & $0.4 \%$ & $4.1 \%$ & $3.0 \%$ & $28.4 \%$ \\
\hline & \multirow{2}{*}{ Domestic } & & $223(32.1 \%)$ & 46 (39.0\%) & 33 (24.4\%) & $13(16.0 \%)$ & 315 (30.4\%) \\
\hline & & & $21.5 \%$ & $4.4 \%$ & $3.2 \%$ & $1.3 \%$ & $30.4 \%$ \\
\hline & \multirow{2}{*}{ International } & & $262(37.7 \%)$ & 68 (57.6\%) & 60 (44.4\%) & 37 (45.7\%) & 427 (41.2\%) \\
\hline & & & $25.3 \%$ & $6.6 \%$ & $5.8 \%$ & $3.6 \%$ & $41.2 \%$ \\
\hline & \multirow{2}{*}{ Total } & $8(100.0 \%)$ & $695(100.0 \%)$ & $118(100.0 \%)$ & 35 (100.0\%) & $81(100.0 \%)$ & $1,037^{(*)}(100 \%)$ \\
\hline & & $0.8 \%$ & $67.0 \%$ & $11.4 \%$ & $13.0 \%$ & $7.8 \%$ & $100.0 \%$ \\
\hline
\end{tabular}

$\left.{ }^{*}\right)$ The total does not sum up 1,076 (total number of questionnaires collected) as relevant information to classify the passenger in terms of his place of origin or nodal function was not filled in. 
A number of interesting characteristics can be identified in Table 3.4. One of them is the nodal function distribution in Wellington and Picton. In Wellington the various nodal functions are reasonably well distributed, ranging from $17.4 \%$ in the case of the origin function to $31.7 \%$ for stopover visitors. These results reflect the importance of Wellington at the national level, especially in relation to the origin and destination functions. The same pattern is not identified in Picton, with two-thirds of passengers $(67 \%)$ being gateway travellers. At the other extreme, only $7.8 \%$ of passengers have Picton as a destination and less than $1 \%$ originated in Picton.

With a distinction between gateway travellers, overnight gateway visitors, stopover visitors and destination tourists accomplished, it is then possible to analyse in Chapter 7 what the key differences between the different travellers are in terms of their behaviour while at the gateways and also to identify which of these groups are more likely to extend their stays in Wellington and Picton.

As stakeholders also have an impact on passengers' decision to stay and visit gateways, data collected from these players are treated separately in Chapter 8. A content analysis approach is taken in the case of the stakeholders' interviews. According to Wilkinson (2004), content analysis is based on examination of the data for recurrent instances that are systematically identified across the data set and grouped together by means of a coding system. Some of these management issues were identified in Chapter 5 and include: the possible interactions and partnerships between transport and tourism companies and organisations, the impact of the fast ferry speed restrictions on tourism, especially in Picton, and the proposal to build another ferry terminal in Clifford Bay. In the case of the Clifford Bay proposal, passengers' reaction to it, in terms of whether they would travel via Picton or Clifford Bay, is also considered.

\subsection{Overall Data Assessment}

Before moving to the data analysis part of this thesis, it is appropriate here to make some comments on the primary data gathered for this thesis.

Some limitations can be found in both the qualitative and quantitative data. In the case of passengers' questionnaire, these are related to the period the survey was conducted (between August and January), which excludes passengers travelling at other times of the year, such as the end of the summer, the Easter holiday time and also the beginning of winter. Another point already mentioned is the fact that no data collection was done during dawn sailings. What is more, the number of questionnaires collected on board the 
Bluebridge ferry is probably below its market share. From Table 3.2, it is possible to count that 82 questionnaires were collected from passengers on this service, which represents $7.6 \%$ of the sample. However, according to non-official data, Bluebridge has $15 \%$ of the Cook Strait market share. Passengers travelling on Sundays, which comprises weekenders and the short break markets, are also underrepresented as according to Figure $3.3 \mathrm{~b}$ only $2.9 \%$ of the questionnaires were collected on Sundays.

In spite of all these considerations, it does not seem that they have greatly affected data reliability, especially when comparing data from this research and the Ka Pai survey (e.g. passengers' place of origin - see Table 3.3). On the other hand, the passenger sample size is sufficiently large (1,076 questionnaires) to allow breakdowns in terms of passengers' nodal functions and places of origin.

For the stakeholders, the main problem was to identify tourism businesses in Wellington that were directly related to the ferry operation. This was not a problem in the case of Picton, a small town whose economy relies on the ferry business. In addition, some important stakeholders declined participation in the research. In a few cases gaining access to the targeted manager was not possible (Thomas, 1993). In these situations interviews were either arranged with other people within the organisation or no interview was possible because there was no one else prepared to talk about the issues related to this research (e.g. Tranz Scenic).

Although the number of semi-structured interviews could have been larger than twenty, especially if funds were available for more trips to be made and also to hire other interviewers and transcribers, it became obvious after a number of interviews, that topics and issues already raised by previous interviewees started to be repeated by other managers and business owners. This can suggest that the major aspects of the Cook Strait tourism transport businesses have been covered during the interviews.

One of the major qualities of data collected for this research is that responses obtained from passengers and managers/owners complement each other. The advantages of the methodology structured for this research are twofold. Firstly, in some cases, the data collected gave the opportunity to consider certain topics taking into consideration the suppliers' and the demand's different perspectives. This is what happened, for example, when they were asked what needs to be done in order to make ferry passengers stay longer in Wellington and Picton, or what would be the impact on Picton if the Clifford Bay proposal went ahead. Understanding what the travellers want and what they would do in these circumstances and comparing it with what the suppliers think that the demand 
wants gives the opportunity to assess whether or not managers/owners have a good understanding of their actual/potential clients. Secondly, data obtained from these two major players also allow complementing information in cases that collecting information from one particular player would be too complicated. This is what happened, for example, when the suppliers explain the different travel patterns of domestic and international travellers. While this was done partially in the questionnaire (e.g. asking the number of nights they have spent in different regions of the country), from the semi-structured interviews it was possible to understand the cases of passengers that visit the nodes two or even three times during their trips.

\subsection{Conclusion}

This chapter has presented the methodology used in this research. As discussed at the beginning of the chapter, a multiple method approach is necessary in order to deal with all theoretical aspects of the research problem.

Perhaps the most challenging issue was the operationalisation of the concept of gateway as it gathers all the different aspects considered in the literature review - Chapter 2 - in order to create a framework to structure the data collection design. The questionnaire designed included all the elements presented for the concept of gateway, in order to collect data about passengers' behaviour while in Wellington and Picton (purpose of trip and length of stay dimensions) or about the rest of their trips (transport intermodality and catchment area dimensions). The understanding of the travellers' trip, as presented in the models of tourist flow, was a prerequisite to better comprehend how easy or difficult would be for passengers to extend their stay in the gateways, as this would impact on the rest of their trips. Other issues relevant for this research, which were also included in the questionnaire, are the passengers' socio-demographic characteristics, their ferry journeys and the proposal to build a new ferry terminal in Clifford Bay.

Collecting data from ferry passengers was crucial for this research as they are the ones who make the ultimate decision to stop and visit the gateways. However, in order to complement the data collected from the passengers, qualitative semi-structured interviews were used to gather information from the suppliers. There were eleven basic questions whose topics included the importance of Cook Strait ferries, the interaction between transport and tourism providers, comparison between the two ferry operators, impacts of the Tory Channel speed restriction on tourism in Picton and the proposal to build a ferry terminal in Clifford Bay. Understanding the relationship between transport and tourism 
firms/organisations and also how managerial and operational decisions impact on travellers can create potential avenues to develop way to facilitate and enhance the stay of ferry passengers in the gateways.

Figure 3.4 summarises the primary data collection process, the target samples and the main data collected for this research. It also stresses the different sorts of data required leading to the use of different data collection methods. During the data collection process it was possible to gather a total of 1,076 questionnaires during 35 ferry trips across Cook Strait. From the supply, 20 semi-structured interviews were conducted. Apart from the two ferry operators, the other stakeholders included a range of accommodation providers in Wellington and Picton; other transport companies such as one regional airline and rental car businesses; government and support organisations; tourism operators and attractions.

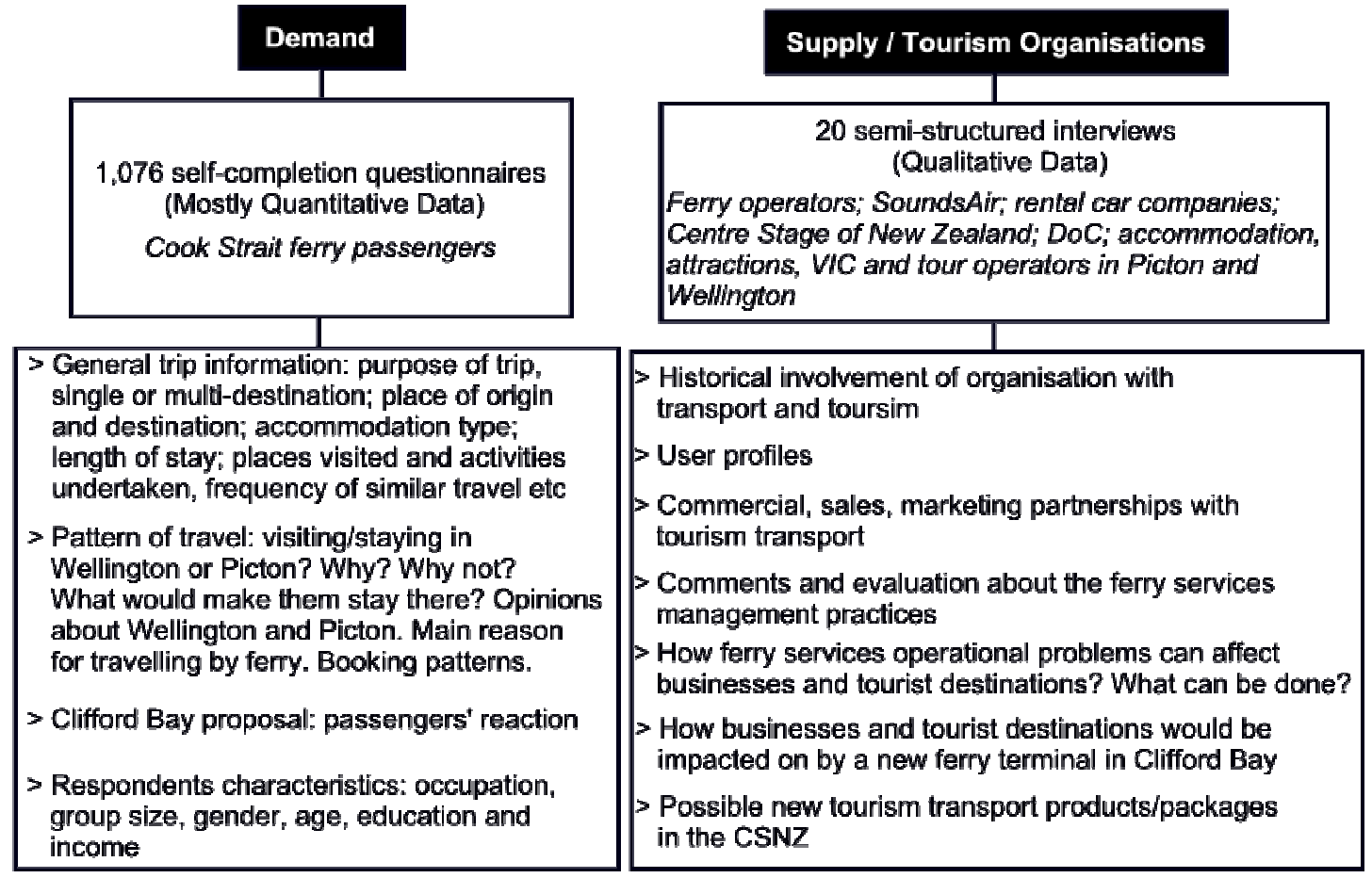

Figure 3.4 Primary data collection methodology and target samples.

Secondary data were also gathered in order to provide a better understanding of the key nodal functions existing in Wellington and Picton (Chapter 4) and of the Cook Strait ferry businesses (Chapter 5). They were particularly useful in understanding the background of the study area, so that the questions developed either in the questionnaires and interviews could take into consideration current key issues associated with the Cook Strait ferry market. The quality of the data obtained from secondary sources permitted, for 
example, to bring into the discussion with the providers the recent tourism development that Picton and Wellington have been passing through and also to consider possible future scenarios, such as the Clifford Bay proposal.

To sum up, this chapter can be seen as a link between the concepts presented in Chapter 2 and the data analysis done, either from secondary data (Chapters 4 and 5) or from primary sources (Chapters 6, 7 and 8 ). In the case of Chapter 7, for example, the operationalisation of the concepts developed in Figure 3.2 was used as the basic framework to analyse the passengers' decision to extend or shorter their stays in the gateways. 


\section{Nodal Functions in the Study Area}

\subsection{Introduction}

This chapter aims to describe the key nodal functions associated with the study area. To this end, it is divided into four main parts. The first focuses on tourism on the national scale. It starts by analysing the domestic and international markets in New Zealand and the main traveller patterns. It is particularly relevant to analyse the circuit travel usually undertaken by international tourists as well the degree of mobility amongst domestic tourists. In many of the more peripheral destinations, domestic tourists are virtually the mainstay of the tourism industry, as few international visitors venture off the major tourist circuits (Oppermann \& Cooper, 1999). These two facts, associated with the geography of the country, i.e. the North-South Islands, are extremely important to understand the role of the ferries and the gateways of Wellington and Picton. The second part of this chapter concentrates on the Centre Stage region of New Zealand, and the four macro-regions are presented, i.e. Wellington, Wairarapa, Nelson and Marlborough. They are the catchment areas of the gateways. This helps to contextualise the last two sections, which deal specifically with the analysis of the key nodal functions existing in Wellington (origin, gateway and destination) and Picton (gateway and destination).

Although most of the information provided in this chapter was obtained from the literature, the description of tourism in the study area also benefited from field trips conducted by the researcher.

\subsection{Tourism in New Zealand}

New Zealand's tourism potential has been recognised since the first European arrivals (early to mid-1850s), and this led to the development of the first government-sponsored national tourism office in the world, in 1901 (McClure, 2004). From its early beginnings with explorers and mountaineers, and some package tours, New Zealand tourism entered the modern era with the arrival of jet engined aircraft in the late 1950s and the subsequent breaking of distance barriers to major markets. Growth in international tourist demand has averaged nine per cent over much of the past two decades, ahead of both Pacific regional and world tourism trends (Simmons, 2000).

Major tourism products in New Zealand include nature, adventure tourism and cultural experiences. The country provides tourists with a wide choice of activities, from the sedate to the adventurous. A feature of the New Zealand tourism industry is the way it caters for 


\section{NOdAl Functions IN THE StUdy AREA}

all budgets, with accommodation ranging from world-class hotels to motels, camping grounds and a network of backpacker hostels.

\subsubsection{International Tourism in New Zealand}

In 2003, New Zealand received 2,104,420 international visitors (TRCNZ, 2004a). While trans-Tasman arrivals accounted for $33 \%$ of New Zealand's inbound tourist market (702,162 visitors), a key trend in the growth of tourism to New Zealand, in recent years, has been the diversification of the market. Australia has always been New Zealand's primary source of overseas visitors but the country is less dependent on this market today than it was 25 years ago, when it was responsible for $60 \%$ of international arrivals (Collier, 2003). Nowadays, seven different countries account for $67 \%$ of the international market. They are Australia, UK, USA, Japan, South Korea, China and Germany.

The length of stay of international visitors has changed over in the last few years. It jumped from an average of 18 days in 1998 to 26 days in 2003 (TRCNZ, 2004b). Figure 4.1 shows that the percentage of short stays (less than 10 days) has decreased in the last few years, while the percentage of longer stays (more than 20 days) increased. This can possibly be attributed to the fact that New Zealand is attracting more long haul travellers, including international students that tend to stay longer in the country. For example, in 2003, while Australians stayed on average thirteen days in New Zealand, travellers from the UK stayed 33 days; and residents from South Korea 44 days. Despite that, half of international visitors stayed less than 10 days in the country, with the most frequent length of stay being between five and seven days (almost 20\%). Pearce (1990a: p.38) argues that short staying visitors 'opt for the most accessible sites and what are perceived to be the main tourist attractions. Those on longer visits take in other secondary and small centres and attractions'.

There are several characteristics of international visitors that are relevant to this research. They are the average length of stay, the way visitors travel (e.g. fully independent travellers - FIT and semi-independent travellers - SIT), the top destinations visited, among others. The main variables will be considered with regard to the seven most important inbound markets ${ }^{8}$. Visitors from Australia exhibit many characteristics that are different from New Zealand's other main visitor markets. For example, they spend less money (on average), stay less time and have higher participation rates for VFR than most other nations. In many ways, this is a function of the relative proximity of Australia to New

\footnotetext{
${ }^{8}$ In the analysis of these markets, when no period of time or date is mentioned, data refer to the year to December 2003.
} 
Zealand, with Australian visitors treating a trip to New Zealand as a short break destination, rather than a major overseas holiday. Furthermore, only $12.6 \%$ of Australian visitors use the ferries while travelling in New Zealand.

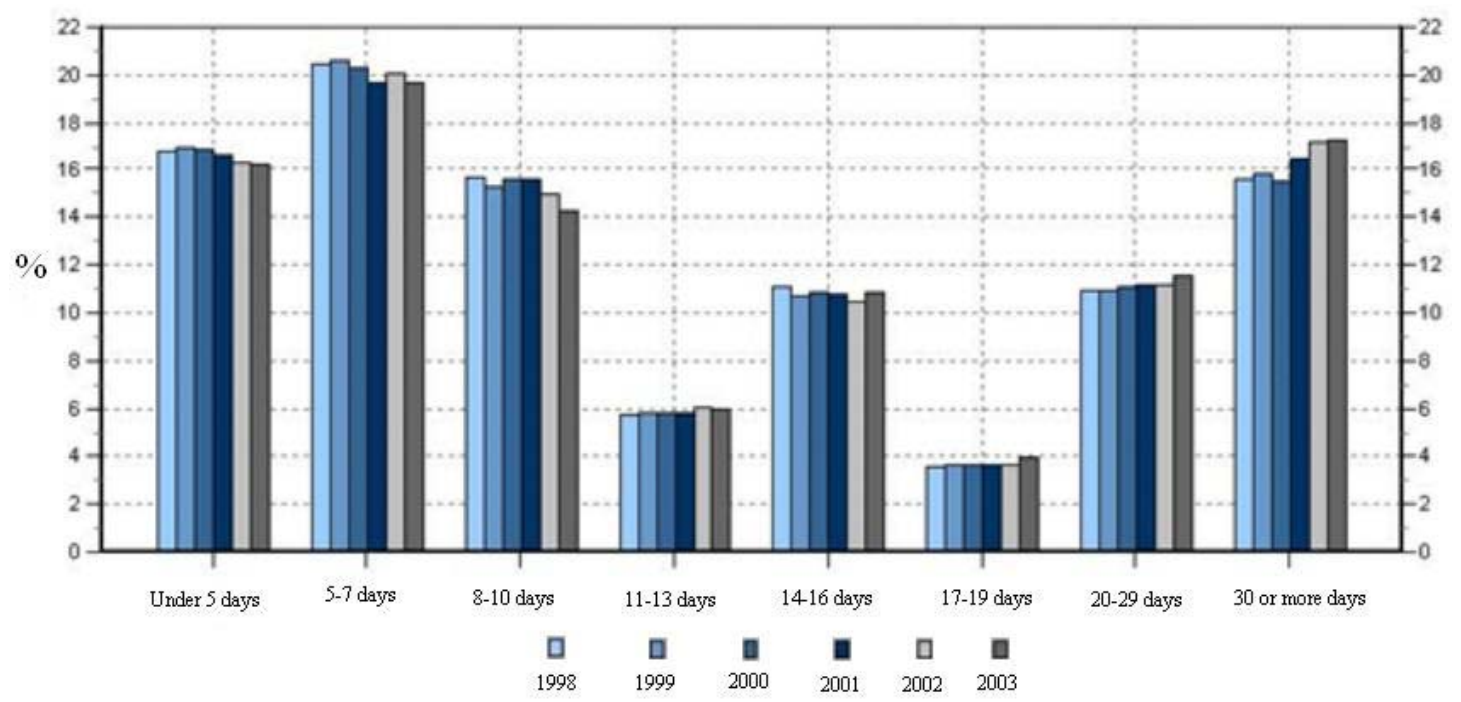

(Source: TRCNZ, 2004a)

Figure 4.1 International visitors' intended length of stay.

With few exceptions, the other major international markets to New Zealand present some common trends. The average visitor expenditure and the average length of stay of international visitors are higher than the total average compared to domestic. The only exception is the Americans who stay 18 days on average (against 26 days of the total average), which ends up impacting on the average visitor expenditure (NZ\$3,257 against $\mathrm{NZ} \$ 3,344$ - see Table 4.1). The longer length of stay is a common pattern amongst long haul visitors. Particularly among the Asian markets, notably the Chinese visitors, the results from Table 4.1 are influenced by the fact that the education market has been included in the data provided there. This has a significant influence in some of the information presented in Table 4.1, such as the average length of stay and average visitor expenditure.

The main reason for visiting New Zealand is another characteristic with similar pattern among the top international markets. Except in the case of China, where $35 \%$ of visitors come for business / conference, holidays are the main reason for international visitors travelling to New Zealand, ranging from $20.6 \%$ in the case of China to $76 \%$ for the German market (see Table 4.1).

Finally, in terms of the use these markets make of the different modes of transport while travelling in New Zealand, it is possible to identify similarities with the way visitors travel: 
i.e. tour groups, package travellers, FIT and SIT. The higher share of FITs and SITs, such as in the case of travellers from Australia, UK and Germany, is associated with a higher use of rental cars. Domestic air is the main mode of transport for American and Japanese visitors - usually on shorter stays - while Chinese and South Korean travellers prefer the coach tour. With the exception of the Australian market, which has the shortest length of stay and does not present a touring pattern around the country (see Figure 4.2), the participation of international travellers using the ferry is very much linked to the way the various markets travel. In the case of German travellers, $42.6 \%$ of them use the ferries, which is in fact the second most used mode of transport. For travellers from the UK, the ferries are the third most important mode of transport, with three out of ten making use of them. International markets that make less use of the ferries include those from Asia, such as Japan, South Korea and China. Only $1 \%$ of Chinese visitors use the ferries while in New Zealand.

Apart from the variables previously presented, it is also relevant to consider some of the characteristics of international visitors in relation to their pattern of travel while in New Zealand. In this regard, it is appropriate to mention that New Zealand is marketed internationally as a touring destination and most trips are multi-destination (Pearce, 2001b). The country's geography, narrow and long - divided into two islands, and the location of the main international gateways, Auckland and Christchurch, means that international tourists tend to engage in circuit travel. They usually enter and leave through the same gateway or arrive in Auckland and depart from Christchurch or vice-versa. Auckland is still the gateway for more than half of international visitor arrivals (Warren \& Taylor, 2003) and, because of this, tourist flow is usually Southbound, even though some travellers also fly back to Auckland in order to leave the country.

Various factors can influence the mobility and circuit travel pattern presented by international travellers whilst in New Zealand, such as the purpose of trip, length of stay and country of residence. For example, Pearce (1995) states that international holidaymakers in New Zealand stayed overnight, on average, in almost seven places each, twice the number of the VFR market, while the mean for business visitors was just two. The greater mobility recorded for holidaymakers reflects a long-standing pattern of sightseeing tourism. Although related to data collected more than a decade ago, meaning that some of the new trends and markets are not considered, Figure 4.2 shows the intraregional flow of tourists for five different international markets. 
Table 4.1 Characteristics of the major international markets to New Zealand - Year Ended 2003.

\begin{tabular}{|c|c|c|c|c|c|c|c|}
\hline & $\begin{array}{l}\text { Number of } \\
\text { visitors }\end{array}$ & $\begin{array}{c}\text { Average visitor } \\
\text { expenditure (NZ\$) }\end{array}$ & $\begin{array}{c}\text { Average length } \\
\text { of stay }^{(*)}\end{array}$ & $\begin{array}{l}\text { Way of } \\
\text { travelling }\end{array}$ & $\begin{array}{l}\text { Top destinations (average } \\
\text { number of nights spent) }\end{array}$ & $\begin{array}{l}\text { Main reason for visiting NZ } \\
\text { (\% of visitors) }\end{array}$ & $\begin{array}{l}\text { Modes of transport used (top } 3+ \\
\text { the ferries - } \% \text { of users) }\end{array}$ \\
\hline Australia & 702,162 & $1,837.90$ & 13.3 days & $\begin{array}{l}90 \% \text { either } \\
\text { FIT or SIT }\end{array}$ & $\begin{array}{l}\text { Auckland (5.1); Wellington (5.0); } \\
\text { Christchurch (2.6); Queenstown (3.3) }\end{array}$ & $\begin{array}{l}\text { Holiday (37.7); VFR (30.3); } \\
\text { Business/Conference (23.7) }\end{array}$ & $\begin{array}{l}\text { Rental cars (30.4); private cars (25.1); } \\
\quad \text { domestic air (20.9); ferries (12.6) }\end{array}$ \\
\hline UK & 264,819 & $3,809.00$ & 33 days & $\begin{array}{l}94 \% \text { either } \\
\text { FIT or SIT }\end{array}$ & $\begin{array}{c}\text { Auckland (8); Wellington (5); } \\
\text { Christchurch (6); Queenstown (4) }\end{array}$ & Holiday (60.4); VFR (27.8) & $\begin{array}{c}\text { Rental cars (37.4); private cars (31.4); } \\
\text { ferries (30.7) }\end{array}$ \\
\hline USA & 211,624 & $3,257.00$ & 18 days & $\begin{array}{l}78 \% \text { either } \\
\text { FIT or SIT }\end{array}$ & $\begin{array}{c}\text { Auckland (4); Wellington (6); } \\
\text { Christchurch (4); Queenstown (3) }\end{array}$ & $\begin{array}{c}\text { Holiday (62.3); } \\
\text { Business/Conference (14.2); } \\
\text { VFR (13.1) }\end{array}$ & $\begin{array}{l}\text { Domestic air (39.7); rental car (32.8); } \\
\quad \text { coach tour (25.7); ferries (17.1) }\end{array}$ \\
\hline Japan & 150,851 & $4,200.00$ & 31 days & $\begin{array}{l}\text { 70.5\% either } \\
\text { FIT or SIT }\end{array}$ & $\begin{array}{l}\text { Auckland (13); Wellington (19); } \\
\text { Christchurch (7); Queenstown (3) }\end{array}$ & $\begin{array}{c}\text { Holiday (57.7); Education } \\
\text { (14.8); VFR (12.6); } \\
\text { Business/Conference (11.7) } \\
\end{array}$ & $\begin{array}{l}\text { Domestic air (56.9); coach tour (36.7); } \\
\text { private cars (16.5); ferries (9.4) }\end{array}$ \\
\hline South Korea & 112,658 & $6,243.00$ & 44 days & $\begin{array}{l}73.9 \% \text { either } \\
\text { FIT or SIT }\end{array}$ & $\begin{array}{l}\text { Auckland (22); Wellington (4); } \\
\text { Christchurch (14); Queenstown (6) }\end{array}$ & $\begin{array}{l}\text { Holiday (57.9); VFR (22.2); } \\
\text { Education (13.0) }\end{array}$ & $\begin{array}{l}\text { Coach tour (42.3); domestic air (33.8); } \\
\text { private cars (21.3); ferries (9.2) }\end{array}$ \\
\hline China & 65,989 & $6,720.00$ & 57 days & $\begin{array}{l}71.3 \% \text { either } \\
\text { FIT or SIT }\end{array}$ & $\begin{array}{l}\text { Auckland (36); Wellington (9); } \\
\text { Christchurch (15); Queenstown (2) }\end{array}$ & $\begin{array}{c}\text { Business/Conference (35.0); } \\
\text { Holiday (20.6); VFR (18.4); } \\
\text { Education (14.6) }\end{array}$ & $\begin{array}{l}\text { Coach tour (36.6); private cars (26.1); } \\
\text { domestic air (25.3); ferries (1.0) }\end{array}$ \\
\hline Germany & 52,534 & $4,421.00$ & 32 days & $\begin{array}{l}\text { 92.3\% either } \\
\text { FIT or SIT }\end{array}$ & $\begin{array}{l}\text { Auckland (4); Wellington (4); } \\
\text { Christchurch (3); Queenstown (2) }\end{array}$ & $\begin{array}{c}\text { Holiday (76.0); } \\
\text { Business/Conference (7.9); } \\
\text { VFR (7.2) } \\
\end{array}$ & $\begin{array}{l}\text { Rental car (47.3); ferries (42.6); } \\
\text { domestic air (24.4) }\end{array}$ \\
\hline Total & $2,104,420$ & $3,344.00$ & 26 days & $\begin{array}{l}87.1 \% \text { either } \\
\text { FIT or SIT }\end{array}$ & $\begin{array}{l}\text { Auckland (10); Wellington (6); } \\
\text { Christchurch (6); Queenstown (3) }\end{array}$ & $\begin{array}{c}\text { Holiday (47); VFR (18); } \\
\text { Business/Conference (13); } \\
\text { Education (13) }\end{array}$ & $\begin{array}{l}\text { Domestic air (36.8); rental cars }(35.1) \text {; } \\
\text { private cars }(29.8) \text {; ferries }(21.4)\end{array}$ \\
\hline
\end{tabular}

(Source: TRCNZ, 2004b)

\section{Key:}

FIT: Fully Independent Traveller SIT: Semi Independent Traveller

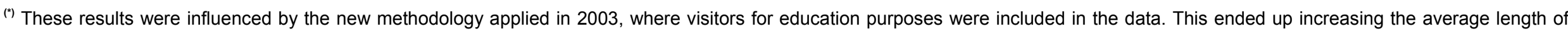
stay for some of the Asian markets, particularly in Auckland, Christchurch and Wellington where there is a concentration of education providers. 

As can be noted, in most of these cases flows from and between the main international gateways of Auckland, Wellington and Christchurch are outstanding, with the intensity of dispersal beyond these nodes varying considerably from country to country. When comparing the Japanese and German markets it is possible to identify that while the former is heavily focused on four key centres, i.e. Auckland, Rotorua, Christchurch and Queenstown (Pearce, 2001b), the latter presents a more complex structure, with flows showing far greater dispersal to the remote regions of New Zealand, such as Northland and the West Coast of the South Island. From the visual presentation offered by Figure 4.2 , it is possible to assume that most of the Japanese inter-island travel is undertaken by air, especially between Auckland-Christchurch and Auckland-Queenstown. However, for the German market, a convergent influx of travellers to and from Wellington can be identified. This can explain the high use of the ferries (43\%) obtained in the International Visitor Survey (IVS) data presented in Table 4.1. This pattern is repeated, to a lesser extent, by British travellers, in which nearly one in three visitors used the ferries to travel between the Islands. Australians and Americans exhibit an intermediate pattern of travel behaviour (Pearce, 1995). In all these cases, such differences reflect, in a great part, variations in length of stay, as has already been mentioned.

From this analysis it is possible to achieve a two-fold conclusion about the likelihood of travellers extending their stays in Wellington and Picton. Firstly, the different travel patterns of the various international markets suggest that some of them are more likely to travel through the ferry gateways. Those that present a more dispersed travel pattern around the country (e.g. Germany and UK) are usually on longer trips. The German and British markets also include a higher proportion of visitors travelling either as FIT or SIT (see Table 4.1). Secondly, considering the dispersion of these specific markets throughout the country, it is reasonable to assume that persuading these travellers to stay longer in Wellington and Picton will only happen at the expense of them spending less time in other destinations. Therefore, the appeal to attract ferry passengers has to be sufficient to convince them that is worthwhile taking advantage of the trip to the gateways to stop and visit them. 

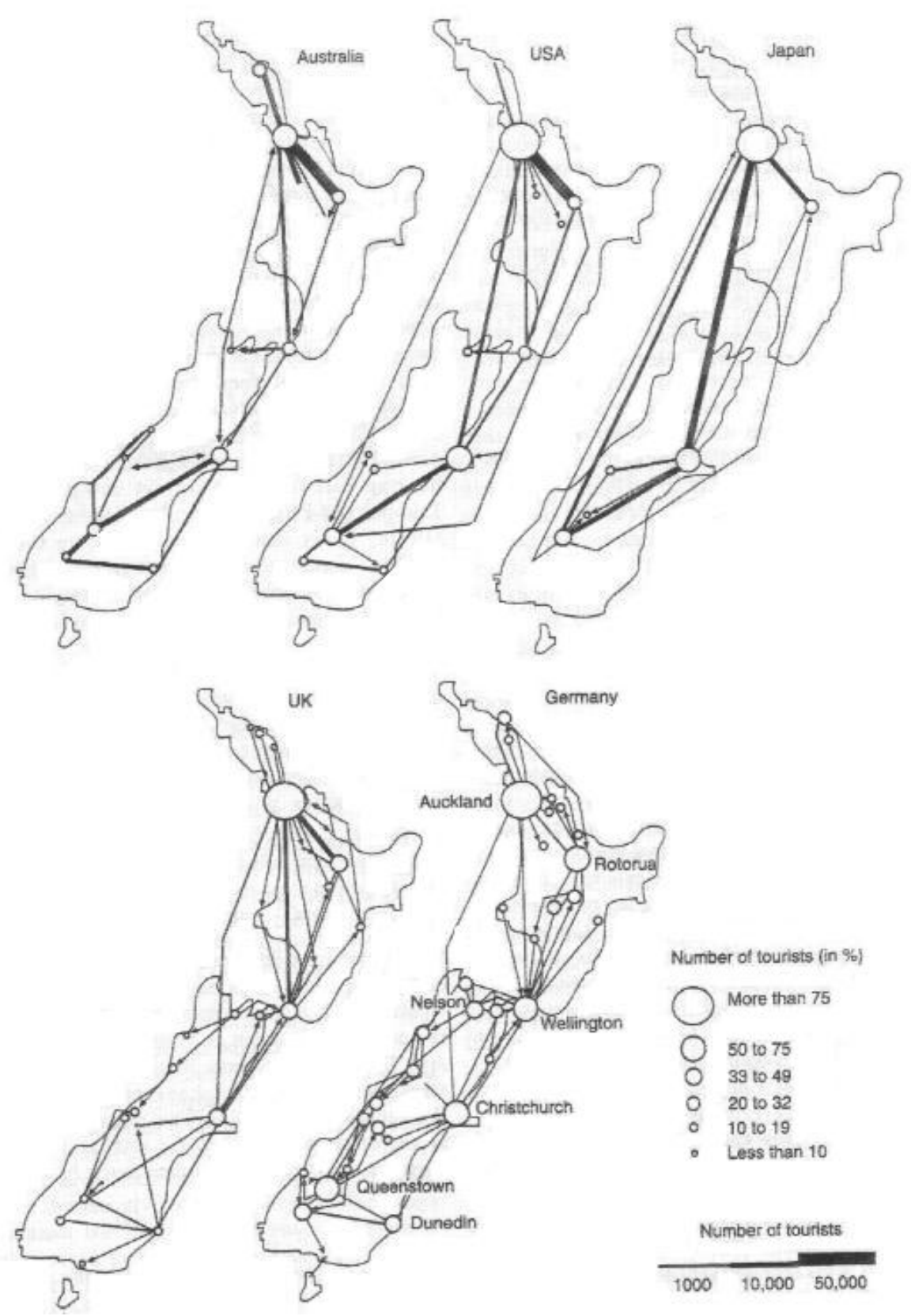

Number of tourists (in \%)

More than 75

50 to 75

O 39 to 48

- 20 to 32

- 10 to 19

- Less than 10

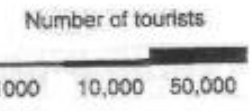

(Source: Pearce, 1995)

Figure 4.2 Intra-national flows in New Zealand by country of residence - 1991.

\subsubsection{Domestic Travellers in New Zealand}

Although international tourism remains the highly visible side of the tourism industry, and in terms of expenditure per visitor per day is more valuable to New Zealand than domestic tourists, domestic tourism is in fact the mainstay of the industry and earned an estimated 
$\$ 9.1$ billion for the year ending March 2003 (Statistics New Zealand, 2004). This represents $55 \%$ of the total tourism expenditure ( $\$ 16.5$ billion), with the remaining $45 \%$ attributed to international tourists. In $2001,37 \%$ of the domestic expenditure was credited to people on day trips, with tourists (overnight travellers) representing the remaining $67 \%$ (Statistics New Zealand, 2003). In addition, without a viable domestic market, it is doubtful whether the infrastructure and facilities required to support international tourism would be available in all regions of the country.

In the period from 1982 to 2001, the total number of domestic overnight trips increased from 13.4 million to 16.6 million. In spite of this growth, the actual number of visitor nights spent by domestic tourists decreased from 54.5 million to 50.3 million (TRCNZ, 2002). This decline in person nights was due to a decrease in the average length of stay from 4.1 days, in 1982, to 3.1 days, in 2001. According to Hall and Kearsley (2001), although traditional holidays and long weekends have been prominent, holiday patterns are changing and short breaks, frequently associated with sporting events, are on the increase.

In terms of domestic tourism on a regional scale, it is relevant to consider the number of tourists generated and received in each region. Pearce (1995) presents some data related to the distribution of domestic tourism in New Zealand at the end of the 1980s. As can be seen from Figure 4.3, Auckland, Bay of Plenty, Waikato, Wellington and Canterbury were the top five regions regarding number of bed-nights. This figure also presents the number of bed-nights received and generated by each region. It is interesting to note that Auckland, Wellington and Coastal North Otago had a significant negative balance, with these three regions being a common place of origin for tourists in New Zealand. Partially, this can be explained by the large population that lives in the three urban areas of the country. According to Pearce (1995: p.68) "the pattern of bed-nights spent in each region is slightly more dispersed than for bed-nights generated". However, despite their net deficit, Auckland and Wellington are still the leading regions for the absolute number of bed-nights spent there. More updated data related to tourist expenditure shows, however, that Auckland, Waikato and Canterbury are the top three regions in terms of domestic tourism. According to Table 4.2, these three regions are responsible for almost $46 \%$ of all the domestic expenditure generated in the country. The same table shows an interesting aspect of Auckland and Otago regions because both are the only regions in the country to have more tourism expenditure from international than domestic visitors. All other regions have more than half of their total expenditure from the domestic market (see column '\% of domestic'). 


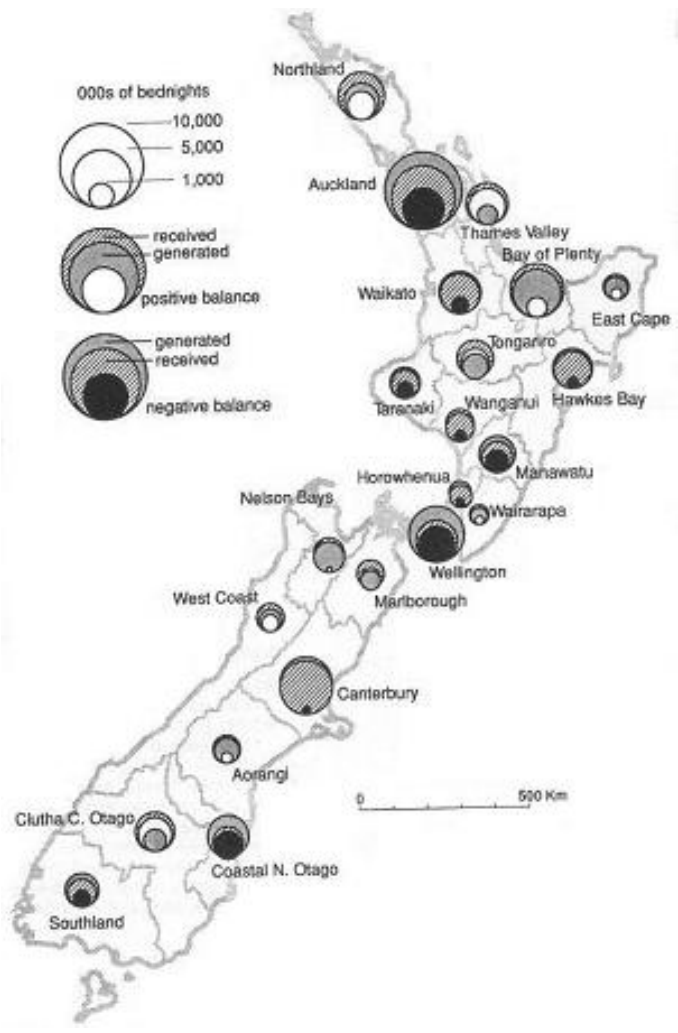

(Source: Pearce, 1995)

Figure 4.3 Distribution of domestic tourism in New Zealand in regional scale - three year average 1987/88 - 1989/90.

Table 4.2 Regional distribution of international and domestic tourism expenditure (1998).

\begin{tabular}{|c|c|c|c|c|c|c|c|}
\hline & \multicolumn{2}{|c|}{ International } & \multicolumn{2}{|c|}{ Domestic } & \multicolumn{2}{|c|}{ Total } & \multirow{2}{*}{$\%$ domestic } \\
\hline & $\$ m$ & $\%$ & $\$ m$ & $\%$ & $\$ m$ & $\%$ & \\
\hline Northland & 103.2 & 3.4 & 394 & 8.4 & 497.2 & 6.4 & 79.2 \\
\hline Auckland & $1,073.1$ & 35.0 & 709.9 & 15.1 & $1,783.1$ & 23.0 & 39.8 \\
\hline Waikato & 187 & 6.1 & 623.2 & 13.3 & 819.2 & 10.5 & 76.1 \\
\hline Bay of Plenty & 175.1 & 5.7 & 337.6 & 7.2 & 512.7 & 6.6 & 65.8 \\
\hline Gisborne & 17.6 & 0.6 & 116.6 & 2.5 & 134.1 & 1.7 & 87.0 \\
\hline Taranaki & 24.8 & 0.8 & 105.7 & 2.3 & 130.5 & 1.7 & 81.0 \\
\hline Manawatu-Wanganui & 75.6 & 2.5 & 326 & 6.9 & 401.6 & 5.2 & 81.2 \\
\hline Hawke's Bay & 45.6 & 1.5 & 190.9 & 4.1 & 236.5 & 3.0 & 80.7 \\
\hline Wellington & 176.4 & 5.8 & 339.6 & 7.2 & 516 & 6.6 & 65.8 \\
\hline Marlborough & 34.5 & 1.1 & 96.2 & 2.1 & 130.7 & 1.7 & 73.6 \\
\hline Nelson & 61.2 & 2.0 & 151.7 & 3.2 & 212.9 & 2.7 & 71.3 \\
\hline Tasman & 14.7 & 0.5 & 36.3 & 0.8 & 51 & 0.7 & 71.2 \\
\hline West Coast & 86.1 & 2.8 & 110.7 & 2.4 & 196.9 & 2.5 & 56.2 \\
\hline Canterbury & 516.1 & 16.8 & 818.8 & 17.5 & $1,334.8$ & 17.2 & 61.3 \\
\hline Otago & 390.6 & 12.7 & 216.2 & 4.6 & 606.7 & 7.8 & 35.6 \\
\hline Southland & 84.8 & 2.8 & 118.8 & 2.5 & 203.6 & 2.6 & 58.3 \\
\hline New Zealand & $3,066.4$ & 100.0 & $4,692.2$ & 100.0 & $7,767.5$ & 100.0 & 60.4 \\
\hline
\end{tabular}

(Source: TIA apud Pearce, 2001c) 
In comparison to international tourism, there has been little research on the structure of domestic tourism in New Zealand. Various reasons can be attributed to this, including the costs to conduct large-scale household surveys as there is no other way to record travel across regional boundaries (Pearce, 1993). The Domestic Travel Survey (DTS) conducted in 2001 (TRCNZ, 2002) interviewed 12,055 New Zealand residents aged 15+ years. Data from the DTS are presented at a regional scale of the 15 Regional Council boundaries. Table 4.3 shows the 15 -by-15 origin-destination matrix for inter-regional overnight trips. Although an analysis of the whole matrix is beyond the scope of this research, it is appropriate at least to consider the inter-island flow of tourists, even though the ferries and domestic airlines share this traffic.

According to Table 4.3, from the total number of 16,563,000 inter-regional overnight trips taken by New Zealand residents in 2001, 1,653,000 were done between the North and South Islands, which represents nearly $10 \%$ of all trips. Traffic originating from the North Island accounted for $6.10 \%$ of all trips, with the remaining $3.88 \%$ originating in the South Island. The top four inter-island regional flows are Auckland $\rightarrow$ Canterbury, Wellington $\rightarrow$ Canterbury, Canterbury $\rightarrow$ Wellington and Canterbury $\rightarrow$ Auckland, which represent the inter-island traffic between the three largest cities in the country. On a secondary scale there are other pairs of links, such as Auckland $\rightarrow$ Otago, Wellington $\rightarrow$ Marlborough and Wellington $\rightarrow$ Nelson/Tasman, the latter two falling within the CSNZ boundaries.

Although using previous data (three year average 1987/88-1989/90) with different regional boundaries, Figure 4.3 illustrates the major domestic travel flows in New Zealand (Figure $4.3 \mathrm{a}$ ) and also the dominant destination and origin flows (respectively Figure 4.3b and Figure $4.3 \mathrm{c}$ ). From Figure 4.3 it is possible to compare the inter-island's regional traffic with those within the North and South Islands.

\subsection{The Hinterland of the Ferry Gateways: the Centre Stage of New Zealand Region}

As mentioned in Chapter 2, the gateways' privileged position usually influences the hinterlands from which they are likely to attract passengers or freight. In the case of the Wellington and Picton, the Centre Stage of New Zealand (hereafter mentioned as CSNZ) Region was chosen mainly due its location around the two ferry ports and due to the fact that the CSNZ brand was created especially to promote tourism in the central part of the country. Another important reason for defining the hinterland for this case study is related 


\section{NOdAL FunCtiONS IN THE STUdY AREA}

to the analytical framework that among other considerations aims to analyse passengers' travel pattern in terms of their place of origin. Using the CSNZ region as the catchment area permits the domestic traveller sample to be split into those living inside and outside it.

In New Zealand, a recent trend in the activities of RTOs has been the creation of macroregions for marketing purposes in co-operation with tourism operators. In 1998, in order to develop and promote internationally the central part of the country, which includes Wellington, Wairarapa, Marlborough and Nelson, a working group named 'Centre Stage of New Zealand' was formed, being a joint venture between a range of tourism organisations including Tourism Wellington, Latitude Nelson, Destination Marlborough, Go Wairarapa plus Tranz Rail, Te Papa and Wellington International Airport (Collier, 2003). The main theme related to the CSNZ Region is culture and heritage.

This section briefly describes each of the four macroregions that have their own unique features. Although the role of the two gateways will be contextualised in the description of these regions, due to their importance to this research, the key nodal functions associated with Wellington and Picton will be described in separate sections.

The Wellington Region is located on the southwestern tip of the North Island. It encompasses four cities (Wellington, Lower Hutt, Upper Hutt and Porirua) and the rural districts of Tararua and the Kapiti Coast. The region offers all the facilities and advantages of a vibrant city, yet it is very easy to escape to some remote areas of coastline, bush and mountain terrain. This region has a usual resident population of 445,400 people (June 2002), accounting for around $11 \%$ of New Zealand's population (Greater Wellington Regional Council, 2003). Urban Wellington is the regional focus, service centre and transport hub (WRC, 2002). Wellington's GDP is $\$ 14.4 \mathrm{bn}$ or $13 \%$ of national economic activity, with the regional economy based on government services, manufacturing, commerce and some tourism. Regional Wellington is an established tourism destination whose strengths lie in its position as a major transport hub (the ferries and the busiest domestic airport in the country), its attractions and its tourism infrastructure. The purpose of visit for travellers to Wellington is a lot more diverse than in some other New Zealand regions. Holidaying in Wellington is still popular amongst international visitors, as is VFR. However for domestic visitors, business travel is also one of the main purposes of travel. This is understandable due to Wellington's position as the seat of Government and a major centre of commerce. Popular attractions and activities in the Wellington Region are largely city based and include museum/gallery, business/conference and eating out (restaurants and cafés). 
Table 4.3 Inter-Regional Overnight Trips 2001.

\begin{tabular}{|c|c|c|c|c|c|c|c|c|c|c|c|c|c|c|c|c|c|}
\hline \multicolumn{17}{|c|}{ Taole $4.3 \mathrm{In}$} & \multirow[b]{3}{*}{ Total } \\
\hline & \multirow{2}{*}{$\begin{array}{l}\text { (000s) } \\
\text { From: To: }\end{array}$} & \multicolumn{9}{|c|}{ North Island (NI) } & \multicolumn{6}{|c|}{ South Island (SI) } & \\
\hline & & $\begin{array}{l}\text { North- } \\
\text { land }\end{array}$ & Auckland & Waikato & $\begin{array}{l}\text { Bay of } \\
\text { Plenty }\end{array}$ & Gisborne & $\begin{array}{c}\text { Hawkes } \\
\text { Bay }\end{array}$ & Taranaki & $\begin{array}{l}\text { Manawatu- } \\
\text { Wanganui }\end{array}$ & Wellington & $\begin{array}{c}\text { Marl- } \\
\text { borough }\end{array}$ & $\begin{array}{c}\text { Nelson } \\
\text { Tasman }\end{array}$ & Canterbury & $\begin{array}{l}\text { West } \\
\text { Coast }\end{array}$ & Otago & Southland & \\
\hline \multirow{9}{*}{ 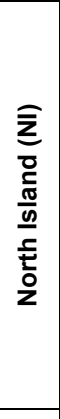 } & Northland & 180 & 285 & 22 & 34 & 7 & 6 & 0 & 5 & 20 & 6 & 9 & 0 & 5 & 2 & 0 & 581 \\
\hline & Auckland & 684 & 564 & 1,191 & 804 & 56 & 141 & 99 & 228 & 361 & 18 & 18 & 220 & 14 & 84 & 22 & 4,504 \\
\hline & Waikato & 81 & 493 & 562 & 458 & 10 & 74 & 61 & 68 & 99 & 9 & 11 & 35 & 0 & 12 & 8 & 1,981 \\
\hline & Bay of Plenty & 37 & 329 & 446 & 271 & 15 & 37 & 22 & 62 & 115 & 0 & 2 & 28 & 0 & 9 & 0 & 1,373 \\
\hline & Gisborne & 0 & 32 & 24 & 56 & 25 & 21 & 3 & 5 & 23 & 0 & 0 & 1 & 0 & 1 & 0 & 191 \\
\hline & Hawkes Bay & 2 & 80 & 58 & 60 & 48 & 72 & 1 & 49 & 85 & 7 & 0 & 8 & 0 & 5 & 0 & 475 \\
\hline & $\begin{array}{l}\text { Taranaki } \\
\text { Manawatu / }\end{array}$ & 3 & 81 & 76 & 67 & 7 & 7 & 65 & 59 & 44 & 0 & 3 & 7 & 0 & 4 & 2 & 425 \\
\hline & Wanganui & 31 & 102 & 202 & 76 & 22 & 130 & 34 & 240 & 245 & 10 & 11 & 28 & 5 & 12 & 17 & 1,165 \\
\hline & Wellington & 24 & 340 & 218 & 65 & 16 & 154 & 71 & 348 & 287 & 75 & 72 & 151 & 4 & 44 & 32 & 1,901 \\
\hline \multirow{7}{*}{$\begin{array}{l}\widehat{\overline{0}} \\
\overline{0} \\
\overline{\frac{\sigma}{0}} \\
\frac{0}{0} \\
\bar{f} \\
\overline{5} \\
\dot{0}\end{array}$} & Marlborough & 0 & 4 & 0 & 4 & 0 & 1 & 0 & 8 & 26 & 25 & 49 & 100 & 25 & 15 & 16 & 273 \\
\hline & Nelson Tasman & 0 & 24 & 0 & 0 & 0 & 8 & 14 & 13 & 17 & 20 & 78 & 75 & 28 & 24 & 0 & 301 \\
\hline & Canterbury & 14 & 100 & 39 & 28 & 2 & 7 & 9 & 17 & 111 & 94 & 172 & 771 & 160 & 335 & 89 & 1,948 \\
\hline & West Coast & 0 & 6 & 2 & 0 & 0 & 3 & 0 & 7 & 9 & 10 & 39 & 68 & 11 & 7 & 0 & 162 \\
\hline & Otago & 4 & 37 & 20 & 8 & 0 & 5 & 4 & 29 & 27 & 24 & 19 & 262 & 9 & 362 & 106 & 916 \\
\hline & Southland & 0 & 15 & 0 & 8 & 0 & 0 & 0 & 4 & 8 & 0 & 5 & 77 & 2 & 170 & 78 & 367 \\
\hline & Total & 1,060 & 2,492 & 2,860 & 1,939 & 208 & 666 & 383 & 1,142 & 1,477 & 298 & 488 & 1,831 & 263 & 1,086 & 370 & 16,563 \\
\hline
\end{tabular}

\begin{tabular}{|c|c|c|c|}
\hline $\mathrm{NI} \rightarrow \mathrm{NI}$ & 11,585 & $69.95 \%$ & \\
\hline $\mathrm{SI} \rightarrow \mathrm{NI}$ & 642 & $3.88 \%$ & Inter-island: $9.98 \%$ \\
\hline $\mathrm{NI} \rightarrow \mathrm{SI}$ & 1,011 & $6.10 \%$ & \\
\hline $\mathrm{SI} \rightarrow \mathrm{SI}$ & 3,325 & $20.07 \%$ & \\
\hline Total & 16,563 & & \\
\hline
\end{tabular}

(Source: TRCNZ, 2002) 


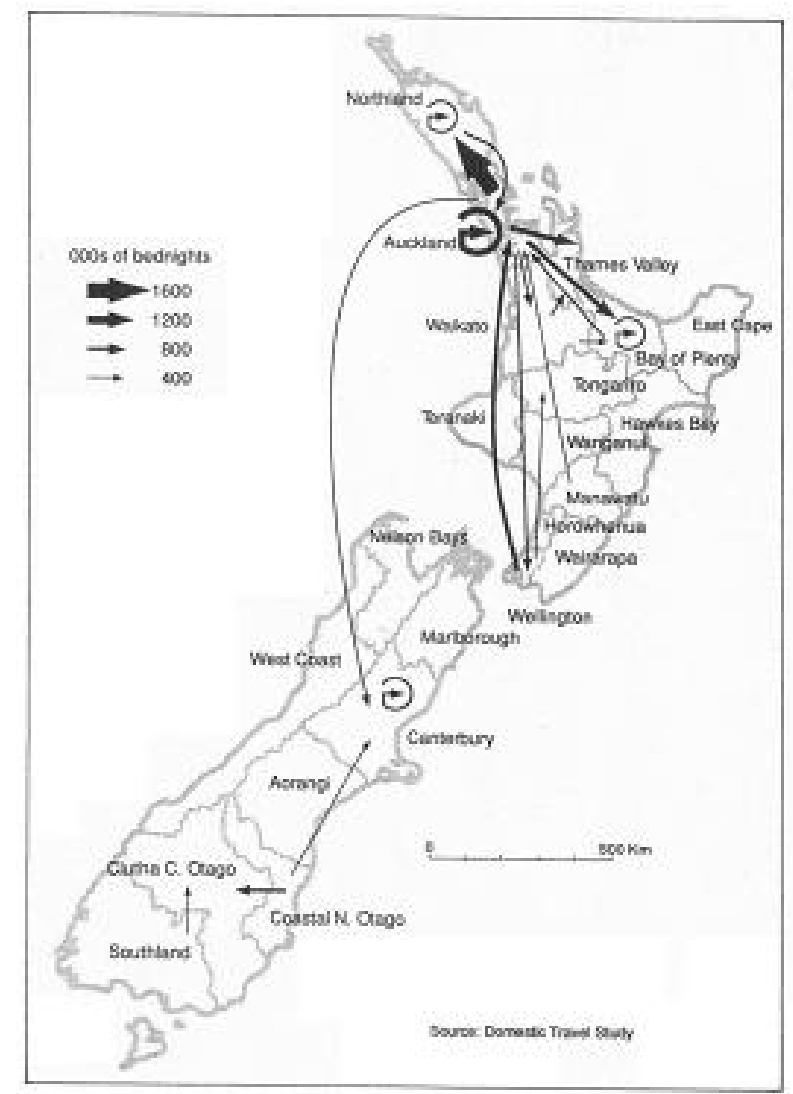

(A) Majof domestic travel flows in New Zealand (over 1\%)

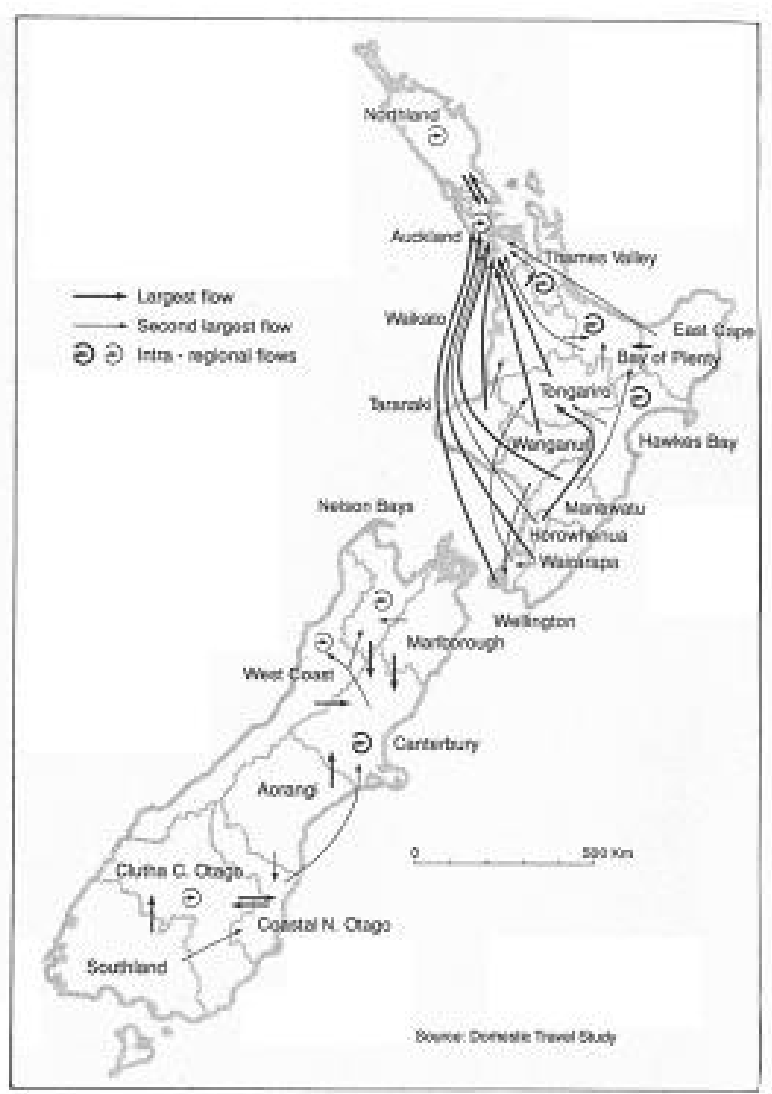

(8) Dominant destination flows in New Zealand

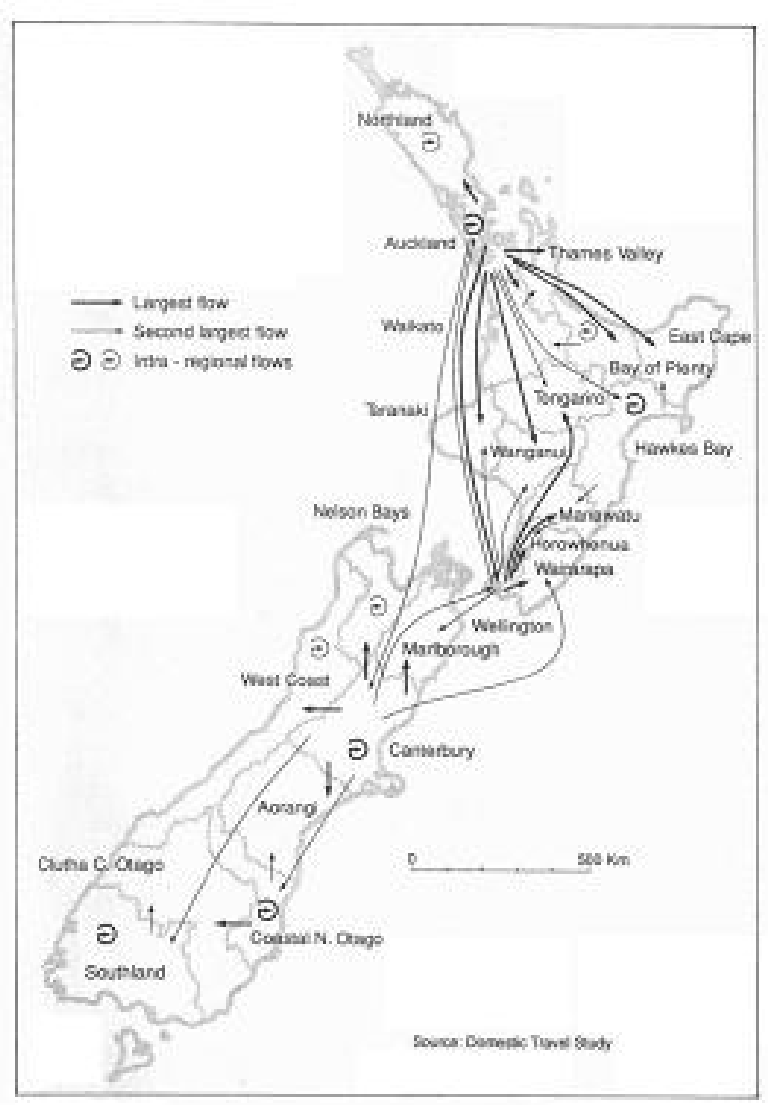

(C) Dominant origin flows in New Zealand

(Source: Pearce, 1993)

Figure 4.4 Domestic travel flows in New Zealand - three year average 1987/88 - $1989 / 90$ 


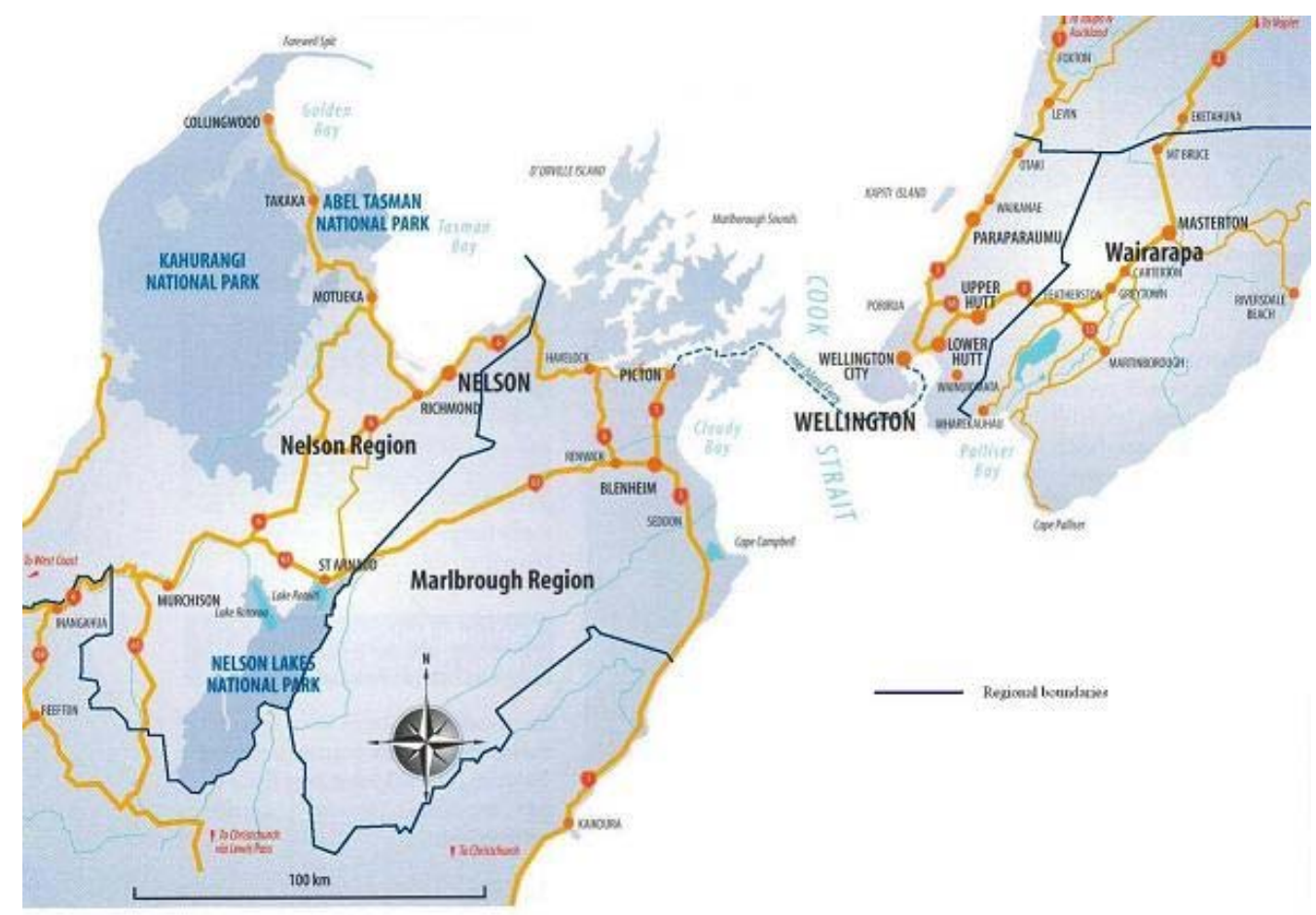

(Source: Centre Stage of New Zealand, 2003)

Figure 4.5 The Centre Stage of New Zealand Region.

Wairarapa, also known as the 'Capital Country Escape', is situated in the South East of the North Island, being an hour's drive away from Wellington (98 km from Masterton to the capital city). The region is a flat rural valley surrounded by mountains and coastline, with many small towns that have retained their charm and character from a friendlier past. The total population, according to the 2001 census, is 38,250 of which 29,673 live in the districts of Masterton (22,797) and Carterton (6,876) (BERL, 2001). Wairarapa has enjoyed solid economic growth in rural sector incomes. The forestry and wood processing sectors have been a significant contributor to lifting output in the manufacturing industry (WRC, 2002). In addition, land use is intensifying at the margins with a steady increase in the area being planted in grape vines and olive trees. There are two activities that are particularly relevant to tourism in the Wairarapa Region. Firstly, fruit growing and wine manufacturing - or 'food and wine' businesses in general - provide a base of activities and attractions. Secondly, homestays which are in many cases a complementary part of agricultural and horticultural businesses, make an important contribution to accommodation for tourists in the region (BERL, 2001). Popular tourist activities in 
Wairarapa include walking and tramping; fishing, diving, hunting and mountain biking; heritage tourism; conference and corporate outings.

The Nelson Region as a tourist destination has an abundance of tourism resources ranging from the city centre of Nelson to Kahurangi and Abel Tasman National Parks. Nelson has one of the best climates in the country and it is one of New Zealand's sunniest regions (Latitude Nelson, 2002). It has an habitual resident population of 84,873 (June 2001). Nelson City is the centre of the province. It is the main commercial, service and transport centre, being very popular with travellers as it is the gateway to many superb beaches on the northern coast and to the scenic West coast Region. The city is also a magnet for artisans - jewellers, potters, glassworkers and sculptors - who create some of New Zealand's finest craftworks (Aitken-Turner et al., 1996). World of Wearable (WoW) Art \& Collectable Cars museum is also located there. To the west of Nelson there are three National Parks. The nearest and most popular is the Abel Tasman National Park, a wonderful area of golden beaches, steep granite formations, limestone caves and native forest. The Abel Tasman Track is the main track through the park and is the most popular tramp in the country. Cars are the most commonly used form of transportation for international visitors travelling to or within the Nelson Region, with $40 \%$ of visitors using either a rental or private car. The ferry is the next most popular form of transportation followed by coach tour and campervan (TRCNZ, 2004b). The predominant use of these independent forms of transport reflects the region's appeal to independent European and American travellers.

The fourth and last region in CSNZ is Marlborough. It is situated in the northeastern corner of the South Island. The region consists of tussock-covered 'high-ground', the alluvial-rich Wairau Plains, and the Marlborough Sounds (of which Picton is the principal township). In 2001, Marlborough Region had a resident population of 39,561 persons, with Blenheim $(26,550)$ the main urban centre, and Picton $(3,003)$ and Havelock $(471)$ as secondary centres. The region's economy is rurally based with pastoral and horticultural farming providing major sources of income. Marlborough's inhabitants utilise marine resources, with fishing and mussel farming also being important. Grape growing has been one of the fastest growing industries and Marlborough is now New Zealand's largest wine producing region. Boutique and large corporate wineries produce some of the best wines in New Zealand. Tourism is a major contributor to the Marlborough Region's economy, with the Marlborough Sounds in particular being promoted as a popular area for water activities and bush walking. In common with most other regions, Marlborough experiences a distinct holiday season, with an influx of tourists during the summer. The average day 
stay in Marlborough is of 1.6 days, suggesting that the region has a more transient role than a true destination. Marlborough's tourist trade is boosted by the fact that Picton is the South Island terminal for the inter-island ferry, which transports passengers and cargo between Wellington and Picton several times a day. The sunny, pleasant climate has attracted people to the region, either as holidaymakers or as permanent settlers. The majority of domestic and international visitors to Marlborough travel for the purpose of holiday. When travelling to and within the Marlborough Region visitors will predominantly travel independently using either a rental/private car or the ferry.

From this brief description of the CSNZ region, it is possible to make some general comments about the nodal functions of each of these macro-regions. Wellington region is by far the most relevant in terms of its origin, destination and gateway functions. Almost three out of four inhabitants of the CSNZ region (73.2\%) live there, it contains some of the key attractions (e.g. Te Papa) and institutions (e.g. the parliament) in the country; and major transport infrastructure (e.g. ferry terminals and the busiest domestic airport in the country) is based in the region. Therefore, Wellington generates and also attracts a significant amount of visitor traffic. Wairarapa is probably the least relevant region, with an incipient tourism industry and few people living there. In some aspects Wairarapa serves as an escape area for people living in Wellington. In the South Island, the Nelson region is probably the one to have the most prominent origin and destination function. The region provides visitors with a wide range of attractions, from national parks, such as Abel Tasman, to museums like WoW. Finally, the Marlborough region has a key gateway function with the ferry port in Picton and some attractions that include the Queen Charlotte track and the vineyards near Blenheim. With a smaller population, the region has a small origin function, with the gateway function being predominant.

\subsection{Wellington's Nodal Functions}

This section focuses on tourism at the local level in Wellington, one of the two ferry gateways. The next section is about Picton. Both sections are structured in order to emphasize and describe the main nodal functions in each place. In the case of Wellington they are origin, destination and gateway. In Picton only the destination and gateway functions are relevant as with a population of only 3,000 inhabitants its origin function is a very small one. 


\subsubsection{Wellington as an origin}

Various factors contribute to Wellington being one of the country's most important sources of tourists with a high propensity of outbound domestic travels (see Figure 4.3). In terms of population numbers, in 2001, Wellington City was the home of 163,824 people, being the sixth largest of the 74 Territorial Authorities in New Zealand. In addition, the median income of people living in Wellington is $\$ 27,000$, considerably higher than the country as a whole at $\$ 18,500$, as well as higher than Auckland and Christchurch. What is more, the median age of the population in Wellington is younger (33 years) than the national median (35 years), with a correspondingly smaller proportion of people aged 65 years and over at $7 \%$ compared with $12 \%$ nationally (Warren \& Taylor, 2003).

\subsubsection{Wellington as a tourist destination}

Since 1865 Wellington City has been New Zealand's capital city, which due to its central location is an ideal place to seat the government. As a consequence of this, Wellington is one of New Zealand's most cultured and vibrant cities. This is supported by the presence of the New Zealand Symphony Orchestra, Royal New Zealand Ballet and the NBR New Zealand Opera. Every two years, during three weeks at the end of the summer, Wellington hosts the New Zealand International Arts Festival. This is the country's most extensive and celebrated multi-arts event. It also offers a wide range of galleries, museums, theatres and cinemas. Other regular events held in Wellington include The Interisland Line Summer City Festival (with more than 60 free outdoor entertainment activities and events), the Cuba Street Carnival, the New Zealand International Sevens (a two-day rugby competition in February), the Jazz Festival, the International Film Festival, among others. In its 2002 edition, the New Zealand International Sevens was responsible for more than 10,000 visitors, generating $\$ 8.5$ million to businesses in Wellington (Weir, 2003).

Various attractions have helped to develop Wellington as a destination, including the National Museum of New Zealand - Te Papa Tongarewa (Leask, 2005). Inaugurated in February 1998, the stunning new building is placed in the heart of the city, within walking distance of the CBD (Tramposch, 1998). Housing exhibits that explore and explain many aspects of New Zealand's history and culture, the museum also plays host to travelling exhibitions from other parts of the country and the world. During its first three years, it had nearly five million visitors. Supporting Te Papa is a wide range of other New Zealand heritage attractions, the arts, shopping, restaurants and cafés and a variety of events. Some of these attractions are: 
- Wellington Botanic Garden: the Botanic Garden is situated $1 \mathrm{~km}$ from the CBD and can be accessed via the Cable Car (see description below);

- Karori Wildlife Sanctuary: situated outside the inner city (only two kilometres from the city centre), the sanctuary has 252 hectares of native forest. Currently it is developing the 'Gateway Project' which aims to make the sanctuary a top visitor attraction in the country and lift visitor numbers to 150,000 per annum;

- The Westpac Trust Stadium: opened in January 2000, this state-of-the-art multipurpose stadium has the capacity to accommodate 34,500 spectators. Being the home for cricket and rugby in the capital city and thanks to the proximity to Wellington $\mathrm{CBD}$, the exciting atmosphere easily spills out into the city;

- Cable Car: opened in 1902, the cable car runs past the Victoria University of Wellington's Kelburn campus and Kelburn Park to the top of the Botanic Gardens where the many walks through the gardens begin. The planetarium and Carter Observatory are also located there;

- Parliament Buildings: Parliament is composed of three buildings: the Beehive, opened in 1981, Parliament House and the Parliamentary Library. Guided tours lasting one hour focus visitors on architecture, the 1992-1995 refurbishment process and engineering work, artworks and the history of this cultural heritage site. In addition, many aspects of the Parliamentary process are covered, providing visitors with an insight into how Parliament operates;

- Museum of Wellington City \& Sea: this recently refurbished museum explores the history of the harbour capital through both traditional and interactive exhibitions;

- National Archives of New Zealand: the original Treaty of Waitangi, symbolising the nation's founding and the 1893 Women's Suffrage Petition are just two of the documents on display in the Archive's Constitution Room. Other galleries have changing exhibitions on a variety of historical and cultural topics.

Most of these attractions are located in the city's central business district that extends along the shore of Port Nicholson and is built on reclaimed land between the steep hills and the sea. Central Wellington is very compact - only two kilometres in diameter - so it is 
possible to walk from one side to the other in under twenty minutes (WellingtonNZ, 2004). In addition, Wellington's four quarters - Lambton, Willis, Cuba and Courtenay - offer an extensive and diverse range of shops, restaurants, bars, cafés and entertainment (see Figure 4.6). Lambton Quarter is the traditional shopping area but also encompasses the Wellington waterfront. Willis Quarter crosses Wellington's Civic Square, the heart of the city. Cuba Quarter is home to an eclectic mix of cafés, bars, shops and dealer galleries. Finally, Courtenay Quarter extends from Courtenay Place - Wellington's theatre, cinema and nightlife precinct - to Te Papa on the waterfront.

One of the main objectives of the recent reconstruction of the waterfront has been the attractiveness of the city to both domestic and international tourists (Page, 1993; Ruggia, 2003; Wellington City Council, 2001). In fact, urban waterfront redevelopment has been a well-established phenomenon internationally (Gospodini, 2001b; Warren \& Taylor, 2003). In Wellington, the scheme has thus been accompanied by more aggressive marketing of the city as a place to visit. It aims to catch up the ground lost when the city missed out on being included on the international "golden route" between Auckland, Rotorua, Christchurch and Queenstown in the late seventies and early eighties (Doorne, 2000). The promotion of Wellington as the cultural centre of the country has been aided by the government's decision to include Te Papa Museum as part of the redeveloped waterfront. This gave Wellington a unique resource funded by the national, rather than the local, taxpayer (Doorne, 1998). Other amenities in the area include the City and Sea Museum, the City Gallery, Civic Square, Town Hall and various restored buildings which house apartments, commercial spaces, bars and restaurants (Warren \& Taylor, 2003).

In terms of imaging, promotion and selling of Wellington, according to Hall et al. (1997), its marketing has a quality-oriented policy, with efforts being made in order to offer a better quality of facilities for consumers. Apparently, all these strategies have been successful. According to Totally Wellington (2000), Wellington City Council's tourism agency, Wellington outstripped New Zealand's other major tourism destinations to enjoy the highest (23\%) growth in guest nights between 1998 and 2000. In 1999, Wellington increased its lead over Rotorua as the fourth busiest tourism destination in the country, for total guest nights. The top three destinations are Auckland, Christchurch and Queenstown. 


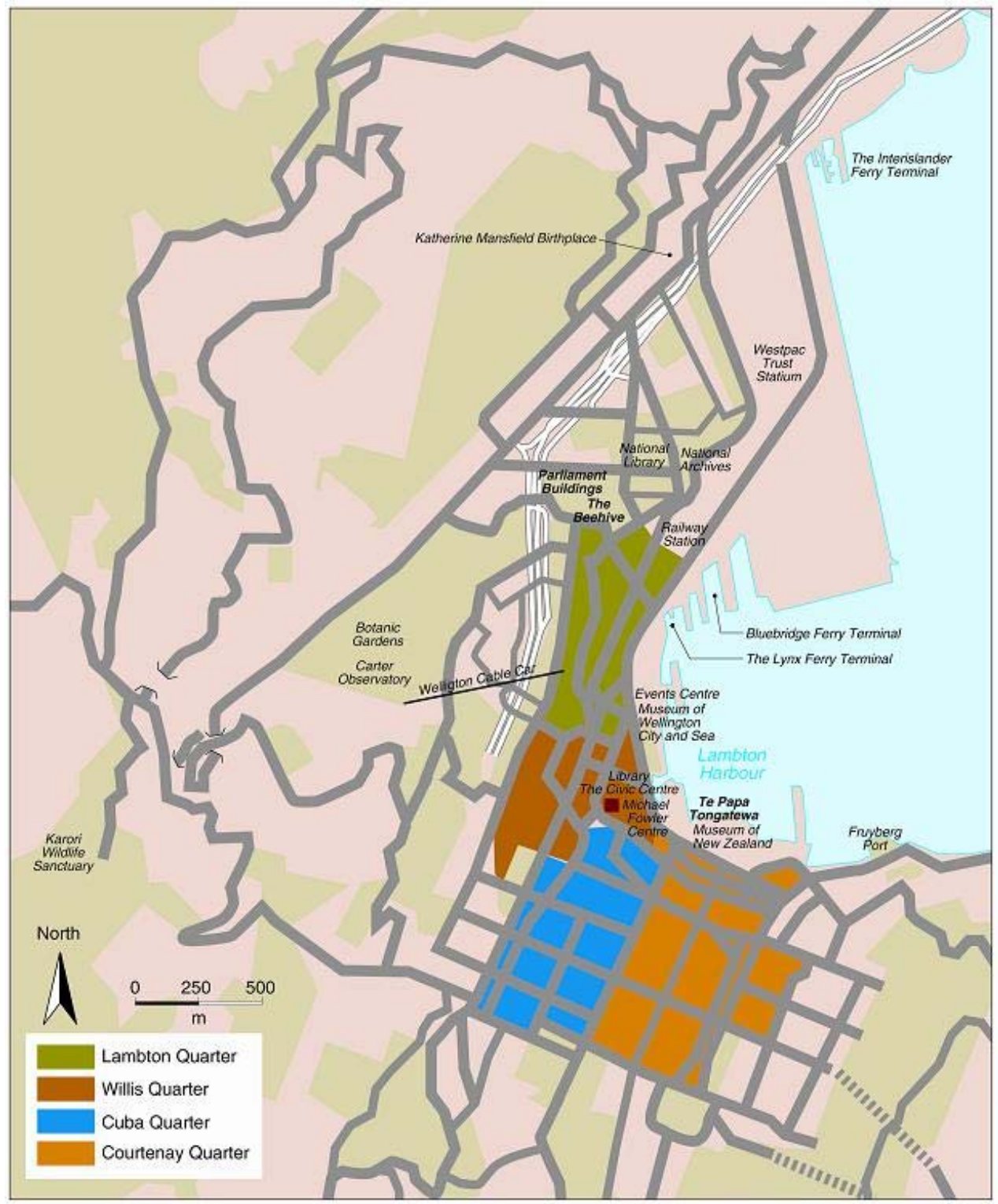

(Source: AA, 2003)

Figure 4.6 Map of Wellington Central Business District.

\subsubsection{The role of Wellington as a gateway}

Wellington's central location and its role as capital city make it an important domestic air transport gateway to the CSNZ region. It has the busiest domestic airport in New Zealand for scheduled flights. Although Wellington Airport provides international direct flights to some Australian cities, such as Sydney, Melbourne and Brisbane (WIAL, 2004) and other destinations in the Pacific, it does not have a full-length runway able to receive long-haul aircraft from Asia and North America. Due to this, the capital city has been unable to 
secure a place as a major international gateway. This role is better serviced by Auckland and Christchurch, the two main international airports in New Zealand.

Apart from the airport, Wellington also serves as the gateway to the North Island for tourists travelling across Cook Strait. In a survey prepared for Te Papa and Totally Wellington, inter-island ferries were identified as the most common reason for international visitors coming to Wellington, accounting for $27 \%$ of visitors who stayed longer than four hours or who were not just passing through (Aalst \& Daly, 2002).

There are three ferry terminals in Wellington, all operating very close to each other. They are The Interislander Terminal, The Lynx Terminal and Bluebridge Terminal. The Interislander Terminal is located five minutes driving north of the railway station. For drivers using the State Highway 1, the terminal is placed before Wellington's CBD, which in some cases makes them go straight to the ferry terminal without taking the opportunity to visit the inner city. Although the terminal is not far from the CBD, no public transport links the inner city and the ferry terminal. The options available are taxis or a free shuttle bus that operates exclusively prior to and after the ferries' arrival and departure. Rental car offices such as Apex, Avis, Budget and Hertz are located in the arrivals terminal. The Lynx Terminal was located 100 metres from the railway station, which is also near some important sites in the capital city, including the Parliament and the Beehive. The terminal's facilities are very limited. Most of the passengers picking up a car need to catch the free shuttle bus and go to the Interislander Terminal. Bluebridge Terminal is a brand new facility built in 2003 , just opposite the railway station.

\subsection{Picton's Nodal Functions}

\subsubsection{Picton as a tourist destination}

Nestled among high hills, at the heart of Queen Charlotte Sound, Picton is ideally placed as a holiday resort, although industries such as boat building, fishing and forestry share its beautiful setting (see Figure 4.7). Inter-island freight also pours through Picton, but tourism is its heartbeat.

The Marlborough Sounds, and Picton in particular, have long been a popular holiday destination. The peace and seclusion of the bays and coves, offering natural privacy, were disturbed little by the casual placement of the numerous weekend cottages built by visitors in the late 1880s. Today, these attributes are still there, although a small resident population and the addition of holiday homes have accumulated over the years. 


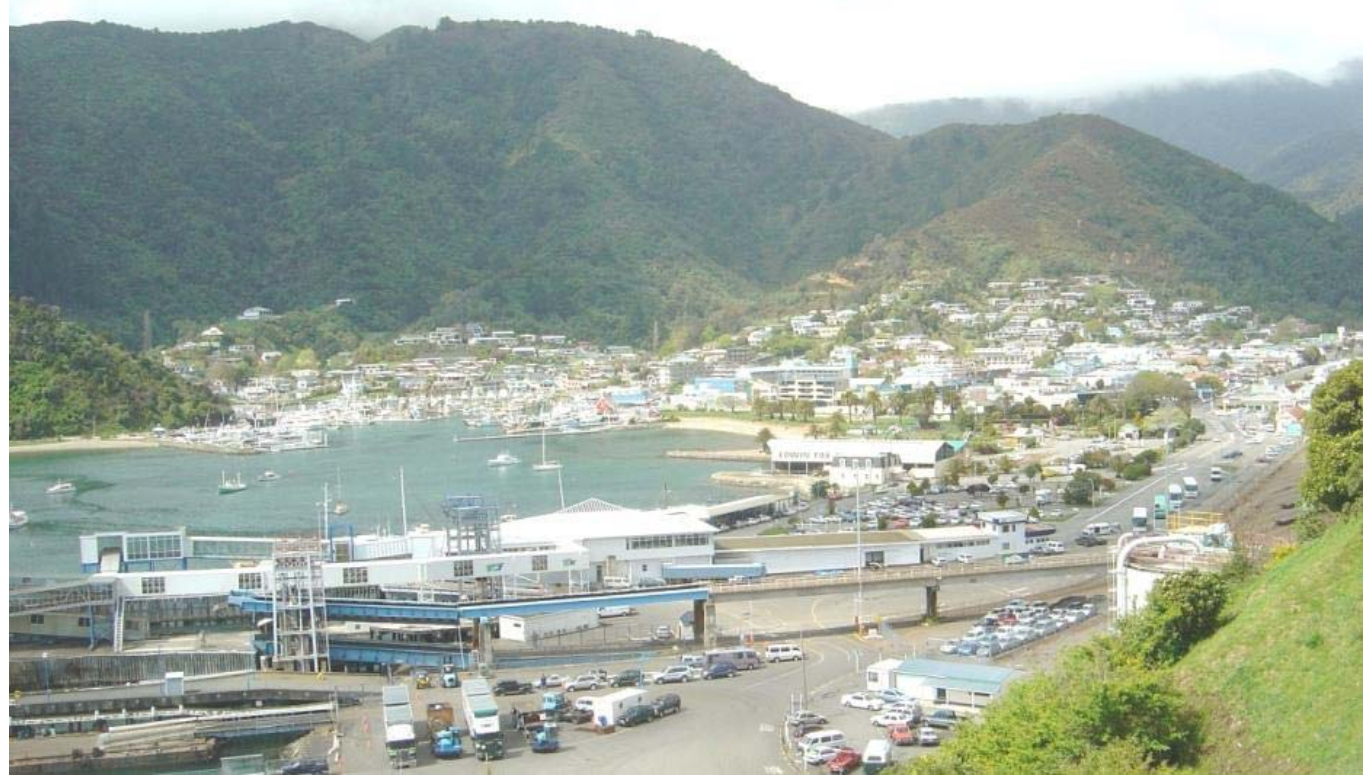

(Photo by Kieron Iveson)

Figure 4.7 The ferry terminal infrastructure (front) and the town of Picton (back).

Nowadays, there are a variety of hotels and accommodation complexes to cater for the flow of visitors that come to Picton to catch the ferry or to admire the Sounds. They range from motels, hotels, bed \& breakfasts, holiday parks, homestays, backpackers and camping facilities. Picton also offers some cafés, restaurants and other businesses to cater for tourists and transit passengers, including a mall, a supermarket and two service stations. In addition, a number of tourist activities are available in Picton, notably those that offer land and water adventures. Some of the popular activities include walking, mountain biking and sea kayaking. Although most of the activities can be done in one day in an independent way, some tourist operators provide options that require 3,4 or even 5 days to be completed, going much beyond Picton and Waikawa, usually reaching the Queen Charlotte Track (see description below). Some of the key tourist activities and attractions located in the Picton area include:

- Seahorse World Aquarium: previously known as the Aquarium of the Marlborough Sounds, it is located on the foreshore. It is a type of boutique aquarium providing visitors with an exceptional educational presentation, emphasising a top quality experience, with free guided tours and two daily feeding sessions. Seahorses are the main attraction with more than 400 on display. They are supplemented by the six-meter preserved giant squid (just one of two on 
display in the Southern hemisphere), sharks, jellyfish, octopus and hundreds of New Zealand saltwater and freshwater species;

- Edwin Fox Maritime Museum: the Edwin Fox is considered to be the ninth oldest ship afloat in the world and is located on the Picton foreshore, fifty metres from the ferry terminal. It is the only remaining East Indiaman vessel of all the hundreds built in the $18^{\text {th }}$ and $19^{\text {th }}$ centuries. This period of international sea-going trade was one of the most remarkable periods in the world's history. Thousands of people visit the museum and the ship each year. Built in 1853, it has been in Picton since 1897. The vessel is part of the Edwin Fox Maritime Museum where visitors can see a large collection of artefacts found on board the ship;

- Picton Museum: particular items of interest to the whaling industry, which operated from Tory Channel and Port Underwood, can be found there. There are also interesting displays on Maori, early settlers and mythical interest from around the area;

- Kiwi Golf: this mini golf is a popular attraction located on the foreshore;

- Queen Charlotte Track: located on the East coast tip of the South Island, this $71 \mathrm{~km}$ track stretches between Queen Charlotte and Kenepuru Sound in the Marlborough Sounds. The walk shows off some spectacular coastal and bush scenery. Visitors can walk the entire track, which takes three or four days or simply walk a portion of it;

- Bush walks: Picton offers a wide range of walks, from short ones lasting less than an hour (Victoria Domain), to all day walks and also long walks, including the Queen Charlotte Track;

- Water activities: including scenic boat cruises, sailing, fishing and kayaking. As some of the areas in the Marlborough Sounds are only accessible by water, visitors can explore the islands existing in the Marlborough Sounds, visit bird sanctuaries, marine reserves and salmon farms, while watching out for dolphins and seals. There is also the possibility of wreck diving, wind surfing and water skiing.

Situated at the northern end of the Marlborough Region and occupying approximately 2,304 square kilometres in area, the Marlborough Sounds is an extensive system of 
drowned river valleys and islands that extend some 96 kilometres along the seacoast (see Figure 4.8). Formed by tectonic submergence of a complex fluvial drainage system, these river valleys provide the underlying structure for this landscape and create a variety of characteristic landforms. The steep slopes abruptly descend towards the water and narrow ridges form a system of long land spurs, enclosing a large number of fiord-like inlets, bays and coves. The Marlborough Sounds is a popular tourist destination for travellers and has long been recognised as a recreation area of important value. This credit is based on the distinct character of the landscape, which gives the Sounds their unique appeal for many forms of recreation. The convoluted coastline of the sounds includes numerous bays, peninsulas and inlets, many of which can be explored only by boat.

Within this system of waterways are two major 'arms': Queen Charlotte Sound (Picton's sound) and Pelorus Sound, each stretching inland for more than 48 kilometres and broken up into hundreds of small bays (see Figure 4.8). The Marlborough Sounds is also popular for camping and tramping. This scenery makes the Cook Strait journey across Tory Channel one of the main attractions of the ferry journey.

The responsibility for promoting and attracting visitors to the Marlborough Region, as a whole, falls to Marlborough's regional tourism organisation; Destination Marlborough. Based in Blenheim, Destination Marlborough is a centralised agency whose aim is to attract visitors to Marlborough through the marketing and promotion of the region (although this falls short of actively promoting one particular location within Marlborough over another). Situated on the foreshore of Picton is the Picton Visitor Information Centre (VIC). Picton's VIC, which is funded and staffed by Marlborough District Council, is actively involved in promoting Picton and the surrounding region through the provision of information services. Picton is also represented by several organised groups of residents. These include various ratepayers and business associations, as well as the Picton-based promotional organisation Picton Sounds Paradise (PSP). The establishment of PSP was a consequence of The Interisland Line's proposal to build a new ferry port in Clifford Bay. This spurred interest by the tourism industry to improve Picton for visitors. With the support of local tourism operators, PSP successfully promoted the redevelopment of Picton's town centre and now Picton offers an attractive townscape to visitors (Destination Marlborough, 2002).

Among the array of development initiatives being undertaken in Picton and neighbouring Waikawa, there is the project to create a 'pedestrian-friendly' town centre, the refurbishment of older, established buildings, and the construction of new waterfront 
apartments. The most striking of these has been the foreshore redevelopment project, which saw this area transformed into an attractive recreational area for both tourists and local residents (Marlborough Economic Development Trust, 2001).

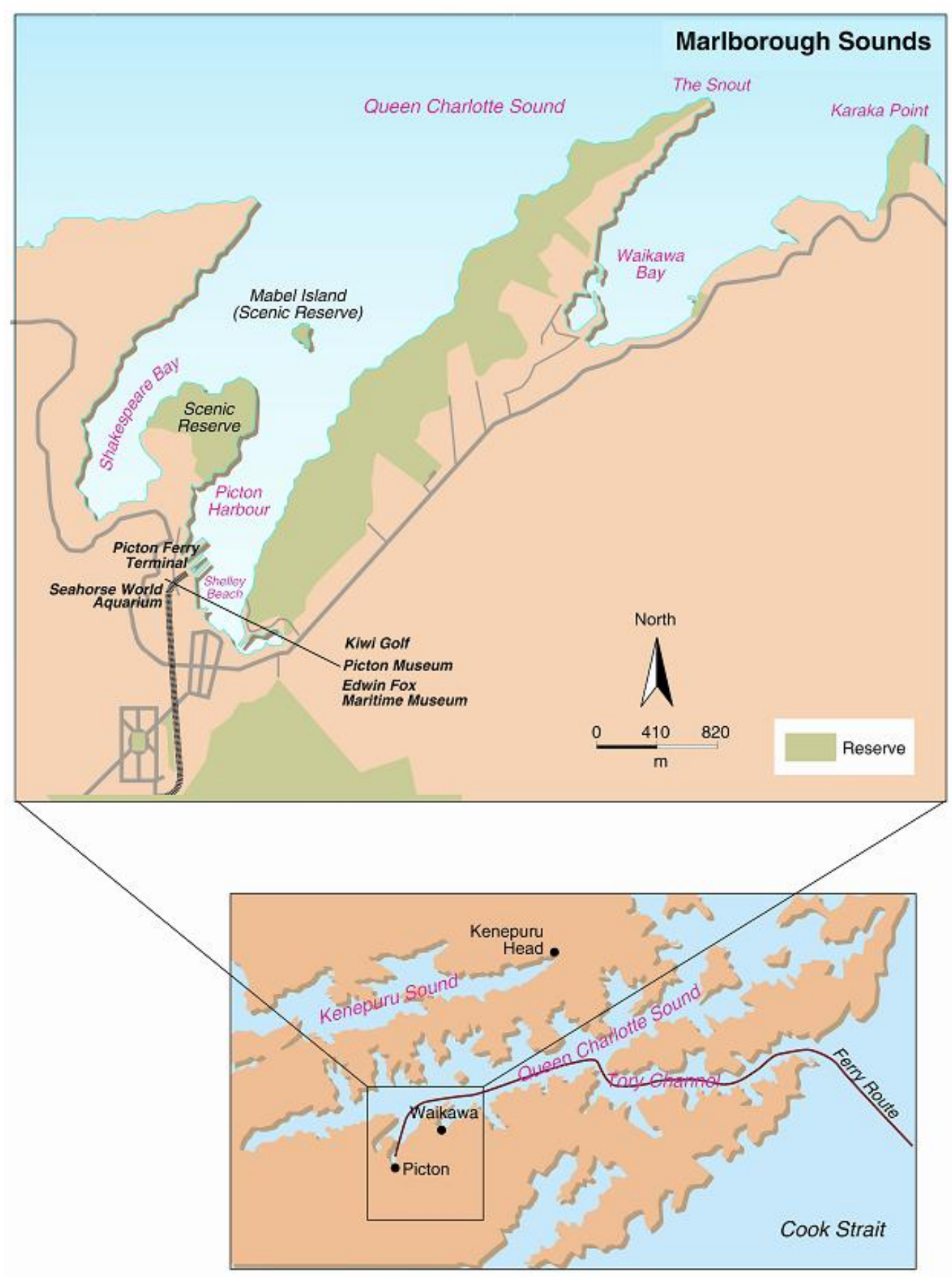

Figure 4.8 Map of Picton Region. 


\subsubsection{The role of Picton as the ferry gateway}

In the case of Picton, the gateway function overshadows the destination one. Its gateway importance goes beyond the local (Marlborough Sounds) and regional (Marlborough) levels and reaches the whole South Island when considering sea (through the ferries connection) and land (road and rail) transport.

Picton acts as the principal port of the Marlborough Region and serves as the commercial and social centre of the Marlborough Sounds. Its large deep-water port is a transport terminus for the South Island main trunk railway, the Cook Strait ferries, the local land and water taxis, the various mail and milk runs, pleasure trips in fishing launches and the usual assortment of sightseeing facilities. Regular sailings of the ferry service transport an estimated 1.265 million passengers, 253,000 vehicles and over 2.5 million tonnes of freight per annum (Marlborough Economic Development Trust, 2001).

As highlighted in the literature review, intermodality is one of the key characteristics of a gateway. Particularly in Picton, this role is very prominent. Located in the ferry terminal there are a number of rental car branches, including local and international companies. In terms of intermodality, Figure 4.9 shows the rental car offices and the West Bay Water Transport office, one of the various water transport providers located in and near the ferry terminal. Other rental car offices are situated in the railway station, which is a few metres from the terminal. In addition, there is one passenger train service called Tranz Coastal that operates daily between Picton and Christchurch. This service arrives in Picton at $12.50 \mathrm{pm}$ and departs at $1.40 \mathrm{pm}$ and is integrated with one of The Interisland Line ferries, so passengers travelling to and from Wellington can have a faster connection in Picton. Picton air travellers are serviced by SoundsAir, which operates regular daily scheduled services to Wellington from the Koromiko airstrip, located six kilometres south of Picton. Picton has just one taxi. In summer, another driver joins the existing one.

When analysing Picton in terms of the Resort Development Spectrum Framework proposed by Prideaux (see Table 2.2) it is clear that, apart from transport, in all other characteristics (markets, accommodation, promotion and tourism infrastructure) Picton is located between the phases One (local tourism) and Two (regional tourism). However, when considered with the transport characteristics, Picton is similar to a national tourism destination, which includes scheduled air services, sea terminals and rail services. This emphasizes that the gateway function is outstanding in comparison to the destination one. 


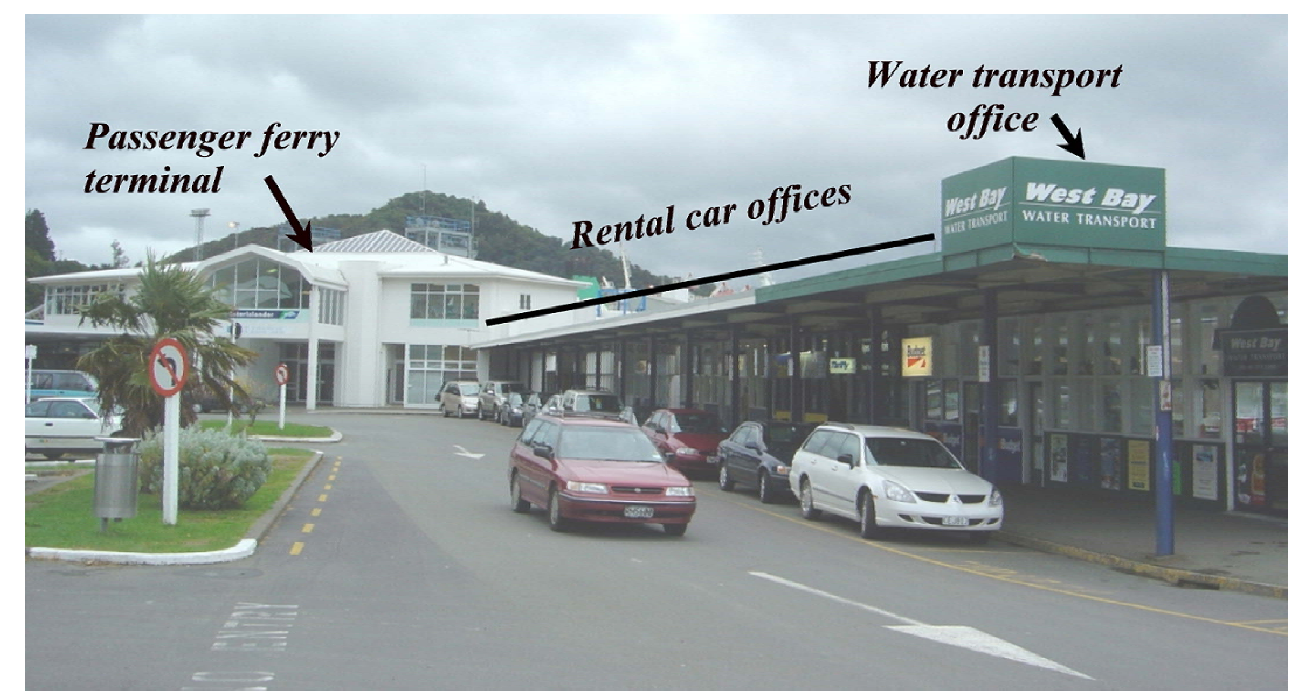

(Photo by Kieron Iveson)

Figure 4.9 Transport integration in Picton.

\subsection{Conclusion}

This chapter presented the key nodal functions existing in the study area. This section considered the major conclusions of three scales analysed: national, regional and local.

At the national level the focus was on presenting the major trends of international and domestic travellers in the country. International tourism has been particularly prominent, with increases in tourist demand averaging nine per cent per year over much of the past two decades, ahead of both Pacific regional and world tourism trends. Domestic tourism, on the other hand, evidenced little if any real growth during the 1980s and 1990s. Key differences were identified between international and domestic visitors in terms of their length of stay. While international travellers are staying longer in the country - according to TRCNZ (2004b), 18 days in 1998 in comparison to 26 days in 2003 - the average length of stay of domestic visitors has dropped from 4.1 in 1982 to 3.1 days in 2001 (TRCNZ, 2002). The consequence of this is that in terms of persuading travellers to stay longer in the gateways, international travellers might be more likely to do so simply because they are spending significantly more days in New Zealand (26 days in 2003) than domestic visitors take for their holidays (3.1 days in 2001). What is more, due to their travel patterns, some international markets seem to be even more likely to do so. They include, for example, visitors from the UK, who have independent travel styles and long lengths of stay (33 days), with $31 \%$ of them using the ferries while in New Zealand. 
In terms of the regional scale, it is possible to conclude that while Wellington is one of the key destinations located at the south of the North Island, Picton on the other hand faces competition with many other destinations, including Nelson which also boasts national parks, beaches and cultural attractions, and Blenheim with the vineyards. On the other hand, the destination function of Picton benefits from the existence of Wellington, which is a large metropolitan area that provides many visitors that use the ferries.

This chapter has also described the two main ferry gateways in New Zealand, Wellington and Picton. Wellington was presented as an important transport centre, having the busiest domestic airport in the country and being the ferry gateway to the North Island. In the last few years, Wellington has been transformed from a monotonous government centre into a vibrant urban destination. Some attractions such as the Karori Wildlife Sanctuary, the Westpac Trust Stadium, Te Papa and the expansion of the harbour have contributed to this. The scheme has thus been accompanied by more aggressive marketing of the city as a place to visit, aiming to catch up the ground lost when the city missed out on being included on the international "blue ribbon" between Auckland, Rotorua, Christchurch and Queenstown in the late seventies and early eighties. As a result, Wellington is now in a better condition to attract ferry passengers to stay and visit as a destination than it was a few years ago. However, as it usually takes time for the image of a given destination to be changed and potential visitors to hear about it, it can be expected that in the next few years the capital city will become a must see destination for the major international markets as well.

In Picton, some changes in the last decade have also tried to improve and promote it as a destination. The Interisland Line's proposal to establish a new ferry port in Clifford Bay ended up launching some interest by the tourism industry to improve Picton for visitors. With the support of local tourism operators, groups such as PSP successfully promoted the redevelopment of Picton's town centre and now Picton offers an attractive townscape to visitors. This includes the foreshore redevelopment project, which saw Picton's foreshore area transformed into an attractive recreational area for both tourists and local residents. This redevelopment has been extended to incorporate the main street in Picton's central business district. Although the subject of some derision amongst local residents, this project has succeeded in creating a 'pedestrian-friendly' town centre. Included in this redevelopment of the town centre have been the refurbishment of older, established buildings, and the construction of new waterfront apartments. However, when compared to other destinations located in the CSNZ region, it becomes clear that Picton has fewer attractions in order to attract potential visitors from the ferry passengers. What 
is more, its major activities are oriented to a particular market, particularly those interested in tramping and water activities.

Exploring the research problem in Wellington and Picton provides key advantages, the main one being the opportunity to consider the gateway function in two nodes where the destination function present different scales and levels of importance. The fact that Picton is a small resort town and Wellington is the capital city and a major urban destination makes it possible to compare and contrast travellers' behaviour in terms of extending their stays in both nodes and also to analyse what sort of different strategies tourism business managers and owners need to undertake in order to attract visitors in Wellington and Picton.

The next chapter will set the scene for transport in the study area, specifically describing the development and the current market of the Cook Strait ferries. 


\section{The Cook Strait Ferries}

\subsection{Introduction}

As seen from the description of the various nodal functions existing in the study area (Chapter 4), the ferry gateway function is a prominent one both in Wellington and Picton. Apart from considering the nodal functions, it is also necessary to present the transport component, which is the dynamic part of the nodal function. Ultimately, the provision of the ferry service is what makes the existence of the gateway function in both ferry ports possible. What is more, the dominant presence of the ferries in the gateways, particularly in Picton where the origin and destination functions are less noteworthy, has a significant impact on tourism in both nodes. Hence, understanding the ferry service also helps to better comprehend the destination functions.

This chapter aims to present the Cook Strait ferry services in order to consider how they have evolved in recent years and what the key issues relevant to tourism in Wellington and Picton are. It is particularly pertinent to identify core aspects of the ferries' management and operation that can impact on the tourism stakeholders located in both ferry ports and on the likelihood of ferry passengers staying longer there. Some of the analysis considered here was used to elaborate a number of questions included in the passenger questionnaire and more importantly in the interviews with the owners and managers of businesses / organisations. The theme of this chapter also helps to better understand some of the discussion presented in Chapter 8, where data from the interviews with the stakeholders are analysed. With the constant evolution of the Cook Strait ferry market, it is worth mentioning that all considerations made in this thesis and notably in this Chapter refer only until August, 2004, the date the data collection process finished.

Three major topics are considered in this chapter. Firstly, the recent development of the Cook Strait ferries, particularly the fast ferry era and the attempt of several companies to compete with the Interisland Line. Secondly, this chapter describes the decision to reduce the speed of the fast ferries along the Tory Channel in order to minimise the environmental impacts of the ferries' waves on the shoreline. Thirdly, this part of the thesis discusses the proposal to build a new ferry terminal at Clifford Bay, in the South Island. As will be shown later on in Chapter 8 , all these three subjects are relevant to understand some of the key issues presented by the tourism stakeholders, particularly those located in Picton. In the case of the ferry speed restriction and the Clifford Bay proposal, these 
topics ended up damaging the relationship between some businesses in Picton and the Interisland Line.

\subsection{The Recent Development of Ferry Services in Cook Strait}

\subsubsection{The 1994-2004 Period}

The ferries' operation in Cook Strait started in August 1962 and for more than thirty years there was only one ferry operator, NZ Rail, which used to operate the rail services and the Cook Strait ferries. Between 1962 and 1994, the incumbent ferry company only used conventional roll-on roll-off ferry vessels. At the end of 1994, fast ferry technology was introduced both by the incumbent company, known today as The Interisland Line, and its first competitor, Sea Shuttles. Since then, four other different ferry companies have tried to operate ferries across Cook Strait and compete with the Interisland Line. Four of them, all using fast ferry vessels, failed to succeed and the fifth and current, Strait Shipping, started a conventional ferry service in January 2003. The previous four were:

- Sea Shuttles: December 1994 - March 1995;

- North by South Ferries: December 1995 - May 1996;

- Sea Cat Ferries: February 1998 - May 1999;

- $\quad$ Fast Cat Ferries Ltd: May 1999 - November 2000.

At the beginning of 1994 , three possible competitors were planning to start a fast ferry service across Cook Strait. Only one, Sea Shuttles NZ Ltd (hereafter to be referred as Sea Shuttles), became a real competitor with the vessel Albayzin. This coincided with the decision by NZ Rail to introduce a fast ferry service. Competition started during the month of December, with the arrival of two fast ferries within days of each other. The 96-metre brand-new Spanish-built monohull Albayzin arrived in Wellington on 8 December, followed by the eighteen-month-old Australian-built Condor 10 on 15 December. The former was operated by Sea Shuttles and the latter by NZ Rail. During their delivery trips both vessels suffered damage. The Albayzin had a log sucked into a waterjet and the Condor 10 hit a whale one day out of Wellington, damaging her starboard T-foil stabiliser (Berry, 1995). The log incident, crew training and various safety concerns delayed the introduction of Albayzin into service by almost two weeks. On the other hand, the Condor 10, known in New Zealand as 'The Lynx', made her debut as scheduled on 21 December (Finnegan, 1994). 


\section{THE COOK STRAIT FERRIES}

Following ten frustrating days in dry-dock at Lyttelton for repairs and several route familiarisation trips later, Albayzin finally left for Picton with fare-paying passengers on Christmas Eve. However, within a few days, it was clear that Albayzin's sailing schedule was too ambitious, despite warnings that it had allowed insufficient time for the turnaround at Wellington. The company was forced to reduce its services from four a day to three. In addition, after striking the wharf heavily at Picton while berthing on 29 December, it became evident that Albayzin's hard-chine hull shape lacked the necessary fendering and was very susceptible to wharf damage (Berry, 1995).

Meanwhile, by the beginning of January, speed restrictions within Wellington Harbour had been placed on both fast ferries after concerns about the effect of their wakes on other ships (Bell, 1995a). The new restrictions added an extra 15 minutes to the passage. In addition, protests were coming from Tory Channel residents about adverse effects of fastferry wakes. Sea animals such as paua and kina were being tossed on the shore to die, rocks were being thrown on to beaches, and erosion was eating away at banks and boat sheds.

On 4 January, following an incident at Picton when Albayzin nearly grounded while berthing with three engines shut down, the Maritime Safety Authority cancelled Sea Shuttles' operating certificate citing serious concerns about the ship's safety and seaworthiness (Berry, 1995). The Picton incident was the latest in a run of steering problems experienced by the vessel, including two on Boxing Day when the ship made two involuntary 360 degree turns at high speed. Although the Albayzin was considered smoother and more luxurious than The Lynx, the crew had struggled with engine and berthing difficulties. According to Currie (1995c), the Maritime Safety Authority suspended Albayzin's certificate for several reasons, including: difficulties in berthing, particularly in heavy winds; occasional steering problems in heavy seas; continuous engine problems, including failures; smouldering in gearbox equipment in one of the four engines; and bow thruster (the machinery used to help it berth) failure.

On January 25, the Albayzin did her first trip after a two-week layoff for dry-docking in Nelson (Currie \& Kayes, 1995). Albayzin's woes did not end there, however, and during January and early February, sailings were regularly cancelled due to ongoing engineering problems. As if these troubles were not enough, it was evident that all was not well on Sea Shuttles' industrial front. On 31 January, one of Albayzin's masters resigned, followed two days later, by the company's Picton manager, both citing major safety and operational disagreements. 


\section{THE COOK STRAIT FERRIES}

A few days later, further industrial troubles were revealed when the Seafarers Union threatened legal action over concerns that Albayzin's cabin crew were working excessive hours on poor pay and insufficient rest periods. These had apparently arisen with the late arrival of several sailings due to the continuing problems with the ship's systems. Following union talks, plans to hire fifteen extra crew to alleviate the problem were already at hand when on 1 March, Sea Shuttles sacked its eighty staff members due to the Albayzin's lamentable mechanical condition. Albayzin's last trip was on 14 February (Bell, 1995c). If anything could go wrong with Sea Shuttle's venture, it did.

Nevertheless, the first fast ferry season was also difficult for the incumbent carrier. During The Lynx's first summer season (21/12/94 - 18/4/95), NZ Rail lost millions of dollars. The main reason was due to the lack of a flexible reservations and pricing system, similar to airlines, with a range of fares. Moreover, apart from competing against Sea Shuttles, NZ Rail had problems finding a balance between the price of The Lynx ${ }^{9}$ and an airline flight ${ }^{10}$ (Currie, 1994, 1995a). During this time, The Lynx carried 212,334 passenger and 34,195 vehicles, making 686 crossings - 96 per cent of planned sailings were made (Berry, 1995), which can be considered quite significant.

On 28 November 1995, The Lynx arrived back in Wellington for a second summer season. In order to avoid the financial losses of the previous season and despite the imminence of a new competitor, North by South Ferries, standard prices increased 31 per cent ( $\$ 59$ an adult and $\$ 29.50$ for a child fare). However, the new thrifty fares included savings up to fifteen, thirty and fifty per cent, requiring early booking and ticket pick-up (Taylor, 1995c).

During the summer of 1995-6, North by South operated a passenger-only ferry, The Straitrunner (196 seats), from Paremata (25km north of downtown Wellington) to Picton. On 7 December, the new entrant had planned to start a service between the city of Porirua (north of Wellington) and Picton, but it was blocked by Tranz Rail which refused access to its original terminal site across Tranz Rail land (Shelton, 1995b). The company had to look for a new site and one was found near the Mana Sailing Club, but it was necessary to build its own facilities. When the work was finished the StraitRunner, a brand new 31-metre monohull passenger ferry, was finally able to make her first trip, on 19 December. The crossing time was one hour and 45 minutes and a one-way adult ticket

\footnotetext{
${ }^{9} \mathrm{~A}$ standard one-way adult fare was $\$ 45$, although a special $\$ 35$ return was offered toward the end of the summer (Currie, 1995a).

$10 \$ 45$ a standard Wellington-Picton fare (Currie, 1995a).
} 


\section{THE COOK STRAIT FERRIES}

cost $\$ 35$ - cheaper than The Interlsland's fast and conventional ferries. The Lynx started its second season two weeks earlier, on 4 December.

At the beginning of 1996, the Maritime Safety Authority decided to investigate North by South, because it was operating the StraitRunner during heavy seas. Ferry sailings often proved to be unreliable because the StraitRunner's limited operating parameters prevented it from sailing in seas higher than 2.5 metres. In February, a drop in passenger numbers forced the company to revise its timetable, close its Wellington office and lay off four staff (Finnegan, 1996a, b).

In the first days of May, North by South went into receivership owing nearly $\$ 1.5$ million, ending its service across Cook Strait (Shelton, 1996). The company had made substantial losses, with most of the money spent on promotion and set-up costs. In the end 21 staff lost their jobs. It was estimated that StraitRunner carried between 35,000 and 40,000 passengers, considering that it lost eighteen days in March due to mechanical repairs and maintenance (Finnegan, 1996c), during its five month service. Breakdowns and bad weather can be considered as the main cause for the service closure.

From the Tranz Rail perspective, once again, the second summer season was not so successful for The Lynx. According to Finnegan (1996c), during the four-month period, passenger numbers on the ship fell 37,000 when compared to the same period the previous season $(212,000$ against 175,000$)$. The Lynx also lost about 3.5 per cent of sailings, due mainly to bad weather and mechanical reasons.

The Lynx's third summer season started on December 13, 1996, and ended on April 2, 1997. This was the first season it ran without a competitor.

On 6 December 1997, Condor 10 arrived back in Wellington for The Lynx's fourth season of operation. As in previous years, the service ran from the beginning of December until Easter (Pryce, 1997). In early 1998, another operator, Cook Strait Sea Cat Ferries, started the first year-round, passenger-only, fast-ferry service between Porirua and Picton (Collier \& Harraway, 1999).

The ferry Te Hukatai commenced commercial service on 21 February. It was a $24 \mathrm{~m}$ long, 208-gross-tonnage, able to carry 150 passengers and had a bar, café and outdoor viewing decks (see Figure 5.1). Services started with three daily Porirua-Picton return sailings (Green, 1998). Opening fares undercut those of Tranz Rail's fast ferry The Lynx by $\$ 14$ (adults fares were $\$ 45$ each way and children $\$ 25$ ).

Due to her small size, Te Hukatai was barred from operating if wave height exceeded three metres and it was subject to speed restrictions. According to Pryce (1998a), she 
started to have problems two days after her inaugural trip. On 23 February, all trips were cancelled because of strong northerlies in Cook Strait. The following day, the same happened due to heavy rain. Many other cancellations happened, due to technical problems (25 March), storm force northerlies in Cook Strait (29 March) and propeller repairs (31 March-4 April). Further problems were experienced again from 30 April to 4 May.

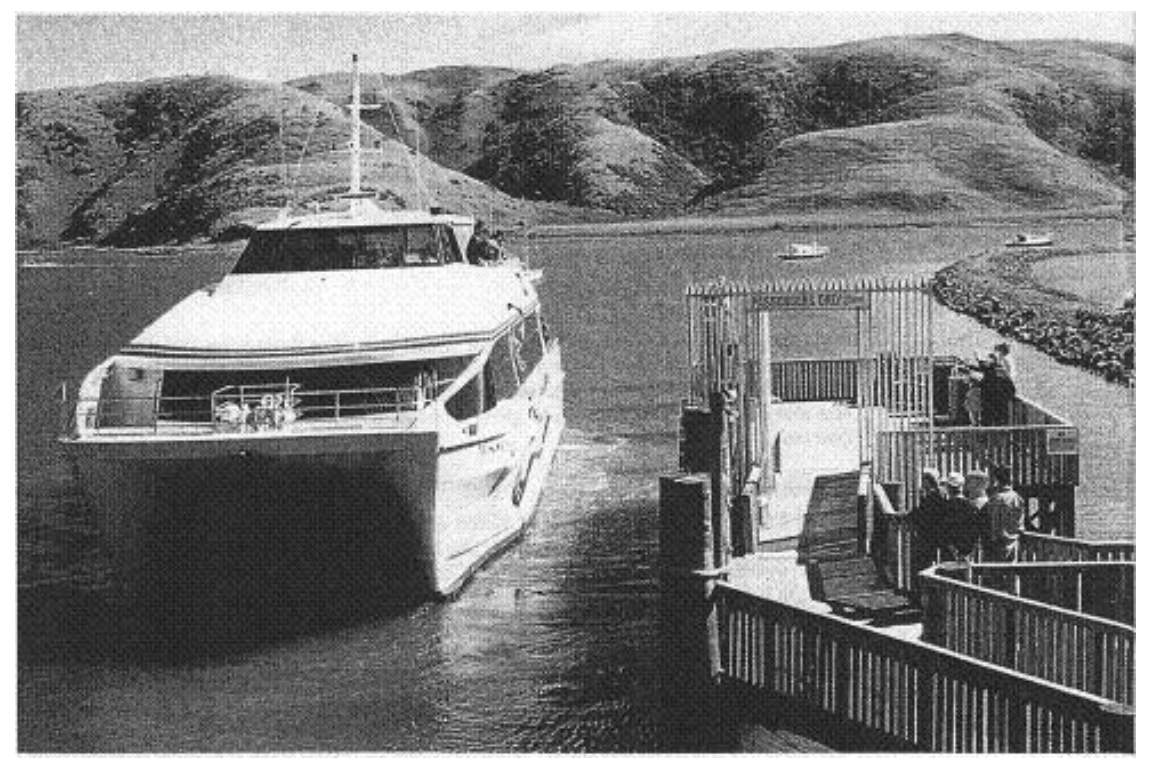

(Photo by M. H. Pryce)

Figure 5.1 The Porirua-Picton fast ferry Te Hukatai.

When Te Hukatai resumed service, Cook Strait Sea Cat Ferries introduced the winter timetable, which consisted of two return sailings per day, instead of three as in summer (Pryce, 1998b). The vessel became the first fast ferry to run during the winter season. However, bad weather and mechanical problems kept preventing the vessel from sailing on several days. On 4 December, the summer timetable was introduced again, with a service of three return sailings per day (Pryce, 1998c). One year after Te Hukatai's first trip, it was estimated that it had carried over 50,000 passengers and made well over one thousand commercial crossings of Cook Strait (Pryce, 1999a). On 19 April 1999, the service reverted to its winter timetable of only two return crossings daily.

The beginning of 1999 brought many changes to the ferry scenario. Firstly, Te Hukatai succeeded in being the first all year round fast ferry. Secondly, on 1 February 1999, The Interisland Line introduced a new conventional ferry Aratere ("Quick Path") on its 'The Interislander' service (see Figure 5.2). The vessel cost US\$ 106 million and was able to carry 350 passengers and 150 cars. Passengers were carried on two decks with a full 
range of retail food and beverage outlets and some entertainment facilities, such as a cinema, gaming machines and TV lounges. Three other decks were designated to store RoRo cargo, including 425m of rolling stock (Pryce, 1999a). Finally, another competitor, Fast Cat Ferries, started to operate a fast ferry - TopCat - on 10 May 1999.

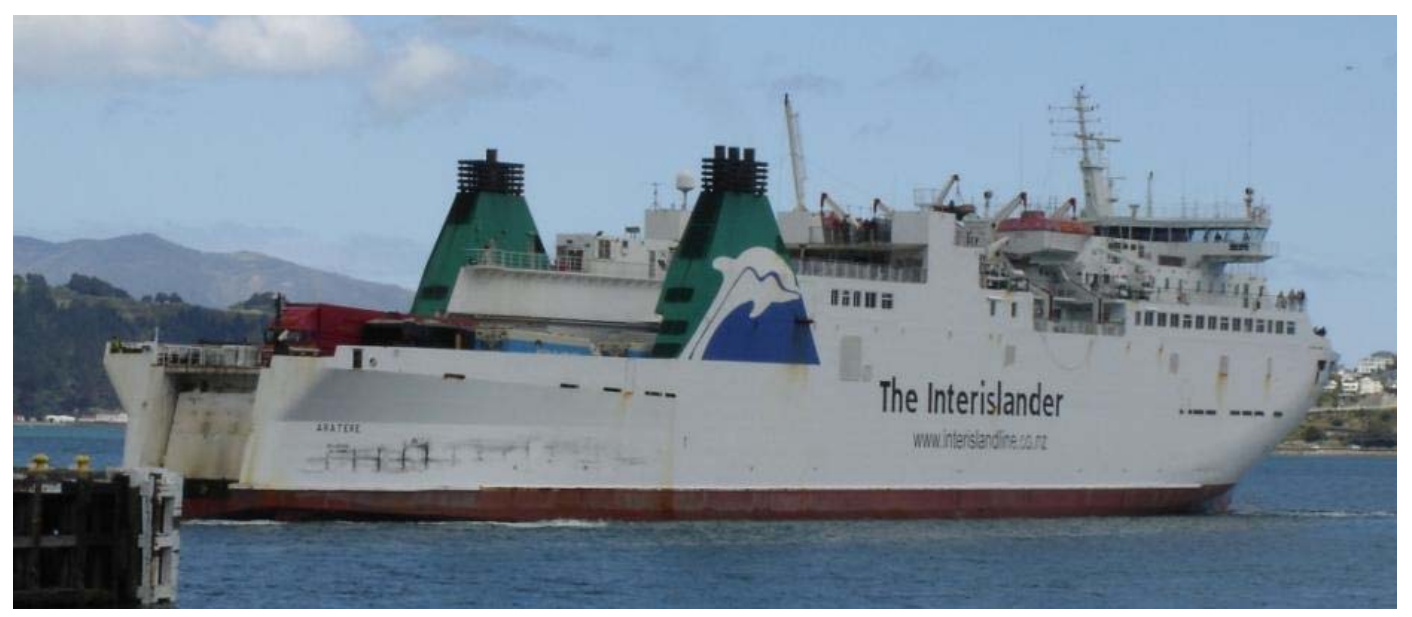

Figure 5.2 Aratere arriving in Wellington.

(Photo by Gui Lohmann)

Fast Cat Ferries Ltd was a company registered in Christchurch with Roger Swolf and Brooke McKenzie as directors (Pryce, 1999a). They were the same businessmen that in 1994-5 operated the Albayzin in partnership with Buquebus (Currie, 1995b, d). Fast Cat put in operation the vessel called Incat 050 (see Figure 5.3) whose trading name was TopCat (much as Condor 10 was 'The Lynx'). Incat 050 weighed 550 tonnes and travelled at more than 47 knots, which meant a Wellington-Picton trip in one and a half hours. Fully laden, it could carry 180 cars, a dozen semi-trailers and 550 passengers. Table 5.1 shows some of the attributes for the different vessels in operation by the end of May 1999.

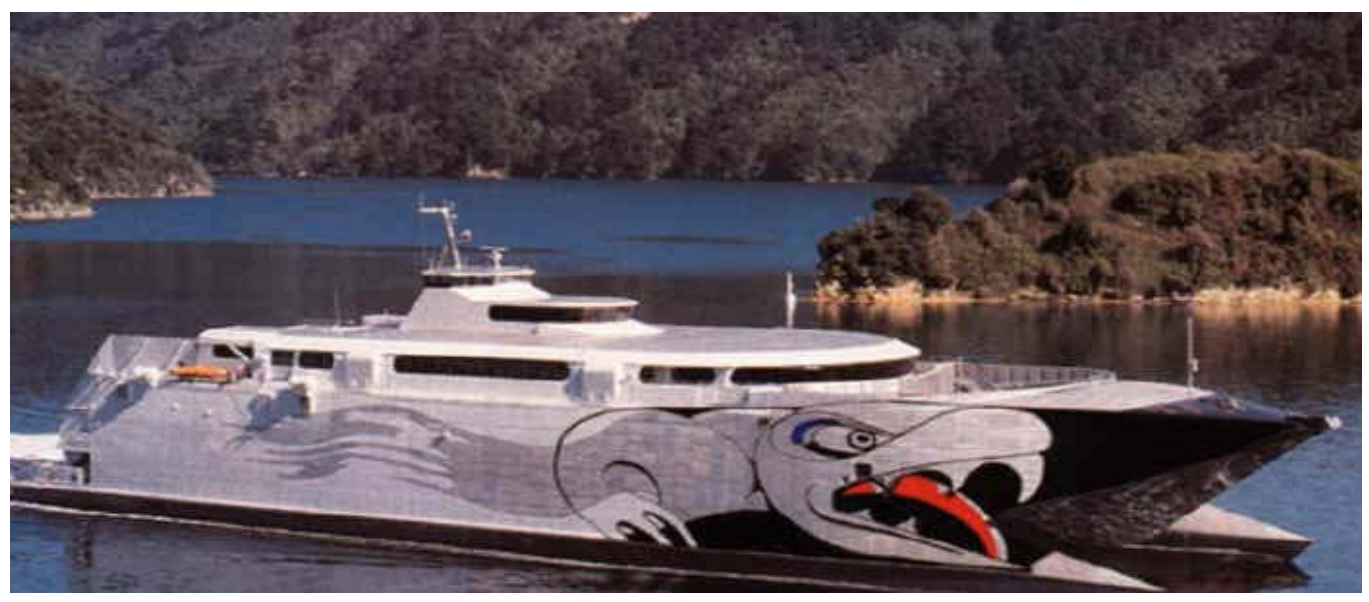

(Photo by Fast Cat Ferries)

Figure 5.3 Incat 050, Fast Cat Ferries' TopCat ferry. 
With the arrival of Top Cat, Tranz Rail announced that it would retain Condor 10 for a limited winter service, operating the vessel at weekends only (Pryce, 1999b). By this time, there were three fast ferry vessels (Te Hukatai, Incat 050 and Condor 10) running during the low season, in addition to the three conventional ferries. All together, the fast ferries offered more than 3,000 seats daily across Cook Strait, for an average demand of about 400-passenger crossings a day. As a result, Sea Cat Ferries ceased operations and closed down the service on 1 June 1999 (Pryce, 1999b). Although Sea Cat Ferries was well financed and had a carefully run operation, the company could not compete with the two other bigger vessels during the off-peak season, and the introduction of a new airline, CityJet $^{11}$, linking Wellington to Blenheim and Nelson (O'Connor, 2000).

Table 5.1 Ferry services comparison - May 1999.

\begin{tabular}{|l|l|l|l|l|}
\cline { 2 - 5 } \multicolumn{1}{c|}{} & \multicolumn{1}{c|}{ Top Cat } & \multicolumn{1}{c|}{ The Lynx } & \multicolumn{1}{c|}{ Te Hukatai } & \multicolumn{1}{c|}{ Aratere } \\
\hline Frequency & Two daily returns & $\begin{array}{l}\text { Two returns Friday, } \\
\text { Saturday and Sunday }\end{array}$ & $\begin{array}{l}\text { Two daily returns } \\
\text { except Tuesdays in } \\
\text { winter }\end{array}$ & $\begin{array}{l}\text { The Interislander service had } \\
\text { nine returns a day, with } \\
\text { Aratere doing three of them }\end{array}$ \\
\hline $\begin{array}{l}\text { Total } \\
\text { journey time }\end{array}$ & $1 \mathrm{hr} 30 \mathrm{~min}$ & $1 \mathrm{hr} 45 \mathrm{~min}$ & $1 \mathrm{hr} 45 \mathrm{~min}$ & Three hours \\
\hline Fares (\$) & $\begin{array}{l}\text { Standard adult one } \\
\text { way 39-59; return 60- } \\
80 ; \text { car one way 125- } \\
190\end{array}$ & $\begin{array}{l}\text { Standard adult one way 30- } \\
\text { 59; return 60-80; car one } \\
\text { way 95-190 }\end{array}$ & $\begin{array}{l}\text { Standard adult one } \\
\text { way 49; return 59- } \\
89 ; \text { no vehicles }\end{array}$ & $\begin{array}{l}\text { Standard adult one way 23- } \\
\text { 46; return 45-64; car one way } \\
83-165\end{array}$ \\
\hline Facilities & $\begin{array}{l}\text { VIP lounge for invited } \\
\text { guests }\end{array}$ & $\begin{array}{l}\text { Computer games } \\
\text { machines, play area for } \\
\text { children, souvenir and } \\
\text { confectionary shop, } \\
\text { information centre, travel } \\
\text { and accommodation } \\
\text { booking etc. }\end{array}$ & none & $\begin{array}{l}\text { Movie screening, computer } \\
\text { games machines, two } \\
\text { television rooms, play room } \\
\text { for children, souvenir and } \\
\text { confectionary shop, } \\
\text { information centre, travel and } \\
\text { accommodation booking etc. }\end{array}$ \\
\hline
\end{tabular}

(Source: O'Connor, 1999)

In June 1999, however, Condor 10's owners came up with a better deal and accepted a lease for the vessel on the east coast of the United States, leaving Incat 050 as the only fast ferry over the winter, with two return trips each day. Consequently, Top Cat heralded the introduction of fast freight during wintertime and soon proved popular with freight operators and the travelling public alike. Moreover, the off-season establishment coincided with The Lynx's annual closedown, giving Fast Cat a ready-made pool of welltrained staff to be hired.

During the following summer season, Tranz Rail introduced the Condor Vitesse $(5,007$ gross tonnage, 800 passengers and 173 cars), substituting the Condor 10 after five summer seasons. Condor Vitesse was newer and slightly larger (by $12 \mathrm{~m}$ ) than the former

\footnotetext{
11 In December 1999, CityJet closed after being grounded by the Civil Aviation Authority.
} 


\section{THE COOK STRAIT FERRIES}

"The Lynx" fast ferry Condor 10. The vessel commenced commercial sailings on 8 December 1999 (Rails, 2000a). 26 April 2000 was her last scheduled sailing for this season (Pryce, 2000a).

At the same time, Fast Cat Ferries was considering starting a freight-only service across Cook Strait, so it could operate more often in rough weather. The rationale was to cut cancellations (from four to two per cent) in order to improve reliability for freight movers (Pryce, 2000a). This was one of the signs that the operation was not satisfactory.

In July 2000, Marlborough District Council passed a bylaw halving the speed of fast ferries to 18 knots (33kph) through the Sounds because of concern that their wakes were damaging the environment and private property. As a result, fast ferries would cruise Cook Strait in two hours and fifteen minutes (half an hour longer than they used to travel), in comparison to the three-hour trip of a conventional ferry. The advantages of a fast ferry journey became less appealing taking into account its more expensive fare. These new speed restrictions imposed on the fast ferries soon spelt the end for Fast Cat Ferries and it too withdrew from service (O'Connor, 2000). Incat 050's last commercial sailing was on 3 November 2000, after having completed 2,334 commercial voyages across Cook Strait (Pryce, 2000b). Other reasons for this decision included a rise in fuel costs, the weak New Zealand dollar and intense competition on Cook Strait (Rails, 2000b).

The ending of sailings by Fast Cat Ferries was the catalyst for some significant changes to The Interisland Line service. Firstly, on 10 December 2000 when the fast ferry service resumed, a new vessel, The Lynx - 6,581 tons, 800 passengers and 260-car capacity -, was brought into operation. Unlike its predecessor, The Lynx was able to carry commercial vehicles (trucks) and to cruise at 38 knots. Because of the eighteen-knot speed limit in the Marlborough Sounds, The Lynx was scheduled to make two return sailings per day, instead of three as in the past. Secondly, The Lynx became a year-round service (Rails, 2000b, 2001). Thirdly, on 27 March 2001 Arahanga, a conventional ferry, was withdrawn from service, after 28 years and 32,664 crossings of Cook Strait (Pryce, 2000c).

\subsubsection{The Cook Strait Market during the Data Collection Process}

During the years 2001-2002, The Interisland Line was, once again, the only ferry operator. However, at the beginning of 2003, Strait Shipping introduced a passenger and vehicle service called Bluebridge. The company was founded in March 1992 and it started transporting livestock and general cargo between Wellington, Picton and Nelson (Strait Shipping, 2003). On 3 January, the 137-metre-long Santa Regina (see Figure 5.4) began 
to operate two or three return Wellington-Picton sailings a day, depending on the time of the year. Later, this French ferry, initially carrying 100 passengers and 300 cars or sixty commercial trucks, was upgraded in order to accommodate 370 passengers (Pryce, 2003a). This eighteen-knot conventional ferry has an estimated crossing time of three hours and twenty minutes. At the end of September, Bluebridge opened a new passenger terminal on Waterloo Quay, just opposite the railway station. Previously Bluebridge had just a small office in the port of Wellington, which was difficult to find.

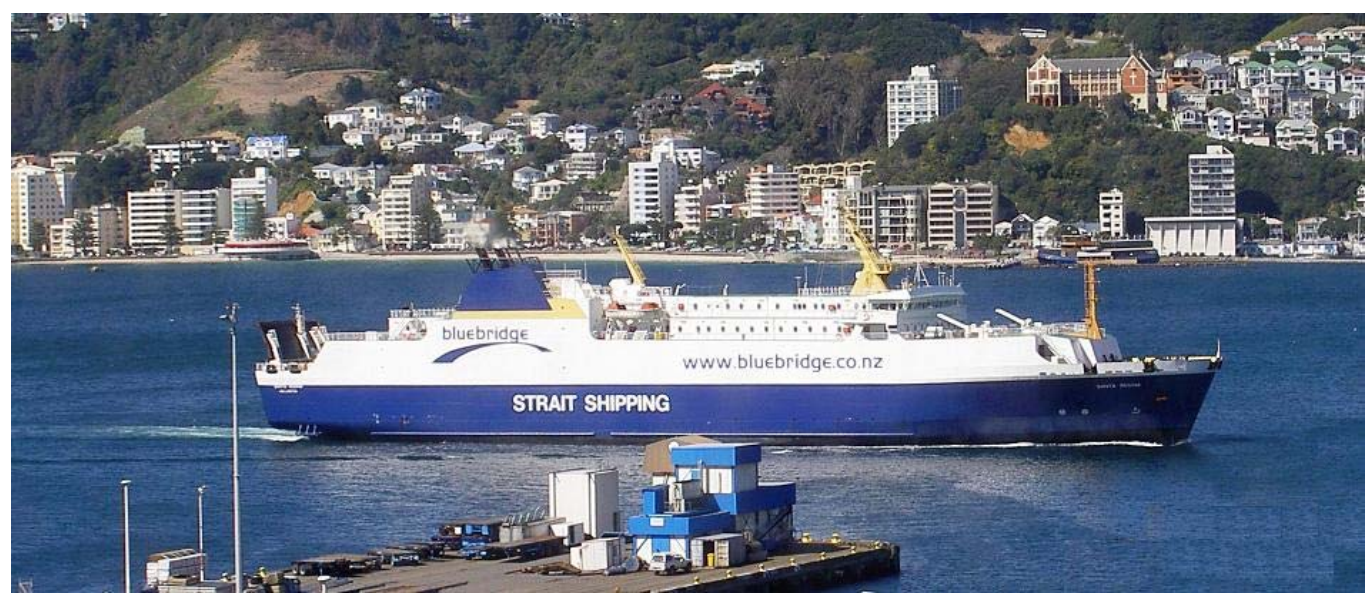

(Photo by Gui Lohmann)

\section{Figure 5.4 The Bluebridge ferry, Santa Regina.}

The new competitor forced The Interisland Line to make some changes in its operations. Firstly the new The Lynx, a 91-metre catamaran known as 'The Cat' (see Figure 5.5) was introduced. The two storey fast ferry offered a range of seating options, an attractive central skylight atrium and plenty of outdoor sundeck areas, being able to accommodate approximately 760 passengers and 175 vehicles. It also featured a separate bar and café; children's play area and shop. The wave-piercing catamaran was slightly shorter than the previous 98-metre vessel, but it had comparatively more power. The main difference between the two vessels was that the replacement fast ferry focused purely on the needs of the passenger market. Back in 2000, the decision to operate a regular daily service of The Lynx year-round was introduced partly to address the freight customers' needs, as it was the first freight-capable fast ferry. But with the introduction of the freight ship Purbeck in The Interisland Line's fleet, there was less business requirement to operate a daily passenger fast ferry service over the winter (Pryce, 2003b). Savings of $\$ 8$ million per annum were made from this re-tonnage exercise, which went someway to mitigate the effects of increased competition on the Cook Strait in recent months. The replacement fast ferry was operated year-round, but with a reduced weekend-only schedule over the 
winter months. Its service commenced at the beginning of August 2003 - in time to cover the period when Aratere was in Auckland for her regular dry-dock maintenance check.

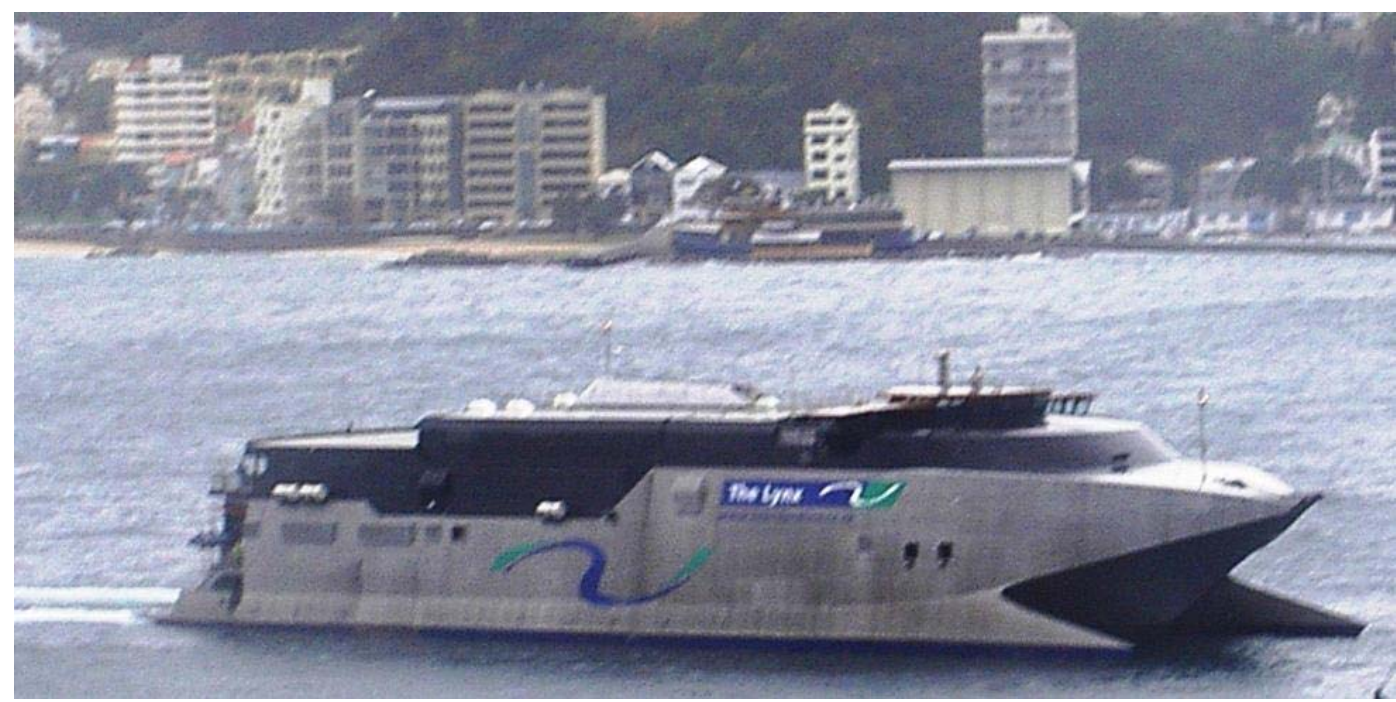

(Photo by Gui Lohmann)

Figure 5.5 The Lynx ferry, The Cat, in Wellington - September 2004.

In February 2003, The Interisland Line began advertising in national newspapers with new special fares on The Lynx, clearly designed to undercut Strait Shipping. A car would cost only $\$ 103$ one-way and a passenger only $\$ 34$, compared with Strait Shipping's fares of $\$ 110$ for a car and $\$ 40$ for a passenger. However, only a relatively small percentage of bookings were available at this special rate (Pryce, 2003c). Table 5.2 shows the timetable and price structure of the various ferry services operated by both companies.

Despite this new attempt to break The Interisland Line's monopoly, the company has always had a competitive advantage over newcomers. Firstly, it has had a long association with the ferry service - over forty years - so most people, when they think "Cook Strait ferries" think "The Interisland Line". Potential users will, therefore, approach the company first rather than an operator they know little about. Secondly, that company also has an equally long-established network of booking offices and contacts in New Zealand and abroad. A new operator, starting from scratch, will find it difficult to build up such an easily accessible nationwide booking service, and will certainly not have The Interisland Line's deep knowledge of what the market wants. Thirdly, it can offer an unrivalled frequency of service through its various services and vessels. A rival firm, starting off with just one ship does not have that advantage (Rails, 1994).

Among The Interisland Line's previous competitors, however, Strait Shipping was not only the first to have more experienced background knowledge of the Cook Strait, but also the first to operate a conventional ferry service. This fact could make a substantial difference 


\section{THE COOK STRAIT FERRIES}

for the company, because, as the next section will show, environmental issues regarding the fast ferries on Marlborough Sounds have escalated in the last few years. As a result, fast ferries are not as appealing as they used to be. In fact, after more than ten years of operation, the Lynx service ceased to exist in April 2005, with the Interisland Line substituting it for a large conventional ferry, Kaitaki. This vessel carries approximately 1,600 passengers and 600 cars.

Table 5.2 Ferry services comparison - Spring 2003 and Summer 2004.

\begin{tabular}{|c|c|c|c|c|}
\hline Operator & Strait Ship Company & \multicolumn{3}{|c|}{ The Interisland Line } \\
\hline Service & Bluebridge & The Lynx & \multicolumn{2}{|c|}{ The Interislander } \\
\hline Vessel & Santa Regina & The Cat & Arahura & Aratere \\
\hline Frequency & $\begin{array}{l}\text { Two daily returns - up to } \\
\text { three returns some days } \\
\text { during peak season } \\
\text { (Dec-Jan) }\end{array}$ & $\begin{array}{l}\text { Low season: two returns } \\
\text { Friday, Saturday and } \\
\text { Sunday. High season: } \\
\text { two returns daily }\end{array}$ & Two to three daily returns & $\begin{array}{l}\text { Two to three daily } \\
\text { returns except } \\
\text { Mondays in winter }\end{array}$ \\
\hline $\begin{array}{l}\text { Total } \\
\text { journey time }\end{array}$ & $3 \mathrm{hr} 20 \mathrm{~min}$ & $2 \mathrm{hr} 15 \mathrm{~min}$ & Three hours & Three hours \\
\hline $\begin{array}{l}\text { Fares }(\$)- \\
\text { one way }\end{array}$ & $\begin{array}{l}\text { Standard adult } 40 \text {; car } \\
110\end{array}$ & $\begin{array}{l}\text { Standard adult 30-55; } \\
\text { car 100-180 }\end{array}$ & $\begin{array}{l}\text { Standard adult } 30-55 \text {; car } \\
100-180\end{array}$ & $\begin{array}{l}\text { Standard adult 30- } \\
55 \text {; car } 100-180\end{array}$ \\
\hline Capacity & $\begin{array}{l}370 \text { passengers and } \\
150 \text { cars (before the } \\
\text { refurbishment in } \\
\text { October could fit } 100 \\
\text { passengers only) }\end{array}$ & $\begin{array}{l}760 \text { passengers and } 175 \\
\text { cars }\end{array}$ & 997 passengers and 126 cars & $\begin{array}{l}369 \text { passengers } \\
\text { and } 130 \text { cars }\end{array}$ \\
\hline Facilities & $\begin{array}{l}\text { Café, TV lounge, quite } \\
\text { area. }\end{array}$ & $\begin{array}{l}\text { Video games, play room } \\
\text { for children, bar, café } \\
\text { and information counter. }\end{array}$ & $\begin{array}{l}\text { The same as The Lynx, plus } \\
\text { movie screening, travel and } \\
\text { accommodation booking, } \\
\text { workstations and VIP Club } \\
\text { Class. }\end{array}$ & $\begin{array}{l}\text { The same as } \\
\text { Arahura }\end{array}$ \\
\hline
\end{tabular}

\subsubsection{Fast Ferries and Environmental Issues}

Shortly after the fast ferries' service began before Christmas 1994, residents in Tory Channel noted that the wash produced by Condor 10 and Albayzin was substantially more powerful than the wash created by conventional ferries (Bell, 1994, 1995b). They became aware of numerous changes and effects along the Tory Channel shorelines, i.e. substantial erosion, the stranding and destruction of marine and bird life, the washing up of large boulders onto the shore, disturbance of ancient burial grounds, potential damage to moored boats and structures like boat sheds and ramps, and danger to individuals, particularly small children (Pardy, 1995). Soon, some residents formed a local organisation called Save the Sounds - Stop the Wash (STS) that applied for an interim enforcement order (Taylor, 1995a). STS, Te Atiawa Trust and the Minister of Conservation's applications were heard together before the Planning Tribunal in March and April 1995 (Pardy, 1995). 


\section{THE COOK STRAIT FERRIES}

In early May, the judge decided not to make an enforcement order restricting the operation of the fast ferries. The Tribunal was unable to find that the effects were of a nature serious enough for a cessation order. Moreover, ferry service was considered of national importance and should not be the subject of a cessation order on the basis of inconclusive and subjective evidence (Milne \& Tiller, 1995). Finally, although the impact of the wash along the shore was found to have been severe enough to alter the equilibrium of the ecosystem, a new 'ecological equilibrium' would be established (Pardy, 1995; Taylor, 1995b). It was decided, however, that the area would be monitored by scientists during the winter when fast ferries were not running, in order to compare data with the next summer season (Shelton, 1995a; Taylor, 1995c).

On the other side of the strait, however, speed restrictions within Wellington Harbour had been placed on both fast ferries (Condor 10 and Albayzin). According to Bell (1995a), they had to stop at the first leading light at Ward Island to allow the pressure wave of their wakes to dissipate before continuing at the $37 \mathrm{kph}$ speed restriction for ships west of Point Jerningham. In the Albayzin's case, the situation was even worse. Because the vessel entered the inner harbour to berth at Waterloo Quay, it had to travel at $18 \mathrm{kph}$ from Point Jerningham.

Even so, the fast ferry introduction has clearly raised the environmental issue in recent years; arguments over the effects of ferry wash go back to the start of the service in 1962. These complaints tend to surface each time there is a change in the service, either the introduction of a new ship or a change in the pattern of sailings. In 1986, for example, the Marlborough Sounds Maritime Park Board was concerned about the problem of erosion caused by ferry wash. On this occasion, it was claimed that the ferries were continuing to travel at up to 21 knots in the Sounds and that their wash had undermined a lighthouse in the area (Rails, 1995).

In January 2000 , the issue of the fast ferry wash was raised again. By this time, seven vessels, Aratere, Arahura, Arahanga, Condor Vitesse, Incat 050, Suilven and Straitsman $^{12}$, could combined make up to eighteen sailings daily from Picton during the summer months (Pryce, 2000c). For this reason in July 2000, Marlborough District Council decided to pass a bylaw restricting the speed of fast ferries from thirty-five knots (64kph) to eighteen knots (33kph) in the Sounds (Marlborough District Council, 2000; Pryce, 2000a). On 12 September, the decision was made official, to take effect from 15 December 2000 (Marlborough District Council, 2000; Pryce, 2000b). Hence, fast ferries'

\footnotetext{
12 The last two operating as freight ferries.
} 
trip time increased from one hour and forty-five minutes to two hours and fifteen minutes. Moreover, the frequency of their services changed from three to two round trips per day. Table 5.3 presents some of the negative and positive aspects of the operation of large, high-speed ferries in the Marlborough Sounds.

Table 5.3 Positive and negative impacts of large, high-speed vessels on Marlborough Sounds.

\begin{tabular}{|l|l|}
\hline \multicolumn{1}{|c|}{ Positive impacts } & \multicolumn{1}{c|}{ Negative impacts } \\
\hline National transport & Land form: significant changes to beach form \\
\cline { 2 - 2 } $\begin{array}{l}\text { issues: large, high } \\
\text { speed vessels, fast } \\
\text { ferries in particular, } \\
\text { provide an important link } \\
\text { between the North and }\end{array}$ & Property: damage to structures such as jetties, loss in property values brought about by \\
changes in beach form and land instability
\end{tabular}

(Source: Marlborough District Council, 2000)

\subsubsection{The Clifford Bay proposal}

Apart from environmental issues, another relevant topic for the ferries is the proposal to build a ferry terminal in Clifford Bay (see Figure 5.7). If one day it becomes a reality it could have a great impact on the transport and tourism sectors across Cook Strait.

The establishment of an inter-island ferry terminal in Clifford Bay, near Lake Grassmere, has been discussed off and on for many years, at least since 1979. Some of the reasons for proposing a new terminal location there include (Rails, 1995):

1) Clifford Bay is 43 nautical miles from Wellington, while Picton is 52 nautical miles;

2) The run to Clifford Bay can be done at normal speed all the way, whereas on the Picton run there are some limitations, due to environmental impacts (as seen in the previous section);

3) Because of the shorter distances and higher speeds, journey times at sea would be reduced by 40 minutes. Total time by rail to Christchurch would be reduced by two hours;

4) The grade the railway climbs between Hauwai and Picton is the steepest on the Auckland-Christchurch trunk through route by a wide margin, being 1 in 37 . The route between Clifford Bay and Christchurch would avoid it; 
5) The Interisland Line would operate its own terminal in the South Island, not having to pay wharfage and harbour dues to Marlborough Harbour Board. It was not a coincidence that in 1992, when the 30-year leases on the two terminals came up for renewal, the issue of a terminal in Clifford Bay was raised again.

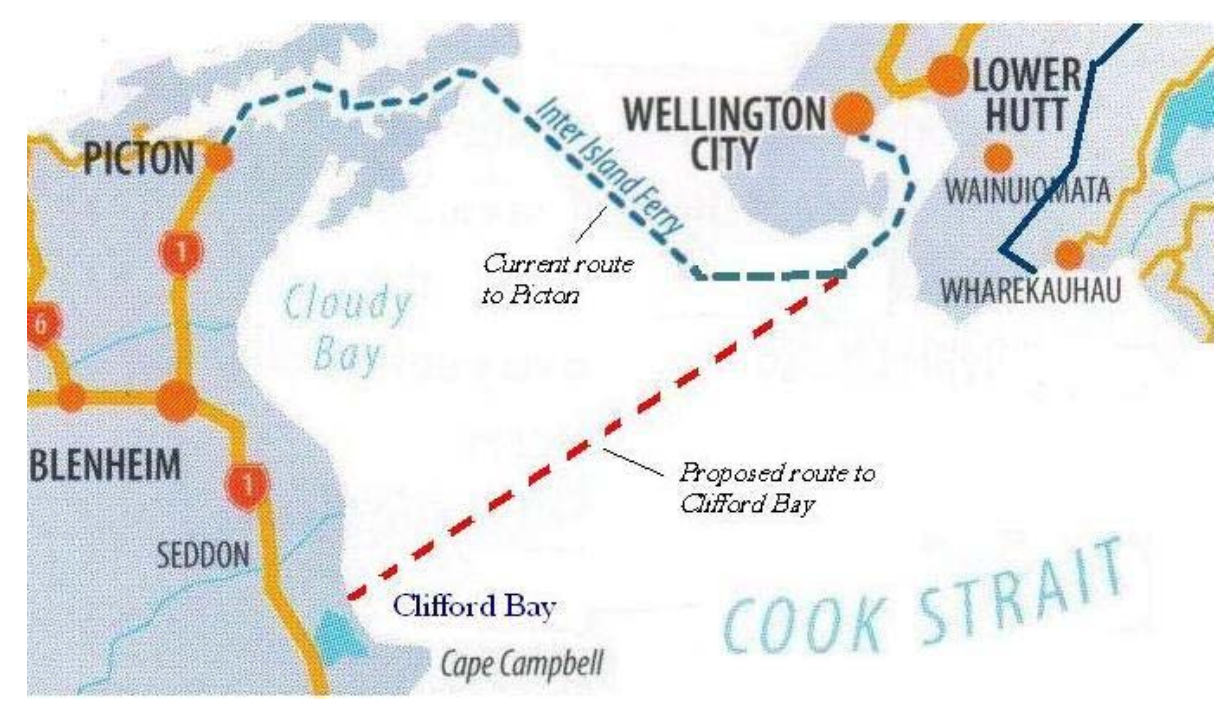

\section{Figure 5.6 Proposed route to Clifford Bay.}

Although the Clifford Bay proposal has a lot more to do with rail and freight than ferries and passengers, this would also benefit the latter. For example, The Lynx fast ferry should be able to make a Cook Strait crossing in a little more than an hour. With the rail trip on to Christchurch reduced by another hour, it might be possible to run a combined WellingtonChristchurch fast ferry Lynx Express trip within six hours. Similar reductions in driving times would also be enjoyed by passengers travelling by car. Overall, the travelling times from Wellington to Blenheim and Nelson would also be less than they are now. Another factor is that by reducing the crossing time, more trips could be made each day using the same fleet. In addition, having the exclusive use of Clifford Bay would give The Interisland Line a competitive advantage that other competitors operating to Picton would not have.

Some of the questions that may be raised from the Clifford Bay proposal and that need to be addressed in this research are:

1. Which places will benefit from it? Which will not?

2. How broad will these changes be? In general, how will they benefit or harm tourism in the Centre Stage region?

3. Will these changes be only beneficial from a transport point of view? How can they also benefit the tourism sector? 
4. What will be the impact of having a new gateway in the Marlborough region? What will happen to Picton as a destination and gateway if another ferry terminal was built in Clifford Bay?

5. What would the passenger reaction be? What would be the percentage of passengers that would choose to travel via Clifford Bay?

\subsection{Conclusion}

This chapter has described the evolution of the Cook Strait ferries in recent years. Currently, two ferry companies provide ferry services across Cook Strait. They are the Interisland Line, which has been in the market for more than forty years, and Bluebridge, which started its operations in 2003. In the last decade, several companies have tried to compete with the incumbent ferry company, all of them operating fast ferries. Bluebridge is the first to use conventional ferries and it is also the Interisland Line's longest-lasting competitor. With previous experience transporting freight across Cook Strait, Bluebridge's parental company, Strait Shipping, is most likely to establish a new scenario in the market with two co-existing companies.

The presence of two ferry operators increases the competition and offers passengers more options in terms of timetable. In addition, marketing and promotional campaigns stimulate traffic through the gateways, enhancing the possibility of passengers staying longer in the gateways. What is more, partnerships with tourism providers can be stimulated, even though by the time of the data collection Bluebridge had a flat rate policy, with bookings being made without intermediaries. Maybe in the future Bluebridge will be able to have the same competitive advantage as the Interisland Line, which has a widespread network of booking agents located in New Zealand and overseas.

Apart from the ferry competition, this chapter also presented two other key issues relevant to the ferry market: the fast ferry speed restrictions and the proposal to build a new terminal in Clifford Bay. As commented on Chapter 4, the threat of a new ferry terminal in the South Island stimulated local businesses and organisations to promote and develop Picton more as a tourist destination. The Clifford Bay proposal topic is particularly relevant to be considered in the data to be collected from passengers and stakeholders. For the passengers it is necessary to analyse their reaction in terms of which gateway (Picton or Clifford Bay) would be more appealing. From the supplier's perspective it is appropriate to consider what would the impacts on Picton be if part of the passenger traffic were diverted to Clifford Bay. 


\section{THE COOK STRAIT FERRIES}

Another consequence of these two issues is to analyse how they have created obstacles between the Interisland Line and the tourism suppliers in Picton. This can have significant

effects on the likelihood of both sides being prepared to work together in order to set up strategies to increase the passengers' stay in both gateways, particularly in Picton. Most of these issues will be considered further in Chapter 8 . 


\section{Patterns of Demand}

\subsection{Introduction}

This chapter introduces a set of chapters that analyse the data collected for this research. This first chapter, which provides an overview of the main characteristics of the ferry passengers is presented in order to better understand the main socio-economic characteristics of the passengers, how they travel in New Zealand and what are the key issues associated with the ferry journey. Hence, this chapter aims to provide background information about the passengers so that the analysis of the nodal functions (Chapter 7) and the gateway and destination issues (Chapter 8) can be better understood. Passengers are the players who ultimately make the decision to stop, visit and stay longer in the gateways, where the need to understand their motivations.

In order to present such broad information about the ferry passengers, it was decided to segment the sample by passengers' place of origin, rather than using the various nodal functions presented in Figure 3.2. This analytical framework is used for three main reasons:

1. In order to compare the key characteristics of the ferry passengers by using information collected from the questionnaires as well as other sources of information, it is necessary to segment the data in a conventional format. Traditionally tourism research separates samples into domestic and international visitors. Doing the same in this part of the data analysis permits a comparison not only with the official statistical data and previous surveys, such as those conducted by the ferry companies, but also with the information provided by the suppliers in Chapter 8 where they analyse the passengers' behaviour while in the gateways;

2. As seen in the literature review, one particular variable to explain travellers' decision to stop and stay in gateways is the place of origin (Janin, 1982). In fact, it may be expected that ferry passengers living near the gateways will feel less inclined to stay longer in the gateways. There are two major reasons for this. The first is that the short distance between their place of origin and the gateway will not require them to use the gateway as a stopover in their trips. The second is that as these passengers live close to the gateways they are usually more familiar with it, perhaps because they already had the chance to visit Wellington and Picton on other occasions, so they might be less likely to take advantage of the novelty of passing through the gateways to enjoy a visit. Therefore, among domestic 


\section{PATTERNS OF DEMAND}

travellers it is necessary to establish certain regions in the country around the gateways so that a distinction can be made. One possibility is to consider the CSNZ region as a boundary for this market segmentation. Due to the proximity to the gateways, it is expected that passengers living in the CSNZ region will have different patterns with regards to the gateways when compared to domestic passengers living outside the CSNZ region (hereafter to be considered as domestic passengers). Considering these two sub-groups in only one category would probably not allow a more detailed understanding of the differences existing between them. Therefore, the analysis will be done segmenting the sample into international travellers, which according to Table 3.3, represent nearly $41 \%$ of the sample, CSNZ passengers (28.6\%) and domestic passengers (30.5\%);

3. The concepts of gateway travellers, overnight gateway visitors, stopover visitors and destination tourists proposed in Chapter 3 will be applied in Chapter 7 which will deal more precisely with the likelihood to convert these various functions into destination tourists. Nevertheless, this analysis will segment each of these functions in terms of the passengers' place of origin, i.e. CSNZ, domestic and international so that differences can be identified within the level of each function. Therefore considering the characteristics of the three groups of origin here will help to understand the differences when analysing data on Chapter 7.

This chapter is structured into three main sections, each addressing particular aspects of the demand. In most cases these sections coincide with one or more specific sections of the questionnaire. For example, the first is devoted to the passengers' profile (Section $G$ of the questionnaire). The second part of the chapter is about the passengers' trip and the information presented there derives from Sections $A, B$ and $C$ of the questionnaire. Finally, the third part focuses more specifically on the ferry journey using some of the data collected in Section D. Passengers' activities and opinions about Wellington and Picton (Section E) will be analysed in Chapter 7. The impact of the proposed Clifford Bay ferry terminal on the passengers' trip (Section F) will be considered, among other topics, in Chapter 8.

\subsection{Passengers' Profile}

This section presents the passengers' profile and their main socio-demographic characteristics. Its objective is to describe the type of passenger that travels on the Cook Strait ferries and relate this information to the key aspects relevant to this research. Some of the data presented here include a more detailed analysis of the passengers' place of 


\section{PATTERNS OF DEMAND}

origin (by country or region in New Zealand), age, occupation and annual household income of the respondents and the number of people in the respondents' travel group, as well as the relationship with them.

In terms of the passengers' place of origin, the map ${ }^{13}$ presented in Figure 6.1 illustrates the distribution of the passengers living in New Zealand (CSNZ and domestic), while Table 6.1 shows the participation of international passengers by country of origin. As the analyses done in this research segregate the demand in these three sub-groups, it is appropriate to explore them in more detail.

For passengers living in New Zealand, according to Figure 6.1, it is possible to make two principal comments about the regions where ferry passengers live. The first is that the regions where the three main cities in the country are located (Auckland, Wellington and Christchurch) represent approximately $45 \%$ of the passengers living in New Zealand. However, a second point to be made is that some of the regions located in the ferries' catchment areas (e.g. Wellington, Hutt Valley, Blenheim and Nelson \& Tasman) are overrepresented when compared with the country's population. One example is the case of Wellington, which accounts for one out of five passengers, while its population represents less than $10 \%$ of people living in the country. Another example is Blenheim which represents $6.2 \%$ of the passengers living in New Zealand, but accounts for only $0.66 \%$ of the country's population. Hence, proximity is a factor in the decision to choose or not choose the ferry as a transport option. Travellers undertaking an inter-island trip who live far from the ferry gateways may prefer to fly to their destinations as it will definitely represent a faster trip and, in some cases, a more affordable option. As the regions presented in the map used in the questionnaire do not match with the political regional division of New Zealand (see Chapter 3), a precise and further comparison between data presented in Figure 6.1 with the population living in each of these regions is not possible.

When comparing data from Figure 6.1 to those of the Ka Pai survey (see Chapter 3), it is still possible to note that they have the same order of importance. In this other survey, Wellington represents $19.1 \%$ of the passengers living in New Zealand, Canterbury $11.8 \%$ and Northland \& Auckland $10.6 \%$. The top three regions in the country represent respectively $45.4 \%$ and $41.5 \%$ for the survey conducted in this research and the Ka Pai survey.

\footnotetext{
${ }^{13}$ Note that the regions' boundaries are not the same as those used for official purposes.
} 


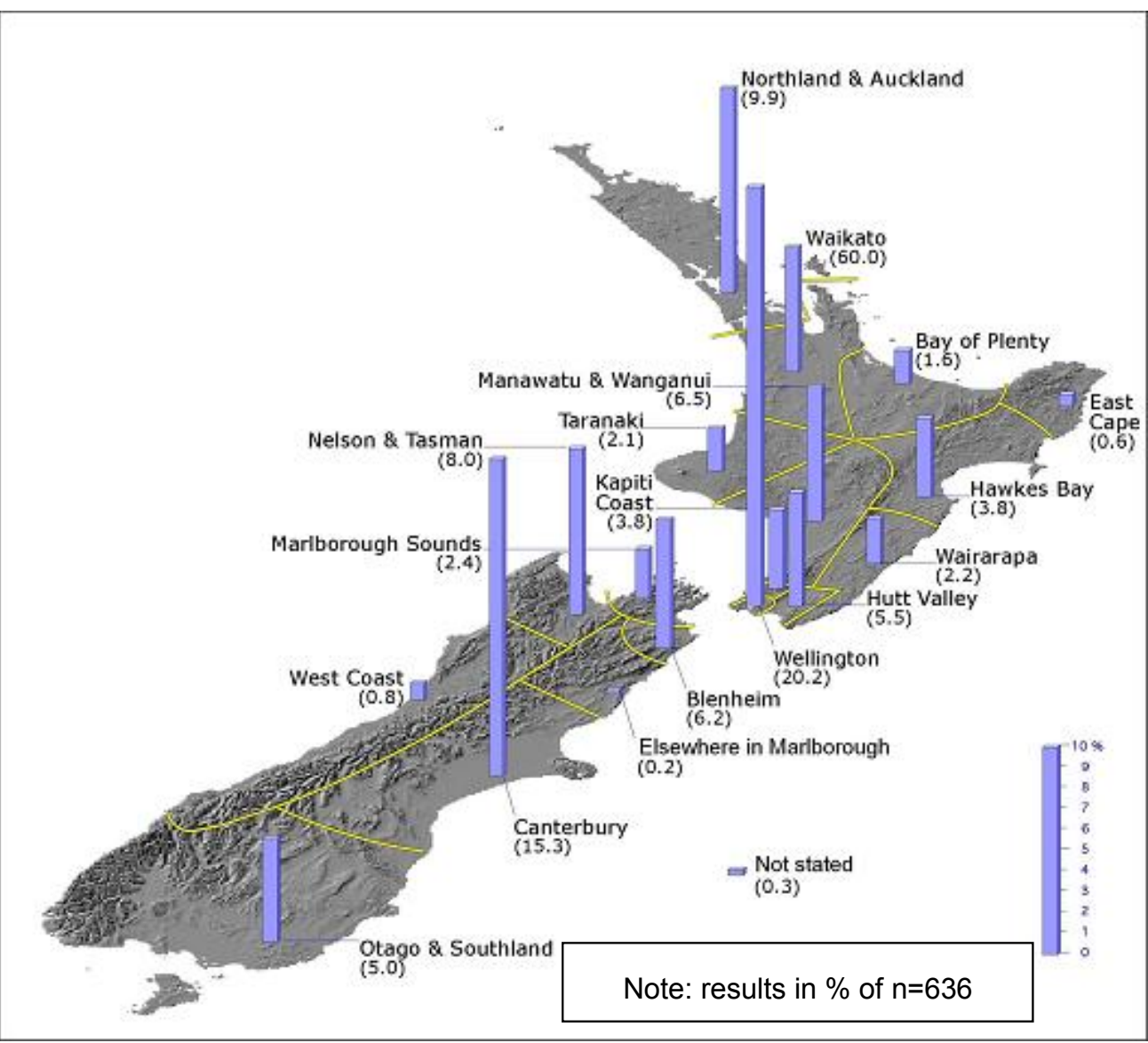

Figure 6.1 Place of origin for ferry passengers living in New Zealand.

For passengers living overseas, those coming from the UK, Australia, the USA and Germany correspond to two-thirds (67\%) of the international sub-group (see Table 6.1). When the country of origin of the international passengers is compared with the arrival of international travellers in New Zealand, it is then possible to identify some interesting trends. From Table 6.1 it becomes clear that there are certain markets that tend to use the ferries more than others. For example, while one in three international visitors to New Zealand is from Australia, they represent only one in five international passengers travelling onboard Cook Strait ferries. There are two major explanations for the mobility of Australians while travelling in New Zealand (see Figure 4.2). The first is that this market has a shorter length of stay - 13.3 days - with New Zealand being a short haul destination. The second is the existence of direct air flights to several gateways in the North Island and a couple in the South Island, which require them to travel less to reach their primary destinations. Asian countries are also under-represented, such as in the case of the Japanese market $(1.8 \%$ of passengers in comparison to $7.6 \%$ of total international visitors). China and South Korea are also two countries that are underrepresented in the passenger sample (each accounting for $0.7 \%$ of the passengers) when 
compared to their participation in visitor arrivals. As described in Chapter 4, particularly for the Japanese market (see Figure 4.2), the Asian market tend to tour less in New Zealand, making more use of domestic air transport and less use of the ferries, in comparison to other markets (see Table 4.1).

Table 6.1 Country of origin for international ferry passengers and total number of international arrivals - year ended September 2004.

\begin{tabular}{|c|c|c|c|c|c|}
\hline Country & $\begin{array}{c}\% \text { ferry } \\
\text { passengers } \\
(n=440)\end{array}$ & $\begin{array}{l}\text { Total visitor } \\
\text { arrivals (\%) }\end{array}$ & Country & $\begin{array}{c}\% \text { ferry } \\
\text { passengers } \\
(n=440)\end{array}$ & $\begin{array}{l}\text { Total visitor } \\
\text { arrivals (\%) }\end{array}$ \\
\hline UK & 29.5 & 11.3 & Ireland & 1.8 & 0.7 \\
\hline Australia & 20.7 & 32.6 & Japan & 1.8 & 7.6 \\
\hline USA & 10.2 & 10.1 & France & 1.6 & 0.7 \\
\hline Germany & 6.6 & 2.5 & Spain & 1.6 & 0.3 \\
\hline Canada & 4.1 & 1.9 & Switzerland & 1.4 & 0.8 \\
\hline Holland & 4.1 & 1.3 & China & 0.7 & 3.2 \\
\hline Sweden & 3.9 & 0.6 & South Korea & 0.7 & 5.5 \\
\hline Europe: other & 3.4 & -- & Other & 2.3 & -- \\
\hline Asia: other ${ }^{\left({ }^{*}\right)}$ & 2.7 & -- & Not informed & 0.9 & -- \\
\hline Denmark & 2.0 & 0.4 & (*) Singap & aysia & \\
\hline
\end{tabular}

(Source: TRCNZ, 2004a)

On the other hand, some markets are over-represented regarding the use they make of the ferries. Among them are travellers from the UK, Germany, Canada and other European countries, such as Holland, Sweden and Denmark. Of particular interest is the case of the UK. It accounts for almost three out of ten international ferry passengers, but represents just over one in ten international visitors. Germans are also another market that deserves some attention. Germany is the fourth largest international market for ferry passengers $(6.6 \%)$, despite accounting for only $2.5 \%$ of international arrivals. Americans are one of the few markets that have similar representation when comparing ferry passengers and the IVA (International Visitor Arrival) survey, with nearly $10 \%$ of in both cases.

Although language barriers could have been a factor leading to an under-representation of non-English speaking passengers, the pattern presented here is very much in line with the discussion from Chapter 4 , including the analysis of intra-national flows of five different countries of residence (see Figure 4.2). This figure describes the top seven international markets in New Zealand and considers the use of the ferries (Table 4.1). The results of Table 6.1 can also be compared to the research conduct by one of the ferry operators ( $\mathrm{Ka}$ 
Pai survey mentioned in Chapter 3). For the top five countries, the results are very much similar to those obtained for this research, with travellers from the UK and Ireland representing $37.9 \%$, Australia $22.2 \%$, the USA $11.8 \%$, Germany $6 \%$ and Canada $5.4 \%$.

Passengers' age is in general reasonably well balanced among the various groups (see Figure 6.2). The main age group for CSNZ (35.2\% of total) and domestic passengers $(32.7 \%)$ is from 35 to 49 years old. In the case of international passengers, the main age group ranges from 25 to 34 years old $(34.0 \%)$. International passengers are younger than those from New Zealand because the majority of them (53\%) are aged between 15 and 34 years old. This can explain why international travellers have a higher length of stay in New Zealand in comparison to travellers living in the country (see Table 6.6) and also the lower participation of international passengers travelling with dependants (see Table 6.4).

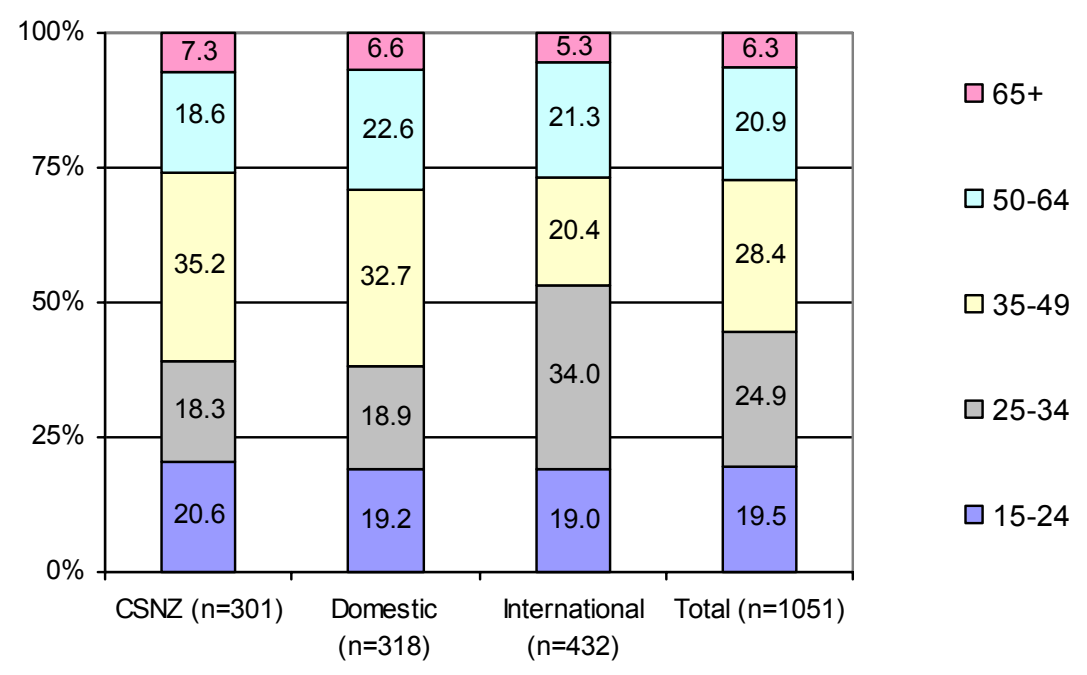

Figure 6.2 Age group of respondents.

As can be seen in Table 6.2, two main types of occupation can be identified among ferry passengers: professionals (39\%) and students (15.6\%). These two categories account for the majority of nearly all the sub-groups, representing $55.4 \%$ of CSNZ passengers, $49.3 \%$ of domestic passengers and $58.1 \%$ of international passengers. Two other categories that deserve some comment are those international passengers with agricultural/trade/manual employment and those who are not in paid employment. In the first case, their participation $(2.9 \%)$ is below the average of $8.1 \%$. This can be explained by the fact that New Zealand has a strong agricultural economy, which is not reflected in the diversity of the countries that compose the international market. In the second case, international passengers without paid employment at the time of their trip $(7.1 \%)$ were higher than domestic (1.9\%) and CSNZ (3\%) passengers. In this case it is relevant to mention that is 
common for overseas travellers to be taking time off work when they are travelling around the world for several months. As presented in Table 6.6, 19\% of international travellers are staying more than 36 nights away from home during their trips, with $9.4 \%$ being away more than two months.

Table 6.2 Respondents' occupation.

\begin{tabular}{|c|c|c|c|c|}
\hline & $\begin{array}{c}\text { CSNZ } \\
(n=298)\end{array}$ & $\begin{array}{c}\text { Domestic } \\
(n=318)\end{array}$ & $\begin{array}{l}\text { International } \\
\quad(n=420)\end{array}$ & $\begin{array}{c}\text { Total } \\
(n=1,036)\end{array}$ \\
\hline Professional & $36.9 \%$ & $35.8 \%$ & $42.9 \%$ & $39.0 \%$ \\
\hline Student & $18.5 \%$ & $13.5 \%$ & $15.2 \%$ & $15.6 \%$ \\
\hline Retired / Superannuitant & $11.1 \%$ & $7.9 \%$ & $11.4 \%$ & $10.2 \%$ \\
\hline Agricultural / Trade / Manual & $10.1 \%$ & $13.2 \%$ & $2.9 \%$ & $8.1 \%$ \\
\hline Managerial & $3.7 \%$ & $8.8 \%$ & $9.0 \%$ & $7.4 \%$ \\
\hline Clerical / Sales & $6.7 \%$ & $5.7 \%$ & $5.2 \%$ & $5.8 \%$ \\
\hline Semi technical / Skilled & $4.4 \%$ & $7.5 \%$ & $4.8 \%$ & $5.5 \%$ \\
\hline Not currently in paid employment & $3.0 \%$ & $1.9 \%$ & $7.1 \%$ & $4.3 \%$ \\
\hline Homemaker & $5.7 \%$ & $5.7 \%$ & $1.4 \%$ & $4.0 \%$ \\
\hline Total & $100.0 \%$ & $100.0 \%$ & $100.0 \%$ & $100.0 \%$ \\
\hline
\end{tabular}

Regarding the passengers' income, nearly half of all passengers (49\%) have a total annual household of over $\$ 65,000$ (Figure 6.3). Forty-seven percent of CSNZ passengers and $39.3 \%$ of domestic passengers were in this category. According to Statistics New Zealand, in the year ended June 2004, the average annual household income was $\$ 60,433$. In the case of international travellers, $58.4 \%$ of passengers' annual household income was higher than $\$ 65,000$. In part, this can be attributed to two main reasons. The first is that some of the major international markets to New Zealand, such as Australia, the UK, the USA and Germany, have a stronger currency than the New Zealand dollar. Secondly, for most international markets New Zealand is a long haul destination, more easily afforded by wealthier tourists. No major difference has been detected between CSNZ and domestic passengers regarding this variable.

In terms of the number of passengers travelling together with the respondents, $87.2 \%$ were accompanied by someone else and $12.8 \%$ were travelling alone (see Table 6.3). Passengers travelling with one other person represent the norm for all three sub-groups, being the majority in the case of international passengers (57\%). It is also possible to note some other trends, notably between international travellers and the other two sub-groups of New Zealand residents. Firstly, the proportion of international passengers travelling alone $(9.5 \%)$ is lower than that of the whole sample $(12.8 \%)$. Secondly, large groups (four 
or more passengers) are predominantly made up of passengers from New Zealand (both domestic and CSNZ), with the only exception being those with 21 or more passengers (more likely to be tour groups), where the percentage of international travellers $(4.3 \%)$ is larger than the two other sub-groups.

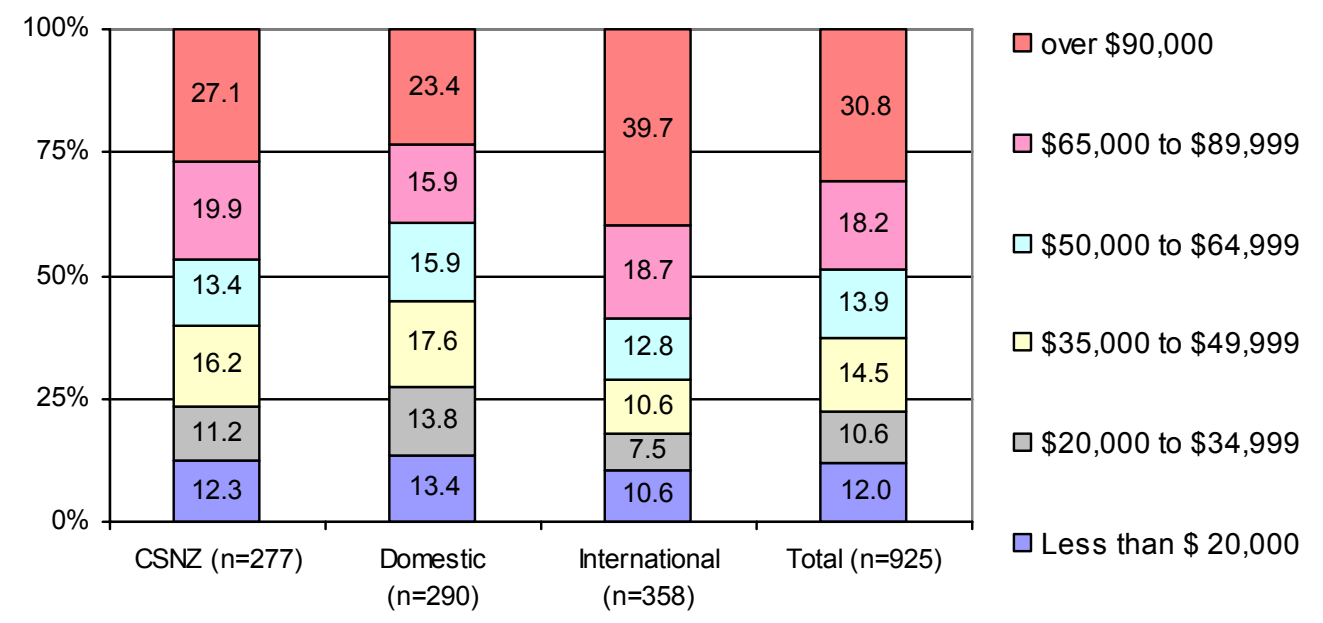

Figure 6.3 Respondents' total annual household (in NZ\$).

Table 6.3 Number of passengers in respondent's group.

\begin{tabular}{|r|r|r|r|r|}
\cline { 2 - 5 } \multicolumn{1}{c|}{} & $\begin{array}{c}\text { CSNZ } \\
\text { (n=308 })\end{array}$ & $\begin{array}{c}\text { Domestic } \\
(\mathrm{n}=328)\end{array}$ & $\begin{array}{c}\text { International } \\
(\mathrm{n}=440)\end{array}$ & $\begin{array}{c}\text { Total } \\
(\mathrm{n}=1,076)\end{array}$ \\
\hline 1 & $14.9 \%$ & $15.2 \%$ & $9.5 \%$ & $12.8 \%$ \\
\hline 2 & $35.7 \%$ & $39.6 \%$ & $57.0 \%$ & $45.6 \%$ \\
\hline 3 & $15.3 \%$ & $13.1 \%$ & $13.0 \%$ & $13.7 \%$ \\
\hline 5 & $13.6 \%$ & $13.4 \%$ & $9.3 \%$ & $11.8 \%$ \\
\hline $6-10$ & $7.1 \%$ & $8.5 \%$ & $2.7 \%$ & $5.8 \%$ \\
\hline $11-20$ & $7.5 \%$ & $7.0 \%$ & $2.3 \%$ & $5.2 \%$ \\
\hline $21+$ & $3.9 \%$ & $0.6 \%$ & $1.8 \%$ & $2.0 \%$ \\
\hline Total & $1.9 \%$ & $2.4 \%$ & $4.3 \%$ & $3.1 \%$ \\
\hline & $100.0 \%$ & $100.0 \%$ & $100.0 \%$ & $100.0 \%$ \\
\hline
\end{tabular}

When considering the relationship of the accompanying passengers, Table 6.4 shows that the majority of passengers are travelling with their spouse or partner $(54.9 \%)$, this pattern being identical among the three sub-groups. In addition, dependants and other family members are the two other most important types of accompanying passengers for those living in New Zealand. In the case of international passengers, friends, other family members and dependants are respectively the second $(21.1 \%)$, third $(16.3 \%)$ and fourth 
(15.9\%) most common groups. Another point to mention is the lower participation of passengers travelling with business associates. This is in line with the information presented in Table 6.5 regarding passengers' purpose of trip, where there is a clear predominance of "leisure/holidays" and VFR (visiting friends and relatives), in comparison to other purposes of trip, including "business/working".

Table 6.4 Relationship of respondents' accompanying passengers.

\begin{tabular}{|lr|r|r|r|}
\cline { 2 - 5 } \multicolumn{1}{c|}{} & \multicolumn{1}{c|}{\begin{tabular}{c} 
CSNZ \\
\multicolumn{1}{c|}{$(\mathrm{n}=308)$}
\end{tabular}} & $\begin{array}{c}\text { Domestic } \\
(\mathrm{n}=328)\end{array}$ & $\begin{array}{c}\text { International } \\
(\mathrm{n}=440)\end{array}$ & $\begin{array}{c}\text { Total } \\
(\mathrm{n}=1,076)\end{array}$ \\
\hline Spouse / partner & $51.9 \%$ & $54.0 \%$ & $57.7 \%$ & $54.9 \%$ \\
\hline Dependants & $21.8 \%$ & $21.6 \%$ & $7.5 \%$ & $15.9 \%$ \\
\hline Other family members & $24.7 \%$ & $22.3 \%$ & $17.7 \%$ & $21.1 \%$ \\
\hline Friends & $15.6 \%$ & $11.9 \%$ & $20.0 \%$ & $16.3 \%$ \\
\hline Educational trip & $2.6 \%$ & $0.9 \%$ & $0.2 \%$ & $1.1 \%$ \\
\hline Tour group & $0.6 \%$ & $4.9 \%$ & $4.1 \%$ & $3.3 \%$ \\
\hline Business associates & $1.0 \%$ & $1.8 \%$ & $0.2 \%$ & $0.9 \%$ \\
\hline Other & $0.3 \%$ & $1.2 \%$ & $0.2 \%$ & $0.6 \%$ \\
\hline
\end{tabular}

\subsection{Passengers' Trip}

This section focuses on the passengers' trip as a whole. As the ferry journey is part of a broader trip which involves the link between the North and the South Islands, to better understand the likelihood of these passengers staying longer in the ferry gateways, it is necessary to consider some aspects of their trip as a whole. There are several issues associated with the passengers' trip that can impact on the decision to stop or not in gateways. One of them is related to the earlier discussion about the flow of travellers around the country. Travellers on long journeys with a multiple destination pattern are more likely to visit the gateways than those travellers in a short single destination trip. Some data collected from the questionnaires help to understand the travellers' movement patterns, such as the length of the trip (in terms of number of nights), the regions in New Zealand where they have stayed at least one night, the regions where they have stayed overnight before and after passing through each gateway, and in the case of international travellers, the gateways they have used to enter and leave the country. Apart from the travellers' flow, other information such as the purpose of trip, the trip's flexibility and the time when decision to undertake the trip was made will be considered. 


\subsubsection{General Information about the Passengers' Trip}

The importance of analysing the various reasons for passengers undertaking a trip is twofold. Firstly, passengers with different purposes of trip are expected to present different likelihoods to modify their plans. Passengers whose main purpose of trip is business/work, event or conference/seminar are usually more restricted as they are travelling with a given itinerary established by the companies and organisations they work for. Those who purpose of trip is leisure and VFR may be expected to have more flexibility to change their itineraries in order to stay longer in the gateways. Secondly, if the destination is seen or made to be appealing to leisure/holiday and VFR groups, the chances of attracting these two types of passengers are higher. In the case of the ferry passengers, according to Table 6.5, they have two main purposes for undertaking their trips: leisure/holiday and VFR. Leisure/holiday represents two-thirds of the total number of passengers and the reason for nine out of ten international passengers. Trips with the main purpose of visiting friends and relatives (VFR) correspond to $20 \%$ of passengers' main purpose of trip, being especially significant to CSNZ (27.4\%) and domestic (30.8\%) passengers. Other purposes of trip such as business/working, education, 'moving house', event or 'conference/seminar' account for less than $15 \%$ of the total main purpose of trip and less than $3 \%$ in the case of international passengers.

Table 6.5 Main purpose of respondents' trip.

\begin{tabular}{|l|r|r|r|r|}
\cline { 2 - 5 } \multicolumn{1}{c|}{} & \multicolumn{1}{c|}{$\begin{array}{c}\text { CSNZ } \\
(\mathrm{n}=307)\end{array}$} & $\begin{array}{c}\text { Domestic } \\
(\mathrm{n}=328)\end{array}$ & $\begin{array}{c}\text { International } \\
(\mathrm{n}=439)\end{array}$ & $\begin{array}{c}\text { Total } \\
(\mathrm{n}=1,074)\end{array}$ \\
\hline Leisure / Holiday & $52.1 \%$ & $48.2 \%$ & $90.0 \%$ & $66.4 \%$ \\
\hline VFR & $27.4 \%$ & $30.8 \%$ & $7.1 \%$ & $20.1 \%$ \\
\hline Business / Working & $4.6 \%$ & $4.9 \%$ & $1.1 \%$ & $3.3 \%$ \\
\hline Moving house & $3.6 \%$ & $5.5 \%$ & -- & $2.7 \%$ \\
\hline Education & $3.3 \%$ & $2.4 \%$ & $0.9 \%$ & $2.0 \%$ \\
\hline Event & $4.2 \%$ & $2.1 \%$ & -- & $1.9 \%$ \\
\hline Conference / Seminar & $1.0 \%$ & $2.4 \%$ & $0.5 \%$ & $1.2 \%$ \\
\hline Other & $3.9 \%$ & $3.7 \%$ & $0.5 \%$ & $2.4 \%$ \\
\hline \multicolumn{1}{|c|}{ Total } & $100.0 \%$ & $100.0 \%$ & $100.0 \%$ & $100.0 \%$ \\
\hline
\end{tabular}

Data related to when the decision was made to undertake the current trip are shown in Figure 6.4. Together with the information regarding the passenger's trip flexibility (see Figure 6.5) this information helps to understand the level of planning undertaken by travellers before making a trip. This is central to identifying travellers' behaviour and likelihood to change their plans in case they decide to stay and visit gateways. According 
to the discussion in Chapter 2 (see Figure 2.9), travellers can be influenced by information at three different moments: before deciding to take the trip; after deciding to travel, but prior to departure; and after departure but before returning home. Therefore, the longer the period between the trip decision and the departure, the higher the chance of travellers influenced by information and probably being more open to changes.

As shown in Figure 6.4, for more than half of the passengers (55.1\%), the decision to undertake the ferry journey is made at least nine or more weeks in advance, with $31.4 \%$ deciding between 9 and 24 weeks before the journey was made and $23.7 \%$ deciding it more than 24 weeks ( 6 months) in advance. When comparing this information for the three groups of passengers, it is possible to associate the time of the decision with the distance the passenger lives from the gateway. Almost seven out of ten international passengers $(69.8 \%)$ took their decision nine or more weeks prior to their departure, with the percentage decreasing to $51.1 \%$ in the case of domestic passengers and $38.3 \%$ for those living in the CSNZ. One explanation for this can be the fact that the further the traveller lives from the gateway, the longer the trip will be, hence the need for more preparation.

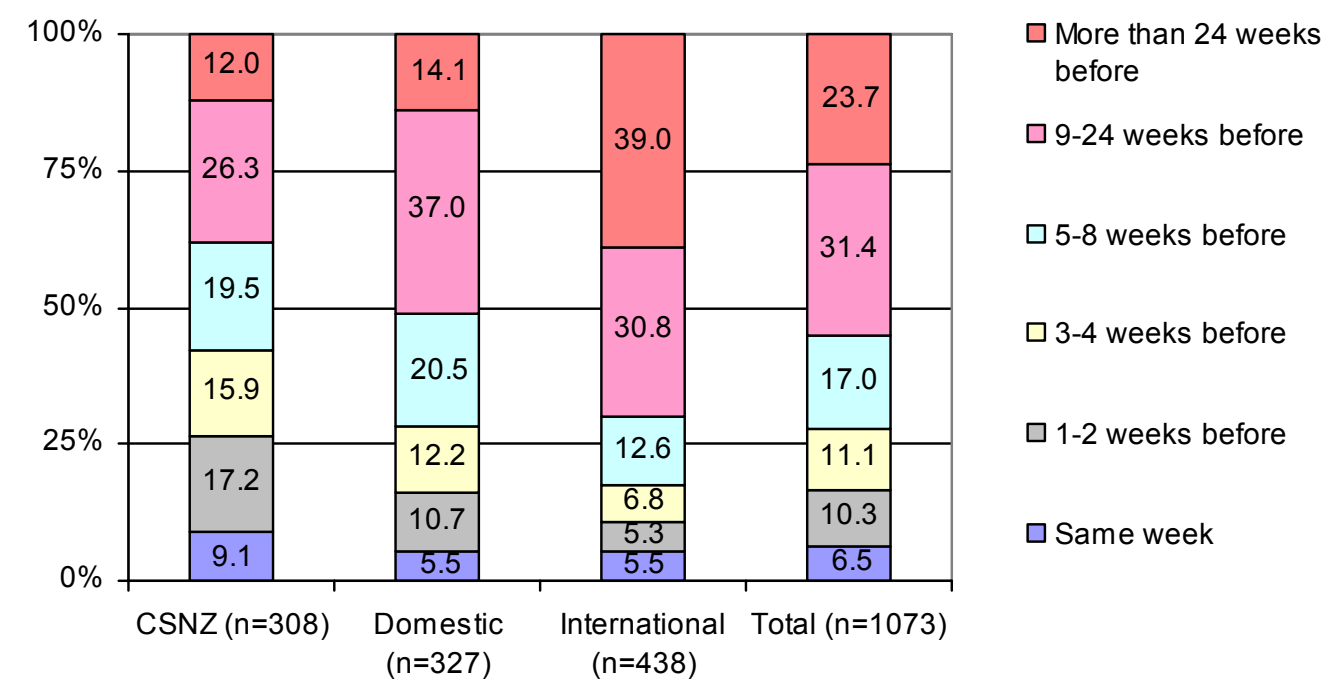

Figure 6.4 How far in advance the decision to travel was made.

If an international trip to New Zealand usually requires more planning than a domestic trip (Figure 6.4), it seems counter-intuitive to suggest from Figure 6.5 that international travellers tend to be more flexible regarding their travel arrangements than residents from New Zealand. Yet, only $19.2 \%$ of international travellers stated that they had everything in their trips well planned, while $51.8 \%$ of CSNZ travellers and $45.1 \%$ of domestic travellers had the same level of planning. When comparing the three sub-groups in Figure 6.5 , it is 
possible to note that the closer the sub-groups are to the gateways, the more planned their trips seem to be. There are some explanations for this behaviour. The first is that the further the traveller lives from the gateway the longer the trip will be (see Table 6.6). This means that in longer trips while travellers need more planning to undertake the trip, they still need to be flexible as it is not an easy task to book in advance all the activities, accommodation and transport required. The second reason is that the touring multiple destination pattern presented by travellers in New Zealand (see next sub-section), particularly among international visitors make them more open to decide where to go and how long to stay in these destinations while they are travelling around. Finally, as discussed earlier, the key international markets are made up of visitors who prefer to travel in an independent way (e.g. Australians, Americans, British and Germans), while the Asian market tends to be less independent, choosing to travel in package tours where the whole itinerary has been previously organised.

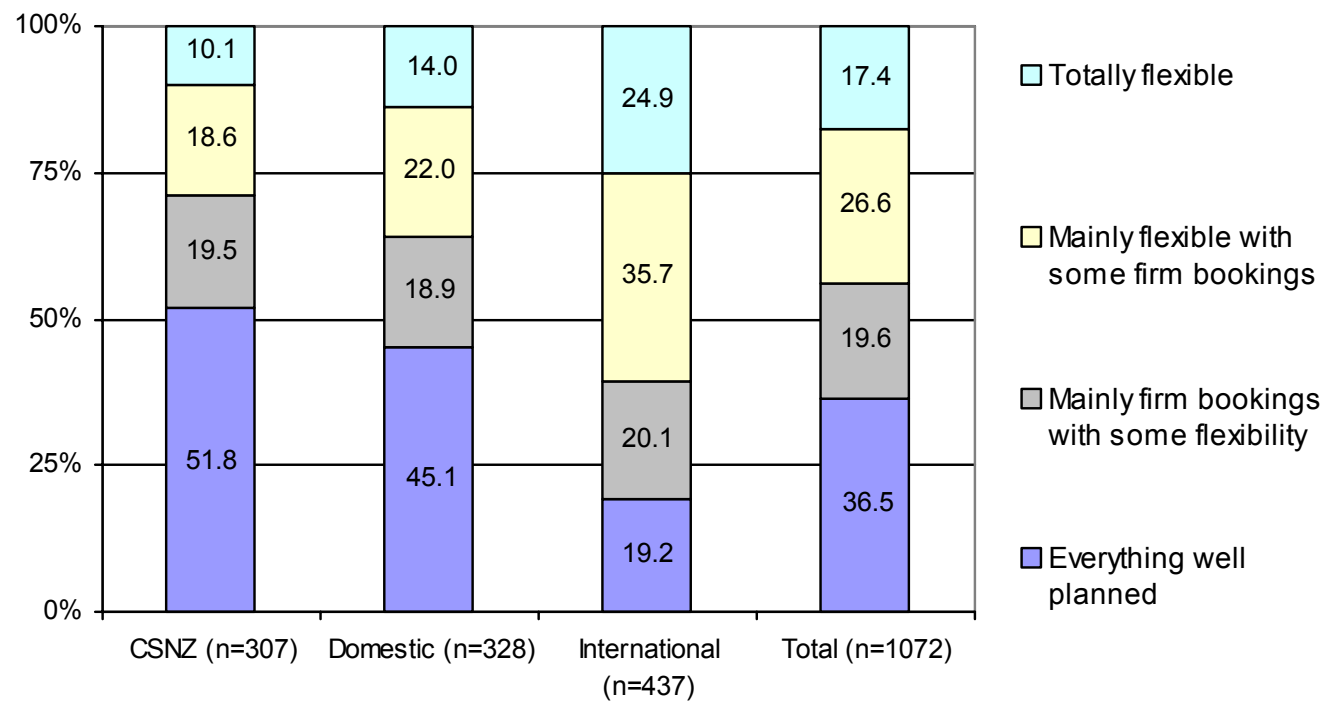

Figure 6.5 Respondents' trip flexibility and previous bookings.

Regarding the number of nights spent on their trips, the three sub-groups of passenger present quite different patterns. In the case of international travellers, only the time spent in New Zealand was considered. Trips with six or fewer days represent $55.5 \%$ of CSNZ travellers, $28.3 \%$ of domestic passengers and only $0.7 \%$ of international tourists (see Table 6.6). On the other hand, trips lasting more than three weeks (22 days or more) corresponded respectively to $7.3 \%, 12.2 \%$ and $53.2 \%$ of these sub-groups. From this brief analysis and also comparing the arithmetic means $(x)$ of CSNZ, domestic and international passengers it is possible to state that trip's length of stay is directly associated with the distance from the gateways. In fact, because of the extreme values 
existing in the sample, such as the cases of passengers that were travelling for 730 days ( 2 years), the median is probably a better method for measuring central tendency. Median values for the three sub-groups are respectively 5, 10 and 23 nights. For passengers living in the CSNZ, the ferries can be the mode of transport to take them on day trips or short breaks across Cook Strait, while domestic passengers generally tend to use the ferries as part of longer trips. Comparisons between international travellers and those living in the country from Table 6.6 only reflect data presented in Chapter 4, with overseas visitors spending more time in their trips than those living in New Zealand.

Table 6.6 Number of nights spent in trip (in New Zealand for international visitors).

\begin{tabular}{|c|c|c|c|c|}
\hline & $\begin{array}{c}\begin{array}{c}\text { CSNZ } \\
(n=300)\end{array} \\
\end{array}$ & $\begin{array}{c}\text { Domestic } \\
(n=317)\end{array}$ & $\begin{array}{c}\text { International } \\
(n=415)\end{array}$ & $\begin{array}{c}\text { Total } \\
(n=1,032)\end{array}$ \\
\hline 0 & $4.0 \%$ & $0.6 \%$ & -- & $1.4 \%$ \\
\hline 1 & $6.0 \%$ & $0.3 \%$ & -- & $1.8 \%$ \\
\hline 2 & $14.2 \%$ & $4.7 \%$ & -- & $5.6 \%$ \\
\hline 3 & $12.0 \%$ & $8.2 \%$ & -- & $6.0 \%$ \\
\hline 4 & $10.3 \%$ & $5.4 \%$ & -- & $4.7 \%$ \\
\hline $5-6$ & $9.0 \%$ & $9.1 \%$ & $0.7 \%$ & $5.7 \%$ \\
\hline $7-8$ & $10.0 \%$ & $10.8 \%$ & $1.5 \%$ & $6.8 \%$ \\
\hline $9-11$ & $9.4 \%$ & $17.3 \%$ & $5.0 \%$ & $9.9 \%$ \\
\hline $12-14$ & $8.0 \%$ & $13.3 \%$ & $8.7 \%$ & $9.8 \%$ \\
\hline $15-17$ & $5.0 \%$ & $7.9 \%$ & $11.8 \%$ & $8.6 \%$ \\
\hline $18-21$ & $4.8 \%$ & $10.2 \%$ & $19.1 \%$ & $12.1 \%$ \\
\hline $22-28$ & $4.4 \%$ & $5.8 \%$ & $19.1 \%$ & $10.8 \%$ \\
\hline $29-35$ & $2.6 \%$ & $2.5 \%$ & $14.9 \%$ & $12.4 \%$ \\
\hline $36-49$ & -- & $1.5 \%$ & $6.7 \%$ & $3.2 \%$ \\
\hline $50-63$ & -- & $1.5 \%$ & $2.9 \%$ & $1.6 \%$ \\
\hline $64-365$ & $0.3 \%$ & $0.3 \%$ & $9.4 \%$ & $4.0 \%$ \\
\hline $366+$ & -- & $0.6 \%$ & $0.2 \%$ & $0.3 \%$ \\
\hline Mean $(x)$ & 8.35 & 16.75 & 35.91 & 13.06 \\
\hline Std. Deviation & 9.9 & 51.3 & 54.2 & --- \\
\hline Median & 5 & 10 & 23 & 5 \\
\hline Minimum & 0 & 0 & 5 & 0 \\
\hline Maximum & 120 & 730 & 730 & 730 \\
\hline
\end{tabular}

What can be expected is that passengers travelling on short trips might be less inclined to spend extra time in the gateways, as they would prefer to concentrate on those places that are their primary destinations. Alternatively, visitors on longer trips have more days to spend in different destinations, which increase, in a certain way, the chances of extending their stays in the gateways if they are perceived as interesting destinations. Combining the information presented in Table 6.6 about the length of stay with the results from Figure 6.5 
regarding the passengers' trip flexibility, it seems that international passengers are more likely to stay longer in the gateways than passengers living in New Zealand. The reason for this is that overseas passengers are more flexible and are travelling for longer.

\subsubsection{The Travellers' Flow Pattern around New Zealand}

The previous sub-section presented some of the general characteristics of the ferry passengers' trip. This one sheds light on the travellers' flow pattern while travelling around New Zealand.

Before discussing the tourist flow of overseas visitors and those living in New Zealand, it is necessary to present what are the international gateways used by foreign visitors to enter and leave New Zealand. As can be noted from Table 6.7, Auckland is the main gateway for travellers arriving $(74.5 \%$ of total) and leaving $(65.5 \%)$ the country. Christchurch is the second most important international gateway, being responsible for $15.7 \%$ of total arrivals and $25 \%$ of departures. It is interesting to note that the percentage of passengers entering Auckland is higher than those using this gateway to leave New Zealand. The opposite happens to Christchurch where the number of outbound passengers is higher than the inbound flow. This suggests that international visitors tend to travel south, entering through Auckland and leaving via Christchurch. In fact, while $50.9 \%$ of Cook Strait international passengers enter and leave New Zealand through Auckland, $20.9 \%$ arrive in Auckland and leave via Christchurch and $12.3 \%$ go the other way round. As discussed in Chapter 5, Wellington has a secondary role as an international gateway to New Zealand. Only $7.5 \%$ of international passengers use it to enter the country and $6.1 \%$ leave New Zealand through it.

Table 6.7 Major international gateways to enter and leave New Zealand.

\begin{tabular}{|c|c|c|c|c|c|}
\hline \multirow{2}{*}{ Entered } & \multicolumn{4}{|c|}{ Will leave } & \multirow{2}{*}{$\begin{array}{c}\text { Total } \\
(n=440)\end{array}$} \\
\hline & Auckland & Wellinaton & Christchurch & Other & \\
\hline Auckland & $50.9 \%$ & $1.1 \%$ & $20.9 \%$ & $1.6 \%$ & $74.5 \%$ \\
\hline Wellington & $1.4 \%$ & $4.5 \%$ & $1.4 \%$ & $0.2 \%$ & $7.5 \%$ \\
\hline Christchurch & $12.3 \%$ & $0.5 \%$ & $2.7 \%$ & $0.2 \%$ & $15.7 \%$ \\
\hline Other & $0.9 \%$ & -- & -- & $1.4 \%$ & $2.3 \%$ \\
\hline Total $(n=440)$ & $65.5 \%$ & $6.1 \%$ & $25.0 \%$ & $3.4 \%$ & $100.0 \%$ \\
\hline
\end{tabular}

When considering the regions in New Zealand where travellers spend at least one night during their trips, Figure 6.6 shows that there is only one region where international passengers do not stay overnight more than the other two sub-groups: Kapiti Coast. Only $10 \%$ of overseas passengers stay there, compared to $12.2 \%$ of domestic passengers. In regions located outside the ferry catchment areas, such as Otago \& Southland, West 
Coast and Northland \& Auckland, the participation of international passengers can be five or even six times higher than CSNZ and domestic passengers. This difference is smaller in those regions located closer to the ferries' gateways, such as Marlborough Sounds, Blenheim, Nelson Tasman, Wellington, Kapiti Coast and Hutt Valley.

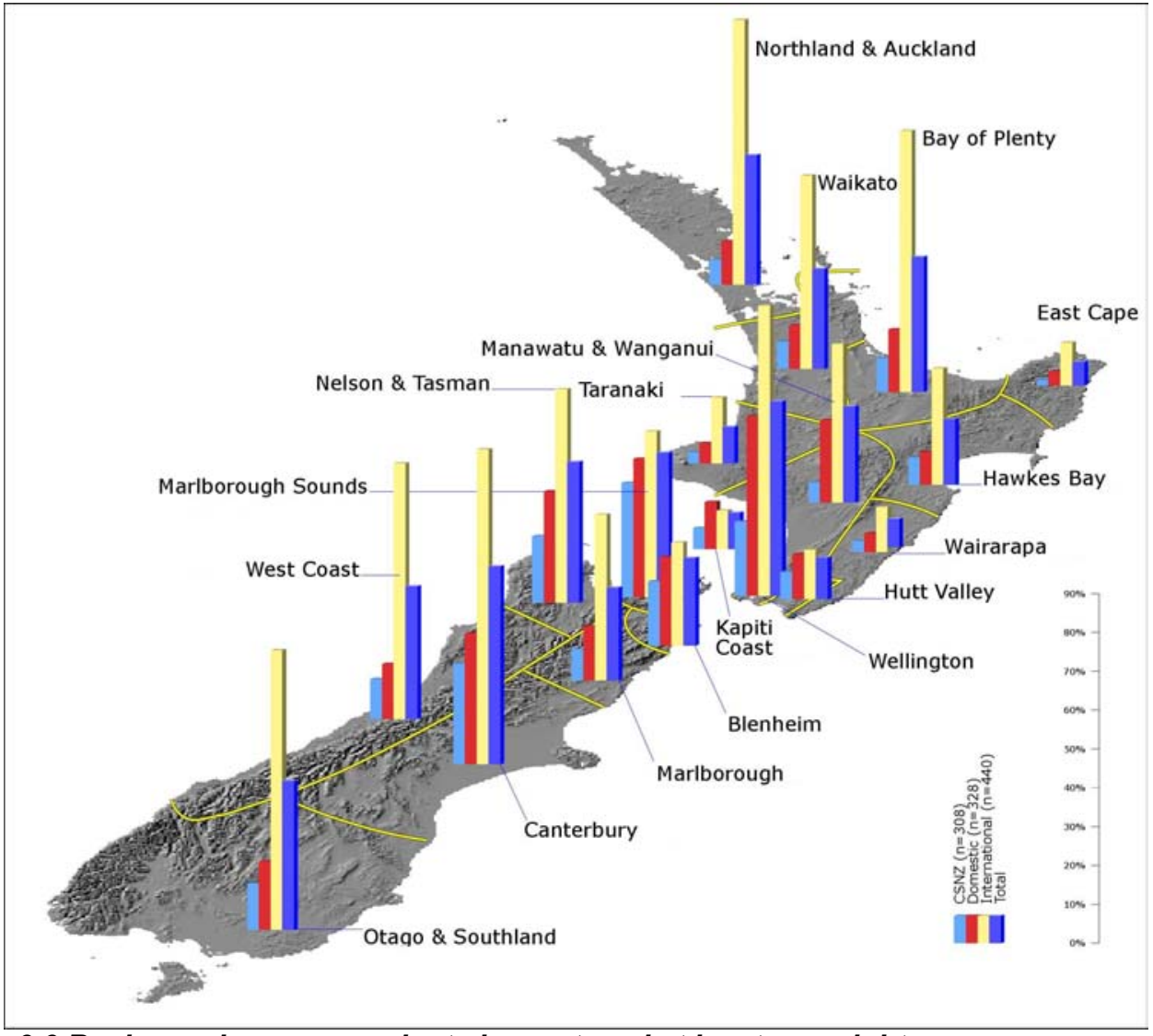

Figure 6.6 Regions where respondents have stayed at least one night.

There are several reasons to explain the pattern presented in Figure 6.6, which are supported by other information sources, such as the number of nights passengers spend in their trips (Table 6.6), the gateways used by international travellers to enter and leave the country (Table 6.7) and the number of regions where they stay at least one night (Figure 6.7).

Firstly, Figure 6.7 complements the visual representation presented in Figure 6.6 showing the number of different regions where the three sub-groups of passengers have stayed at least one night. According to Figure 6.7, on average CSNZ passengers stay overnight in 1.94 regions, with over half of CSNZ passengers (51\%) staying in only one region (single destination trip). Four percent of CSNZ passengers (see Table 6.6) do not stay overnight in any region. Domestic passengers present an intermediary position between CSNZ and international passengers. On average they stay overnight in 3.2 regions, with $21 \%$ staying 
in only one region, $25.6 \%$ in two regions and $19.2 \%$ in three different regions. International passengers are characterised by a multiple destination pattern, with nights spent on average in 7.8 different regions. Very few international passengers $(1.1 \%)$ stay overnight in only one region, while over $15 \%$ of them stayed overnight in more than ten different regions in New Zealand.

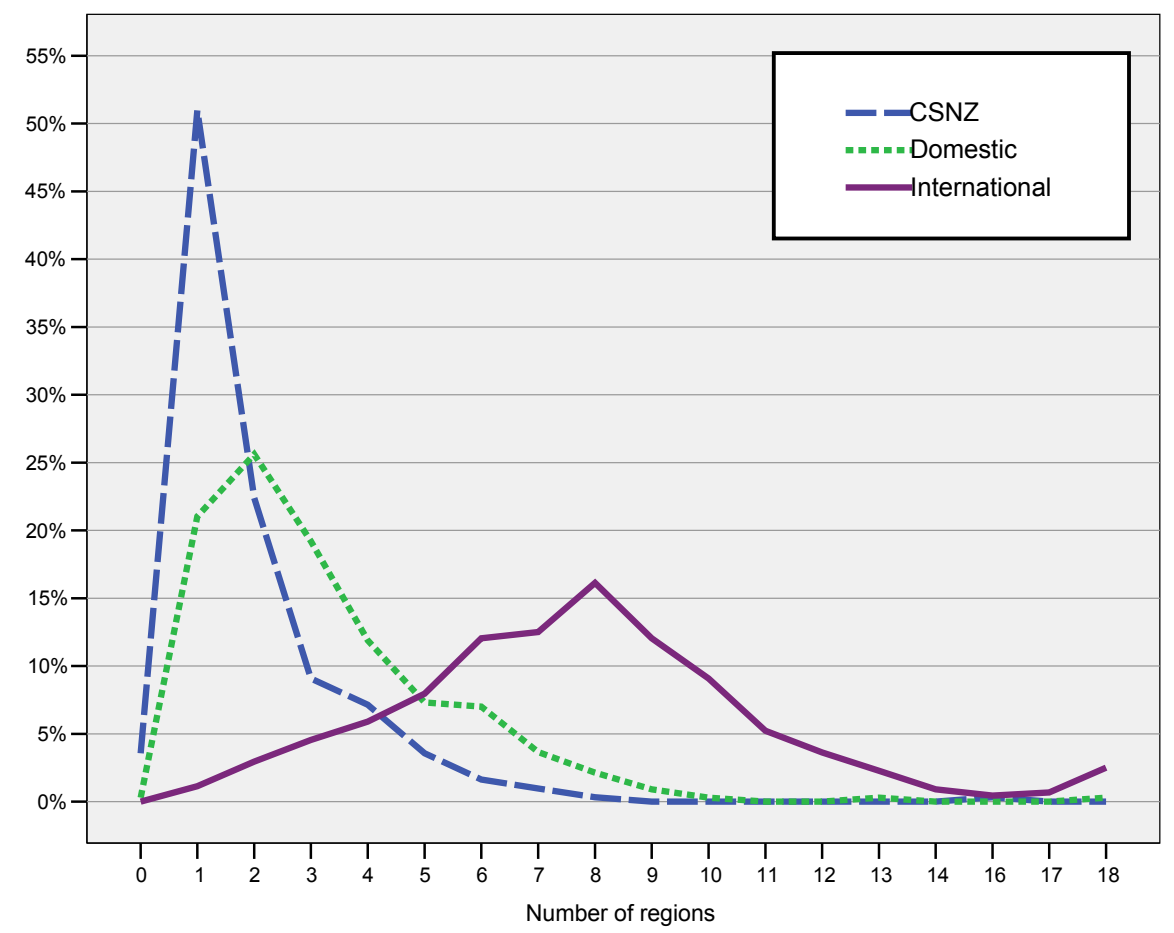

Figure 6.7 Number of different regions in which passengers stayed at least one night.

Secondly, the higher participation of international travellers in regions such as Auckland and Canterbury is related to the fact that these are the two major international gateways. They are among the top three regions where international passengers spend at least one night (Northland \& Auckland 68.2\%; Canterbury 81.1\%). Regions located near these two gateways also have more international passengers staying overnight than those passengers living in New Zealand. Therefore, it is possible to conclude that international passengers take advantage of the fact that they are arriving or departing through Auckland or Christchurch to visit the attractions and destinations located near these two international gateways. The same pattern is not followed by CSNZ and domestic passengers. In the case of the Northland \& Auckland region, only $6.5 \%$ of CSNZ and $11.3 \%$ of domestic passengers stayed at least one night there. Information on the precise number of nights that ferry passengers spend in each region would be interesting, but 
collecting this sort of data would require a more elaborate questionnaire, which would be beyond the focus of the research question.

Thirdly, the pattern presented in Figure 6.6 can be explained by the average length of stay of international passengers (nearly 36 nights), which is four times greater than CSNZ (8.35 nights) and twice that of domestic (16.75) passengers. With longer trips, a desire to visit as many different destinations and attractions as possible in order to take advantage of the fact that New Zealand is an isolated destination and a flow pattern that is influenced by the location of international gateways, international passengers tend to spread their stays around the country, while those living in New Zealand tour less or just undertake a single destination trip. Although the behaviour of international passengers seems to facilitate their stays in different regions, including those where the gateways are located, extending their stays there might occur at the expense of them not staying overnight somewhere else.

Regarding the areas where passengers stay overnight before or after taking the ferry in Wellington and Picton, these are presented in Figures 6.8 to 6.11. In order to persuade passengers to stay in the gateways, it is relevant to know where they are staying overnight before and after catching the ferries. If passengers are already staying overnight near the gateways, particularly if there are plenty of attractions and popular destinations in the ferries' catchment areas, it may be harder to attract these passengers as they will probably have no need to stay overnight in the gateway, as they can reach it fairly easily, or the gateway will need to compete with other destinations for the passenger's choice. In the following maps the region where the passenger stayed overnight before or after taking the ferries is their own place of origin in some cases.

In Wellington, the areas that comprise the two CSNZ's macro-regions near the gateway (Wellington and Wairarapa) account for the majority of places where passengers stay overnight before departing from Wellington (Figure 6.8) and after arriving in the capital city (Figure 6.9). The four areas that comprise the Wellington and Wairarapa macro-regions (Wellington, Hutt Valley, Kapiti Coast and Wairarapa) represent respectively $78.2 \%$ and $69.2 \%$ of the places where passengers stay overnight before and after using the ferries in Wellington. It is appropriate to mention that the Manawatu \& Wanganui region, which is half-way out the Auckland-Wellington route, is the third (in the case of nights before taking the ferry in Wellington) and the second (in the case of nights spent after arriving in Wellington) most popular region for overnight stays, especially among domestic travellers - corresponding, respectively, to $21.7 \%$ and $23.2 \%$ of this sub-group. The Wellington area, together with the Hutt Valley area, which can be considered as part of the same 
urban region, is the preponderant choice for international travellers to stay overnight before and after their Cook Strait journey departing from/arriving in Wellington, with a total of $80.1 \%$ and $68.1 \%$ respectively of the overnight stays.

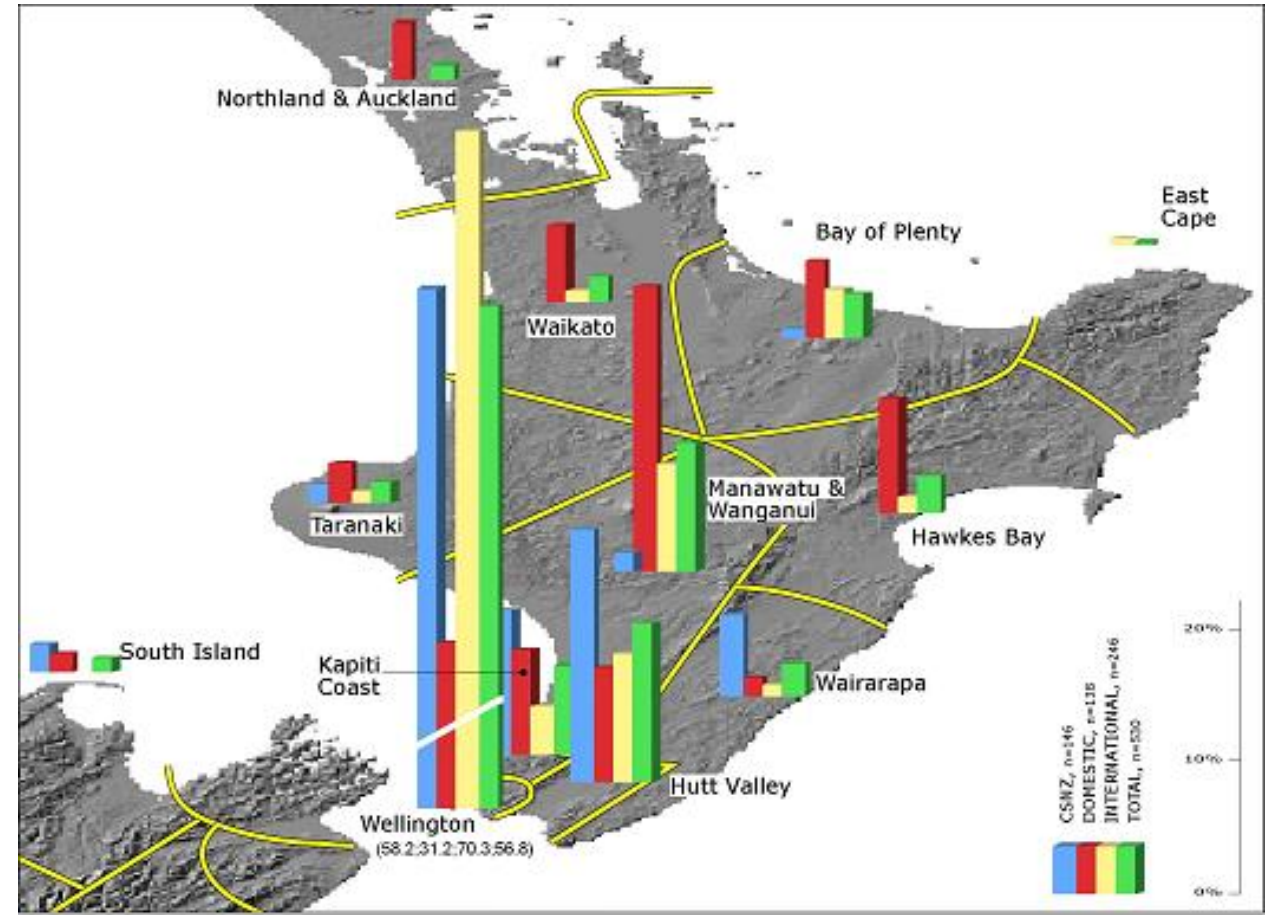

Figure 6.8 Area where passengers stayed overnight before departing from Wellington.

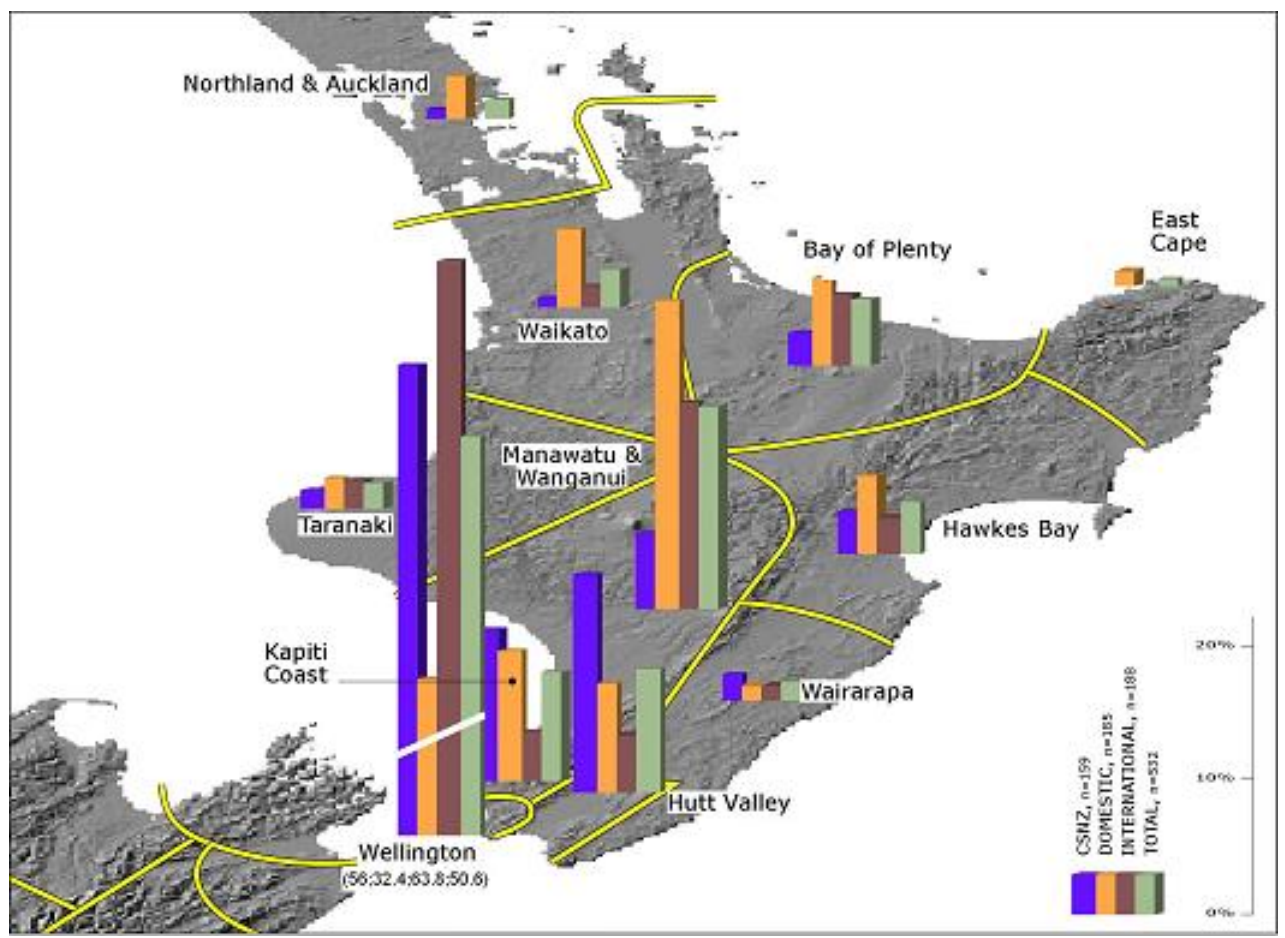

Figure 6.9 Area where passengers stayed overnight after having arrived in Wellington. 
In the case of Picton, $77.1 \%$ of passengers stay overnight in the Marlborough and Nelson regions (Marlborough Sounds, Blenheim, Nelson \& Tasman and Marlborough), which are the two CSNZ's macro-regions in the South Island. Although this trend could be expected for CSNZ's passengers (84.5\%), an even higher percentage of international passengers $(90.5 \%)$ stay overnight in these regions. In fact, $45 \%$ of international passengers stay overnight in the Marlborough Sounds area before crossing to Wellington (see Figure 6.10). Domestic passengers present a slightly different pattern. Although the majority of them $(57.1 \%)$ stay overnight in these regions, Canterbury represents the main region with $38 \%$. In terms of the regions where passengers stay overnight after arriving in Picton (see Figure 6.11), again the same level of participation can be found in the four areas Marlborough Sounds, Blenheim, Marlborough and Nelson \& Tasman - comprising the two CSNZ regions in the South Island. Approximately three-quarters $(75.7 \%)$ of passengers stay in one of these areas the night after crossing the Cook Strait.

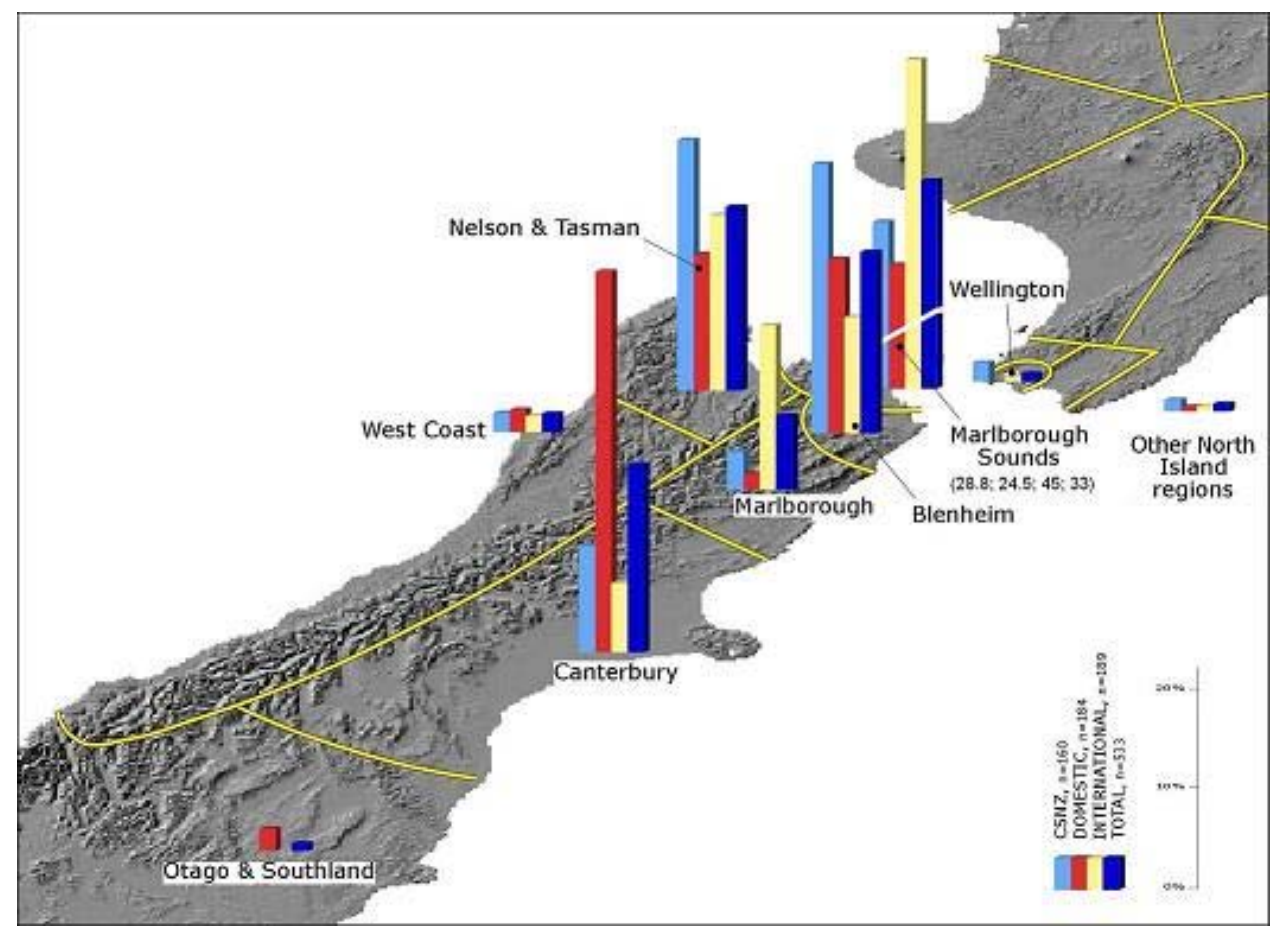

Figure 6.10 Area where passengers stayed overnight before departing from Picton. 


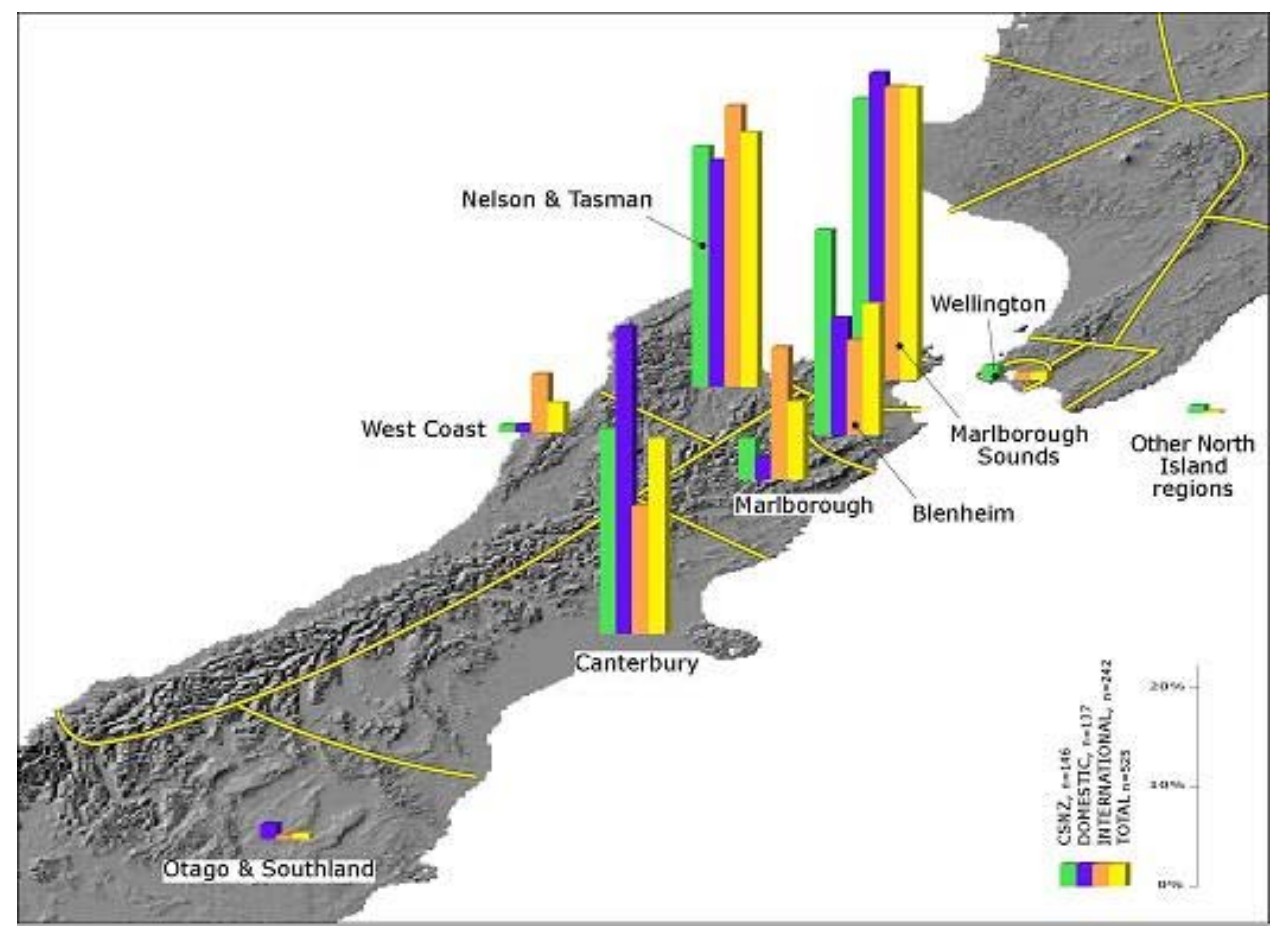

Figure 6.11 Area where passengers stayed overnight after having arrived in Picton.

Data presented in the previous maps provide some interesting conclusions. Nearly eight out of ten international passengers are already staying overnight either in Wellington or in the Hutt Valley before or after taking the ferry. As the Hutt region can be considered as part of Wellington's metropolitan area, it becomes clear that in Wellington the effort is not on attracting international passengers to stay overnight there, but to extend their stays. While the higher participation of CSNZ in Wellington is to be expected due to Wellington's strong origin function, nearly half of domestic passengers are staying overnight either in Wellington or in surrounding regions before / after catching the ferry. It might be the case that the option to stay overnight in Hutt Valley and Kapiti Coast is associated with the cheaper costs of accommodation in these areas. In addition, one in four domestic passengers stay overnight in Manawatu \& Wanganui, that is half way between Auckland and Wellington. If this is the case, it would be harder to attract those passengers to stay overnight in Wellington simply because travellers would not be prepared to drive long hours just to stay overnight in the capital city, unless they could take an extra day to visit the city.

In Picton, while on average three out of ten passengers are staying overnight in the Marlborough Sounds, it seems that the short distance between Picton and other more developed destinations such as Blenheim and Nelson / Tasman makes it more difficult to attract the same level of stays than in Wellington. Therefore, Picton has not only to attract 


\section{PATTERNS OF DEMAND}

passengers to stay there, but also to increase their length of stay as there is comparatively more competition with other destinations. Chapter 7 will discuss more specifically the passengers' nodal functions, their stays and behaviours while in Wellington and Picton and the information provided here will help to better understand where passengers stay overnight before and after catching the ferries.

\subsection{Passengers' Ferry Journey}

This section deals with topics associated with the passengers' ferry journey. A wide range of different attributes related to the ferry journey will be presented here. They include, for example, the means of transport used to arrive in and depart from the gateways, the frequent use passengers living in New Zealand make of the ferries, the main reason for travelling by ferry and information related to the ferry bookings. In the latter case, understanding how, when and where the passengers book their ferry crossing helps to consider the possibility of promoting the gateways at the same time that the ferries are booked.

Regarding the means of transport used to arrive in the gateways, although passengers have a wide range of options such as airplanes, train and the various types of road transport, some particular means of transport predominate among the three sub-groups. For example, domestic passengers mostly use private cars/campervans to/from Wellington (24.9\%) or Picton (26\%) - see Table 6.8. Among international passengers, on the other hand, rental cars (22\%) and campervans $(24.4 \%)$ represent the main means of transport to reach and leave the two ferry ports. Other forms of transport also relevant to this sub-group include private cars/campervans and scheduled buses. For passengers living in the CSNZ, a significant part of passengers live in Wellington (17.5\%). This creates a biased situation because as Wellington works as an origin node, it does not give a proper idea of the modes of transport used by this sub-group to arrive in and leave from Wellington. However, in the case of Picton, as its origin function can be ignored due to the small population living there, nearly every passenger will be using a mode of transport to arrive there. As in the case of domestic passengers, private cars/campervans account for more than two-thirds of the mode of transport used by CSNZ passengers to arrive or depart from Picton.

The high use of cars and campervans (private or rental) emphasizes one aspect of the Cook Strait ferries. As the ferries are able to take vehicles on board, this is one of the key factors for passengers using this service (see Table 6.11). In the case of passengers travelling with rental cars/campervans, not every passenger will bring their vehicle on 
board as the rental car companies prefer to keep separate fleets in the North and South Islands and do not encourage their vehicles being taken on the ferries. The high use of rental cars among international passengers can be attributed to the level of flexibility they offer in comparison to other modes of transport. Tour coaches are also used more by international passengers than by travellers living in New Zealand. In the case of the South Island, the train has also a certain appeal to international passengers. This can be attributed to the scenic nature of the journey between Picton and Christchurch.

Table 6.8 Means of transport to arrive in or depart from Wellington and Picton.

\begin{tabular}{|l|r|r|r|r|r|r|r|r|}
\cline { 2 - 9 } \multicolumn{1}{c|}{} & \multicolumn{3}{c|}{ Wellington $(\mathrm{n}=1,067)$} & \multicolumn{3}{c|}{ Picton (n=1,052) } \\
\cline { 2 - 10 } & CSNZ & \multicolumn{1}{c|}{ Dom } & \multicolumn{1}{c|}{ Int'l } & \multicolumn{1}{c|}{ Total } & CSNZ & Dom & Int'l & Total \\
\hline Live in Wellington/Picton & $17.5 \%$ & -- & -- & $17.5 \%$ & $0.7 \%$ & -- & -- & $0.7 \%$ \\
\hline Rental car / campervan & $0.2 \%$ & $0.6 \%$ & $22.0 \%$ & $22.8 \%$ & $1.7 \%$ & $1.0 \%$ & $24.4 \%$ & $27.2 \%$ \\
\hline Private car / campervan & $8.0 \%$ & $24.9 \%$ & $5.3 \%$ & $38.2 \%$ & $20.1 \%$ & $26.0 \%$ & $5.9 \%$ & $52.1 \%$ \\
\hline Scheduled bus & $0.5 \%$ & $0.5 \%$ & $2.2 \%$ & $3.1 \%$ & $1.1 \%$ & $0.7 \%$ & $2.0 \%$ & $3.8 \%$ \\
\hline Tour coach & $0.1 \%$ & $0.6 \%$ & $4.2 \%$ & $4.9 \%$ & -- & $0.3 \%$ & $3.9 \%$ & $4.2 \%$ \\
\hline Train & $0.4 \%$ & $1.3 \%$ & $0.9 \%$ & $2.6 \%$ & $0.3 \%$ & $0.9 \%$ & $2.9 \%$ & $4.0 \%$ \\
\hline Ferry & $1.8 \%$ & $1.3 \%$ & $0.4 \%$ & $3.5 \%$ & $2.3 \%$ & $0.6 \%$ & $0.7 \%$ & $3.5 \%$ \\
\hline Airplane & $0.2 \%$ & $0.8 \%$ & $5.2 \%$ & $6.2 \%$ & -- & -- & $0.2 \%$ & $0.2 \%$ \\
\hline Other & $0.3 \%$ & $0.4 \%$ & $0.6 \%$ & $1.2 \%$ & $2.5 \%$ & $1.0 \%$ & $0.9 \%$ & $4.3 \%$ \\
\hline & $29.0 \%$ & $30.4 \%$ & $40.8 \%$ & $100.0 \%$ & $28.7 \%$ & $30.5 \%$ & $40.9 \%$ & $100.0 \%$ \\
\hline
\end{tabular}

Comparing the modes of transport used in Wellington and Picton it is possible to note that most passengers are quite consistent, using the same means of transport before and after taking the ferries. Table 6.9 shows that among CSNZ passengers $(n=303)$, private vehicles are used by $28.1 \%$ of this sub-group's passengers to arrive in/depart from Wellington and Picton. Even more relevant is the percentage of passengers (36.3\%) living in Wellington but using private vehicles to leave Picton. Most probably they also used a private vehicle from their homes in Wellington to the ferry terminal and were taking their vehicles onboard the ferries. Within the domestic sub-group $(n=319), 78.1 \%$ use private vehicle (car or campervan) both in Wellington and Picton. Finally, among international passengers $(n=424)$, three main types of transport are used in both ferry gateways: rental vehicles $(50.7 \%)$, private vehicles $(10.6 \%)$ and tour coaches $(8.3 \%)$. International passengers, who arrived in Wellington by plane, took the ferry and then left Picton using a rental vehicle represents $6.4 \%$ of this group. 


\section{PATTERNS OF DEMAND}

Table 6.9 Means of transport to arrive in or depart from Wellington and Picton by place of origin.

\begin{tabular}{|c|c|c|c|c|c|c|c|c|c|c|c|}
\hline \multirow{2}{*}{\multicolumn{2}{|c|}{$\begin{array}{l}\text { Means of transport to } \\
\text { arrive in / depart from } \\
\text { Wellington (\%) }\end{array}$}} & \multicolumn{10}{|c|}{ Means of transport to arrive in / depart from Picton (\%) } \\
\hline & & $\begin{array}{l}\text { Live in } \\
\text { Picton }\end{array}$ & $\begin{array}{c}\text { Renta } \\
\text { car }\end{array}$ & $\begin{array}{c}\text { Private } \\
\text { car }\end{array}$ & $\begin{array}{c}\text { Scheduled } \\
\text { bus }\end{array}$ & $\begin{array}{l}\text { Tour } \\
\text { coach }\end{array}$ & Train & Ferry & $\begin{array}{c}\text { Air- } \\
\text { plane }\end{array}$ & Other & Total \\
\hline \multirow{10}{*}{ 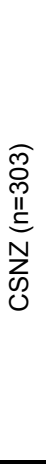 } & Live in Wellington & & 5.9 & 36.3 & 2.6 & & 0.7 & 7.3 & & 7.6 & 60.4 \\
\hline & Rental car & 0.3 & & 0.3 & & & & & & & 0.7 \\
\hline & Private car & 1.0 & & 25.4 & 0.7 & & & 0.7 & & 0.3 & 28.1 \\
\hline & Scheduled bus & & & 1.7 & & & & & & & 1.7 \\
\hline & Tour coach & & & 0.3 & & & & & & & 0.3 \\
\hline & Train & & & 0.7 & 0.3 & & 0.3 & & & & 1.3 \\
\hline & Ferry & 1.0 & & 4.6 & 0.3 & & & & & & 5.9 \\
\hline & Airplane & & & 0.7 & & & & & & & 0.7 \\
\hline & Other & & & 0.3 & & & & & & 0.7 & 1.0 \\
\hline & Total & 2.3 & 5.9 & 70.3 & 4.0 & & 1.0 & 7.9 & & 8.6 & 100.0 \\
\hline \multirow{9}{*}{ 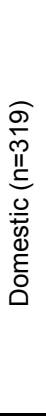 } & Rental car & & 1.3 & 0.6 & & & & & & & 1.9 \\
\hline & Private car & & 0.6 & 78.1 & 0.3 & & 0.3 & 1.6 & & 1.3 & 82.1 \\
\hline & Scheduled bus & & 0.3 & 0.3 & 0.3 & & 0.3 & & & 0.3 & 1.6 \\
\hline & Tour coach & & & 0.3 & & 0.6 & & 0.3 & & 0.6 & 1.9 \\
\hline & Train & & & 1.6 & 0.6 & & 1.9 & & & 0.3 & 4.4 \\
\hline & Ferry & & 0.3 & 3.4 & 0.3 & & & & & & 4.1 \\
\hline & Airplane & & 0.9 & 0.6 & 0.6 & 0.3 & 0.3 & & & & 2.8 \\
\hline & Other & & & 0.6 & & & & & & 0.6 & 1.3 \\
\hline & Total & & 3.4 & 85.6 & 2.2 & 0.9 & 2.8 & 1.9 & & 3.1 & 100.0 \\
\hline \multirow{9}{*}{ 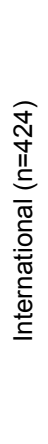 } & Rental car & & 50.7 & 1.4 & 0.2 & & 0.9 & 0.5 & 0.2 & 0.7 & 54.7 \\
\hline & Private car & & 0.9 & 10.6 & 0.2 & 0.2 & 0.5 & 0.7 & & & 13.2 \\
\hline & Scheduled bus & & 0.7 & & 2.6 & 0.7 & 0.9 & & & & 5.0 \\
\hline & Tour coach & & & 0.2 & & 8.3 & 2.1 & & & & 10.6 \\
\hline & Train & & 0.9 & & 0.2 & 0.2 & 0.5 & & & 0.5 & 2.4 \\
\hline & Ferry & & 0.2 & 0.5 & 0.2 & & & & & & 0.9 \\
\hline & Airplane & & 6.4 & 1.7 & 0.9 & 0.2 & 2.1 & 0.5 & 0.2 & & 12.0 \\
\hline & Other & & & & 0.2 & & & & & 0.9 & 1.2 \\
\hline & Total & & 59.9 & 14.4 & 4.7 & 9.7 & 7.1 & 1.7 & 0.5 & 2.1 & 100.0 \\
\hline
\end{tabular}

The questionnaire had a specific question in section B regarding the frequency of use that passengers living in New Zealand make of the Cook Strait ferries. This was a relevant question because some of the passengers' patterns in the ferry gateways can be related to the frequency with which they pass through. It may be expected that for frequent travellers the novelty appeal of the gateway would be less relevant in attracting them to stay and visit the gateways, as they are probably more familiar with them. When comparing the use CSNZ and domestic passengers make of the ferries (see Table 6.10), it becomes clear that passengers from the CSNZ tend to travel on the ferries more frequently than those living outside it. For example, while only $2.9 \%$ of CSNZ passengers were travelling on the ferries for the first time, $11.6 \%$ of the domestic passengers were 
making their first trip on this transport service. While the percentage of passengers travelling once or twice a year is very similar for both sub-groups, $21.8 \%$ of CSNZ passengers travel three or four times on the ferries each year, compared to only $8.2 \%$ of domestic passengers (Table 6.10).

Table 6.10 Frequent use of ferry for New Zealand resident passengers.

\begin{tabular}{|lr|r|r|}
\cline { 2 - 4 } \multicolumn{1}{c|}{} & CSNZ $(\mathrm{n}=308)$ & Domestic $(\mathrm{n}=328)$ & Total $(\mathrm{n}=636)$ \\
\hline This is my first trip & $2.9 \%$ & $11.6 \%$ & $7.4 \%$ \\
\hline Once a year & $18.2 \%$ & $18.9 \%$ & $18.6 \%$ \\
\hline Twice a year & $16.9 \%$ & $15.5 \%$ & $16.2 \%$ \\
\hline $3-4$ times per year & $21.8 \%$ & $8.2 \%$ & $14.8 \%$ \\
\hline $5-8$ times per year & $11.0 \%$ & $4.0 \%$ & $7.4 \%$ \\
\hline Every month & $3.9 \%$ & $0.3 \%$ & $2.0 \%$ \\
\hline Twice a month & $1.6 \%$ & $0.6 \%$ & $1.1 \%$ \\
\hline Every week & $0.3 \%$ & -- & $0.2 \%$ \\
\hline I don't travel on a regular basis & $23.1 \%$ & $40.9 \%$ & $32.2 \%$ \\
\hline Not informed & $0.3 \%$ & -- & $0.2 \%$ \\
\hline \multicolumn{1}{r|}{ Total } & $100.0 \%$ & $100.0 \%$ & $100.0 \%$ \\
\hline
\end{tabular}

In terms of the main reason for travelling by ferry, three major attributes can be identified in Table 6.11: ability to take vehicle on board the ferries (46.8\%), price/fare $(23.3 \%)$ and scenery $(17.8 \%)$. Ability to take vehicle on board the ferry is the major attribute for CSNZ $(48.2 \%)$ and domestic $(67.2 \%)$ passengers, which is not a surprise considering the high use of private vehicles to arrive in or depart from Wellington and Picton. In the case of international passengers, taking the vehicle onboard the ferry is not as important as for the other two sub-groups, simply because they can drop their cars in Wellington and pick up another one in Picton (or vice-versa). The most important attribute for international passengers $(33.3 \%)$ is the scenery aspect of the ferry trip, with the journey being considered as part of the tourism experience while travelling in New Zealand. Price/fare was the second most important reason for CSNZ and domestic passengers when deciding to travel by ferry. For international passengers price / fare was only the third most important reason, yet the proportion of international passengers $(21.6 \%)$ considering it the most important reason was higher than for domestic passengers $(18.8 \%)$. 
Table 6.11 Main reason for travelling by ferry.

\begin{tabular}{|l|r|r|r|r|}
\cline { 2 - 5 } \multicolumn{1}{c|}{} & \multicolumn{1}{c|}{\begin{tabular}{c} 
CSNZ \\
\multicolumn{1}{c|}{$(\mathrm{n}=303)$}
\end{tabular}} & $\begin{array}{c}\text { Domestic } \\
(\mathrm{n}=320)\end{array}$ & $\begin{array}{c}\text { International } \\
(\mathrm{n}=430)\end{array}$ & $\begin{array}{c}\text { Total } \\
(\mathrm{n}=1,053)\end{array}$ \\
\hline Ability to take vehicle & $48.2 \%$ & $67.2 \%$ & $30.7 \%$ & $46.8 \%$ \\
\hline Price / fare & $30.4 \%$ & $18.8 \%$ & $21.6 \%$ & $23.3 \%$ \\
\hline Scenery & $7.3 \%$ & $6.9 \%$ & $33.3 \%$ & $17.8 \%$ \\
\hline Time of departure & $2.6 \%$ & $0.6 \%$ & $0.7 \%$ & $1.2 \%$ \\
\hline Total travel time & $0.7 \%$ & $0.6 \%$ & $1.9 \%$ & $1.1 \%$ \\
\hline Comfort & $1.0 \%$ & $0.9 \%$ & $1.4 \%$ & $1.1 \%$ \\
\hline Vessel facilities / technology & $0.3 \%$ & $0.3 \%$ & $0.5 \%$ & $0.4 \%$ \\
\hline Safety & $0.7 \%$ & -- & -- & $0.2 \%$ \\
\hline Other & $8.9 \%$ & $4.7 \%$ & $10.0 \%$ & $8.1 \%$ \\
\hline & $100.0 \%$ & $100.0 \%$ & $100.0 \%$ & $100.0 \%$ \\
\hline
\end{tabular}

Apart from considering the main reason for travelling by ferry, the questionnaire also asked passengers to rate five key attributes when deciding to travel by ferry: the total travel time, the ferry price/fare, smooth crossing, the scenic aspect of the journey and cancellation / trip delay. A 'Likert' type of scale was suggested, with the respondents being able to rate the attributes with five different options: not important (1), less important (2), neutral (3), important (4) and very important (5). In order to compare these attributes among CSNZ, domestic and international passengers, the means were used (see Figure 6.12). According to Veal (1997), means are an appropriate form of analysis when using the 'Likert' scale.

From the results presented in Figure 6.12, it is possible to identify certain common patterns for most of the attributes. Except in the case of scenic trip, the means for the total sample and the means for each individual sub-group were usually very close, with variations no higher than 0.4 . Price/fare had the highest mean for all cases considered (total mean equal to 4.0), followed by cancellation/trip delay (3.7), smooth crossing (3.2) and total travel time (3.0). In comparison to international passengers, CSNZ and domestic passengers gave more importance to total travel time, price/fare and cancellation/trip delay. Even though the scenic trip attribute presented a similar overall mean (3.2) in comparison to total travel time and smooth crossing, the differences presented by the means of the individual groups have a spread of 1.0 point between international (3.8) and CSNZ (2.8) passengers. 


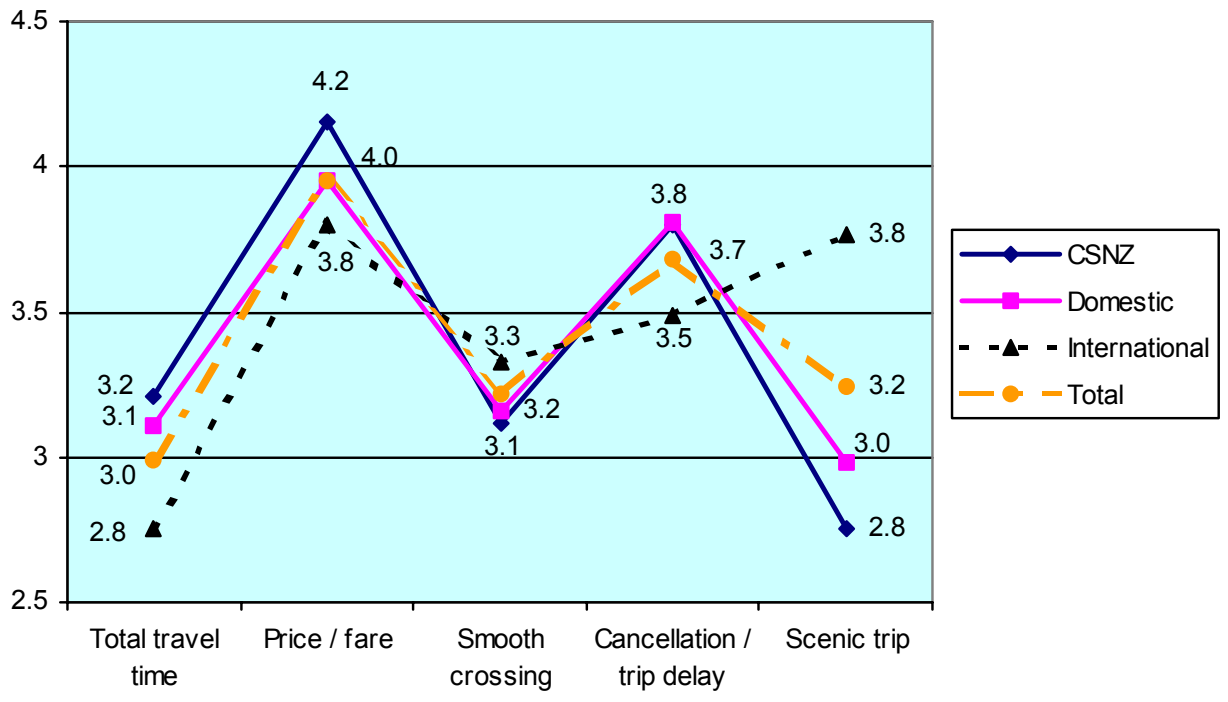

Figure 6.12 Average importance of five main attributes when using Cook Strait ferries.

The chart on Figure 6.12 permits an interesting interpretation of key attributes related to the ferry journey. In general, while CSNZ and domestic passengers gave more value to total travel time and price/fare, international passengers were particularly interested in the scenic attributes of the ferry journey. While passengers living in New Zealand considered the ferry as just another means of transport to take them as fast and cheaply as possible from one place to the other, international passengers perceived the ferry journey as part of their tourist experience. As one of the most scenic parts of the journey is the travel through the Tory Channel, where Picton is located, this gives this ferry port a competitive advantage when discussing the proposal to build a new terminal in Clifford Bay. Particularly among international passengers, this can be a significant reason to keep them going to Picton, while passengers living in New Zealand may prefer a fast travel option. The Clifford Bay proposal will be discussed in more detail in Chapter 8.

Finally, in terms of the ferries' booking procedures, three main characteristics can be identified. They are the distribution channels used to book the ferry (Table 6.12), when (Table 6.13) and where (Figure 6.13) the booking was made. The importance of knowing more about how passengers book the ferries can help to identify ways to promote the gateways when the reservation is being made, so travellers can consider the possibility to visit and stay in Wellington and Picton before they arrive there.

The main channels used to book the ferry service were telephone $(29.9 \%)$, the internet $(29.4 \%)$ and travel agents (19.2\%). In the case of CSNZ and domestic passengers, telephone and internet are, respectively, the first and second most common distribution 
channel. For international passengers, travel agents are the most used type of distribution channel (29.7\%).

Table 6.12 Ferry bookings' distribution channels.

\begin{tabular}{|l|r|r|r|r|}
\cline { 2 - 5 } \multicolumn{1}{c|}{} & $\begin{array}{r}\text { CSNZ } \\
(n=305)\end{array}$ & $\begin{array}{c}\text { Domestic } \\
(n=325)\end{array}$ & $\begin{array}{c}\text { International } \\
(n=438)\end{array}$ & $\begin{array}{c}\text { Total } \\
(n=1,068)\end{array}$ \\
\hline Telephone (0800) & $38.0 \%$ & $36.9 \%$ & $18.9 \%$ & $29.9 \%$ \\
\hline Internet & $33.1 \%$ & $36.6 \%$ & $21.5 \%$ & $29.4 \%$ \\
\hline Travel agent & $12.5 \%$ & $11.4 \%$ & $29.7 \%$ & $19.2 \%$ \\
\hline Ferry terminal & $5.9 \%$ & $4.9 \%$ & $12.1 \%$ & $8.1 \%$ \\
\hline Railway station & $5.2 \%$ & $2.8 \%$ & $1.6 \%$ & $3.0 \%$ \\
\hline I don't know & $3.9 \%$ & $2.5 \%$ & $2.7 \%$ & $3.0 \%$ \\
\hline Other & $1.3 \%$ & $4.9 \%$ & $13.5 \%$ & $7.4 \%$ \\
\hline Total & $100.0 \%$ & $100.0 \%$ & $100.0 \%$ & $100.0 \%$ \\
\hline
\end{tabular}

In terms of how far in advance the ferry ticket was booked before the journey was made, it is possible to note some similarities between this information and that provided by Figure 6.5 regarding passengers' flexibility. For example, according to Table 6.13 , bookings within the six previous days of the ferry journey account for $22 \%$ of CSNZ passengers, $21.6 \%$ of domestic and $48.3 \%$ of international passengers. As seen in Figure 6.5, international passengers were the sub-group with the most flexibility among ferry passengers, emphasizing that this group is making decisions about their trips as they travel, with most deciding whether or not a given destination, attraction or activity is worthwhile visiting/staying. This characteristic of international passengers can make them more likely to stay longer in the gateways, particularly if they like both nodes when they arrive there.

While the questionnaire did not ask whether or not passengers were bringing a vehicle aboard the ferries, it is possible to speculate that the more advanced bookings made by travellers living in New Zealand is due to the fact that they are travelling with vehicles onboard. The ferries have a more constrained capacity in terms of carrying vehicles than passengers, hence the need for early bookings. As shown in Table 6.8, a high proportion of CSNZ and domestic passengers make use of private vehicles to arrive in or depart from Wellington and Picton. International passengers tend to use more rental cars or campervans which rental car companies do not encourage to be taken onboard. 
Table 6.13 How far in advance ferry ticket was booked before the day of crossing.

\begin{tabular}{|l|r|r|r|r|}
\cline { 2 - 5 } \multicolumn{1}{c|}{} & \multicolumn{1}{c|}{\begin{tabular}{c} 
CSNZ \\
\multicolumn{1}{c|}{}
\end{tabular}} & $\begin{array}{c}\text { Domestic } \\
(\mathrm{n}=308)\end{array}$ & $\begin{array}{c}\text { International } \\
(\mathrm{n}=437)\end{array}$ & $\begin{array}{c}\text { Total } \\
(\mathrm{n}=1,069)\end{array}$ \\
\hline Same day & $0.6 \%$ & $4.0 \%$ & $8.0 \%$ & $4.7 \%$ \\
\hline $1-2$ days before & $8.4 \%$ & $7.4 \%$ & $24.7 \%$ & $14.8 \%$ \\
\hline 3-6 days before & $13.0 \%$ & $10.2 \%$ & $15.6 \%$ & $13.2 \%$ \\
\hline 7-14 days before & $15.9 \%$ & $12.0 \%$ & $9.2 \%$ & $12.0 \%$ \\
\hline $15-30$ days before & $20.5 \%$ & $15.4 \%$ & $10.1 \%$ & $14.7 \%$ \\
\hline $1-3$ months before & $25.6 \%$ & $28.4 \%$ & $20.6 \%$ & $24.4 \%$ \\
\hline $4-8$ months before & $14.9 \%$ & $21.6 \%$ & $10.8 \%$ & $15.2 \%$ \\
\hline More than 8 months before & $1.0 \%$ & $0.9 \%$ & $1.1 \%$ & $1.0 \%$ \\
\hline
\end{tabular}

A factor associated with when the ferry was booked is the information about where the booking was made (see Figure 6.13). Basically, three possibilities can be considered. The first occurs when passengers book the ferry before leaving their place of origin. The second is when the ferry is booked after having left their place of origin, but before arriving in the gateways. This happens when travellers book the ferry en route to one of the gateways. Thirdly, passengers can book the ferry after arriving in the ferry ports (Wellington or Picton). This information provides some clues about the flexibility the passengers have in their own trips, as those booking the ferry crossing en route or after arriving in the gateways are probably prepared to stay shorter or longer in the nodes they visit before catching the ferry according to their need to stay longer or shorter in their trips. CSNZ and domestic passengers have a higher percentage of bookings before leaving their place of residence, respectively $76.4 \%$ and $85.6 \%$. As they tend to book the ferry trip later, international passengers have a lower percentage of booking from their place of residence $(39.7 \%)$, while bookings en route represent $38.1 \%$ and in the ferry ports (Wellington and Picton) $22.2 \%$. It is likely that in the case of CSNZ passengers, who often live in Wellington, they were referring to Wellington/Picton as their place of residence, rather than the ferry port. 


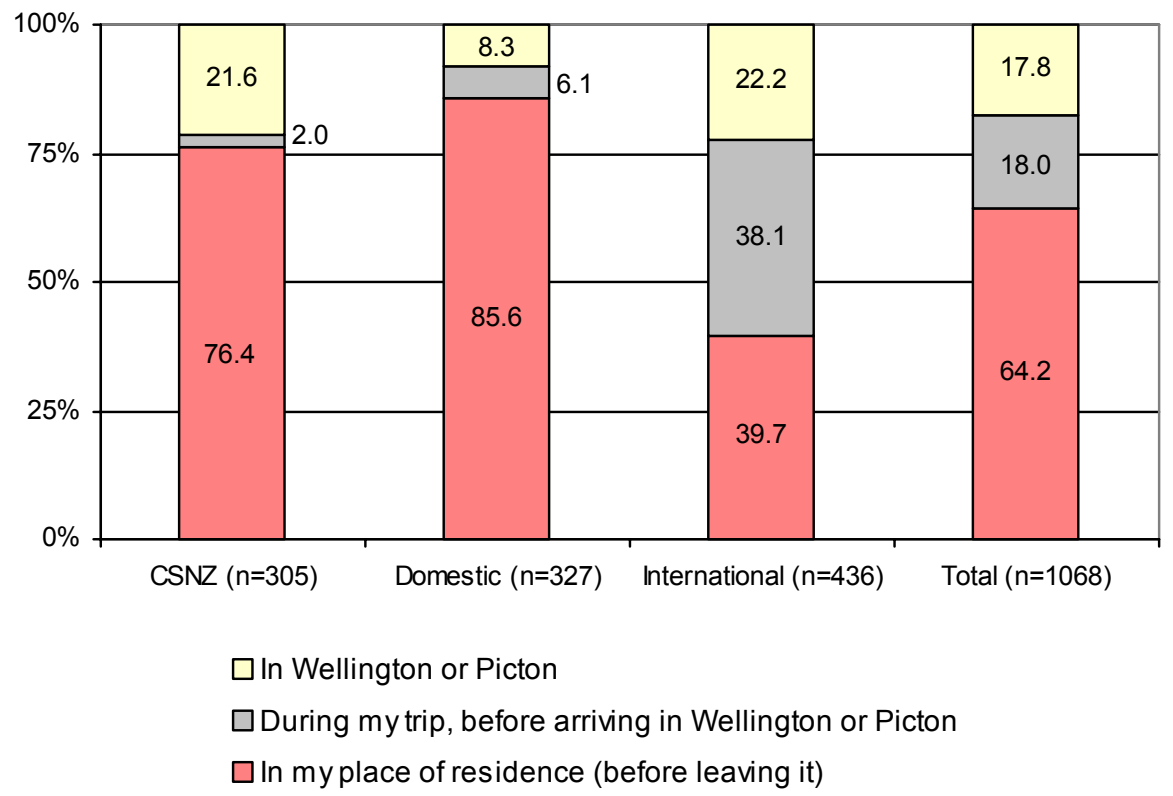

Figure 6.13 Place where ferry ticket was booked.

\subsection{Conclusion}

This chapter has presented a wide range of information related to the passengers' socioeconomic profile, their trips and the ferry journey. While the data presented in this chapter help to better segment the ferry passengers, it also highlighted that there are some characteristics of the demand that make certain passengers more likely to use the ferry gateways as tourist destinations. As data were presented segmenting passengers in terms of their place of origin, it is then possible to anticipate what are the chances of CSNZ, domestic and international passengers extending their stays in the gateways.

Some general conclusions about the passengers across sub-groups are that distance from the gateways influences the use of the ferries, with passengers living in the CSNZ region being over-represented in comparison to the population residing in these regions. In terms of international passengers, as was suggested on Chapter 4, certain nationalities have a higher participation rate than others, particularly those from the UK, Germany and other countries in Europe. On the other hand, passengers from the Asian market are underrepresented. Even though this is not the purpose of this research, the identification of the key markets that use the ferries can help to set up marketing campaigns targeting those segments of passengers that are more likely to stay longer in the gateways. Another 
general characteristic of the three sub-groups of passengers is the predominance of passengers travelling with the main purpose of trip either as leisure/holiday or VFR.

When considering other relevant data presented in this chapter, it was possible to conclude that international passengers are those who present the most favourable preconditions to extend their stays in Wellington in Picton. For example, they are those travelling with a higher degree of flexibility regarding the number of firm bookings (see Figure 6.5), with one out of four passenger travelling in a totally flexible manner and $35 \%$ of them being mainly flexible with some firm bookings. Adding this information to that about the ferry booking procedure, where international passengers tend to book the ferry within a week of the departure usually either in Wellington or Picton (22\%) or during their trip before arriving in the gateway (38\%), it is possible to affirm that in comparison to CSNZ and domestic passengers they are more prepared to make changes in their trips while travelling. This increases the chances of international passengers staying longer in the gateways if they perceive them as tourist destinations.

Other variables such as the number of nights spent on the trip and the number of regions visited also help to increase the likelihood of international passengers converting gateways into destinations. As the median values of their trips are higher (23 nights) than CSNZ (5 nights) and domestic (10 nights) passengers, it means simply that increasing one extra night at the gateways will have a different impact on the overall trip in comparison to those passengers on short trips. Taking into consideration that half of CSNZ passengers have a single destination trip, adding an extra night in the gateway may be considered as unnecessary as they can perhaps easily reach their final destination. Additionally, CSNZ passengers are probably more familiarised with the gateways perhaps from previous trips across Cook Strait or from previous trips to Wellington and Picton as tourist destinations. Therefore, the ferries can be considered more as a mode of transport to reach their final destinations rather than an opportunity to visit another destination, as happens with international passengers. On average they stay overnight in nearly eight different regions, which a significant part of them already staying overnight in the gateways, particularly in Wellington. However, it is true that extending their stays in the gateways would only happen at the expense of passengers staying shorter in other destinations.

Most of the assumptions made in terms of the information presented in this chapter need to be confirmed by the passengers' behaviour in terms of the gateways and their likelihood to stay longer there, which will be done in the next chapter. 


\section{Ferry Passengers in Wellington and Picton}

\subsection{Introduction}

Making more passengers extending their stay in both ferry gateways is part of the main aim of this research, which is to understand the relationship between gateway and destination functions. As discussed in Chapter 1 and the rest of this thesis, two key sets of players are involved in this task: the passengers and the suppliers. The former, however, have the ultimate decision to stay longer in the gateways, hence their opinions about the gateways and the analysis of their behaviour while there are crucial in understanding how to convert gateway travellers into destination tourists. This chapter analyses data from the passengers' point of view, while the next chapter deals with the suppliers' perspective.

The discussion in Chapter 2 about the concepts of gateways and destinations led in Chapter 3 to the operationalisation of four different functions; i.e. gateway travellers, overnight gateway visitors, stopover visitors and destination tourists. These functions encapsulate two core variables that help to understand the use passengers make of the nodes. They are the number of nights spent there and the main reason for going to the node (see Figure 3.2). The key characteristics of these functions are summarised below:

- Gateway Travellers (GT): passengers who do not stay overnight in the node and whose main reason for going there is the inter-island connection;

- Overnight Gateway Visitor (OGV): passengers who stay one night in the node, as long as this is not the only night they are spending on their trip ( $T \mid \neq 100)$, and whose main reason for going there is the inter-island connection;

- Stopover Visitors (SV): passengers who do not stay overnight and whose main reason for going to the node is other than the inter-island connection (case $S_{1}$ ) or those passengers that stay one night, as long as this is not the only night they are spending on their trip $(T \mid \neq 100)$, whose main reason for going there is not the interisland connection (case $\mathrm{SV}_{2}$ );

- Destination Tourists (DT): passengers who are staying two or more consecutive nights in the gateway during one leg of the journey (case $\mathrm{DT}_{1}$ ); or those who are staying only one night, but who are spending only one night on the current trip this particular node is their sole destination (case $\mathrm{DT}_{2}$ ).

While segmenting the sample by place of origin, as employed in Chapter 6, was a useful way to explore some of the general features of the demand, in order to fully analyse the 


\section{FERRY PASSENGERS IN WELLINGTON AND PICTON}

likelihood of passengers staying longer in the gateways it is necessary to incorporate their nodal functions in the analysis. Then it will be possible to consider what types of functions are more likely to extend their stays in the gateways and what the different functions require to do so.

In the case of Wellington, due to the high proportion of passengers living there (182 or $17.4 \%$ of the sample - see Table 3.4 ) and also excluding those passengers without a nodal function classification (e.g. those who did not state the number of nights they were spending in Wellington or the main reason for going there), a total of 861 passengers were classified. As can be seen from Figure 7.1, gateway travellers are the major segment (280 passengers or $32.5 \%$ of the sample), followed by destination tourists $(n=251$ or $29.1 \%)$, stopover visitors $(n=212$ or $24.6 \%)$ and overnight gateway visitor $(n=118$ or $13.7 \%)$. Stopover visitors comprise two sub-groups of passengers; i.e. $\mathrm{SV}_{1}$ $(\mathrm{n}=74$ or $8.6 \%)$ and $\mathrm{SV}_{2}(\mathrm{n}=138$ or $16 \%)$. Finally, most of the 251 passengers that are destination tourists in Wellington consist of passengers staying two or more nights there $\left(D_{1}\right)$. Only eight passengers $(0.9 \%)$ were spending only one night in Wellington with the capital city as their sole destination - $\mathrm{Ti}=100\left(\mathrm{DT}_{2}\right)$.

In Picton, due to its small population, less than $1 \%$ of the sample $(n=8)$ lived there (see Table 3.4). A total of 30 passengers omitted information about their length of stay in Picton or their main reason for going there and they could not be classified in terms of their nodal functions. A total of 1,029 passengers were classified in terms of the four nodal functions proposed in this research. As Table 3.4 has already shown, the gateway function in Picton is very predominant, with 695 passengers $(67 \%)$ being classified as gateway travellers. Overnight gateway visitors account for 118 passengers (11.4\%). According to Figure 7.1, stopover visitors in Picton ( $n=135$ or $13.1 \%$ ), consist of 99 passengers $(9.6 \%)$ in the case $\mathrm{SV}_{1}$ and 36 passengers $(3.5 \%)$ in the case $\mathrm{SV}_{2}$. Finally, destination tourists are almost all made up of passengers in the case $\mathrm{DT}_{1}(\mathrm{n}=78$ or $7.6 \%$ ), with only three passengers $(0.3 \%)$ in the case $\mathrm{DT}_{2}$.

After passengers have been classified in terms of their nodal functions for Wellington and Picton, it is then possible to identify their main characteristics for each of these nodes and the likelihood of them extending their stays in both nodes. For most of the data presented in this chapter the analysis will compare the different passengers' behaviour for the various functions. However, in order to enrich the level of detail of the analysis undertaken data will also be segmented in terms of the passengers' place of origin (CSNZ, domestic and international). This aims to help explain some of the passengers' behaviour while in the node based on their place of origin. 
WELLINGTON $\mathrm{n}=86 !(100.0 \%)$

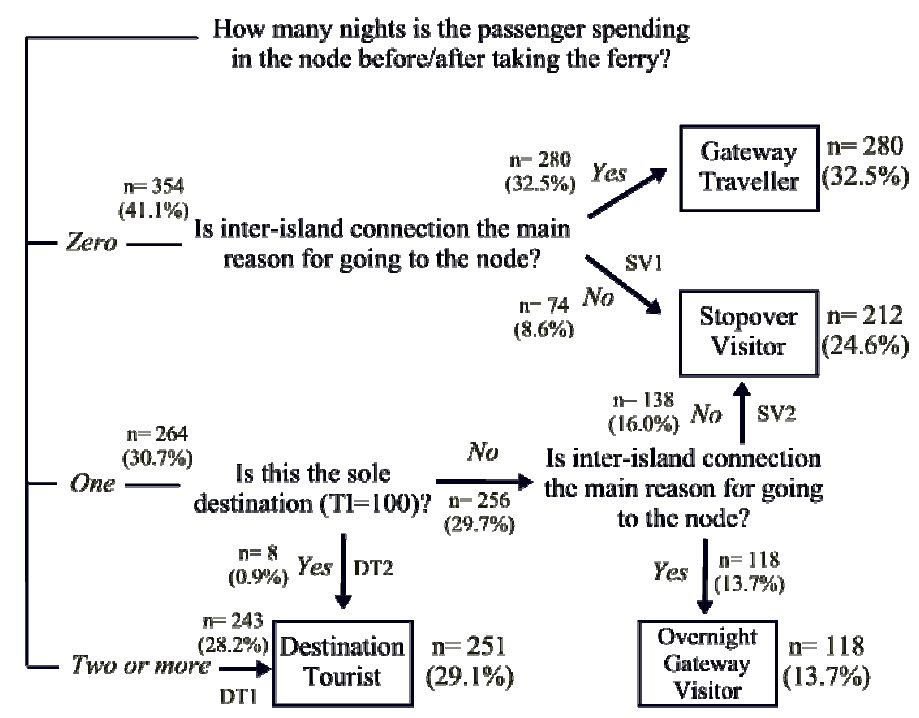

PICTON $\mathrm{n}=1,029(100.0 \%)$

How many nights is the passenger spending

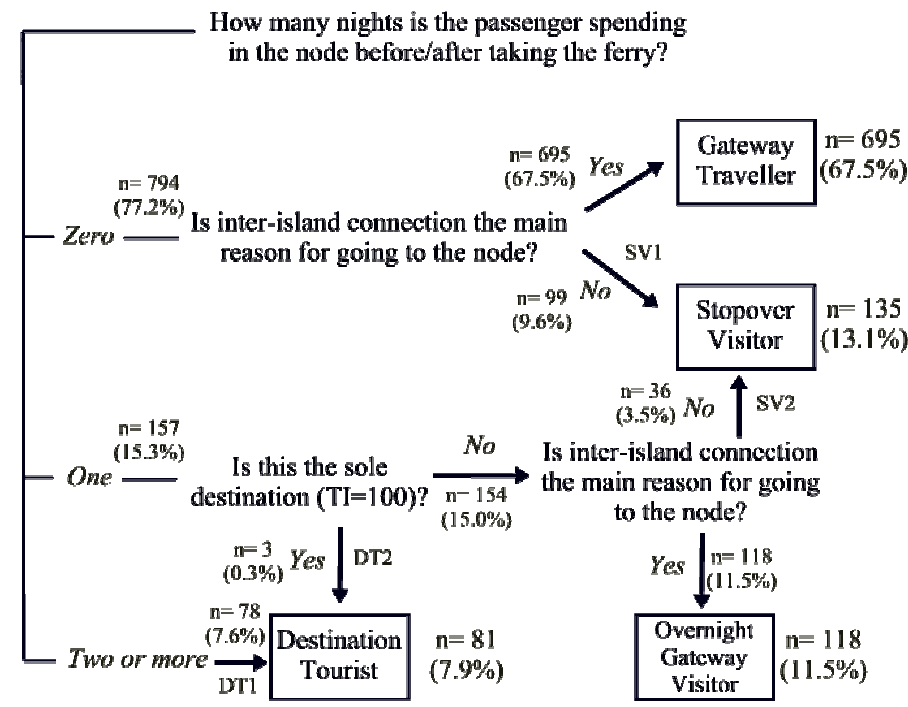

Figure 7.1 Distribution of nodal functions for respondents in Wellington and Picton.

Data presented, analysed and interpreted in this chapter come from Section E - About Wellington and Picton - of the questionnaire (see Appendix Two). This includes, for example, the main reason for going to the node, the length of stay, the type of accommodation used, the activities undertaken there and the sources of information used by the passengers to know more about Wellington and Picton and the actual change of plans in terms of their stays in both nodes. Data obtained in Section $D$ of the questionnaires - About current ferry journey - was also considered. More precisely, it 


\section{FERRY PASSENGERS IN WELLINGTON AND PICTON}

analyses the impact that more information and a special deal would have had on passengers' decision to stay longer or shorter in the nodes. It is worth mentioning that in most of the cases data presented in this chapter refers to the total percentage of the sample for each nodal function (gateway traveller, overnight gateway visitor, stopover visitor or destination tourist). For each nodal function, data is then segmented in terms of the passengers' place of origin so that comparisons in terms of what are the main values/characteristics for CSNZ, domestic or international passengers can be made between the various nodal functions (e.g. Table 7.6). In the cases where multiple responses were allowed (e.g. Table 7.10), the percentage presented in each cell refers to the column's total sample.

This chapter is divided into two major sections: one about Wellington; the other about Picton. The main reason for this is that there are major differences between Wellington and Picton in terms of their tourism development and characteristics (urban capital city vs. small resort town), which may influence the same passenger to have different category functions for both nodes. Table 7.1 shows a cross-tabulation of nodal functions for Wellington and Picton. Apart from passengers that are concomitantly gateway travellers in Wellington and Picton (18.1\% of the total sample), for all other functions this participation is less expressive (overnight gateway visitor: $0.6 \%$; stopover visitor: $3.9 \%$; destination tourist: $1.8 \%)$. Gateway travellers in both nodes are the most common case, followed by DT in Wellington / GT in Picton (15.7\%), SV in Wellington / GT in Picton (12.5\%) and OGV in Wellington / GT in Picton (9.7\%). Even in the situations that passengers have the same function in both nodes it is still appropriate to consider the cases of Wellington and Picton separately, as they will be interacting with different tourism products and activities existing in each place. At the end of each section there is a summary that draws together the key passenger patterns associated with both nodes.

While an individual analysis of each node is useful in order to recognize what sort of behaviour passengers have while at individual nodes, from an academic point of view this research can also benefit from a comparison between passengers' behaviour in both nodes. There are two benefits in doing this. Firstly, to identify the passengers' characteristics that are common to both Wellington and Picton. These two nodes have rather different features - Wellington being the capital city with a well established destination function; Picton a small seaport town with a strong gateway function - that passengers behaving in a similar way in both places may indicate that these are characteristics intrinsic to the passengers, not to the nodes. Secondly, there is the advantage of being able to identify how different nodes can influence passengers to have 


\section{FERRY PASSENGERS IN WELLINGTON AND PICTON}

different behaviours depending on whether they are in Wellington or Picton. Therefore, a comparative section is presented at the end of this chapter.

Table 7.1 Cross-tabulation of nodal function in Wellington and Picton.

\begin{tabular}{|c|c|c|c|c|c|c|c|}
\hline & & \multicolumn{6}{|c|}{ PICTON } \\
\hline & & Origin & $\begin{array}{l}\text { Gateway } \\
\text { traveller }\end{array}$ & $\begin{array}{r}\text { Overnight } \\
\text { gateway visitor }\end{array}$ & $\begin{array}{r}\text { Stopover } \\
\text { visitor }\end{array}$ & $\begin{array}{r}\text { Destination } \\
\text { tourist }\end{array}$ & Total \\
\hline \multirow{12}{*}{ 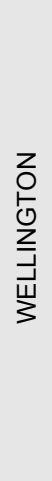 } & \multirow{2}{*}{ Origin } & & 112 & 2 & 34 & 30 & 178 \\
\hline & & & $11.1 \%$ & $0.2 \%$ & $3.4 \%$ & $3.0 \%$ & $17.6 \%$ \\
\hline & \multirow{2}{*}{ Gateway traveller } & & 183 & 52 & 26 & 16 & 277 \\
\hline & & & $18.1 \%$ & $5.2 \%$ & $2.6 \%$ & $1.6 \%$ & $27.5 \%$ \\
\hline & \multirow{2}{*}{$\begin{array}{r}\text { Overnight } \\
\text { gateway visitor }\end{array}$} & 2 & 98 & 6 & 4 & 4 & 114 \\
\hline & & $0.2 \%$ & $9.7 \%$ & $0.6 \%$ & $0.4 \%$ & $0.4 \%$ & $11.3 \%$ \\
\hline & \multirow{2}{*}{ Stopover visitor } & 2 & 126 & 25 & 39 & 11 & 203 \\
\hline & & $0.2 \%$ & $12.5 \%$ & $2.5 \%$ & $3.9 \%$ & $1.1 \%$ & $20.1 \%$ \\
\hline & \multirow{2}{*}{ Destination tourist } & 4 & 158 & 29 & 28 & 18 & 237 \\
\hline & & $0.4 \%$ & $15.7 \%$ & $2.9 \%$ & $2.8 \%$ & $1.8 \%$ & $23.5 \%$ \\
\hline & \multirow{2}{*}{ Total } & 8 & 677 & 114 & 131 & 79 & 1,009 \\
\hline & & $0.8 \%$ & $67.1 \%$ & $11.3 \%$ & $13.0 \%$ & $7.8 \%$ & $100.0 \%$ \\
\hline
\end{tabular}

Finally, two other factors that need to be considered before focusing the analysis on Wellington and Picton is to identify whether or not there are differences in the passengers' functions in terms of seasonality (low or high seasons) and regarding the directions they are travelling (northbound or southbound).

When a cross-tabulation between passengers' nodal function and when they were travelling in the ferries (low or high season) is considered (see Table 7.2) different results are obtained. As considered earlier in Chapter 3, passengers travelling between July and October 2003 fall within the winter season, with those travelling between December 2003 and January 2004 forming part of the summer season. When comparing the total participation of passengers in both seasons (approximately $36 \%$ for the low season and $64 \%$ for the high season), it is possible to note that the participation of gateway travellers in Wellington is higher during the summer time (80\%). On the other hand, the participation of destination tourists in Wellington during the high season $(56.6 \%)$ is below the total participation of passengers during this season (64.4\%). In Picton, even though the participation of the various functions are very similar to the distribution between the low and high seasons, the participation of destination tourists during the winter time is higher than the total participation $(43.2 \%$ against $35.8 \%)$. While at first glance these results contradict what may have been expected, further analysis would be necessary to try to explain them, which is beyond the scope of this research. 


\section{FERRY PASSENGERS IN WELLINGTON AND PICTON}

Table 7.2 Cross-tabulation between passengers' nodal functions and time of ferry journey.

\begin{tabular}{|l|r|r|r|r|r|r|}
\cline { 2 - 7 } \multicolumn{1}{c|}{ Nodal Function } & \multicolumn{3}{c|}{ Wellington } & \multicolumn{3}{c|}{ Picton } \\
\cline { 2 - 7 } & Low season & High season & Total & Low season & High season & Total \\
\hline Origin & 78 & 104 & 182 & 5 & 3 & 8 \\
\hline Gateway & $42.9 \%$ & $57.1 \%$ & $100.0 \%$ & $62.5 \%$ & $37.5 \%$ & $100.0 \%$ \\
traveller & 56 & 224 & 280 & 239 & 456 & 695 \\
\hline Overnight & $20.0 \%$ & $80.0 \%$ & $100.0 \%$ & $34.4 \%$ & $65.6 \%$ & $100.0 \%$ \\
gateway visitor & 52 & 66 & 118 & 37 & 81 & 118 \\
\hline Stopover visitor & $31.4 \%$ & $68.6 \%$ & $100.0 \%$ & $31.4 \%$ & $68.6 \%$ & $100.0 \%$ \\
\hline Destination & 76 & 136 & 212 & 55 & 80 & 135 \\
tourist & $35.8 \%$ & $64.2 \%$ & $100.0 \%$ & 40.7 & $59.3 \%$ & $100.0 \%$ \\
\hline \multicolumn{1}{|c}{ Total } & 109 & 142 & 251 & 35 & 46 & 81 \\
& $43.4 \%$ & $56.6 \%$ & $100.0 \%$ & $43.2 \%$ & $56.8 \%$ & $100.0 \%$ \\
\hline
\end{tabular}

As seen earlier, particularly in the case of international passengers, tourist flows in New Zealand are characterised by a touring pattern with multiple destination visits, with a slightly higher participation of visitors travelling southbound. If there are major variations in the passengers' nodal functions according to the direction they are travelling, then this might help to set up strategies to increase their stays in the gateways before they reach Wellington or Picton. Tables 7.3 and 7.4 respectively present the nodal functions according to whether they are departing from Wellington or Picton. As the same number of questionnaires was collected in both directions (538 - i.e. 50\% each way), significant differences between directions for the same nodal function can emphasize a certain tendency towards one particular place of departure. According to both tables presented below, in some cases it seems that no major difference was identified, such as SV in Wellington $(49.1 \%$ for passengers departing from Picton vs. $50.9 \%$ for those departing from Wellington). In most cases, the differences are not higher than $10 \%$. The only case where major differences are found is OGV. Both in Wellington and Picton there is a higher proportion of passengers in this category of those passengers who are leaving the node rather than arriving there. In Wellington, for example, nearly two-thirds of the OGV $(66.1 \%)$ are made up of passengers who were leaving Wellington. The same happens in the case of Picton, where $61 \%$ of OGV there consist of passengers who were departing from Picton. As seen on Table 3.2, time of departure can explain this pattern as nearly half of all questionnaires were collected in ferry services that departed in the morning. Hence, these travellers most likely stayed overnight in the gateway in order to catch the ferry the next morning. 
Table 7.3 Nodal functions in Wellington by place of departure.

\begin{tabular}{|c|c|c|c|c|c|c|c|c|c|c|c|c|c|c|c|c|}
\hline \multirow[b]{2}{*}{$\begin{array}{l}\text { Place of } \\
\text { departure }\end{array}$} & \multicolumn{4}{|c|}{ Gateway Travellers } & \multicolumn{4}{|c|}{ Overnight Gateway Visitors } & \multicolumn{4}{|c|}{ Stopover Visitors } & \multicolumn{4}{|c|}{ Destination Tourists } \\
\hline & $\begin{array}{l}\text { CSNZ } \\
(n=39)\end{array}$ & $\begin{array}{c}\text { Dom } \\
(n=157)\end{array}$ & $\begin{array}{c}\text { Int'l } \\
(n=84)\end{array}$ & $\begin{array}{c}\text { Total } \\
\text { (n=280; }\end{array}$ & $\begin{array}{l}\operatorname{CSNZ} \\
(n=6)\end{array}$ & $\begin{array}{c}\text { Dom } \\
(n=41)\end{array}$ & $\begin{array}{c}\text { Int'l } \\
(n=71)\end{array}$ & $\begin{array}{c}\text { Total } \\
\text { (n=118; }\end{array}$ & $\begin{array}{l}\text { CSNZ } \\
(n=21)\end{array}$ & $\begin{array}{l}\text { Dom } \\
(n=55)\end{array}$ & $\begin{array}{c}\text { Int'l } \\
(n=136)\end{array}$ & $\begin{array}{c}\text { Total } \\
\text { [n=212; }\end{array}$ & $\begin{array}{l}\text { CSNZ } \\
(n=46)\end{array}$ & $\begin{array}{c}\text { Dom } \\
(n=63)\end{array}$ & $\begin{array}{c}\text { Int'l } \\
(n=142)\end{array}$ & $\begin{array}{c}\text { Total } \\
(n=251\end{array}$ \\
\hline Picton & $\begin{array}{r}22 \\
56.4 \%\end{array}$ & $\begin{array}{r}93 \\
59.2 \%\end{array}$ & $\begin{array}{r}43 \\
51.2 \%\end{array}$ & $\begin{array}{r}158 \\
56.4 \%\end{array}$ & $\begin{array}{r}2 \\
33.3 \%\end{array}$ & $\begin{array}{r}20 \\
48.8 \%\end{array}$ & $\begin{array}{r}18 \\
25.4 \%\end{array}$ & $\begin{array}{r}40 \\
33.9 \%\end{array}$ & $\begin{array}{r}12 \\
57.1 \%\end{array}$ & $\begin{array}{r}32 \\
58.2 \%\end{array}$ & $\begin{array}{r}60 \\
44.1 \%\end{array}$ & $\begin{array}{r}104 \\
49.1 \%\end{array}$ & $\begin{array}{r}30 \\
65.2 \%\end{array}$ & $\begin{array}{r}37 \\
58.7 \%\end{array}$ & $\begin{array}{r}67 \\
47.2 \%\end{array}$ & $\begin{array}{r}134 \\
53.4 \%\end{array}$ \\
\hline Wellington & $\begin{array}{r}17 \\
43.6 \% \\
\end{array}$ & $\begin{array}{r}64 \\
40.8 \% \\
\end{array}$ & $\begin{array}{r}41 \\
48.8 \% \\
\end{array}$ & $\begin{array}{r}122 \\
43.6 \% \\
\end{array}$ & $\begin{array}{r}4 \\
66.7 \\
\end{array}$ & $\begin{array}{r}21 \\
51.2 \% \\
\end{array}$ & $\begin{array}{r}53 \\
74.6 \% \\
\end{array}$ & $\begin{array}{r}78 \\
66.1 \% \\
\end{array}$ & $\begin{array}{r}9 \\
42.9 \% \\
\end{array}$ & $\begin{array}{r}23 \\
41.8 \% \\
\end{array}$ & $\begin{array}{r}76 \\
55.9 \% \\
\end{array}$ & $\begin{array}{r}108 \\
50.9 \% \\
\end{array}$ & $\begin{array}{r}16 \\
34.8 \% \\
\end{array}$ & $\begin{array}{r}26 \\
41.3 \% \\
\end{array}$ & $\begin{array}{r}75 \\
52.8 \% \\
\end{array}$ & $\begin{array}{r}117 \\
46.6 \% \\
\end{array}$ \\
\hline Total & $100 \%$ & $100 \%$ & $100 \%$ & $100 \%$ & $100 \%$ & $100 \%$ & $100 \%$ & $100 \%$ & $100 \%$ & $100 \%$ & $100 \%$ & $100 \%$ & $100 \%$ & $100 \%$ & $100 \%$ & $100 \%$ \\
\hline
\end{tabular}

Table 7.4 Nodal functions in Picton by place of departure.

\begin{tabular}{|c|c|c|c|c|c|c|c|c|c|c|c|c|c|c|c|c|}
\hline \multirow[b]{2}{*}{$\begin{array}{l}\text { Place of } \\
\text { departure }\end{array}$} & \multicolumn{4}{|c|}{ Gateway Travellers } & \multicolumn{4}{|c|}{ Overnight Gateway Visitors } & \multicolumn{4}{|c|}{ Stopover Visitors } & \multicolumn{4}{|c|}{ Destination Tourists } \\
\hline & $\begin{array}{c}\text { CSNZ } \\
(\mathrm{n}=210)\end{array}$ & $\begin{array}{c}\text { Dom } \\
(\mathrm{n}=223)\end{array}$ & $\begin{array}{c}\text { Int'l } \\
(n=262)\end{array}$ & $\begin{array}{c}\text { Total } \\
(n=695\end{array}$ & $\begin{array}{l}\text { CSNZ } \\
(n=4)\end{array}$ & $\begin{array}{c}\text { Dom } \\
(n=46)\end{array}$ & $\begin{array}{c}\text { Int'l } \\
(n=68)\end{array}$ & $\begin{array}{r}\text { Total } \\
(n=11 \varepsilon\end{array}$ & $\begin{array}{l}\mathrm{CSN} Z \\
(\mathrm{n}=42)\end{array}$ & $\begin{array}{l}\text { Dom } \\
(n=33)\end{array}$ & $\begin{array}{c}\text { Int'l } \\
(n=60)\end{array}$ & $\begin{array}{c}\text { Total } \\
(n=135\end{array}$ & $\begin{array}{l}\text { CSNZ } \\
(n=31)\end{array}$ & $\begin{array}{l}\text { Dom } \\
(n=13)\end{array}$ & $\begin{array}{c}\text { Int'l } \\
(n=37)\end{array}$ & $\begin{array}{r}\text { Total } \\
(\mathrm{n}=81\end{array}$ \\
\hline Picton & $\begin{array}{r}108 \\
51.4 \% \\
\end{array}$ & $\begin{array}{r}122 \\
54.7 \% \\
\end{array}$ & $\begin{array}{r}103 \\
39.3 \% \\
\end{array}$ & $\begin{array}{r}333 \\
47.9 \% \\
\end{array}$ & $\begin{array}{r}3 \\
75.0 \% \\
\end{array}$ & $\begin{array}{r}26 \\
56.5 \% \\
\end{array}$ & $\begin{array}{r}43 \\
63.2 \% \\
\end{array}$ & $\begin{array}{r}72 \\
61.0 \% \\
\end{array}$ & $\begin{array}{r}21 \\
50.0 \% \\
\end{array}$ & $\begin{array}{r}21 \\
63.6 \% \\
\end{array}$ & $\begin{array}{r}20 \\
33.3 \% \\
\end{array}$ & $\begin{array}{r}62 \\
45.9 \% \\
\end{array}$ & $\begin{array}{r}16 \\
51.6 \% \\
\end{array}$ & $\begin{array}{r}6 \\
46.2 \% \\
\end{array}$ & $\begin{array}{r}20 \\
54.1 \% \\
\end{array}$ & $\begin{array}{r}42 \\
51.9 \% \\
\end{array}$ \\
\hline Wellington & $\begin{array}{r}102 \\
48.6 \% \\
\end{array}$ & $\begin{array}{r}101 \\
45.3 \% \\
\end{array}$ & $\begin{array}{r}159 \\
60.7 \% \\
\end{array}$ & $\begin{array}{r}362 \\
52.1 \% \\
\end{array}$ & $\begin{array}{r}1 \\
25.0 \% \\
\end{array}$ & $\begin{array}{r}20 \\
43.5 \% \\
\end{array}$ & $\begin{array}{r}25 \\
36.8 \% \\
\end{array}$ & $\begin{array}{r}46 \\
39.0 \% \\
\end{array}$ & $\begin{array}{r}21 \\
50.0 \% \\
\end{array}$ & $\begin{array}{r}12 \\
36.4 \% \\
\end{array}$ & $\begin{array}{r}40 \\
66.7 \% \\
\end{array}$ & $\begin{array}{r}73 \\
54.1 \% \\
\end{array}$ & $\begin{array}{r}15 \\
48.4 \% \\
\end{array}$ & $\begin{array}{r}7 \\
53.8 \% \\
\end{array}$ & $\begin{array}{r}17 \\
45.9 \% \\
\end{array}$ & $\begin{array}{r}39 \\
48.1 \% \\
\end{array}$ \\
\hline Total & $100 \%$ & $100 \%$ & $100 \%$ & $100 \%$ & $100 \%$ & $100 \%$ & $100 \%$ & $100 \%$ & $100 \%$ & $100 \%$ & $100 \%$ & $100 \%$ & $100 \%$ & $100 \%$ & $100 \%$ & $100 \%$ \\
\hline
\end{tabular}




\section{FERRY PASSENGERS IN WELLINGTON AND PICTON}

\subsection{Ferry Passengers in Wellington}

From the definitions used to classify passengers into their nodal functions (see Figure 7.1), three key variables were used. They are the number of nights spent in the node, the main reason for going there and the Trip Index. They will be presented before discussing other pertinent data to understand the passengers' behaviour while in Wellington.

In terms of the main reason for going to Wellington, clear differences are found between the four different nodal functions (see Table 7.5). By definition, gateway travellers (GT) and overnight gateway visitors (OGV) are exclusively made up of passengers whose main reason for going to the node is the inter-island connection. Stopover visitors (SV) and destination tourists (DT) present different characteristics, with leisure/holiday being the most important reason for SV going to Wellington (61.6\%), followed by VFR $(25.6 \%)$. These are the two major reasons for DT going to Wellington, but the difference here is very narrow, with leisure/holiday accounting for $38.3 \%$ and VFR for $36.3 \%$. For both functions, however, international passengers on leisure/holiday comprise the single most important sub-group ( $53.6 \%$ for SV and $33.5 \%$ for DT). On the other hand, VFR accounts for the most important reason for CSNZ and domestic passengers going to Wellington (see Table 7.5). This is in line with the urban tourism literature (Chapter 2) that shows VFR is one the most important reasons for visiting cities, especially for domestic travellers. Finally it is worth mentioning that for both functions (SV and DT) the number of passengers using the ferry for business/work trips to Wellington was very small. One of the reasons for this can be attributed to the long journey a ferry trip represents, taking at least two hours and a quarter in addition to the time spent to/from the ferry ports to their place of origin/final destination. Being less price sensitive, business travellers are usually more likely to fly to Wellington rather than use the ferries. As seen in Chapter 4, Wellington has the busiest airport for domestic traffic in the country.

For the variable 'number of nights spent in the node' (Table 7.6), the analysis is even more straightforward than in the previous case. For the number of nights spent in Wellington, it is worthwhile examining only the SV and DT functions, as the other two functions are a constant. While $65 \%$ of SV stay one night in Wellington, the remaining $35 \%$ do not stay overnight. In the case of DT, nearly half of the passengers (47.4\%) stay two nights in Wellington, followed by three nights (17.5\%) and six or more nights $(14.7 \%)$.

While the Trip Index is very much associated with the number of nights spent in the node, it compares the importance that the node analysed has in relation to the other nodes the traveller has spent at least one night in. Gateway travellers consist exclusively of transit or 


\section{FERRY PASSENGERS IN WELLINGTON AND PICTON}

day trip $(\mathrm{Ti}=0)$, as by definition these passengers do not stay overnight in the node. For the other three functions (OGV, SV and DT) the single most important Trip Index is the short stopover $(0<\mathrm{Ti} \leq 10)$, even though its weight decreases from $87.3 \%$ in the case of OGV to $33.8 \%$ for DT. For SV, short stopovers account for over half of this sample $(54 \%)$, with transit or day trip representing over one-third of the passengers (34.8\%). Destination tourists present an even distribution in terms of the Trip Index, with the short stopover accounting for $33.8 \%$ of its passengers, sole destination $(\mathrm{Ti}=100)$ representing nearly a quarter of its passengers $(23.8 \%)$, followed narrowly by medium stopover $(10<\mathrm{Ti} \leq 20)$. It is interesting to highlight that while for all three other functions the majority of CSNZ and domestic passengers have a transit / short stopover approach towards the capital city, in terms of destination tourists the sole destination is the main Trip Index for these two places of origin. This emphasizes that while for most of CSNZ and domestic passengers Wellington has only a transient role in order to reach other destinations across the country, for those passengers that are staying more than two nights (DT) they are catching the ferry to reach Wellington as a destination, rather than just passing through. International passengers, on the other hand, appear to take more advantage of their circuit travel around New Zealand (see discussion presented in Chapters 2, 4 and 6) to stop in Wellington before or after crossing the Cook Strait. While they do not tend to stay long in the capital city, nor do they see it as a simple transit point.

Associated with the number of nights spent in Wellington is the type of accommodation used by travellers. By definition, gateway travellers do not stay overnight in the node (see Figure 7.1); therefore they were not included in this analysis (see Table 7.8). According to Table 7.8, travellers mostly used four types of accommodations while in Wellington: hotels, motels, backpackers and private homes. It is interesting to note that in the case of longer stays - destination tourists - private homes are the most common type of accommodation. Apparently DTs seem to take advantage of the free stay with friends and relatives in their private homes to afford longer stays in the capital city, with VFR travel being less associated with circuit travel. Private homes are also the main type of accommodation for CSNZ and domestic passengers for all nodal functions as CSNZ and domestic passengers are more likely to have more friends and relatives living in the capital city in comparison to travellers from overseas. For international travellers, backpackers / youth hostels are the preferred type of accommodation, followed by hotels, for the three types of nodal functions (OGV, SV and DT). 
Table 7.5 Main reason for going to Wellington.

\begin{tabular}{|c|c|c|c|c|c|c|c|c|c|c|c|c|c|c|c|c|c|}
\hline \multirow[b]{2}{*}{ Main reason } & \multirow{2}{*}{$\begin{array}{c}\text { Origin } \\
(n=182)\end{array}$} & \multicolumn{4}{|c|}{ Gateway Travellers $(n=280)$} & \multicolumn{4}{|c|}{ Overnight Gateway Visitors $(n=118)$} & \multicolumn{4}{|c|}{ Stopover Visitors $(n=211)$} & \multicolumn{4}{|c|}{ Destination Tourists $(n=248)$} \\
\hline & & CSNZ & Dom & Int'l & Total & CSNZ & Dom & Int'l & Total & CSNZ & Dom & Int'l & Total & CSNZ & Dom & Int'I & Total \\
\hline Live in Wellington & $100.0 \%$ & & & & & & & & & & & & & & & & \\
\hline Inter-island connection & & $13.9 \%$ & $56.1 \%$ & $30.0 \%$ & $100.0 \%$ & $5.1 \%$ & $34.7 \%$ & $60.2 \%$ & $100.0 \%$ & & & & & $0.8 \%$ & $3.2 \%$ & $7.3 \%$ & $11.3 \%$ \\
\hline Leisure/Holiday & & & & & & & & & & $0.5 \%$ & $7.6 \%$ & $53.6 \%$ & $61.6 \%$ & $2.4 \%$ & $2.4 \%$ & $33.5 \%$ & $38.3 \%$ \\
\hline VFR & & & & & & & & & & $4.7 \%$ & $12.8 \%$ & $8.1 \%$ & $25.6 \%$ & $10.5 \%$ & $13.7 \%$ & $12.1 \%$ & $36.3 \%$ \\
\hline Business/work & & & & & & & & & & $0.9 \%$ & $3.8 \%$ & $0.5 \%$ & $5.2 \%$ & $0.8 \%$ & $0.8 \%$ & $0.4 \%$ & $2.0 \%$ \\
\hline Other & & & & & & & & & & $3.8 \%$ & $1.9 \%$ & $1.9 \%$ & $7.6 \%$ & $4.0 \%$ & $5.2 \%$ & $2.8 \%$ & $12.1 \%$ \\
\hline Total & $100.0 \%$ & $13.9 \%$ & $56.1 \%$ & $30.0 \%$ & $100.0 \%$ & $5.1 \%$ & $34.7 \%$ & $60.2 \%$ & $100.0 \%$ & $10.0 \%$ & $26.1 \%$ & $64.0 \%$ & $100.0 \%$ & $18.5 \%$ & $25.4 \%$ & $56.0 \%$ & $100.0 \%$ \\
\hline
\end{tabular}

Table 7.6 Number of nights while in Wellington.

\begin{tabular}{|c|c|c|c|c|c|c|c|c|c|c|c|c|c|c|c|c|}
\hline \multirow{2}{*}{$\begin{array}{l}\text { Number of } \\
\text { nights }\end{array}$} & \multicolumn{4}{|c|}{ Gateway Travellers $(n=280)$} & \multicolumn{4}{|c|}{ Overnight Gateway Visitors $(n=118)$} & \multicolumn{4}{|c|}{ Stopover Visitors $(n=212)$} & \multicolumn{4}{|c|}{ Destination Tourists $(n=251)$} \\
\hline & CSNZ & Dom & Int'l & Total & CSNZ & Dom & Int'l & Total & CSNZ & Dom & Int'l & Total & CSNZ & Dom & Int'l & Total \\
\hline 0 & $13.9 \%$ & $56.1 \%$ & $30.0 \%$ & $100.0 \%$ & & & & & $6.1 \%$ & $13.2 \%$ & $15.6 \%$ & $34.9 \%$ & & & & \\
\hline 1 & & & & & $5.1 \%$ & $34.7 \%$ & $60.2 \%$ & $00.0 \%$ & $3.8 \%$ & $12.7 \%$ & $48.6 \%$ & $65.1 \%$ & $2.8 \%$ & $0.4 \%$ & & $3.2 \%$ \\
\hline 2 & & & & & & & & & & & & & $4.4 \%$ & $9.6 \%$ & $33.5 \%$ & $47.4 \%$ \\
\hline 3 & & & & & & & & & & & & & $4.4 \%$ & $4.8 \%$ & $8.4 \%$ & $17.5 \%$ \\
\hline 4 & & & & & & & & & & & & & $2.8 \%$ & $3.2 \%$ & $6.0 \%$ & $12.0 \%$ \\
\hline 5 & & & & & & & & & & & & & $1.6 \%$ & $2.0 \%$ & $1.6 \%$ & $5.2 \%$ \\
\hline $6+$ & & & & & & & & & & & & & $2.4 \%$ & $5.2 \%$ & $7.2 \%$ & $14.7 \%$ \\
\hline Total & $13.9 \%$ & $56.1 \%$ & $30.0 \%$ & $100.0 \%$ & $5.1 \%$ & $34.7 \%$ & $60.2 \%$ & $00.0 \%$ & $9.9 \%$ & $25.9 \%$ & $64.2 \%$ & $100.0 \%$ & $18.3 \%$ & $25.1 \%$ & $56.6 \%$ & $100.0 \%$ \\
\hline
\end{tabular}


Table 7.7 Trip index for Wellington.

\begin{tabular}{|c|c|c|c|c|c|c|c|c|c|c|c|c|c|c|c|c|}
\hline \multirow[b]{2}{*}{ Trip Index } & \multicolumn{4}{|c|}{ Gateway Travellers $(n=280)$} & \multicolumn{4}{|c|}{ Overnight Gateway Visitors $(n=118)$} & \multicolumn{4}{|c|}{ Stopover Visitors $(\mathrm{n}=212)$} & \multicolumn{4}{|c|}{ Destination Tourists $(n=251)$} \\
\hline & CSNZ & Dom & Int'l & Total & CSNZ & Dom & Int'l & Total & CSNZ & Dom & Int'l & Total & CSNZ & Dom & Int'l & Total \\
\hline $\mathrm{Ti}=0$ (transit or day trip) & $13.9 \%$ & $56.1 \%$ & $30.0 \%$ & $100.0 \%$ & & & & & $4.5 \%$ & $13.6 \%$ & $16.7 \%$ & $34.8 \%$ & & & & \\
\hline $0<\mathrm{Ti} \leq 10$ (short stopover) & & & & & $2.7 \%$ & $24.5 \%$ & $60.0 \%$ & $87.3 \%$ & $1.0 \%$ & $7.1 \%$ & $46.0 \%$ & $54.0 \%$ & $0.4 \%$ & $4.2 \%$ & $29.2 \%$ & $33.8 \%$ \\
\hline $10<\mathrm{Ti} \leq 20$ (medium stopover) & & & & & $1.8 \%$ & $6.4 \%$ & $0.9 \%$ & $9.1 \%$ & $1.5 \%$ & $3.0 \%$ & $2.0 \%$ & $6.6 \%$ & $1.3 \%$ & $3.8 \%$ & $17.9 \%$ & $22.9 \%$ \\
\hline $20<\mathrm{Ti} \leq 50$ (long stopover) & & & & & $0.9 \%$ & $2.7 \%$ & & $3.6 \%$ & $1.0 \%$ & $3.0 \%$ & & $4.0 \%$ & $1.3 \%$ & $5.0 \%$ & $6.7 \%$ & $12.9 \%$ \\
\hline $50<\mathrm{Ti}<100$ (principal stopover) & & & & & & & & & & & & & $1.7 \%$ & $3.3 \%$ & $1.7 \%$ & $6.7 \%$ \\
\hline $\mathrm{Ti}=100$ (principal destination) & & & & & & & & & $0.5 \%$ & & & $0.5 \%$ & $14.2 \%$ & $9.6 \%$ & & $23.8 \%$ \\
\hline Total & $13.9 \%$ & $56.1 \%$ & $30.0 \%$ & $100.0 \%$ & $5.5 \%$ & $33.6 \%$ & $60.9 \%$ & $100.0 \%$ & $8.6 \%$ & $26.8 \%$ & $64.6 \%$ & $100.0 \%$ & $18.8 \%$ & $25.8 \%$ & $55.4 \%$ & $100.0 \%$ \\
\hline
\end{tabular}

Table 7.8 Type of accommodation used in Wellington.

\begin{tabular}{|c|c|c|c|c|c|c|c|c|c|c|c|c|}
\hline \multirow[b]{2}{*}{ Type of accommodation } & \multicolumn{4}{|c|}{ Overnight Gateway Visitors ( $n=117)$} & \multicolumn{4}{|c|}{ Stopover Visitors $(n=136)$} & \multicolumn{4}{|c|}{ Destination Tourists $(n=247)$} \\
\hline & CSNZ & Dom & Int'l & Total & CSNZ & Dom & Int'l & Total & CSNZ & Dom & Int'l & Total \\
\hline Private home & $4.3 \%$ & $13.7 \%$ & $0.9 \%$ & $18.8 \%$ & $3.7 \%$ & $12.5 \%$ & $5.1 \%$ & $21.3 \%$ & $11.3 \%$ & $17.4 \%$ & $12.1 \%$ & $40.9 \%$ \\
\hline Backpacker / Youth hostel & & $4.3 \%$ & $22.2 \%$ & $26.5 \%$ & $0.7 \%$ & $0.7 \%$ & $27.9 \%$ & $29.4 \%$ & $2.8 \%$ & $2.0 \%$ & $16.2 \%$ & $21.1 \%$ \\
\hline Hotel & & $5.1 \%$ & $14.5 \%$ & $19.7 \%$ & $0.7 \%$ & $2.2 \%$ & $23.5 \%$ & $26.5 \%$ & $2.4 \%$ & $2.4 \%$ & $13.0 \%$ & $17.8 \%$ \\
\hline Motel & $0.9 \%$ & $4.3 \%$ & $13.7 \%$ & $18.8 \%$ & $0.7 \%$ & $2.9 \%$ & $7.4 \%$ & $11.0 \%$ & & $1.2 \%$ & $6.9 \%$ & $8.1 \%$ \\
\hline Touring caravan / campervan & & $0.9 \%$ & $2.6 \%$ & $3.4 \%$ & & $0.7 \%$ & $4.4 \%$ & $5.1 \%$ & $0.4 \%$ & $0.4 \%$ & $2.4 \%$ & $3.2 \%$ \\
\hline Other (e.g. camping, self catering cottage/flat, B\&B) & & $6.0 \%$ & $6.8 \%$ & $12.8 \%$ & & $0.7 \%$ & $5.9 \%$ & $6.6 \%$ & $1.6 \%$ & $1.6 \%$ & $5.7 \%$ & $8.9 \%$ \\
\hline Total & $5.1 \%$ & $34.2 \%$ & $60.7 \%$ & $100.0 \%$ & $5.9 \%$ & $19.9 \%$ & $74.3 \%$ & $100.0 \%$ & $18.6 \%$ & $25.1 \%$ & $56.3 \%$ & $100.0 \%$ \\
\hline
\end{tabular}




\section{FERRY PASSENGERS IN WELLINGTON AND PICTON}

It is also appropriate to analyse the reasons why other passengers decide not to stay overnight in the node (by definition only gateway travellers and stopover visitors). Table 7.9 presents the main reasons that were gathered from the passengers' responses (open question). The categories presented there emphasize a wide variety of explanations for passengers' behaviour. They include the fact that some passengers have no need to stay as they can easily drive from where they were to where they were going to, or simply because they opted to stay overnight somewhere else near Wellington. These two reasons encapsulate the notion of a gateway use of Wellington. Others can be explained from a destination perspective, such as the case of passengers who decided not to stay in Wellington as this was not their type of destination - they might not be interested in cities. Finally, other reasons are related to time, for example where passengers mentioned that they did not stay due to a "time constraint", which in fact implies that they have preferred other destinations rather than Wellington, or when the passengers were on a day trip and were "returning the same day". Regarding the reasons for not staying in Wellington the number of SV was particularly small (see Table 7.9) as most passengers stay at least one night in Wellington. According to Figure 7.1, passengers not staying overnight in Wellington consist of all GT $(n=280)$ and some SV $(n=74)$. As a consequence of this when broken down by place of origin some sub-groups have only a few respondents (e.g. CSNZ stopover visitors; $n=13)$. Therefore, it was decided to use a column percentage approach in Table 7.9, which allows a comparison among the various reasons given for each nodal function without mixing very different sample sizes.

From Table 7.9 it is possible to notice that three particular reasons have been highly considered by all sub-groups: "no need", "no reason given" and "staying overnight somewhere else near Wellington". The option for staying overnight near Wellington might be related to a decision to spend the night in a more affordable accommodation, especially motels, located along the State Highway 1 on the way to the capital city. Regarding the high number of answers left blank ("no reason given"), this might be associated with the fact that some passengers feel less inclined to answer open questions as they require more effort to answer in comparison to the closed question format, where it usually involves only ticking one of the options provided.

As discussed in the literature review (Fesenmaier \& Jeng, 2000), information obtained by travellers can have a significant impact on the decision making process to visit or not visit a certain destination. Table 7.10 presents various types of possible sources of information, including examples from the different classifications (commercial vs. noncommercial; impersonal vs. personal). Most of these sources are external, except "past visits" which is an internal source of information. In addition, according to the stage of 


\section{FERRY PASSENGERS IN WELLINGTON AND PICTON}

information sourcing (see Figure 2.9), all types of source of information presented in Table 7.10 can be obtained before the departure, with the exception of the visitor information centres (VIC) in New Zealand where travellers got information "en route". Visitor information centres are located in the major destinations throughout New Zealand and apart from free information they also provide bookings for major transport providers, accommodation and attractions in different parts of the country. Multiple responses were allowed for this question, hence there is no "total" row for Table 7.10. Before analysing the data, it is worth considering the small size of some CSNZ cells, particularly OGV $(n=6)$.

Several characteristics can be identified for the sources of information used to find out more about Wellington. As international passengers and those living in New Zealand usually make use of different sources of information, the total distribution of the sources of information used by the different nodal functions is then very much influenced by the participation of the passengers' place of origin in each nodal function. This is what happened for passengers living in New Zealand in the case of gateway travellers, while international passengers dominate the other functions. As past visits are by far the most important source of information used by CSNZ and domestic passengers, this resulted in four out of ten gateway travellers relying on their previous experience to gain information about the capital city. This is followed by commercial guide books (18.6\% of GT passengers), as $57 \%$ of international GT made use of this source of information. On the other hand, OGV and SV main sources of information were commercial guide books (respectively $47.5 \%$ and $46.2 \%$ ) and free visitor guides (respectively $27.1 \%$ and $34 \%$ ), as these are the major sources used by international passengers. Even though international passengers correspond to the majority of destination tourists, a great number of CSNZ and domestic passengers use their previous visits (respectively $76.1 \%$ and $66.7 \%$ ) as a source of information about the capital city. As a consequence, this is the most common type of information source, accounting for four out of ten DT.

One of the reasons for overseas travellers needing more information is that they are not as familiar with Wellington as passengers who live in New Zealand. It is also related to the discussion mentioned in Chapter 2, where overseas travellers want to minimise the risks of expensive international trips (Snepenger \& Snepenger, 1993). Although not as important as past visits, commercial guide books and free visitor guides, word of mouth, internet and VIC can also be identified as relevant sources of information for all nodal functions.

When comparing the distribution channels used to book the ferries (see Table 6.12) with the sources of information about Wellington (Table 7.10), one particular channel deserves 


\section{FERRY PASSENGERS IN WELLINGTON AND PICTON}

some attention, namely travel agents. Travel agents are the most used channel for booking the ferries, with nearly three out of ten passengers using it (see Table 6.12). However, depending on the nodal function, travel agents are the second least used source of information or the least used for bookings, in the case of gateway travellers for example. While this may simply reflect differences between information search and booking procedures, travel agents could be used to stimulate more passengers to consider staying in Wellington or Picton when they book their ferry ticket. It would be interesting to know whether passengers do not ask for information about Wellington or whether the travel agents do not have information to give them. As will be developed in Chapter 8 , the lack of commissionable attractions in Wellington does not provide incentives for travel agents to promote the capital city.

Finally, the last aspect of passengers' behaviour considered is the activities undertaken / attractions visited during their stay in Wellington. Activities and attractions can be divided into two main groups. The first is more generic, such as shopping, eating out, going to bars, general/tour sightseeing, walking in the city and enjoying cafés. The second group of activities consists of those with a more touristic appeal, such as visiting Te Papa, Parliament or the Botanic Gardens, or going for a ride on the cable car, among others. According to Table 7.11, Te Papa is by far the most visited tourist attraction in Wellington, followed by the Botanic Gardens and the cable car.

Various trends can be identified in terms of the activities undertaken and attractions visited by ferry passengers in Wellington. Firstly, the length of stay, which is directly related to the passengers' nodal function, influences the percentage of passengers undertaking both general (e.g. eating out) and tourist (e.g. visiting a tourist attraction) activities. Comparing the total percentage of DT, OGV, SV and GT passengers that engage in activities and attractions in Wellington (Table 7.11), for all activities and attractions DT have a higher participation than the other functions. Similarly, OGV also presents a higher involvement in activities and attractions in the capital city in comparison to GT. One example to illustrate this is the percentage of passengers visiting Te Papa. When comparing the total column for each nodal function the proportion of DT passengers visiting this museum is $59 \%$, which is higher than SV (42\%), OGV (33.1\%) and GT $(11.8 \%)$. This means that the longer the passengers are staying in the node, the higher the likelihood of them engaging in attractions and activities. This emphasizes the importance of persuading Cook Strait passengers to stay longer in the ferry gateways or suggesting what attractions and activities might lead them to stay longer. 
Table 7.9 Reason for gateway travellers and destination visitors not staying overnight in Wellington.

\begin{tabular}{|c|c|c|c|c|c|c|c|c|}
\hline \multirow[b]{2}{*}{ Reason } & \multicolumn{4}{|c|}{ Gateway Travellers } & \multicolumn{4}{|c|}{ Stopover Visitors } \\
\hline & $\begin{array}{l}\text { CSNZ } \\
(n=39)\end{array}$ & $\begin{array}{c}\text { Dom } \\
(\mathrm{n}=157)\end{array}$ & $\begin{array}{c}\text { Int'l } \\
(n=84)\end{array}$ & $\begin{array}{l}\text { Total } \\
(n=280)\end{array}$ & $\begin{array}{l}\text { CSNZ } \\
(n=13)\end{array}$ & $\begin{array}{c}\text { Dom } \\
(n=28)\end{array}$ & $\begin{array}{c}\text { Int'l } \\
(\mathrm{n}=33)\end{array}$ & $\begin{array}{l}\text { Total } \\
(\mathrm{n}=74)\end{array}$ \\
\hline No reason given & $23.2 \%$ & $15.3 \%$ & $8.5 \%$ & $14.1 \%$ & $30.7 \%$ & $28.5 \%$ & $15.1 \%$ & $22.9 \%$ \\
\hline No need / passing through & $41.0 \%$ & $38.2 \%$ & $20.2 \%$ & $33.2 \%$ & $23.1 \%$ & $32.1 \%$ & $12.1 \%$ & $21.6 \%$ \\
\hline Staying overnight somewhere else & $17.9 \%$ & $16.6 \%$ & $19.0 \%$ & $17.5 \%$ & $23.1 \%$ & $21.4 \%$ & $21.2 \%$ & $21.6 \%$ \\
\hline Already been there / will be there later & & $2.5 \%$ & $7.1 \%$ & $3.6 \%$ & & $3.6 \%$ & $21.2 \%$ & $10.8 \%$ \\
\hline Live near Wellington & $12.8 \%$ & $11.5 \%$ & $1.2 \%$ & $8.6 \%$ & $15.4 \%$ & $3.6 \%$ & & $4.1 \%$ \\
\hline Time constraint & & $5.1 \%$ & $19.0 \%$ & $8.6 \%$ & & $3.6 \%$ & $6.1 \%$ & $4.1 \%$ \\
\hline Not my type of destination & & $1.3 \%$ & $9.5 \%$ & $3.6 \%$ & & & $6.1 \%$ & $2.7 \%$ \\
\hline Returning the same day & & & $1.2 \%$ & $0.4 \%$ & & $3.6 \%$ & & $1.4 \%$ \\
\hline Too far to travel from where I was & $5.1 \%$ & $2.5 \%$ & $2.4 \%$ & $2.9 \%$ & & & & \\
\hline Other reason & & $7.0 \%$ & $11.9 \%$ & $7.5 \%$ & $7.7 \%$ & $3.6 \%$ & $18.2 \%$ & $10.8 \%$ \\
\hline Total & $100.0 \%$ & $100.0 \%$ & $100.0 \%$ & $100.0 \%$ & $100.0 \%$ & $100.0 \%$ & $100.0 \%$ & $100.0 \%$ \\
\hline
\end{tabular}


Table 7.10 Sources of information used to find out more about Wellington.

\begin{tabular}{|c|c|c|c|c|c|c|c|c|c|c|c|c|c|c|c|c|}
\hline \multirow[b]{2}{*}{ Source of Information } & \multicolumn{4}{|c|}{ Gateway Travellers } & \multicolumn{4}{|c|}{ Overnight Gateway Visitors } & \multicolumn{4}{|c|}{ Stopover Visitors } & \multicolumn{4}{|c|}{ Destination Tourists } \\
\hline & $\begin{array}{l}\text { CSNZ } \\
(n=39)\end{array}$ & $\begin{array}{c}\text { Dom } \\
(n=157)\end{array}$ & $\begin{array}{c}\text { Int'l } \\
(n=84)\end{array}$ & $\begin{array}{c}\text { Total } \\
(\mathrm{n}=280)\end{array}$ & $\begin{array}{l}\text { CSNZ } \\
(n=6)\end{array}$ & $\begin{array}{c}\text { Dom } \\
(n=41)\end{array}$ & $\begin{array}{c}\text { Int'| } \\
(n=71)\end{array}$ & $\begin{array}{c}\text { Total } \\
(\mathrm{n}=118)\end{array}$ & $\begin{array}{l}\text { CSNZ } \\
(n=21)\end{array}$ & $\begin{array}{c}\text { Dom } \\
(n=55)\end{array}$ & $\begin{array}{c}\text { Int'l } \\
(n=136)\end{array}$ & $\begin{array}{c}\text { Total } \\
(n=212)\end{array}$ & $\begin{array}{l}\text { CSNZ } \\
(n=46)\end{array}$ & $\begin{array}{l}\text { Dom } \\
(n=63)\end{array}$ & $\begin{array}{c}\text { Int'l } \\
(n=142)\end{array}$ & $\begin{array}{c}\text { Total } \\
(n=251)\end{array}$ \\
\hline Past visits & $41.0 \%$ & $51.0 \%$ & $21.4 \%$ & $40.7 \%$ & $50.0 \%$ & $41.5 \%$ & $7.0 \%$ & $21.2 \%$ & $38.1 \%$ & $58.2 \%$ & $8.8 \%$ & $24.5 \%$ & $76.1 \%$ & $66.7 \%$ & $17.6 \%$ & $40.6 \%$ \\
\hline Commercial guide book & $2.6 \%$ & $1.9 \%$ & $57.1 \%$ & $18.6 \%$ & & $17.1 \%$ & $69.0 \%$ & $47.5 \%$ & $4.8 \%$ & $9.1 \%$ & $67.6 \%$ & $46.2 \%$ & & $4.8 \%$ & $61.3 \%$ & $35.9 \%$ \\
\hline Free visitor guides & $10.3 \%$ & $5.7 \%$ & $29.8 \%$ & $13.6 \%$ & $16.7 \%$ & $9.8 \%$ & $38.0 \%$ & $27.1 \%$ & $14.3 \%$ & $21.8 \%$ & $41.9 \%$ & $34.0 \%$ & $8.7 \%$ & $17.5 \%$ & $47.9 \%$ & $33.1 \%$ \\
\hline Word of mouth & $7.7 \%$ & $7.6 \%$ & $22.6 \%$ & $12.1 \%$ & $33.3 \%$ & $12.2 \%$ & $19.7 \%$ & $17.8 \%$ & $9.5 \%$ & $23.6 \%$ & $26.5 \%$ & $24.1 \%$ & $23.9 \%$ & $22.2 \%$ & $33.8 \%$ & $29.1 \%$ \\
\hline Internet & $5.1 \%$ & $9.6 \%$ & $17.9 \%$ & $11.4 \%$ & $16.7 \%$ & $2.4 \%$ & $21.1 \%$ & $14.4 \%$ & $14.3 \%$ & $12.7 \%$ & $22.1 \%$ & $18.9 \%$ & $15.2 \%$ & $14.3 \%$ & $24.6 \%$ & $20.3 \%$ \\
\hline VIC (in New Zealand) & $2.6 \%$ & $3.8 \%$ & $26.2 \%$ & $10.4 \%$ & & $4.9 \%$ & $22.5 \%$ & $15.3 \%$ & $9.5 \%$ & $14.5 \%$ & $22.8 \%$ & $19.3 \%$ & $8.7 \%$ & $7.9 \%$ & $24.6 \%$ & $17.5 \%$ \\
\hline Travel agent & $5.1 \%$ & $0.6 \%$ & $7.1 \%$ & $3.2 \%$ & & & $16.9 \%$ & $10.2 \%$ & $9.5 \%$ & & $16.2 \%$ & $11.3 \%$ & $6.5 \%$ & $3.2 \%$ & $15.5 \%$ & $10.8 \%$ \\
\hline Media & $5.1 \%$ & $3.2 \%$ & $4.8 \%$ & $3.9 \%$ & & $4.9 \%$ & & $1.7 \%$ & $14.3 \%$ & $5.5 \%$ & $2.9 \%$ & $4.7 \%$ & $8.7 \%$ & $7.9 \%$ & $4.2 \%$ & $6.0 \%$ \\
\hline
\end{tabular}

(Multiple responses allowed - ranked in terms of importance to DT international passengers) 
Table 7.11 Activities undertaken and attractions visited in Wellington.

\begin{tabular}{|c|c|c|c|c|c|c|c|c|c|c|c|c|c|c|c|c|}
\hline \multirow[b]{2}{*}{ Activities / Attractions } & \multicolumn{4}{|c|}{ Gateway Travellers } & \multicolumn{4}{|c|}{ Overnight Gateway Visitors } & \multicolumn{4}{|c|}{ Stopover Visitors } & \multicolumn{4}{|c|}{ Destination Tourists } \\
\hline & $\begin{array}{l}\text { CSNZ } \\
(n=39)\end{array}$ & $\begin{array}{c}\text { Dom } \\
(n=157)\end{array}$ & $\begin{array}{c}\text { Int'l } \\
(n=84)\end{array}$ & $\begin{array}{c}\text { Total } \\
(\mathrm{n}=280)\end{array}$ & $\begin{array}{l}\text { CSNZ } \\
(n=6)\end{array}$ & $\begin{array}{c}\text { Dom } \\
(n=41)\end{array}$ & $\begin{array}{c}\text { Int'l } \\
(n=71)\end{array}$ & $\begin{array}{c}\text { Total } \\
(n=118)\end{array}$ & $\begin{array}{l}\text { CSNZ } \\
(n=21)\end{array}$ & $\begin{array}{l}\text { Dom } \\
(n=55)\end{array}$ & $\begin{array}{c}\text { Int'l } \\
(n=136)\end{array}$ & $\begin{array}{c}\text { Total } \\
(n=212)\end{array}$ & $\begin{array}{l}\text { CSNZ } \\
(n=46)\end{array}$ & $\begin{array}{c}\text { Dom } \\
(n=63)\end{array}$ & $\begin{array}{c}\text { Int'l } \\
(n=142)\end{array}$ & $\begin{array}{c}\text { Total } \\
(n=251)\end{array}$ \\
\hline Walk in the city & $10.3 \%$ & $7.0 \%$ & $26.2 \%$ & $13.2 \%$ & $16.7 \%$ & $34.1 \%$ & $67.6 \%$ & $53.4 \%$ & $33.3 \%$ & $25.5 \%$ & $66.2 \%$ & $52.4 \%$ & $41.3 \%$ & $65.1 \%$ & $83.1 \%$ & $70.9 \%$ \\
\hline Shopping & & $7.6 \%$ & $15.5 \%$ & $8.9 \%$ & $16.7 \%$ & $31.7 \%$ & $29.6 \%$ & $29.7 \%$ & $42.9 \%$ & $32.7 \%$ & $43.4 \%$ & $40.6 \%$ & $71.7 \%$ & $60.3 \%$ & $65.5 \%$ & $65.3 \%$ \\
\hline Eating out / restaurant & $5.1 \%$ & $5.7 \%$ & $11.9 \%$ & $7.5 \%$ & $16.7 \%$ & $34.1 \%$ & $43.7 \%$ & $39.0 \%$ & $28.6 \%$ & $21.8 \%$ & $41.9 \%$ & $35.4 \%$ & $54.3 \%$ & $50.8 \%$ & $67.6 \%$ & $61.0 \%$ \\
\hline Te Papa Museum & $7.7 \%$ & $8.9 \%$ & $19.0 \%$ & $11.8 \%$ & $16.7 \%$ & $22.0 \%$ & $40.8 \%$ & $33.1 \%$ & $23.8 \%$ & $27.3 \%$ & $50.7 \%$ & $42.0 \%$ & $34.8 \%$ & $50.8 \%$ & $70.4 \%$ & $59.0 \%$ \\
\hline Café & $2.6 \%$ & $10.2 \%$ & $17.9 \%$ & $11.4 \%$ & $16.7 \%$ & $22.0 \%$ & $47.9 \%$ & $37.3 \%$ & $33.3 \%$ & $38.2 \%$ & $47.8 \%$ & $43.9 \%$ & $39.1 \%$ & $49.2 \%$ & $59.9 \%$ & $53.4 \%$ \\
\hline Bar & & $2.5 \%$ & $4.8 \%$ & $2.9 \%$ & & $12.2 \%$ & $19.7 \%$ & $16.1 \%$ & $9.5 \%$ & $20.0 \%$ & $27.2 \%$ & $23.6 \%$ & $30.4 \%$ & $33.3 \%$ & $40.1 \%$ & $36.7 \%$ \\
\hline Botanic Garden & & & $8.3 \%$ & $2.5 \%$ & & $2.4 \%$ & $22.5 \%$ & $14.4 \%$ & & $7.3 \%$ & $22.8 \%$ & $16.5 \%$ & $15.2 \%$ & $17.5 \%$ & $46.5 \%$ & $33.5 \%$ \\
\hline Cable Car & & $1.9 \%$ & $7.1 \%$ & $3.2 \%$ & & $2.4 \%$ & $33.8 \%$ & $21.2 \%$ & $9.5 \%$ & $7.3 \%$ & $24.3 \%$ & $18.4 \%$ & $8.7 \%$ & $15.9 \%$ & $44.4 \%$ & $30.7 \%$ \\
\hline General/tour sightseeing & & $1.3 \%$ & $6.0 \%$ & $2.5 \%$ & $16.7 \%$ & $4.9 \%$ & $15.5 \%$ & $11.9 \%$ & $14.3 \%$ & $14.5 \%$ & $22.1 \%$ & $19.3 \%$ & $23.9 \%$ & $19.0 \%$ & $37.3 \%$ & $30.3 \%$ \\
\hline Parliament & & $1.9 \%$ & $3.6 \%$ & $2.1 \%$ & & $7.3 \%$ & $16.9 \%$ & $12.7 \%$ & $4.8 \%$ & $14.5 \%$ & $22.1 \%$ & $18.4 \%$ & $2.2 \%$ & $17.5 \%$ & $31.7 \%$ & $22.7 \%$ \\
\hline Scenic drive & & $1.3 \%$ & $8.3 \%$ & $3.2 \%$ & $16.7 \%$ & $2.4 \%$ & $18.3 \%$ & $12.7 \%$ & $4.8 \%$ & $9.1 \%$ & $22.8 \%$ & $17.5 \%$ & $15.2 \%$ & $17.5 \%$ & $26.1 \%$ & $21.9 \%$ \\
\hline Cultural events & & $0.6 \%$ & $2.4 \%$ & $1.1 \%$ & & & $1.4 \%$ & $0.8 \%$ & & $14.5 \%$ & $7.4 \%$ & $8.5 \%$ & $13.0 \%$ & $23.8 \%$ & $12.7 \%$ & $15.5 \%$ \\
\hline City Gallery Wellington & & $0.6 \%$ & $1.2 \%$ & $0.7 \%$ & & & $1.4 \%$ & $0.8 \%$ & & $5.5 \%$ & $4.4 \%$ & $4.2 \%$ & $2.2 \%$ & $9.5 \%$ & $17.6 \%$ & $12.7 \%$ \\
\hline Bush walk & & $0.6 \%$ & & $0.4 \%$ & & $2.4 \%$ & & $0.8 \%$ & $4.8 \%$ & $7.3 \%$ & $2.2 \%$ & $3.8 \%$ & $10.9 \%$ & $14.3 \%$ & $12.0 \%$ & $12.4 \%$ \\
\hline Museum of City \& Sea & $2.6 \%$ & $1.3 \%$ & $1.2 \%$ & $1.4 \%$ & & & $2.8 \%$ & $1.7 \%$ & $4.8 \%$ & $9.1 \%$ & $8.8 \%$ & $8.5 \%$ & $4.3 \%$ & $9.5 \%$ & $14.8 \%$ & $11.6 \%$ \\
\hline
\end{tabular}

(Multiple responses allowed - ranked in terms of importance to DT international passengers) 


\section{FERRY PASSENGERS IN WELLINGTON AND PICTON}

Another trend that can be highlighted is that international passengers usually undertake more activities and visit more attractions than passengers living in New Zealand. This pattern can be identified for most activities/attractions, including a visit to parliament, Te Papa Museum, cafés, bars and the Botanic Gardens. Using once again the example of Te Papa, the participation rates for international, domestic and CSNZ passengers for the various nodal functions are: GT (19.0\%; 8.9\%; 7.7\%), OGV $(40.8 \% ; 22.0 \% ; 16.7 \%)$, SV $(50.7 \% ; 27.3 \% ; 23.8 \%)$ and DT $(70.4 \% ; 50.8 \% ; 34.8 \%)$. The higher participation of international passengers in activities and attractions in Wellington has a two-fold explanation. The first is due to the longer length of stay of international passengers in the capital city. As seen in Table 7.6, international passengers represent more than half of destination tourists, with domestic passengers accounting for the majority of gateway travellers. The second reason is due to the novelty that a trip to New Zealand may represent to this group of passengers, who are usually more inclined to have as many experiences as possible. Passengers living in New Zealand are more likely to have had the opportunity to visit the capital's main attractions on a previous visit.

So far, this section has presented and discussed the passengers' behaviour while in Wellington. The next sub-section will deal particularly with the possibility of extending the passengers' stay in the capital city. While the main aim is to understand what needs to be done to persuade passengers to stay longer, it will also consider what has made some passengers change their plans, either staying for a longer or shorter period of time.

\subsubsection{Changing passengers' length of stay in Wellington}

This section analyses one of the core parts of this research, which is the possibility of extending passengers' stay in one of the gateways. As previously considered in the literature (see Chapter 2), this is not an easy task, as persuading passengers to stay longer in one place will usually happen at the expense of them spending less time in other destinations. This is particularly an issue in New Zealand where trips are characterised by multiple destination touring patterns with travellers tending to have short stays in various places rather than concentrating on one particular destination.

A twofold analysis is presented here. The first considers those passengers that actually changed their plans before or during their stays in Wellington. This can happen, for example, if the traveller decides to stay longer than planned in a gateway, because s/he enjoyed the place and would like to explore it further. The second uses an induced approach and tries to create a scenario where some sort of incentive is offered to the traveller. The traveller could then decide whether or not this incentive would have affected their decision to stay longer. In the questionnaire, two types of incentives were 


\section{FERRY PASSENGERS IN WELLINGTON AND PICTON}

considered; more information (question 20) and a special deal (question 21) which consisted of transport (ferry) and accommodation. In addition, an open question (number 22) was included so passengers could state anything else that would make them stay longer than planned in Wellington.

\section{Actual change of plans in Wellington}

This sub-section and the next one deal with actual and potential change of plans of Cook Strait passengers in relation to their stays in Wellington. Here the approach taken is to analyse the actual change of plans. Basically, three results are possible: passengers did not change their plans, passengers decided to stay longer, or passengers chose to stay shorter than planned. As shown in Table 7.12, the majority of passengers stated that they did not change their initial plans about their length of stay in Wellington, with the participation for individual nodal functions ranging from $62.5 \%$ to $77 \%$. Except in the case of destination tourists, where $10.8 \%$ of passengers either stayed longer or shorter than planned in Wellington, in all other functions approximately $5 \%-6 \%$ of passengers changed their plans. In most of the cases passengers tend to extend their stays in the capital city, rather than reducing them. The nodal function with the highest number of passengers staying longer than planned is destination tourists, with $8.8 \%$ (22 passengers). It is worth commenting on the high number of respondents who did not answer this question. Approximately one-quarter of the respondents (221 passengers) left this question blank. The only reasonable explanation for this is that as most passengers did not change their plans, they did not answer this question because they thought only passengers changing their plans had to answer it.

While Table 7.12 presents a more quantitative analysis of this behaviour, it is also important to understand the reasons behind these changes. In the case of passengers staying shorter than planned it is relevant to identify the causes of these decisions so that, if possible, it can be avoided and in the future fewer passengers will have the same behaviour. On the other hand, passengers staying longer can provide some useful information in terms of what has made them stay longer than planned. This can help to set up some strategies to improve the length of stay among ferry passengers. However, it is important to notice that a decision to change plans is not always within the passengers' control, as some external factors can make them change their original plans. In these situations very little can be done by tourism organisations and businesses in order to improve travellers' stay.

Table 7.13 classifies the reasons for passengers staying longer or shorter than planned in Wellington in terms of whether these decisions are beyond or within their control. In some 


\section{FERRY PASSENGERS IN WELLINGTON AND PICTON}

cases, the same type of event can have different consequences. For example, ferry cancellation can make passengers stay either longer than planned (in the case where the ferry was cancelled while they were in Wellington) or shorter (if passenger is forced to stay in Picton and then decided not to stay in Wellington, after the ferry service has been resumed, in order to keep previous arrangements and plans) depending on the situation. In cases where the decision is made due to circumstances beyond the travellers' control, there is little that can be done to make passengers stay longer in Wellington. Therefore, it is more relevant to analyse the circumstances within the travellers' control that affect their length of stay. A final comment about Table 7.13 is that due to the various possible combinations in terms of the nodal functions and the reasons giving, only the qualitative aspects of the results are presented.

Time, preference to visit another destination and no need to stay longer are the three main reasons for shortening the stay in Wellington (see Table 7.13). In the first case, some travellers ended up spending more time in other destinations while touring in the North Island and arrived in Wellington only to catch the ferry. In other cases it took longer for passengers to reach Wellington, either because they did not plan their trip carefully or due to traffic on their way there. As nearly $80 \%$ of passengers book their ferry ticket prior to their arrival in Wellington or Picton (see Figure 6.13) if by any reason they arrived later in Wellington than originally planned, one possibility was to shorten their stay in the capital city in order to keep their previous trip arrangements, such as the ferry journey and other already booked accommodations/attractions in the South Island. In other circumstances, travellers did not like Wellington and preferred to go somewhere else or they realised after visiting it that there was no need to spend the time they had originally planned.

On the other hand, some factors made Cook Strait passengers opt to stay longer than planned in Wellington. According to Table 7.13 they include a special event, such as the Lord of the Rings Exhibition at Te Papa Museum; extra time during their journey making travellers decide to spend it in an urban environment or in the capital city; bad weather in other destinations and also the fact that the traveller enjoyed the stay in Wellington and decided that it was worth spending more time there.

The next sub-section will discuss, in a scenario perspective, how to make potential travellers change their length of stay in Wellington. 
Table 7.12 Number of passengers that changed original length of stay in Wellington.

\begin{tabular}{|c|c|c|c|c|c|c|c|c|c|c|c|c|c|c|c|c|}
\hline \multirow[b]{2}{*}{ Change of plans } & \multicolumn{4}{|c|}{ Gateway Travellers } & \multicolumn{4}{|c|}{ Overnight Gateway Visitors } & \multicolumn{4}{|c|}{ Stopover Visitors } & \multicolumn{4}{|c|}{ Destination Tourists } \\
\hline & $\begin{array}{l}\text { CSNZ } \\
(n=39)\end{array}$ & $\begin{array}{c}\text { Dom } \\
(n=157)\end{array}$ & $\begin{array}{c}\text { Int'l } \\
(n=84)\end{array}$ & $\begin{array}{l}\text { Total } \\
(n=280)\end{array}$ & $\begin{array}{l}\text { CSNZ } \\
(n=6)\end{array}$ & $\begin{array}{l}\text { Dom } \\
(n=41)\end{array}$ & $\begin{array}{c}\text { Int'l } \\
(n=71)\end{array}$ & $\begin{array}{c}\text { Total } \\
(n=118 ;\end{array}$ & $\begin{array}{l}\text { CSNZ } \\
(n=21)\end{array}$ & $\begin{array}{l}\text { Dom } \\
(n=55)\end{array}$ & $\begin{array}{c}\text { Int'l } \\
(n=136)\end{array}$ & $\begin{array}{l}\text { Total } \\
(n=212 ;\end{array}$ & $\begin{array}{l}\text { CSNZ } \\
(n=46)\end{array}$ & $\begin{array}{l}\text { Dom } \\
(n=63)\end{array}$ & $\begin{array}{c}\text { Int'l } \\
(n=142)\end{array}$ & $\begin{array}{c}\text { Total } \\
(n=251\end{array}$ \\
\hline \multirow[t]{2}{*}{ Plans were not changed } & 20 & 108 & 54 & 182 & 4 & 30 & 57 & 91 & 11 & 38 & 107 & 156 & 29 & 42 & 86 & 157 \\
\hline & $51.3 \%$ & $68.8 \%$ & $64.3 \%$ & $65.0 \%$ & $66.7 \%$ & $73.2 \%$ & $80.3 \%$ & $77.1 \%$ & $52.4 \%$ & $69.1 \%$ & $78.7 \%$ & $73.6 \%$ & $63.0 \%$ & $66.7 \%$ & $60.6 \%$ & $62.5 \%$ \\
\hline \multirow[t]{2}{*}{ Stayed longer } & 2 & 1 & 1 & 4 & & 2 & 1 & 3 & 2 & 2 & 2 & 6 & 2 & 5 & 15 & 22 \\
\hline & $5.1 \%$ & $0.6 \%$ & $1.2 \%$ & $1.4 \%$ & & $4.9 \%$ & $1.4 \%$ & $2.5 \%$ & $9.5 \%$ & $3.6 \%$ & $1.5 \%$ & $2.8 \%$ & $4.3 \%$ & $7.9 \%$ & $10.6 \%$ & $8.8 \%$ \\
\hline \multirow[t]{2}{*}{ Stayed shorter } & & 7 & 1 & 8 & & & 2 & 2 & & & 4 & 4 & & 1 & 4 & 5 \\
\hline & & $4.5 \%$ & $1.2 \%$ & $2.9 \%$ & & & $2.8 \%$ & $1.7 \%$ & & & $2.9 \%$ & $1.9 \%$ & & $1.6 \%$ & $2.8 \%$ & $2.0 \%$ \\
\hline \multirow[t]{2}{*}{ Did not answer this question } & 17 & 41 & 28 & 86 & 2 & 9 & 11 & 22 & 8 & 15 & 23 & 46 & 15 & 15 & 37 & 67 \\
\hline & $43.6 \%$ & $26.1 \%$ & $33.3 \%$ & $30.7 \%$ & $33.3 \%$ & $22.0 \%$ & $15.5 \%$ & $18.6 \%$ & $38.1 \%$ & $27.3 \%$ & $16.9 \%$ & $21.7 \%$ & $32.6 \%$ & $23.8 \%$ & $26.1 \%$ & $26.7 \%$ \\
\hline Total & $100 \%$ & $100 \%$ & $100 \%$ & $100 \%$ & $100 \%$ & $100 \%$ & $100 \%$ & $100 \%$ & $100 \%$ & $100 \%$ & $100 \%$ & $100 \%$ & $100 \%$ & $100 \%$ & $100 \%$ & $100 \%$ \\
\hline
\end{tabular}

\section{Table 7.13 Reasons for passengers changing original plans in Wellington.}

\begin{tabular}{|c|c|c|}
\hline & Circumstances beyond traveller's control & Circumstances within traveller's control \\
\hline $\begin{array}{l}\text { Reason to } \\
\text { stay shorter } \\
\text { than planned }\end{array}$ & $\begin{array}{l}\text { - Time: journey to Wellington took longer than expected (e.g. traffic); } \\
\text { - Ferry cancellation: passenger is forced to stay in Picton and in order to catch } \\
\text { up with his/her plans/arrangements decides to skip Wellington; } \\
\text { - Missed ferry or the shuttle to the ferry terminal; } \\
\text { - Accommodation: booked up. }\end{array}$ & $\begin{array}{l}\text { - Time: traveller ended up spending more time in other destinations and did not have } \\
\text { much time in Wellington; } \\
\text { - Preferred to go to other destination: "preferred to camp in South Island tonight and } \\
\text { got earlier crossing by standby"; "see more of the South"; } \\
\text { - No need: "we realized we could see everything in a day". }\end{array}$ \\
\hline $\begin{array}{l}\text { Reason to } \\
\text { stay longer } \\
\text { than planned }\end{array}$ & $\begin{array}{l}\text { - Ferry cancellation: passenger is forced to stay extra time in Wellington (most } \\
\text { fill spare time at Te Papa); } \\
\text { - Transport arrangement: "Had to stay an extra night due to Kiwi Experience } \\
\text { bus timetable"; car broke down; } \\
\text { - Tiredness/sickness }\end{array}$ & $\begin{array}{l}\text { - Event: Te Papa Lord of the Rings Exhibition; } \\
\text { - Time: "Had extra time for our trip and decided the capital city would be a good place } \\
\text { to spend extra time"; } \\
\text { - Bad weather: either due to the forecast at the destination the traveller was planning } \\
\text { to go to before/after Wellington; } \\
\text { - Enjoyed Wellington as a destination: "enjoyed the city very much"; "enjoyed } \\
\text { Wellington so much and decided to spend } 2 \text { nights on the way back"; "sightseeing } \\
\text { places l' ve missed and wanted to visit needed an extra day". }\end{array}$ \\
\hline
\end{tabular}




\section{FERRY PASSENGERS IN WELLINGTON AND PICTON}

\section{Potential change of plans in Wellington}

As presented in Table 7.12, the number of passengers that currently change their plans in Wellington is relatively low. For those that have changed, the majority stayed longer. What is more, in some cases the motivation for this change was beyond the passenger's control, while in others the traveller was responsible for this change of plans. This research is interested not only in the reasons why passengers actually change their plans, but also in understanding how to induce passengers to stay longer than planned in the gateways. This can create an opportunity for various sectors of the tourism industry to increase their revenues from the longer stays of ferry passengers while in Wellington. For this purpose, the questionnaire asked whether or not more information (Table 7.14), a special deal (Table 7.15) or any other incentive (Table 7.16) would make passengers change their length of stay. This section analyses data collected from these three questions.

Regarding the impact that more information would have on passengers changing their stay in Wellington, two major comments can be made. Firstly, when considering the total number of passengers for each nodal function, gateway travellers are the group of passengers less inclined to stay longer. According to Table 7.14 , only $6.1 \%$ of GT would have stayed longer if they had more information. On the other hand, OGV $(21.2 \%)$, SV $(23.1 \%)$ and DT $(15.1 \%)$ have a higher response. This shows how difficult it can be to convert gateway travellers into destination tourists, as apparently gateway travellers are not at all interested in extending their stay. Secondly, despite being the group of passengers that looks for more information in various different sources (Table 7.10), international passengers are by far the group most likely to stay longer in Wellington if they had more information. For example, one-third of international OGV would have stayed longer if they had more information about the capital city, with the other nodal functions of international passengers accounting for $31.6 \%$ (SV), 19\% (DT) and $14.3 \%$ (GT). Passengers living in New Zealand appear to be less affected by information. It seems that they believe that they are familiar with the destination and have sufficient information to decide whether or not to extend their stay in the capital city. Few passengers also mentioned that if they had more information about Wellington they would have stayed less time than planned. Apparently these passengers were disappointed with Wellington or they simply realised that they had allocated more time to stay there than was really necessary.

Respondents indicate that a special deal, including transport (ferry) and accommodation in the capital city, would potentially have a higher impact on passengers' decision to stay 


\section{FERRY PASSENGERS IN WELLINGTON AND PICTON}

longer than providing more information (see Table 7.15). While the passengers' response to a special deal is higher for all passengers regardless of place of origin (compare data between Table 7.14 and Table 7.15) passengers living in New Zealand seem to be even more sensitive to a special deal. For example, while none of the domestic OGV would potentially stay longer than planned in Wellington if they had more information (see Table 7.14), a special deal would have made $19.5 \%$ of them stay longer. The same happens to CSNZ and domestic passengers for all other nodal functions. In the case of gateway travellers, the decision to stay in Wellington due to a special deal could perhaps be an incentive to make them change from an overnight stopover somewhere else near Wellington, to more affordable accommodation in the capital city. Therefore, in the case of gateway travellers, as they do not stay overnight, making them stay longer will increase the total number of visitor-nights in Wellington, benefiting particularly the accommodation providers. This has a twofold impact. Firstly, the chances of travellers staying in a commercial accommodation increases as the accommodation sector benefits only when visitors stay overnight. Secondly, the extra time they will have in Wellington will lead them to spend more money either in restaurants, bars and shops, or in tourist attractions and activities.

Even though passengers living in New Zealand might be more sensitive to a deal than to extra information, international passengers are still the group with the highest response to extend their stays in Wellington. For example, international OGV was the most sensitive sub-group (36.6\%), followed by international SV (35.3\%). Due to the nature of the touring pattern trip of international travellers in New Zealand, increasing the stay in one destination might happen at the expense of another destination, with the deal representing a strategy to increase the price competitiveness among destinations in the country. However, the fact that international tourists are prepared to stay longer might be a sign that they like the type of destination Wellington is and they believe it is worth spending more time there as it has more for them to do and explore than they originally thought.

When considering the various nodal functions, once again travellers already staying in Wellington seem to be more sensitive to a special deal than those not staying overnight. Comparing, for example, the reaction between GT and DT when a deal is offered, the latter group of passengers is more likely to stay longer than the former. This shows, once again, how difficult is to persuade gateway travellers to stay longer in the nodes they are just passing through. With their aim to reach a certain destination, they are less likely to be persuaded than destination tourists who can take advantage of the deal just to afford an extra night in a destination, as this will make their stay cheaper. 


\section{FERRY PASSENGERS IN WELLINGTON AND PICTON}

Surprisingly, a few international passengers mentioned that they would have spent less time in Wellington if they had a special deal. Most likely these passengers misunderstood the question and thought that the proposed deal would be to stay in another destination, so they would stay shorter than planned in the capital city and spend more time somewhere else.

Finally, Table 7.16 presents information obtained from an open question asking what should be offered to passengers to make them stay longer than planned in Wellington. A total of 113 passengers answered this question, accounting for approximately $10 \%$ of the total sample. Most of these passengers were international $(n=63)$, followed by domestic $(n=41)$ and CSNZ $(n=9)$. The few responses for this question can be attributed to the fact that this was an open question, requiring an extra effort of the respondents than just ticking a box, and also due to some passengers not really wanting to stay longer in Wellington, regardless of what is offered to them.

More time (28 passengers), accommodation related issues (20), a special event/festival (19) and a "must see" attraction (11) are some of the most mentioned factors. While some of the motivators mentioned by passengers are beyond the control of tourism organisations and businesses (e.g. more time, weather, family/friends living there), others can be addressed. These include accommodation related issues, more events and attractions, more information and better shopping. One passenger mentioned the Lord of the Rings exhibition, which proved to be a popular temporary attraction at Te Papa (see Chapter 4).

A summary of the data analysed here will be presented in the next section. This will be useful to understand the key issues associated with the passengers' behaviour while in Wellington and also what can be done to make them stay longer there. It will also help to compare data between Wellington and Picton, which will be done at the end of the chapter. 
Table 7.14 Change of plans in case passenger had more information about Wellington.

\begin{tabular}{|c|c|c|c|c|c|c|c|c|c|c|c|c|c|c|c|c|}
\hline \multirow{2}{*}{$\begin{array}{l}\text { Change of plans in case } \\
\text { passenger had more } \\
\text { information }\end{array}$} & \multicolumn{4}{|c|}{ Gateway Travellers } & \multicolumn{4}{|c|}{ Overnight Gateway Visitors } & \multicolumn{4}{|c|}{ Stopover Visitors } & \multicolumn{4}{|c|}{ Destination Tourists } \\
\hline & $\begin{array}{l}\text { CSNZ } \\
(n=39)\end{array}$ & $\begin{array}{c}\text { Dom } \\
(n=157)\end{array}$ & $\begin{array}{c}\text { Int'l } \\
(n=84)\end{array}$ & $\begin{array}{c}\text { Total } \\
\text { ' } n=280 \text {; }\end{array}$ & $\begin{array}{l}\text { CSNZ } \\
(n=6)\end{array}$ & $\begin{array}{c}\text { Dom } \\
(n=41)\end{array}$ & $\begin{array}{c}\text { Int'l } \\
(n=71)\end{array}$ & $\begin{array}{c}\text { Total } \\
\text { 'n=118; }\end{array}$ & $\begin{array}{l}\text { CSNZ } \\
(n=21)\end{array}$ & $\begin{array}{l}\text { Dom } \\
(n=55)\end{array}$ & $\begin{array}{c}\text { Int'l } \\
(n=136)\end{array}$ & $\begin{array}{c}\text { Total } \\
(n=212 ;\end{array}$ & $\begin{array}{l}\text { CSNZ } \\
(n=46)\end{array}$ & $\begin{array}{l}\text { Dom } \\
(n=63)\end{array}$ & $\begin{array}{c}\text { Int'l } \\
(n=142)\end{array}$ & $\begin{array}{c}\text { Total } \\
(n=251\end{array}$ \\
\hline No changes made & $\begin{array}{r}38 \\
97.4 \% \\
\end{array}$ & $\begin{array}{r}147 \\
93.6 \% \\
\end{array}$ & $\begin{array}{r}57 \\
67.9 \% \\
\end{array}$ & $\begin{array}{r}242 \\
86.4 \% \\
\end{array}$ & $\begin{array}{r}4 \\
66.7 \% \\
\end{array}$ & $\begin{array}{r}40 \\
97.6 \% \\
\end{array}$ & $\begin{array}{r}43 \\
60.6 \% \\
\end{array}$ & $\begin{array}{r}87 \\
73.7 \% \\
\end{array}$ & $\begin{array}{r}19 \\
90.5 \% \\
\end{array}$ & $\begin{array}{r}51 \\
92.7 \% \\
\end{array}$ & $\begin{array}{r}86 \\
63.2 \% \\
\end{array}$ & $\begin{array}{r}156 \\
73.6 \% \\
\end{array}$ & $\begin{array}{r}43 \\
93.5 \% \\
\end{array}$ & $\begin{array}{r}52 \\
82.5 \% \\
\end{array}$ & $\begin{array}{r}102 \\
71.8 \% \\
\end{array}$ & $\begin{array}{r}197 \\
78.5 \% \\
\end{array}$ \\
\hline Passenger would stay longer & $\begin{array}{r}1 \\
2.6 \% \\
\end{array}$ & $\begin{array}{r}4 \\
2.5 \% \\
\end{array}$ & $\begin{array}{r}12 \\
14.3 \% \\
\end{array}$ & $\begin{array}{r}17 \\
6.1 \% \\
\end{array}$ & $\begin{array}{r}1 \\
16.7 \% \\
\end{array}$ & & $\begin{array}{r}24 \\
33.8 \% \\
\end{array}$ & $\begin{array}{r}25 \\
21.2 \% \\
\end{array}$ & $\begin{array}{r}2 \\
9.5 \% \\
\end{array}$ & $\begin{array}{r}4 \\
7.3 \% \\
\end{array}$ & $\begin{array}{r}43 \\
31.6 \% \\
\end{array}$ & $\begin{array}{r}49 \\
23.1 \% \\
\end{array}$ & $\begin{array}{r}3 \\
6.5 \% \\
\end{array}$ & $\begin{array}{r}8 \\
12.7 \% \\
\end{array}$ & $\begin{array}{r}27 \\
19.0 \% \\
\end{array}$ & $\begin{array}{r}38 \\
15.1 \% \\
\end{array}$ \\
\hline Passenger would stay shorter & & & $\begin{array}{r}2 \\
2.4 \% \\
\end{array}$ & $\begin{array}{r}2 \\
0.7 \% \\
\end{array}$ & & & & & & & & & & $\begin{array}{r}1 \\
1.6 \% \\
\end{array}$ & $\begin{array}{r}2 \\
1.4 \% \\
\end{array}$ & $\begin{array}{r}3 \\
1.2 \% \\
\end{array}$ \\
\hline Did not answer & & $\begin{array}{r}6 \\
3.9 \% \\
\end{array}$ & $\begin{array}{r}13 \\
15.4 \% \\
\end{array}$ & $\begin{array}{r}19 \\
6.8 \% \\
\end{array}$ & $\begin{array}{r}1 \\
16.6 \% \\
\end{array}$ & $\begin{array}{r}1 \\
2.4 \% \\
\end{array}$ & $\begin{array}{r}4 \\
5.6 \% \\
\end{array}$ & $\begin{array}{r}6 \\
5.1 \% \\
\end{array}$ & & & $\begin{array}{r}7 \\
5.2 \% \\
\end{array}$ & $\begin{array}{r}7 \\
3.3 \% \\
\end{array}$ & & $\begin{array}{r}2 \\
3.2 \% \\
\end{array}$ & $\begin{array}{r}11 \\
7.8 \\
\end{array}$ & $\begin{array}{r}13 \\
5.2 \\
\end{array}$ \\
\hline Total & $100 \%$ & $100 \%$ & $100 \%$ & $100 \%$ & $100 \%$ & $100 \%$ & $100 \%$ & $100 \%$ & $100 \%$ & $100 \%$ & $100 \%$ & $100 \%$ & $100 \%$ & $100 \%$ & $100 \%$ & $100 \%$ \\
\hline
\end{tabular}

Table 7.15 Change of plans in Wellington in case passenger was offered a special deal.

\begin{tabular}{|c|c|c|c|c|c|c|c|c|c|c|c|c|c|c|c|c|}
\hline \multirow{2}{*}{$\begin{array}{c}\text { Change of plans in case } \\
\text { passenger was offered a special } \\
\text { deal }\end{array}$} & \multicolumn{4}{|c|}{ Gateway Travellers } & \multicolumn{4}{|c|}{ Overnight Gateway Visitors } & \multicolumn{4}{|c|}{ Stopover Visitors } & \multicolumn{4}{|c|}{ Destination Tourists } \\
\hline & $\begin{array}{l}\text { CSNZ } \\
(n=39)\end{array}$ & $\begin{array}{c}\text { Dom } \\
(n=157)\end{array}$ & $\begin{array}{c}\text { Int'l } \\
(n=84)\end{array}$ & $\begin{array}{c}\text { Total } \\
(n=280)\end{array}$ & $\begin{array}{l}\text { CSNZ } \\
(n=6)\end{array}$ & $\begin{array}{c}\text { Dom } \\
(n=41)\end{array}$ & $\begin{array}{c}\text { Int'l } \\
(n=71)\end{array}$ & $\begin{array}{c}\text { Total } \\
(n=118 ;\end{array}$ & $\begin{array}{l}\text { CSNZ } \\
(n=21)\end{array}$ & $\begin{array}{c}\text { Dom } \\
(n=55)\end{array}$ & $\begin{array}{c}\text { Int'l } \\
(n=136)\end{array}$ & $\begin{array}{c}\text { Total } \\
\text { (n=212) }\end{array}$ & $\begin{array}{l}\text { CSNZ } \\
(n=46)\end{array}$ & $\begin{array}{c}\text { Dom } \\
(n=63)\end{array}$ & $\begin{array}{c}\text { Int'l } \\
(n=142)\end{array}$ & $\begin{array}{c}\text { Total } \\
(n=251\end{array}$ \\
\hline No changes made & $\begin{array}{r}33 \\
84.6 \%\end{array}$ & $\begin{array}{r}114 \\
72.6 \%\end{array}$ & $\begin{array}{r}53 \\
63.1 \%\end{array}$ & $\begin{array}{r}200 \\
71.4 \%\end{array}$ & $\begin{array}{r}4 \\
66.7 \%\end{array}$ & $\begin{array}{r}32 \\
78.0 \%\end{array}$ & $\begin{array}{r}40 \\
56.3 \%\end{array}$ & $\begin{array}{r}76 \\
64.4 \%\end{array}$ & $\begin{array}{r}16 \\
76.2 \%\end{array}$ & $\begin{array}{r}41 \\
74.5 \%\end{array}$ & $\begin{array}{r}82 \\
60.3 \%\end{array}$ & $\begin{array}{r}139 \\
65.6 \%\end{array}$ & $\begin{array}{r}38 \\
82.6 \%\end{array}$ & $\begin{array}{r}43 \\
68.3 \%\end{array}$ & $\begin{array}{r}83 \\
58.5 \%\end{array}$ & $\begin{array}{r}164 \\
65.3 \%\end{array}$ \\
\hline Passenger would stay more time & $\begin{array}{r}4 \\
10.3 \% \\
\end{array}$ & $\begin{array}{r}30 \\
19.1 \% \\
\end{array}$ & $\begin{array}{r}19 \\
22.6 \% \\
\end{array}$ & $\begin{array}{r}53 \\
18.9 \% \\
\end{array}$ & $\begin{array}{r}1 \\
16.7 \% \\
\end{array}$ & $\begin{array}{r}8 \\
19.5 \% \\
\end{array}$ & $\begin{array}{r}26 \\
36.6 \% \\
\end{array}$ & $\begin{array}{r}35 \\
29.7 \% \\
\end{array}$ & $\begin{array}{r}4 \\
19.0 \% \\
\end{array}$ & $\begin{array}{r}11 \\
20.0 \% \\
\end{array}$ & $\begin{array}{r}48 \\
35.3 \% \\
\end{array}$ & $\begin{array}{r}63 \\
29.7 \% \\
\end{array}$ & $\begin{array}{r}8 \\
17.4 \% \\
\end{array}$ & $\begin{array}{r}16 \\
25.4 \% \\
\end{array}$ & $\begin{array}{r}40 \\
28.2 \% \\
\end{array}$ & $\begin{array}{r}64 \\
25.5 \% \\
\end{array}$ \\
\hline Passenger would stay less time & & & $\begin{array}{r}1 \\
1.2 \% \\
\end{array}$ & $\begin{array}{r}1 \\
0.4 \% \\
\end{array}$ & & & $\begin{array}{r}1 \\
1.4 \% \\
\end{array}$ & $\begin{array}{r}1 \\
0.8 \% \\
\end{array}$ & & & & & & & $\begin{array}{r}4 \\
2.8 \% \\
\end{array}$ & $\begin{array}{r}4 \\
1.6 \% \\
\end{array}$ \\
\hline Did not answer & $\begin{array}{r}2 \\
5.1 \% \\
\end{array}$ & $\begin{array}{r}13 \\
8.3 \% \\
\end{array}$ & $\begin{array}{r}11 \\
13.1 \% \\
\end{array}$ & $\begin{array}{r}26 \\
9.3 \% \\
\end{array}$ & $\begin{array}{r}1 \\
16.7 \% \\
\end{array}$ & $\begin{array}{r}1 \\
2.5 \% \\
\end{array}$ & $\begin{array}{r}4 \\
5.7 \% \\
\end{array}$ & $\begin{array}{r}6 \\
5.1 \% \\
\end{array}$ & $\begin{array}{r}1 \\
4.8 \% \\
\end{array}$ & $\begin{array}{r}3 \\
5.5 \% \\
\end{array}$ & $\begin{array}{r}6 \\
4.4 \% \\
\end{array}$ & $\begin{array}{r}10 \\
4.7 \% \\
\end{array}$ & & $\begin{array}{r}4 \\
6.3 \% \\
\end{array}$ & $\begin{array}{r}15 \\
10.5 \% \\
\end{array}$ & $\begin{array}{r}19 \\
7.6 \% \\
\end{array}$ \\
\hline Total & $100 \%$ & $100 \%$ & $100 \%$ & $100 \%$ & $100 \%$ & $100 \%$ & $100 \%$ & $100 \%$ & $100 \%$ & $100 \%$ & $100 \%$ & $100 \%$ & $100 \%$ & $100 \%$ & $100 \%$ & $100 \%$ \\
\hline
\end{tabular}


Table 7.16 What should be offered to make passengers stay longer than planned in Wellington.

\begin{tabular}{|l|c||l|l|}
\hline \multicolumn{1}{|c|}{ What should be offered to passenger } & $\begin{array}{c}\text { Number of } \\
\text { Respondents }\end{array}$ & What should be offered to passenger \\
\hline More time & 28 & Family/friends living there & Respondents \\
\hline $\begin{array}{l}\text { Accommodation (availability, cheaper, located near ferry terminal, } \\
\text { camping grounds, free parking in motel/hotel, home stay with Maori } \\
\text { family) }\end{array}$ & 20 & $\begin{array}{l}\text { Transport to/from Wellington (direct flights, Air NZ not } \\
\text { limiting cheap fare to 30 days stay) }\end{array}$ & 3 \\
\hline $\begin{array}{l}\text { An event/festival (e.g. music Cuba St Carnival, Chinese New Year, } \\
\text { family oriented, more information) }\end{array}$ & 19 & Better shopping (including more money to shop) \\
\hline A "must see" attraction (include activities as well) & 11 & Work & 3 \\
\hline Weather (better, guaranteed good weather) & 8 & Nature & Deals including offers for children \\
\hline \begin{tabular}{l|l|l|} 
More information about Wellington (including suburbs, knowing where \\
things are)
\end{tabular} & 6 & 5 & Lord of the Rings related attractions \\
\hline Ferries (Cheaper, cancellation, time of crossing) & & 1 \\
\hline
\end{tabular}




\section{FERRY PASSENGERS IN WELLINGTON AND PICTON}

\subsubsection{Summary of passengers' behaviour in Wellington}

This section, which summarises the passengers' behaviour in Wellington, can be divided into four main parts, each one devoted to one nodal function, i.e. gateway travellers, overnight gateway visitors, stopover visitors and destination tourists. For each of these groups, two main subparts will follow, one related to what ferry passengers do while in Wellington, the other about what would make them stay longer in the capital city. In most cases only general aspects of each group will be presented, with few considerations of the most relevant functions highlighted.

In Wellington, gateway travellers are dominated by domestic passengers $(56.1 \%$ according to Table 3.4), followed by international passengers (30\%) and CSNZ (13.9\%). By definition, GT do not stay overnight, hence their Trip Index is a constant equal to zero and their only main reason for going to Wellington is the inter-island connection. According to Table 7.9 the main reasons for GT not staying in Wellington are "no need / passing through" $(33.2 \%)$ and the fact that they were "staying overnight somewhere else" $(17.5 \%)$. Due to the large presence of domestic passengers among gateway travellers, the main sources of information used to find out more about Wellington are past visits (40.7\% - see Table 7.10$)$, followed by commercial guide books (18.8\%), especially due to the fact that $57.1 \%$ of international GT used this source of information. Due to the short stay of GT in Wellington, their participation in attractions and activities in the capital city is very small, except in few cases (see Table 7.11). According to Table 7.12, gateway travellers are the only group of passengers that the change of plans made more passengers stay shorter $(2.9 \%)$ than longer $(1.4 \%)$. More information would have made $6.1 \%$ of GT staying longer (Table 7.14 ), while a special deal would have increased the length of stay of $18.9 \%$ (Table 7.15 ).

As in the case of the next two functions, OGVs in Wellington are dominated by international passengers. According to Table 3.4, six out of ten OGV are from overseas, with CSNZ passengers accounting for only $5 \%$ of the sample. By definition, the main reason for OGT to go to a node is the inter-island connection and all travellers in this group stay one night there. In the case of Wellington, short stopovers $(0<\mathrm{Ti} \leq 10)$ represent $87.3 \%$ of the passengers (Table 7.7). The choice of accommodation used by OGT while in Wellington is fairly equally divided among a set of different types of accommodation, ranging from backpackers / youth hostels $(26.5 \%)$, hotels $(19.7 \%)$, motels $(18.8 \%)$ and private homes (18.8\%). The explanation for the higher use of backpackers / youth hostels is due to international travellers dominating this nodal function, who tend to stay in this type of accommodation (22.2\% of the sample). The sources of information used to find out 


\section{FERRY PASSENGERS IN WELLINGTON AND PICTON}

more about Wellington are also highly influenced by the presence of international travellers among this group. Commercial guidebooks are the most used source of information, with $47.5 \%$ of OGV using them to know more about the capital city, followed by free visitor guides $(27.1 \%$ - see Table 7.10$)$. In the case of commercial guidebooks, nearly seven out of ten international passengers make use of this source of information, which explains the high participation of this source of information among OGV. For all activities and attractions listed in Table 7.11 the participation of OGV is higher than GT. Apart from Te Papa museum (33.1\%), all major activities undertaken by OGV are general ones, such as walk in the city (53.4\%), eating out (39\%) and going to a café (37.3\%). The actual change of plans for OGV in Wellington was very small, with $2.5 \%$ of passengers staying longer and $1.7 \%$ of them staying shorter (Table 7.12 ). Over one-fifth of OGV would have stayed longer in Wellington if they had more information (Table 7.14), with nearly three out of ten passengers doing the same if they had been offered a special deal (Table 7.15).

In terms of passengers' place of origin, overseas travellers represent $64.2 \%$ of stopover visitors (Table 3.4). Domestic passengers represent over a quarter of the sample $(25.9 \%)$ and CSNZ only one out of ten passengers (9.9\%). According to Table 7.5, two major reasons bring SV to Wellington. They are leisure / holiday (61.6\%) and VFR (25.6\%), with the largest sub-group consisting of international passengers on leisure / holiday purpose (53.6\%). By definition, SV either do not stay overnight or stay one night. The former case represents $34.9 \%$ of the sample, with $65.1 \%$ staying one night. Those SV who were not staying overnight $(n=74)$ gave two main reasons for doing so - excluding passengers who did not give a reason. They were "no need" or because they were staying overnight somewhere else near Wellington. Both answers were stated by $21.6 \%$ of the sample. Those SV who stayed overnight in Wellington $(n=136)$ used three main types of accommodation: backpacker / hostels $(29.4 \%)$, hotels $(26.5 \%)$ and private homes $(21.3 \%)$. Due to the higher participation of international travellers, once again the most used sources of information to find out more information about Wellington were commercial guidebooks $(46.2 \%)$, followed by free visitor guides $(34 \%)$ and past visits $(24.5 \%)$, in this case due to the strong participation of CSNZ $(38.1 \%)$ and domestic (58.2\%) passengers (Table 7.10). In comparison to other tourist attractions, such as cable car (18.4\%), Parliament (18.4\%), Botanic Gardens (16.5\%) and Museum of City \& Sea $(8.5 \%)$, Te Papa museum is the most visited by SV, with $42 \%$ of this group of passengers going there (Table 7.11). Actual change of plans by SV passengers in Wellington was very similar to OGV, with $2.8 \%$ of passengers staying longer and $1.9 \%$ of them staying shorter (Table 7.12). SV had the higher participation of passengers staying longer if they 


\section{FERRY PASSENGERS IN WELLINGTON AND PICTON}

had had more information about Wellington (23.1\% according to Table 7.14$)$ and, together with OGV, the higher percentage of passengers staying longer $(29.7 \%)$ if they had had a special deal (Table 7.15).

Finally, in terms of the destination tourists' place of origin, once again the majority are from overseas $(56.6 \%)$, with CSNZ passengers accounting for $18.3 \%$ and domestic passengers for a quarter of the sample (25.1\%). Leisure / holiday (38.3\%) and VFR (36.3\%) also account for the two most important reasons for DT going to Wellington. Nearly half of DT stayed two nights in Wellington, with $17.5 \%$ of them staying three nights and approximately $15 \%$ staying six or more nights (Table 7.6). Regarding the Trip Index, the two major types of passengers include the short stopover $(0<\mathrm{Ti} \leq 10)$ with $33.8 \%$ and the single destination $(\mathrm{Ti}=100)$ with $23.8 \%$. In the first case this behaviour is very much influenced by international passengers in a short stopover, with this single sub-group accounting for nearly three out of ten passengers $(29.2 \%)$. In the second case, only CSNZ $(14.2 \%)$ and domestic $(9.6 \%)$ passengers make a single destination trip to Wellington. Three major types of accommodation are used by destination tourists while staying in Wellington (Table 7.8). Private homes are the most used (40.9\%), which can be associated with longer stays as tourists do not need to pay for accommodation and also the higher participation of VFR, as tourists will usually stay with their friends and relatives. As in the case of stopover visitors, commercial guidebooks, free visitor guides and past visits are the most used source of information. The main difference is that due to the higher participation of CSNZ and domestic passengers among DT, past visits (see Table 7.10) are the most used source of information (40.6\%) rather than commercial guide books (35.9\%). In terms of activities and attractions, Table 7.11 presents how important DTs are for a destination as they have higher participation than any other nodal function. Apart from general activities such as walk in the city (70.9\%), shopping (65.3\%), eating out $(61 \%)$ and going to a café $(53.4 \%)$, destination tourists comprehend the main function visiting tourist attractions, such as Te Papa (59\%). In terms of the actual change in the length of stay, DT had the highest number of passengers staying longer, with 22 passengers $(8.8 \%$ - see Table 7.12$)$. More information would have made $15.1 \%$ of passengers staying longer (Table 7.14) and a special deal would have increased the length of stay of one-quarter of the sample (Table 7.15).

The next section presents data related to passengers' behaviour in regards to Picton. 


\section{FERRY PASSENGERS IN WELLINGTON AND PICTON}

\subsection{Ferry Passengers in Picton}

This section presents the analysis of data collected for the passengers' behaviour while in Picton. The same data analysis structure used in the previous section will be employed here. It is important to consider the analysis done in this section with the results presented in Figure 7.1 , where Picton is very much dominated by gateway travellers $(67.5 \%)$ - more than two-thirds of the sample and twice as many as Wellington - followed by stopover visitors $(13.1 \%)$, overnight gateway visitors $(11.5 \%)$, with very few destination tourists $(7.9 \%)$.

In Picton, ferry passengers in general present mainly one reason for going there, i.e. the inter-island connection (see Table 7.17). Nearly $82 \%$ of the total sample has the interisland connection as the main reason for going to Picton, while leisure and holiday corresponding to $12.5 \%$. Other reasons for going to Picton, such as business/work and VFR, are, in general, less important. Picton has a small economy to attract business tourists and a small population, therefore fewer friends and relatives to be visited. These facts emphasize a very strong gateway function in Picton with a secondary leisure/holiday destination role.

As a consequence of the strong gateway function identified in Picton, the majority of passengers do not stay overnight in the ferry port of the South Island. A total of 794 passengers ( $G T=695+\mathrm{SV}=99)$ did not stay overnight, which represents $77.2 \%$ of the sample (Table 7.18). A total of $7.3 \%$ of passengers stayed one night there, with the remaining passengers staying two or more nights. CSNZ usually do not stay overnight in Picton if they do not have this node as a destination. This can be explained by the fact that their place of residence is usually a few hours from Picton. On the other hand, CSNZ passengers are the second most important place of origin within the destination tourist function $(38.3 \%)$. This seems to reflect the short break market, especially for travellers living in Wellington. Apparently domestic passengers do not tend to stay in Picton and when they do it is as part of a stopover in their inter-island trips. In the case of international passengers, the higher proportion of passengers staying at least one night just emphasizes the stopover-touring pattern that characterises this group of passengers.

Regarding the reasons for not staying overnight in Picton, apart from passengers that did not answer this question ("no reason given"), two major types of motives were stated and grouped in the categories "staying overnight somewhere near Picton" and "no need / passing through". These two reasons account for at least half of each function of the various places groups. The higher response of passengers staying overnight somewhere 


\section{FERRY PASSENGERS IN WELLINGTON AND PICTON}

else near Picton can be attributed to the relatively high number of established and wellknown tourist destinations that are located close to Picton, such as Blenheim, Nelson and Kaikoura. Within easy access to Picton, in approximately 2 hours drive, a majority of passengers prefer to stay in these places rather than Picton (see Figures 6.10 and 6.11). Passengers stating that there was no need to stay overnight, because they were just passing through, were usually referring to the fact that they could easily drive between the places they were to the destinations they were going to.

Respondents not answering this question ("no reason given") were particularly high in some cases (e.g. CSNZ gateway travellers and domestic SV) which, as cited earlier, has been a common pattern for this research in the case of open questions. Other notable reasons include the fact that passengers live near Picton, especially in the case of CSNZ gateway travellers $(23.3 \%)$. Some international passengers also mentioned that time constraints did not allow them to stay overnight in Picton. Initially this may seem a bit contradictory especially knowing that international passengers spend more time on their trips in New Zealand than do passengers who live in the country (Table 6.6). However, it is important to consider two issues in order to interpret this piece of information. The first is that the time constraint may particularly affect some segments of international travellers, especially those spending less time in the country. The second is that as international travellers tend to tour around the country, trying to visit as many places as they can, they may feel that their time is limited in order to visit and stay in all the places they would like to. Passengers living in New Zealand are more familiar with a wide range of places existing in their own country, therefore they know they will not stay overnight in Picton because either they do not have a need to or simply because they would prefer to stay somewhere else. Time constraints are less important to them as they already know what to expect from different places.

The Trip Index reflects the two characteristics presented earlier, i.e. the main reason for going to Picton and the number of nights spent there. Results for the transit / day trip type of traveller $(\mathrm{Ti}=0)$ is similar to passengers not staying overnight in Picton (see Table 7.18). Therefore, it is more interesting to analyse the different types of stopovers (short, medium and long) and destination (principal and sole) travellers. OGV and SV are made mostly of transit / day-trippers or short stopovers, as these passengers, by definition, do not stay more than one night. On the other hand, destination tourists present a different pattern, with the main Trip Index category being the sole destination type of traveller $(\mathrm{Ti}=100)$, which accounts for nearly $40 \%$. It is interesting to note that $34.2 \%$ of destination tourists consist of CSNZ travellers that are only going to Picton. Once again this highlights the use passengers living in Wellington make of Picton, considering it as a destination to escape 


\section{FERRY PASSENGERS IN WELLINGTON AND PICTON}

from the urban environment, with the ferries serving as the means of transport to give accessibility.

Associated with the number of nights spent in Picton and the Trip Index is the type of accommodation used there. It is appropriate to mention that the small number of respondents in Table 7.21 is a consequence of the strong gateway function that exists in Picton with over three-quarters of the passenger sample not staying overnight there (see Table 7.18). From Table 7.21 it is possible to notice that the different nodal segments stay in various types of accommodation. For OGV, the single most important type of accommodation is motel (35.6\%); with SV predominantly choosing backpacker / youth hostels (25\%); and DT preferring private homes (34.6\%). In the latter case, this can be explained by the fact that travellers staying longer in Picton usually take advantage of private homes to afford longer stays, particularly with CSNZ passengers $(22.2 \%)$. The use of backpacker / youth hostels by stopover visitors can be linked to the number of international travellers staying in this type of accommodation (16.7\%). This can suit travellers on long international holidays when they are more budget oriented. Finally, the use of motels by OGV is, most probably, related to the use of vehicles while touring around the country, especially among domestic and international passengers (private cars in the first case; rental cars in the second group - see Table 6.9). In Picton, quite a number of passengers also stay in "other" types of accommodation. The reason for this is that in Picton there are few traditional types of commercial accommodations (e.g. hotels), with a higher number of touring campervan/caravan, camping and $B \& B$, rather than hotels. In fact this is exactly what the Resort Development Spectrum suggests for resorts classified in the Phase 1, where usually the accommodation sector is less developed, with few hotel facilities and more caravan parks, motels and backpacker hostels (see Table $2.2)$. 
Table 7.17 Main reason for going to Picton.

\begin{tabular}{|c|c|c|c|c|c|c|c|c|c|c|c|c|c|c|c|c|c|}
\hline \multirow[b]{2}{*}{ Main reason } & \multirow{2}{*}{$\begin{array}{l}\text { Origin } \\
(n=8)\end{array}$} & \multicolumn{4}{|c|}{ Gateway Travellers ( $n=695)$} & \multicolumn{4}{|c|}{ Overnight Gateway Visitors $(n=118)$} & \multicolumn{4}{|c|}{ Stopover Visitors $(n=134)$} & \multicolumn{4}{|c|}{ Destination Tourists $(n=80)$} \\
\hline & & CSNZ & Dom & Int'l & Total & CSNZ & Dom & Int'l & Total & CSNZ & Dom & Int'l & Total & CSNZ & Dom & Int'l & Total \\
\hline Live in Picton & $100.0 \%$ & & & & & & & & & & & & & & & & \\
\hline Leisure / Holiday & & & & & & & & & & $20.1 \%$ & $11.9 \%$ & $40.3 \%$ & $72.3 \%$ & $21.3 \%$ & $6.3 \%$ & $12.5 \%$ & $40.0^{c}$ \\
\hline Inter-island connection & & $30.2 \%$ & $32.1 \%$ & $37.7 \%$ & $100.0^{c}$ & $3.4 \%$ & $39.0 \%$ & $57.6 \%$ & $100.0 \%$ & & & & & $3.8 \%$ & $2.5 \%$ & $27.5 \%$ & $33.8^{c}$ \\
\hline VFR & & & & & & & & & & $4.5 \%$ & $9.0 \%$ & $2.2 \%$ & $15.7 \%$ & $11.3 \%$ & $7.5 \%$ & $5.0 \%$ & $23.8^{c}$ \\
\hline Business / work & & & & & & & & & & $2.2 \%$ & $0.7 \%$ & & $3.0 \%$ & $1.3 \%$ & & & $1.3^{c}$ \\
\hline Other & & & & & & & & & & $4.5 \%$ & $3.0 \%$ & $1.5 \%$ & $9.0 \%$ & $1.3 \%$ & & & $1.3^{\prime}$ \\
\hline Total & $100.0 \%$ & $30.2 \%$ & $32.1 \%$ & $37.7 \%$ & $100.0^{c}$ & $3.4 \%$ & $39.0 \%$ & $57.6 \%$ & $100.0 \%$ & $31.3 \%$ & $24.6^{c}$ & $44.0 \%$ & $100.0 \%$ & $38.8^{\circ}$ & $16.3 \%$ & $45.0 \%$ & $100.0^{c}$ \\
\hline
\end{tabular}

\section{Table 7.18 Number of nights while in Picton.}

\begin{tabular}{|c|c|c|c|c|c|c|c|c|c|c|c|c|c|c|c|c|}
\hline \multirow{2}{*}{$\begin{array}{l}\text { Number } \\
\text { of nights }\end{array}$} & \multicolumn{4}{|c|}{ Gateway Travellers ( $n=695)$} & \multicolumn{4}{|c|}{ Overnight Gateway Visitors $(n=118)$} & \multicolumn{4}{|c|}{ Stopover Visitors $(n=135)$} & \multicolumn{4}{|c|}{ Destination Tourists $(n=81)$} \\
\hline & CSNZ & Dom & Int'l & Total & CSNZ & Dom & Int'l & Total & CSNZ & Dom & Int'l & Total & CSNZ & Dom & Int'l & Total \\
\hline 0 & $30.2 \%$ & $32.1 \%$ & $37.7 \%$ & $100.0 \%$ & & & & & $27.4 \%$ & $20.7 \%$ & $25.2 \%$ & $73.3 \%$ & & & & \\
\hline 1 & & & & & $3.4 \%$ & $39.0 \%$ & $57.6 \%$ & $100.0 \%$ & $3.7 \%$ & $3.7 \%$ & $19.3 \%$ & $26.7 \%$ & $3.7 \%$ & & & $3.7 \%$ \\
\hline 2 & & & & & & & & & & & & & $17.3 \%$ & $1.2 \%$ & $34.6 \%$ & $53.1 \%$ \\
\hline 4 & & & & & & & & & & & & & $3.7 \%$ & $2.5 \%$ & & $6.2 \%$ \\
\hline 5 & & & & & & & & & & & & & & & & \\
\hline $6+$ & & & & & & & & & & & & & $4.9 \%$ & $3.7 \%$ & $4.9 \%$ & $13.6 \%$ \\
\hline Total & $30.2 \%$ & $32.1 \%$ & $37.7 \%$ & $100.0 \%$ & $3.4 \%$ & $39.0 \%$ & $57.6 \%$ & $100.0 \%$ & $31.1 \%$ & $24.4 \%$ & $44.4 \%$ & $100.0 \%$ & $38.3 \%$ & $16.0 \%$ & $45.7 \%$ & $100.0 \%$ \\
\hline
\end{tabular}


Table 7.19 Reason for gateway travellers and stopover visitors not staying overnight in Picton.

\begin{tabular}{|c|c|c|c|c|c|c|c|c|}
\hline \multirow[b]{2}{*}{ Reason } & \multicolumn{4}{|c|}{ Gateway Travellers } & \multicolumn{4}{|c|}{ Stopover Visitors } \\
\hline & $\operatorname{CSNZ}(n=210)$ & Dom $(n=223)$ & Int'I $(n=262)$ & Total $(n=695$ & $\operatorname{csNZ}(n=37)$ & Dom $(n=28)$ & Int'l $(n=34)$ & Гotal $(n=99)$ \\
\hline Staying overnight somewhere else & $29.5 \%$ & $31.8 \%$ & $31.3 \%$ & $30.9 \%$ & $40.5 \%$ & $60.7 \%$ & $14.7 \%$ & $37.4 \%$ \\
\hline No reason given & $15.7 \%$ & $13.5 \%$ & $16.8 \%$ & $15.4 \%$ & $24.3 \%$ & $14.3 \%$ & $20.6 \%$ & $20.2 \%$ \\
\hline No need / passing through & $23.3 \%$ & $39.0 \%$ & $19.1 \%$ & $26.8 \%$ & $18.9 \%$ & $10.7 \%$ & $23.5 \%$ & $18.2 \%$ \\
\hline Time constraint & $2.9 \%$ & $2.2 \%$ & $12.2 \%$ & $6.2 \%$ & $2.7 \%$ & & $17.6 \%$ & $7.1 \%$ \\
\hline Returning the same day & & & & & $5.4 \%$ & $3.6 \%$ & $5.9 \%$ & $5.1 \%$ \\
\hline Not my type of destination & $3.3 \%$ & $2.7 \%$ & $3.8 \%$ & $3.3 \%$ & $2.7 \%$ & & $5.9 \%$ & $3.0 \%$ \\
\hline Already been there / will be there later & & $2.2 \%$ & $1.1 \%$ & $1.2 \%$ & & $3.6 \%$ & $2.9 \%$ & $2.0 \%$ \\
\hline Live near Picton & $23.3 \%$ & $3.1 \%$ & & $8.1 \%$ & $5.4 \%$ & & & $2.0 \%$ \\
\hline Other reason & $1.9 \%$ & $5.4 \%$ & $15.6 \%$ & $8.2 \%$ & & $7.1 \%$ & $8.8 \%$ & $5.1 \%$ \\
\hline Total & $100.0 \%$ & $100.0 \%$ & $100.0 \%$ & $100.0 \%$ & $100.0 \%$ & $100.0 \%$ & $100.0 \%$ & $100.0 \%$ \\
\hline
\end{tabular}

\section{Table 7.20 Trip index for Picton.}

\begin{tabular}{|c|c|c|c|c|c|c|c|c|c|c|c|c|c|c|c|c|}
\hline \multirow[b]{2}{*}{ Trip Index } & \multicolumn{4}{|c|}{ Gateway Travellers $(n=690)$} & \multicolumn{4}{|c|}{ Overnight Gateway Visitors $(n=113)$} & \multicolumn{4}{|c|}{ Stopover Visitors $(n=104)$} & \multicolumn{4}{|c|}{ Destination Tourists $(n=76$ ) } \\
\hline & CSNZ & Dom & Int'l & Total & CSNZ & Dom & Int'l & Total & CSNZ & Dom & Int'l & Total & CSNZ & Dom & Int'l & Total \\
\hline $\mathrm{Ti}=0$ (transit or day trip) & $29.9 \%$ & $32.2 \%$ & $38.0 \%$ & $100.0^{c}$ & & & & & $8.7 \%$ & $26.0 \%$ & $31.7 \%$ & $66.3 \%$ & & & & \\
\hline $0<\mathrm{Ti} \leq 10$ (short stopover) & & & & & $0.9 \%$ & $26.5 \%$ & $55.8 \%$ & $83.2 \%$ & $1.0 \%$ & $3.8 \%$ & $21.2 \%$ & $26.0 \%$ & $1.3 \%$ & & $28.9 \%$ & $30.3 \%$ \\
\hline $10<\mathrm{Ti} \leq 20$ (medium stopover) & & & & & & $3.5 \%$ & $0.9 \%$ & $4.4 \%$ & $1.9 \%$ & & $2.9 \%$ & $4.8 \%$ & & $3.9 \%$ & $7.9 \%$ & $11.8 \%$ \\
\hline $20<\mathrm{Ti} \leq 50$ (long stopover) & & & & & $1.8 \%$ & $10.6 \%$ & & $12.4 \%$ & $1.9 \%$ & $1.0 \%$ & & $2.9 \%$ & $2.6 \%$ & $2.6 \%$ & $5.3 \%$ & $10.5 \%$ \\
\hline $50<\mathrm{Ti}<100$ (principal destination) & & & & & & & & & & & & & $2.6 \%$ & $5.3 \%$ & & $7.9 \%$ \\
\hline $\mathrm{Ti}=100$ (sole destination) & & & & & & & & & & & & & $34.2 \%$ & $5.3 \%$ & & $39.5 \%$ \\
\hline Total & $29.9 \%$ & $32.2 \%$ & $38.0 \%$ & $100.0^{c}$ & $2.7 \%$ & $40.7 \%$ & $56.6 \%$ & $100.0 \%$ & $13.5 \%$ & $30.8 \%$ & $55.8 \%$ & $100.0 \%$ & $40.8 \%$ & $17.1 \%$ & $42.1 \%$ & $100.0 \%$ \\
\hline
\end{tabular}




\section{FERRY PASSENGERS IN WELLINGTON AND PICTON}

In terms of the sources of information used by travellers to find out more about Picton (Table 7.22), different patterns can be identified between overseas passengers and those living in New Zealand. For example, while commercial guide books, visitor information centres and free visitor guides were the most used source of information for international passengers, passengers living in New Zealand relied particularly on past visits for information about Picton. In general terms it is possible to say that passengers staying longer in Picton usually make more use of sources of information to know more about it. This is the case particularly among DT and OGV. As suggested in the literature review, the search for information may be a way to minimise the risks associated with the decision to visit a certain destination. In the case of GT and SV passengers, while their lack of interest to search for information might be associated with a previous decision not to visit and stay in Picton, if tourism businesses and organisations are interested in promoting Picton more as a destination they might need to make more use of information channels which target large audiences, such as the media.

Adopting the same approach taken to analyse passengers' activities and attractions visited in Wellington, two major groups can be identified in Picton. The first is composed of more generic activities, such as walking in town, shopping and eating out, while other attractions are more specifically designed for tourists, such as visiting the Aquarium of the Marlborough Sounds or the Picton Museum and taking a scenic drive or boat cruise. According to Table 7.23, the most popular activities in Picton are walking in town, general sightseeing and eating out. Activities and attractions with a more touristic appeal that are also of interest to passengers include scenic drives, bush walks and the Queen Charlotte Track.

Except in a very few cases, including those with small size cells (e.g. CSNZ overnight gateway visitors and domestic DT), the participation of passengers in activities and attractions increase with their length of stay. When comparing the overall results from DT to GT, it is clear that the longer the stay the higher the chance of travellers doing more activities and visiting more attractions. This emphasizes the need to make passengers stay longer in Picton, so that they can participate in more activities and visit more attractions, or encourage them to do more activities and visit more attractions so they will stay longer. In addition, when comparing the three different places of origin, it is possible to identify that in most cases international passengers are the sub-group with the highest participation (e.g. general sightseeing). For some activities and attractions such as walking in town, travelling by water taxi, eating out and the Aquarium of the Marlborough Sounds, passengers from CSNZ have higher interest than the other groups. 
Table 7.21 Type of accommodation used in Picton.

\begin{tabular}{|c|c|c|c|c|c|c|c|c|c|c|c|c|}
\hline \multirow[b]{2}{*}{ Type of accommodation } & \multicolumn{4}{|c|}{ Overnight Gateway Visitors $(n=118)$} & \multicolumn{4}{|c|}{ Stopover Visitors $(n=36)$} & \multicolumn{4}{|c|}{ Destination Tourists $(n=81)$} \\
\hline & CSNZ & Dom & Int'l & Total & CSNZ & Dom & Int'l & Total & CSNZ & Dom & Int'l & Total \\
\hline Private home & & $1.7 \%$ & $0.8 \%$ & $2.5 \%$ & $2.8 \%$ & & & $2.8 \%$ & $22.2 \%$ & $8.6 \%$ & $3.7 \%$ & $34.6 \%$ \\
\hline Backpacker / Youth hostel & $1.7 \%$ & $5.1 \%$ & $15.3 \%$ & $22.0 \%$ & $2.8 \%$ & $5.6 \%$ & $16.7 \%$ & $25.0 \%$ & $2.5 \%$ & & $14.8 \%$ & $17.3 \%$ \\
\hline Motel & & $18.6 \%$ & $16.9 \%$ & $35.6 \%$ & $2.8 \%$ & $8.3 \%$ & $5.6 \%$ & $16.7 \%$ & $3.7 \%$ & $1.2 \%$ & $6.2 \%$ & $11.1 \%$ \\
\hline Hotel & & $0.8 \%$ & & $0.8 \%$ & $2.8 \%$ & & $11.1 \%$ & $13.9 \%$ & $4.9 \%$ & $1.2 \%$ & $3.7 \%$ & $9.9 \%$ \\
\hline Touring caravan / campervan & $0.8 \%$ & $5.1 \%$ & $8.5 \%$ & $14.4 \%$ & & & $11.1 \%$ & $11.1 \%$ & $1.2 \%$ & & $6.2 \%$ & $7.4 \%$ \\
\hline Other (includes camping and B\&B) & $0.8 \%$ & $7.6 \%$ & $16.1 \%$ & $24.6 \%$ & $2.8 \%$ & & $27.8 \%$ & $30.6 \%$ & $3.7 \%$ & $4.9 \%$ & $11.1 \%$ & $19.8 \%$ \\
\hline Total & $3.4 \%$ & $39.0 \%$ & $57.6 \%$ & $100.0 \%$ & $13.9 \%$ & $13.9 \%$ & $72.2 \%$ & $100.0 \%$ & $38.3 \%$ & $16.0 \%$ & $45.7 \%$ & $100.0 \%$ \\
\hline
\end{tabular}


Table 7.22 Sources of information used to find out more about Picton.

\begin{tabular}{|c|c|c|c|c|c|c|c|c|c|c|c|c|c|c|c|c|}
\hline \multirow[b]{2}{*}{ Source of Information } & \multicolumn{4}{|c|}{ Gateway Travellers } & \multicolumn{4}{|c|}{ Overnight Gateway Visitors } & \multicolumn{4}{|c|}{ Stopover Visitors } & \multicolumn{4}{|c|}{ Destination Tourists } \\
\hline & $\begin{array}{c}\text { CSNZ } \\
(n=210)\end{array}$ & $\begin{array}{c}\text { Dom } \\
(n=223)\end{array}$ & $\begin{array}{c}\text { Int'l } \\
(n=262)\end{array}$ & $\begin{array}{c}\text { Total } \\
(n=695)\end{array}$ & $\begin{array}{l}\text { CSNZ } \\
(n=4)\end{array}$ & $\begin{array}{l}\text { Dom } \\
(n=46)\end{array}$ & $\begin{array}{c}\text { Int'l } \\
(n=68)\end{array}$ & $\begin{array}{c}\text { Total } \\
(n=118 ;\end{array}$ & $\begin{array}{l}\text { CSNZ } \\
(n=42)\end{array}$ & $\begin{array}{c}\text { Dom } \\
(n=33)\end{array}$ & $\begin{array}{c}\text { Int'l } \\
(\mathrm{n}=60)\end{array}$ & $\begin{array}{c}\text { Total } \\
(n=135\end{array}$ & $\begin{array}{l}\text { CSNZ } \\
(n=31)\end{array}$ & $\begin{array}{c}\text { Dom } \\
(n=13)\end{array}$ & $\begin{array}{c}\text { Int'l } \\
(n=37)\end{array}$ & $\begin{array}{c}\text { Total } \\
(n=81)\end{array}$ \\
\hline Past visits & $39.0 \%$ & $44.8 \%$ & $11.5 \%$ & $30.5 \%$ & $50.0 \%$ & $47.8 \%$ & $11.8 \%$ & $27.1 \%$ & $31.0 \%$ & $51.5 \%$ & $15.0 \%$ & $28.9 \%$ & $61.3 \%$ & $46.2 \%$ & $13.5 \%$ & $37.0 \%$ \\
\hline Commercial guide book & $2.9 \%$ & $4.5 \%$ & $44.3 \%$ & $19.0 \%$ & $25.0 \%$ & $4.3 \%$ & $70.6 \%$ & $43.2 \%$ & $7.1 \%$ & $9.1 \%$ & $45.0 \%$ & $24.4 \%$ & $9.7 \%$ & $15.4 \%$ & $67.6 \%$ & $37.0 \%$ \\
\hline VIC (in New Zealand) & $6.7 \%$ & $5.4 \%$ & $12.6 \%$ & $8.5 \%$ & & $8.7 \%$ & $19.1 \%$ & $14.4 \%$ & $7.1 \%$ & $12.1 \%$ & $18.3 \%$ & $13.3 \%$ & $16.1 \%$ & $15.4 \%$ & $32.4 \%$ & $23.5 \%$ \\
\hline Free visitor guides & $6.7 \%$ & $6.3 \%$ & $19.5 \%$ & $11.4 \%$ & $25.0 \%$ & $8.7 \%$ & $29.4 \%$ & $21.2 \%$ & $11.9 \%$ & $6.1 \%$ & $30.0 \%$ & $18.5 \%$ & $9.7 \%$ & $23.1 \%$ & $29.7 \%$ & $21.0 \%$ \\
\hline Word of mouth & $10.5 \%$ & $8.1 \%$ & $14.5 \%$ & $11.2 \%$ & & $15.2 \%$ & $26.5 \%$ & $21.2 \%$ & $4.8 \%$ & $9.1 \%$ & $21.7 \%$ & $13.3 \%$ & $29.0 \%$ & $7.7 \%$ & $18.9 \%$ & $21.0 \%$ \\
\hline Internet & $7.1 \%$ & $8.5 \%$ & $10.7 \%$ & $8.9 \%$ & & $6.5 \%$ & $16.2 \%$ & $11.9 \%$ & $11.9 \%$ & $21.2 \%$ & $18.3 \%$ & $17.0 \%$ & $9.7 \%$ & $23.1 \%$ & $16.2 \%$ & $14.8 \%$ \\
\hline Media & $5.2 \%$ & $4.0 \%$ & $1.1 \%$ & $3.3 \%$ & & & $1.5 \%$ & $0.8 \%$ & $4.8 \%$ & $15.2 \%$ & $3.3 \%$ & $6.7 \%$ & $3.2 \%$ & $7.7 \%$ & $2.7 \%$ & $3.7 \%$ \\
\hline Travel agent & $1.9 \%$ & $0.9 \%$ & $9.5 \%$ & $4.5 \%$ & & & $4.4 \%$ & $2.5 \%$ & $2.4 \%$ & $3.0 \%$ & $13.3 \%$ & $7.4 \%$ & & & $5.4 \%$ & $2.5 \%$ \\
\hline
\end{tabular}

(Multiple responses allowed - ranked in terms of importance to total DT passengers) 
Table 7.23 Activities undertaken and attractions visited in Picton.

\begin{tabular}{|c|c|c|c|c|c|c|c|c|c|c|c|c|c|c|c|c|}
\hline \multirow[b]{2}{*}{ Activities / Attractions } & \multicolumn{4}{|c|}{ Gateway Travellers } & \multicolumn{4}{|c|}{ Overnight Gateway Visitors } & \multicolumn{4}{|c|}{ Stopover Visitors } & \multicolumn{4}{|c|}{ Destination Tourists } \\
\hline & $\begin{array}{c}\text { CSNZ } \\
(n=210)\end{array}$ & $\begin{array}{c}\text { Dom } \\
(n=223)\end{array}$ & $\begin{array}{c}\text { Int'l } \\
(n=262)\end{array}$ & $\begin{array}{c}\text { Total } \\
(\mathrm{n}=695\end{array}$ & $\begin{array}{c}\text { CSNZ } \\
(n=4)\end{array}$ & $\begin{array}{c}\text { Dom } \\
(n=46)\end{array}$ & $\begin{array}{c}\text { Int'l } \\
(n=68)\end{array}$ & $\begin{array}{c}\text { Total } \\
(n=11 \varepsilon\end{array}$ & $\begin{array}{l}\text { CSNZ } \\
(n=42)\end{array}$ & $\begin{array}{c}\text { Dom } \\
(n=33)\end{array}$ & $\begin{array}{c}\text { Int'| } \\
(n=60)\end{array}$ & $\begin{array}{c}\text { Total } \\
(n=135\end{array}$ & $\begin{array}{l}\text { CSNZ } \\
(n=31)\end{array}$ & $\begin{array}{c}\text { Dom } \\
(n=13)\end{array}$ & $\begin{array}{c}\text { Int'| } \\
(n=37)\end{array}$ & $\begin{array}{c}\text { Total } \\
(n=81)\end{array}$ \\
\hline Walk in town & $22.4 \%$ & $18.8 \%$ & $18.3 \%$ & $19.7 \%$ & $50.0 \%$ & $54.3 \%$ & $58.8 \%$ & $56.8 \%$ & $31.0 \%$ & $42.4 \%$ & $48.3 \%$ & $41.5 \%$ & $54.8 \%$ & $46.2 \%$ & $70.3 \%$ & $60.5 \%$ \\
\hline Eating out & $18.1 \%$ & $15.2 \%$ & $11.1 \%$ & $14.5 \%$ & $25.0 \%$ & $32.6 \%$ & $33.8 \%$ & $33.1 \%$ & $33.3 \%$ & $24.2 \%$ & $36.7 \%$ & $32.6 \%$ & $48.4 \%$ & $23.1 \%$ & $45.9 \%$ & $43.2 \%$ \\
\hline General sightseeing & $7.6 \%$ & $9.9 \%$ & $12.6 \%$ & $10.2 \%$ & $25.0 \%$ & $30.4 \%$ & $36.8 \%$ & $33.9 \%$ & $11.9 \%$ & $3.0 \%$ & $36.7 \%$ & $20.7 \%$ & $22.6 \%$ & $46.2 \%$ & $59.5 \%$ & $43.2 \%$ \\
\hline Sailing & $2.4 \%$ & $0.9 \%$ & & $3.3 \%$ & & $2.2 \%$ & $11.8 \%$ & $7.6 \%$ & $7.1 \%$ & $6.1 \%$ & $13.3 \%$ & $9.6 \%$ & $3.2 \%$ & $7.7 \%$ & $8.1 \%$ & $34.6 \%$ \\
\hline Mini golf & $4.8 \%$ & $1.3 \%$ & $1.1 \%$ & $1.4 \%$ & & & $8.8 \%$ & $5.1 \%$ & $2.4 \%$ & $12.1 \%$ & $1.7 \%$ & $4.4 \%$ & $16.1 \%$ & $15.4 \%$ & $10.8 \%$ & $29.6 \%$ \\
\hline Picton museum & $1.0 \%$ & $1.3 \%$ & & $5.5 \%$ & & $8.7 \%$ & $16.2 \%$ & $12.7 \%$ & $9.5 \%$ & & $11.7 \%$ & $8.1 \%$ & $9.7 \%$ & $7.7 \%$ & $8.1 \%$ & $28.4 \%$ \\
\hline Queen Charlotte Track & $4.3 \%$ & $1.3 \%$ & $2.7 \%$ & $2.7 \%$ & & $2.2 \%$ & $8.8 \%$ & $5.9 \%$ & $9.5 \%$ & $9.1 \%$ & $10.0 \%$ & $9.6 \%$ & $9.7 \%$ & $15.4 \%$ & $29.7 \%$ & $19.8 \%$ \\
\hline Scenic boat cruise & $1.0 \%$ & $0.9 \%$ & $0.8 \%$ & $0.9 \%$ & & $4.3 \%$ & $1.5 \%$ & $2.5 \%$ & $4.8 \%$ & & $3.3 \%$ & $3.0 \%$ & $22.6 \%$ & $7.7 \%$ & $16.2 \%$ & $17.3 \%$ \\
\hline Trekking / tramping & $2.9 \%$ & & $1.5 \%$ & $1.4 \%$ & & & $5.9 \%$ & $3.4 \%$ & $7.1 \%$ & $3.0 \%$ & $6.7 \%$ & $5.9 \%$ & $9.7 \%$ & $7.7 \%$ & $24.3 \%$ & $16.0 \%$ \\
\hline Water taxi & $1.9 \%$ & & $0.4 \%$ & $0.7 \%$ & & $2.2 \%$ & $4.4 \%$ & $3.4 \%$ & $11.9 \%$ & $9.1 \%$ & $5.0 \%$ & $8.1 \%$ & $12.9 \%$ & & $13.5 \%$ & $11.1 \%$ \\
\hline Aquarium of the Marlborough Sounds & $1.4 \%$ & $2.7 \%$ & $0.8 \%$ & $1.6 \%$ & & & $1.5 \%$ & $0.8 \%$ & $7.1 \%$ & $3.0 \%$ & $3.3 \%$ & $4.4 \%$ & $12.9 \%$ & $7.7 \%$ & $5.4 \%$ & $8.6 \%$ \\
\hline Beach & $5.2 \%$ & $3.1 \%$ & $1.9 \%$ & $0.7 \%$ & & $2.2 \%$ & $1.5 \%$ & $1.7 \%$ & $2.4 \%$ & $3.0 \%$ & $5.0 \%$ & $3.7 \%$ & $35.5 \%$ & $38.5 \%$ & $32.4 \%$ & $8.6 \%$ \\
\hline Bush walk & $2.9 \%$ & $0.4 \%$ & $1.1 \%$ & $1.0 \%$ & & & & & & $3.0 \%$ & $1.7 \%$ & $1.5 \%$ & $29.0 \%$ & $23.1 \%$ & $32.4 \%$ & $6.2 \%$ \\
\hline
\end{tabular}

(Multiple responses allowed - ranked in terms of importance to total DT passengers) 


\subsubsection{Changing passengers' length of stay in Picton}

Similarly to Section 7.2.1, this section analyses the actual and potential change of plans of ferry passengers in Picton.

\section{Actual change of plans in Picton}

When considering the actual change of plans by passengers in Picton, some general comments can be made. Firstly, as in the case of Wellington there was a large percentage of passengers, at least $60 \%$ for each function, that did not change their plans, in addition to another large group of passengers that did not answer this question. Secondly, according to Table 7.24 , only 3\% (31 passengers) of the total sample changed their plans in terms of the length of stay in Picton. Of these, 25 passengers stayed longer ( $G T n=3$; OGV n=10; SV n=4; DT n=8), only six stayed shorter (GT $n=5$; DT $n=1$ ). Therefore, Picton ended up benefiting from the passengers' change of plans. Thirdly, while to some particular sub-groups the proportion of passengers changing plans is very small (e.g. CSNZ and domestic GT with less than 1\%), in some cases it can reach over $10 \%$ of the sub-group, such as domestic OGV and international DT (not considering 50\% of CSNZ OGV due to the small cell size $-n=4$ ). Finally, it is worth mentioning that a significant number of passengers (351 or approximately $34 \%$ of the total sample) did not answer this question. This number is particularly high, in fact more than ten times higher than the actual number of passengers who changed their plans, and could have a significant impact on the total results, especially if a considerable number of passengers who did not answer this question had in fact changed their plans for Picton. While this is very unlikely, the only reasonable explanation for this is that passengers who did not change their plans did not bother about answering this question.

Apart from the number of passengers changing their plans for Picton, it is also necessary to consider the reasons why they made these changes. According to Table 7.25, ferry cancellation was one the most important reasons to make passengers alter their plans. From the total 25 passengers staying longer in Picton, nearly half of them (12) ended up staying longer due to the cancellation of ferry services. In most cases this was a consequence of the bad weather and rough sea. Apart from the influence on the ferry cancellation, bad weather also impacted on passengers' decision in different ways. As seen in Table 7.25, in some cases it made passengers stay shorter than planned, as they would prefer to go to another destination where they could do an indoor activity or visit certain types of attractions. In fact, as presented in Chapter 4, there are very few indoor attractions to visit and activities to do in Picton. Other passengers preferred to stay longer 


\section{FERRY PASSENGERS IN WELLINGTON AND PICTON}

because they wanted to avoid a ferry journey in a rough sea, especially if they get seasick easily.

In terms of passengers' perception of Picton as a destination, positive and negative impressions made passengers change their minds (circumstances within the travellers' control). In both cases where passengers extended their stays in Picton they mentioned the quality of the accommodation they were in, in addition to other reasons, such as convenience of the ferry and more activities to do than initially thought. On the other hand, one passenger changed his/her mind en route to Picton and decided to stay shorter. This passenger stated that this was a good decision, as Picton appeared to be boring. Another preferred to travel in the North Island rather than doing the Queen Charlotte track.

\section{Potential change of plans in Picton}

This section makes use of the scenario technique to analyse what would happen to the passengers' length of stay in case they had more information or had been offered a special deal before they arrived in Picton. It also considers, from an open question, what need to be offered to passengers in order to make them stay longer.

Table 7.27 presents the results of passengers' reaction to more information. Overall, the proportion of passengers staying longer is higher than those deciding to stay shorter, even though these differences vary considerably for different sub-groups (e.g. CSNZ and international passengers). As can be seen in Table 7.27, more information would have diverse impacts on different groups of passengers, with OGV (11.9\%) and SV (11.1\%) being more sensitive than GT $(5.3 \%)$ and DT $(6.2 \%)$. When comparing the differences between the various places of origin groups, it is also possible to identify variations in the passengers' response. For example, while $18.3 \%$ of international SV would stay longer, only $1.9 \%$ of CSNZ gateway tourists would do the same. In fact, in terms of the passenger's reaction to extending their stay in Picton if they had more information, apparently the closer they live to Picton the less influenced they would be. The proportion of international passengers staying longer is higher than those living in New Zealand. In the same way, the proportion of domestic passengers is higher than those from the CSNZ region. This indicates that the closer the passenger lives to the node the more familiar they seem to be with it, implying that they need less information to make their plans. It is interesting to note that none of the CSNZ and domestic destination tourists mentioned that they would stay longer than planned if they had had more information about Picton. Apparently destination tourists living in New Zealand consider themselves very much aware of what Picton has to offer and they are already taking advantage of this to spend as much time as they can in this destination. Even though the number of passengers 


\section{FERRY PASSENGERS IN WELLINGTON AND PICTON}

deciding to stay shorter in Picton is lower than those staying longer, the fact that some of them would spend less time in Picton if they had more information implies that they were disappointed with Picton and the image they had of Picton did not match the impression obtained when they arrived there.

In comparison to information, the respondents' reaction to a special deal, which includes transport and accommodation, was more positive for most sub-groups of passengers (see Table 7.27). In general, the response was proportionally more important for passengers living in New Zealand than those from overseas, with stopover visitors being the group of passengers with the highest likelihood to extend their stay in Picton. In fact, over a quarter of international SV (28.3\%) stated that they would have stayed longer in Picton if a special deal had been offered to them, with over $19 \%$ of CSNZ and domestic stopover visitors having the same pattern. After stopover visitors, OGV were the group of passengers with higher chances of staying longer, followed, in the case of the three places of origin subgroups, by gateway travellers and destination tourists. The only situation where a special deal would be less appealing than information is international DT (10.8\% - Table 7.27 against $13.5 \%$ - Table 7.26 ). Apparently as international travellers are less familiar with New Zealand, they tend to obtain more information about its destinations, so they can minimise the risk of spending time in a place where they would not like to be. In the case of passengers living in New Zealand, the response to the deal was significantly higher than the one from information. In this latter case it seems that the deal would attract passengers that were staying overnight somewhere else to stay in Picton. As seen in Table 7.19, staying overnight somewhere else near Picton was the main reason given by passengers living in New Zealand to not stay in Picton.

Finally, considering passengers' own suggestions of what would make them stay longer than planned in Picton, a wide variety of factors were mentioned (see Table 7.28). Before analysing these factors it is worth mentioning that only $7 \%$ of the total sample answered this question. At least two reasons can be attributed for the low response rate. Firstly, as seen previously for other open questions, the response rate is usually lower than the closed questions. Secondly, passengers might have considered that the previous items, information and special deal, were enough to persuade them whether or not to stay longer and that there was nothing else that would make them extend their length of stay in Picton. 


\section{Table 7.24 Change of plans in Picton.}

\begin{tabular}{|c|c|c|c|c|c|c|c|c|c|c|c|c|c|c|c|c|}
\hline \multirow[b]{2}{*}{$\begin{array}{c}\text { Change of } \\
\text { Plans }\end{array}$} & \multicolumn{4}{|c|}{ Gateway Travellers } & \multicolumn{4}{|c|}{ Overnight Gateway Visitors } & \multicolumn{4}{|c|}{ Stopover Visitors } & \multicolumn{4}{|c|}{ Destination Tourists } \\
\hline & $\begin{array}{c}\text { CSNZ } \\
(n=210)\end{array}$ & $\begin{array}{c}\text { Dom } \\
(n=223)\end{array}$ & $\begin{array}{c}\text { Int'l } \\
(n=262)\end{array}$ & $\begin{array}{c}\text { Total } \\
(n=695)\end{array}$ & $\begin{array}{l}\text { CSNZ } \\
(n=4)\end{array}$ & $\begin{array}{l}\text { Dom } \\
(n=46)\end{array}$ & $\begin{array}{c}\text { Int'l } \\
(n=68)\end{array}$ & $\begin{array}{c}\text { Total } \\
(n=118\end{array}$ & $\begin{array}{l}\text { CSNZ } \\
(n=42)\end{array}$ & $\begin{array}{c}\text { Dom } \\
(n=33)\end{array}$ & $\begin{array}{c}\text { Int'| } \\
(n=60)\end{array}$ & $\begin{array}{c}\text { Total } \\
(n=135\end{array}$ & $\begin{array}{l}\text { CSNZ } \\
(n=31)\end{array}$ & $\begin{array}{c}\text { Dom } \\
(n=13)\end{array}$ & $\begin{array}{c}\text { Int'| } \\
(n=37)\end{array}$ & $\begin{array}{c}\text { Total } \\
(n=81)\end{array}$ \\
\hline \multirow{2}{*}{$\begin{array}{l}\text { Plans were not } \\
\text { changed }\end{array}$} & 144 & 161 & 129 & 434 & 1 & 29 & 49 & 79 & 30 & 28 & 28 & 86 & 23 & 8 & 17 & 48 \\
\hline & $68.6 \%$ & $72.2 \%$ & $49.2 \%$ & $62.4 \%$ & $25.0 \%$ & $63.0 \%$ & $72.1 \%$ & $66.9 \%$ & $71.4 \%$ & $84.8 \%$ & $46.7 \%$ & $63.7 \%$ & $74.2 \%$ & $61.5 \%$ & $45.9 \%$ & $59.3 \%$ \\
\hline \multirow[t]{2}{*}{ Stayed longer } & & 2 & & 3 & 2 & 6 & 2 & 10 & & 1 & 3 & 4 & 3 & & 5 & 8 \\
\hline & $0.5 \%$ & $0.9 \%$ & & $0.4 \%$ & $50.0 \%$ & $13.0 \%$ & $2.9 \%$ & $8.5 \%$ & & $3.0 \%$ & $5.0 \%$ & $3.0 \%$ & $9.7 \%$ & & $13.5 \%$ & $9.9 \%$ \\
\hline \multirow[t]{2}{*}{ Stayed shorter } & 1 & 1 & 3 & 5 & & & & & & & & & & & 1 & 1 \\
\hline & $0.5 \%$ & $0.4 \%$ & $1.1 \%$ & $0.7 \%$ & & & & & & & & & & & $2.7 \%$ & $1.2 \%$ \\
\hline \multirow{2}{*}{$\begin{array}{l}\text { Did not answer } \\
\text { this question }\end{array}$} & 64 & 59 & 130 & 253 & 1 & 11 & 17 & 29 & 12 & 4 & 29 & 45 & 5 & 5 & 14 & 24 \\
\hline & $30.5 \%$ & $26.5 \%$ & $49.6 \%$ & $36.4 \%$ & $25.0 \%$ & $23.9 \%$ & $25.0 \%$ & $24.6 \%$ & $28.6 \%$ & $12.1 \%$ & $48.3 \%$ & $33.3 \%$ & $16.1 \%$ & $38.5 \%$ & $37.8 \%$ & $29.6 \%$ \\
\hline Total & $100.0 \%$ & $100.0 \%$ & $100.0 \%$ & $100.0 \%$ & $100.0 \%$ & $100.0 \%$ & $100.0 \%$ & $100.0 \%$ & $100.0 \%$ & $100.0 \%$ & $100.0 \%$ & $100.0 \%$ & $100.0 \%$ & $100.0 \%$ & $100.0 \%$ & $100.0 \%$ \\
\hline
\end{tabular}

\section{Table 7.25 Reasons for change of plans in Picton.}

\begin{tabular}{|c|c|c|}
\hline & Circumstances beyond travellers' control & Circumstances within travellers' control \\
\hline $\begin{array}{l}\text { Stayed shorter } \\
\text { than planned }\end{array}$ & $\begin{array}{l}\text { - Time: "took longer than we anticipated driving up from Wanaka"; } \\
\text { - Ferry cancellation: "ferry cancellation and work commitments"; } \\
\text { - Bad weather: "bad weather so that we could not go kayaking". }\end{array}$ & $\begin{array}{l}\text { - Time: "end of holiday, wanted to get home and were able to change } \\
\text { ferry booking to today"; } \\
\text { - Preferred to go to other destination: "luckily we changed our mind } \\
\text { en route - Picton was boring"; "planned Queen Charlotte walk but } \\
\text { changed plans and went to North Island instead"; } \\
\text { - Other: "found a job". }\end{array}$ \\
\hline $\begin{array}{l}\text { Stayed longer } \\
\text { than planned }\end{array}$ & $\begin{array}{l}\text { - Ferry cancellation: this is the most cited motive for change of plans, with at least } \\
12 \text { passengers mentioning it; } \\
\text { - Transport arrangement: "had to stay } 4: 30 \text { hours because of time between bus } \\
\text { and ferry"; "transport from Christchurch to Picton too limited - I wanted to stay in } \\
\text { Kaikoura but there is no main transport for a morning crossing to Wellington"; } \\
\text { - Ferry booked: "had to wait } 7 \text { hours for first available seat"; } \\
\text { - Weather: "the weather was very rough and I easily get seasick". }\end{array}$ & $\begin{array}{l}\text { - Attraction: "allowed extra time to see Edwin Fox on } \\
\text { recommendation of relatives"; } \\
\text { - Good weather: "the weather was great"; } \\
\text { - Enjoyed Picton as a destination: "convenience to ferry and good } \\
\text { accommodation"; "quality of camping site, more activities than } \\
\text { initially thought". }\end{array}$ \\
\hline
\end{tabular}




\section{FERRY PASSENGERS IN WELLINGTON AND PICTON}

Most of the various reasons presented by passengers to stay longer in Picton (see Table 7.28) can be grouped into three main categories: factors beyond the supply control (e.g. more time available, better weather), the need for more infrastructure (such as a "must see" attraction, picture theatre and parking facilities) and product development (event/festival, cheaper accommodation and guided walks). Some passengers also mentioned other types of deals including accommodation and fishing activities or the Queen Charlotte track. Two passengers also stated that more information would make them stay longer. This shows that in fact they did not pay attention to the question being asked as they were expected to answer what, apart from more information and a special deal, would make them stay longer in Picton.

Infrastructure and product development are perhaps the two categories that local business and tourism organisations can do something to persuade travellers to stay longer in Picton. Apparently what those passengers were stating is that there is a general disappointment in terms of the lack of facilities and attractions (including temporary ones such as events and festivals) to capture the travellers' attention and time.

A summary of the data analysed here will be presented in the next section. This is useful to understand the key issues associated with the passengers' behaviour while in Picton and also what can be done to make them stay longer there. It will also help to compare data between Picton and Wellington, which will be done in Section 7.4.

\subsubsection{Summary of passengers' behaviour in Picton}

This section summarises the passengers' behaviour in Picton using a similar structure to the summary for Wellington.

Gateway travellers in Picton represent over two-thirds of the passengers' nodal function in Wellington. In terms of their place of origin there is a reasonably equal distribution among CSNZ (30.2\%), domestic (32.1\%) and international (37.7\%) passengers (Table 3.4 ). According to Table 7.19 the main reasons for GT not staying in Picton are the fact that they were "staying overnight somewhere else" (30.9\%) and "no need / passing through" (26.8\%). CSNZ and domestic passengers make past visits to Picton the main source of information for GT (30.5\%), with commercial guidebooks coming in second place with $19 \%$ (Table 7.22). Due to the short stay of GT in Picton, their participation in attractions and activities in the node is very small. According to Table 7.23, very few activities attract more than one out of ten passengers, such as in the case of walking in town $(19.7 \%)$, eating out $(14.5 \%)$ and general sightseeing (10.2\%). According to Table 7.24 , the actual change of plans in Picton is very small with five passengers $(0.7 \%)$ staying shorter and 
three passengers $(0.4 \%)$ deciding to stay longer. Even though only $5.3 \%$ of GT stated that they would have stayed longer in Picton if they had more information, which in fact is the smaller percentage, the total number of passengers doing so is the highest (37), according to Table 7.26. In fact, this number of passengers represents more than the other three nodal functions together (34). A special deal would have made $11.4 \%$ of GT to extend their stay in Picton, which represents a total of 79 passengers, once again more than all the other functions together. Due to the large sample of GT in Picton $(n=695)$ even a smaller participation of passengers increasing their stays in Picton can have a significant impact on the actual number of passengers staying longer.

According to Table 3.4, OGV are basically made up of domestic (39.0\%) and international (57.6\%), with very few CSNZ passengers (3.4\%). In the case of Picton, short stopovers $(0<\mathrm{Ti} \leq 10)$ represent $83.2 \%$ of its passengers (Table 7.20$)$. Apart from other types of accommodation used by OGT while in Picton (24.6\%), motels (35.6\%) and backpackers/youth hostels $(22.0 \%)$ are the two most preferred. The sources of information used to know about Picton are commercial guidebooks (43.2\%), past visits (27.1\%), free visitor guides and word of mouth, both accounting for $21.2 \%$ of passengers. In terms of the activities undertaken in Picton by OGV, the top three are walking in town $(56.8 \%)$, general sightseeing (33.9\%) and eating out (33.1\%). Tourist attractions have little appeal among this group of passengers, with the most visited one being the Picton Museum (12.7\%). According to Table 7.24, ten OGV passengers decided to stay longer in Picton $(8.5 \%)$, with nearly $12 \%$ of passengers staying longer if they had had more information about Picton (Table 7.26 ) and $18.6 \%$ doing the same if they had a special deal (Table 7.27). 
Table 7.26 Change of plans in Picton in case passenger had more information.

\begin{tabular}{|c|c|c|c|c|c|c|c|c|c|c|c|c|c|c|c|c|}
\hline \multirow{2}{*}{$\begin{array}{l}\text { Change of plans } \\
\text { in case } \\
\text { passenger had } \\
\text { more information }\end{array}$} & \multicolumn{4}{|c|}{ Gateway Travellers } & \multicolumn{4}{|c|}{ Overnight Gateway Visitors } & \multicolumn{4}{|c|}{ Stopover Visitors } & \multicolumn{4}{|c|}{ Destination Tourists } \\
\hline & $\begin{array}{c}\text { CSNZ } \\
(n=210)\end{array}$ & $\begin{array}{c}\text { Dom } \\
(n=223)\end{array}$ & $\begin{array}{c}\text { Int'l } \\
(n=262)\end{array}$ & $\begin{array}{c}\text { Total } \\
(n=695)\end{array}$ & $\begin{array}{l}\text { CSNZ } \\
(n=4)\end{array}$ & $\begin{array}{l}\text { Dom } \\
(n=46)\end{array}$ & $\begin{array}{c}\text { Int'l } \\
(n=68)\end{array}$ & $\begin{array}{c}\text { Total } \\
(n=118\end{array}$ & $\begin{array}{l}\text { CSNZ } \\
(n=42)\end{array}$ & $\begin{array}{l}\text { Dom } \\
(n=33)\end{array}$ & $\begin{array}{c}\text { Int'l } \\
(n=60)\end{array}$ & $\begin{array}{c}\text { Total } \\
(n=135)\end{array}$ & $\begin{array}{l}\text { CSNZ } \\
(n=31)\end{array}$ & $\begin{array}{c}\text { Dom } \\
(n=13)\end{array}$ & $\begin{array}{c}\text { Int'l } \\
(n=37)\end{array}$ & $\begin{array}{c}\text { Total } \\
(n=81)\end{array}$ \\
\hline \multirow{2}{*}{$\begin{array}{l}\text { No changes } \\
\text { made }\end{array}$} & 193 & 205 & 175 & 573 & 4 & 42 & 45 & 91 & 38 & 31 & 36 & 105 & 30 & 10 & 29 & 69 \\
\hline & $91.9 \%$ & $91.9 \%$ & $66.8 \%$ & $82.4 \%$ & $100 \%$ & $91.3 \%$ & $66.2 \%$ & $77.1 \%$ & $90.5 \%$ & $93.9 \%$ & $60.0 \%$ & $77.8 \%$ & $96.8 \%$ & $76.9 \%$ & $78.4 \%$ & $85.2 \%$ \\
\hline \multirow[t]{2}{*}{ Stay longer } & 4 & 9 & 24 & 37 & & 2 & 12 & 14 & 2 & 2 & 11 & 15 & & & 5 & 5 \\
\hline & $1.9 \%$ & $4.0 \%$ & $9.2 \%$ & $5.3 \%$ & & $4.3 \%$ & $17.6 \%$ & $11.9 \%$ & $4.8 \%$ & $6.1 \%$ & $18.3 \%$ & $11.1 \%$ & & & $13.5 \%$ & $6.2 \%$ \\
\hline \multirow[t]{2}{*}{ Stay shorter } & 2 & & 2 & 4 & & 2 & 2 & 4 & & & & & 1 & & 1 & 2 \\
\hline & $1.0 \%$ & & $0.8 \%$ & $0.6 \%$ & & $4.3 \%$ & $2.9 \%$ & $3.4 \%$ & & & & & $3.2 \%$ & & $2.7 \%$ & $2.5 \%$ \\
\hline \multirow[t]{2}{*}{ Did not answer } & 11 & 9 & 61 & 81 & & & 9 & 9 & 2 & & 13 & 15 & & 3 & 2 & 5 \\
\hline & $5.2 \%$ & $4.1 \%$ & $23.2 \%$ & $11.7 \%$ & & & $13.2 \%$ & $7.6 \%$ & $4.8 \%$ & & $21.7 \%$ & $11.1 \%$ & & $23.1 \%$ & $5.4 \%$ & $6.2 \%$ \\
\hline Total & $100 \%$ & $100.0 \%$ & $100.0 \%$ & $100.0 \%$ & $100.0 \%$ & $100.0 \%$ & $100.0 \%$ & $100.0 \%$ & $100.0 \%$ & $100.0 \%$ & $100.0 \%$ & $100.0 \%$ & $100.0 \%$ & $100.0 \%$ & $100.0 \%$ & $100.0 \%$ \\
\hline
\end{tabular}

\section{Table 7.27 Change of plans in Picton in case passenger was offered a special deal.}

\begin{tabular}{|c|c|c|c|c|c|c|c|c|c|c|c|c|c|c|c|c|}
\hline \multirow{2}{*}{$\begin{array}{l}\text { Change of plans in } \\
\text { case passenger } \\
\text { was offered a } \\
\text { special deal }\end{array}$} & \multicolumn{4}{|c|}{ Gateway Travellers } & \multicolumn{4}{|c|}{ Overnight Gateway Visitors } & \multicolumn{4}{|c|}{ Stopover Visitors } & \multicolumn{4}{|c|}{ Destination Tourists } \\
\hline & $\begin{array}{c}\text { CSNZ } \\
(n=210)\end{array}$ & $\begin{array}{c}\text { Dom } \\
(n=223)\end{array}$ & $\begin{array}{c}\text { Int'l } \\
(n=262)\end{array}$ & $\begin{array}{c}\text { Total } \\
(n=695\end{array}$ & $\begin{array}{c}\text { CSNZ } \\
(n=4)\end{array}$ & $\begin{array}{c}\text { Dom } \\
(n=46)\end{array}$ & $\begin{array}{c}\text { Int'l } \\
(\mathrm{n}=68)\end{array}$ & $\begin{array}{c}\text { Total } \\
(n=118)\end{array}$ & $\begin{array}{l}\text { CSNZ } \\
(n=42)\end{array}$ & $\begin{array}{l}\text { Dom } \\
(n=33)\end{array}$ & $\begin{array}{c}\text { Int'l } \\
(n=60)\end{array}$ & $\begin{array}{c}\text { Total } \\
(n=135\end{array}$ & $\begin{array}{l}\text { CSNZ } \\
(n=31)\end{array}$ & $\begin{array}{l}\text { Dom } \\
(n=13)\end{array}$ & $\begin{array}{c}\text { Int'l } \\
(n=37)\end{array}$ & $\begin{array}{l}\text { Total } \\
(n=81)\end{array}$ \\
\hline No changes made & $\begin{array}{r}168 \\
80.0 \%\end{array}$ & $\begin{array}{r}159 \\
71.3 \%\end{array}$ & $\begin{array}{r}154 \\
58.8 \%\end{array}$ & $\begin{array}{r}481 \\
69.2 \%\end{array}$ & $\begin{array}{r}4 \\
100 \%\end{array}$ & $\begin{array}{r}33 \\
71.7 \%\end{array}$ & $\begin{array}{r}40 \\
58.8 \%\end{array}$ & $\begin{array}{r}77 \\
65.3 \%\end{array}$ & $\begin{array}{r}34 \\
81 \%\end{array}$ & $\begin{array}{r}25 \\
75.8 \%\end{array}$ & $\begin{array}{r}30 \\
50.0 \%\end{array}$ & $\begin{array}{r}89 \\
65.9 \%\end{array}$ & $\begin{array}{r}29 \\
93.5 \%\end{array}$ & $\begin{array}{r}10 \\
76.9 \%\end{array}$ & $\begin{array}{r}30 \\
81.1 \%\end{array}$ & $\begin{array}{r}69 \\
85.2 \%\end{array}$ \\
\hline $\begin{array}{l}\text { Passenger would } \\
\text { stay longer }\end{array}$ & $\begin{array}{r}20 \\
9.5 \% \\
\end{array}$ & $\begin{array}{r}25 \\
11.2 \% \\
\end{array}$ & $\begin{array}{r}34 \\
13.0 \% \\
\end{array}$ & $\begin{array}{r}79 \\
11.4 \% \\
\end{array}$ & & $\begin{array}{r}7 \\
15.2 \% \\
\end{array}$ & $\begin{array}{r}15 \\
22.1 \% \\
\end{array}$ & $\begin{array}{r}22 \\
18.6 \% \\
\end{array}$ & $\begin{array}{r}8 \\
19.0 \% \\
\end{array}$ & $\begin{array}{r}7 \\
21.2 \% \\
\end{array}$ & $\begin{array}{r}17 \\
28.3 \% \\
\end{array}$ & $\begin{array}{r}32 \\
23.7 \% \\
\end{array}$ & $\begin{array}{r}1 \\
3.2 \% \\
\end{array}$ & $\begin{array}{r}1 \\
7.7 \% \\
\end{array}$ & $\begin{array}{r}4 \\
10.8 \% \\
\end{array}$ & $\begin{array}{r}6 \\
7.4 \% \\
\end{array}$ \\
\hline $\begin{array}{l}\text { Passenger would } \\
\text { stay shorter }\end{array}$ & $\begin{array}{r}1 \\
0.5 \% \\
\end{array}$ & & & $\begin{array}{r}1 \\
0.1 \%\end{array}$ & & $\begin{array}{r}2 \\
4.3 \% \\
\end{array}$ & $\begin{array}{r}1 \\
1.5 \%\end{array}$ & $\begin{array}{r}3 \\
2.5 \% \\
\end{array}$ & & & & & & & & \\
\hline Did not answer & $\begin{array}{r}21 \\
10.0 \% \\
\end{array}$ & $\begin{array}{r}39 \\
17.5 \% \\
\end{array}$ & $\begin{array}{r}74 \\
28.2 \% \\
\end{array}$ & $\begin{array}{r}134 \\
19.3 \% \\
\end{array}$ & & $\begin{array}{r}4 \\
8.7 \% \\
\end{array}$ & $\begin{array}{r}12 \\
17.6 \% \\
\end{array}$ & $\begin{array}{r}16 \\
13.6 \% \\
\end{array}$ & & $\begin{array}{r}1 \\
3.0 \%\end{array}$ & $\begin{array}{r}13 \\
21.7 \% \\
\end{array}$ & $\begin{array}{r}14 \\
10.4 \% \\
\end{array}$ & $\begin{array}{r}1 \\
3.3 \% \\
\end{array}$ & $\begin{array}{r}2 \\
15.4 \% \\
\end{array}$ & $\begin{array}{r}3 \\
8.1 \% \\
\end{array}$ & $\begin{array}{r}6 \\
7.4 \% \\
\end{array}$ \\
\hline Total & $100.0 \%$ & $100.0 \%$ & $100.0 \%$ & $100.0 \%$ & $100.0 \%$ & $100.0 \%$ & $100.0 \%$ & $100.0 \%$ & $100.0 \%$ & $100.0 \%$ & $100.0 \%$ & $100.0 \%$ & $100.0 \%$ & $100.0 \%$ & $100.0 \%$ & $100.0 \%$ \\
\hline
\end{tabular}


Table 7.28 What should be offered to make passengers stay longer than planned in Picton.

\begin{tabular}{|l|c||c|c|}
\hline \multicolumn{1}{|c|}{ What should be offered to passenger } & $\begin{array}{c}\text { Number of } \\
\text { Respondents }\end{array}$ & What should be offered to passenger \\
\hline More time & 23 & Walks (guided, scenic) & Respondents \\
\hline An event/festival & 13 & Parking facilities (better secure, cheap overnight parking) & 2 \\
\hline Accommodation (free, cheaper) & 12 & More information & 2 \\
\hline A "must see" attraction (include activities as well) & 4 & Ferries (cancellation, time of crossing) \\
\hline Weather (better) & 3 & Better ancillary services (restaurants, shops) & 2 \\
\hline Picture theatre & 3 & Whale watch & 2 \\
\hline Deal (including accommodation and fishing; Queen Charlotte Track) & 3 & Nature & 1 \\
\hline Work & 2 & Family/friends living there \\
\hline
\end{tabular}




\section{FERRY PASSENGERS IN WELLINGTON AND PICTON}

Stopover visitors have a reasonably well spread distribution in terms of passengers' place of origin, with $44.4 \%$ being international passengers, $31.1 \%$ of CSNZ and $24.4 \%$ of domestic passengers (Table 3.4). According to Table 7.17, the major reason for SV to go to Picton is leisure / holiday, with VFR coming second with $15.7 \%$. By definition, SV either do not stay overnight or stay one night. The former case represents $73.3 \%$ of the sample, with $26.7 \%$ staying one night (Table 7.18 ). Those SV who were not staying overnight $(n=99)$ gave three main reasons for doing so. They had no need - or the fact that the passenger was just passing by - $(18.2 \%)$; because they were staying overnight somewhere else near Picton (37.4\%); or no reason given. Those SV who stayed overnight in Picton $(n=36)$ used two main types of accommodation, i.e. "other" types of accommodation (three out of ten visitors), which includes camping and $B \& B$, and backpacker/hostels, accounting for one-quarter of the sample (Table 7.21). Most used sources of information to know more about Picton were past visits (28.9\%) and commercial guidebooks (24.4\%). As in the case of OGV, the three activities that SV undertake most are walking in town (41.5\%), eating out (32.6\%) and general sightseeing (20.7\%). According to Table 7.24, fifteen SV decided to stay longer in Picton (11.1\%), with nearly $24 \%$ of passengers staying longer if they had had more information about Picton (Table 7.26 ) and $23.7 \%$ doing the same if they had a special deal (Table 7.27). In both cases they were the highest responses among all four nodal functions.

Finally, in terms of the DT's place of origin, CSNZ (38.3\%) and international $(45.7 \%)$ passengers account for most of this nodal function, with domestic passengers $(16 \%)$ having the smallest participation among the four nodal functions (Table 3.4). Leisure / holiday (40.0\%), inter-island connection (33.8\%) and VFR (23.8\%) account for the three most important reasons for DT going to Picton. The higher participation of inter-island connection passengers staying at least two nights in Picton is mainly due to international passengers. Over a quarter of DT are made up of international passengers whose main reason for going to Picton is the inter-island connection (Table 7.17). Over half of DT stayed two nights in Picton, with a third of the sample consisting of international passengers staying two nights. Approximately a quarter of DT stay three nights (Table 7.18). Regarding the Trip Index, the two major types of passengers include the sole destination $(\mathrm{Ti}=100)$ with $39.5 \%$ and the short stopover $(0<\mathrm{Ti} \leq 10)$ with $30.3 \%$ (see Table 7.20). In the first case this behaviour is very much influenced by the fact that $34.2 \%$ of DT consist of CSNZ only staying in Picton during their trips. In the second case, international passengers $(28.9 \%)$ are responsible for the large number of short stopovers in Picton. Three major types of accommodation are used by destination tourists while staying in Picton (Table 7.21 ). Private homes are the most used one $(34.6 \%)$, which can be 


\section{FERRY PASSENGERS IN WELLINGTON AND PICTON}

associated with the longer stays as tourists do not need to pay for accommodation, especially CSNZ passengers staying in private homes (22.2\%). Other types of accommodation (Table 7.21) include "other" (19.8\%) and backpacker / youth hostel $(17.3 \%)$. DT is probably the nodal function that makes more use of a wide range of sources of information to know more about Picton. This can be associated with wanting to know all the attractions and activities available in Picton before arriving there. Past visits and commercial guidebooks are used by $37 \%$ of DT (Table 7.22 ), followed by VIC $(23.5 \%)$ and free visitor guides and word of mouth, each representing $21 \%$ of this nodal function. Destination tourists make far more use of several activities and attractions in Picton. According to Table 7.23 , nearly $30 \%$ of DT play mini-golf and visit Picton Museum, with one out of five DT walking the Queen Charlotte Track. In terms of the actual and potential change in the length of stay of DT in Picton, nearly one out of ten DT passengers ended up staying longer in Picton (Table 7.24). Destination tourists in Picton would be less influenced by more information in order to extend their stays there, when compared to the other functions (Table 7.26). This can be explained by the fact that they got plenty of information about Picton before arriving there. Only $6 \%$ of DT passengers said that they would have stayed longer. A special deal would also not have encouraged as many DT (7.4\%), being the nodal function less sensitive to a special deal (Table 7.27).

\subsection{Comparison between passengers' behaviour in Wellington and Picton}

So far, this chapter has presented and discussed separately the behaviour of Cook Strait passengers while in Wellington and Picton. The main advantage of this approach is to be able to understand what the individual characteristics of the various groups of passengers are in each of these nodes. This section, however, aims to identify the similarities and differences existing in both nodes for the various nodal functions. In most cases the comparison will be made using the passengers' place of origin. There are two main reasons for analysing the data this way. Firstly, place of origin is a characteristic intrinsic to the passenger, not the node, which can make the comparison more consistent. Secondly, this helps to provide different marketing strategies in terms of the geographical location of the passengers.

The differences between Wellington and Picton were firstly pointed out in this research in the literature review (Chapter 2), with regard to the particularities of urban destinations and small resorts. Later, in Chapter 4, the context of these two nodes was also described, 


\section{FERRY PASSENGERS IN WELLINGTON AND PICTON}

with the structure of the different nodal functions being identified and the scale and range of tourist attractions highlighting the differences existing between these two nodes.

The most evident difference existing between Wellington and Picton is the distribution of functions in both nodes. According to Figure 7.1, in Wellington, except in the case of the OGV function, there is a fairly equal distribution between the various functions (GT $32.5 \%$; OGV 13.7\%; SV 24.6\%; DT 29.1\%). Picton, on the other hand, is dominated by the gateway function (67.5\% of passengers), with few destination tourists $(7.9 \%)$. In part, this is a consequence of nearly $82 \%$ of the passengers having the inter-island connection as the main reason for going there. While the inter-island connection is also the most important reason for passengers going to Wellington (40.8\%), the capital city also offers other motives for people travelling there, notably leisure/holiday (21.6\%), especially for international passengers (see Table 7.5), and VFR (13.8\%). What is more, while Picton has a very strong transit role (Table 7.20), Wellington exhibits various Trip Index values. Particularly in the case of international passengers, there is a clear difference between their approaches toward Wellington and Picton. In the former case, for most of international passengers the capital city is seen as a necessary stopover during their touring trips around the country. While international passengers account for the majority of the short stopover visitors, seven out of ten international passengers are only passing through Picton.

Two other key features to influence passengers while in Wellington and Picton are the structure of the accommodation sector and the activities and attractions available at both nodes. In Picton, while nearly half of CSNZ passengers stay in private homes, domestic passengers tend to use more motels and other types of accommodation (e.g. camping and $\mathrm{B} \& \mathrm{~B})$. International passengers prefer a wide range of accommodations, including camping, B\&B, backpacker/youth hostels and motels. As discussed before, due to the current stage of development of Picton (Phase 1 of the Resort Development Spectrum see Table 2.2), it has fewer hotel facilities and more caravan parks, motels and backpacker hostels. In Wellington, most of passengers from New Zealand stay in private homes, with international passengers choosing primarily backpackers/youth hostels and hotels. The fact that CSNZ passengers tend to stay more in private homes in both nodes and that domestic passengers use the same type of non-commercial accommodation in Wellington, it may be less attractive to accommodation providers to spend money on promotion in order to persuade these passengers to stay longer. They may prefer to focus on international passengers as this subgroup has a higher proportion of travellers staying in commercial types of accommodation. 


\section{FERRY PASSENGERS IN WELLINGTON AND PICTON}

Regarding the activities undertaken and attractions visited by passengers while in Wellington and Picton, it became clear that the nature and the range of activities and attractions offered by these two nodes are associated with the major type of nodal function existing there. In Picton most of the activities and attractions available are located near the ferry terminal, most of them in easy walking distance. This emphasizes the fact that even the tourist attractions and activities in Picton are primarily available to serve the strong gateway function existing there, especially to attract passengers waiting for the ferry journey. For example, when comparing the amount of GT passengers eating out in Wellington and Picton, their numbers are higher in the latter case than in the former one. The same happens to walking in town/city, shopping and taking a scenic drive. Wellington, on the other hand, has a number of attractions that suit destination tourists more, including Te Papa, the Botanic Gardens, the Cable Car and the parliament. The proportion of DT, especially international ones, visiting these attractions is particularly high. One aspect to limit the participation of gateway travellers in many activities and attractions in Wellington is the location of the ferry terminal of the Interislander service, which is near the exit of $\mathrm{SH} 1$ and before the entrance to the Wellington CBD. As will be discussed in the next chapter, the location of the ferry terminal and the lack of regular public transport between the terminal and the CBD discourage gateway travellers to visit Wellington even if for only a few hours before taking the ferries.

Despite the differences that exist in terms of scale and stage of development of the destination function in Wellington and Picton, it is clear that there are some similarities in terms of how passengers behave while at both nodes. One of these similarities includes how passengers obtain information about Wellington and Picton. This can be expected as the way travelers obtain information is more related to their own behaviour rather than the node. In the case of passengers living in New Zealand, past visits and word of mouth were the most common used source of information for them to know more about these two places. Considering themselves familiar with both nodes, CSNZ and domestic passengers tend to rely more on their own experiences or on someone else's experiences, rather than making use of other sources of information, such as guide books, visitor guides, VIC and travel agents. This means that in order to persuade passengers to stay and visit both places it is important that Wellington and Picton businesses and organizations provide the best experience as possible, as potential visitors will rely particularly on their own previous experiences or their friends' and relatives' experiences to decide whether or not to visit these nodes. Even though there is a slight difference in terms of the number of international passengers looking for information in Wellington and Picton, with the capital city receiving more attention, in general terms overseas travelers 


\section{FERRY PASSENGERS IN WELLINGTON AND PICTON}

use the same sources of information for both nodes. In the case of international passengers, the most used source of information is commercial guide books with at least half of respondents making use of them to search for information about Wellington and Picton. Other very important sources of information include free visitor guides, word of mouth and VIC. These suggest, especially in the case of guidebooks and visitor guides, the need for tourism businesses and organizations to provide accurate information in these sources. Travel agents are one channel of information that deserve some attention in terms of increasing the amount of information been sent to travelers. This was one of the least used sources of information for passengers to find out more about Wellington and Picton, despite the importance of travel agents as a distribution channel for passengers booking the ferry journey.

Reasons for passengers not staying overnight in Wellington and Picton were also very similar. Staying overnight somewhere else near the gateways or the fact that the passenger did not have a particular need were the most commented by all groups of passengers. Some particular groups also gave similar reasons for not staying overnight, such as the cases of GT stating that they live near Wellington / Picton or international passengers that pointed out time constraints as one argument for not staying overnight in both nodes.

Another similar feature in both places is the fact that very few travelers actually change their plans. This shows that passengers are usually not flexible in terms of extending their stay while in the node. It is probably necessary to persuade them to do it in advance. In addition, the reasons given for changing plans in both nodes were also very similar. Passengers ended up staying shorter in both ferry ports due to lack of time, usually associated with road travel, unforeseen situations, ferry cancellation or the decision to go to another destination. Arguments used to extend their stays included ferry cancellation, transport arrangements and the fact that the passenger enjoyed the node as a destination.

One last similar characteristic of passengers in both places is the impact of information and a special deal on their decision to stay longer in Wellington and Picton. Even though more passengers stated that they would have stayed longer in Wellington than Picton, there is a fairly similar reaction to how information and a special deal would influence passengers. In comparison to information, a special deal would definitively be more effective in making passengers stay longer. This would be particularly true in the case of CSNZ and domestic passengers who, as mentioned earlier, do not value information so much. GT and SV in particular would take more advantage of a special deal; most 


\section{FERRY PASSENGERS IN WELLINGTON AND PICTON}

probably transferring to Wellington or Picton for the night where they were staying somewhere else near it. To international passengers a special deal would still make them stay longer in comparison to more information. However, neither in Wellington nor Picton would the impact of a deal be as high as information. International passengers do value the importance of information and they believe information can be as valuable as a deal.

Similar issues for what would increase the passengers' length of stay were obtained for Wellington and Picton. Most of them are related to having more time, a "must see" attraction, better weather or topics related to accommodation. These factors show what the basic needs of passengers are in order to extend their stays. While some of them are beyond tourism businesses' and organizations' control (e.g. more time and better weather), others are directly related to tourism, such as accommodation and attractions, and they should be examined further.

\subsection{Conclusions}

After analysing and interpreting data presented in this chapter, it is possible to make some general conclusions regarding the passengers' behaviour while at the gateways as well as about their actual and potential decisions to extend their stays in Wellington and Picton. Before doing this, it is worth mentioning that the analytical framework chosen in this research achieved its aims in order to identify similarities and differences for the four groups of nodal functions in Wellington and Picton. It was possible to understand that in some situations the context of the nodes shaped the behaviour of the passengers while in other cases the characteristics of the various groups of passengers were similar both in Wellington and Picton.

One of the main conclusions from this chapter is that passengers do not necessarily want to take advantage of the fact that they are passing through a node to stop and visit it. Even if some type of incentive is offered, such as a special deal including accommodation and transport, the percentage of passengers not inclined to stay longer than planned is still significant. In the case of Wellington, depending on the sub-group, passengers not changing their plans can range from nearly $56 \%$ to almost $85 \%$ (Table 7.15 ). In Picton these values vary from $55 \%$ to nearly $94 \%$, depending on the sub-group of passenger (Table 7.26). On the other hand, some specific sub-groups, notably international travellers, are more sensitive to some forms of incentive, such as information and deals. In general, passengers are more likely to stay longer in the node if they were offered a deal than information. This is particularly evident in the case of passengers living in New Zealand. 


\section{FERRY PASSENGERS IN WELLINGTON AND PICTON}

Differences were identified between Wellington and Picton, even though in general international passengers are the group of passengers more inclined to stay longer than planned and that participate in a higher number of activities and attractions. What is more, visitors from overseas tend to stay more in commercial accommodation than those living in New Zealand. In Wellington, it was possible to identify a clear pattern linking nodal function (GT, OGV, SV and DT) and place of origin (CSNZ, domestic and international) with the likelihood of passengers staying longer and the degree of interest in tourist activities, attractions and accommodation. The longer the passengers are staying $(\mathrm{DT}>\mathrm{SV}>\mathrm{OGV}>\mathrm{GT}$ ) and the further they come from (international $>$ domestic $>\mathrm{CSNZ}$ ) the higher the chances of passengers extending their stays in the gateways, especially when more information or a special deal is offered. In fact, these results just confirm what was predicted in Chapter 6, where international passengers were presented as having many characteristics that would make them more available and keener to stay longer in the gateways. Among the key variables to influence them are the way they travel with few firm bookings (including the ferry booking that is usually done only a few days in advance), the number of nights spent in their trips and the fact that they stay in different regions around the country.

Two basic characteristics may be associated with the difficulty to attract passing through traffic to stop and visit gateways. One is related to passengers living near the nodes who are not interested in visiting them anyway. They are familiar with the nodes and are only concerned about going to their final destination or returning home. The second is linked to the travel pattern of most tourists in New Zealand, particularly international ones. As conceptualised in Chapter 2 and contextualised in Chapter 4, tourists tend to have a circuit pattern while visiting New Zealand, making them stay only a few days in each destination. This means that persuading passengers to extend their stay in one particular node may only occur at the expense of them staying shorter or not visiting other destinations. With major and well-known destinations clustered near the major international gateways in the North (e.g. Auckland and Rotorua) and South (Christchurch and Queenstown) Islands, being both quite apart from each other, the CSNZ region does not have the appeal to capture the travellers attention for such a long period of time, especially when this implies missing other attractive destinations.

Another important conclusion from this chapter is that while passengers may display some very similar behaviour either in Wellington or in Picton, such as information use, reasons for not staying overnight there and the likelihood to stay longer in case more information and a special deal had been offered, in other situations the stage of development of the node can have a significant impact on them. The latter case includes the type of 


\section{FERRY PASSENGERS IN WELLINGTON AND PICTON}

accommodation used, the attractions visited and activities undertaken and the main reason for going to Wellington and Picton. Wellington has a stronger and more developed destination function; while in Picton the activities and the whole tourism sector tend to gravitate around the ferry terminal and the gateway function. In fact, this is what the description of the tourism functions presented in Chapter 4 has highlighted. It seems than that the more developed the destination function the higher the chances to attract passing travellers to extend their stays. Picton presents very few tourist services and infrastructures in order to create a reasonable impression on travellers so they can consider worth spending more time there.

This chapter has dealt with the demand perspective on how to convince passengers to stay longer in Wellington and Picton. While the ultimate decision to do so depends entirely on the travellers, providers can also be responsible to facilitate, encourage and promote ways to attract passengers to extend their stays in both nodes. The supply-side point of view will be discussed in the next chapter. It is particularly important to examine the relationship between the ferry companies with the tourism sector in both ferry ports. It is especially relevant to discuss how the suppliers imagine that these deals could be set up and how better ways to better promote and disseminate information about Wellington and Picton might be found. 


\section{Gateway and Destination Issues: the Suppliers' Perspective}

\subsection{Introduction}

The analysis of the nodal functions undertaken in this research brings together information from different sources and players. So far, this thesis has discussed some of the concepts and models presented in the literature (Chapter 2), it has described tourism (Chapter 4) and transport (Chapter 5 ) in the study area and data collected from the ferry passengers (Chapters 6 and 7). While data obtained from the ferry passengers are crucial to understand how to improve the destination function of gateways, mainly because the ultimate decision to stop and visit a gateway is made by the passengers, there are several benefits in considering the suppliers' perspective as well.

Firstly, due to the day-to-day contact the suppliers have with the visitors they can provide extra information about the visitors' behaviour. This is particularly crucial to cover areas where the questionnaire presents some limitations, such as in understanding the multiple stops and visits some travellers make in Wellington and Picton. The suppliers' experience and knowledge about the study area can contribute to this research with a general understanding of the impacts and implications that the tourist flow has on the analysis of the nodes, particularly considering that collecting this type of information from the questionnaires (Question 24) did not prove to be successful.

Secondly, the contribution of the suppliers allows an understanding of the current stage of tourism development existing in Wellington and Picton using another source of information apart from the literature. Chapter 4 discussed the changes that have occurred in Wellington and Picton over the last few years, and suppliers can comment on this development, particularly considering the elements of the attractiveness of a destination; i.e. attractions, amenities, accessibility, image and price (Cho, 2000). It may be the case that the gateway nodes lack one or more of these elements in order to persuade ferry passengers to stay longer and the suppliers are able to identify these gaps. What is more, as both gateways are particularly sensitive to the ferry companies' management and policies, it is also relevant to consider the existing relationship that tourism providers in Wellington and Picton have with the ferry operators. One of the aspects considered by the demand in order to stay longer either in Wellington and Picton was the existence of a special deal involving transport and accommodation. These packages can only be set up if transport and tourism providers are able to work together. Additionally, increasing 
competition with other destinations, particularly those located in the ferry's catchment areas, makes it vital to understand which major issues need to be tackled in order to improve their destination functions, such as, product development and promotion strategies (Bonnett, 1982).

Thirdly, analysing the suppliers' point of view creates an opportunity to assess whether or not the perception they have of the demand matches the information collected in the questionnaires, particularly on the topics related to how to encourage ferry passengers to stop, visit and stay longer in Wellington and Picton. This can be done by comparing the statements from the suppliers with the data obtained from the questionnaires in previous chapters.

This chapter, therefore, presents the main findings obtained from the semi-structured interviews conducted with key tourism and transport stakeholders. Some of the topics discussed during the interviews were identified in the literature review (Chapter 2) and in the secondary data collection process (Chapters 4 and 5). They were included among the questions posed to the interviewees, while other topics were raised during the course of the interviews. The interviews included conceptual and contextual aspects of the research theme and most of the conversation was about how to convert gateway travellers into destination tourists. In some instances, such as discussion about ferry cancellation and speed restriction, the emphasis was on how to overcome some of the problems that impede the development of the destination function. Interviewees were also asked to comment on the impacts a possible terminal in Clifford Bay would have on tourism in Picton and Wellington.

Data collected from the suppliers are used in two different ways. In the first place, business managers and owners suggested ways to attract passengers to stay longer in the gateways. Most commonly, they focused on attractions and activities that need to exist in Wellington and Picton to capture the passing travellers' attention. Secondly, the suppliers tried to identify ways to implement the suggestions made by passengers, particularly in terms of providing more information and special deals in order to stimulate passengers to stay longer in these two places. It is particularly interesting to compare these issues in terms of Wellington and Picton. As the former is more developed as a tourist destination (see Chapter 4), with the latter having a strong gateway function (see Table 3.4), it is appropriate to consider how this can impact on the suppliers' perspectives in order to make passengers stay longer in the gateways.

Before discussing these points, it is appropriate to comment briefly on how the suppliers perceive the importance of the ferries to their own businesses and to Wellington and 
Picton as a whole. This was the first question raised during the interviews. The degree of relevance that they identified reflects the magnitude of the topics discussed by them during the interviews. In addition to the significance of the ferries, the respondents were also asked to comment on the types of benefits that the ferries bring to both places. All twenty of the interviewees (see Table 3.1) mentioned that the ferries are important to Wellington and Picton. Despite the differences existing between these two places, respondents agreed on the value of having the ferries there. This emphasizes not only the significance of the research topic, but also confirms that the selection of respondents was appropriate, as the interviewees considered this subject important to them.

Many interviewees regarded the ferries as being vital to Wellington and Picton. It is in Picton, however, that the ferries were considered even more important, particularly due to the size of the town and the way its economy is primarily focused on the gateway role. For example, one rental car manager from Picton estimated that $90-95 \%$ of his business comes from ferry passengers. The question about the importance of the ferries to Picton was, on some occasions, discussed by the interviewees together with other issues such as seasonality and the possibility of the ferries going to Clifford Bay. The manager of a tourist attraction in Picton said that "if they [the ferries] weren't here it would really hurt". Another manager stated that the ferry service was vital to the development of Picton, representing the vast majority of the people coming to Picton during ten to eleven months of the year. The only exception would be during mid-summer when travellers from the South Island holiday in the Sounds. All these topics will be discussed in more detail during this chapter, including the possible impacts of the ferries moving to Clifford Bay.

This chapter is divided into three main sections. The first describes how the suppliers consider the current situation of the gateway and destination functions in Wellington and Picton. It also analyses future trends that need to be addressed in order to develop the destination function in these two nodes. Topics such as possible product development to attract ferry passengers, packaging and information distribution and promotion will be considered. In this section the discussion will be done separately for Wellington and Picton. The second main section presents the main limitations and constraints in developing the destination function in the two gateways. Some of the themes discussed include the travel flow pattern, lack of funding and the relationship between the tourism operators and the ferry companies. Due to the higher number of interviewees from Picton, the importance of the ferries to this node and the fact that some of the issues raised are specifically related to Picton (e.g. the speed restriction and Clifford Bay proposal), most of the emphasis will be on this particular node. Finally, the third section discusses the Clifford Bay proposal and what would happen if a second gateway were developed in the 
South Island. This section brings together the analysis from the demand and the suppliers in order to discuss what would be the impacts on Picton if this becomes a reality. The reason for including data from the passengers' questionnaire in a chapter on the supplier's perspective is due to the fact that the Clifford Bay proposal is a very specific topic and having this information presented either in Chapter 6 or 7 would not be as effective there as having it together with the opinions collected from the semi-structured interviews. The conclusion will draw together all these topics, comparing and contrasting the differences existing between Wellington and Picton.

\subsection{Current situation and future trends for developing the destination function}

This section presents and discusses the current transport and tourism situation in both nodes predominantly from the suppliers' perspective. It also takes into consideration the ideas and suggestions that the suppliers believe are needed in order to develop the destination function in Wellington and Picton. The discussion focuses on the two major topics suggested in the passengers' questionnaires to make them stay longer in the nodes; i.e. more information and a special deal. Therefore, product development, information and packaging are the key themes covered here.

As earlier developed in this thesis, this section will deal separately with issues related to Wellington and Picton in order to consider what pertains to each individual location in particular. However, in some cases the discussion and proposals debated go beyond the local level, including the regions where the nodes are located (e.g. in the case of Picton the Marlborough Region) or the whole CSNZ region. Within each sub-section, one for Wellington the other for Picton, the issues referring to the products and the promotion will be analysed separately. Firstly the products and packages will be considered, followed by how to promote them and the destinations to ferry passengers. However, as these two issues are very much related to each other, in some parts they will be considered together.

Finally, in the cases of the proposed packages combining ferry journeys with accommodation and/or attractions, it is worth commenting that so far only The Interisland Line has offered this type of product, with the 'Escape' packages being associated with The Lynx and the vineyards in the Marlborough region. At the time of data collection, these packages were not in operation, even though the ferry operator later re-introduced them. These packages continued to be offered with the conventional ferries when The Lynx service ceased to exist in 2005 . Bluebridge, so far, has not set up anything similar 
even though, according to its marketing manager, it is "absolutely in the plan to have the range of sort of packages on offer that would include accommodation and make it really easy for people to choose to come to Wellington or to go to Picton or Marlborough for a break".

\subsubsection{Wellington}

As described in Chapter 4, Wellington has changed significantly in the last few years and has developed an image of a vibrant and cultural city. While all the suppliers interviewed in Wellington share this impression, in some cases the results of these changes to their own businesses can be diametrically opposed. The reason for this is that apart from the overall changes at the destination level, businesses operating within the same market can be differently prepared to benefit from these changes. This became clear when considering the following statements of two hostel managers:

I have been managing the hostel for nine years and the average stay has gone up a bit and the average length of stay used to be one night, but I think as travellers start to know about Wellington, they know that is better to stay more than one night. [General Manager of the YHA in Wellington]

I think stays are getting shorter, compared to 10 years ago. There weren't so many tourists coming, but those who were coming tended to stay longer. That is the impression I got, anyway. Whereas now, everybody is here [in New Zealand] for three weeks or one month and they have cramped everything in and Wellington doesn't rate high with all the other stuff in the rest of the country. [General Manager of Downtown Backpackers]

On the other hand, the interviewees were unanimous in affirming that the driving force behind the development of Wellington as a destination was the establishment of Te Papa. In fact, the suppliers' perception is very much supported by data presented in Chapter 7 , with Te Papa being the most visited attraction by ferry passengers (see Table 7.11). Together with Te Papa, many other events, attractions and facilities are sustaining the new image Wellington is promoting, as commented by two interviewees:

Wellington city itself is really changing its focus in the last five years, from being seen as a city for bureaucrats, recreated city as a vibrant place to be. Courtenay Place is now an attraction, development of the waterfront, undoubtedly combined with Te Papa are reasons to stay. We are getting around about half a million international visitors in Te Papa a year. We are having incredible growth in our visitor-nights stopover and both indicate that most people that come to Wellington that stay are visiting Te Papa. [Manager of a tourist attraction in Wellington]

We are really getting out there. Wellington is becoming probably more the place to be because we are putting stuff on the map. With large events, such as the international rugby Sevens, the Lord of the Rings has just done wonderful for Wellington, we are 
hosting the world premiere in a few weeks time, which is quite scary; this has done a lot for Wellington. [Manager ${ }^{14}$ of a tourism organisation in Wellington]

Other interviewees also commented about parliament, the café culture and the nightlife, which is supporting all the major attractions. Some suppliers are also confident that the Karori Wildlife Sanctuary will become another major attraction in the city once the whole project has been completed in the next few years. Combining these activities with the opportunity to enjoy "a luxury or any reasonable accommodation, a wealth of bars, restaurants, cafes, entertainments, movies, opera or the theatre" (manager of a tourist attraction) give Wellington a privileged position to attract a wide range of visitors.

In spite of the recent growth in the number and scale of the attractions existing in Wellington, some interviewees considered that the capital city still faces two major challenges. The first is that apart from Te Papa there are no other major attractions. Most of those that exist in Wellington (as described in Chapter 4) are of medium or small size. The second problem is associated with the fact that most of the tourist attractions in Wellington are free. While to some interviewees this is an advantage that encourages people to visit Wellington, to others it represents a significant disadvantage as the lack of commissionable products do not make travel agents and tour operators promote Wellington as much as possible. They would prefer to broker Rotorua, Waitomo Caves or Queenstown because they can earn extra revenue from the commission they will receive for the bookings they make in these destinations.

Another fact that leads to ferry passengers not visiting the capital city is the location of the Interislander terminal, outside the CBD. Some interviewees said that for travellers driving south on the $\mathrm{SH}-1$, the ferry terminal is located before entering into the city. This makes a lot of travellers go straight to the ferry terminal. As explained by the manager of a rental car company:

It is pretty easy to get to the ferry terminal when you are heading to Wellington. If you are heading down that highway and you see the indication of the ferry, even if you have a couple of hours to spend, the temptation is to go straight to the ferry terminal. When you get there you realise there is nothing there. Getting them to go into town is a bit of a barrier. There are a lot of things to do in a walking distance in Wellington.

Even though The Interisland Line provides a free shuttle from the railway station to the ferry terminal thirty minutes before the ferry departure, there is no regular public transport to the Interislander ferry terminal. Travellers arriving earlier at the ferry terminal, either to drop off their rental cars or to check-in, are not usually encouraged to visit the main

\footnotetext{
${ }^{14}$ Consent was not given to attribute information to herself and her organisation.
} 
attractions in the CDB. In addition, the area around the Interislander terminal is not very pleasant, being located under an overpass and surrounded by rail tracks and highways.

What can be concluded from these comments is that while the interviewees believe that Wellington has transformed itself in the last few years, especially with the urban development that took place in the waterfront with the building of Te Papa, it relies too much on public funds to establish its main attractions, particularly those that are free of charge. This makes it more difficult for wholesalers to promote Wellington as a destination as they do not receive commission for sending tourists there.

In terms of marketing and promotion, two main aspects were raised during the interviews. The first is the impression that some tourists have of Wellington as it being a boring capital city "like Canberra", as one interviewee mentioned. The marketing manager of a tourist attraction in Wellington commented:

There are people who have been in Wellington six or seven years ago and still think that we are a grey, boring, city of bureaucrats [...]. We have to get these people back to Wellington again so they can know what is happening here, because it is a really exciting, beautiful, vibrant city.

The second is that Wellington is not part of the 'golden route', undertaken primarily by international visitors, which consists essentially of Auckland, Rotorua, Christchurch and Queenstown. In the words of one interviewee, "the biggest challenge is changing people's frequent ideas of what we have to offer". Basically the suppliers identified two major types of international tourists who travel in New Zealand. Those on a short stay trip around the country, probably doing the 'golden route'; and those with more time and flexibility. The second group has also been defined by Tourism New Zealand as the 'interactive traveller'. One of the interviewees explained this term as follows:

People who want to travel a little bit slower and have a more in-depth experience. Then the Auckland-Rotorua-Christchurch-Queenstown flying out again is not that attractive. They are looking for a more real experience with education, tasting flavours, learning about where the travel and tourism are important than quick sightseeing. With more of this type of traveller coming to New Zealand the more they will be happy to spend longer in areas and hopefully Wellington will have a share of that. I believe we have the products to attract those customers.

Interviewees from Wellington commented that the 'interactive traveller' is more sensitive to the promotion and marketing campaigns they have set up in the past. Persuading the first type of traveller is more expensive and not always successful, as commented by the manager of the Centre Stage marketing organisation: 
They have had their whole itinerary sewn up for the next country so they know they have got to be in Rotorua by this day and they have got to be you know, there's only so much that we can do when they get off the ferries to influence them, do you know what I mean? They can't, they know that they've got to fly to Auckland on this date or whatever, so we can put brochures out and we can put posters out on the ferries and do all sorts of things [...] That is why we try to hit them when they are deciding to come to New Zealand through the guidebooks, through the wholesalers and try to reach these markets through that.

It seems, however, that the type of international traveller who uses the ferry resembles more the 'interactive traveller' as the average length of stay of the international market using the ferries is longer (35.9 nights - see Table 6.6), than that of all international travellers who visit New Zealand (26 days, according to Table 4.1). What is more, as shown on Table 4.1 and Figure 4.2, the international markets that are more likely to use the ferries - notably Germany and UK - are those that present a higher degree of mobility and dispersal to remote regions while travelling in the country. It seems then the promotion and marketing campaign targeting the 'interactive traveller' may be favourable in terms of increasing the participation of those travellers using the ferries. The question is how to create mechanisms to induce them to stay in both nodes. The suppliers did not answer this. However, the above-mentioned quote identifies two key sources of information that can be appropriate to target international travellers. They confirm the earlier discussion about both guidebooks and travel agents being relevant distribution channels or sources of information for ferry passengers. Travel agents were the most commonly used channel of distribution for international passengers booking the ferries (nearly $30 \%$ according to Table 6.12), with guidebooks being the most used source of information for passengers to know more about Wellington (Table 7.10). Considering the results obtained in Chapter 7 about international passengers being sensitive to more information and a special deal in order to stay longer in Wellington, and the comments from the manager of the Centre Stage marketing organisation, guidebooks and travel agents should be then used as principal channels to promote these two nodes more and also to advertise the deals which the suppliers might decide to create.

\subsubsection{Picton}

Picton faces similar problems to Wellington, even though they are generally of a different magnitude. Apart from competing with the major national destinations (e.g. Auckland, Rotorua, Wellington, Christchurch and Queenstown), Picton also struggles with the destinations and attractions located in the Marlborough and Nelson regions, such as the vineyards in Blenheim, the whale watching in Kaikoura, and Abel Tasman National Park in Nelson. Some of the interviewees reported that a lot of their clients comment that they did 
not know how pretty Picton was before they arrived there. One of them explained this matter in the following way:

By the time they [the passengers] are booking the ferry ticket it is too late [to attract them to stay in Picton]. They have already planned where they want to go. And they are saying we are catching the ferry, we have heard about Abel Tasman National Park, so we are going there, or we have heard about whale watching in Kaikoura, so we are going straight away there. [...] Some tourists have asked in the visitor information centre in Auckland what they have to do in Marlborough; they said there is nothing to do unless you want to go to a vineyard [...] The other thing is that we have so many visitors that only have ten days [in New Zealand]. And they have to include Queenstown, and they have to include the whale watch, Abel Tasman National Park, Rotorua, and Auckland. There is not enough time.

In terms of the range of attractions that Picton provides, these are very much concentrated on the outdoor and marine type of activities, which are particularly appealing during summer time. With the exception of the Aquarium and the Picton Museum (see Chapter 4), which are both very small attractions, all the remaining tourist activities in Picton are only suitable during fine weather. In the words of the manager from the Department of Conservation:

There are problems with Picton and I have seen this for a number of years. There are very few things to do in Picton if it is raining because Picton is so outdoor orientated, not everyone wants to walk in a mudded area [...] The other thing is that years ago Picton used to have its own picture theatre, had a lot of services that we don't see now because of the way people now travel straight through. Places that pick up the biggest slice of the pie in Picton are really the motel and the hotel owners and the food suppliers and the restaurants and petrol stations to a certain extent.

In-door family type of activities, such as a fun park, were considered during two interviews. This sort of attraction would probably fit well with the markets that Picton already has. The key argument was that "there are a lot of families that come here because it is affordable for families and there is not enough for their kids to do. If you had an indoor attraction with water you would make a goldmine. I would love to do it." (Tour operator in Picton). This idea could appeal not only to gateway travellers, but also to the domestic market, especially the family type of tourists from the South Island that visit Picton during summer. In addition, an indoor aquatic centre would also suit local residents. As the owner of a hostel explains, "our kids need somewhere to swim rather than going 30 kilometres into Blenheim and our high school needs a better pool, that sort of thing [...] There are four main holiday parks [in Picton]. Those kids will be in the pool all the time". Others also suggested a bungee jump, movie theatre, a cable car or gondola (suggested by three different interviewees) and more events (particularly conventions and conferences). In the case of the activities that are related to adventure tourism, it seems 
that the interviewees were just trying to benchmark the success of other destinations in the country, rather than really proposing attractions that would fit with the needs, the themes and the markets already existing in Picton. In addition, a general comment was that at night there is nowhere to go apart from pubs and restaurants. Except for the movie theatre, no other activity or attraction proposed by the interviewees would address this issue.

Most interviewees commented that the recent development of Picton is a reaction to the proposal to build another ferry terminal in Clifford Bay. Destination Marlborough has been promoting Picton more and the council has also undertaken major improvements, particularly on the port shore. Private investment has also been made, especially with old hotels being replaced with more modern ones. The challenge, however, from the town perspective, is to get buildings which are in a similar shape or design or colour which tries to unify the town rather than split it apart. As suggested by the manager of the Department of Conservation for the Sounds area, town planning is also important in order to attract visitors:

Tourists that have no real set route when they arrive in the South Island they will turn around and have a look and say "this place looks interesting and I will stay here" [...] It is a whole thing about attitude and town planning and landscape and seascape and a whole lot of things that are rolled into one.

In spite of the recent investments and improvements in the town, the suppliers believe that the regional council can do more in Picton. They think the Marlborough Regional Council has never invested in Picton as much as it should. One statement from a hostel owner in Picton explains the situation:

Picton has always been the poor relation to Blenheim. This is the gateway to the Sounds; this is the gateway to the South Island. I think that we need to have more investment by the people of this region into Picton rather than into Blenheim. But Blenheim is the centre of power. That is where the population is; it is where the council is. We have tried to break these barriers down, through the chamber of commerce, but it is still hard.

In fact, what they suggest is that the tourism products in the Marlborough region could be integrated with the phrase "wilderness, water and wine" ( $3 \mathrm{Ws).} \mathrm{While} \mathrm{Blenheim} \mathrm{and} \mathrm{the}$ rest of the Marlborough region could provide the wine products, Picton could offer the water activities and the Marlborough Sounds provides the wilderness, especially with the Queen Charlotte track and the Lidia Track, or even the local walks in Picton. As suggested by some of the interviews, the 'quiet wilderness experience' could be promoted: 
Tourists can see nature that is not plastic, that is very uncrowded, like we have say 30,000 people a year walking Queen Charlotte track as oppose to 200,000 on the Abel Tasman [...] And people don't see millions of people walking in all directions, so they are getting a very, very quiet wilderness experience, with birds and occasional companions, but not hordes. With the water, they can be out on a boat, they can sea kayak, more and more sea kayaking is happening here, they can see dolphins, they can even hire a horse on the water, one of the horse track places around the Sounds, and they can swim with horses and dolphins. And the wine, of course, wine goes together with food, with the best wineries in the country here, they are friendly to our market. Our people come here and they want to know some of the wines they see in the UK, they want to know more about Cloudy Bay, Montana or something like that. [Hostel owner in Picton].

In effect, most of the existing packages that combine the ferries with other tourist products in the South Island are already associated with the $3 \mathrm{Ws}$ marketing approach suggested by the interviewees. These packages were particularly popular when there was no speed restriction to the fast ferries with travellers from the North Island doing, in some cases, a day trip to the vineyards in the Marlborough regions. Most of them were designed and marketed by The Interisland Line and operated by tour operators located in Picton. Later, The Interisland Line also incorporated other types of activities, such as kayaking, mountain biking and hiking on its packages. These packages appeal predominantly to travellers living in Wellington and aim to attract particularly the short break market. So far, no other market has been targeted, such as international travellers, which seem to be the most sensitive market to stay longer in Picton. Therefore, the current packages use the ferry to provide access to some destinations and products, while the proposed type of deal suggested to the passengers would be a way to increase the stay of those travellers already using the ferries.

In spite of the current packages already promoting the key products and activities in the Marlborough region (wilderness, water and wine), the passengers stating that they would stay longer if a deal including transport and accommodation was offered (Table 7.27) and the arguments considered above, in general the suppliers do not believe the packages provided by the ferries are necessarily an alternative. As one hostel owner in Picton commented:

I don't necessarily see that they [The Interisland Line] have to provide a package, but they have to make it user friendly so there are transport links there. The information centres, the accommodation places they can do packages as long as people have access to the ferry ticket at a reasonable price. For our sector of the market we were never involved in those packages anyway.

The problem Marlborough and Picton face is that if the ferries are not involved in these packages, which businesses or organisations in the region would be able to afford the 
promotion and the marketing necessary to attract potential customers? The ferries, particularly The Interisland Line, already have the distribution channels to make these products available, including the overseas markets. In addition, attracting the existing ferry demand who already mentioned that would be keen to stay longer if they were offered a special deal seems to be easier than trying to promote Picton to new markets. In this case, no other supplier is more relevant to be involved in these packages than the ferry providers, as they are the ones who already have a market to whom these packages could be offered. However, the view of one ferry manager is that the packages are only just something extra that the ferries provide. He stated that this is not the ferries' main role. He also said that they "provide a large number of people into that region [Marlborough]; it is over to the region to then decide how they best persuade those people to stay". In other words, he considers that the main job of the ferries is to transport people and that it is up to the region to develop its own strategies to capture this demand. In this regard, it seems that tourism and transport managers do not believe that integration between the two sectors is crucial, as they have clearly different roles.

The other problem with the current packages is that many tourism businesses in Picton complain about the strong emphasis on marketing the wine product in the region, as exemplified by the manager of a tourism operator in Picton:

They are focusing on the wines that in fact market themselves. They don't market the fishing, because it is considered an old-fashioned activity and the wine is marketed heavily. It doesn't benefit us much because it markets Blenheim, not Picton. I would look at the historical, cultural and natural attributes of the area. But they are not really promoted. So therefore people don't show an interest in it. Other areas in New Zealand are more heavily promoted historically and culturally than Marlborough. The wine is dominating everything.

The problem with Picton, however, is that there is no major commercial tourism product to be marketed, which makes the wine tourism in Blenheim an unrivalled competitor. As a result, the only option for some tour operators in Picton is to provide transport services between Picton and the vineyards, even though many travellers are not aware of this possibility, as Picton is not promoted as part of the wine tourism.

When asked to discuss whether Picton needs more products or more marketing efforts, some interviewees commented that promoting what Picton already has is more important as most travellers are simply not aware of the attractions and activities existing there. It was very common for the interviewees to give examples of tourists that have told them how surprised they were with the beauties of the region and the fact they were not aware of it. However, promoting Picton in the media can be an expensive way to send 
information to potential travellers, especially considering the lack of resources in such a small economy. Another option would be to use travel agents to disseminate information to passengers when they book or buy their ferry journey. As seen in Table 7.22, travel agents were, among passengers living in New Zealand, the least used source of information to know more about Picton. In the case of international passengers they were the second least used. The international sales manager of Centre Stage commented on the importance of the internet and wholesalers: "it's the website, what's the information on the website about, it's what the wholesalers are saying about the destination, it's how educated the travel agent is in talking to the consumers".

\subsection{Major constraints and limitations to develop the destination function}

The previous section presented, from the suppliers' perspective, the characteristics of both nodes that can help enhance their destination function, particularly in order to attract gateway travellers. This section, on the other hand, discusses what the constraints and limitations are that the suppliers foresee as major obstacles to improving the destination function from gateway travellers. Five main topics will be covered here, most of which have also been touched on earlier parts of this thesis: the tourist flow pattern; ferry cancellations; speed restrictions and environmental impacts; the relationship between tourism operators and ferry companies; and lack of funding.

\subsubsection{Tourist flow pattern}

This section presents the suppliers' perceptions of the impact of the flow patterns on tourism in Wellington and Picton. As previously mentioned, international travellers are those whose travel patterns are more spread throughout the country, being also characterised by a circular type of itinerary. When compared to domestic travellers, the suppliers clearly identified this feature with international visitors, which made them comment exclusively on overseas travellers when referring to the tourist flow pattern.

The flow of international travellers while travelling in New Zealand is very much influenced by the fact that Auckland is the main gateway for travellers entering in New Zealand. According to Table 6.7 , nearly three-quarters $(74.5 \%)$ of international ferry passengers present this characteristic. While approximately $21 \%$ of international passengers will enter via Auckland and depart from Christchurch, 50.9\% - half of all international ferry passengers - will go back to Auckland to leave the country. There are two major consequences related to this pattern. The first is a higher percentage of ferry passengers 
travelling southbound (55\% according to one of the ferry managers). The second is that part of those international travellers returning to Auckland - circuit trip - will use the ferries on their way back, creating an opportunity for another visit to the gateways. One hostel manager in Wellington, which at the time of the interview was the major accommodation provider for Kiwi Experience, Magic Bus and Strait Tours, describes the backpacker tourist pattern as follows:

A lot of people are doing a round the country trip and are coming back through Wellington. And we are seeing more and more people on their way back staying longer because they have been here, have seen it, had a taste of Wellington and they stay longer on their return trip.

In terms of how Picton is affected by the international traveller circuit pattern, another hostel owner in Picton said that over $24 \%$ of his business are repeat guests. He explained the movement of tourists in the following way:

So we get one bite when they come in to the South Island. They get round the South Island and they generally choose to stop with us again before going [back to the North Island]. Sometimes we even get three bites when they come in here, we tell them about the Queen Charlotte track, for example, or maybe a kayaking trip on the Sounds, they do that, finish that trip, stay one night or two, then go to [visit the rest of] the South Island, then stay another night or two and then carry on back up to the North Island.

Unfortunately the question designed in the questionnaire to understand the eventual behaviour of the ferry passengers on their second leg of the journey did not yield the expected data (Question 24), thus not permitting a comparison between these two pieces of information from the suppliers and the response from the passengers. However, the information provided by the suppliers indicate that travellers will make different use of the nodes according to the various circumstances and experiences they are facing each time they have the opportunity to go there.

The manager of the Visitor Information Centre in Picton also pointed out another characteristic associated with international travellers, namely that international visitors using the ferries usually arrive in Picton without a planned itinerary, and they are prepared to stay if Picton appeals to them. She illustrates her argument with the following example:

They arrive here, they are on the ferry, they like the view of the town [...] and they will stay. Sometimes they will leave it until the last minute. Sometimes they arrive on the ferry and walk around the afternoon and at about 5 to 5 , we are due to close at 5 o'clock, they will come in and say we are staying. They want me to book an accommodation, the track and I stay here until 6 o'clock in the night. 
While it is true that international passengers are more flexible in terms of planning and booking their trips (see Figure 6.5), when comparing her quote with the information provided by the passengers in Chapter 7 , it seems that there is not much evidence of this. Even though international passengers were the group with more travellers spontaneously deciding to stay longer in Picton (see Table 7.24), it was still a small number of passengers (only ten passengers $-0.97 \%$ ) that presented this behaviour. What is more, despite Table 7.25 not quantifying the reasons passengers gave to change their plans in Picton, those who stayed longer gave several motives apart from the fact that they have enjoyed Picton as a destination, including, for example, ferry cancellation and transport arrangement, which are circumstances beyond the travellers' control. It seems that in this case the supplier has been a bit optimistic about the overall ferry market.

\subsubsection{Cancellation}

As presented in Chapter 7, cancellation of the ferry is usually due to weather conditions; this is one of the main reasons to make passengers change their length of stay either in Wellington or in Picton. According to Table 7.13 and Table 7.25, it influenced passengers either to stay longer or shorter in both nodes. Therefore, it is also appropriate to consider the suppliers' perspectives on the subject. Due to its size and how the accommodation sector is structured in Picton (see previous Chapters), the impact of ferry cancellations is usually higher in Picton than Wellington. In fact, when asked about the consequences of the ferry cancellation in Wellington, none of the interviewees located in this node perceived that as a major issue. It seems that the capital city is able to easily accommodate the needs of extra hundreds of travellers, with these passengers being divided between a large number of accommodation providers, catering facilities and tourist attractions in a way that none of them is hugely affected.

From the analysis of the transcripts of the interviews, basically three types of reactions regarding ferry cancellations can be identified from businesses managers and owners in Picton. Some were positive about the impact the ferry cancellation has on Picton, others believe that it does not make much difference and to some businesses it is a real "nightmare".

The first group of interviewees has the view that the cancellations, which sometimes last for a day or two, are good for Picton as they make travellers stay in the town until the ferry operations resume. Due to the size of the vessels, these circumstances can create a need for accommodation, activities and catering for hundreds of passengers, which is usually enough to make most tourism providers fully booked. One interviewee commented that in some cases, where there is no accommodation available, passengers are put on a marae 
or in local halls so they can have somewhere to sleep. While some people get frustrated because their holiday is delayed, others take the opportunity to look around Picton and engage in activities.

A second group of interviewees thinks that while, on the one hand, the ferry cancellation makes passengers waiting in Picton stay longer in the town, on the other hand most businesses end up losing the bookings from passengers that are coming from Wellington. As passengers in Wellington also cannot cross the Cook Strait in these circumstances, once the services resume they usually prefer to move on, in order to keep up with their itineraries, rather than to stay in Picton. The owner of a holiday park in Picton explained the situation in this way:

Let's say that if they [passengers] have planned on staying in Picton tonight, and their sailings have been cancelled. Their itinerary says they are in Picton tonight; they are in Hamner [Springs] tomorrow or Fox Glacier the following night. If they don't do the Picton thing tonight, they will carry on the next day to keep up with their itinerary. Generally if there is a problem with the cancellation of the ferry, we lose from the North, you gain a little bit from the South. And so it goes on.

Finally, there are those businesses that only lose with the ferry cancellation. Apart from the ferry operators, rental car companies also face challenging situations, as the manager of a rental car company in Picton explains:

It is a nightmare, really. It is a bit chaotic. We rely on fleet management, logistics for us is essential. For a start we will have people that arrived from the ferries turning up to pick their car. But then clients who already have a car and are not able to catch the ferry because it has been cancelled they may ask: 'can I keep my car for an extra day?'. And we never turn down an extension. If someone has already got a car, and they say I will need it for a couple of days, we will let them have it. It is our customer policy service. Then people start doing strange things like flying to Christchurch or Wellington because they don't want to wait. If someone says a ferry was cancelled and they are going to Picton and we are expecting them to turn up, but they couldn't turn up because the ferry was cancelled, we wouldn't charge that person for the rental. We are not going to penalize them for something that is out of their control. Generally we don't charge for people that don't turn up, no-show.

While it seems that some interviewees consider the cancellation as an opportunity to have the town packed with visitors and their businesses running in full capacity, it is clear that the experience of being forced to stay in any destination is not the best. In addition, even though some passengers will take advantage of the cancellation to visit and enjoy Picton, it is sure that this experience will not be the best one, as all places and attractions in Picton will be full of people. To sum up, while the cancellation can bring some extra revenue for some particular businesses, the overall tourist experience does not seem to 
be the best, with some of the tourism and transport businesses struggling to cope with its impacts.

\subsubsection{Speed Restrictions and Environmental Impacts}

As presented in Chapter 5, after the introduction of the fast ferries in the Cook Strait market and during several periods after that, the community of Picton and the Marlborough Sounds was concerned about the environmental impacts caused by these vessels. The wash produced by the fast ferries impacted on the Tory Channel shoreline creating substantial erosion, the destruction of marine and bird life and the washing up of large boulders onto the shore. The situation culminated with the fast ferries' speed being restricted to $18 \mathrm{knots}(33 \mathrm{~km} / \mathrm{h})$ in the year 2000 . This episode created a strained relationship between the fast ferry operators of that time and the community in Picton, who set up an organisation called Save the Sounds - Stop the Wash. After the threat of the Clifford Bay proposal, in addition to the juridical battle regarding the ferries' speed, part of the tourism sector and the community of Picton were very upset with The Interisland Line. Two different interviewees summarised the situation taking into account both perspectives, i.e. the ferry providers and the rest of the community. These issues of relationship between the tourism sector and the ferry operators will be discussed in the next sub-section and these topics will be considered once again.

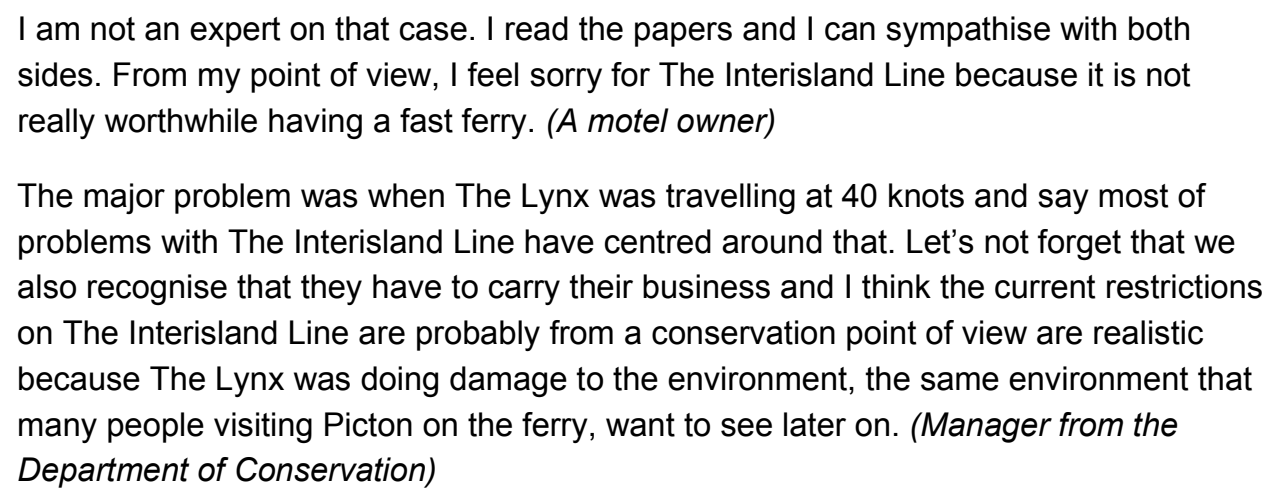
problems with The Interisland Line have centred around that. Let's not forget that we also recognise that they have to carry their business and I think the current restrictions on The Interisland Line are probably from a conservation point of view are realistic because The Lynx was doing damage to the environment, the same environment that many people visiting Picton on the ferry, want to see later on. (Manager from the Department of Conservation)

The introduction of fast ferry technology created a market for day trips and short breaks across the Cook Strait, especially favoured by those living in Wellington. Visitors could leave the city in the morning and arrive back in the evening. The wineries in Marlborough and the tour operators in Picton particularly benefited from day-trippers and the convenience of a fast ferry travelling at 35 knots. When the survey was conducted for this research these packages were not in operation as The Interisland Line was restructuring them. Therefore, it was not possible to obtain precise information on whether or not a given passenger was involved with this sort of product. However, what can be said, 
considering the number of nights passengers spent on their trips, is that among CSNZ passengers nearly one-quarter of them (24.2\% - see Table 6.6) are staying two nights or fewer away from home. This suggests that particularly among this group of passengers there is a demand for short break holidays, which are the major target market of these packages. However, it is important to highlight that this is boosting destination traffic from Wellington, rather than converting gateway passengers.

With the advent of speed restrictions in Tory Channel, however, and a proliferation of cheap airfares, Wellingtonians opted to fly directly to Blenheim, the heart of the wine country. Tourism businesses in Picton, especially wine tour operators, ended up losing this niche market. Despite this loss, all the tourism business people interviewed in Picton support the decision to reduce the ferries' speed. Their point of view is that it is important to protect the environment and beauty of the Marlborough Sounds. One tour operator in Picton made the following comment:

If it took one hour and fifteen minutes, like it did, it would be fantastic. People would be doing it all the time. But, in saying that, I hate what it did to the environment, because it is a jewel and we don't want to ruin it.

From the ferry point of view, the manager of one of the ferry operators said that more important than the total travel time is the time travellers leave, how they maximise their day with activities when they arrive and what time they get back. He said that if travellers are particularly concerned with travel time the air transport option is probably the most suitable one. In fact, from Table 6.11 it is possible to see that only $1.1 \%$ of passengers stated that the total travel time was the main reason for travelling by ferry. Time of departure, however, had nearly the same importance, with $1.2 \%$ of the sample considering it as the main reason for travelling by ferry. Total travel time also had one of the lowest averages in terms of the five attributes rated by passengers when using the ferries (see Figure 6.12).

A twofold conclusion can be drawn from this episode. The first is that despite the loss of a niche market, particularly day-trippers, businesses in Picton supported the decision about the speed restriction in the Marlborough Sounds in order to protect the environment. The second is that the situation only aggravated the relationship between the tourism businesses and organisations in Picton with the main ferry provider, The Interisland Line. This topic will be discussed in the next section. 


\subsubsection{Relationship between Tourism and Ferry Operators}

Since 1962, the ferries have been part of the day-to-day life of Wellington and Picton. Apart from the fact that these nodes benefit indirectly from the flow of traffic that the ferries generate, many tourism businesses also earn an extra income making reservations for The Interisland Line services. As the manager of a hostel in Wellington mentioned "a large part of our travel sales products is ferry tickets and it is a good income for us". On the other hand, the ferry companies also provide bookings for their passengers for accommodation and rental cars on both sides of the Cook Strait, either through the packages they provide or onboard the vessels at the information desk.

In Wellington, the suppliers seem to have a good relationship with the ferry operators, particularly the Interisland Line. The manager of a large tourist attraction in the capital city said that the initiatives they have put forward in the past have been warmly received by the ferries. From the analysis of the interviews it is possible to state that managers of tourism businesses and organisations in Wellington do not have any major issues with the ferry companies. All of them, in one way or another, mentioned two things about the ferries. The first was the importance of selling tickets for the Interisland Line and the commission they receive from this transaction. The second was that they see the competition with other ferry providers as good for tourism in Wellington, particularly with the ferry fares going down.

In Picton, on the other hand, it was possible to note that some of the suppliers have reservations about The Interisland Line, while being very supportive of Bluebridge. This can be associated with the issues they had in the past with the incumbent ferry operator (e.g. the Clifford Bay proposal and the dispute to slow down the fast ferries), trying to be sympathetic to any new entrant so they are less vulnerable than dealing with only one big player. All these questions are aggravated by the fact that in Picton and the Marlborough Region there "is a bunch of very, very small operators besides some of the large wineries that are just starting to think that they are part of the tourism industry, like Montana" (hostel owner in Picton), dealing with only one major operator, The Interisland Line. It is interesting to consider the comment of the owner of a holiday park in Picton where he clearly speaks about his preference towards Bluebridge without really explaining why exactly the new ferry provider is more "sympathetic" to Picton:

Bluebridge seems to be a little better to get on with. We actually push a bit of traffic their way. Over the years, The Interisland Line has basically stood on whoever they wanted to and done whatever they wanted to. They have not really given a hoot about us [...]. They come into port and drop their load and drop their freight, and they don't really care about anyone else. Whereas Bluebridge is a little bit more sympathetic. 
To understand the relationship between small Picton-based tourism operators and the ferry companies, especially The Interisland Line, it is important to consider the various issues and points of view. Speed restriction, the Clifford Bay proposal, and the amount of commission paid are the main concerns. In most cases, these matters are related to each other, as one rental car manager stated:

The Interisland Line wanted the fast ferries to be able to increase their speed through the Sounds. It was going to the Environment Court and, in the newspaper at the time, they said if they couldn't go as fast as they wanted to, they would have to look at Clifford Bay. Later on, there was another issue: if they didn't get what they wanted, they would be going to look at Clifford Bay.

Other interviewees summarize their relationship with the ferry companies, notably The Interisland Line, as a "love-hate relationship because in the past the ferries dominated the people. They say 'your town will be nothing when we go. You have to do this". Even some tourism businesses that have formal partnerships with The Interisland Line, such as packages or business agreements, find it hard to cope with the Clifford Bay threat. One manager says:

We do have a good relationship with them. We don't like the fact that every time they want to put pressure on the Council or want something for themselves they say Clifford Bay is a very viable option. The relationship would be a lot better, if they didn't do that to the community.

Businesses that have not formally been involved with The Interisland Line in the past and that have tried to establish partnerships with its competitors are more supportive of the arrival of Bluebridge into the market. The main problem with Bluebridge is that, at the time the research was conducted, it had a flat rate system i.e. direct booking only, with no commission.

Apart from the Clifford Bay proposal and the dispute related to speed restriction, another issue that has created problems between tourism and ferry operators is the commission paid between them. The major complaint from the tourism businesses in Picton was about the fact that The Interisland Line charges 10\% commission when they book their passengers into another product (e.g. accommodation or activities in Picton), but only pay $5 \%$ when another business books a ferry to its clients (e.g. someone staying in a backpacker wanting to travel by ferry to Wellington). As the small businesses in Picton rely on the commission they receive to increase their revenues, they consider this practice a bit unfair. As a tour operator in Picton explains:

They [The Interisland Line] have slashed the commission that they pay so we have a $10 \%$ commission rule among this community. If I book my costumers on a boat trip, 
they pay me $10 \%$ commission. The Interisland Line cut the commission they pay now to $5 \%$. If I book a costumer on the Escape [package], they only pay $5 \%$. But they still take $10 \%$. It has become a very unequal relationship. That does upset the community. Cutting the commissions plus holding this harmful Clifford Bay around our neck. It is really unfair and it does not need to be like that.

In Wellington, none of the interviewees raised this issue, even though the topic about commission was considered.

What could be noticed was that the Interisland Line has reduced its agents' commission incentives on the basis that this would become a contribution rather than a commission. By way of comparison, it is possible to consider that while the average value for an Interisland Line booking may be several hundred dollars, an activity or one night accommodation might be $\$ 80$. If that has a $10 \%$ margin on it that is an $\$ 8$ contribution. On the other hand, if one ferry booking is $\$ 300$ and the company has a $5 \%$ commission on it; that's $\$ 15$, which is nearly two times the contribution of the accommodation provider. Another issue to be considered is the fact that the ferry companies, particularly the Interisland Line, invest a significant amount of money promoting the region and its products.

These problems of relationship in some cases create a difficulty for promoting and developing products that can help to increase the length of stay of travellers passing by. This explains, in part, why it is not an easy task to convert gateway travellers into destination tourists, especially in the case of Picton where the majority of tourism enterprises are composed of small businesses. Two managers, one of a rental car company, the other from one of the ferry operators, also mentioned the difficulties of developing new products with tourism providers because there is no common booking system. The manager of the ferry operator said that the company runs "an application which is an inventory tool which requires a significant amount of technological upgrade for it to be compatible with other central reservation systems". So this is a big limitation, especially in order to set up packages combining transport with accommodation and activities.

\subsubsection{Lack of Funding}

The last issue to be discussed in terms of the constraints and limitations to improve the destination function in both nodes is the lack of funding. In spite of the differences existing between Wellington and Picton, both nodes face similar problems, even if in different degrees. 
Despite the size of Wellington as a destination, being the capital city and one of the largest cities in the country, most of the promotion relies on the government agencies and organisations, as there are no major private attractions there. Combined promotion which involves the ferry operators and tourism providers in Wellington is not always easy, especially considering that Te Papa, despite being the biggest attraction in the capital city, does not have the funds for this sort of partnership. For example, according to Te Papa's Marketing Manager, when the museum was hosting the Lord of the Rings exhibition, which was a paid exhibition, The Interisland Line was "more than happy to have a poster displayed in the terminals". However, as he stated, "there is a financial limit in terms of money [as] they need to earn money through advertisements onboard the ferries and we don't have the budget to afford that. Although we are a huge organization, we don't have the budget'. This emphasizes, once again, the negative aspects of not having major paid attractions in Wellington. As the manager of one of the ferry companies commented:

The weakness in Wellington is that we don't have the same degree of development as wholesalers, as there are in the other regions. Queenstown, Christchurch, Auckland or Rotorua. They have, you know they may not be based there, but the wholesalers and inbound tour operators in New Zealand are primarily focused around those regions and they're not doing enough to actually provide the product, the combined package product for Wellington.

Suppliers in Wellington also highlighted the importance of the wholesalers as a key distribution channel. The image that the wholesalers have about the destination will make them influence the travellers in a positive or negative way. In addition to that, the lack of commissionable products was pointed out as another factor to contribute to wholesalers not to broker the tourism products available in the capital city. On the top of that, much of the wholesalers' business has traditionally come from tour groups, which are not as prominent among the ferry passengers as independent travellers.

Picton has significantly fewer resources to attract tourists than Wellington. With a smaller economy, fewer residents and a tourism sector comprised exclusively of small businesses, there are not many opportunities and funds to promote it. The suppliers consider that the marketing and promotion are usually below the ideal. As a motel owner explained, "the problem of Marlborough is that it is a very small region. It is only 44,000 people in the region. Destination Marlborough doesn't have the budget for marketing". Other issues which make it more difficult to market Picton and the Sounds are the fact that most of the businesses located there are small enterprises usually run by couples. One of the members of the organisation called Picton Sounds Paradise (PSP) put in this way: 
I guess part of this is that we are all trying to run very busy small businesses. We don't have the funds for a full time coordinator or someone that would do the job. There is not enough money for the salary. Before I came to PSP, there was a full time coordinator. But the financial constraints didn't allow us to keep with that.

Apart from the lack of resources, owners and managers also lack the time and ability to explore further possibilities existing in the region. The owner of a hostel illustrated this with the following example:

The Queen Charlotte track is a big feature for Picton. I don't believe we've made the most of it yet, I wrote the tourism award entry for in 1998 for New Zealand Tourism Awards and that won the best visitor attraction. The operators were perhaps too smallminded to capitalize on the benefits.

With the exception of the wineries in the Marlborough region that have sufficient funds to market themselves, most of the marketing initiatives rely on Destination Marlborough and the transport providers, i.e. the ferry companies, notably The Interisland Line, and SoundsAir. However, as discussed earlier, due to the importance the vineyards have, they end up dominating the promotion done by these other organisations. Wine tourism is the main product for the Marlborough region and this does not help much in terms of the other 'W' products (water and wild) that Picton is able to offer.

\subsection{Clifford Bay proposal: threats and opportunities}

\subsubsection{The Impacts of Multiple Gateways}

Section 5.2.4 briefly presented the key aspects of the proposal to build a new ferry terminal in Clifford Bay (South Island). From a transport point of view, the main advantage of this project would be a more direct and faster ferry link between the North and South Islands. The route proposed would operate in open sea with no speed restriction imposed (see earlier section in this chapter), although more cancellations might be expected, as the Cook Strait can be quite rough sometimes, especially outside the sheltered waters of Tory Channel. On the other hand, losing the exclusiveness as the gateway ferry to the South Island could have significant impacts on Picton as part of the passing through traffic would be diverted to Clifford Bay.

This section presents data collected from both the passengers (through Section $\mathrm{F}$ of the questionnaire) and the stakeholders (semi-structured interviews). On the whole, it is going to answer the research questions made at the end of Section 5.2.4 regarding the Clifford Bay proposal. As this is a very specific topic, it was decided to include data from the passengers' questionnaires together with those from the stakeholders in order to facilitate the analysis of these two different players. 
The Passengers' reaction

Ultimately, the impact of the Clifford Bay proposal would depend directly on the passengers' choices regarding whether they would travel via Picton or Clifford Bay. This sub-section presents the results from data collected through the questionnaires in terms of which route passengers would prefer (Figure 8.1) and the reasons for them deciding to travel through Picton (Table 8.1) or Clifford Bay (Table 8.2).

A total of 924 passengers answered Question 41 regarding whether they would prefer to travel via Picton or Clifford Bay. There was a slightly higher proportion of passengers preferring to travel via Clifford Bay (51.3\%) in comparison to Picton (48.7\%). Even though a general analysis of the passengers' reaction is relevant for this study, it is also important to consider the choices for the individual nodal functions. The reason for this is that, as seen earlier in this thesis, the various types of passengers make different use of the node according to their functions. Losing gateway travellers, for example, is probably less relevant for Picton than having destination visitors opting to travel via Clifford Bay. Figure 8.1 presents the results obtained for the four nodal functions. For example, while $42.2 \%$ of gateway travellers would prefer to travel via Picton, $74.3 \%$ of destination tourists would take the same option. As can be seen from Figure 8.1, the chance of passengers preferring to travel to Picton would increase with the type of function they have in Picton, mainly being associated with the reason for going to Picton and their original length of stay. What can be concluded from these results is that Picton would lose predominantly gateway travellers, which represents over three-quarters of the passengers travelling via Picton. As seen in Table 7.23, gateway travellers are the group of passengers that have the lowest level of interaction with activities and attractions in Picton, meaning that their loss would be less important for the tourism sector. Even though Picton would lose nearly half of the passing through traffic, it would still be able to keep those passengers that tend to be more highly involved in tourist activities and attractions. It would be interesting to have considered what would be the impact to other businesses, such as gas stations and general retail stores as they usually benefit more from purchases made by gateway travellers. Apart from the choice of gateway presented in Figure 8.1, it is also appropriate to consider the reasons that would make the various groups of passengers travel via Picton or Clifford Bay. This is considered in the following section. 


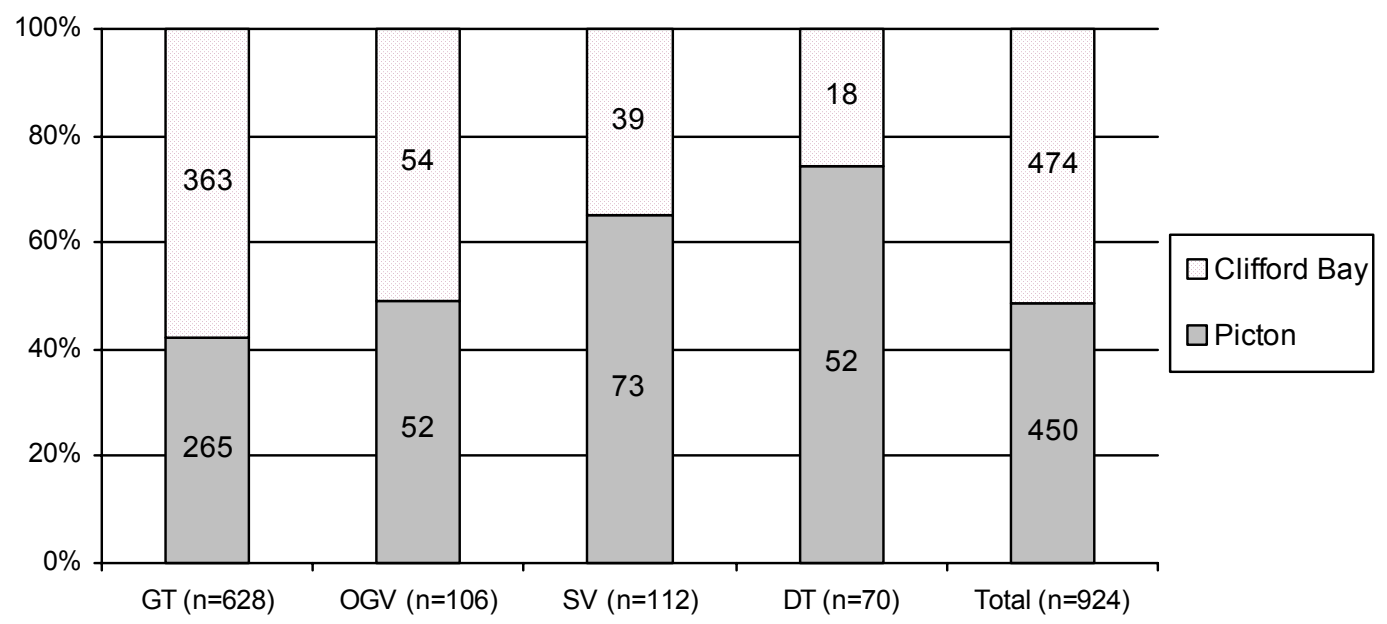

Figure 8.1 Passenger choice of route - comparison between Picton and Clifford Bay.

When considering the reasons for passengers preferring to travel via Picton (Table 8.1), three main aspects could be identified: the fact that Picton is closer to where the passenger was going; the scenery and the tourist experience of travelling through the Marlborough Sounds; and simply that Picton was the passenger's destination. Approximately $47 \%$ of the passengers deciding to travel via Picton $(n=414)$ state that proximity to the place they were going as their main reason for opting to travel via Picton. This argument was the most important one for two nodal functions, i.e. gateway travellers and stopover visitors. Scenery and the tourist experience to travel through the Marlborough Sounds was the second most common argument for travelling via Picton, with over a quarter of the total number of passengers stating it $(27.8 \%)$. What is more, this was the number one reason for overnight gateway travellers $(41.3 \%)$ and destination tourists $(32 \%)$ to travel via Picton. Finally, the fact that Picton was the passenger's destination represented the option of $7 \%$ of the total number for passengers choosing to travel via Picton, being a strong argument particularly to destination visitors (32\%). Other reasons presented by passengers for deciding to travel via Picton include the fact that Picton is an established port and it is important to keep the ferries there; and the passenger is familiar with Picton and it is easier to get to.

While the geographical location was overall the most important attribute for passengers deciding to travel via Picton, scenery / tourist experience and the fact that Picton was the passenger's destination had different meaning for the various groups of passengers. One explanation for the interest of OGV and DT in the scenery aspect of the cruise can be associated with the higher participation of international passengers among these two groups of passengers. As seen in Table 6.11, the scenic appeal was the main reason for 
international travellers choosing to travel by ferry, and accounts for one-third of this group of passengers. This emphasizes the aspect of the ferries being not only a means of transport between the North and South Islands, but also part of the tourist experience while travellers move around New Zealand.

Table 8.1 Reasons for passenger travelling via Picton.

\begin{tabular}{|c|c|c|c|c|c|}
\hline & $\begin{array}{c}\mathrm{GT} \\
(\mathrm{n}=249)\end{array}$ & $\begin{array}{c}\text { OGV } \\
(n=46)\end{array}$ & $\begin{array}{c}\text { SV } \\
(n=69)\end{array}$ & $\begin{array}{c}\text { DT } \\
(n=50)\end{array}$ & $\begin{array}{c}\text { Total } \\
(n=414)\end{array}$ \\
\hline Closer to where I am going & $53.8 \%$ & $37.0 \%$ & $43.5 \%$ & $24.0 \%$ & $46.6 \%$ \\
\hline Scenery / tourist experience & $24.1 \%$ & $41.3 \%$ & $29.0 \%$ & $32.0 \%$ & $27.8 \%$ \\
\hline Picton is my destination & & $2.2 \%$ & $11.6 \%$ & $32.0 \%$ & $7.0 \%$ \\
\hline Important to keep the ferry in Picton & $3.2 \%$ & $10.9 \%$ & $2.9 \%$ & $2.0 \%$ & $3.9 \%$ \\
\hline I am used to / It is easier to get there & $1.6 \%$ & & $5.8 \%$ & $2.0 \%$ & $2.2 \%$ \\
\hline Convenient & $2.4 \%$ & $4.3 \%$ & $1.4 \%$ & & $2.2 \%$ \\
\hline More than one of the above reasons & $7.2 \%$ & $2.2 \%$ & $2.9 \%$ & $2.0 \%$ & $5.3 \%$ \\
\hline Other & $8.6 \%$ & $2.2 \%$ & $2.9 \%$ & $6.0 \%$ & $5.0 \%$ \\
\hline Total & $100.0 \%$ & $100.0 \%$ & $100.0 \%$ & $100.0 \%$ & $100.0 \%$ \\
\hline
\end{tabular}

In the case of passengers opting to travel via Clifford Bay, two major reasons could be identified. Having a faster and shorter sea crossing would be the most significant factor, accounting for nearly two-thirds of the total number of passengers preferring this route (see Table 8.2). This result is very much similar for the four different functions, ranging from $61.1 \%$ in the case of DT to $67.3 \%$ for GT. The second most relevant reason for choosing a ferry trip via Clifford Bay would be the fact the new terminal would be located closer to where the passenger was going. This is associated with the need for shorter land travel distance, which contrasts to shorter sea crossing. Other reasons for travelling via Clifford Bay were less weighty than these first two. They include, for example, the novelty of visiting Clifford Bay, which was mentioned particularly by CSNZ passengers who are probably more used to travelling via Picton and just want to visit a new location.

The next sub-section presents the point of view of the suppliers about the Clifford Bay proposal. In some cases the information discussed here will be used to assess whether or not business managers and owners know what sort of behaviour passengers would have if the Clifford Bay proposal were implemented. 
Table 8.2 Reasons for passenger travelling via Clifford Bay.

\begin{tabular}{|l|r|r|r|r|r|}
\cline { 2 - 5 } \multicolumn{1}{c|}{} & $\begin{array}{c}\text { GT } \\
(n=249)\end{array}$ & $\begin{array}{c}\text { OGV } \\
(n=46)\end{array}$ & $\begin{array}{c}\text { SV } \\
(n=69)\end{array}$ & $\begin{array}{c}\text { DT } \\
(n=50)\end{array}$ & $\begin{array}{c}\text { Total } \\
(n=414)\end{array}$ \\
\hline Faster / shorter sea crossing & $67.3 \%$ & $64.8 \%$ & $62.2 \%$ & $61.1 \%$ & $66.4 \%$ \\
\hline Closer to where I am going & $20.7 \%$ & $29.6 \%$ & $18.9 \%$ & $27.8 \%$ & $21.9 \%$ \\
\hline Novelty of visiting Clifford Bay & $2.3 \%$ & & $5.4 \%$ & $11.1 \%$ & $2.7 \%$ \\
\hline More than one of the above reasons & $0.9 \%$ & $3.7 \%$ & $8.1 \%$ & & $1.8 \%$ \\
\hline Other & $6.1 \%$ & $1.9 \%$ & $5.4 \%$ & & $5.3 \%$ \\
\hline Total & $2.6 \%$ & & & & $2.0 \%$ \\
\hline
\end{tabular}

\section{Suppliers' Perspective}

Although the possibility of the ferries starting to operate between Wellington and Clifford Bay seems to be less probable each year, no other topic made the interviewees talk more passionately than this one. Several reasons contribute to this reaction, but the paramount one is the question of Picton's survival without the ferries. In fact, on this matter, the respondents' opinions were quite diverse, with some managers and owners believing that Picton would struggle to maintain its economy, while others think that not having the ferries would be the real opportunity for Picton to grow as a destination. Other aspects, which contribute to this lively debate, include the fact that some interviewees consider that The Interisland Line uses the Clifford Bay proposal to threaten Picton and the Marlborough Regional Council in order to obtain whatever they want. According to them, this situation happened during the debate about the fast ferries' speed restriction, with the ferry operator mentioning that Clifford Bay was a viable option to consider if a speed restriction was imposed. As will be discussed later in this chapter, this ended up damaging the relationship between some tourism businesses operators in Picton and The Interisland Line.

The general impression from the interviews was that no one really believed that the proposal would go ahead, but if it did happen Picton would probably be better prepared to cope with the move, especially now that Bluebridge has started a passenger ferry service. In fact, one of the reasons why Picton's community support new ferry entrants such as Bluebridge is to minimise the threat of the proposed move of The Interisland Line to Clifford Bay. Compared with previous ferry operators (see Chapter 5), the interviewees seemed to be more confident with Bluebridge as Strait Shipping, the ferry company that provides this service, has been operating freight services from Picton for more than a decade and they have survived the competition in the cargo market against The 
Interisland Line. One manager also mentioned that if The Interisland Line leaves Picton, some independent operators using smaller boats might enter into this market. A backpacker owner from Picton sums up the situation thus:

If it did go ahead [the Clifford Bay proposal], I would now be more confident of Picton's development as a tourist destination than I used to be. Simply because: a) we have a convenient ferry line that is going to last [B/uebridge] and b) Picton and the Sounds have developed their own identity - the Queen Charlotte track, the Sounds and its waterways, and accommodation properties have all created a demand. [...] People usually like Picton. So, a momentum has started, which will continue.

Another aspect raised during the interviews is the real feasibility of the Clifford Bay proposal. Several points were raised. Firstly, if the Marlborough Regional Council were to fund the project, some stakeholders were worried about what would happen if, with continuing developments in fast ferry technology, a direct route between Wellington and Christchurch (the main city in the South Island) opened up, and ferry companies no longer needed to go to Clifford Bay. This would mean that all the investment made by the regional council in the development of the Clifford Bay area would be lost. Secondly, environmental impacts were also perceived as an important issue. The argument was that after thirty years of servicing ferries, Picton and the ecosystem have developed and modified themselves, except for the damage caused by the fast ferries. A new terminal in Clifford Bay could potentially cause an environmental disaster due to the huge infrastructure that would need to be constructed there. Finally, the coast where Clifford Bay is located is very exposed with the risk of the route Wellington-Clifford Bay being less serviceable during the year. The same backpacker owner made the following statement:

\begin{abstract}
We came here ten years ago with the knowledge that this was a possibility. We learnt quite a lot about the proposal of Clifford Bay. I was a transport operator, heavy trucks, before getting into tourism. From that aspect I would like the aspect of a more stream line of transport system from Wellington to Clifford Bay, less time travelling on the way. As a tourism operator in Picton, I would obviously much prefer to see that 1.2 million people keep coming passing the door. I don't believe [Clifford Bay is going to happen]... I don't want to see the wheel being recreated. In Clifford Bay, there is no room for any infrastructure.
\end{abstract}

Losing the exclusiveness of the ferry gateway to the South Island would have significant ramifications for Picton. Some managers and business owners estimate that Picton would take between three and five years to recover if the ferries move to Clifford Bay. A number of businesses might never recover, such as restaurants, cafes and roadside motels on the way out of Picton. One accommodation manager said his business "would never be as good again. Its income would be cut in half, or quarter. The overall takings would be like winter takings all year round, which is really low. It wouldn't be worth being here". 
Probably the only time of the year when the absence of the ferries would not be felt would be during summer with very few businesses being able to survive only with the summer incomes.

For some interviewees, the Clifford Bay threat has been a wake-up call for the whole town. One of PSP's board members states that:

Motels, particularly, never belonged to anything, they never contributed to anything. Now they think: I have an investment here, I have to protect it. I have to create a market for myself and I have to think about my business.

This is a very interesting criticism as apparently some businesses have just been relying on the ferries in order to have their clients coming, but never tried to develop new markets so they could be less dependent on the ferries. The Clifford Bay threat forced them to promote themselves and to develop Picton. In fact, the general opinion among the interviewees was not only that Picton would suffer, but also Blenheim and the whole Marlborough region. With the move to Clifford Bay, there will be no need for the trains to go to Blenheim and Picton and few passengers would drive back to visit these two destinations. Other places on the route between Clifford Bay and Christchurch would benefit from it, especially Kaikoura and Hamner Springs. In the view of a manager of a tourist attraction in Wellington, the capital city could also benefit from a move of the ferries to Clifford Bay. His argument is that the shorter trip across the Cook Strait could make people leave later or arrive earlier in the capital city.

Several interviewees hold the view that the town could benefit if the ferries moved away from Picton. They believe Picton would be able to develop itself as a true tourist destination without the ferry infrastructure and its gateway function. Supporters of this argument say the absence of a ferry terminal would make the Marlborough Sounds the focus for people visiting Picton. And, in fact, the interface between land and sea, with native bush rolling down to the water's edge, makes the region quite unique. Beautiful beaches for swimming are also available, as well as fishing and a host of other activities. However, Picton will need to market itself and also to have other attractions and activities to sustain the tourism industry outside the summer season.

\subsection{Conclusions}

This chapter brings together several issues related to the suppliers and the gateways studied, providing a common background to analyse the possibility of increasing the ferry passenger stay both in Wellington and Picton. Before discussing the major conclusions of this chapter in more detail, it is appropriate to consider that the suppliers interviewed in 
both nodes share a certain enthusiasm about what is happening in terms of tourism in their nodes. They confirm the changes that are occurring both in Wellington and Picton and that were earlier described in Chapter 4. Particularly in Wellington, the managers interviewed seemed to be confident of what is happening in the capital city, predominantly the consolidation of Wellington as the most important cultural city of New Zealand, heavily supported by Te Papa, a range of events and small attractions, in addition to the regeneration process that is happening in the waterfront. What is more, they believe Wellington has now an appeal to attract holidaymakers, as the perception of a grey, boring capital city is something of the past. Picton also suffered some restructuring works, particularly on the seafront. Interviewees in Picton are now more confident about the coastal resort, particularly in coping with the very unlikely possibility of the ferry service moving to Clifford Bay.

In spite of the recent changes that have occurred in Wellington and Picton in the last few years, it seems, however, that both nodes still struggle to take advantage of the ferry passengers to generate more visitors. The major problem in Wellington is being deficient in large private enterprises with the funds to promote the destination. In addition, the lack of commissionable products does not help travel agents and tour operators to broker the capital city, as Wellington does not offer attractions and activities where a significant amount of commission can be earned by these intermediaries. Therefore, they tend to encourage tourists to go to other destinations, particularly Rotorua, Waitomo Caves and Queenstown. Apart from this, many intermediaries, as well as the visitors, are not always fully aware of the changes that are happening in Wellington, with the previous perception of a city of bureaucrats still very much strong in people's minds. Picton, on the other hand, struggles due to the lack of indoor facilities and also places where people can go at night. The interviewees suggested several types of possible attractions and activities, ranging from movie theatres to a gondola. One facility suggested that would probably benefit Picton from the perspective of all its nodal functions is an indoor aquatic centre. Firstly because it could be available in every weather condition, suiting the family market that is very strong particularly during summer as well as the gateway travellers that could use it even in order to kill time while waiting for the ferry. In addition, it would also benefit the local community, as there are few public swimming pools in Picton. Interviewees in Picton also complain about the lack of funds to promote the destination, with most of the marketing in the region being directed to the vineyards that are located around Blenheim.

There are several conclusions from this chapter, all of them leading to the difficulties that exist in order to provide ferry passengers with the opportunities for them to stay longer in both nodes. As concluded from Chapter 7 , more information and a special deal would 
have made some travellers stay longer in Wellington and Picton. However, while the suppliers agree with the need for more information to be sent out, particularly through some specific channels such as travel agents, they struggle with the lack of resources to do that and in the case of Wellington the absence of major commissionable products to make them encourage travellers to visit and stay in the capital city. A special deal involving transport and accommodation, which in fact was considered by the ferry passengers as the most appealing way to increase their length of stay in both ferry ports, was seen as something that needs to exist, but not necessarily being provided by the ferries. The ferries are by far the biggest private operators in the region and those that already have the marketing and the distribution network to sell its products to all markets. Therefore, without their involvement it would be hard to make these deals attractive in order to influence the travellers planning to include an overnight stay in Wellington or Picton while they book their Cook Strait crossing.

Particularly in Picton, where the tourism industry is made up of several small businesses dealing with the big ferry providers, various issues have led to the tourism operators experiencing difficulties in dealing with The Interisland Line. They involved the speed restriction campaign in the 1990s, commission paid and the threat of the Clifford Bay proposal. Even those businesses that have some sort of partnership with the ferry operator find it hard to cope with these issues. As a consequence, it does not seem that there is an adequate business environment to discuss ways that could make the ferry passengers stay longer. Due to Wellington's larger scale and not being over dependent on the ferries as Picton is, no major issues were identified between the tourism providers in the capital city with the ferry operators, notably the Interisland Line.

A clear example of how the opportunities are not maximised in Picton is that the suppliers there suggested that the Marlborough region should be promoted as a place for wine, water and wild activities. In fact, the only player that uses this strategy in full is The Interisland Line, with its packages that offered the visitors the possibility to visit either the vineyards in Blenheim ("Marlborough Wine Trails") or to undertake outdoor activities ("Marlborough Outdoor Adventures") in Picton. In spite of that and the marketing campaign that the ferry provider promoted for these packages, the businesses in Picton did not take advantage of this in order to create more ways to bring travellers into the area, particularly targeting visitors outside the CSNZ region.

In this regard, the suppliers were able to identify the "interactive traveller", which the national tourist organisation and some of the RTOs are already promoting New Zealand to, as a potential type of traveller to visit places like Wellington and Picton. In fact, from 
the description portrayed by some of the interviewees and the information presented in Chapters 4 and 6 , it is possible to say that travellers from the UK and Germany would fit into this category, as they usually travel at a different pace, being more inclined to visit places outside the beaten track. What the suppliers were not able to do was to provide strategies to bring these travellers to their destinations. In terms of the special deals that would make passengers to stay longer, not only could they not come up with any ideas on how to do that, but also in some cases this was considered unnecessary, at least in a way that would involve the ferry operators.

Another conclusion from this chapter is that although a new ferry terminal in Clifford Bay would have a significant impact on Picton's economy in the short term, simply the prospect of it even occurring has galvanized Picton into being less reliant on its gateway function and notably trying to develop itself as a tourist destination. From the passenger point of view, while nearly half of the sample stated that they would prefer to travel via Clifford Bay, when considering the current nodal function of these passengers it is possible to realise that the intention to travel via Clifford Bay decreases proportionally to the degree of involvement that the passengers have with Picton. In other words, gateway travellers would be keener to go via Clifford Bay, while destination tourists would still choose Picton. The opinion of the suppliers was that while it is true that those businesses that serve primarily the gateway travellers in Picton, which in fact is the majority, would suffer with the loss of the passenger traffic to Clifford Bay, tourism in Picton could be improved if most of the ferry and transport infrastructure that currently exist there was relocated to Clifford Bay. 


\section{Conclusion}

\subsection{Introduction}

This thesis has examined the relationships that exist between the gateway and destination functions in the Centre Stage of New Zealand Region (CSNZ). From the study of the Cook Strait ferries and their importance to Wellington and Picton it was possible to look at their role as a vital transport provider, not only to bring visitors to these two nodes and their catchment areas, but also to link travellers between the North and South Islands. With over 1.2 million passengers being carried each year, the ferries also provide a huge potential number of tourists passing by these two nodes, who could stop and visit Wellington and Picton.

From the literature review (Chapter 2), it is possible to affirm that this research has helped to contribute to a better understanding of the tourism and transport interface, advancing the concept of gateways and the various nodal functions passengers can have while travelling to a destination gateway. Previous studies related to the geography of transport have concentrated particularly on hubs, even though not many have aimed to explain how to improve tourism in this type of node. Few studies have linked tourism to gateways, such as the research of Janin (1982) and Mistilis and Dwyer (1999). However, in general the research about gateways and tourism lacks two major features. Firstly, to explain how gateways can take advantage of the passing traffic to increase the number of tourists they receive. This would help to improve the destination function of gateways. Secondly, the concept of gateway has not been operationalised in a way that travellers can be classified in terms of their interaction with the node. These two issues were considered in this research. Another contribution of this thesis was to study the tourism transport relationship using ferries as a mode of transport. Most of the tourism-transport research concentrates on other modes of transport, particularly air transport and cruise tourism.

Intermodality is one characteristic of the ferries that is particular relevant to develop tourism in a particular node. As ferry passengers are required to change from one mode of transport to another, this creates an opportunity for the passengers to visit the gateway as a tourist destination. Gateways where there is no need for a change of mode of transport can be less likely to present the same benefits. Another aspect of the ferries, which distinguish them for air transport, is the fact that they provide point-to-point linkages to a restricted number of ferry ports. While in Europe and some other regions of the world ferries have a wider network, in the case of the study area only one point in the North and 


\section{CONCLUSION}

South Islands are connected to the ferries. This gives Wellington and Picton the opportunity to potentially target all the inter-island traffic to visit their nodes, hence providing both nodes with more opportunities to develop their destination functions.

In order to understand the relationship different passengers have with Wellington and Picton, it was necessary to establish attributes that could be linked to the various passengers' functions. The operationalisation of passengers' nodal functions was undertaken using previous research about gateways in order to identify what factors make a certain node a gateway. From the four dimensions identified in Figure 3.1, i.e. transport intermodality, catchment area, purpose of trip and length of stay, the last two were used to propose, in an empirical way, four categories of functions: gateway travellers, stopover visitors, overnight gateway visitors and destination tourists. This classification proved to be useful in understanding the level of interaction ferry passengers have with Wellington and Picton, particularly in terms of their likelihood to extend their stays in both destinations.

The classification of the passengers in the nodes will be influenced by several factors. As discussed in the literature review, one of them is the travellers' place of origin (Janin, 1982). Another is related to the characteristics of tourism in New Zealand, which has a high proportion of circuit travel. One of the drawbacks of circuit travel is that as most travellers have a limited length of stay, extending the stay in one particular destination can only happen at the expense of them staying for a shorter time somewhere else. As Wellington and particularly Picton have not been traditionally considered as major destinations in the country, persuading passengers to extend their stays is not an easy task. The comment below from the manager of a tourism organisation reflects this:

I have been here [in Picton] for 13 years and the most common debate is how can we get people coming off the ferry just to stop and stay a couple of days and really there is no easy answer to that because many times people are using the ferry to get from $A$ to $B$ and a lot of the time Picton does not become part of that equation [...]. The main thing they want to do, when they arrive in Picton or in some cases Wellington, is perhaps fill up and go to their destination. [...] So nobody has found an easy answer to it. In fact, some people say, well, a quite critical effect that the ferries have been, the infrastructure around the ferries, in terms of roading, it encourages people to just pass it rather than coming to it.

On the other hand, considering that the CSNZ does not have a major international airport to bring tourists directly into this area, capturing part of the passengers' traffic can be seen as one of the best strategies to increase visitors' stay in Wellington and Picton.

Another issue when dealing with the "tourism transport" and "destination gateway" topics in this research is the differences existing between Wellington and Picton. As presented in 


\section{CONCLUSION}

Chapter 5, one can see that although the ferries are a major tourism provider for Wellington and Picton, these places do not rely exclusively on the ferries to bring visitors in, as many visitors arrive by car and in the case of Wellington by airplane, as the capital city has the busiest domestic airport in the country. Chapter 4 described Wellington as having some large attractions such as Te Papa, the Karori Wildlife Sanctuary and the Westpac Trust Stadium, which give visitors a reason to visit this destination. Despite having the largest marina in the South Island, Picton is a small resort town whose tourism businesses are exclusively made up of small enterprises. Most tourist activities in Picton are oriented to the outdoors, notably water activities and the Queen Charlotte track, which make Picton a very seasonal destination. As most of the economy in Picton exists as a consequence of the ferries' presence, usually any issues involving the ferries have a significantly higher impact on Picton than Wellington. This was the case, for example, with the Clifford Bay proposal and the dispute related to the fast ferries speed restriction. In addition to other issues arising in Chapter 8 (e.g. commission), this led to lack of support by the tourism community in Picton for the Interisland Line. This situation creates difficulties in setting up common strategies between the ferry providers and the tourism businesses in Picton to make ferry passengers stay longer. What is more, offering fewer options in terms of attractions and activities, Picton is naturally less appealing than Wellington as an extended stay destination. The differences between Wellington and Picton provided the opportunity to compare how different nodes are influenced by the "tourism transport" and "destination gateways" topics.

This chapter brings together the major conclusions obtained in this research and it explores some of the research issues more deeply. In order to achieve this, it is structured into four main sections: theoretical contribution, methodological issues, objectives revisited, and further research suggestions. In the first two parts the core discussion is related to the concept of nodal functions, focusing particularly on explaining how different factors influence the functions existing on a given node and how the nodal functions used in this research were operationalised. The chapter then revisits the research objectives, by answering the three major questions associated with this thesis. Finally, suggestions for further research are made. This is done considering either options that would utilise what was concluded from this thesis or proposing other studies that would compare the results obtained here with other situations where different modes of transports or types of nodal functions exist. 


\section{CONCLUSION}

\subsection{Theoretical Contribution}

The discussion of gateways presented by the literature has been, in some ways, dispersed and fragmented. As was shown in Chapter 2, even the definition of what a gateway is remain unclear in most cases with many scholars using it to refer to any large city. Pearce (2001f) is one of the few authors to propose one definition that clearly states the key role of gateways as points of entry/exit to reach a given place. Another aspect usually not well explored is the fact that places usually have more than one nodal function and that these functions are inter-related to each other (Lew \& McKercher, 2002; Pearce, 2001f). Finally, no previous research has aimed to operationalise the concept of gateways, especially when trying to create mechanisms to classify visitors according to their nodal function.

From the theoretical point of view, this thesis has contributed in two major areas regarding the concept of gateways. Firstly, in an empirical way, it has operationalised four different functions: gateway travellers, overnight gateway visitors, stopover visitors and destination tourists. Secondly, it has enhanced the understanding of nodal functions, particularly by explaining how they evolve through time and also which characteristics can influence them. The first topic will be discussed in this section while the second will be evaluated in the following section, which deals with methodological issues.

\subsubsection{Dimensions of nodal functions}

One theoretical contribution of this study was to enhance the understanding of what influences the functions existing in a node. Studies presented in the literature usually consider this topic from two major perspectives; i.e. the place and the travellers. The first is the more common practice, with a certain place being classified, for example, as a gateway or a destination (Ley \& Murphy, 2001; Mistilis \& Dwyer, 1999; Page, 2001). Some authors go further and discuss the existence of multiple functions in a given place (Caves \& Gosling, 1999; Lew \& McKercher, 2002; Pearce, 2001f), with the classifications (e.g. gateway destination or destination gateway) changing from author to author. Usually these studies classify a certain node in terms of its role and position in the transport network (e.g. Dredge, 1999a). A second way of considering the nodal function is from the travellers' perspective, even though few studies seem to use this approach (e.g. Lew \& McKercher, 2002; Pearce \& Elliott, 1983). While it is a fact that, particularly among the more complex types of nodes such as gateways and hubs, nodes do depend on the transport configuration, it is also true that classifying a given node in terms of the use travellers make of it provides a more useful approach. From what has been presented, 


\section{CONCLUSION}

analysed and discussed in this thesis, it is possible to take the discussion about nodal functions further in order to add new dimensions associated with this topic.

In terms of the characteristics of the place that can affect its nodal function, it seems that the development of its urban infrastructure, touristic facilities and transport connections can lead to a change in its functions over time. The literature review commented on Butler's (1980) destination lifecycle model and the development of the destination function, showing how it evolves through its different stages. Later, in Chapter 4, a description of the different functions existing in Wellington and Picton was offered. Particularly in the case of Wellington, the waterfront redevelopment and the construction of Te Papa helped to increase its destination function. When the analysis was done considering primary data collected from the interviews as well (Chapter 8), it was possible to understand, notably in the case of Picton, how the town is becoming less reliant on the gateway function to strengthen its destination function. This was done mainly as a response to the Clifford Bay proposal, with the foreshore redevelopment and some extra initiatives to market Picton. Hence, what can be concluded is that apart from the growth that a node can have in terms of the number of visitors it attracts, its functions may also change over time. Figure 9.1 (a) shows a hypothetical node where these two major transformations are illustrated. Firstly, the size of the node is growing, which means that more and more travellers, visitors and tourists are going there. Secondly, the participation of each function in the node is also changing, with the case illustrated suggesting a significant increase in the destination function (D).

Apart from changing its functions in a constant way over a certain period of time, a cyclical pattern can also be identified (Figure 9.1b), as suggested particularly in the analysis of the interviews from the managers in Picton where a change in the town's functions between summer and winter seasons was noted. Due to its scale and geographical characteristics, Picton is more sensitive to the seasonal disparity in visitor numbers, with its outdoor and marine activities being more appealing during the warmer weather. According to the owner of a backpacker hostel in Picton, in summer the town grows from 4,000 people to about 20,000 , especially with visitors attracted by holiday homes and the marina, the biggest in the South Island. Therefore, summer is the time of the year when businesses rely, not only on ferry passengers to visit Picton, but also on other travellers, predominantly from the South Island. The foreshore and holiday homes in particular attract a lot of families. The change in the type of traveller can also be noted by accommodation providers. From January to March the length of stay is longer than during the rest of the year when the common pattern is guests staying only one night, which is a characteristic of the gateway/stopover function. In fact the owner of a motel conveyed that 


\section{CONCLUSION}

"Picton becomes more of a destination at that time of the year". Winter, on the other hand, is the season that the town relies particularly on gateway travellers from the ferries. According to the manager of a rental car company in Picton, the absence of destination tourists and the decrease in the visitors' numbers make some shops close down during the four months of the off-peak season. The same manager made the following statement from his own business experience, which can illustrate how seasonal Picton can be:

In the summer we do 30-35 reservations per day. In the winter, 3 or 5 cars hired a day. So it is very seasonal. In the summer we are very tight on cars, our fleet is very utilized we don't have many idle cars sitting around and we need to be able at some stage to say sorry we can't take any more bookings, we are fully booked. In the winter, usually no problem, however demand isn't that nice, the weather isn't nice. Winter is our quiet time that is when we will try anything to get people to rent a car.

(a)
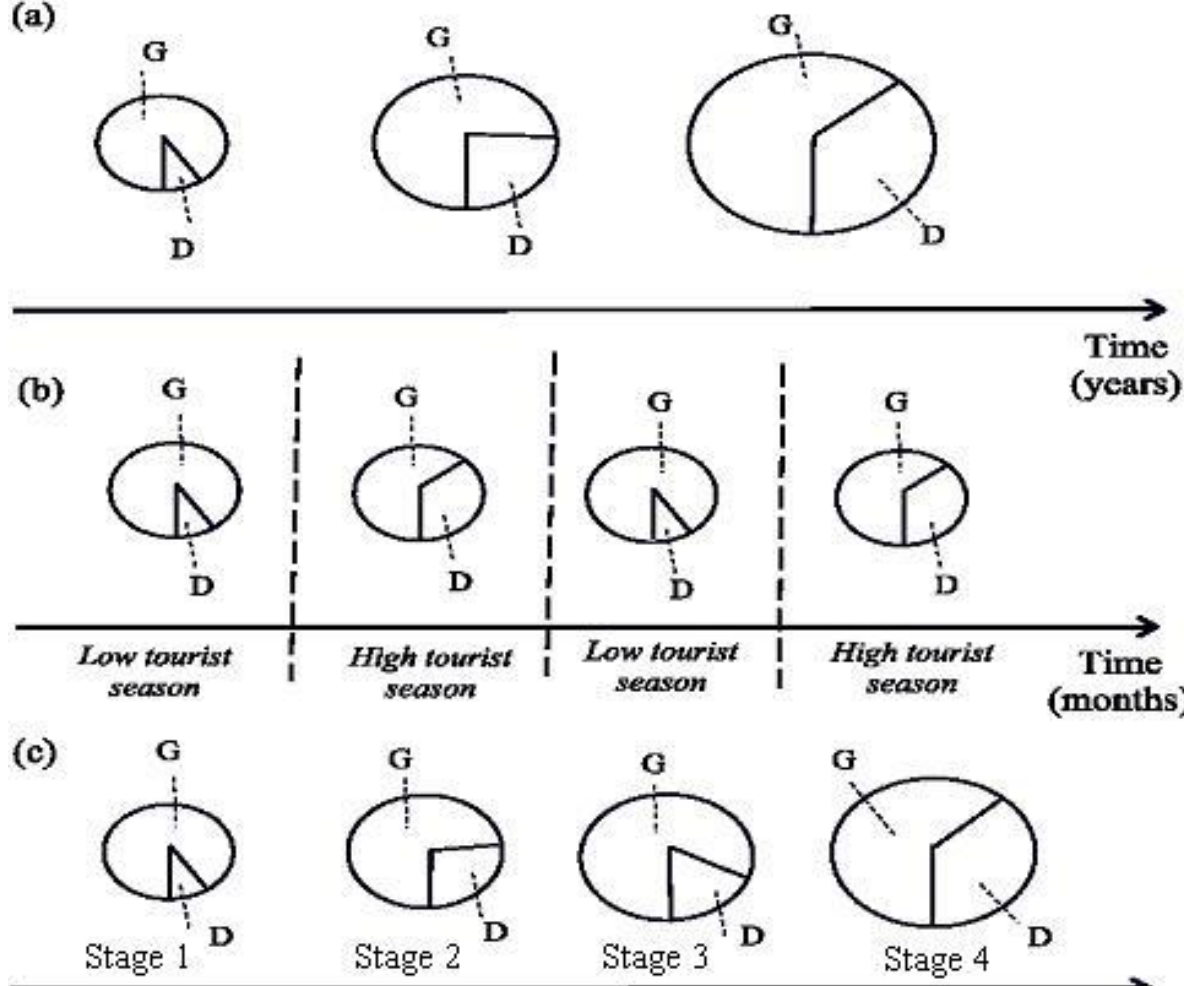

(months)

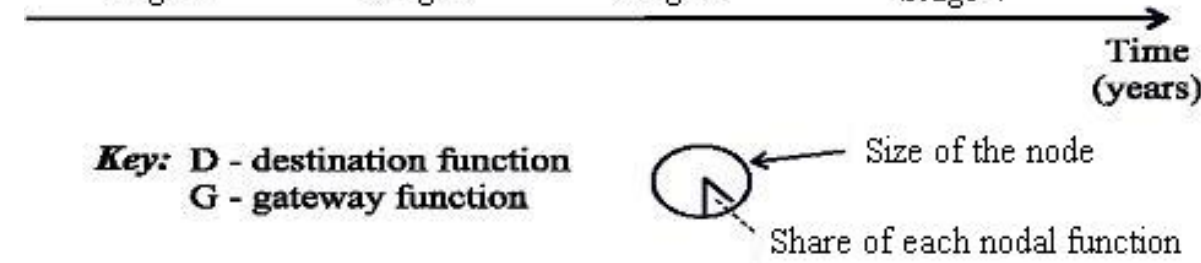

Figure 9.1 Hypothetical nodal function variation: evolvement through the time and seasonal change. 


\section{CONCLUSION}

What can be concluded from the suppliers' comments is that nodal functions can increase and decrease in a cyclical way. In Picton, for example, the destination function increases during the summer or high season, when tourists from the South Island holiday there. With its activities and attractions oriented towards the outdoors and marine environment, there is a significant decrease in the number of destination tourists visiting Picton during the winter or low season.

In order to better explain the evolutionary and seasonal evolvement of a node, Figure 9.1 presents these variations separately (Figure $9.1 \mathrm{a}, \mathrm{b}$ ). In practical terms, however, what can be expected to happen is a combination of these two patterns; with one place constantly increasing or decreasing its size and functions distribution through time, while some sort of seasonal variation also occurs (Figure $9.1 \mathrm{c}$ ). Part (c) of the diagram presented in Figure 9.1 shows four stages of development where the size of the node grows throughout time with the increase in visitor numbers. At the same time, it also illustrates that there are variations in the participation of the various functions existing in the node. In this particular example it only shows the destination and gateway functions. The increase in the destination function (stage 2) is a consequence of the high season, particularly in Picton, where more tourists arrive there, using not only the ferries, but also driving from the South Island. In the third stage, the destination contribution then decreases, but its participation is probably higher in comparison to the first stage as more visitors are going there as time passes by. In stage four, the high season is back bringing more tourists which makes the node grow, with a higher participation of destination tourists.

With respect to the characteristics of the passengers that influence how they will be classified in terms of their nodal function to a certain place, two factors seem to be relevant after analysing the data collected for this research: passenger's place of origin and the direction they are travelling. The results presented here confirm what Janin (1982) pointed out about the link between the traveller's place of origin and the use made of a node. In his study about the Aosta Valley in Italy he noticed that the further the travellers live from the gateway, the higher the chances of them staying overnight there. Similarly, what can be concluded from the analysis of data presented in previous chapters is that not only is the participation of destination tourists higher among international passengers, but they also tend to engage in more activities and attractions in the nodes studied, as well as being among those passengers more likely to stay longer if they had had more information or had been offered a special deal. It seems though that a successful way to attract ferry passengers to stay longer in Wellington and Picton is to start targeting overseas passengers. In terms of the influence of the direction the passengers are 


\section{CONCLUSION}

travelling on their functions in Wellington and Picton, no major differences were identified, except in the case of overnight gateway visitors (OGV). Both in Wellington and Picton at least six out of ten OGV were classified as such when leaving the node, rather than arriving there. This suggests a pattern where passengers would tend to stay overnight in the node before taking the ferry the next day.

\subsection{Methodological Issues}

With regards to the methodology designed for this research, it is worth making two major general comments. The first is related to how secondary data were gathered in order to support the development of the questionnaires and semi-structured interviews. The analytical description of the study area in terms of the various nodal functions existing in Wellington and Picton (Chapter 4) and the development of the Cook Strait ferries (Chapter 5) helped to contextualise the key issues about transport and tourism into the instruments of data collection. Particularly in Picton, some of the current issues about the Cook Strait ferries, such as the Clifford Bay proposal and the speed restriction, were useful to understand why a possible partnership between tourism businesses and the major ferry provider to improve visitors' stays from ferry passengers is not an easy task. What is more, in the case of the Clifford Bay proposal, it gave the opportunity to discuss the results considering the perspectives of both sides, i.e. tourism businesses/organisations and passengers.

The second major contribution of the methodology used in this thesis is precisely the possibility of considering the debate about gateways from the suppliers' as well as the passengers' points of view. In many tourism studies data are collected from only one set of stakeholders involved in the process, which in fact limits the analysis that can be developed. The analytical framework concentrated particularly on the passengers' responses as they make the ultimate decision to stay longer than planned in the nodes. Whereas Chapter 6 considered exclusively the passengers' profile, Chapter 7 focused on the behaviour of passengers while in the gateways and the likelihood of them extending their stays there. The interviews conducted with tourism businesses and organisations were structured in such a way as to give support to the passengers' responses, particularly trying to identify from the suppliers' perspectives ways to make travellers stay longer in the gateways. As shown in this research, while a small but significant number of passengers would be keen to extend their stays in the nodes if more information or a special deal had been offered to them, the suppliers were not prepared to make any suggestions on how this could be done. 


\section{CONCLUSION}

Particularly in terms of the primary data collection process, some of the limitations encountered in this research were:

- The passenger questionnaire was only provided in English. Some Asian (mainly Japanese) and European (French and German) travellers declined to take part in the research due to language constraints;

- Passenger data collection occurred between the months of July and January. No surveys were conducted between February and June. Passengers travelling between midnight and 8am were also not interviewed. Due to this, a small but different type of traveller is probably not represented in this research;

- Identifying tourism businesses in Wellington whose operation was intrinsically related to the Cook Strait ferries was not an easy task. Hence the higher number of interviewees from Picton;

- Only gateway ports were considered in this thesis. Different outcomes may be possible for inland gateways, such as airports and land transport (road and rail);

- Residents' perspectives were not included in this study.

Apart from these more general issues about the methodology, this research has also made an important contribution in terms of the operationalisation of the travellers' functions. This is going to be discussed in more detail in the next sub-section.

\subsubsection{Operationalisation of travellers' functions}

From the review of the literature it was possible to note that while some studies have established definitions of gateways and destinations, very few have tried to operationalise these concepts. Pearce and Elliott (1983) developed the Trip Index which compared the number of nights the traveller is spending in a certain place with the total number of nights spent in the whole trip. While the Trip Index is useful in order to better understand the importance one particular node has in comparison with the other nodes existing in a traveller's trip, it only provides a classification that is relative to how travellers perceive a given node. The reference is in terms of how the total number of nights is distributed among the various nodes. For example, two travellers staying three nights in a node, going there for identical reasons, doing the same activities and staying in similar types of accommodation can have different classifications according to the total number of nights spent in the whole trip. If one of the travellers in the previous example was staying five nights away from home, this particular node would be his principal destination ( $\mathrm{Ti}=60)$, while the other traveller, who was away from home for 30 nights, would have this 


\section{CONCLUSION}

particular node classified as a short stopover $(\mathrm{Ti}=10)$. From the travellers' perspective towards each individual node it is clear that there is a need to find other ways to set up a classification that takes into consideration other variables and classifies the nodes in a more autonomous way. Another study that aimed to look into the relationship between travellers and the places visited by them was done by Lew and McKercher (2002). Based on Oppermann's (1995) tourist flow patterns model, it classifies destinations based on the order they have in the traveller's trip. For example, a gateway destination is classified exclusively on the basis that this is the point of access/entry, regardless of the number of nights, the purpose for going there or any other variable. These definitions are very limited, particularly for two gateways located in the middle of a country such as New Zealand that is characterised by circuit travel.

Both classifications presented above use only one variable to classify the nodes: the number of nights, in the case of the Trip Index, and the position of the node in the route itinerary, for Lew and McKercher's forms of relationship. However, from the literature review of the concept of gateway it was possible to identify many more dimensions and elements that are associated with the concept of a gateway. Figure 3.1, for example, presented four dimensions that are related to gateways; i.e. transport intermodality, catchment area, purpose of trip and length of stay. Using mainly the last two, Figure 3.2, in an empirical approach, proposed four different types of nodal functions: gateway traveller, stopover visitor, overnight gateway visitor and destination tourist. While the term 'gateway traveller' and 'destination tourist' have already been used in the literature, there was no previous attempt to operationalise these concepts. Gateway travellers were classified as those passengers that present little interaction with the node; i.e. they are not staying overnight and the inter-island connection is their main reason for going there. Destination tourists, on the other hand, present a high level of interaction, with two or more nights being spent there. In the case of single destination trips, $\mathrm{Ti}=100$, as travellers were staying only one night away from home, they were also classified as a destination tourist for this particular node. The other two concepts, stopover visitors (SV) and overnight gateway visitors (OGV), were developed as intermediary functions between gateway travellers and destination tourists.

A deductive approach was used in the operationalisation of these four categories of passengers. Basically two factors were considered in the development of the travellers' functions: the main reason for going to the node and their length of stay there. From the results obtained from the questionnaires it seems that the different functions were useful in order to understand the passengers' behaviour while in Wellington and Picton and the likelihood of them extending their stay. Even though other ways of operationalising these 


\section{CONCLUSION}

functions could have been proposed (e.g. including the activities done by travellers while in the node), the one presented here is simple and was able, in some cases, to identify strong features for each function. One clear example is the participation of passengers in terms of activities undertaken and attractions visited for the various nodal functions proposed. Tables 7.11 and 7.23 show that in general destination tourists are those passengers more involved in activities and attractions in both nodes, followed by stopover visitors, overnight gateway visitors and finally gateway travellers. It seems that the key variables chosen to operationalise the four nodal functions, length of stay and purpose of trip, are fundamental in establishing the relationship the travellers have with the node, as the reason for going to the node and the time spent there can significantly impact on what travellers do while at the gateway.

The four categories of functions also helped to better understand the nodal functions existing in the two nodes studied in this research. According to Figure 3.4, in both nodes gateway travellers were the major group of passengers, even though their presence in Picton $(67 \%$ of the sample) was significantly larger than in Wellington $(26.8 \%$ of the sample). Conversely, it was possible to conclude that the destination function in Wellington is nearly three times bigger than Picton, as $24.1 \%$ of passengers were classified as destination tourists in the capital city, in comparison to only $7.8 \%$ passengers in Picton.

In addition, the four nodal functions proposed in this research were also useful when analysing the core research question, which is how to make ferry passengers stay longer in Wellington and Picton. Having more nodal functions than just gateway travellers and destination tourists permitted a detailed analysis of the sample in terms of their likelihood of extending their stays in Wellington and Picton if they had more information on destinations or a special deal. From the results presented in Chapter 7 one could note that overnight gateway visitors and stopover visitors were the two functions where passengers were keener to stay longer in both nodes, particularly international passengers among these two functions. Gateway travellers, on the other hand, may be more determined to reach their final destinations rather than visiting the gateways and their level of interaction with the tourism industry.

\subsection{Objectives Revisited}

Chapter 1 presented three main research questions associated with the topic of gateways. This section deals with each of them in a separate sub-section. 


\section{CONCLUSION}

\subsubsection{How can passing by passengers be persuaded to visit and stay in gateways?}

The questionnaires delivered to passengers on board the ferries asked them whether or not more information, a special deal or any other factor would make them extend their stays in Wellington or Picton. While some passengers stated that better or more accommodation, an event/festival or a "must-see" attraction would have increased their length of stay in both nodes, more information and particularly a special deal would have made spending more time in the nodes more appealing.

Three major conclusions can be obtained from these results. The first is that considering the passengers' response to the incentives offered to encourage them stay longer in Wellington and Picton, at least $80 \%$ of ferry passengers would not have spent more time in either of these two gateways. This shows that persuading travellers to extend their stay in gateways is not an easy task. This means that these passengers are more interested in reaching their final destinations or simply returning home, rather than extending their stays in the gateways.

The second conclusion is that those passengers that are keen to stay longer in the gateways can make a significant contribution to boost the destination function of Wellington and Picton. For example, if more information had been provided, $12 \%$ of the ferry passengers would have stayed longer in Wellington; while in Picton 6.6\% would do the same. Considering that approximately 1.2 million passengers use the Cook Strait ferries every year, this means that the capital city could have 144,000 visitors extending their stays, while Picton would have 79,200. A special deal would have made one out of five passengers to stay longer in Wellington and $13 \%$ of passengers to do the same in Picton. This represents 240,000 visitors staying longer than planned in Wellington and nearly 155,000 in Picton. This shows the potential increase in visitor stays if each individual strategy (more information or a special deal) were offered. If both strategies were used, the increase in visitor stays would not be the sum of each individual strategy as some passengers stated that either a deal or more information would have made them stay longer. Table 9.1 shows the number of passengers who stated that they would have stayed longer in Wellington or Picton if more information, a special deal or both were offered. It also shows for each case the percentage in terms of the total sample and the number of passengers this would represent in terms of the total ferry passenger population. 


\section{CONCLUSION}

Table 9.1 Impact of more information, a special deal or both strategies would have on visitor numbers in Wellington and Picton.

\begin{tabular}{|c|c|c|c|c|}
\hline & \multirow[b]{2}{*}{$\begin{array}{c}\text { If only more } \\
\text { information was } \\
\text { offered }\end{array}$} & \multirow[b]{2}{*}{$\begin{array}{c}\text { If only a special } \\
\text { deal was } \\
\text { offered }\end{array}$} & \multirow[b]{2}{*}{$\begin{array}{l}\text { If more information } \\
\text { and a special deal } \\
\text { was offered }\end{array}$} \\
\hline & & & & \\
\hline \multirow{2}{*}{ 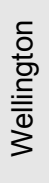 } & $\begin{array}{l}\text { Passengers' response }(\% \text { in } \\
\text { terms of total sample } n=1,076)\end{array}$ & $131(12.2 \%)$ & 220 (20.4\%) & 254 (23.6\%) \\
\hline & $\begin{array}{l}\text { Total visitors extending their } \\
\left.\text { length of stay - per year }{ }^{*}\right)\end{array}$ & 144,000 & 240,000 & 283,200 \\
\hline \multirow{2}{*}{$\frac{.}{0}$} & $\begin{array}{l}\text { Passengers' response }(\% \text { in } \\
\text { terms of total sample } n=1,076)\end{array}$ & $71(6.6 \%)$ & $140(13.0 \%)$ & $156(14.5 \%)$ \\
\hline & $\begin{array}{l}\text { Total visitors extending their } \\
\text { length of stay - per year }{ }^{(*)}\end{array}$ & 79,200 & 155,000 & 173,977 \\
\hline
\end{tabular}

${ }^{(*)}$ Considering the percentage of passengers extending their stays in terms of the total passenger ferry traffic per year of 1.2 million passengers.

One aspect to explain the higher chance of passengers deciding to stay longer in Wellington than in Picton can be associated with the fact that Wellington has a more developed destination function, with more attractions and activities to appeal to potential tourists. What is more, the capital city probably has more funds to promote itself and provide passengers with more information, in case the arrangement of deals with the ferry operators does not work.

The third major conclusion is that there are some segments of passengers that are keener to extend their stays in the nodes than others. In terms of the nodal functions, overnight gateway visitors and stopover visitors are the two functions that are more likely to have passengers extending their stays both in Wellington and Picton if more information or a special deal were offered to them. As passengers classified in these two functions are not staying more than one night in the nodes, the fact that these are the two groups of passengers with the highest chances of increasing their stays in the gateways is very promising, particularly emphasizing that they believe the nodes have more to offer, hence the need to stay longer. Regarding the passengers' place of origin, it is interesting to notice that those from overseas are keener to extend their stays in both nodes than passengers living in New Zealand (CSNZ and domestic). According to Table 3.3, approximately $41 \%$ of the sample is made of international passengers. However, international passengers account for $82.1 \%$ of those extending their stays if they had more information about Wellington (Table 7.14), 61.8\% if a special deal was offered in the capital city (Table 7.15), 73.2\% if they had more information about Picton (Table 7.26) and $50.4 \%$ if they had a special deal in Picton (Table 7.27 ).

Even though creating marketing strategies to target some of these markets is beyond the scope of this research, it is possible to affirm from data collected in the questionnaires and 


\section{CONCLUSION}

from the interview with the manager of the Centre Stage of New Zealand marketing organisation, that travel agents have a key role in the booking of ferry tickets (see Table 6.12), even though they are one of the least used source of information for Wellington (Table 7.10) and Picton (Table 7.22). Therefore, one recommendation that can be made to marketing managers of tourism businesses and organisations is to identify together with the ferry operators who the major travel agents that book and sell this mode of transport are so that more information about both ferry ports could be disseminated to them. Travel agents could then pass this information to ferry passengers which could make them more interested in Wellington and Picton and hopefully make them stay longer there.

\subsubsection{How can partnerships among transport and tourism suppliers improve tourism in gateways?}

In spite of the positive response obtained from some segments of passengers who would be keen to extend their stays in the gateways, it seems, from the suppliers' perspective, that there are major difficulties and constraints in improving the partnership between ferry operators and tourism providers to achieve this aim. A common problem in Wellington and Picton is that the tourism sector in both places lacks the necessary resources to develop specific combined products that could make passengers stay longer.

In general terms, Wellington relies particularly on attractions that are funded by the public sector. What is more, due to the lack of commissionable products in the capital city, travel agents and tour operators prefer to broker other destinations and products, where they can earn extra revenues from the recommendations they are making.

Picton faces similar problems, even though they are on a different scale. Firstly, the Marlborough region has a smaller population, with fewer resources to be spent on marketing campaigns, with most of the promotion and investments being made in Blenheim and the vineyards located around the region's major town. Secondly, most of the businesses located in Picton are very small ones, usually being owned by couples. Thirdly, some current and past issues seem to have affected the relationship between the tourism industry in Picton and the ferry companies, notably the Interisland Line. The dispute about the ferries' speed restriction that took place several years ago, the constant threat of the ferries moving to Clifford Bay and the discussion about the fact that the Interisland Line charges a higher commission compared to businesses that book a ferry for their clients make it difficult to propose partnerships that would involve the ferries and the whole community in Picton.

Apart from these more specific issues, there are two other difficulties in terms of developing possible partnerships between transport and tourism providers. This difficulty 


\section{CONCLUSION}

occurs despite the huge importance both sectors have for each other, with transport providing destinations with tourists and tourism enterprises and organisations stimulating the use of several means of transport so visitors can reach and visit tourist destinations. The first is that both tourism providers and ferry companies do not consider that it is necessary that the ferries have to be involved in the organisation of strategies to increase the length of stay of traffic passing by the gateways. Some interviewees think that the role of the ferries is to provide travellers with access between the islands, with possible packages being developed by the information centres or the accommodation sectors. One of the ferry managers interviewed also stated something similar, when he considered that his company provides Marlborough and Wellington regions with thousands of passengers every week, it being up to these regions to do their best to attract visitors to stay and visit their main attractions and destinations. The second main difficulty associated with the elaboration of possible packages that would attract passengers to stay in the gateways, particularly in Picton, is that usually when the interviewees heard about deals or packages they thought about the current packages that the Interisland Line has. The problem is that these packages are primarily designed to attract people living in Wellington to have a short break in the Marlborough region, rather then aiming to persuade the passing through traffic to stay longer in the gateways. None of the interviewees was able to suggest any ideas for increasing the length of stay. In fact, some of them said that the best way to improve the passengers' stay in Wellington and Picton would be simply more promotion of what these nodes have to offer, rather than creating anything new. However, as mentioned earlier, it is not an easy task to set up marketing and promotional strategies to improve the travellers' length of stay in the gateways if there are no funds to support it. It seems that while they have proposed some answers to this question, in practical terms they do not foresee a way to implement them.

\subsubsection{How would passengers and business react if a second gateway existed? What would happen to Picton if the Clifford Bay proposal becomes a reality?}

As discussed in the review of the literature, one of the characteristics of a gateway is the influence it has over a certain catchment area. Gateways provide access to and from their catchment areas, usually offering some sort of advantage for the travellers using it. When there is only one gateway to a given region, visitors and local population have no choice but to use this particular point of entry/exit. This is the case of inter-island traffic in New Zealand for those who decide not to fly, as there is only one gateway to the North (Wellington) and South (Picton) Islands. In a situation like this, when a new gateway is created, travellers then have an option to choose which gateway is better for them. In the 


\section{CONCLUSION}

case of the South Island, a proposal to establish a ferry port in Clifford Bay has been discussed for many years. The key advantages of the Clifford Bay proposal are the possibility of ferries crossing without a speed limit and the proximity to Christchurch, the most important city in the South Island. Losing the exclusiveness of the ferry gateway in the South Island would have various implications for Picton, as the decrease in the passenger traffic would certainly mean less potential customers for a number of businesses located there.

The analysis of the impacts on Picton of the Clifford Bay proposal for this research was done from the passengers' and the suppliers' perspectives, as indicated by the two research questions posed above. While a general consideration of the passengers' responses shows that nearly half of the passengers would prefer to travel via each of the two gateways (see Figure 8.1), differences can be clearly identified among the various nodal functions. For example, nearly $60 \%$ of current gateway travellers to Picton would have preferred to travel via Clifford Bay. On the other hand, approximately $70 \%$ of destination tourists would still travel to Picton. The appeal of a faster sea crossing being closer to where the passenger is going (Table 8.2) would make Clifford Bay the best option for those passengers that are just interested in travelling from one place to another. Nonetheless, destination tourists seem to be more interested in Picton, particularly due to the proximity to their destination, the scenery of the trip and the fact that Picton is their destination. Despite the strong gateway function existing in Picton (according to Table 3.4 two-thirds of passengers were classified as gateway travellers in Picton), it seems that Picton would appeal more to destination tourists than gateway travellers if the ferries were also operating to Clifford Bay.

In fact, one of the consequences of the Clifford Bay proposal threat has been that many tourism businesses and organisations, together with Marlborough Regional Council, have undertaken some urban development and marketing initiatives to improve and promote Picton as a destination.

The opinion of the interviewees regarding the impact the Clifford Bay proposal would have on Picton was quite diverse. A group of managers and owners believe that some businesses such as restaurants, cafes and roadside motels will close as they rely particularly on the traffic passing by to make profit. They said that Picton without the ferries would be like the winter season and that it would take between three to five years for the place to recover. Another group of interviewees mentioned that not having the ferries and the port facilities and infrastructure would make Picton an even more appealing destination. 


\section{CONCLUSION}

\subsection{Further Research Suggestions}

A number of significant issues have arisen as a result of this research, which would contribute to and enhance understanding of the conversion of gateway travellers into destination visitors. However, the characteristics of the study area might have affected the results obtained here. There are several factors that may have influenced the outcomes of this research. They include, for example, the fact that the Centre Stage of New Zealand region is not part of the 'golden route' usually visited by most international visitors, the short duration of the ferry trip between the two gateways (nearly three hours) and the way the tourism sector is structured in Wellington - with a small number of commissionable products with some of the major attractions relying on public funds - and Picton - with mostly small businesses operating there. Other issues that are specifically related to the study case area are:

1. The touring pattern followed by international visitors while in New Zealand, which increases competition among various destinations for the visitors' stay;

2. The relationship between the main ferry provider, the Interisland Line, and some business owners and managers located in Picton could have decreased the chances of involving the whole community there to work together in order to set up strategies to improve the passengers' length of stay.

It would be interesting to compare the results obtained from this research to other ferry and non-ferry gateways around the world. In the case of other ferry gateways or any other pair of gateways where a mode of transport connects both nodes, it is possible that the different spatial and nodal configurations of the gateways can impact on the results obtained. For example, in the case of this research, the configuration of Wellington and Picton according to Figure 2.6 is similar to the situation " $f$ " presented there. It might be interesting to analyse whether or not the possibilities of persuading passengers to stay longer would increase for the other six configurations proposed there. In the cases where both gateways have similar type of functions, such as the examples "e" (strong gateway and destination functions in both nodes) and " $g$ " (strong origin, gateway and destination functions in both nodes) illustrated on Figure 2.6, it could happen that either both nodes will compete between themselves for the passengers' stay or simply the opposite, as the presence of two nodes with strong destination functions can stimulate travellers to visit both locations if they are promoted together as a cluster.

Another aspect to be considered in the case of ferry gateways is the ferry service itself. Variables such as the length of the journey may have an impact on the decision to stay 


\section{CONCLUSION}

overnight in the gateway. For example, considering the case of the ferry linking Melbourne to Tasmania, in Australia, where journeys take at least 10 hours and the vessels have cabins for passengers to sleep in, it may happen that the transport provider becomes a competitor with the accommodation sectors located in both ferry ports.

Other possible types of gateways where investigation could be done in order to identify whether or not similar patterns exist in comparison with those obtained in this thesis are nodes where travellers are either not required to change to another mode of transport (e.g. road transport) or only one change is necessary (e.g. airports). In the case of ferry gateways, two nodes exist with travellers having to change modes of transport at least twice, even if they are taking their vehicles on board the ferries. The presence of two gateways may or may not facilitate travellers to stay longer there. In fact, it may happen that the existence of two gateways, as in the case of Wellington and Picton, make them compete with each other for the travellers' stay. In the case of gateways that exist as a consequence of air transport, it would be interesting to consider whether airlines are better prepared to stimulate traffic to stay in the gateways. In fact, in some countries such as Singapore and the United Arab Emirates, the national airlines are used to induce passengers passing through their main airports to stop and visit them as tourist destinations by providing travellers with incentives. In some cases it may involve huge discounts on accommodation, as in the case of Dubai, or a free sightseeing service such as that provided by Changi Airport to those passengers staying at least five hours in Singapore. Are airlines, as large organisations with the budget and the distribution network to promote their services and main destinations through the world, better prepared to convert gateway travellers into destination tourists than other modes of transports, such as the ferries? With respect to road gateways, as travellers do not need to change modes of transport, what sort of challenges does this pose to these nodes in order to make passing through traffic stop and visit them? It would be fascinating to compare the answers for these questions with the results obtained in this research.

Another possible situation to be studied is to consider what happens to previous single gateways when a new gateway is developed in its catchment area. In the case of this research this issue has been dealt within a scenario perspective, as the Clifford Bay terminal is only a proposal. An analysis of what happened with previous experiences in other places could benefit the suppliers, particularly those located in Picton, as they would be better prepared to consider whether losing the gateway function could in fact benefit the node with a strong destination function where visitors would tend to stay longer rather than just pass through. 


\section{CONCLUSION}

\subsection{Conclusion}

This study is an initial contribution to a more applied analysis of the concept of gateways and how to make passing by passengers stay and visit this kind of node. Two major conclusions can be made about this topic regarding the studied area. The first is that even though most travellers are not interested in taking advantage of the fact that they are passing through a gateway to visit and stay, there is a small but still significant part that would be prepared to do so if they had been offered more information about the nodes or a special deal including the ferry trip and some sort of accommodation. As the Centre Stage of New Zealand region is not part of the 'golden-route' that consists of AucklandRotorua-Christchurch-Queenstown, which is particularly popular for international visitors, it seems that a good strategy to boost the destination function of Wellington and Picton is to target ferry passengers to take advantage of passing by these nodes to visit them. The second main conclusion from this research is that creating the conditions to attract these potential visitors is not always easy, particularly due to the lack of funds in both nodes and the difficulties of relationship that exist, notably in Picton, between the local tourism businesses and the major ferry operator, the Interisland Line. Nevertheless, what this research was able to show is that there are some markets that are interested in extending their stays in both nodes if they had more information or a special deal including transport and accommodation. 
Appendix One:

Stakeholders' Semi-structured interview 


\section{INFORMATION SHEET}

My name is Gui Lohmann and I am a Brazilian PhD student from Tourism Management at Victoria University of Wellington. I am doing my thesis on Cook Strait ferries and the development of tourism in Wellington and Picton. At this stage of the project I am interviewing key tourism and transport stakeholders on the Centre Stage of New Zealand region.

The purpose of this interview is to find out how tourism in Wellington, Picton and the whole Centre Stage of New Zealand region can be improved by attracting transient Cook Strait ferry passengers to visit and stay in the Centre Stage region. This interview is designed to take no more than one hour.

The project will contribute to an understanding of passengers' behaviour in gateway destinations and consequently to maximise the possibilities to convert a gateway into a destination. This project is being supported by Victoria Management School, the Brazilian Ministry of Education and the ferry operators.

All information provided will be accessed only by members of the research project (supervisor and researcher), stored securely and used only for the purposes of this project. The results of this research will be reported in academic publications and at academic workshops and conferences. A copy of the finished thesis will be held in the University archive. Consent is sought to attribute information to the organisations that individuals represent. All data collected will be destroyed 2 years from the completion of the project. Feedback from the thesis is available after completion of the project in Winter 2005.

If you have any questions or would like to receive further information about the project, please contact either me, Gui Lohmann at (04) 463-6972 - e-mail gui.lohmann@vuw.ac.nz, or my supervisors, Dr Mondher Sahli at (04) 463-5718 - e-mail mondher.sahli@vuw.ac.nz and Prof Doug Pearce at (04) 463-5715 - e-mail douglas.pearce@vuw.ac.nz.

Thank you for taking the time to participate in this interview! 


\section{CONSENT FORM}

I have read and understood the accompanying information sheet and I agree to provide information under these conditions.

I $\square$ allow / $\square$ do not allow * the attribution of information to my organisation in the reporting of this research (If you do not have authority to give consent for use of the organisation's name, please refer the name of someone that does).

$I \square$ allow $/ \square$ do not allow * the attribution of information to myself.

I understand that I may withdraw from participating in this project at any stage, before the final analysis of data (March 2004), without any penalty or requirement to provide any reason. I understand that if I withdraw from the project any data I have provided will be returned to me or destroyed.

Moreover, I understand that the information I have provided will be used only for this research project (and related academic publications/conferences) and that any further use will require my written consent. In addition, I understand that when this research is completed the information obtained will be destroyed within 2 years.

Finally, I $\square$ would / $\square$ would not like * to receive a summary of the results of this project.

${ }^{*}$ Please tick as appropriate

Name:

Position:

Organisation:

Address:

Date:

Signed: 


\section{SEMI-STRUCTURED INTERVIEW CHECKLIST}

1. How important is the ferry service to the tourism development in Wellington and Picton?

2. Do you think the Cook Strait ferry benefits the Wellington / Picton tourism sector? What are the main benefits?

3. How well are Wellington and Picton benefiting or not from their gateway functions to the North and South Islands to develop tourism in their catchment areas?

4. What is the current importance of the ferry passenger to your own business / organization? Does your company/organization have any kind of partnership with any of the ferry companies? What can be improved?

5. What can be done, in terms of partnership between the ferry operators and the tourism sector (suppliers and organizations), in order to develop more tourism in Wellington and Picton?

6. Which are the main issues to overcome in order to achieve a better integration between the ferry services and the tourism / transport suppliers and organizations?

7. How can your organization contribute to this integration? In which areas can your organization have a greater impact in terms of helping to convert the gateway function of Wellington and Picton into a destination one? What have you been doing until now?

8. Which of the ferry operators (The Interisland Line and Bluebridge) is better prepared for this kind of partnership? Why?

9. The Interisland Line used to offer 'The Lynx Weekend Escape' package which included transport, accommodation and attractions (e.g. fishing trips, vineyards, ski etc) in one single product. Since last year, this product is not offered any more. One of the main reasons for that is the lack of a common reservation system to integrate all the suppliers. What can be done in the future in order to promote and stimulate products like that? Are they viable? What are the main problems?

10. What is your opinion about the speed restriction applied on the fast ferries? Do you think tourism / short break trips are impacted by it?

11. What is your general opinion about the proposal to build another ferry terminal in Clifford Bay (South Island)? Do you believe it is going to happen? Which regions within the Centre Stage of New Zealand (Wellington, Wairarapa, Nelson and Marlborough) will benefit from it? Which will not? What would happen specifically to Picton? 
Appendix Two:

Passenger Self-completion questionnaire 


\section{The Role of Transport in Tourism Development}

Hello!

My name is Gui Lohmann and I am a Brazilian PhD student from Tourism Management at Victoria University of Wellington. I am doing my thesis on Cook Strait ferries and the development of tourism in Wellington and Picton. At this stage of the project I am interviewing ferry passengers to get details about their trip.

This project is being supported by Victoria Management School, the Brazilian Ministry of Education and the ferry operator.

This questionnaire is designed to take about 10-15 minutes. Your responses are anonymous and will be used as part of my research. Aggregate data will be published in the form of the PhD thesis, conference and journal papers and seminars. All information provided will be accessed only by my supervisors and me, stored securely and used only for the purposes of this project. Moreover, all data collected will be destroyed two years from the completion of the project. Please, remember that you do not have to answer all the questions, especially the personal ones at the end of the questionnaire. Completion of the questions implies consent to take part under these conditions.

If you have any questions or would like to receive further information about the project, please contact either me, Gui Lohmann at (04) 463-6972 - e-mail gui.lohmann@vuw.ac.nz, or my supervisor, Prof Doug Pearce at (04) 463-5715 - e-mail douglas.pearce@vuw.ac.nz.

Thank you for taking the time to answer the questionnaire!

Once you have completed it, please, return it directly to me or leave it on the questionnaire box located on the Information Desk.
1. Herthlamed \& Aucchand

2 Waild

Comondel Pertinusula, Thanes Valey

3. Bay of Plenty

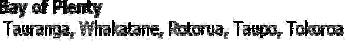

4. Eastcape

Tarmated

New Ptymouth, Taunaranusis Hawera

6. Haskes Bay

7. Manawates \& Wangani

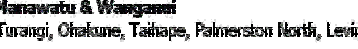

8. Whirarapa

9. Kaplat Coast

, Wikanas, Otat

16. Hutt Yalley \& Waldulcomata

11. Weltingten

12. Harthorough Soumrats

Pitsen Haveladk

13. Blemplom

14. Mdson $\mathrm{s}$ Tasman

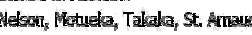

15. Matheraugh

Grassmere, kaklous

16. West Caast

Wespart, Murchiesn, Geynouth, Hasidila

17. Cantertury

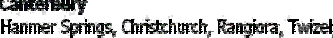

Timanu 2 Southand

Camern, Dunedit, Queentomi, Imercargil, 


\section{SECTION A: ABOUT YOUR TRIP}

1 What is the main purpose of your current trip? (tick only one box)

1. $\square$ Leisure / Holiday 4. $\square$ Conference / Seminar 7. $\square$ Moving house

2. $\square$ Education $\quad$ 5. $\square$ Visiting Friends / Relatives $\quad$ 8. $\square$ Othe

3. $\square$ Business / Working 6. $\square$ Event (Art, Sport etc)

2 When did you decide to make this journey? (tick only one box)

1. $\square$ This week $\quad$ 3. $\square$ Within the last 4 weeks $\quad$ 5. $\square$ Within the last 6 months

2. $\square$ Within the last 2 weeks $\quad$ 4. $\square$ Within the last 2 months $\quad$ 6. $\square$ Six or more months ago

3 How much planning have you undertaken for this trip? (tick only one box)

1. $\square$ Everything well planned / pre-booked $\quad$ 3. $\square$ Mainly flexible with some firm bookings

2. $\square$ Mainly firm bookings with some flexibility $\quad$ 4. $\square$ Totally flexible

\section{To answer questions 4 to 7 , please use the map on opposite page}

4 Please, write in the number of the area where you stayed last night

5 Please, write in the number of the area where you are staying tonight
6 Please, tick the boxes of the regions where you spent / are going to spend at least one night during this trip.
1. $\square$
4. $\square \quad 5$.
5. $\square$
. 7
8.
9.
11. $\square \quad 12$
13.
14.
15.
16.
17.
18.

10.

\section{SECTION B: FOR PASSENGERS LIVING IN NEW ZEALAND ONLY}

7 Please, write in the number of the area where you live

8 How many nights away from home are you spending on your current trip?

9 How often do you take the Cook Strait ferry? (tick only one box)

1. $\square$ This is my first trip! $\quad \square_{\text {3-4 times per year } \quad \text { 7. } \square \text { Twice a month }}$

2. $\square$ Once a year $\quad$ 5. $\square$ 5-8 times per year $\quad$ 8. $\square$ Every week

3. $\square$ Twice a year $\quad$ 6. $\square$ Every month $\quad$ 9. $\square$ I don't travel on a regular basis

Now go to question 13

SECTION C: FOR PASSENGERS LIVING OVERSEAS ONLY

10 What country do you live in? 
11 At which cities did you enter New Zealand and will you leave New Zealand?

\begin{tabular}{|lrc|}
\hline \multicolumn{1}{|l}{ Entered NZ } & Will leave NZ \\
Wuckland & 1. $\square$ & 1. $\square$ \\
Christchurch & 2. $\square$ & 2. $\square$ \\
Other & 3. $\square$ & 3. $\square$ \\
\hline
\end{tabular}

12 How many nights do you expect to stay in New Zealand? nights.

\section{SECTION D: ABOUT YOUR CURRENT FERRY JOURNEY (This Section Is For Everyone)}

13 Which means of transport did you use to arrive in Wellington? (tick only one box)
1. $\square$ I live in Wellington $\quad$ 4. $\square$ Scheduled Bus 7. $\square$ Ferry
2. $\square$ Rental Car / Campervan $\quad$ 5. $\square$ Tour Coach $\quad$ 8. $\square$ Airplane
3. $\square$ Private Car / Campervan $\quad$ 6. $\square$ Train 9. $\square$ Other

14 Which means of transport are you using to depart from Picton? (tick only one box)
1. $\square$ I live in Picton
4. $\square$ Scheduled Bus 7. $\square$ Ferry
2. $\square$ Rental Car / Campervan $\quad$ 5. $\square$ Tour Coach $\quad$ 8. $\square$ Airplane
3. $\square$ Private Car / Campervan 6. $\square$ Train 9. $\square$ Other

15 What is the main reason for travelling by ferry? (tick only one box)

$$
\begin{array}{lll}
\text { 1. } \square \text { Price / fare } & \text { 4. } \square \text { Ability to take vehicle } & \text { 7. } \square \text { Scenery / experience } \\
\text { 2. } \square \text { Comfort } & \text { 5. } \square \text { Total travel time } & \text { 8. } \square \text { Vessel facilities / technology } \\
\text { 3. } \square \text { Safety } & \text { 6. } \square \text { Time of departure } & \text { 9. } \square \text { Other }
\end{array}
$$

16 Please, rate (1-5) the following factors when using the Cook Strait ferry. (tick

\begin{tabular}{|l}
\begin{tabular}{|lccccc|}
\hline \multicolumn{1}{|c}{$\begin{array}{c}\text { Not } \\
\text { Important }\end{array}$} & Neutral & $\begin{array}{c}\text { Very } \\
\text { Important }\end{array}$ \\
a) Total travel time & $1 \square$ & $2 \square$ & $3 \square$ & $4 \square$ & $5 \square$ \\
b) Fare & $1 \square$ & $2 \square$ & $3 \square$ & $4 \square$ & $5 \square$ \\
c) Smooth crossing & $1 \square$ & $2 \square$ & $3 \square$ & $4 \square$ & $5 \square$ \\
d) Scenic trip & $1 \square$ & $2 \square$ & $3 \square$ & $4 \square$ & $5 \square$ \\
e) Cancellation / trip delay & $1 \square$ & $2 \square$ & $3 \square$ & $4 \square$ & $5 \square$ \\
\hline
\end{tabular}
\end{tabular}

17 How did you book your ferry ticket? (tick only one box)

$$
\begin{aligned}
& \text { 1. } \square \text { Travel agent 3. } \square \text { Railway station } \quad \text { 5. } \square \text { Internet } \quad \square \text { Other } \\
& \text { 2. } \square \text { Ferry terminal } \quad \text { 4. } \square \text { Telephone (0800) 6. } \square \text { I don't know. }
\end{aligned}
$$

18 How long in advance did you book your ferry ticket? (tick only one box)

$$
\begin{array}{llll}
\text { 1. } \square \text { Today } & \text { 3. } \square_{3-6} \text { days } & \text { 5. } \square_{15-30 \text { days }} \text { 7. } \square_{4-8} \text { months } \\
\text { 2. } \square_{1-2 \text { days }} & \text { 4. } \square_{7-14 \text { days }} \text { 6. } \square_{1-3} \text { months } & \text { 8. } \square>8 \text { months }
\end{array}
$$


19 Where did you book your ferry ticket? (use the appropriate column whether you live in NZ or overseas)

\begin{tabular}{|c|c|}
\hline For passengers living in NZ & For passengers living overseas \\
\hline $\begin{array}{l}\text { 1. } \square \text { Before leaving my place of residence } \\
\text { 2. } \square \text { During my trip, before arriving in } \\
\text { Wellington or Picton } \\
\text { 3. } \square \text { In Wellington or Picton }\end{array}$ & $\begin{array}{l}\text { 4. } \square \text { In my home country } \\
\text { 5. } \square \text { While on route to New Zealand } \\
\text { 6. } \square \text { In NZ, before arriving in Wellington or Picton } \\
\text { 7. } \square \text { In Wellington or Picton }\end{array}$ \\
\hline
\end{tabular}

20 Had you had more information about Wellington or Picton during the reservation process, would you have changed your length of stay in Wellington or Picton? (tick as many as appropriate)

a. $\square$ Not at all!

b. $\square_{\text {I could have stayed longer in Wellington. } \quad \text { d. }} \square_{\text {I could have stayed longer in Picton. }}$

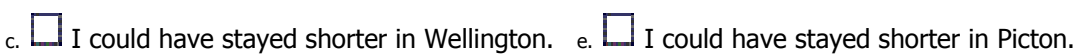

21 Had you had a special deal (including transport and accommodation) for Wellington or Picton during the reservation process, would you have changed your length of stay in Wellington or Picton? (tick as many as appropriate)

a. $\square$ Not at all!

b. $\square_{\text {I could have stayed longer in Wellington. d. }} \square_{\text {I could have stayed longer in Picton. }}$

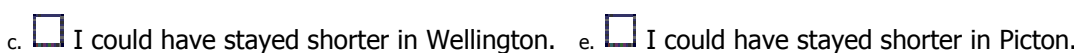

22 Apart from more information and a special deal, is there anything else that, if offered to you, would make you stay longer in Wellington or Picton during your current trip?

\section{In Wellington:}

In Picton:

\section{SECTION E: ABOUT WELLINGTON AND PICTON}

23 What sources of information did you use to find out more about Wellington, Picton and the ferry? (tick as many as appropriate)

\begin{tabular}{|lcrrr|}
\hline & Wellington & Picton & Ferry \\
\hline Commercial guide books (e.g. Lonely Planet) & a. $\square$ & a. $\square$ & a. $\square$ \\
Free visitor guides (e.g. Wellington Visitor Guide) & b. $\square$ & b. $\square$ & b. $\square$ \\
Travel Agent & c. $\square$ & c. $\square$ & c. $\square$ \\
Visitors Information Centres (in NZ) & d. $\square$ & c. $\square$ & c. $\square$ \\
Word of mouth & e. $\square$ & e. $\square$ & e. $\square$ \\
Internet & f. $\square$ & f. $\square$ & f. $\square$ \\
TV, magazine, newspaper \& radio & g. $\square$ & g. $\square$ & g. $\square$ \\
Past experiences / visits & h. $\square$ &.$\square$ & h. $\square$ \\
\hline
\end{tabular}


24 How many times are you travelling - by ferry or plane - between the North and South islands while you are away from home? (tick only one box)

1. $\square$ Only one time (the current Cook Strait crossing only)

2. $\square$ Two times (the current Cook Strait crossing + another Interisland trip by ferry or by airplane)

If you passed / are passing through Wellington and Picton during the other inter-island crossing, please write down the number of nights you spent / are spending there during that occasion:

Wellington: nights Picton: nights

\section{Questions 25 to 32 are about Wellington}

25 What is the main reason for going to Wellington? (tick only one box)

1. $\square$ I live in Wellington $\quad 4 . \square$ Inter-island connection $\quad$ 7. $\square$ Conference / Seminar

2. $\square$ Leisure / Holiday $\quad$ 5. $\square$ Visiting Friends / Relatives $\quad$ 8. $\square$ Shopping

3. $\square$ Business / Working 6. $\square$ Event (Art, Sport etc) 9. $\square$ Other

26 Did you stay last night in Wellington? (tick only one box)

$\square$ No $\rightarrow$ Why did you not stay overnight in Wellington?

Now go to question 28

$\square$ Yes $\rightarrow$ How many nights did you spend there? nights
27 What type of accommodation did you stay in Wellington? (tick only one box)

1. $\square$ Hotel $\quad$ 4. $\square$ Backpacker / Youth hostel $\quad \square$ Private home

2. $\square$ Motel $\quad$ 5. $\square$ Touring caravan / campervan $\quad$ 8. $\square$ Bed and Breakfast

3. $\square$ Camping $\quad$ 6. $\square$ Self catering cottage / flat $\quad$ 9. $\square$ Other

28 What kind of activities did you undertake in Wellington? (please, tick as many as appropriate)

\begin{tabular}{|ll|}
\hline Cultural Activities in Wellington: \\
$\begin{array}{ll}\text { a. } \square \text { Katherine Mansfield Birthplace } & \text { d. } \square \text { Te Papa Museum } \\
\text { b. } \square \text { Parliament / Beehive } & \text { e. } \square \text { Museum of Wellington, City \& Sea } \\
\text { c. } \square \text { City Gallery Wellington } & \text { f. } \square \text { Any other museum }\end{array}$
\end{tabular}

\begin{tabular}{|lll|}
\hline General Activities in Wellington: \\
$\begin{array}{lll}\text { a. } \square_{\text {Café }} & \text { e. } \square \text { Shopping } & \text { i. } \square \text { City Circular (yellow bus) } \\
\text { b. } \square \text { Bar } & \text { f. } \square \text { Walk in the city } & \text { j. } \square \text { Westpac Trust Stadium } \\
\text { c. } \square \text { Nightclub } & \text { g. } \square \text { Business / conference } & \text { k. } \square \text { General / tour sightseeing } \\
\text { d. } \square \text { Cable Car } & \text { h. } \square \text { Eating out / restaurant } & \text { ।. } \square \text { Other }\end{array}$ \\
\hline
\end{tabular}

\begin{tabular}{|lll|}
\hline Outdoor Activities in Wellington: \\
\begin{tabular}{lll} 
a. $\square$ Red Rocks Seal Tour & c. $\square$ Botanic Garden & e. $\square$ Scenic drive \\
b. $\square$ Wellington Zoo & d. $\square$ Kapiti Coast & f. $\square$ Bush walk \\
\hline
\end{tabular}
\end{tabular}

\begin{tabular}{|c|c|c|}
\hline Events in Wellington & 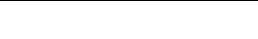 & 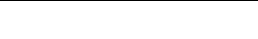 \\
\hline a. $\square$ Sporting events & b. $\square$ Cultural events & c. $\square$ Business events \\
\hline
\end{tabular}


29 Please, rate (1-5) the following statements for Wellington. (tick only one box per line).

\begin{tabular}{|c|c|c|c|c|c|}
\hline & $\begin{array}{l}\text { Strongly } \\
\text { Disagree }\end{array}$ & Disagree & Neutral & Agree & $\begin{array}{c}\text { Strongly } \\
\text { Agree }\end{array}$ \\
\hline a) Wellington fulfilled my expectations & $1 \square$ & $2 \square$ & $3 \square$ & $4 \square$ & $5 \square$ \\
\hline $\begin{array}{l}\text { b) I am satisfied with the quality and } \\
\text { range of attractions in Wellington }\end{array}$ & ${ }_{1} \square$ & $2 \square$ & $3 \square$ & $4 \square$ & $5 \square$ \\
\hline $\begin{array}{l}\text { c) I regret not spending more time in } \\
\text { Wellington }\end{array}$ & $1 \square$ & $2 \square$ & $3 \square$ & $4 \square$ & $5 \square$ \\
\hline $\begin{array}{l}\text { d) Wellington is just a place to take the } \\
\text { ferry }\end{array}$ & $1 \square$ & $2 \square$ & $3 \square$ & $4 \square$ & $5 \square$ \\
\hline
\end{tabular}

30 Did you change your initial plans about your length of stay in Wellington?

1. $\square$ No, my plans were not changed. $\rightarrow$ Go to Question 31

2. $\square$ Yes, I stayed longer. $\rightarrow$ Explain below what made you change them $\downarrow$

3. $\square$ Yes, I stayed for a shorter period. $\rightarrow$ Explain below what made you change them $\downarrow$

31 What is your general impression of Wellington?

32 Please, suggest any potential attractions / events you would like to see developed in Wellington.

\section{Questions 33 to 40 are about Picton}

33 What is the main reason for going to Picton? (tick only one box)

$\begin{array}{ll}\text { 1. } \square \text { I live in Picton } & \text { 3. } \square \text { Inter-island connection } \\ \text { 2. } \square \text { Leisure / Holiday } & \text { 4. } \square \text { Visiting Friends / Relatives } \\ \text { Business / Working } & \text { 6. } \square \text { Other }\end{array}$

34 Are you going to spend this night in Picton?

$\square$ No $\rightarrow$ Why will you not stay overnight in Picton?

Now go to question 36

Yes $\rightarrow$ How many nights are you spending there? nights

35 What type of accommodation are you staying in Picton? (tick only one box)

1. $\square$ Hotel $\quad$ 4. $\square$ Backpacker / Youth hostel $\quad$ 7. $\square$ Private home

2. $\square$ Motel $\quad$ 5. $\square$ Touring caravan / campervan $\quad$ 8. $\square$ Bed and Breakfast

3. $\square$ Camping 6. $\square$ Static caravan / chalet $\quad \square$ Other 
36 What kind of activities are you undertaking in Picton?

\begin{tabular}{ll}
\hline General Activities in Picton: \\
$\begin{array}{lll}\text { a. } \square \text { Walk in the town } & \text { c. } \square \text { Shopping } & \text { e. } \square \text { Eating out / restaurant } \\
\text { b. } \square \text { General sightseeing } & \text { d. } \square \text { Water taxi } & \text { f. } \square \text { Other }\end{array}$
\end{tabular}

\begin{tabular}{|c|c|c|}
\hline \multicolumn{3}{|l|}{ Outdoor Activities in Picton: } \\
\hline a. $\square$ Aquarium of the Marlborough Sounds & e. $\square$ Scenic drive & i. $\square$ Bush walk \\
\hline b. $\square$ Queen Charlotte Track & f. $\square$ Picton museum & j. $\square$ Mini golf \\
\hline c. $\square$ Trekking/tramping & g. $\square$ Beaches & \\
\hline d. $\square$ Scenic boat cruise & h. $\square$ Sailing & \\
\hline
\end{tabular}

37 If you have already been in Picton during this current trip, regardless of the time you have spent there, rate the following statements. (tick only one box per line). Otherwise, go to Section F.

\begin{tabular}{|l|llllll|}
\hline & $\begin{array}{c}\text { Strongly } \\
\text { Disagree }\end{array}$ & & & & $\begin{array}{c}\text { Strongly } \\
\text { Disagree }\end{array}$ \\
& Neutral & Agree & Agree \\
\hline a) Picton fulfilled my expectations & 1 & $2 \square$ & $3 \square$ & $4 \square$ & $5 \square$ \\
\hline $\begin{array}{l}\text { b) I am satisfied with the quality and range of } \\
\text { attractions in Picton }\end{array}$ & $1 \square$ & $2 \square$ & $3 \square$ & $4 \square$ & $5 \square$ \\
\hline c) I regret not spending more time in Picton & $1 \square$ & $2 \square$ & $3 \square$ & $4 \square$ & $5 \square$ \\
\hline \begin{tabular}{l} 
d) Picton is just a place to take the ferry \\
\hline
\end{tabular} & $1 \square$ & $2 \square$ & $3 \square$ & $4 \square$ & $5 \square$ \\
\hline
\end{tabular}

38 Did you change your initial plans about your length of stay in Picton?

1. $\square$ No, my plans were not changed. $\rightarrow$ Go to question 39

2. $\square$ Yes, I stayed longer. $\rightarrow$ Explain below what made you change them. $\downarrow$

3. $\square$ Yes, I stayed for a shorter period. $\rightarrow$ Explain below what made you change them.

39 What is your general impression of Picton?

40 Please, suggest any potential attractions / events you would like to see developed in Picton.

\section{SECTION F: ABOUT A NEW FERRY TERMINAL IN CLIFFORD BAY (SOUTH ISLAND)}

In the last few years, it has been proposed to build another ferry terminal in the South Island. The area chosen is Clifford Bay (see map below). Some of the reasons for this proposal include:

- Clifford Bay is 43 nautical miles from Wellington, while Picton is 52 nautical miles;

- Journey times at sea would be reduced by 30-45 minutes;

- Road travel distance to Christchurch will be shorter, although it will increase for some other destinations (see Table below). However, even to those destinations where the land distance will increase, total travel time (including the ferry trip) will definitively be shorter. 


\begin{tabular}{|l|c|}
\hline Destination & (km) \\
\hline Nelson & +24.1 \\
\hline Blenheim & +16.3 \\
\hline Christchurch & -55.9 \\
\hline
\end{tabular}

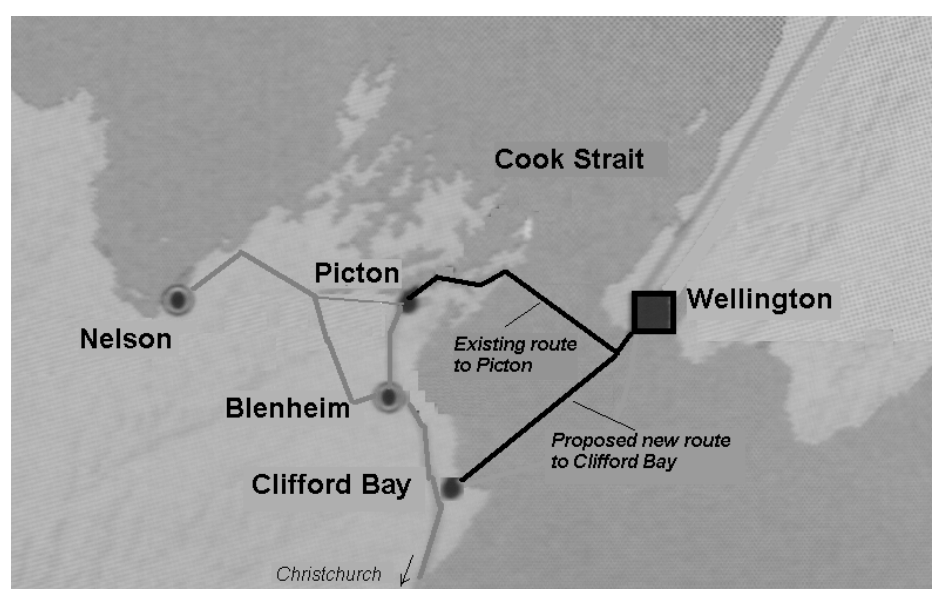

41 Considering the information above and the possibility that ferries would be operating to Picton and Clifford Bay, which route would you have taken in your current trip? (choose only one option)

1. $\square$ Picton $\rightarrow$ Why would you choose to travel via Picton? $\downarrow$

\section{Go to question 42}

2. $\square$ Clifford Bay $\rightarrow$ Why would you choose to travel via Clifford Bay? $\downarrow$ 41.a Which places would you or would you not be visiting in case you had travelled through Clifford Bay?

I would visit:

I would not visit:

\section{SECTION G: ABOUT YOURSELF AND YOUR FAMILY}

42 What is your main occupation? (tick only one box)
1. $\square$ Agricultural/Trade/Manual 4. $\square$ Professional
7. $\square$ Managerial
2. $\square$ Semi Technical / Skilled 5. $\square$ Clerical / Sales
8. $\square$ Student
3. $\square$ Retired / Super annuitant $\quad$ 6. $\square$ Not currently in paid employment $\quad$ 9. $\square$ Homemaker

43 Are you travelling alone?

$\square$ No $\quad \square$ Yes $\rightarrow$ go to question 44

$\downarrow$

43.a How many people (including yourself) are in your group?

43.b With whom are you travelling? (tick as many as appropriate)
a. $\square$ Spouse / Partner
d. $\square$ Friends
g. $\square$ Business associates
๖. $\square$ Dependants
e. $\square$ Educational trip
c. $\square$ other family members
f. $\square$ Tour group
h. $\square$ Club or Organisation
i. $\square$ Other 
45 Which age group do you fall into?
$\begin{array}{ll}\text { 1. } \\ 15-24 & \text { 2. } \square 25-34\end{array}$
3. $\square$ 35-49
4. $\square 50-64$
5. $\square 65+$

46 Please, indicate your highest educational attainment. (tick only one box)
1. $\square$ Primary School
3. $\square$ Vocational / trade qualification
2. $\square$ Secondary School
4. $\square$ Tertiary (university)
5. $\square$ Post-graduate degree
6. $\square$ Other

47 Approximately what is your total annual household income in \$NZ (before tax)?
1. $\square$ less than $\$ 20,000$
3. $\square \$ 35,000$ to $\$ 49,999$
5. $\square \$ 65,000$ to $\$ 89,999$
2. $\square \$ 20,000$ to $\$ 34,999$
4. $\square \$ 50,000$ to $\$ 64,999$
6. $\square$ over $\$ 90,000$

Thank you for taking the time to answer the questionnaire!

Have a nice and safe trip!

For the researcher's use:

Place of Departure: 2. Wellington

Questionnaire \#:

Date:

Time of vessel departure:

1. $\square$ The Lynx

$\begin{array}{ll}\text { 2. } \square \text { The Interislander } & \text { 3. } \square \text { BlueBridge }\end{array}$ 


\section{References}

AA. (2003). Wellington - Wairarapa - Marlborough - Nelson Visitor Guide 2004. Auckland: New Zealand Automobile Association.

Aalst, I. v., \& Daly, C. (2002). International visitors to Wellington. Wellington: PS...Services.

Abeyratne, R. I. R. (1993). Air transport tax and its consequences on tourism. Annals of Tourism Research, 20, 450-460.

Abeyratne, R. I. R. (1999). Management of the environmental impact of tourism and air transport on small island developing states. Journal of Air Transport Management, 5, 31-37.

Aitken-Turner, A., Campbell, K., Chang, A., Davidson, L., \& Roddick, M. (1996). A profile of the arts and crafts and their economic impact in the Nelson region. Wellington: Victoria University of Wellington.

Ashworth, G. J. (1992). Is there an urban tourism? Tourism Recreation Research, 17(2), 3-8.

Avraham, E. (2000). Cities and their news media images. Cities, 17(5), 363-370.

Baird, A. (1999). A comparative study of the ferry industry in Japan and the UK. Transport Reviews, 19(1), 33-55.

Baird, A. (2000). The Japan coastal ferry system. Maritime Policy Management, 27(1), 316.

Baloglu, S., \& Mangaloglu, M. (2001). Tourism destination images of Turkey, Egypt, Greece, and Italy as perceived by US-based tour operators and travel agents. Tourism Management, 22(1), 1-9.

Baloglu, S., \& McCleary, K. W. (1999). A model of destination image formation. Annals of Tourism Research, 26(4), 868-897.

Beerli, A., \& Martin, J. D. (2004a). Factors influencing destination image. Annals of Tourism Research, 31(3), 657-681.

Beerli, A., \& Martin, J. D. (2004b). Tourists' characteristics and the perceived image of tourist destinations: a quantitative analysis--a case study of Lanzarote, Spain. Tourism Management, 25(5), 623-636.

Bell, C. (1994, 29/12). Trouble in fast ferries' wake. The Dominion, p. 1.

Bell, C. (1995a, 4/1). Hundreds hit by holdups. The Dominion, p. 1;3.

Bell, C. (1995b, 5/1). Sounds residents may take action over ferries. The Dominion, p. 3.

Bell, C. (1995c, 20/4). Speeding across the Strait. The Dominion, p. 4.

BERL. (2001). Economic profile of the Wairarapa region. Wellington: Business and Economic Research Limited.

Berry, S. (1995). Fast ferries on Cook Strait - the first season reviewed. The Transportant, April/May, 6-10.

Bertazzi, L., \& Grazia Speranza, M. (1999). Inventory control on sequences of links with given transportation frequencies. International Journal of Production Economics, 59(1-3), 261-270. 
Bieger, T., \& Laesser, C. (2004). Information sources for travel decisions: toward a source process model. Journal of Travel Research, 42, 357-371.

Blank, U., \& Petkovich, M. (1987). Research on urban tourism destinations. In J. R. B. Ritchie \& C. R. Goeldner (Eds.), Travel, tourism and hospitality research: a handbook for managers (pp. 165-177). New York: Wiley.

Boberg, K., \& Choy, D. J. L. (1988). Emerging Trends in Trans-Pacific Air Routes. Journal of Travel Research, 26(3), 15-23.

Boniface, B., \& Cooper, C. (2001). Worldwide Destinations: The Geography of Travel and Tourism (3 ed.). Oxford: Butterworth Heinemann.

Bonnett, J. (1982). Implications of marketing and promotion for the development of tourism. Tourism Management, 3(4), 242-247.

Bowen, J. (2000). Airline hubs in Southeast Asia: national economic development and nodal accessibility. Journal of Transport Geography, 8, 25-41.

Braun, B. M., Xander, J. A., \& White, K. R. (2002). The impact of the cruise industry on a region's economy: a case study of Port Canaveral, Florida. Tourism Economics, 8(3), 281-288.

Brown, J., Tompkins, K., Chaney, E., \& Donovan, R. (1998). Family member ride-alongs during interfacility transport. Air Medical Journal, 17(4), 169-173.

Burghardt, A. F. (1971). A hypothesis about gateway cities. Annals of the Association of American Geographers, 61(2), 269-285.

Burrell, G., \& Morgan, G. (1979). Sociological paradigms and organisational analysis. London: Heinemann.

Butler, R. W. (1980). The concept of the tourist area cycle of evolution: implications for management of resources. Canadian Geographer, 24(1), 5-12.

Carson, D., Waller, I., \& Scott, N. (Eds.). (2002). Drive Tourism: up the wall and around the bend. Altona: Common Ground.

Cartwright, R., \& Baird, C. (1999). The development and growth of the cruise industry. Oxford: Butterworth-Heinemann.

Caruana, V., \& Simmons, C. (1995). The role of traffic growth and capital investment from 1918-82 in the development of Manchester Airport as an international gateway. Journal of Transport Geography, 3(3), 199-219.

Cavana, R. Y., Delahaye, B. L., \& Sekaran, U. (2001). Applied business research: qualitative and quantitative methods: John Wiley \& Sons Australia.

Caves, R. E., \& Gosling, G. D. (1999). Strategic Airport Planning. Oxford: Pergamon.

Centre Stage of New Zealand. (2003). Centre Stage of New Zealand Trade Directory 2003. Wellington: Centre Stage of New Zealand.

Chen, J. S., \& Gursoy, D. (2000). Cross-cultural comparison of the information sources used by first-time and repeat travelers and its marketing implications. International Journal of Hospitality Management, 19(2), 191-203.

Chew, J. (1987). Transport and tourism in the year 2000. Tourism Management., 8(2), 8385.

Cho, B.-H. (2000). Destination. In J. Jafari (Ed.), Encyclopedia of Tourism (pp. 144-145). London and New York: Routledge.

Collier, A. (2003). Principles of Tourism: A New Zealand Perspective (6 ed.). Auckland: Pearson Education. 
Collier, A., \& Harraway, S. (1999). The New Zealand Tourism Industry (2nd ed.). Auckland: Addison Wesley Longman.

Currie, S. (1994, 10/10). NZ Rail expects ferry losses. The Evening Post, p. 3.

Currie, S. (1995a, 26/4). Lynx loss runs to millions. The Evening Post, p. 1.

Currie, S. (1995b, 24/9). Plans for two more fast-ferry operators. The Evening Post, p. 1.

Currie, S. (1995c, 5/1). Safety fears tie up Albayzin. The Evening Post, p. 1.

Currie, S. (1995d, 1/7). Seat Shuttles gets set for strait sailings. The Evening Post, p. 3.

Currie, S., \& Kayes, J. (1995, 25/1). Fast ferry back in business. The Evening Post, p. 1.

Dann, G., Nash, D., \& Pearce, P. L. (1988). Methodology in Tourism Research. Annals of Tourism Research, 15(1), 1-28.

Dean, C. J. (1993). Travel by Excursion Coach in the United Kingdom. Journal of Travel Research, Spring, 59-64.

Decrop, A. (2004). Trustworthiness in qualitative tourism research. In J. Phillimore \& L. Goodson (Eds.), Qualitative research in tourism. London: Routledge.

Dennis, N. (1994). Airline hub operations in Europe. Journal of Transport Geography, 2(4), 219-233.

Deshpande, R. (1983). "Paradigms Lost": on theory and method in research in marketing. Journal of Marketing, 47(4), 101-110.

Destination Marlborough. (2002). Sustainable Tourism Strategy for Marlborough - Part Two - Marlborough's Tourism Resources and Issues and Opportunities for Sustainable Development and Marketing. Blenheim: Destination Marlborough.

Dickison, R. H., \& Vladimir, A. N. (1997). Selling the sea: an inside look at the cruise industry. New York: John Wiley \& Sons.

Doorne, S. M. (1998). The last resort: a study of tourism policy, power and participation on the Wellington waterfront. Unpublished Doctor of Philosophy, Victoria University of Wellington, Wellington.

Doorne, S. M. (2000). Tourism the capital city. In J. McConchie, D. Winchester \& R. Willis (Eds.), Dynamic Wellington: a contemporary synthesis and explanation of Wellington (pp. 217-232). Wellington: Victoria University of Wellington.

Douglas, N. (1997). Applying the life cycle model to Melanesia. Annals of Tourism Research, 24(1), 1-22.

Douglas, N., \& Douglas, N. (1999). Cruise Consumer Behavior: A Comparative Study. In A. Pizam \& Y. Mansfeld (Eds.), Consumer Behavior in Travel and Tourism (pp. 369-392). Binghamton: The Haworth Hospitality Press.

Douglas, N., \& Douglas, N. (2001a). The cruise experience. In N. Douglas, N. Douglas \& R. Derrett (Eds.), Special interest tourism - Context and cases (pp. 330-351). Milton: John Wiley \& Sons Australia.

Douglas, N., \& Douglas, N. (2001b). The short, unhappy life of an Australia-based cruise line. Pacific Tourism Review, 5, 131-142.

Douglas, N., \& Douglas, N. (2004a). The cruise experience. Frenchs Forest: Pearson.

Douglas, N., \& Douglas, N. (2004b). Cruise Ship Passenger Spending Patterns in Pacific Island Ports. International Journal of Tourism Research, 6, 251-261.

Downward, P., \& Lumsdon, L. (2004). Tourism Transport and Visitor Spending: A Study in The North York Moors National Park, UK. Journal of Travel Research, 42(May), 415-420. 
Dredge, D. (1999a). Destination place planning and design. Annals of Tourism Research, 26(4), 772-791.

Dredge, D. (1999b). Destination place planning and design, Annals of Tourism Research (Vol. 26, pp. 772-791).

Dunlop, G. (2002). The European ferry industry - challenges and changes. International Journal of Transport Management, 1, 115-116.

Dwyer, L., Douglas, N., \& Livaic, Z. (2004). Estimating the Economic Contribution of a Cruise Ship Visit. Tourism in Marine Environments, 1(1), 1-16.

Dwyer, L., \& Forsyth, P. (1998). Economic significance of cruise tourism. Annals of Tourism Research, 25(2), 393-415.

Dwyer, L., Forsyth, P., \& Rao, P. (2000). The price competitiveness of travel and tourism: a comparison of 19 destinations. Tourism Management, 21, 9-22.

Eby, D. W., \& Molnar, L. J. (2002). Importance of scenic byways in route choice: a survey of driving tourists in the United States. Transportation Research Part A, 36, 95106.

Fallon, L. D., \& Kriwoken, L. K. (2003). Community involvement in tourism infrastructure-the case of the Strahan Visitor Centre, Tasmania. Tourism Management, 24(3), 289-308.

Faulkner, B., \& Russell, R. (1997). Chaos and complexity in tourism: In search of a new perspective, Pacific Tourism Review (Vol. 1, pp. 93-102).

Fesenmaier, D. R., \& Jeng, J. (2000). Assessing structure in the pleasure trip planning process. Tourism Analysis, 5, 13-27.

Feyer, A.-M., \& Williamson, A. M. (1995). The influence of operational conditions on driver fatigue in the long distance road transport industry in Australia. International Journal of Industrial Ergonomics, 15(4), 229-235.

Fidgeon, P. R. (1983). Holiday caravaning in Wales: Construction of a national site inventory. Tourism Management, 4(3), 199-208.

Finn, M., Elliott-White, M., \& Walton, M. (2000). Tourism and Leisure Research Methods data collection, analysis and interpretation. Essex: Longman.

Finnegan, A. (1994, 21/12). Queasy first trip for ferry. The Evening Post, p. 1.

Finnegan, A. (1996a, 9/5). Fast-ferry debt may go beyond \$1.5 million. The Evening Post, p. 3.

Finnegan, A. (1996b, 2/5). Ferry collapse threatens other. The Evening Post, p. 1.

Finnegan, A. (1996c, 15/4). Fewer passengers ride Lynx. The Evening Post, p. 1.

Fleming, D. K., \& Hayuth, Y. (1994). Spatial characteristics of transportation hubs: centrality and intermediacy. Journal of Transport Geography, 2(1), 3-18.

Fodness, D., \& Murray, B. (1997). Tourist information search. Annals of Tourism Research, 24(3), 503-523.

Foster, G. M. (1986). South seas cruise: A case study of a short-lived society. Annals of Tourism Research, 13, 215-238.

Garnett, C. (1993). Impact of the Channel Tunnel on the tourism industry - A sea of change in cross-Channel travel. Tourism Management, 14(6), 436-439.

Gnoth, J. (1999). Tourism expectation formation: the case of campervan tourists in New Zealand. In A. Pizam \& Y. Mansfeld (Eds.), Consumer behavior in travel and tourism (pp. 245-266). New York, London, Oxford: The Haworth Hospitality Press. 
González-Savignat, M. (2004). Will the High-speed Train Compete against the Private Vehicle? Transport Reviews, 24(3), 293-316.

Goodson, L., \& Phillimore, J. (2004). The inquiry paradigm in qualitative tourism research. In J. Phillimore \& L. Goodson (Eds.), Qualitative research in tourism: ontologies, epistemologies and methodologies (pp. 30-45). London: Routledge.

Gospodini, A. (2001a). Urban Design, Urban Space Morphology, Urban Tourism: An Emerging New Paradigm Concerning Their Relationship. European Planning Studies, 9(7), 925-934.

Gospodini, A. (2001b). Urban Waterfront Redevelopment in Greek Cities: A Framework for Redesigning Space. Cities, 18(5), 285-295.

Greater Wellington Regional Council. (2003). Our profile. Wellington: Greater Wellington Regional Council.

Grech, J. C. (2000). The relationship between tourism and air transport and the importance of this partnership for island destinations: the case of Malta. Paper presented at the Tourism and Air Transport, Funchal.

Green, S. (1998, 6/2). Fast ferry arrives; 'end of the beginning'. The Evening Post, p. 3.

Guba, E. G., \& Lincoln, Y. S. (1998). Competing paradigms in qualitative research. In L. Y.S (Ed.), The Landscape of Qualitative Research: Theories and Issues. Thousand Oaks: Sage Publications.

Gursoy, D., \& Chen, J. S. (2000). Competitive analysis of cross cultural information search behavior. Tourism Management, 21(6), 583-590.

Gursoy, D., \& McCleary, K. (2004). An integrative model of tourists' information search behavior. Annals of Tourism Research, 31(2), 353-373.

Haitovsky, Y., Salomon, I., \& Silman, L. A. (1987). The economic impact of charter flights on tourism to Israel. Journal of Transport Economics and Policy, May, 111-134.

Hall, C. M., Jenkins, J., \& Kearsley, G. (1997). Tourism Planning and Policy in Urban Areas: Introductory Comments. In C. M. Hall, J. Jenkins \& G. Kearsley (Eds.), Tourism planning and policy in Australia and New Zealand: cases, issues and practice (pp. 198-208). Sydney: Irwin.

Hall, C. M., \& Kearsley, G. (2001). Tourism in New Zealand: an introduction. Oxford: Oxford University Press.

Hensher, D. A., Hooper, P. G., \& Smith, N. C. (1991). Developments in surface passenger transport: implications for tourism. Sydney, NSW: University of Sydney Institute of Transport Studies.

Henthorne, T. L. (2000). An Analysis of Expenditures by Cruise Ship Passengers in Jamaica. Journal of Travel Research, 38, 246-250.

Heung, V. C. S., \& Qu, H. (1998). Tourism shopping and its contributions to Hong Kong. Tourism Management, 19(4), 383-386.

Hobson, J. S. P. (1993). Analysis of the US cruise line industry. Tourism Management, 14(6), 453-462.

Hollings, D. (1997). Europe's railways in the 21st century. Travel \& Tourism Analyst, 4, 422.

Holloway, J. C. (1985). The business of tourism (2 ed.). Plymouth, Eng.: Macdonald and Evans.

Holloway, J. C. (2002). The business of tourism (6 ed.). Essex: Pearson Education. 
Hoyle, B., \& Knowles, R. (1998). Transport geography: an introduction. In B. Hoyle \& R. Knowles (Eds.), Modern transport geography (2 ed., pp. 1-12). Chichester: John Wiley \& Sons.

Jaakson, R. (2004). Beyond the tourist bubble? Cruiseship Passengers in Port. Annals of Tourism Research, 31(1), 44-60.

Jang, S. S., Bai, B., Hong, G.-S., \& O'Leary, J. T. (2004). Understanding travel expenditure patterns: a study of Japanese pleasure travelers to the United States by income level. Tourism Management, 25(3), 331-341.

Janic, M. (1999). Aviation and externalities: the accomplishments and problems. Transportation Research Part D, 4, 159-180.

Janin, B. (1982). Circulation touristique internationale et tourisme étranger en Val d'Aoste. Revue de Géographie Alpine, 70(4), 415-430.

Janiskee, R. L. (1990). Resort camping in America. Annals of Tourism Research, 17, 385407.

Jansen-Verbeke, M., \& van Rekom, J. (1996). Scanning museum visitors: Urban tourism marketing. Annals of Tourism Research, 23(2), 364-375.

Jennings, G. (2001). Tourism research. Milton: John Wiley.

Jobes, P. C. (1984). Old timers and new mobile lifestyles. Annals of Tourism Research, $11,181-189$.

Kissling, C. (1998a). International tourism and civil aviation in the South Pacific: issues and innovations. GeoJournal, 19(3), 309-315.

Kissling, C. (1998b). Liberal aviation agreements - New Zealand. Journal of Air Transport Management, 4, 177-180.

Kosters, M. J. (1995). Tourism by Train: Its Role in Alternative Tourism. In V. L. Smith \& W. R. Eadington (Eds.), Tourism Alternatives: potentials and problems in the development of tourism (pp. 180-193). Chichester: John Wiley \& Sons.

Lafferty, G., \& Fossen, A. (2001). Integrating the tourism industry: Problems and strategies. Tourism Management, 22, 11-19.

Latitude Nelson. (2002). Nelson region tourism trade directory 2002. Nelson: Latitude Nelson.

Law, C. M. (2002). Urban Tourism - the visitor economy and the growth of large cities. London and New York: Continuum.

Lawton, L. J., \& Butler, R. W. (1987). Cruise ship industry - patterns in the Caribbean 1880-1986. Tourism Management, 8(4), 329-343.

Leask, A. (2005). The nature and purpose of visitor attractions. In A. Fyall, B. Garrod \& A. Leask (Eds.), Managing visitor attractions: new directions. Oxford: Elsevier.

Leiper, N. (1990). Tourism Systems: An Interdisciplinary Perspective. Palmerston North: Department of Management Systems, Massey University, New Zealand.

Lew, A. A., \& McKercher, B. (2002). Trip destinations, gateways and itineraries: the example of Hong Kong. Tourism Management, 23, 609-621.

Ley, D., \& Murphy, P. (2001). Immigration in gateways cities: Sydney and Vancouver in comparative perspective. Progress in Planning, 55, 119-194.

Li, Y. (2000). Ethnic tourism: A Canadian experience. Annals of Tourism Research, 27(1), 115-131.

Loverseed, H. (1999). Rail travel in North America. Travel \& Tourism Analyst, 4, 1-19. 
Low, L., \& Heng, T. M. (1997). Singapore: Development of gateway tourism. In F. M. Go \& C. L. Jenkins (Eds.), Tourism and economic development in Asia and Australasia (pp. 237-254). London: Cassell.

Lue, C.-C., Crompton, J. L., \& Fesenmaier, D. R. (1993). Conceptualization of multidimensional pleasure trips. Annals of Tourism Research, 20, 289-301.

Lumsdon, L., \& Page, S. (2003). Progress in Transport and Tourism Research: Reformulating the Transport-Tourism Interface and Future Research Agendas. In L. Lumsdon \& S. Page (Eds.), Tourism and Transport: Issues and Agenda for the New Millennium. Oxford: Elsevier.

Mangan, J., Lalwani, C., \& Gardner, B. (2002). Modelling port/ferry choice in RoRo freight transportation. International Journal of Transport Management, 1, 15-28.

Marlborough District Council. (2000). Use of large, high speed vessels in the Marlborough Sounds. Blenheim: Marlborough District Council.

Marlborough Economic Development Trust. (2001). Marlborough Today: An Analysis of the Current Economic Base of the Region. Blenheim: Marlborough Economic Development Trust.

Matthiessen, C. W. (2004). International air traffic in the Baltic Sea Area: hub-gateway status and prospects. Copenhagen in focus. Journal of Transport Geography, 12, 197-206.

McClure, M. (2004). The wonder country - making New Zealand tourism. Auckland: Auckland University Press.

Mescon, T. S., \& Vozikis, G. S. (1985). The economic impact of tourism at the port of Miami. Annals of Tourism Research, 12, 515-528.

Milne, P., \& Tiller, J. (1995). Making waves: the fast ferries decision. Resource Management Bulletin(11), 146-148.

Mings, R. C., \& McHugh, K. E. (1992). The spatial configuration of travel to Yellowstone National Park. Journal of Travel Research, 30(4), 38-46.

Mistilis, N., \& Dwyer, L. (1999). Tourism Gateways and Regional Economies: the Distributional Impacts of MICE. International Journal of Tourism Research, 1, 441457.

Morley, C. L. (2003). Impacts of international airline alliances on tourism. Tourism Economics, 9(1), 31-51.

Nelson, R., \& Wall, G. (1986). Transportation and Accommodation Changing Interrelationships on Vancouver Island. Annals of Tourism Research, 13, 239-260.

Nueman, W. L. (2000). Social research methods, quantitative and qualitative approaches. London: Sage Publications.

Oborne, D. J. (1977). Vibration and passenger comfort. Applied Ergonomics, 8(2), 97-101.

O'Connor, J. (1999, 29/5). Which ferry? The Evening Post, p. 7;10.

O'Connor, J. (2000, 28/10). Cat fight. The Evening Post, p. 28.

O'Kelly, M. E. (1998). A geographer's analysis of hub-and-spoke networks. Journal of Transport Geography, 6(3), 171-186.

O'Kelly, M. E., \& Miller, H. J. (1994). The hub network design problem: a review and synthesis. Journal of Transport Geography, 2(1), 31-40.

Okumus, F., Altinay, M., \& Arasli, H. (2005). The impact of Turkey's economic crisis of February 2001 on the tourism industry in Northern Cyprus. Tourism Management, 26(1), 95-104. 
Okumus, F., \& Hemmington, N. (1998). Management of the change process in hotel companies: An investigation at unit level. International Journal of Hospitality Management, 17(4), 363-374.

Olsen, V. L. (2000). Feminism and qualitative research at and into the millenium. In L. Y.S. (Ed.), Handbook of qualitative research (2nd ed.). Thousand Oaks: Sage.

Oppermann, M. (1995). A model of travel itineraries. Journal of Travel Research, 33(4), 57-61.

Oppermann, M., \& Cooper, M. (1999). Outbound Travel and Quality of Life: The Effect of Airline Price Wars. Journal of Business Research, 44(3), 179-188.

Owen, C. (1991). Improving multi-modal passenger interchanges - an urgent need. Tourism Management, 12(2), 97-100.

Page, S. (1993). Perspectives on Urban Heritage Tourism in New Zealand Wellington in the 1990s. In C. M. Hall \& S. McArthur (Eds.), Heritage Management in New Zealand and Australia: visitor management, interpretation, and marketing. Oxford: Oxford University Press.

Page, S. (1994). Transport for tourism. London; New York: Routledge.

Page, S. (1995). Urban Tourism. London: Routledge.

Page, S. (1999). Transport and tourism. Harlow: Longman.

Page, S. (2001). Gateways, hubs and transport interconnections in Southeast Asia: implications for tourism development in the twenty-first century. In P. Teo, T. C. Chang \& K. C. Ho (Eds.), Interconnected Worlds: Tourism in Southeast Asia (pp. 84-99). Oxford: Pergamon.

Page, S., Brunt, P., Busby, G., \& Connell, J. (2001). Tourism: a modern synthesis. London: Thomson Learning.

Page, S., \& Hall, C. M. (2003). Managing urban tourism. Essex: Prentice Hall.

Palhares, G. L. (2002). Transportes Turísticos. São Paulo: Aleph.

Pardy, B. (1995). Fast ferries: new equilibrium versus ecological sustainability. New Zealand Law Journal, June, 202-204.

Pearce, D. G. (1981). L'Éspace Touristique de la Grande Ville: Éléments de Synthèse et Application à Christchurch (Nouvelle-Zélande). L'Espace Géographique, 10, 207213.

Pearce, D. G. (1989). Tourist development (2 ed.). Harlow \& New York: Wiley.

Pearce, D. G. (1993). Domestic Tourist Travel Patterns in New Zealand. GeoJournal, 29(3), 225-232.

Pearce, D. G. (1995). Tourism today: a geographical analysis (2 ed.). Harlow \& New York: Wiley.

Pearce, D. G. (2001a). An integrative framework for urban tourism research. Annals of Tourism Research, 28(4), 926-946.

Pearce, D. G. (2001b). Réflexions sur une géographie nationale du tourisme: le cas de la Nouvelle-Zélande. Annales de Géographie(619), 266-283.

Pearce, D. G. (2001c). Tourism. Asia Pacific Viewpoint, 42(1), 75-84.

Pearce, D. G. (2001d). Tourism and urban land use change: Assessing the impact of Christchurch's tourist tramway. Tourism and Hospitality Research, 3(2), 132-148.

Pearce, D. G. (2001e). Tourism, trams and local government policy-making in Christchurch, New Zealand. Currents Issues in Tourism, 4(2-4), 331-354. 
Pearce, D. G. (2001f). Towards a regional analysis of tourism in Southeast Asia. In P. Teo, T. C. Chang \& K. C. Ho (Eds.), Interconnected Worlds: Tourism in Southeast Asia (pp. 27-43). Oxford: Pergamon.

Pearce, D. G., \& Elliott, J. M. C. (1983). The Trip Index. Journal of Travel Research, 22(1), 6-9.

Pearce, D. G., \& Priestley, G. K. (1998). Tourism in Spain: a spatial analysis and synthesis. Tourism Analysis, 2, 185-205.

Pearce, P. L. (1991). Analysing Tourist Attractions. Journal of Tourism Studies, 2(1), 4655.

Peisley, T. (1992). Ferries, short sea cruises and the Channel Tunnel. Travel \& Tourism Analyst, 4, 5-26.

Peisley, T. (1997). The cross-channel ferry market. Travel \& Tourism Analyst, 1, 4-20.

Petrick, J. F. (2003). Measuring cruise passengers' perceived value. Tourism Analysis, 7 , 251-258.

Petrick, J. F. (2004). Are loyal visitors desired visitors? Tourism Management, 25(4), 463470.

Phang, S.-Y. (2003). Strategic development of airport and rail infrastructure: the case of Singapore. Transport Policy, 10, 27-33.

Prideaux, B. (1999a). The Role of the Transport System in the Growth of Coastal Resorts - an examination of resort development in South Queensland. Unpublished PhD, University of Queensland, Brisbane.

Prideaux, B. (1999b). Tracks to tourism: Queensland Rail joins the tourist industry. International Journal of Tourism Research, 1, 73-86.

Prideaux, B. (2000a). Links between transport and tourism - past, present and future. In B. Faulkner, G. Moscardo \& E. Laws (Eds.), Tourism in the 21st Century: Reflection on Experience (pp. 91-109). London: Cassells Academic.

Prideaux, B. (2000b). The resort development spectrum -- a new approach to modeling resort development. Tourism Management, 21(3), 225-240.

Prideaux, B. (2000c). The role of the transport system in destination development. Tourism Management, 21, 53-63.

Prideaux, B., Wei, S., \& Ruys, H. (2001). The senior drive tour market in Australia. Journal of Vacation Marketing, 7(3), 209-219.

Prosser, E., \& Leisen, B. (2003). Determinants of on-Board Retail Expenditures in the Cruise Industry. Journal of American Academy of Business, 3, 304-310.

Pryce, M. (1997). Nautical News. New Zealand Marine News, 46, 191-222.

Pryce, M. (1998a). Nautical News. New Zealand Marine News, 47, 24-58.

Pryce, M. (1998b). Nautical News. New Zealand Marine News, 47, 83-110.

Pryce, M. (1998c). Nautical News. New Zealand Marine News, 47, 213-239.

Pryce, M. (1999a). Nautical News. New Zealand Marine News, 48, 21-51.

Pryce, M. (1999b). Nautical News. New Zealand Marine News, 48, 78-102.

Pryce, M. (2000a). Nautical News. New Zealand Marine News, 49, 27-50.

Pryce, M. (2000b). Nautical News. New Zealand Marine News, 49, 129-155.

Pryce, M. (2000c). Nautical News. New Zealand Marine News, 49, 186-205.

Pryce, M. (2003a). Nautical News. New Zealand Marine News, 51, 79-102. 
Pryce, M. (2003b). Nautical News. New Zealand Marine News, 51(4), 182-206.

Pryce, M. (2003c). Nautical News. New Zealand Marine News, 51(3), 129-154.

Punter, M. S. (1999). Tourist Transport Systems with Particular Reference to the South West of England. Unpublished PhD, University of Exeter, Exeter.

Pyle, T. I. (1985). The International Air Transport Association and Tourism. Annals of Tourism Research, 12(4), 648-650.

Qu, H., \& Ping, E. W. Y. (1999). A service performance model of Hong Kong cruise travelers' motivation factors and satisfaction. Tourism Management, 20, 237-244.

Raguraman, K. (1997). Estimating the net economic impact of air services. Annals of Tourism Research, 24(3), 658-674.

Rails. (1994). NZ Rail plans fast ferry for Cook Strait this summer. Rails, 24, 28-29.

Rails. (1995, August). New ferries, new terminals - possible futures for NZ Rail's Interisland Line. Rails, 25, 4-9.

Rails. (2000a). Bigger, brighter Lynx fast ferry enters service. Rails, 29, 124-126.

Rails. (2000b). Bigger, year-round Lynx coming, Top Cat and Arahanga depart. Rails, 30, 111.

Rails. (2001). Tranz Rail's Lynx is bigger, better, and all-year-round. Rails, 30, 128.

Riley, R. W., \& Love, L. L. (2000). The state of qualitative tourism research, Annals of Tourism Research (Vol. 27, pp. 164-187).

Rogerson, C. M. (2002). Urban tourism in the developing world: the case of Johannesburg. Development Southern Africa, 19(1), 169-190.

Rowley, G., \& Purcell, K. (2001). 'As cooks go, she went': is labour churn inevitable? International Journal of Hospitality Management, 20(2), 163-185.

Ruggia, J. (2002). Marketing Middle-Earth. TravelAgent, January 28, 67.

Ruggia, J. (2003). New Zealand's Harbor Capital. TravelAgent, May 26, 72-74.

Russell, P. (2000). Rail travel in Europe. Travel \& Tourism Analyst, 2, 3-33.

Russell, R., \& Faulkner, B. (1999). Movers and shakers: chaos makers in tourism development. Tourism Management, 20(4), 411-423.

Rutner, S. M., \& Mundy, R. A. (1996). Hub versus hub-nots: a comparison of various US airports. Journal of air Transportation World Wide, 1(1), 81-90.

Rutz, W. O. A., \& Coull, J. R. (1996). Inter-island passenger shipping in Indonesia: development of the system. Journal of Transport Geography, 4(4), 275-286.

Sharpley, R. (2000). The influence of the accommodation sector on tourism development: lessons from Cyprus. International Journal of Hospitality Management, 19(3), 275293.

Shaw, S. (1982). Airline deregulation and the tourist industry. Tourism Management, 3(1), 40-51.

Shelton, A. (1995a, 21/12). Faster ferries' teething problems. The Dominion, p. 13.

Shelton, A. (1995b, 4/12). North by South service to start next week at Paremata site. The Dominion, p. 1.

Shelton, A. (1996, 2/5). StraitRunner firm in receivership. The Dominion, p. 1.

Short, J. R., Breitbach, C., Buckmand, S., \& Essex, J. (2000). From world cities to gateway cities: Extending the boundaries of globalization theory. City, 4(3), 317340 . 
Shoval, N., \& Raveh, A. (2004). Categorization of tourist attractions and the modeling of tourist cities: based on the co-plot method of multivariate analysis. Tourism Management, 25(6), 741-750.

Simmons, D. G. (2000). New Zealand. In J. Jafari (Ed.), Encyclopedia of Tourism (pp. 413-414). London and New York: Routledge.

Simons, M. S. (1992). Competition law, air transport deregulation and the tourism industry in the European Economic Community Part I A legal perspective. International Journal of Hospitality Management, 11(1), 33-45.

Singleton Jr, R., Straits, B. C., Straits, M. M., \& McAllister, R. J. (1988). Approaches to Social Research. Oxford: Oxford University Press.

Smith, V. L., Hetherington, A., \& Brumbaugh, M. D. D. (1986). California's highway 89 a regional tourism model. Annals of Tourism Research, 13, 415-433.

Snepenger, D., \& Snepenger, M. (1993). Information Search by Pleasure Travelers. In M. A. Kahn, M. D. Olsen \& T. Var (Eds.), Encyclopedia of Hospitality and Tourism. New York: Van Nostrand Reinhold.

Statistics New Zealand. (2003). Provisional tourism satellite account 2000-2002. Wellington: Statistics New Zealand.

Statistics New Zealand. (2004). Tourism Satellite Account 2000-2003. Wellington: Statistics New Zealand.

Strait Shipping. (2003). Strait Shipping History. Retrieved 17/03, 2003, from http://www.bluebridge.co.nz/history.html

Stubbs, J., \& Jegede, F. (1998). The integration of rail and air transport in Britain. Journal of Transport Geography, 6(1), 53-67.

Subrémon, A. (2000). La libéralisation du transport aérien dans l'Union européene et les perspectives pour le tourisme. Paper presented at the Tourism and Air Transport, Funchal.

Sypher:Mueller International Inc. (1990). Air Transportation and Tourism: Competing and Complementary Needs: Tourism Canada.

Taylor, A. (1995a, 13/2). All agree on damage, says Sounds group. The Evening Post, p. 3.

Taylor, A. (1995b, 1/4). Ferry decision 'backward step' - law lecturer. The Evening Post, p. 8.

Taylor, A. (1995c, 29/11). Lynx wake tests could avoid speed restriction. The Evening Post, p. 1.

Teye, V. B. (1992). Land transportation and tourism in Bermuda. Tourism Management, 13(4), 395-405.

Thomas, R. J. (1993). Interviewing important people in big companies. Journal of contemporary ethnography, 22(1), 80-96.

Timothy, D. J. (1995). Political boundaries and tourism: borders as tourist attractions. Tourism Management, 16(7), 525-532.

Timothy, D. J. (2000). Gateway. In J. Jafari (Ed.), Encyclopedia of Tourism (pp. 246). London and New York: Routledge.

Tooman, L. A. (1997). Applications of the life-cycle model in tourism. Annals of Tourism Research, 24(1), 214-234.

Totally Wellington. (2000). It all adds up. Wellington: Totally Wellington. 
Tramposch, W. J. (1998). Te Papa: reinventing the museum. Museum Management and Curatorship, 17(4), 339-350.

TRCNZ. (2002). New Zealand Domestic Travel Survey 2001. Wellington: Tourism Research Council of New Zealand.

TRCNZ. (2004a). International Visitor Arrivals. Wellington: Tourism Research Council of New Zealand.

TRCNZ. (2004b). International visitor survey. Wellington: Tourism Research Council of New Zealand.

Tribe, J. (2001). Research Paradigms and the Tourism Curriculum. Journal of Travel Research, 39(4), 442-448.

Turton, B. J., \& Mutambirwa, C. C. (1996). Air transport services and the expansion of international tourism in Zimbabwe. Tourism Management, 17, 453-462.

Tyrrell, T. J., \& Devitt, M. F. (1999). Valuing Changes to Scenic Byways. In A. Pizam \& Y. Mansfeld (Eds.), Consumer Behavior in Travel and Tourism (pp. 227-244). Binghamton: The Haworth Hospitality Press.

van Klink, H. A., \& van den Berg, G. C. (1998). Gateways and intermodalism. Journal of Transport Geography, 6(1), 1-9.

Veal, A. J. (1997). Research Methods for Leisure and Tourism (2 ed.). London: Pitman.

Walle, A. H. (1997). Quantitative versus qualitative tourism research. Annals of Tourism Research, 24(3), 524-536.

Wallis, M. (2001). Route 66: the mother road. New York: St Martin's Press.

Walmsley, D. J. (2004). Behavioral Approaches in Tourism Research. In A. A. Lew, C. M. Hall \& A. M. Williams (Eds.), A Companion to Tourism. Oxford: Blackwell.

Wang, J., \& McOwan, S. (2000). Fast passenger ferries and their future. Maritime Policy and Management, 27(3), 231-251.

Wansink, B., \& van Ittersum, K. (2004). Stopping decisions of Travelers. Tourism Management, 25, 319-330.

Ward, J. (1987). Tourism and the private car. Tourism Management, 8(2), 164-165.

Warren, C. A. B. (2002). Qualitative Interviewing. In J. F. Gubrium \& J. A. Holstein (Eds.), Handbook of interview research - context \& method (pp. 83-101). Thousand Oaks: Sage.

Warren, J. A. N., \& Taylor, C. N. (2003). Developing urban tourism in New Zealand. Wellington: Centre for Research, Evaluation and Social Assessment.

Waryszak, R., \& King, B. (2000). Tourists and taxis: an examination of the tourism transport interface. Journal of Vacation Marketing, 6(4), 318-328.

Weigend, G. G. (1958). Some elements in the study of port geography. Geographical Review, 48, 185-200.

Weir, J. (2003, 5/2/2003). Wellington enjoys tourist boom. The Dominion Post, p. A3.

Wellington City Council. (2001). The Wellington Waterfront Framework. Wellington: Wellington City Council.

WellingtonNZ. (2004). About Wellington. Retrieved 25/04/2004, 2004, from http://www.wellingtonnz.com

Wheatcroft, S. (1991). Airlines, tourism and the environment. Tourism Management, 12(2), 119-124. 
Wheatcroft, S. (1994). Aviation and Tourism Policies: Balancing the Benefits. London: World Tourism Organization and Routledge.

WIAL. (2004, 04/07/2004). General airport information. Retrieved 10/08/2004, 2004, from http://www.wlg-airport.co.nz/airport/index.html

Wilkinson, S. (2004). Focus group research. In D. Silverman (Ed.), Qualitative research theory, methods and practice (2 ed., pp. 177-199). London: Sage.

Williams, A. M., \& Patterson, G. (1998). "An empire lost but a province gained": a cohort analysis of British international retirement in the Algarve. International Journal of Population Geography, 4, 135-155.

Williams, S. (2002). Tourism Geography. New York: Routledge.

Wood, R. E. (2000). Caribbean cruise tourism: globalization at sea. Annals of Tourism Research, 27(2), 345-370.

Wood, R. E. (2004). Cruise ships: deterritorialized destinations. In L. Lumsdon \& S. Page (Eds.), Tourism and transport: issues and agenda for the new millenium (pp. 133145). Oxford: Elsevier.

WRC. (2002). Regional Outlook. Wellington: Wellington Regional Council.

Yang, J.-t. (2004). Qualitative knowledge capturing and organizational learning: two case studies in Taiwan hotels. Tourism Management, 25(4), 421-428.

Yang, J.-T., \& Wan, C.-S. C.-S. (2004). Advancing organizational effectiveness and knowledge management implementation. Tourism Management, 25(5), 593-601.

Yang, M. (1998). Transportation and environment in Xiamen. Transportation Research Part D: Transport and Environment, 3(5), 297-307.

Yuksel, A. (2004). Shopping experience evaluation: a case of domestic and international visitors. Tourism Management, 25(6), 751-759. 